\title{
Extended Theories of Gravity
}

\author{
Salvatore Capozziello and Mariafelicia De Laurentis, \\ Dipartimento di Scienze Fisiche, Università di Napoli "Federico II" and INFN Sezione di Napoli, \\ Complesso Universitario di Monte S. Angelo, Edificio G, Via Cinthia, I-80126, Napoli, Italy \&
}

Extended Theories of Gravity can be considered a new paradigm to cure shortcomings of General Relativity at infrared and ultraviolet scales. They are an approach that, by preserving the undoubtedly positive results of Einstein's Theory, is aimed to address conceptual and experimental problems recently emerged in Astrophysics, Cosmology and High Energy Physics. In particular, the goal is to encompass, in a self-consistent scheme, problems like Inflation, Dark Energy, Dark Matter, Large Scale Structure and, first of all, to give at least an effective description of Quantum Gravity. We review the basic principles that any gravitational theory has to follow. The geometrical interpretation is discussed in a broad perspective in order to highlight the basic assumptions of General Relativity and its possible extensions in the general framework of gauge theories. Principles of such modifications are presented, focusing on specific classes of theories like $f(R)$-gravity and scalar-tensor gravity in the metric and Palatini approaches. The special role of torsion is also discussed. The conceptual features of these theories are fully explored and attention is payed to the issues of dynamical and conformal equivalence between them considering also the initial value problem. A number of viability criteria are presented considering the post-Newtonian and the postMinkowskian limits. In particular, we discuss the problems of neutrino oscillations and gravitational waves in Extended Gravity. Finally, future perspectives of Extended Gravity are considered with possibility to go beyond a trial and error approach.

* capozziello@na.infn.it felicia@na.infn.it 


\section{CONTENTS}

Part I : Generalities and open problems

I. Introduction

A. A short history of Theories of Gravity

B. What we mean for a "Good Theory of Gravity"

C. General Relativity and its shortcomings

1. UV scales: the Quantum Gravity Problem

2. Issues and shortcomings in Quantum Gravity

3. IR scales: Dark Energy and Dark Matter

IV. Deformations and conformal transformations 41

\begin{tabular}{l|l|} 
A. Space-time deformations & 42 \\
B. Properties of deforming matrices & 42 \\
C. The propagation of metric deformations & 45 \\
D. Approximate Killing vectors & 46 \\
E. Discussion and conclusions & 48 \\
\hline \hline
\end{tabular}

V. The physical meaning of General Relativity 48

A. The Equivalence Principle 49

B. The geodesic structure $\quad 50$

C. The Einstein field equations in metric and Palatini formalisms

D. The Newtonian limit of General Relativity 54

E. The Minkowskian limit of General Relativity 56

1. Gravitational Waves

Part II: Extended Theories of Gravity 60

VI. Introduction 60

VII. Quantum field theory in curved space-time 61

VIII. Variational principles and field equations in metric formalism 65

A. The Brans-Dicke gravity as the first extension $\quad 65$

B. $f(R)$-gravity in metric formalism 67

1. The case of $f(R)=R+\alpha R^{2}$

2. $f(R)$-gravity: the general case 68 
C. A more general class of Extended Theories of Gravity

IX. The Palatini formalism

A. The Palatini approach and the conformal structure 72

B. Equivalence between $f(R)$ and scalar-tensor gravity 75

1. Equivalence between scalar-tensor and metric $f(R)$-gravity 75

2. Equivalence between scalar-tensor and Palatini $f(R)$ - gravity 76

X. Conformal transformations and Extended Theories of Gravity 76

A. The case of Brans-Dicke gravity 77

B. Scalar-tensor theories

C. $f(R, \phi)$-gravity

D. The interpretation of conformal frames 81

XI. Extended Theories with torsion 83

A. The $f(R)$-field equations with torsion 84

B. Equivalence of $f(R)$-gravity with torsion and scalar-tensor theories 88

XII. The $\mathcal{J}$-bundles framework 90

A. The field equations in the $\mathcal{J}$-bundle formalism 92

B. $f(R)$-gravity within the $\mathcal{J}$-bundle framework 93

C. Symmetries and conserved quantities 94

D. $f(R)$-gravity in presence of matter 9

1. The case of Dirac fields 97

2. The case of Yang-Mills fields 98

3. The case of spin fluid matter $\quad 99$

E. Equivalence with scalar-tensor theories

F. Discussion 101

XIII. The Hamiltonian formulation 101

A. The Hamiltonian constraint of General Relativity

B. The Hamiltonian constraint for $f(R)$-gravity 103

C. The cosmological constant as an eigenvalue 104

D. The transverse traceless (TT) spin 2 operator for the Schwarzschild metric and the WKB
approximation

E. One-loop energy Regularization and Renormalization

F. Concluding remarks

XIV. The initial value problem 113

A. The Cauchy problem of scalar-tensor gravity

B. The initial value problem of $f(R)$-gravity in the ADM formulation

C. The Gaussian normal coordinates approach in General Relativity 118

D. The Cauchy problem of $f(R)$-gravity in Gaussian normal coordinates

\begin{tabular}{ll} 
1. The vacuum case & 120 \\
\hline
\end{tabular}

2. The case with matter

Part III: Applications

XV. Spherical and axial symmetry 125

A. Spherically symmetric solutions in $f(R)$-gravity

1. Generalities of spherical symmetry

2. The Ricci scalar in spherical symmetry

3. Spherical symmetry in $f(R)$-gravity 127

4. Solutions with constant Ricci scalar 128

5. Solutions with Ricci scalar depending on the radial coordinate

B. The Noether approach and spherical symmetry 132

C. From spherical to axial symmetry in $f(R)$-gravity 137

XVI. The Post- Newtonian limit 141 
A. The weak field and small velocities approximations 141

B. General remarks on Newtonian and post-Newtonian approximations

C. Corrections to the Newtonian potential

D. An example: Neutrino oscillation phase dynamically induced by non-Newtonian corrections 151

XVII. The Post- Minkowskian limit 154

A. The weak field limit in Minkowski space-time 154

B. The $f(R)$-gravity energy-momentum pseudo-tensor and gravitational radiation 155

C. Ghosts, massless and massive gravitational modes 157

1. New polarization states of gravitational radiation 162

2. The detector response

D. Concluding remarks 166

XVIII. Conclusions and Perspectives 167

Acknowledgments

Appendix A. Notations 170

Appendix B. Zeta function regularization 171

\begin{tabular}{l|l|}
$I_{+}$computation & 171 \\
\hline
\end{tabular}

\begin{tabular}{l}
$I_{-}$computation \\
172 \\
\hline
\end{tabular}

Appendix C. The field equations and the Noether vector for spherically symmetric $f(R)$-gravity 173

References 174 


\section{PART I : GENERALITIES AND OPEN PROBLEMS}

\section{INTRODUCTION}

Several issues and shortcomings emerged in the last thirty years leading to the conclusion that Einstein's General Relativity is not the final theory of gravitational interaction. The goal of this Report is to take into account some of these problems to show that more general approaches to gravity have to be pursued.

The Review article is organised in three parts. Each part has an introduction, a main body and is self-contained.

Part I is devoted to a general discussion of gravitational interaction under the standard of gauge theories. Our aim is to show the path that led to General Relativity, its self-consistency and successes in addressing open problem at Einstein's time. A summary of shortcomings emerged later is given. In particular we discuss problems at infra-red and ultra-violet scales. After we review the Gauge Theory showing that General Relativity comes out from local gauge transformations, local Poincaré invariance and space-time symmetries. The role of space-time deformations and conformal transformations is discussed in view of Extended Theories of Gravity. We close this part by pointing out the physical meaning of General Relativity, in particular discussing the Equivalence Principle, the geodesic and metric structures, the post-Newtonian and post-Minkowskian limits.

Part II is the main part of this Report. After a general discussion of Extended Theories of Gravity, we provide a summary of their emergence in Quantum Field Theory formulated in curved space-time. We develop the variational principles and the field equations for some classes of Extended Theories in metric and Palatini formalism. Conformal transformations and their physical interpretation are widely discussed. The role of torsion in these theories is analysed. Besides, we develop their jet-bundle representation in order to put in evidence the role of symmetries and conserved quantities. After we present the Hamiltonian formulation showing how it can be connected to the problems of cosmological constant and renormalization at one-loop level. Finally the Initial Value Problem, in various formulations, is addressed showing that the well-formulation and well-position are still not available for any Extended Theory of Gravity.

Part III is devoted to the applications. We consider exact solutions in spherical and axial symmetry. After we develop post-Newtonian and post-Minkowskian limits. The main achievement is the fact that further features as corrections to Newton potential, new polarizations and new gravitational modes emerge with respect to General Relativity. In our opinion, these characteristics will be the test-bed capable of confirming or ruling out Extended Theories of Gravity.

We conclude the Report with some Appendices containing notations, zeta-function regularization and technicalities on Noether symmetries.

\section{A. A short history of Theories of Gravity}

It is remarkable that gravity is probably the fundamental interaction which still remains the most enigmatic, even though it is related to phenomena experienced in everyday life and is the one most easily conceived without any sophisticated knowledge. As a matter of fact, the gravitational interaction was the first one to have been put under the microscope of experimental investigation, obviously due to the simplicity of constructing a suitable experimental apparatus.

Galileo Galilei was the first to introduce pendula and inclined planes to the study of terrestrial gravity at the end of the 16th century [31, 199, 207, 208, 222]. Gravity played an important role in the development of Galileo's ideas about the necessity of experiment in the study of Science, which had a great impact on modern scientific thinking. However, it was not until 1665, when Isaac Newton introduced the now renowned "inverse-square gravitational force law", that terrestrial gravity was actually related to celestial gravity in a single theory [295, 584]. Newton's theory made correct predictions for a variety of phenomena at different scales, including both terrestrial experiments and planetary motion.

Obviously, Newton's contribution to gravity, quite apart from his enormous contribution to physics overall, is not restricted to the expression of the inverse square law. Much attention should be paid to the conceptual basis of his gravitational theory, which incorporates two key ideas:

1. The idea of absolute space, i.e. the view of space as a fixed, unaffected structure; a rigid arena where physical phenomena take place.

2. The idea of what was later called the Weak Equivalence Principle which, expressed in the language of Newtonian theory, states that the inertial and the gravitational mass coincide. 
Asking whether Newton's theory, or any other physical theory, is right or wrong, would be an ill-posed question to begin with, since any consistent theory is apparently "right". A more appropriate way to pose the question would be to ask how suitable this theory is to describe the physical world or, even better, how large a portion of the physical world is sufficiently described by such a theory. Also, one could ask how unique the specific theory is for the description of the relevant phenomena. It was obvious, in the first 20 years after the introduction of Newtonian gravity, that it did manage to explain all of the aspects of gravity known at that time. However, all of the questions above were posed sooner or later.

In 1855, Urbain Le Verrier observed a 35 arc-second excess precession of Mercury's orbit and later on, in 1882, Simon Newcomb measured this precession more accurately to be 43 arc-seconds [5, 39, 302, 394, 431]. This experimental fact was not predicted by Newton's theory. It should be noted that Le Verrier initially tried to explain the precession within the context of Newtonian gravity, attributing it to the existence of another, yet unobserved, planet whose orbit lies within that of Mercury. He was apparently influenced by the fact that examining the distortion of the planetary orbit of Uranus in 1846 had led him, and, independently, John Couch Adams, to the discovery of Neptune and the accurate prediction of its position and momenta. However, this innermost planet was never found.

On the other hand, in 1893, Ernst Mach stated what was later called by Albert Einstein "Mach's principle". This is the first constructive attack to Newton's idea of absolute space after the 18th century debate between Gottfried Wilhelm von Leibniz and Samuel Clarke (Clarke was acting as Newton's spokesman) on the same subject, known as the Leibniz-Clarke Correspondence [1]. Mach's idea can be considered as rather vague in its initial formulation and it was essentially brought into the mainstream of physics later on by Einstein along the following lines:

\section{"...inertia originates in a kind of interaction between bodies...".}

This is obviously in contradiction with Newton's ideas, according to which inertia was always relative to the absolute frame of space. There exists also a later, probably clearer interpretation of Mach's Principle, which, however, also differs in substance. This was given by Dicke 215]:

\section{"The gravitational constant should be a function of the mass distribution in the Universe".}

This is different from Newton's idea of the gravitational constant as being universal and unchanging. Now Newton's basic axioms have to be reconsidered.

But it was not until 1905, when Albert Einstein completed Special Relativity, that Newtonian gravity would have to face a serious challenge. Einstein's new theory, which managed to explain a series of phenomena related to non-gravitational physics, appeared to be incompatible with Newtonian gravity. Relative motion and all the linked concepts had gone well beyond Galileo and Newton ideas and it seemed that Special Relativity should somehow be generalised to include non-inertial frames. In 1907, Einstein introduced the equivalence between gravitation and inertia and successfully used it to predict the gravitational redshift. Finally, in 1915, he completed the theory of General Relativity (GR), a generalisation of Special Relativity which included gravity and any accelerated frame. Remarkably, the theory matched perfectly the experimental result for the precession of Mercury's orbit, as well as other experimental findings like the Lense-Thirring [379, 476, 545, 546] gravitomagnetic precession (1918) and the gravitational deflection of light by the Sun, as measured in 1919 during a Solar eclipse by Arthur Eddington [236].

GR overthrew Newtonian gravity and continues to be up to now an extremely successful and well-accepted theory for gravitational phenomena. As mentioned before, and as often happens with physical theories, Newtonian gravity did not lose its appeal to scientists. It was realised, of course, that it is of limited validity compared to GR, but it is still sufficient for most applications related to gravity. What is more, in weak field limit of gravitational field strength and velocities, GR inevitably reduces to Newtonian gravity. Newton's equations for gravity might have been generalised and some of the axioms of his theory may have been abandoned, like the notion of an absolute frame, but some of the cornerstones of his theory still exist in the foundations of GR, the most prominent example being the Equivalence Principle, in a more suitable formulation of course.

This brief chronological review, besides its historical interest, is outlined here also for a practical reason. GR is bound to face the same questions as were faced by Newtonian gravity and many people would agree that it is actually facing them now. In the forthcoming sections, experimental facts and theoretical problems will be presented which justify that this is indeed the case. Remarkably, there exists a striking similarity to the problems which Newtonian gravity faced, i.e. difficulty in explaining several observations, incompatibility with other well established theories and lack of uniqueness. 


\section{B. What we mean for a "Good Theory of Gravity"}

From a phenomenological point of view, there are some minimal requirements that any relativistic theory of gravity has to match. First of all, it has to explain the astrophysical observations (e.g. the orbits of planets, self-gravitating structures).

This means that it has to reproduce the Newtonian dynamics in the weak-energy limit. Besides, it has to pass the classical Solar System tests which are all experimentally well founded [586]. As second step, it should reproduce Galactic dynamics considering the observed baryonic constituents (e.g. luminous components as stars, sub-luminous components as planets, dust and gas), radiation and Newtonian potential which is, by assumption, extrapolated to Galactic scales.

Thirdly, it should address the problem of large scale structure (e.g. clustering of galaxies) and finally cosmological dynamics, which means to reproduce, in a self-consistent way, the cosmological parameters as the expansion rate, the Hubble constant, the density parameter and so on. Observations and experiments, essentially, probe the standard baryonic matter, the radiation and an attractive overall interaction, acting at all scales and depending on distance: the gravity.

The simplest theory which try to satisfies the above requirements is the GR [233]. It is firstly based, on the assumption that space and time have to be entangled into a single space-time structure, which, in the limit of no gravitational forces, has to reproduce the Minkowski space-time structure. Einstein profitted also of ideas earlier put forward by Riemann, who stated that the Universe should be a curved manifold and that its curvature should be established on the basis of astronomical observations [242].

In other words, the distribution of matter has to influence point by point the local curvature of the space-time structure. The theory, eventually formulated by Einstein in 1915, was strongly based on three assumptions that the Physics of Gravitation has to satisfy.

The "Principle of Relativity", that requires all frames to be good frames for Physics, so that no preferred inertial frame should be chosen a priori (if any exist).

The "Principle of Equivalence", that amounts to require inertial effects to be locally indistinguishable from gravitational effects (in a sense, the equivalence between the inertial and the gravitational mass).

The "Principle of General Covariance", that requires field equations to be "generally covariant" (today, we would better say to be invariant under the action of the group of all space-time diffeomorphisms) [511].

And - on the top of these three principles - the requirement that causality has to be preserved (the "Principle of Causality", i.e. that each point of space-time should admit a universally valid notion of past, present and future).

Let us also recall that the older Newtonian theory of space-time and gravitation, that Einstein wanted to reproduce at least in the limit of weak gravitational forces (what is called today the "post-Newtonian approximation"), required space and time to be absolute entities, particles moving in a preferred inertial frame following curved trajectories, the curvature of which (e.g. the acceleration) had to be determined as a function of the sources (i.e. the "forces").

On these bases, Einstein was led to postulate that the gravitational forces have to be expressed by the curvature of a metric tensor field $d s^{2}=g_{\mu \nu} d x^{\mu} d x^{\nu}$ on a four-dimensional space-time manifold, having the same signature of Minkowski metric, e.g., the so-called "Lorentzian signature", herewith assumed to be $(-,+,+,+)$. He also postulated that space-time is curved in itself and that its curvature is locally determined by the distribution of the sources, e.g., being space-time a continuum, by the four-dimensional generalization of what in Continuum Mechanics is called the "matter stress-energy tensor", e.g. a rank-two (symmetric) tensor $T_{\mu \nu}^{(m)}$.

Hilbert and Einstein [511] proved that the field equations for a metric tensor $g_{\mu \nu}$, related to a given distribution of matter-energy, can be achieved by starting from the Ricci curvature scalar $R$ which is an invariant. We will give details below.

The choice of Hilbert and Einstein was completely arbitrary (as it became clear a few years later), but it was certainly the simplest one both from the mathematical and the physical point of view. As it was later clarified by Levi-Civita in 1919, curvature is not a "purely metric notion" but, rather, a notion related to the "linear connection" to which "parallel transport" and "covariant derivation" refer [380].

In a sense, this is the precursor idea of what in the sequel would be called a "gauge theoretical framework" 359], after the pioneering work by Cartan in 1925 [159]. But at the time of Einstein, only metric concepts were at hands and his solution was the only viable.

It was later clarified that the three principles of relativity, equivalence and covariance, together with causality, just require that the space-time structure has to be determined by either one or both of two fields, a Lorentzian metric $g$ and a linear connection $\Gamma$, assumed at the beginning to be torsionless for the sake of simplicity. 
The metric $g$ fixes the causal structure of space-time (the light cones) as well as its metric relations (clocks and rods); the connection $\Gamma$ fixes the free-fall, i.e. the locally inertial observers. They have, of course, to satisfy a number of compatibility relations which amount to require that photons follow null geodesics of $\Gamma$, so that $\Gamma$ and $g$ can be independent, a priori, but constrained, a posteriori, by some physical restrictions. These, however, do not impose that $\Gamma$ has necessarily to be the Levi-Civita connection of $g$ [464].

This justifies - at least on a purely theoretical basis - the fact that one can envisage the so-called "alternative theories of gravitation", that we prefer to call "Extended Theories of Gravitation" (ETGs) since their starting points are exactly those considered by Einstein and Hilbert: theories in which gravitation is described by either a metric (the so-called "purely metric theories"), or by a linear connection (the so-called "purely affine theories") or by both fields (the so-called "metric-affine theories", also known as "first order formalism theories"). In these theories, the Lagrangian is a scalar density of the curvature invariants constructed out of both $g$ and $\Gamma$.

The choice of Hilbert-Einstein Lagrangian is by no means unique and it turns out that the Hilbert-Einstein Lagrangian is in fact the only choice that produces an invariant that is linear in second derivatives of the metric (or first derivatives of the connection). A Lagrangian that, unfortunately, is rather singular from the Hamiltonian point of view, in much than same way as Lagrangians, linear in canonical momenta, are rather singular in Classical Mechanics (see e.g. [29]).

A number of attempts to generalize GR (and unify it to Electromagnetism) along these lines were followed by Einstein himself and many others (Eddington, Weyl, Schrödinger, just to quote the main contributors; see, e.g., [57]) but they were eventually given up in the fifties of XX Century, mainly because of a number of difficulties related to the definitely more complicated structure of a non-linear theory (where by "non-linear" we mean here a theory that is based on non-linear invariants of the curvature tensor), and also because of the new understanding of physics that is currently based on four fundamental forces and requires the more general "gauge framework" to be adopted (see [347]).

Still a number of sporadic investigations about "alternative theories" continued even after 1960 (see [586] and Refs. quoted therein for a short history). The search for a coherent quantum theory of gravitation or the belief that gravity has to be considered as a sort of low-energy limit of string theories [298], something that we are not willing to enter here in detail, has more or less recently revitalized the idea that there is no reason to follow the simple prescription of Einstein and Hilbert and to assume that gravity should be classically governed by a Lagrangian linear in the curvature.

Further curvature invariants or non-linear functions of them should be also considered, especially in view of the fact that they have to be included in both the semi-classical expansion of a quantum Lagrangian or in the low-energy limit of a string Lagrangian.

Moreover, it is clear from the recent astrophysical observations and from the current cosmological hypotheses that Einstein equations are no longer a good test for gravitation at Solar System, Galactic, extra-galactic and cosmic scale, unless one does not admit that the matter side of field equations contains some kind of exotic matter-energy which is the "dark matter" and "dark energy" side of the Universe.

The idea which we propose here is much simpler. Instead of changing the matter side of Einstein field equations in order to fit the "missing matter-energy" content of the currently observed Universe (up to the 95\% of the total amount!), by adding any sort of inexplicable and strangely behaving matter and energy, we claim that it is simpler and more convenient to change the gravitational side of the equations, admitting corrections coming from non-linearities in the effective Lagrangian. However, this is nothing else but a matter of taste and, since it is possible, such an approach should be explored. Of course, provided that the Lagrangian can be conveniently tuned up (i.e., chosen in a huge family of allowed Lagrangians) on the basis of its best fit with all possible observational tests, at all scales (Solar, Galactic, extragalactic and cosmic).

Something that, in spite of some commonly accepted but disguised opinion, can and should be done before rejecting a priori a non-linear theory of gravitation (based on a non-singular Lagrangian) and insisting that the Universe has to be necessarily described by a rather singular gravitational Lagrangian (one that does not allow a coherent perturbation theory from a good Hamiltonian point of view) accompanied by matter that does not follow the behaviour that standard baryonic matter, probed in our laboratories, usually satisfies.

\section{General Relativity and its shortcomings}

Considering the above discussion it is worth noticing that in the last thirty years several shortcomings came out in the Einstein theory and people began to investigate whether GR is the only fundamental theory capable of explaining the gravitational interaction. Such issues come, essentially, from cosmology and quantum field theory. The shortcomings are related both to many theoretical aspects and to observational results. In this section we will try to summarize these problems. An important issue has to be underlined: even if there are many problems, the reaction of scientific community is not uniform. In a very simple scheme we can summarize the guide lines. 
Many people will agree that modern physics is based on two main pillars: GR and Quantum Field Theory. Each of these two theories has been very successful in its own arena of physical phenomena: GR in describing gravitating systems and non-inertial frames from a classical point of view on large enough scales, and Quantum Field Theory at high energy or small scale regimes where a classical description breaks down. However, Quantum Field Theory assumes that space-time is flat and even its extensions, such as Quantum Field Theory in curved space time, consider space-time as a rigid arena inhabited by quantum fields. GR, on the other hand, does not take into account the quantum nature of matter. Therefore, it comes naturally to ask what happens if a strong gravitational field is present at quantum scales. How do quantum fields behave in the presence of gravity? To what extent are these amazing theories compatible?

Let us try to pose the problem more rigorously. Firstly, what needs to be clarified is that there is no final proof that gravity should have some quantum representation at high energies or small scales, or even that it will retain its nature as an interaction. The gravitational interaction is so weak compared with other interactions that the characteristic scale under which one would expect to experience non-classical effects relevant to gravity, the Planck scale, is $10^{-33}$ $\mathrm{cm}$. Such a scale is not of course accessible by any current experiment and it is doubtful whether it will ever be accessible to future experiments either 1 . However, there are a number of reasons for which one would prefer to fit together GR and Quantum Field Theory [88, 338]. Let us list some of the most prominent ones here and leave the discussion about how to address them for the next subsection. Curiosity is probably the motivation leading scientific research. From this perspective it would be at least unusual if the gravity research community was so easily willing to abandon any attempt to describe the regime where both quantum and gravitational effects are important. The fact that the Planck scale seems currently experimentally inaccessible does not, in any way, imply that it is physically irrelevant. On the contrary, one can easily name some very important open issues of contemporary physics that are related to the Planck scale.

A particular example is the Big Bang scenario in which the Universe inevitably goes through an era in which its dimensions are smaller than the Planck scale (Planck era). On the other hand, space-time in GR is a continuum and so in principle all scales are relevant. From this perspective, in order to derive conclusions about the nature of space-time one has to answer the question of what happens on very small and very large scales.

\section{UV scales: the Quantum Gravity Problem}

One of the main challenges of modern physics is to construct a theory able to describe the fundamental interactions of nature as different aspects of the same theoretical construct. This goal has led, in the past decades, to the formulation of several unification schemes which, inter alia, attempt to describe gravity by putting it on the same footing as the other interactions. All these schemes try to describe the fundamental fields in terms of the conceptual apparatus of Quantum Mechanics. This is based on the fact that the states of a physical system are described by vectors in a Hilbert space $\mathcal{H}$ and the physical fields are represented by linear operators defined on domains of $\mathcal{H}$. Until now, any attempt to incorporate gravity in this scheme has either failed or been unsatisfactory. The main conceptual problem is that the gravitational field describes simultaneously the gravitational degrees of freedom and the background space-time in which these degrees of freedom live.

Owing to the difficulties of building a complete theory unifying interactions and particles, during the last decades the two fundamental theories of modern physics, GR and Quantum Mechanics, have been critically re-analyzed. On the one hand, one assumes that the matter fields (bosons and fermions) come out from superstructures (e.g. Higgs bosons or superstrings) that, undergoing certain phase transitions, have generated the known particles. On the other hand, it is assumed that the geometry (e.g. the Ricci tensor or the Ricci scalar) interacts directly with quantum matter fields which back-react on it. This interaction necessarily modifies the standard gravitational theory, that is, the Lagrangian of gravity plus the effective fields is modified with respect to the Hilbert-Einstein one, and this fact can directly lead to the ETGs.

From the point of view of cosmology, the modifications of standard gravity provide inflationary scenarios of interest. In any case, a condition that must be satisfied in order for such theories to be physically acceptable is that GR is recovered in the low-energy limit.

Although remarkable conceptual progress has been made following the introduction of generalized gravitational theories, at the same time the mathematical difficulties have increased. The corrections introduced into the Lagrangian augment the (intrinsic) non-linearity of the Einstein equations, making them more difficult to study because differential equations of higher order than second are often obtained and because it is impossible to separate the geometric from the matter degrees of freedom. In order to overcome these difficulties and simplify the equations of motion, one often

\footnotetext{
1 This fact does not imply, of course, that imprints of Quantum Gravity phenomenology cannot be found in lower energy experiments.
} 
looks for symmetries of the Lagrangian and identifies conserved quantities which allow exact solutions of dynamics to be discovered. The key step in the implementation of this program consists of passing from the Lagrangian of the relevant fields to a point-like Lagrangian or, in other words, in going from a system with an infinite number of degrees of freedom to one with a finite number of degrees of freedom. Fortunately, this is feasible in cosmology because most models of physical interest are spatially homogeneous Bianchi models and the observed Universe is spatially homogeneous and isotropic to a high degree (Friedmann-Lemaitre-Robertson-Walker (FLRW) models).

The need for a quantum theory of gravity was recognized at the end of the 1950s, when physicist tried for the first time to treat all interactions at a fundamental level and to describe them in terms of Quantum Field Theory. Naturally, the first attempts to quantize gravity used the canonical approach and the covariant approach, which had been applied with remarkable success to Electromagnetism. In the first approach applied to Electromagnetism, one considers the electric and magnetic fields satisfying the Heisenberg Uncertainty Principle and the quantum states are gauge-invariant functionals generated by the vector potential defined on three-surfaces of constant time. In the second approach, one quantizes the two degrees of freedom of the Maxwell field without any $3+1$ decomposition of the metric, while the quantum states are elements of a Fock space [339]. These procedures are equivalent in Electro-magnetism. The former allows for a well-defined measure, whereas the latter is more convenient for perturbative calculations such as, for example, the computation of the $S$-matrix in Quantum Electro Dynamics (QED).

These methods have been applied also to GR, but many difficulties arise in this case due to the fact that Einstein's theory cannot be formulated in terms of a quantum field theory on a fixed Minkowski background. To be more specific, in GR the geometry of the background space-time cannot be given a priori: space-time is the dynamical variable itself. In order to introduce the notions of causality, time, and evolution, one must first solve the equations of motion and then "build" the space-time. For example, in order to know if particular initial conditions will give rise to a black hole, it is necessary to fully evolve them by solving the Einstein equations. Then, taking into account the causal structure of the obtained solution, one has to study the asymptotic metric at future null infinity in order to assess whether it is related, in the causal past, with those initial conditions. This problem becomes intractable at the quantum level. Due to the Uncertainty Principle, in non-relativistic Quantum Mechanics, particles do not move along well-defined trajectories and one can only calculate the probability amplitude $\psi(t, x)$ that a measurement at time $t$ detects a particle around the spatial point $x$. Similarly, in Quantum Gravity, the evolution of an initial state does not provide a specific space-time. In the absence of a space-time, how is it possible to introduce basic concepts such as causality, time, elements of the scattering matrix, or black holes?

The canonical and covariant approaches provide different answers to these questions. The canonical approach is based on the Hamiltonian formulation of GR and aims at using the canonical quantization procedure. The canonical commutation relations are the same that lead to the Uncertainty Principle; in fact, the commutation of certain operators on a spatial three-manifold of constant time is imposed, and this three-manifold is fixed in order to preserve the notion of causality. In the limit of asymptotically flat space-time, the motion generated by the Hamiltonian must be interpreted as temporal evolution (in other words, when the background becomes the Minkowski space-time, the Hamiltonian operator assumes again its role as the generator of translations). The canonical approach preserves the geometric features of GR without the need to introduce perturbative methods [30, 211, 415, 416, 581].

The covariant approach, instead, employs Quantum Field Theory concepts and methods. The basic idea is that the problems mentioned above can be easily circumvented by splitting the metric $g_{\mu \nu}$ into a kinematical part $\eta_{\mu \nu}$ (usually flat) and a dynamical part $h_{\mu \nu}$, as in

$$
g_{\mu \nu}=\eta_{\mu \nu}+h_{\mu \nu}
$$

The geometry of the background is given by the flat metric tensor and is the same as in Special Relativity and ordinary Quantum Field Theory, which allows one to define the concepts of causality, time, and scattering. The quantization procedure is then applied to the dynamical field, considered as a (small) deviation of the metric from the Minkowski background metric. Quanta are discovered to be particles with spin two, called gravitons, which propagate in flat space-time and are defined by $h_{\mu \nu}$. Substituting the metric $g_{\mu \nu}$ into the Hilbert-Einstein action, it follows that the Lagrangian of the gravitational sector contains a sum whose terms represent, at different orders of approximation, the interaction of gravitons and, eventually, terms describing matter-graviton interaction (if matter is present). Such terms are analyzed by using the standard techniques of perturbative Quantum Field Theory.

These quantization programs were both pursued during the 1960s and 1970s. In the canonical approach, Arnowitt, Deser, and Misner [30] provided a Hamiltonian formulation of GR using methods proposed earlier by Dirac and Bergmann. In this Hamiltonian formalism, the canonical variables are the three-metric on the spatial submanifolds obtained by foliating the four-dimensional manifold (note that this foliation is arbitrary). The Einstein equations give constraints between the three-metrics and their conjugate momenta and the evolution equation for these fields, known as the Wheeler-DeWitt (WDW) equation. In this way, GR is interpreted as the dynamical theory of the threegeometries (geometrodynamics). The main difficulties arising from this approach are that the quantum equations involve products of operators defined at the same space-time point and, in addition, they entail the construction of 
distributions whose physical meaning is unclear. In any case, the main problem is the absence of a Hilbert space of states and, as consequence, a probabilistic interpretation of the quantities calculated is missing.

The covariant quantization approach is closer to the known physics of particles and fields in the sense that it has been possible to extend the perturbative methods of QED to gravitation. This has allowed the analysis of the mutual interaction between gravitons and of the matter-graviton interactions. The formulation of Feynman rules for gravitons and the demonstration that the theory might be unitary at every order of the expansion was achieved by DeWitt [212].

Further progress was achieved with Yang-Mills theories, which describe the strong, weak, and electromagnetic interactions of quarks and leptons by means of symmetries. Such theories are renormalizable because it is possible to give the fermions a mass through the mechanism of Spontaneous Symmetry Breaking. Then, it is natural to attempt to consider gravitation as a Yang-Mills theory in the covariant perturbation approach and check whether it is renormalizable. However, gravity does not fit into this scheme; it turns out to be non-renormalizable when one considers the graviton-graviton interactions (two-loops diagrams) and graviton-matter interactions (one-loop diagrams) 2 The covariant method allows one to construct a theory of gravity which is renormalizable at one-loop in the perturbative series [76]. Due to the non-renormalizability of gravity at different orders, its validity is restricted only to the low-energy domain, i.e., to large scales, while it fails at high energy and small scales. This implies that the full unknown theory of gravity has to be invoked near or at the Planck era and that, sufficiently far from the Planck scale, GR and its first loop corrections describe the gravitational interactions. In this context, it makes sense to add higher order terms to the Hilbert-Einstein action as we will do in the second part of this Report. Besides, if the free parameters are chosen appropriately, the theory has a better ultraviolet behavior and is asymptotically free. Nevertheless, the Hamiltonian of these theories is not bounded from below and they are unstable. In particular, unitarity is violated and probability is not conserved.

An alternative approach to the search for a theory of Quantum Gravity comes from the study of the Electroweak interaction. In this approach, gravity is treated neglecting the other fundamental interactions. The unification of the Electromagnetic and the weak interactions suggests that it might be possible to obtain a consistent theory when gravitation is coupled to some kind of matter. This is the basic idea of Supergravity [433]. In this class of theories, the divergences due to the bosons (gravitons in this case) are cancelled exactly by those due to the fermions, leading to a renormalized theory of gravity. Unfortunately, this scheme works only at the two-loop level and for matter-gravity couplings. The Hamiltonian is positive-definite and the theory turns out to be unitary. But, including higher order loops, the infinities re-appear, destroying the renormalizability of the theory.

Perturbative methods are also used in String Theories. In this case, the approach is completely different from the previous one because the concept of particle is replaced by that of an extended object, the fundamental string. The usual physical particles, including the spin two graviton, correspond to excitations of the string. The theory has only one free parameter (the string tension) and the interaction couplings are determined uniquely. It follows that string theory contains all fundamental physics and it is therefore considered as a candidate for the Theory of Everything. String Theory seems to be unitary and the perturbative series converges implying finite terms. This property follows from the fact that strings are intrinsically extended objects, so that ultraviolet divergencies coming from small scales or from large transfer impulses, are naturally cured. In other words, the natural cutoff is given by the string length, which is of Planck size $l_{P l}$. At scales larger than $l_{P l}$, the effective string action can be rewritten in terms of non-massive vibrational modes, i.e., in terms of scalar and tensor fields (tree-level effective action). This eventually leads to an effective theory of gravity non-minimally coupled with scalar fields, the so-called dilaton fields.

To conclude, let us summarize the previous considerations:

- a consistent (i.e., unitary and renormalizable) theory of gravity does not exist yet.

- In the quantization program for gravity, two approaches have been used: the covariant approach and the perturbative approach. They do not lead to a definitive theory of Quantum Gravity.

- In the low-energy regime (with respect to the Planck energy) at large scales, GR can be generalized by introducing into the Hilbert-Einstein action terms of higher order in the curvature invariants and non-minimal couplings between matter and gravity. These lead, at the one-loop level, to a consistent and renormalizable theory.

A part the lack of final theory, the Quantum Gravity Problem already contains some issues and shortcomings which could be already addresses by the today physics. We will summarize them in the forthcoming section.

\footnotetext{
${ }^{2}$ Higher order terms in the perturbative series imply an infinite number of free parameters. At the one-loop level it is sufficient to renormalize only the effective constants $G_{e f f}$ and $\Lambda_{\text {eff }}$ which, at low energy, reduce to Newton's constant $G_{N}$ and the cosmological constant $\Lambda$.
} 


\section{Issues and shortcomings in Quantum Gravity}

Considered the status of art, are some predictions of Quantum Gravity already available? Can remnants of Planck scale be detected at lower energy couplings and masses? As it is well known, only a fine-tuned combination of the low-energy constants leads to an observable Universe like ours. It would thus appear strange if a fundamental theory possessed just the right constants to achieve this. Hogan [322] has argued that Grand Unified Theories constrain relations among parameters, but leave enough freedom for a selection. In particular, he suggests that one coupling constant and two light fermion masses are not fixed by the symmetries of the fundamental theory 3 . One could then determine this remaining free constants only by the (weak form of the) Anthropic Principle: they have values such that a Universe like ours is possible. The cosmological constant, for example, must not be much bigger than the presently observed value, because otherwise the Universe would expand too fast to allow the formation of galaxies. The Universe is, however, too special to be explainable on purely anthropic grounds. We know that the maximal entropy would be reached if all the matter in the observable Universe were collected into a single giant black hole. This entropy would be about $10^{123}$, which is exceedingly more than the observed entropy of about $10^{88}$. The "probability" for our Universe would then be about $\frac{\exp \left(10^{88}\right)}{\exp \left(10^{123}\right)}$. From the Anthropic Principle alone one would not need such a special Universe. As for the cosmological constant, for example, one could imagine its calculation from a fundamental theory. Taking the presently observed value for $\Lambda$, one can construct a mass according to

$$
\left(\frac{\hbar \Lambda^{\frac{1}{2}}}{G}\right) \simeq 15 \mathrm{MeV}
$$

which in elementary particle physics is not an unusally big or small value. The observed value of $\Lambda$ could thus emerge together with medium-size particle mass scales. Since fundamental theories are expected to contain only one dimensionfull parameter, low-energy constants emerge from fundamental quantum fields. An important example in string theory, is the dilaton field from which one can calculate the gravitational constant. In order that these fields mimic physical constants, two conditions have to be satisfied. First, decoherence must be effective in order to guarantee a classical behaviour of the field. Second, this "classical" field must then be approximately constant in large-enough space-time regions, within the limits given by experimental data. The field may still vary over large times or large spatial regions and thus mimic a "time- or space-varying constant". The last word on any physical theory has to be spoken by experiments (observations). Apart from the possible determination of low-energy constants and their dependence on space and time, what could be the main tests for Quantum Gravity?

- Black-hole evaporation: A key test would be the final evaporation phase of a black hole. To this end, it would be useful to observe signatures of primordial black holes. These objects are forming not at the end of stellar collapse, but they can result from strong density perturbations in the early Universe. In the context of inflation, their initial mass can be as small as $1 \mathrm{~g}$. Primordial black holes with initial masses of about $5 \times 10^{14} \mathrm{~g}$ would evaporate at the present age of the Universe. Unfortunately, no such object has yet been observed. Especially promising may be models of inflationary cosmology acting at different scales [89].

- Cosmology: Quantum aspects of gravitational field may be observed in the anisotropy spectrum of the cosmic microwave background. First, future experiments may be able to observe the contribution of the gravitons generated in the early Universe. This important effect was already emphasized in [529]. The production of gravitons by the cosmological evolution would be an effect of Quantum Gravity. Second, quantum-gravitational correction terms from the Wheeler-DeWitt equation or its generalization in loop quantum cosmology may leave their impact on the anisotropy spectrum. Third, a discreteness in the inflationary perturbations could manifest itself in the spectrum [322].

- Discreteness of of space and time: Both in String Theory and Quantum Gravity there are hints of a discrete structure of space-time. This quantum foam could be seen through the observation of effects violating local Lorentz invariance [19], for example, in the dispersion relation of the electromagnetic waves coming from gammaray bursts. It has even be suggested that space-time fluctuations could be seen in atomic interferometry [472]. However, there exist severe observational constraints [475].

- Signatures of higher dimensions: An important feature of String Theory is the existence of additional space-time dimensions. They could manifest themselves in scattering experiments at the Large Hadron Collider (LHC) at CERN. It is also imaginable that they cause observable deviations from the standard cosmological scenario [327].

\footnotetext{
${ }^{3}$ String theory contains only one fundamental dimensionfull parameter, the string length. The connection to low energies may nonetheless be non-unique due to the existence of many different possible "vacua"..
} 
Some of these features are discussed in detail in [358]. Of course, there may be other possibilities which are not yet known and which could offer great surprises. It is, for example, imaginable that a fundamental theory of Quantum Gravity is intrinsically non-linear [471, 515]. This is in contrast to most currently studied theories of Quantum Gravity, which are linear. Quantum Gravity has been studied since the end of the 1920s. No doubt, much progress has been made since then. The final goal has not yet been reached. The belief expressed here is that a consistent and experimentally successful theory of Quantum Gravity will be available in the future. However, it may still take a while before this time is reached. In any case, ETGs could constitute a serious approach in this direction.

\section{IR scales: Dark Energy and Dark Matter}

The revision of standard early cosmological scenarios leading to inflation implies that a new approach is necessary also at later epochs: ETGs could play a fundamental role also in this context. In fact, the increasing bulk of data accumulated over the past few years has paved the way for a new cosmological model usually referred to as the Concordance Model or $\Lambda$ Cold Dark Matter $(\Lambda \mathrm{CDM})$ model.

The Hubble diagram of type Ia supernovae (hereafter SNeIa) measured by both the Supernova Cosmology Project [360, 474] and the High- $z$ Team [487, 549] up to redshift $z \sim 1$, was the first piece of evidence that the Universe is currently undergoing a phase of accelerated expansion. Besides, balloon-born experiments such as BOOMERANG 200] and MAXIMA [536] determined the location of the first two Doppler peaks in the spectrum of Cosmic Microwave Background (CMB) anisotropies, strongly suggesting a Universe with flat spatial sections. When combined with the constraints on the matter density parameter $\Omega_{M}$, these data indicate that the Universe is dominated by an unclustered fluid with negative pressure commonly referred to as dark energy, which drives the accelerated expansion. This picture has been further strengthened by the recent precise measurements of the CMB spectrum by the $W M A P$ satellite experiment [320, 527, 528], and by the extension of the SNeIa Hubble diagram to redshifts larger than one [488].

An overwhelming number of papers appeared following these observational evidences, which present a large variety of models attempting to explain the cosmic acceleration. The simplest explanation would be the well known cosmological constant $\Lambda$ [496]. Although the latter provides the best-fit to most of the available astrophysical data [527], the $\Lambda \mathrm{CDM}$ model fails egregiously in explaining why the inferred value of $\Lambda$ is so tiny (120 orders of magnitude lower) in comparison with the typical value of the vacuum energy density predicted by particle physics, and why its present value is comparable to the matter density, this is the so-called coincidence problem.

As a tentative solution, many authors have replaced the cosmological constant with a scalar field $\phi$ rolling slowly down a flat section of a potential $V(\phi)$ and giving rise to the models known as quintessence 189, 461. Albeit successful in fitting the data with many models, the quintessence approach to dark energy is still plagued by the coincidence problem since the dark energy and dark matter densities evolve differently and reach comparable values only during a very short time of the history of the Universe, coinciding in order of magnitude right at the present era. In other words, the quintessence dark energy is tracking matter and evolves in the same way for a long time; at late times, somehow it changes its behaviouor and no longer tracks the dark matter but begins to dominate in the fashion of a (dynamical) cosmological constant. This is the coincidence problem of quintessence.

Furthermore, the origin of this quintessence scalar field is mysterious, although various (usually rather exotic) models have been proposed, leaving a great deal of uncertainty on the choice of the scalar field potential $V(\phi)$ necessary to achieve the late-time acceleration of the Universe. The subtle and elusive nature of dark energy has led many authors to look for a completely different explanation of the acceleration behaviour of the cosmos without introducing exotic components. To this end, it is worth stressing that the present-day cosmic acceleration only requires a negative pressure component that comes to dominate the dynamics late in the matter era, but does not tell us anything about the nature and the number of the cosmic fluids advocated to fill the Universe. This consideration suggests that it could be possible to explain the accelerated expansion with a single cosmic fluid characterized by an equation of state causing it to act like dark matter at high densities, while behaving as dark energy at low densities. An attractive feature of these models, usually referred to as Unified Dark Energy (UDE) or Unified Dark Matter (UDM) models, is that the coincidence problem is solved naturally, at least at the phenomenological level, with such an approach [441, 442]. Examples are the generalized Chaplygin gas [350], the tachyon field [460], and condensate cosmology [59]. A different class of UDE models with a single fluid has been proposed [116, 153]: its energy density scales with the redshift $z$ in such a way that a radiation-dominated era, followed by a matter era and then by an accelerating phase can be naturally achieved. These models are extremely versatile since they can be interpreted both in the framework of UDE or as two-fluid scenarios containing dark matter and scalar field dark energy. A characteristic feature of this approach is that a generalized equation of state can always be obtained and the fit to the observational data can be attempted. However, such models explain the phenomenology but cannot be addressed to some fundamental physics.

There is another, different, way to approach the problem of the cosmic acceleration. As stressed in 393], it is possible that the observed acceleration is not the manifestation of yet another ingredient of the cosmic pie, but rather the first 
signal of a breakdown, in the infrared limit, of the laws of gravitation as we understand them. From this point of view, it is tempting to modify the Einstein-Friedmann equations to see whether it is still possible to fit the astrophysical data with models containing only standard matter without exotic fluids. Examples are the Cardassian expansion [279] and Dvali-Gabadadze-Porrati (DGP) gravity [227]. Within the same conceptual framework, it is possible to find alternative schemes in which a quintessential behaviour is obtained by incorporating effective models coming from fundamental physics and giving rise to generalized or higher order gravity actions [106] (see Ref. [147, 148, 203, 444]. For instance, a cosmological constant may be recovered as a consequence of a non-vanishing torsion field, leading to a model consistent with both the SNeIa Hubble diagram and observations of the Sunyaev-Zel'dovich effect in galaxy clusters 107]. SNeIa data could also be efficiently fitted by including in the gravitational sector higher order curvature invariants [108, 384, 385, 444]. These alternative models provide naturally a cosmological component with negative pressure originating in the geometry of the Universe, thus overcoming the problematic nature of quintessence scalar fields. Cosmological models coming from ETGs are in the track of this philosophy.

The variety of cosmological models which are phenomenologically viable candidates to explain the observed accelerated expansion is clear from this short review. This overabundance signals that only a limited number of cosmological tests are available to discriminate between competing theories, and it is clear that there is a high degeneracy of models. Let us remark that both the SNeIa Hubble diagram and the angular size-redshift relation of compact radio sources [478] are distance-based probes of the cosmological model and, therefore, systematic errors and biases could be iterated. With this point in mind, it is interesting to search for tests based on time-dependent observables. For example, one can take into account the lookback time to distant objects since this quantity can discriminate between different cosmological models. The lookback time is observationally estimated as the difference between the present-day age of the Universe and the age of a given object at redshift $z$. This estimate is possible if the object is a galaxy observed in more than one photometric band since its colour is determined by its age as a consequence of stellar evolution. Hence, it is possible to obtain an estimate of the galaxy's age by measuring its magnitude in different bands and then using stellar evolutionary codes to choose the model that best reproduces the observed colours [112, 125].

Coming to the weak-field limit, which essentially means considering Solar System scales, any alternative relativistic theory of gravity is expected to reproduce GR which, in any case, is firmly tested only in this limit and at these scales [586]. Even this limit is a matter of debate since several relativistic theories do not reproduce exactly the Einsteinian

results in their Newtonian limit but, in some sense, generalize them. As was first noticed by Stelle 534], $R^{2}$-gravity gives rise to Yukawa-like corrections to the Newtonian potential with potentially interesting physical consequences. For example, it is claimed by some authors that the flat rotation curves of galaxies can be explained by such terms [501]. Others [396] have shown that a conformal theory of gravity is nothing else but a fourth order theory containing such terms in the Newtonian limit. Reports of an apparent anomalous long-range acceleration in the data of the Pioneer 10/11, Galileo, and Ulysses spacecrafts could be accommodated in a general theoretical scheme incorporating Yukawa corrections to the Newtonian potential [27, 70].

In general, any relativistic theory of gravitation yields corrections to the weak-field gravitational potentials (e.g., [480]) which, at the post-Newtonian level and in the Parametrized Post-Newtonian (PPN) formalism, could constitute a test of these theories [586]. Furthermore, the newborn gravitational lensing astronomy [516] is providing additional tests of gravity over small, large, and very large scales which will soon provide direct measurements of the variation of the Newton coupling [367], the potential of galaxies, clusters of galaxies, and several other features of self-gravitating systems. Very likely, such data will be capable of confirming or ruling out as GR or ETGs.

This short overview shows that several shortcomings point out that GR cannot to be the final theory of gravity notwithstanding its successes in addressing a large amount of theoretical and experimental issues. ETGs could be a viable approach to solve some of these problems at IR and UV scales without pretending to be the comprehensive and self-consistent fundamental theory of gravity but in the track outlined by GR and then in the range of gauge theories. This review paper is mainly devoted to the theoretical foundation of ETGs trying to insert them in the framework of gauge theories and showing that they are nothing else but a straightforward extension of GR. The cosmological phenomenology and the genuinely astrophysical aspects of ETGs are not faced here. We refer the readers to the excellent reviews and books quoted in the bibliography [20, 129, 140, 146, 147, 180, 189, 203, 257, 390, 444, 445].

\section{A SUMMARY OF GAUGE SYMMETRIES}

Modern gauge theory has emerged as one of the most significant developments of physics of XX century. It has allowed us to realize partially the issue of unifying the fundamental interactions of nature. We now believe that the electromagnetism, which has been long studied, has been successfully unified with the nuclear weak interaction, the force to which radioactive decay is due. What is the most remarkable about this unification is that these two forces differ in strength by a factor of nearly $10^{5}$. This important accomplishment by the Weinberg-Salam gauge theory [583], and insight gained from it, have encouraged the hope that also the other fundamental forces could be unified 
within a gauge theory framework. At the same time, it has been realized that the potential areas of application for gauge theory extended far beyond elementary particle physics. Although much of the impetus for a gauge theory came from new discoveries in particle physics, the basic ideas behind gauge symmetry have also appeared in other areas as seemingly unrelated, such as condensed matter physics, non-linear wave phenomena and even pure mathematics. This great interest in gauge theory indicates that it is in fact a very general area of study and not only limited to elementary particles.

Gauge invariance was recognized only recently as the physical principle governing the fundamental forces between the elementary particles. Yet the idea of gauge invariance was first proposed by Hermann Weyl in 1919 when only the electron and proton [585] were known as fundamental particles. It required nearly 50 years for gauge invariance to be "rediscovered" and its significance to be understood. The reason for this long hiatus was that Weyl's physical interpretation of gauge invariance was shown to be incorrect soon after he had proposed the theory. Gauge invariance only managed to survive because it was known to be a symmetry of Maxwell's equations and thus became a useful mathematical help in order to simplify equations and thus became a useful mathematical device for simplifying many calculations in the electrodynamics. In view of present success of gauge theory, we can say that gauge invariance was the classical case of a good idea which was discovered long before its time.

In this section, we present a brief summary of gauge theory in view of the fact that any theory of gravity can be considered under the same standard. The early history of gauge theory can be divided into old and new periods where the dividing can be set in the 1950's. The most important question is what motivated Weyl to propose the idea of gauge invariance as a physical symmetry? How did he manage to express it in a mathematical form that has remained almost the same today although the physical interpretation has altered radically? And, how did the development of Quantum Mechanics lead Weyl himself to a rebirth of a gauge theory? The new period of gauge theory begins with the pioneering attempt of Yang and Mills to extend gauge symmetry beyond the narrow limits of electromagnetism [591]. Here we will review the radically new interpretation of gauge invariance required by Yang-Mills theory and the reasons for the failure of original theory. By comparing the new theory with that of Weyl, we can see that many original ideas of Weyl have been rediscovered and incorporated into the modern theory [418].

In these next subsection, our purpose is to present an elementary introduction to a gauge theory in order to show that any relativistic theory of gravity is a gauge theory.

\section{A. What is a gauge symmetry?}

In physics, gauge invariance (also called gauge symmetry) is the property of a field theory where different configurations of the underlying fundamental but unobservable fields result in identical observable quantities. A theory with such a property is called a gauge theory. A transformation from a field configuration to another is called a gauge transformation. Modern physical theories describe nature in terms of fields, e.g., the electromagnetic field, the gravitational field, and fields for the electron and all other elementary particles. A general feature of these theories is that none of these fundamental fields, which are the fields that change under a gauge transformation, can be directly measured. On the other hand, the observable quantities, namely the ones that can be measured experimentally as charges, energies, velocities, etc. do not change under a gauge transformation, even though they are derived from the fields that do change. This (and any) kind of invariance under a transformation is called a symmetry.

For example, in classical electromagnetism the Electric field, $E$, and the magnetic field, $B$, are observable, while the underlying and more fundamental electromagnetic potentials $V$ and $A$ are not. Under a gauge transformation which jointly alters the two potentials, no change occurs either in $E$ or $B$ or in the motion of charged particles. In this example, the gauge transformation was just a mathematical feature without any physical relevance, except that gauge invariance is intrinsically connected to the fundamental law of charge conservation. As shown above, with the advent of Quantum Mechanics in the 1920s, and with successive Quantum Field Theory, the importance of gauge transformations has steadily grown. Gauge theories constrain the laws of physics, because of the fact that all the changes induced by a gauge transformation have to cancel each other out when written in terms of observable quantities. Over the course of the 20th century, physicists gradually realized that all forces (fundamental interactions) arise from the constraints imposed by local gauge symmetries, in which case the transformations vary from point to point in space and time. Perturbative quantum field theory (usually employed for scattering theory) describes forces in terms of force mediating particles called gauge bosons. The nature of these particles is determined by the nature of the gauge transformations. The culmination of these efforts is the Standard Model, a quantum field theory explaining all of the fundamental interactions except gravity. 


\section{B. The Einstein connection}

In 1919, people thought that only two fundamental forces of nature existed, Electromagnetism and Gravitation. In that same year, a group of scientists also made the first experimental observation of starlight bending in the gravitational field of the sun during a total eclipse [576. The brilliant confirmation of Einstein's General Theory of Relativity inspired Weyl to propose his own revolutionary idea of gauge invariance in 1919. To see how this came about, let us first briefly recall some basic ideas on which Relativity was built. The fundamental concept underlying both Special and GR is that are no absolute frames of reference in the Universe. The physical motion of any system must be described relatively to some arbitrary coordinate frame specified by an observer, and the laws of physics must be independent of the choice of frame. In Special Relativity, one usually, defines convenient reference frames, which are called "inertial", in motion with uniform velocity. For example, consider a particle which is moving with constant velocity $v$ with respect to an observer. Let $S$ be the rest frame of the observer and $S^{\prime}$ be an inertial frame which is moving at the same velocity as the particle. The observer can either state that the particle is moving with velocity $v$ in $S$ or that it is at rest $S^{\prime}$. The important point to be noted from this trivial example is that the inertial frame $S^{\prime}$ can always be related by a simple Lorentz transformation to the observer frame $S$. The transformation depends only on the relative velocity between the and observers, not on their positions in space-time. The particle and observer can be infinitesimally close together or at opposite ends of the Universe; the Lorentz transformation is still the same. Thus the Lorentz transformation, or rather the Lorentz symmetry group of Special Relativity, is an example of "global" symmetry. In GR, the description of relative motion is much more complicated because now one is dealing with the motion of a system embedded in a gravitational field. As an illustration, let us consider the following "gedanken" experiment for measuring the motion of a test particle which is moving through a gravitational field. The measurement is to be performed by a physicist in an elevator. The elevator cable as broken so that the elevator and the physicist are falling freely [67]. As the particle moves through the field, the physicist determines its motion with respect to the elevator. Since both particle and elevator are falling in the same field, the physicist can describe the particle motion as if there were no gravitational field. The acceleration of the elevator cancels out the acceleration of particle due to gravity. This example of the Principle of Equivalence, follows from the well-known fact that all bodies accelerate at the same rate given the gravitational field (e.g. $9.8 \mathrm{~m} / \mathrm{s}^{2}$ on the surface of the Earth). Let us now compare the physicist in the falling elevator with the observer in the inertial frame in Special Relativity. It could appear that the elevator corresponds to an accelerating or "non-inertial" frame that is analogous to the frame $S^{\prime}$ in which the particle appeared to be at rest. However, it is not true that a real gravitational field does not produce the same acceleration at every point in space. As one moves infinitely far away from the source, the gravitational field will eventually vanish. Thus, the falling elevator can only be used to define a reference frame within an infinitesimally small region where the gravitational field can be considered uniform. Over a finite region, the variation of the field may be sufficiently large for the acceleration of the particle not to be completely cancelled. We see that an important difference between Special Relativity and GR is that a reference frame can only be defined "locally" or at a single point in a gravitational field. This creates a fundamental problem. To illustrate this difficult point, let us now suppose that there are many more physicists in nearby falling elevators. Each physicist makes an independent measurement so that the path of particle in the gravitational field can be determined. The measurements are made in separate elevators at different locations in the field. Clearly, one cannot perform an ordinary Lorentz transformation between the elevators. If the different elevators were related only by Lorentz transformation, the acceleration would have to be independent of position and the gravitational field could not decrease with distance from the source. Einstein solved the problem of relating nearby falling frames by defining a new mathematical relation known as "connection". To understand the meaning of a connection, let us consider a 4-vector $A_{\mu}$ which represents some physically measured quantity. Now suppose that the physicist in the elevator located at $x$ observes that $A_{\mu}$ changes by an amount $d A_{\mu}$ and a second physicist in a different elevator at $x^{\prime}$ observes a change in $d A_{\mu}^{\prime}$. In Special Relativity, the differential $d A_{\mu}$ is also a vector like $A_{\mu}$ itself. Thus, the differential $A_{\nu}$ in the elevator at $x^{\prime}$ is given by the relation 4

$$
d A_{\nu}^{\prime}=\frac{\partial x^{\mu}}{\partial x^{\prime \nu}} d A_{\mu}
$$

where $\mu, \nu=0,1,2,3$. The relation (2.1) follows from the fact that the Lorentz transformation between $x$ and $x^{\prime}$ is a linear transformation. We can no longer assume that the transformation from $x$ to $x^{\prime}$ is linear in GR. Thus, we must write for $d A_{\nu}^{\prime}$ the general expression

$$
d A_{\nu}^{\prime}=\frac{\partial x^{\mu}}{\partial x^{\prime \nu}} d A_{\mu}+A_{\mu} d\left(\frac{\partial x^{\mu}}{\partial x^{\prime \nu}}\right)=\frac{\partial x^{\mu}}{\partial x^{\prime \nu}} d A_{\mu}+A_{\mu} \frac{\partial^{2} x^{\mu}}{\partial x^{\prime \nu} \partial x^{\prime \lambda}} d x^{\prime \lambda}
$$

\footnotetext{
4 The components of the 4 -vector $A_{\mu}=\left(A^{0}, \mathbf{A}\right)$ and $A^{\mu}=\left(A_{0}, \mathbf{A}\right)$ with $A^{0}=-A_{0}$. Vector components with upper and lower indices are related by $x_{\mu}=g_{\mu \nu} x^{\nu}$, where $g_{\mu \nu}$ is the metric tensor which appears in the definition of the invariant space-time interval $d s^{2}=g_{\mu \nu} d x^{\mu} d x^{\nu}$. The components of $g_{\mu \nu}$ are $g_{11}=g_{22}=g_{33}=1, g_{00}=-1$ and all other components are zero.
} 
Clearly, the second derivatives $\frac{\partial^{2} x^{\mu}}{\partial x^{\prime \nu} \partial x^{\prime \lambda}}$ will vanish if the $x^{\mu}$ are linear functions of the $x^{\prime \nu}$. Such terms are actually quite familiar in physics. They occur in "curvilinear" coordinate systems. These curvilinear coefficients are denoted by the symbol

$$
\Gamma_{\nu \lambda}^{\mu}=\frac{\partial^{2} x^{\mu}}{\partial x^{\prime \nu} \partial x^{\prime \lambda}}
$$

and are called the components of a "connection". They are also called affine connections or Christoffel symbols [414]. It is important to note that the gravitational connection is not simply the result of using a curvilinear coordinate system. The value of the connection at each point in space-time is dependent on the properties of the gravitational field. The field is important in the determination of the relative orientation of the different falling elevators in the same way that the "upward" direction on the surface of the earth varies from one position to another. The analogy with curvilinear coordinate systems merely indicates that the mathematical descriptions of free-falling frames and curvilinear coordinates are similar. Einstein generalized this similarity and arrived at the revolutionary idea of replacing gravity by the curvature of space-time [234]. Let us briefly summarize the essential characteristics of GR that Weyl would have utilized for his new gauge theory. First of all, GR involves a specific force, gravitation, which is not studied in Special Relativity. However, by studying the properties of coordinates frames just as in Special Relativity, one learns that only local coordinates can be defined in a gravitational field. This local property is required by the physical behavior of the field and leads naturally to the idea of a connection between local coordinate frames. Thus the essential difference between Special Relativity and GR is that the former is a global theory while the latter is a local theory. This local property was the key to Weyl's gauge theory [418]. In Sec. [II we will develope extensively this idea.

\section{The Weyl Gauge}

Weyl went a step beyond GR and asked the following question: if the effects of a gravitational field can be described by a connection which gives the relative orientation between local frame in space-time, can other forces of nature such as Electromagnetism also be associated with similar connections? Generalizing the idea that all physical measurements are relative, Weyl proposed that absolute magnitude or norm of a physical vector also should not be an absolute quantity but should depend on its location in space-time. A new connection would then be necessary in order to relate the lengths of vectors at different positions. This is the scale or "gauge" invariance. It is important to note here that the true significance of Weyl's proposal lies on the local property of gauge symmetry and not in a special choice of the norm or "gauge" as a physical variable. The assumption of locality is a powerful condition that determines not only the general structure but many of the detailed features of gauge theory. Weyl's gauge invariance can be easily expressed in mathematical form 592. Let us consider a vector at position $x$ with norm given by $f(x)$. If we shift the vector or transform the coordinates so that the vector is now at $x+d x$, the norm becomes $f(x+d x)$. Expanding to first order in $d x$, we can write the norm as

$$
f(x+d x)=f(x)+\partial_{\mu} f d x^{\mu} .
$$

We now introduce a gauge change through a multiplicative scaling factor $S(x)$. The factor $S(x)$ is defined for convenience to equal unity at the position $x$. The scale factor at $x+d x$ is then given by

$$
S(x+d x)=1+\partial_{\mu} S d x^{\mu} .
$$

The norm of the vector at $x+d x$ is then equal to the product of Eqs. (2.4) and (2.5). Keeping only first order terms in $d x$, we obtain

$$
S f=f+\left(\partial_{\mu} S\right) f d x^{\mu}+\partial_{\mu} f d x^{\mu} .
$$

For a constant vector, we see that the norm has changed by an amount

$$
\left(\partial_{\mu}+\partial_{\mu} S\right) f d x^{\mu} .
$$

Te derivative $\partial_{\mu} S$ is the new mathematical "connection" associated with the gauge change. Weyl identified the gauge connection $\partial_{\mu} S$ with the electromagnetic potential $A_{\mu}$. It is straightforward to show that a second gauge change with a scale factor $\Lambda$ will transform the connection as follows,

$$
\partial_{\mu} S \rightarrow \partial_{\mu} S+\partial_{\mu} \Lambda
$$


From classical Electromagnetism, we known that the potential behaves under gauge transformation like

$$
A_{\mu} \rightarrow A_{\mu}+\partial_{\mu} \Lambda
$$

which leaves the electric and magnetic fields unchanged. Since the forms of (2.8) and (2.9) are identical, it appeared that Weyl's new interpretation of the potential as a gauge connection was perfectly compatible with Electromagnetism. Unfortunately, it was soon pointed out that the basic idea of scale invariance itself would lead to conflict with known physical facts [68]. Some years later, Bergmann noted, that Weyl's original interpretation of gauge invariance would also be in conflict with Quantum Theory. The wave description of matter defines a natural scale for a particle through its Compton wavelength $\lambda=\frac{h}{M c}$. Since the wavelength is determined by the particle mass $M$, it cannot depend on position and thus contradicts Weyl's original assumption about scale invariance. Despite the initial failure of Weyl's gauge theory, the idea of a local gauge symmetry survived. It was well known that Maxwell's equations were invariant under a gauge change. However, without an acceptable interpretation, gauge invariance was regarded as only an "accidental" symmetry of Electromagnetism. The gauge transformation property in Eq. (2.9) was interpreted as just a statement of the well known arbitrariness of the potential in classical physics. Only the electric and magnetic fields were considered to be real and observable. Gauge symmetry was retained largely because it was useful for calculations in both classical and quantum electrodynamics. In fact a lot of problems in electrodynamics can often be most easily solved by first choosing a suitable gauge, such as the Coulomb gauge or Lorentz gauge, in order to make the equations more tractable [418].

\section{Electromagnetism as a Gauge Theory}

It is clear that the electromagnetic interaction of charged particles could be interpreted as a local gauge theory within the context of Quantum Mechanics. In analogy with Weyl's first theory, the phase of a particle wavefunction can be identified as a new physical degree of freedom which is dependent on the space-time position. The value can be changed arbitrarily by performing purely mathematical phase transitions on the wavefunction at each point. Therefore, there must be some connections between phase value nearby points. The role of this connection is payed by the electromagnetic potential. This strict relation between potential and the change in phase is clearly demonstrated by the Aharonov-Bohm effect [9]. Thus by using the phase of wavefunction as the local variable instead of the norm of a vector, Electromagnetism can be interpreted as a local gauge theory very much as Weyl envisioned. Gauge transformations can be viewed as merely phase changes so that they look more like a property of Quantum Mechanics than Electromagnetism. In addition, the symmetry defined by the gauge transformations does not appear to be "natural". The set of all gauge transformations forms a one-dimensional unitary group known as the $U(1)$ group. This group does not arise from any form of coordinate transformation like the more familiar spin-rotation group $S U(2)$ or Lorentz group. Thus, one has lost the original interpretation proposed by Weyl of a new space-time symmetry. The status of gauge theory was also influenced by the historical fact that Maxwell had formulated Electromagnetism long before Weyl proposed the idea of gauge invariance. Therefore, unlike the GR, the gauge symmetry group did not play any essential role in defining the dynamical content of Electromagnetism. This sequence of events was to be completly reversed in the development of modern gauge theory [296, 343, 418].

\section{E. The Yang-Mills Gauge}

In 1954, C. N. Yang and R. Mills proposed that the strong nuclear interaction can be described by a field theory like Electromagnetism. They postulated that the local gauge group was the $S U(2)$ isotopic-spin group. This idea was revolutionary because it changed the very concept of the "identity" of an elementary particle. If the nuclear interaction is a local gauge theory like Electromagnetism, then there is a potential conflict with the notion of how a particle state. For example, let us assume that we can "turn off" the electromagnetic interaction so that we cannot distinguish the proton and neutron by electric charge. We also ignore the small mass difference. We must then label the proton as the "up" state of isotopic spin $\frac{1}{2}$ and the neutron as the "down" state. But if isotopic spin invariance is an independent symmetry at each point in space-time, we cannot assume that the "up" state is the same at any other point. The local isotopic spin symmetry allows to choose arbitrarily which direction is "up" at each point without reference to any other point. Given that the labelling of a proton or a neutron is arbitrary at each point, once the choice has been made at one location, it is clear that some rule is then needed in order to make a comparison with the choice at any other position. The required rule, as Weyl proposed originally, is supplied by a connection. A new isotopic spin potential field was therefore postulated by Yang and Mills in analogy with the electromagnetic potential. 
However, the greater complexity of the $S U(2)$ isotopic-spin group as compared to the $U(1)$ phase group means that Yang-Mills potential will be quite different from the electromagnetic field. In Electromagnetism, the potential provides a connection between the phase values of the wavefunction at different positions. In the Yang-Mills theory, the phase is replaced by a more complicated local variable that specifies the direction of the isotopic spin. In order to understand qualitatively how this leads to a connection, we need only to recall that the $S U(2)$ isotopic-spin group is also the

group of rotations in a 3 -dimensional space 5 . As an example, let us visualize the "up" component of isotopic spin $\frac{1}{2}$ as a vector in an abstract "isotopic spin space". An obvious way to relate the "up" states at different locations $x$ and $y$ is to ask how much the "up" state at $x$ needs to be rotated so that it is oriented in the same direction as the "up" state at $y$. This suggests that the connection between isotopic spin states at different points must act like isotopic spin rotation itself. In other words, if a test particle in the "up" state at $x$ is moved through the potential field to position $y$, its isotopic spin direction must be rotated by the field so that it is pointing in the "up" direction corresponding to the position $y$. We can immediately generalize this idea to states of arbitrary isotopic spin. Since the components of an isotopic spin state can be transformed into another one by elements of the $S U(2)$ group, we can conclude that the connection must be capable of performing the same isotopic spin transformations as the $S U(2)$ group itself. This idea that the isotopic spin connection, and therefore the potential, acts like the $S U(2)$ symmetry group is the most important result of the Yang-Mills theory. This concept lies at the heart of the local gauge theory. It shows explicitly how the gauge symmetry group is built into the dynamics of the interaction between particles and fields. How is ti possible for a potential to generate a rotation in an internal symmetry space? To answer this question, we must define the Yang-Mills potential more carefully in the language of the rotation group. A 3-dimentional rotation $R(\theta)$ of a wavefunction is written as

$$
R(\theta) \psi=e^{i \theta \mathbf{L}} \psi
$$

where $\theta$ is the angle of rotation and $\mathbf{L}$ is the angular momentum operator. Let us compare this rotation with the phase change of wavefunction after a gauge transformation. The rotation has the same mathematical form as the phase factor of the wavefunction. However, this does not mean that the potential itself is a rotation operator like $R(\theta)$. We noted earlier that the amount of phase change must also be proportional to the potential in order to ensure that Schrödinger equation remains gauge invariant. To satisfy this condition, the potential must be proportional to the angular momentum operator $\mathbf{L}$ in (2.10). Thus, the most general form of the Yang-Mills potential is a linear combination of the angular momentum operators

$$
A_{\mu}=\sum_{i} A_{\mu}^{i}(x) L_{i}
$$

where the coefficients $A_{\mu}^{i}(x)$ depend on the space-time position and we explicitly write the sum over the components. This relation indicates that the Yang-Mills is not a rotation, but rather is a "generator" of a rotation. For the case of Electromagnetism, the angular momentum operator is replaced by a unit matrix and $A_{\mu}^{i}(x)$ is just proportional to the phase change $\partial_{\mu} \lambda$. The relation (2.11) explicitly displays the dual role of the Yang-Mills potential as both a field in space-time and an operator in the isotopic-spin space.

We can immediately deduce some interesting properties of the Yang-Mills potential. For example, the potential must have three charge components corresponding to the three independent angular momentum operators $L_{+}, L_{-}$and $L_{3}$. The potential component which acts like a raising operator $L_{+}$can transform a "down" state into a "up" state. We can associate this formal operation with a real process where a neutron absorbs a unit of isotopic spin from the gauge field and turns into a proton. This example indicates that the Yang-Mills gauge field must itself carry electric charge unlike electromagnetic potential. The Yang-Mills field also differs in other respects from the electromagnetic field but they both have one property in common, namely, they have zero mass. The zero mass of the photon is well known from Maxwell's equations, but local gauge invariance requires that the mass of the gauge potential field be identically zero for any gauge theory. The reason is that the mass of the potential must be introduced into a Lagrangian through a term of the form

$$
m^{2} A_{\mu} A^{\mu}
$$

This guarantees that the correct equation of motion for a vector field will be obtained from the Euler-Lagrange equations. Unfortunately, the term given by (2.12) is not invariant under a gauge transformation. The special

\footnotetext{
${ }^{5}$ Technically, the $S U(2)$ group is different from the group of 3-dimensional rotations, $O(3)$; the $S U(2)$ group is the "covering group" of $O(3)$.
} 
transformation property of the potential will introduce extra terms in (2.12) proportional to $A_{\mu}$, which are not cancelled by the transformation of the wavefunction. Thus, the standard mass term is not allowed in the YangMills gauge field must have exactly zero mass like the photon. The Yang-Mills field will therefore exhibit long-range behaviour like Coulomb field and cannot reproduce the observed short range of the nuclear force. Since this conclusion appeared to be an inescapable consequence of a local gauge invariance, the Yang-Mills theory was not considered to be an improvement on the already existing theories of the strong nuclear interaction.

Although the Yang-Mills theory field in its original purpose, it established the foundation for modern gauge theory. The $S U(2)$ isotopic-spin gauge transformation could not be regarded as a mere phase change; it required an entirely new interpretation of a gauge invariance. Yang and Mills showed for the first time that local gauge symmetry was a powerful fundamental principle that could provide new insight into the newly discovered "internal" quantum numbers like isotopic spin. In the Yang-Mills theory, isotopic spin was not just a label for the charge states of particles, but it was crucially involved in determining the fundamental form of the interaction [418].

\section{F. Geometry and Gauge}

The Yang-Mills theory revived the old ideas that elementary particles have degrees of freedom in some "internal" space. By showing how these internal degrees of freedom could be unified in a non-trivial way with the dynamical motion in space-time, Yang and Mills discovered a new type of geometry in physics.

The geometrical structure of a gauge theory can be seen by comparing the Yang-Mills theory with of GR. The essential role of the connection is evident in both gauge theory and relativity. There is an analogy between non-inertial coordinate frame and gauge theory but the local frame has to be is located in an abstract space associated with the gauge symmetry group. To see how the gauge group defines an internal space, let us examine the examples of the $U(1)$ phase group and the $S U(2)$ isotopic spin group. For the $U(1)$ group, the internal space consists of all possible values of the phase of the wavefunction. These phase values can be interpreted as angular coordinates in a 2-dimensional space. The internal symmetry space of $U(1)$ thus looks like a ring, and the coordinate of each point in this space is just the phase value itself. The internal space defined by $S U(2)$ group is more complicated because it describes rotation in a 3 -dimensional space.

We recall that the orientations of an isotopic spin state can be generated by starting from a fixed initial isotopic direction, which can be chosen as the isotopic spin "up" direction, and then rotating to the desired final direction. The values of the three angles which specify the rotation can be considered as the coordinates for a point inside an abstract 3-dimensional space. Each point corresponds to a distinct rotation so that the isotopic spin states themselves can be identified with the points in this angular space. Thus, the internal symmetry space of the $S U(2)$ group looks like the interior of a 3-dimensional sphere.

The symmetry space of a gauge group provides the local non-inertial coordinate frame for the internal degrees of freedom. To an imaginary observer inside this internal space, the interaction between a particle and an external gauge field looks like a rotation of the local coordinates. The amount of the rotation is determined by the strength of the external potential, and the relative change in the internal coordinates between two space-time points is just given by the connection as stated before. Thus, we see that there is a similarity between the geometrical description of relativity and the internal space picture.

The internal space is called a "fiber" by mathematicians [174, 223]. The idea of using a gauge potential to "link" together space-time with internal symmetries space is a new concept in physics. The new space formed by the union of 4-dimensional space time with an internal space is called a "fiber bundle" space. The reason for this name is that the internal spaces or "fibers" at each space-time point can all be viewed as the same space because they can be transformed into each other by a gauge transformation. Hence, the total space is a collection or "bundle" of fibers.

Given that Yang and Mills developed their theory using the same terminology as electrodynamics, it is relevant to ask if there are good reasons to describe gauge theory in geometrical terms, other than to establish a historical link with relativity. The best reason for doing so is that the geometrical picture provides a valuable aid to the standard language of field theory. Most of the pedagogical aids in field theory are based on a long familiarity with electrodynamics. Modern gauge theory, on the other hand, requires a new approach in order to deal with all a of the fundamental forces between elementary particles. The geometrical picture can provide a common arena for discussing electromagnetism, the electromagnetism, the strong and weak nuclear forces, and even gravity, because it depends on only very general properties of gauge theory. The fiber bundle representation will be reconsidered in Sec. XII to discuss ETGs. 


\section{G. Local gauge transformations}

We have described qualitatively how the gauge group is associated with a connection. Any particle or system which is localized in a small volume and carries an internal quantum number like isotopic spin has a direction in the internal symmetry space. This internal direction can be arbitrarily chosen at each point in space-time. In order to compare these internal space directions at two different space-time points $x$ and $x+d x$, we need to define an appropriate connection which can tell us how much the internal direction at $x$ differs from the direction $x+d x$. This connection must be capable of relating all possible directions in the internal space to each other. The most obvious way to relate two directions is to find out how much one direction has to rotate so that it agrees with the other direction. The set of all such rotations forms a symmetry group; thus, the connection between inertial space directions at different points act like a symmetry group as well. Our problem now is to see how a symmetry group transformation can lead to a connection which we identify with a gauge potential field. Let us, begin by writing the general form of a local symmetry transformation for an arbitrary (non Abelian) group,

$$
U \psi=\exp \left(-i q \sum_{k} \theta^{k}(x) F_{k}\right) \psi .
$$

The "local" nature of the transformation is indicated by the parameters $\theta^{k}(x)$ which are continuous of $x$. The constant $q$ is the electric charge for the $U(1)$ gauge group or a general "coupling constant" for an arbitrary gauge group. This is the only way in which the charge enters directly into the calculation. The general transformation (2.13) is identical to the usual form of an ordinary spatial rotation if we identify the position-dependent parameter $\theta^{k}(x)$ with rotation angles. The $F_{k}$ are the generators of the internal symmetry group and satisfy the usual commutation relations

$$
\left[F_{i}, F_{j}\right]=i c_{i j k} F_{k}
$$

where the structure constant $c_{i j k}$ depend on the particular group. For the isotopic-spin rotation group $S U(2)$, the generators, $F_{k}$ are the angular momentum operators. To see how the transformation (2.13) defines a connection, let us consider the following simple operation. We will take a test particle described by a wavefunction $\psi(x)$ and move it between two points $x$ and $x+d x$ in space-time, and analyze how its direction changes in the internal symmetry space. The internal direction at $x$ is initially chosen to have the angles $\theta^{k}(x)$. As the test particle moves away form $x$, the internal direction changes in some continuous way until it reaches $x+d x$ where it has new internal direction given angles $\theta^{k}(x+d x)$. For an infinitesimal distance $d x$, this change can be described by the transformation (2.13) acting on $\psi(x)$ and producing a rotation of the internal direction equal to the difference $d \theta^{k}=\theta^{k}(x+d x)-\theta^{k}(x)$. This rotation gives us what we need, namely, a connection between internal space directions at different points in space-time. We also see that this connection involves the derivative of a quantity just like the connection defined by Weyl. In this case, the quantities are the internal rotation angles $\theta^{k}(x)$. This is a straightforward generalization of the phase of a wavefunction to a set of angles which specify the internal direction.

\section{H. Connections and potentials}

Let us see how to calculate the connection from the symmetry transformation (2.13) by moving the charged test particle through an external potential field. We will explicitly separate the particle wavefunction $\psi(x)$ into external and internal parts. Let us write

$$
\psi(x)=\sum_{\alpha} \psi_{\alpha}(x) u_{\alpha},
$$

where $u_{\alpha}$ form a set o a "basis vectors" in the internal space. The index $\alpha$ is an internal label such as the components of isotopic spin. The basis $u_{\alpha}$ is analogous to the local non-inertial frame in relativity. The external part $\psi_{\alpha}(x)$ is then a "component of $\psi(x)$ in the basis $u_{\alpha}$. Under an inertial symmetry transformation, they transform in the usual way

$$
\psi_{\beta}=U_{\alpha \beta} \psi_{\alpha}
$$

where $U_{\alpha \beta}$ is the matrix representation of the symmetry group. We assume, that the representations is irreducible so that the particle has a unique charge or isotopic spin. The decomposition in Eq. (2.15) is particularly useful because it will allow us to interpret the effect of the external potential field on the particle as a precession of the internal basis. 
Now, when the test particle moves from $x$ to $x+d x$ through the external potential field, $\psi(x)$ changes by an amount $d \psi$ given

$$
d \psi=\psi(x+d x)-\psi(x)
$$

In general, $d \psi$ must contain both the change in the external $x$-dependent part of $\psi_{\alpha}$ and change in the internal space basis $u_{\alpha}$. From Eq. (2.15), we can expand $d \psi$ to first order in $d x$ as

$$
d \psi=\sum_{\alpha}\left[\left(\partial_{\mu} \psi_{\alpha}\right) d x^{\mu} u_{\alpha}+\psi_{\alpha} d u_{\alpha}\right]
$$

The second terms contains the change $d u_{\alpha}$ in the internal space basis. This term is given by the connection which we discussed above; it describes the effect of the external potential field on the internal space direction of the particle.

We now need to calculated the change $d u_{\alpha}$ in the internal space basis. The connection between the internal space direction at different space-time points is given by an internal rotation. In this case, the internal directions are specified by a set of basis vectors, so we must calculate the change $d u_{\alpha}$ from an infinitesimal internal rotation $U(d x)$ which is associated with the external displacement $d x$.

From Eq. (2.13), we calculate the infinitesimal internal rotation $U(d x)$,

$$
\begin{gathered}
U(d x)=\exp \left[-i q \sum_{k} d \theta^{k} F_{k}\right] \\
d \theta^{k}=\left(\partial_{\mu} \theta^{k}\right) d x_{\mu}
\end{gathered}
$$

which rotates the internal basis $u$ by an amount $d u$,

$$
U(d x) u=u+d u \text {. }
$$

The generators $F_{k}$ act like matrix operators on the column basis vector $u_{\alpha}$ so we can write

$$
U(d x) u_{\alpha}=\exp \left[-i q \sum_{k}\left(\partial_{\mu} \theta^{k}\right) d x^{\mu}\left(F_{k}\right)_{\alpha \beta}\right] u_{\beta}
$$

Expanding $U(x)$ to first order in $d x$, we obtain

$$
u_{\alpha}+d u_{\alpha}=\left[\delta_{\alpha \beta}-i q \sum_{k}\left(\partial_{\mu} \theta^{k}\right) d x^{\mu}\left(F_{k}\right)_{\alpha \beta}\right] u_{\beta},
$$

which then gives for the change in the basis,

$$
d u_{\alpha}=-i q \sum_{k}\left(\partial_{\mu} \theta^{k}\right) d x^{\mu}\left(F_{k}\right)_{\alpha \beta} u_{\beta}
$$

The net change $d u_{\alpha}$ will give us the connection that we have been seeking. Let us therefore introduce the new "connection operator"

$$
\left(A_{\mu}\right)_{\alpha \beta}=\sum_{k}\left(\partial_{\mu} \theta^{k}\right)\left(F_{k}\right)_{\alpha \beta}
$$

We thus finally obtain for the total change $d \psi$,

$$
d \psi=\sum_{\alpha \beta}\left[\left(\partial_{\mu} \psi_{\alpha}\right) \delta_{\alpha \beta}-i q\left(A_{\mu}\right)_{\alpha \beta} \psi_{\alpha}\right] d x^{\mu} u_{\beta},
$$


where we have put in $\delta_{\alpha \beta}$ in order to factor out the basis vector $u_{\beta}$. Now, we can factor the change $d \psi$ into its own external and internal parts

$$
d \psi=\sum_{\beta}(d \psi)_{\beta} u_{\beta} \equiv \sum_{\beta}\left(D_{\mu} \psi_{\beta}\right) d x^{\mu} u_{\beta} .
$$

The operator $D_{\mu}$ the gauge covariant derivative which describes the changes in both the external and internal parts of $\psi(x)$. Thus we get from Eq. (2.26)

$$
D_{\mu} \psi_{\beta}=\sum_{\alpha}\left[\delta_{\alpha \beta} \partial_{\mu}-i q\left(A_{\mu}\right)_{\alpha \beta}\right] \psi_{\alpha} .
$$

For the case of the electromagnetic gauge group $U(1)$, the internal space is one dimensional so that Eq. (2.28) reduces to

$$
D_{\mu} \psi=\left(\partial_{\mu}-i q A_{\mu}\right) \psi
$$

This is the "canonical momentum" which is familiar from Electromagnetism. We can also deduce from the example of the $U(1)$ group, that the connection operator defined in Eq. (2.25), should be identified as the generalized version of the vector potential field $A_{\mu}$. Thus, we conclude that the external potential field is indeed a connection in the internal symmetry space.

\section{Choosing a gauge}

We know that the usual reason for selecting a particular gauge is to simplify a calculation or to explicitly display an interesting features of a problem. This freedom to choose a gauge is another example of the arbitrariness in the vector potential.

The choice of a gauge actually involves both gauge invariance and Lorentz invariance simultaneously. A particular gauge usually imposes a constraints on the vector potential, such as $\nabla \cdot \mathbf{A}=0$ for the Coulomb gauge. In general, such equations are obviously not Lorentz invariant. Yet we know that two observers, each in a different inertial frame, can choose the Coulomb gauge for the same electromagnetism problem. The electric and magnetic fields observed in the two different frames can then be related by the usual Lorentz transformations between two frames. This example points out the fact that the space-time location $x$ at which the internal coordinate $\theta(x)$ is evaluated is also not a fixed position. A Lorentz transformation which changes the spatial coordinate in the inertial frame also affects the value of the internal angles ad can be interpreted as an internal rotation. Thus, regardless of whether the coordinate change is associated with Lorentz transformation of observers or an actual movement of the particle in the external field, the effect on the internal space is the same: it is rotated by a gauge transformation.

A Lorentz transformation between two inertial frames is therefore always associated with a gauge transformation. Thus, the vector potential observed in the two frames are related by

$$
A_{\mu}^{\prime}=L_{\mu}^{\nu} A_{\nu}-\partial_{\mu} \lambda
$$

where $L_{\mu}{ }^{\nu}$ is the Lorentz transformation. This shows that the vector potential actually does not transform like an ordinary four-vector under a Lorentz transformation. It picks up an extra term $\partial_{\mu} \lambda$ due to the rotaion in the internal space. This interesting fact is well known in quantum field theory but it is rarely mentioned in ordinary electromagnetism [77].

We can now see exactly what is involved for a particular choice of gauge. In the Lorentz gauge, $\partial^{\mu} A_{\mu}$ is required to be invariant,

$$
\partial^{\prime \mu} A_{\mu}^{\prime}=\partial^{\mu} A_{\mu}
$$

even though $A_{\mu}$ is not a true four-vector. From Eq. (2.30), we see that this is possible only if

$$
\partial^{\mu} \partial_{\mu} \lambda=0
$$

or, equivalently, 


$$
\nabla^{2} \lambda-\frac{\partial^{2} \lambda}{\partial t^{2}}=0,
$$

which is the familiar equation for $\lambda$ in the Lorentz gauge. Thus we see that the choice of Lorentz gauge is a requirement that the effect of the internal space precession be eliminated so that $\partial^{\mu} A_{\mu}$ can be treated as if it were a relativistic invariant. By the same reasoning, other gauges like the Coulomb gauge are not invariant because the gauge condition does not completely eliminate the extra internal precession term. Thus in the Coulomb gauge

$$
\nabla^{\prime} \cdot \mathbf{A}^{\prime}=\nabla \cdot \mathbf{A}=0,
$$

but $\mathbf{A}$ and $\mathbf{A}^{\prime}$ are not related by a simple Lorentz transformation. An additional gauge rotation is required [278].

\section{GRAVITY FROM GAUGE INVARIANTS}

The above considerations on gauge symmetry can be developed for gravity. In this section, before discussing the physical meaning of GR, we will derive in a very general way the field equations considering manifolds equipped with curvature and torsion. Specifically, we want to show the role of global and local Poincaré invariance and the relevance of conservation laws in any theory of gravity. The approach is completely general and suitable for spinor, vector, bivector and tetrad fields independently of their specific physical meaning. Our first issue is what can "generate" gravity.

\section{A. What can "generate" gravity?}

Since the perturbative scheme is unsatisfactory because it fails over one loop level and cannot be renormalized [94], as we have seen above, we can ask what can to produce gravity or in other words if there exists invariance principles leading to the gravitation [137]. Following the prescriptions of GR, the physical space-time is assumed to be a four-dimensional differential manifold. In Special Relativity, this manifold is the Minkwoski flat-space-time $M_{4}$ while, in GR, the underlying space-time is assumed to be curved in order to describe the effects of gravitation.

Utiyama [553] was the first to propose that GR can be seen as a gauge theory based on the local Lorentz group $S O(3,1)$ in much the same way that the Yang-Mills gauge theory [591] was developed on the basis of the internal isospin gauge group $S U(2)$. In this formulation the Riemannian connection is the gravitational counterpart of the Yang-Mills gauge fields. While $S U(2)$, in the Yang-Mills theory, is an internal symmetry group, the Lorentz symmetry represents the local nature of space-time rather than internal degrees of freedom. The Einstein Equivalence Principle, asserted for GR, requires that the local space-time structure can be identified with the Minkowski space-time possessing Lorentz symmetry. In order to relate local Lorentz symmetry to the external space-time, we need to solder the local space to the external space. The soldering tools are the tetrad fields. Utiyama regarded the tetrads as objects given a priori. Soon after, Sciama [506] recognized that space-time should necessarily be endowed with torsion in order to accommodate spinor fields. In other words, the gravitational interaction of spinning particles requires the modification of the Riemann space-time of GR to be a (non-Riemannian) curved space-time with torsion. Although Sciama used the tetrad formalism for his gauge-like handling of gravitation, his theory fell shortcomings in treating tetrad fields as gauge fields. Kibble [356] made a comprehensive extension of the Utiyama gauge theory of gravitation by showing that the local Poincaré symmetry $S O(3,1) \rtimes T(3,1)(\rtimes$ represents the semi-direct product) can generate a space-time with torsion as well as curvature. The gauge fields introduced by Kibble include the tetrads as well as the local affine connection. There have been a variety of gauge theories of gravitation based on different local symmetries which gave rise to several interesting applications in theoretical physics [162, 301, 317, 334, 340 342, 397, 399, 502].

Following the Kibble approach, it can be demonstrated how gravitation can be formulated starting from a pure gauge point of view. In particular, the aim of this section is to show, in details, how a theory of gravitation is a gauge theory which can be obtained starting from the local Poincaré symmetry and this feature works not only for GR but also for ETGs.

A gauge theory of gravity based on a nonlinear realization of the local conformal-affine group of symmetry transformations can be formulated in any case [13]. This means that the coframe fields and gauge connections of the theory can be always obtained. The tetrads and Lorentz group metric have been used to induce a space-time metric. The inhomogenously transforming (under the Lorentz group) connection coefficients give rise to gravitational gauge potentials used to define covariant derivatives accommodating minimal coupling of matter and gauge fields. On the other hand, the tensor valued connection forms can be used as auxiliary dynamical fields associated with the dilation, 
special conformal and deformation (shear) degrees of freedom inherent to the bundle manifold. This allowes to define the bundle curvature of the theory. Then boundary topological invariants have been constructed. They served as a prototype (source free) gravitational Lagrangian. Finally the Bianchi identities, covariant field equations and gauge currents are obtained.

Here, starting from the Invariance Principle, we consider first the Global Poincaré Invariance and then the Local Poincaré Invariance. This approach lead to construct a given theory of gravity as a gauge theory. This point of view, if considered in detail, can avoid many shortcomings and could be useful to formulate self-consistent schemes for any theory of gravity.

\section{B. Invariance Principles and the Noether Theorem}

As it is well-known, field equations and conservation laws can be obtained from a least action principle. The same principle is the basis of any gauge theory so we start from it to develop our considerations. Let us start from a least action principle and the Noether theorem.

Let $\chi(x)$ be a multiplet field defined at a space-time point $x$ and $\mathcal{L}\left\{\chi(x), \partial_{j} \chi(x) ; x\right\}$ be the Lagrangian density of the system. In order to make a distinction between the global transformations and the local transformations, for the moment we use the Latin indices $(i, k=0,1,2,3)$ for the former and the greek indices $(\mu, \nu=0,1,2,3)$ for the latter. The action integral of the system over a given space-time volume $\Omega$ is defined by

$$
I(\Omega)=\int_{\Omega} \mathcal{L}\left\{\chi(x), \partial_{j} \chi(x) ; x\right\} d^{4} x .
$$

Now let us consider the infinitesimal variations of the coordinates

$$
x^{i} \rightarrow x^{i}=x^{i}+\delta x^{i}
$$

and the field variables

$$
\chi(x) \rightarrow \chi^{\prime}\left(x^{\prime}\right)=\chi(x)+\delta \chi(x)
$$

Correspondingly, the variation of the action is given by

$$
\delta I=\int_{\Omega^{\prime}} \mathcal{L}^{\prime}\left(x^{\prime}\right) d^{4} x^{\prime}-\int_{\Omega} \mathcal{L}(x) d^{4} x=\int_{\Omega}\left[\mathcal{L}^{\prime}\left(x^{\prime}\right)\left\|\partial_{j} x^{\prime j}\right\|-\mathcal{L}(x)\right] d^{4} x
$$

Since the Jacobian for the infinitesimal variation of coordinates becomes

$$
\left\|\partial_{j} x^{\prime j}\right\|=1+\partial_{j}\left(\delta x^{j}\right)
$$

the variation of the action takes the form,

$$
\delta I=\int_{\Omega}\left[\delta \mathcal{L}(x)+\mathcal{L}(x) \partial_{j}\left(\delta x^{j}\right)\right] d^{4} x
$$

where

$$
\delta \mathcal{L}(x)=\mathcal{L}^{\prime}\left(x^{\prime}\right)-\mathcal{L}(x)
$$

For any function $\Phi(x)$ of $x$, it is convenient to define the fixed point variation $\delta_{0}$ by,

$$
\delta_{0} \Phi(x):=\Phi^{\prime}(x)-\Phi(x)=\Phi^{\prime}\left(x^{\prime}\right)-\Phi\left(x^{\prime}\right) .
$$

Expanding the function to first order in $\delta x^{j}$ as

$$
\Phi\left(x^{\prime}\right)=\Phi(x)+\delta x^{j} \partial_{j} \Phi(x)
$$

we obtain

$$
\delta \Phi(x)=\Phi^{\prime}\left(x^{\prime}\right)-\Phi(x)=\Phi^{\prime}\left(x^{\prime}\right)-\Phi\left(x^{\prime}\right)+\Phi\left(x^{\prime}\right)-\Phi(x)=\delta_{0} \Phi(x)+\delta x^{j} \partial_{j} \Phi(x),
$$


or

$$
\delta_{0} \Phi(x)=\delta \Phi(x)-\delta x^{j} \partial_{j} \Phi(x)
$$

The advantage to have the fixed point variation is that $\delta_{0}$ commutes with $\partial_{j}$ :

$$
\delta_{0} \partial_{j} \Phi(x)=\partial_{j} \delta_{0} \Phi(x)
$$

For $\Phi(x)=\chi(x)$, we have

$$
\delta \chi=\delta_{0} \chi+\delta x^{i} \partial_{i} \chi
$$

and

$$
\delta \partial_{i} \chi=\partial_{i}\left(\delta_{0} \chi\right)-\partial\left(\delta x^{j}\right) \partial_{i} \chi
$$

Using the fixed point variation in the integrand of (3.6) gives

$$
\delta I=\int_{\Omega}\left[\delta_{0} \mathcal{L}(x)+\partial_{j}\left(\delta x^{j} \mathcal{L}(x)\right)\right] d^{4} x .
$$

If we require the action integral defined over any arbitrary region $\Omega$ be invariant, that is, $\delta I=0$, then we must have

$$
\delta \mathcal{L}+\mathcal{L} \partial_{j}\left(\delta x^{j}\right)=\delta_{0} \mathcal{L}+\partial_{j}\left(\mathcal{L} \delta x^{j}\right)=0
$$

If $\partial_{j}\left(\delta x^{j}\right)=0$, then $\delta \mathcal{L}=0$, that is, the Lagrangian density $\mathcal{L}$ is invariant. In general, however, $\partial_{j}\left(\delta x^{j}\right) \neq 0$, and $\mathcal{L}$ transforms like a scalar density. In other words, $\mathcal{L}$ is a Lagrangian density unless $\partial_{j}\left(\delta x^{j}\right)=0$.

For convenience, let us introduce a function $h(x)$ that behaves like a scalar density, namely

$$
\delta h+h \partial_{j}\left(\delta x^{j}\right)=0 .
$$

We further assume $\mathcal{L}\left(\chi, \partial_{j} \chi ; x\right)=h(x) L\left(\chi, \partial_{j} \chi ; x\right)$. Then we see that

$$
\delta \mathcal{L}+\mathcal{L} \partial_{j}\left(\delta x^{j}\right)=h \delta L
$$

Hence the action integral remains invariant if

$$
\delta L=0
$$

The newly introduced function $L\left(\chi, \partial_{j} \chi ; x\right)$ is the scalar Lagrangian of the system.

Let us calculate the integrand of explicitly. The fixed point variation of $\mathcal{L}(x)$ is a consequence of a fixed point variation of the field $\chi(x)$,

$$
\delta_{0} \mathcal{L}=\frac{\partial \mathcal{L}}{\partial \chi} \delta_{0} \chi+\frac{\partial \mathcal{L}}{\partial\left(\partial_{j} \chi\right)} \delta_{0}\left(\partial_{j} \chi\right)
$$

which can be cast into the form,

$$
\delta_{0} \mathcal{L}=[\mathcal{L}]_{\chi} \delta_{0} \chi+\partial_{j}\left(\frac{\partial \mathcal{L}}{\partial\left(\partial_{j} \chi\right)} \delta_{0} \chi\right)
$$

where

$$
[\mathcal{L}]_{\chi} \equiv \frac{\partial \mathcal{L}}{\partial \chi}-\partial_{j}\left(\frac{\partial \mathcal{L}}{\partial\left(\partial_{j} \chi\right)}\right)
$$

Consequently, we have the action integral in the form

$$
\delta I=\int_{\Omega}\left\{[\mathcal{L}]_{\chi} \delta_{0} \chi+\partial_{j}\left(\frac{\partial \mathcal{L}}{\partial\left(\partial_{j} \chi\right)} \delta \chi-T_{k}^{j} \delta x^{k}\right)\right\} d^{4} x
$$

where

$$
T_{k}^{j}:=\frac{\partial \mathcal{L}}{\partial\left(\partial_{j} \chi\right)} \partial_{k} \chi-\delta_{k}^{j} \mathcal{L}
$$


is the canonical energy-momentum tensor density. If the variations are chosen in such a way that $\delta x^{j}=0$ over $\Omega$ and $\delta_{0} \chi$ vanishes on the boundary of $\Omega$, then $\delta I=0$ gives us the Euler-Lagrange equation,

$$
[\mathcal{L}]_{\chi}=\frac{\partial \mathcal{L}}{\partial \chi}-\partial_{j}\left(\frac{\partial \mathcal{L}}{\partial\left(\partial_{j} \chi\right)}\right)=0 .
$$

On the other hand, if the field variables obey the Euler-Lagrange equation, $[\mathcal{L}]_{\chi}=0$, then we have

$$
\partial_{j}\left(\frac{\partial \mathcal{L}}{\partial\left(\partial_{j} \chi\right)} \delta \chi-T_{k}^{j} \delta x^{k}\right)=0
$$

which gives rise, considering also the Noether theorem, to conservation laws. These very straightforward considerations are at the basis of our following discussion.

\section{The Global Poincaré invariance}

As standard, we assert that our space-time in the absence of gravitation is a Minkowski space $M_{4}$. The isometry group of $M_{4}$ is the group of Poincaré transformations (PT) which consists of the Lorentz group $S O(3,1)$ and the translation group $T(3,1)$. The Poincaré transformations of coordinates are

$$
x^{i} \stackrel{P T}{\rightarrow} x^{i}=a_{j}^{i} x^{j}+b^{i}
$$

where $a_{j}^{i}$ and $b^{i}$ are real constants, and $a_{j}^{i}$ satisfy the orthogonality conditions $a_{k}^{i} a_{j}^{k}=\delta_{j}^{i}$. For infinitesimal variations,

$$
\delta x^{i}=\chi^{\prime}\left(x^{\prime}\right)-\chi(x)=\varepsilon^{i}{ }_{j} x^{j}+\varepsilon^{i}
$$

where $\varepsilon_{i j}+\varepsilon_{j i}=0$. While the Lorentz transformation forms a six parameter group, the Poincaré group has ten parameters. The Lie algebra for the ten generators of the Poincaré group is

$$
\begin{aligned}
& {\left[\Xi_{i j}, \Xi_{k l}\right]=\eta_{i k} \Xi_{j l}+\eta_{j l} \Xi_{i k}-\eta_{j k} \Xi_{i l}-\eta_{i l} \Xi_{j k},} \\
& {\left[\Xi_{i j}, T_{k}\right]=\eta_{j k} T_{i}-\eta_{i k} T_{j}, \quad\left[T_{i}, T_{j}\right]=0,}
\end{aligned}
$$

where $\Xi_{i j}$ are the generators of Lorentz transformations, and $T_{i}$ are the generators of four-dimensional translations. Obviously, $\partial_{i}\left(\delta x^{i}\right)=0$ for the Poincaré transformations (3.27). Therefore, our Lagrangian density $\mathcal{L}$, which is the same as $L$ with $h(x)=1$ in this case, is invariant; namely, $\delta \mathcal{L}=\delta L=0$ for $\delta I=0$.

Suppose that the field $\chi(x)$ transforms under the infinitesimal Poincar é transformations as

$$
\delta \chi=\frac{1}{2} \varepsilon^{i j} S_{i j} \chi
$$

where the tensors $S_{i j}$ are the generators of the Lorentz group, satisfying

$$
S_{i j}=-S_{j i},\left[S_{i j}, S_{k l}\right]=\eta_{i k} S_{j l}+\eta_{j l} S_{i k}-\eta_{j k} S_{i l}-\eta_{i l} S_{j k}
$$

Correspondingly, the derivative of $\chi$ transforms as

$$
\delta\left(\partial_{k} \chi\right)=\frac{1}{2} \varepsilon^{i j} S_{i j} \partial_{k} \chi-\varepsilon_{k}^{i} \partial_{i} \chi
$$

Since the choice of infinitesimal parameters $\varepsilon^{i}$ and $\varepsilon^{i j}$ is arbitrary, the vanishing variation of the Lagrangian density $\delta \mathcal{L}=0$ leads to the identities,

$$
\frac{\partial \mathcal{L}}{\partial \chi} S_{i j} \chi+\frac{\partial \mathcal{L}}{\partial\left(\partial_{k} \chi\right)}\left(S_{i j} \partial_{k} \chi+\eta_{k i} \partial_{j} \chi-\eta_{k j} \partial_{i} \chi\right)=0
$$

We also obtain the following conservation laws

$$
\partial_{j} T_{k}^{j}=0, \partial_{k}\left(S^{k}{ }_{i j}-x_{i} T_{j}^{k}+x_{j} T_{i}^{k}\right)=0,
$$


where

$$
S^{k}{ }_{i j}:=-\frac{\partial \mathcal{L}}{\partial\left(\partial_{k} \chi\right)} S_{i j} \chi
$$

These conservation laws imply that the energy-momentum and angular momentum, respectively

$$
P_{l}=\int T_{l}^{0} d^{3} x, J_{i j}=\int\left[S^{0}{ }_{i j}-\left(x_{i} T^{0}{ }_{j}-x_{j} T^{0}{ }_{i}\right)\right] d^{3} x,
$$

are conserved. This means that the system invariant under the ten parameter symmetry group has ten conserved quantities. This is an example of Noether symmetry. The first term of the angular momentum integral corresponds to the spin angular momentum while the second term gives the orbital angular momentum. The global Poincaré invariance of a system means that, for the system, the space-time is homogeneous (all space-time points are equivalent) as dictated by the translational invariance and is isotropic (all directions about a space-time point are equivalent) as indicated by the Lorentz invariance. It is interesting to observe that the fixed point variation of the field variables takes the form

$$
\delta_{0} \chi=\frac{1}{2} \varepsilon^{j} \Xi_{j}{ }^{k} \chi+\varepsilon^{j} T_{j} \chi
$$

where

$$
\Xi_{j}{ }^{k}=S_{j}{ }^{k}+\left(x^{j} \partial_{k}-x^{k} \partial_{j}\right), T_{j}=-\partial_{j} .
$$

We remark that $\Xi_{j}{ }^{k}$ are the generators of the Lorentz transformation and $T_{j}$ are those of the translations.

\section{The Local Poincaré invariance}

As next step, let us consider a modification of the infinitesimal Poincaré transformations (3.28) by assuming that the parameters $\varepsilon_{k}^{j}$ and $\varepsilon^{j}$ are functions of the coordinates and by writing them altogether as

$$
\delta x^{\mu}=\varepsilon_{\nu}^{\mu}(x) x^{\nu}+\varepsilon^{\mu}(x)=\xi^{\mu},
$$

which we call the local Poincaré transformations (or the general coordinate transformations). As before, in order to distinguish between global transformations and local transformation, we use the Latin indices $(j, k=0,1,2,3)$ for the former and the Greek indices $(\mu, \nu=0,1,2,3)$ for the latter. The variation of the field variables $\chi(x)$ defined at a point $x$ is still the same as that of the global Poincaré transformations,

$$
\delta \chi=\frac{1}{2} \varepsilon_{i j} S^{i j} \chi
$$

The corresponding fixed point variation of $\chi$ takes the form,

$$
\delta_{0} \chi=\frac{1}{2} \varepsilon_{i j} S^{i j} \chi-\xi^{\nu} \partial_{\nu} \chi
$$

Differentiating both sides of (3.41) with respect to $x^{\mu}$, we have

$$
\delta_{0} \partial_{\mu} \chi=\frac{1}{2} \varepsilon^{i j} S_{i j} \partial_{\mu} \chi+\frac{1}{2}\left(\partial_{\mu} \varepsilon^{i j}\right) S^{i j} \chi-\partial_{\mu}\left(\xi^{\nu} \partial_{\nu} \chi\right) .
$$

By using these variations, we obtain the variation of the Lagrangian $L$,

$$
\delta \mathcal{L}+\partial_{\mu}\left(\delta x^{\mu}\right) \mathcal{L}=h \delta L=\delta_{0} \mathcal{L}+\partial_{\nu}\left(\mathcal{L} \delta x^{\nu}\right)=-\frac{1}{2}\left(\partial_{\mu} \varepsilon^{i j}\right) S^{\mu}{ }_{i j}-\partial_{\mu} \xi^{\nu} T_{\nu}^{\mu}
$$

which is no longer zero unless the parameters $\varepsilon^{i j}$ and $\xi^{\nu}$ become constants. Accordingly, the action integral for the given Lagrangian density $\mathcal{L}$ is not invariant under the local Poincaré transformations. We notice that while $\partial_{j}\left(\delta x^{j}\right)=0$ for the local Poincaré transformations, $\partial_{\mu} \xi^{\mu}$ does not vanish under local Poincaré transformations. Hence, as expected $\mathcal{L}$ is not a Lagrangian scalar but a Lagrangian density. As mentioned earlier, in order to define the Lagrangian $L$, we have to select an appropriate non-trivial scalar function $h(x)$ satisfying

$$
\delta h+h \partial_{\mu} \xi^{\mu}=0
$$


Now we consider a minimal modification of the Lagrangian so as to make the action integral invariant under the local Poincaré transformations. It is rather obvious that if there is a covariant derivative $\nabla_{k} \chi$ which transforms as

$$
\delta\left(\nabla_{k} \chi\right)=\frac{1}{2} \varepsilon^{i j} S_{i j} \nabla_{k} \chi-\varepsilon_{k}^{i} \nabla_{i} \chi
$$

then a modified Lagrangian $L^{\prime}\left(\chi, \partial_{k} \chi, x\right)=L\left(\chi, \nabla_{k} \chi, x\right)$, obtained by replacing $\partial_{k} \chi$ of $L\left(\chi, \partial_{k} \chi, x\right)$ by $\nabla_{k} \chi$, remains invariant under the local Poincaré transformations, that is

$$
\delta L^{\prime}=\frac{\partial L^{\prime}}{\partial \chi} \delta \chi+\frac{\partial L^{\prime}}{\partial\left(\nabla_{k} \chi\right)} \delta\left(\nabla_{k} \chi\right)=0
$$

To find such a $k$-covariant derivative, we introduce the gauge fields $A^{i j}{ }_{\mu}=-A^{j i}{ }_{\mu}$ and define the $\mu$-covariant derivative

$$
\nabla_{\mu} \chi:=\partial_{\mu} \chi+\frac{1}{2} A_{\mu}^{i j} S_{i j} \chi
$$

in such a way that the covariant derivative transforms as

$$
\delta_{0} \nabla_{\mu} \chi=\frac{1}{2} S_{i j} \nabla_{\mu} \chi-\partial_{\mu}\left(\xi^{\nu} \nabla_{\nu} \chi\right)
$$

The transformation properties of $A^{a b}{ }_{\mu}$ are determined by $\nabla_{\mu} \chi$ and $\delta \nabla_{\mu} \chi$. Making use of

$$
\delta \nabla_{\mu} \chi=\frac{1}{2} \varepsilon^{i j}{ }_{\mu} S_{i j} \chi+\frac{1}{2} \varepsilon^{i j} S_{i j} \partial_{\mu} \chi-\left(\partial_{\mu} \xi^{\nu}\right) \partial_{\nu} \psi+\frac{1}{2} \delta A_{\mu}^{i j} S_{i j} \chi+\frac{1}{4} A^{i j}{ }_{\mu} S_{i j} \varepsilon^{k l} S_{k l} \chi
$$

and comparing with (3.47) we obtain,

$$
\delta A_{\mu}^{i j} S_{i j} \chi+\varepsilon^{i j}{ }_{\mu} S_{i j} \chi+\frac{1}{2}\left(A_{\mu}^{i j} \varepsilon^{k l}-\varepsilon^{i j} A_{\mu}^{k l}\right) S_{i j} S_{k l} \chi+\left(\partial_{\mu} \xi^{\nu}\right) A_{\nu}^{i j} S_{i j} \chi=0 .
$$

Using the antisymmetry in $i j$ and $k l$ to rewrite the term in parentheses on the rhs of (3.50) as $\left[S_{i j}, S_{k l}\right] A^{i j}{ }_{\mu} \varepsilon^{k l} \chi$, we see the explicit appearance of the commutator $\left[S_{i j}, S_{k l}\right]$. Using the expression for the commutator of Lie algebra generators

$$
\left[S_{i j}, S_{k l}\right]=\frac{1}{2} c^{[e f]}[i j][k l] S_{e f},
$$

where $c_{[i j][k l]}^{[e f]}$ (the square brackets denote anti-symmetrization) is the structure constants of the Lorentz group (deduced below), we have

$$
\left[S_{i j}, S_{k l}\right] A_{\mu}^{i j} \varepsilon^{k l}=\frac{1}{2}\left(A_{\mu}^{i c} \varepsilon_{c}^{j}-A_{\mu}^{c j} \varepsilon_{c}^{i}\right) S_{i j} .
$$

The substitution of this equation and the consideration of the antisymmetry of $\varepsilon_{c}^{b}=-\varepsilon_{c}^{b}$ enable us to write

$$
\delta A_{\mu}^{i j}=\varepsilon^{i}{ }_{k} A_{\mu}^{k j}+\varepsilon^{j}{ }_{k} A_{\mu}^{i k}-\left(\partial_{\mu} \xi^{\nu}\right) A_{\nu}^{i j}-\partial_{\mu} \varepsilon^{i j} .
$$

We require the $k$-derivative and $\mu$-derivative of $\chi$ to be linearly related as

$$
\nabla_{k} \chi=V_{k}{ }^{\mu}(x) \nabla_{\mu} \chi
$$

where the coefficients $V_{k}{ }^{\mu}(x)$ are position-dependent and behave like a new set of field variables. From (3.54) it is evident that $\nabla_{k} \chi$ varies as

$$
\delta \nabla_{k} \chi=\delta V_{k}^{\mu} \nabla_{\mu} \chi+V_{k}^{\mu} \delta \nabla_{\mu} \chi
$$

Comparing with $\delta \nabla_{k} \chi=\frac{1}{2} \varepsilon^{a b} S_{a b} \nabla_{k} \chi-\varepsilon^{j}{ }_{k} \nabla_{j} \chi$ we obtain,

$$
V_{\alpha}^{k} \delta V_{k}^{\mu} \nabla_{\mu} \chi-\xi^{\nu},{ }_{\alpha} \nabla_{\nu} \chi+V_{\alpha}^{k} \varepsilon_{k}^{j} \nabla_{j} \chi=0 .
$$


Exploiting $\delta\left(V_{\alpha}^{k} V_{k}^{\mu}\right)=0$ we find the quantity $V_{k}{ }^{\mu}$ transforms according to

$$
\delta V_{k}{ }^{\mu}=V_{k}{ }^{\nu} \partial_{\nu} \xi^{\mu}-V_{i}{ }^{\mu} \varepsilon_{k}^{i} .
$$

It is also important to recognize that the inverse of $\operatorname{det}\left(V_{k}{ }^{\mu}\right)$ transforms like a scalar density as $h(x)$ does. For our minimal modification of the Lagrangian density, we utilize this available quantity for the scalar density $h$; namely, we let

$$
h(x)=\left[\operatorname{det}\left(V_{k}^{\mu}\right)\right]^{-1} .
$$

In the limiting case, when we consider Poincaré transformations, that are not space-time dependent, $V_{k}^{\mu} \rightarrow \delta_{k}^{\mu}$ so that $h(x) \rightarrow 1$. This is a desirable property. Then we replace the Lagrangian density $\mathcal{L}\left(\chi, \partial_{k} \chi, x\right)$, invariant under the global Poincaré transformations, by a Lagrangian density

$$
\mathcal{L}\left(\chi, \partial_{\mu} \chi ; x\right) \rightarrow h(x) L\left(\chi, \nabla_{k} \chi\right) .
$$

The action integral with this modified Lagrangian density remains invariant under the local Poincaré transformations. Since the local Poincaré transformations $\delta x^{\mu}=\xi^{\mu}(x)$ are nothing else but generalized coordinate transformations, the newly introduced gauge fields $V_{i}^{\lambda}$ and $A^{i j}{ }_{\mu}$ can be interpreted, respectively, as the tetrad (vierbein) fields which set the local coordinate frame and as a local affine connection with respect to the tetrad frame (see also [58]).

\section{E. Spinors, vectors, bivectors and tetrads}

Let us consider first the case where the multiplet field $\chi$ is the Dirac field $\psi(x)$ which behaves like a four-component spinor under the Lorentz transformations and transforms as

$$
\psi(x) \rightarrow \psi^{\prime}\left(x^{\prime}\right)=S(\Lambda) \psi(x),
$$

where $S(\Lambda)$ is an irreducible unitary representation of the Lorentz group. Since the bilinear form $v^{k}=i \bar{\psi} \gamma^{k} \psi$ is a vector, it transforms according to

$$
v^{j}=\Lambda_{k}^{j} v^{k}
$$

where $\Lambda_{i}^{j}$ is a Lorentz transformation matrix satisfying

$$
\Lambda_{i j}+\Lambda_{j i}=0 .
$$

The invariance of $v^{i}$ (or the covariance of the Dirac equation) under the transformation $\psi(x) \rightarrow \psi^{\prime}\left(x^{\prime}\right)$ leads to

$$
S^{-1}(\Lambda) \gamma^{\mu} S(\Lambda)=\Lambda_{\nu}^{\mu} \gamma^{\nu}
$$

where the $\gamma^{\prime} s$ are the Dirac $\gamma$-matrices satisfying the anticommutator,

$$
\gamma_{i} \gamma_{j}+\gamma_{j} \gamma_{i}=\eta_{i j} \mathbf{1}
$$

Furthermore, we notice that the $\gamma$-matrices have the following properties:

$$
\left\{\begin{array}{c}
\left(\gamma_{0}\right)^{\dagger}=-\gamma_{0},\left(\gamma^{0}\right)^{2}=\left(\gamma_{0}\right)^{2}=-1, \gamma_{0}=-\gamma^{0} \text { and } \gamma_{0} \gamma^{0}=1 \\
\left(\gamma_{k}\right)^{\dagger}=\gamma_{k},\left(\gamma^{k}\right)^{2}=\left(\gamma_{k}\right)^{2}=1 ;(k=1,2,3) \text { and } \gamma_{k}=\gamma^{k} \\
\left(\gamma_{5}\right)^{\dagger}=-\gamma_{5},\left(\gamma_{5}\right)^{2}=-1 \text { and } \gamma^{5}=\gamma_{5}
\end{array}\right.
$$

We assume the transformation $S(\Lambda)$ can be put into the form $S(\Lambda)=e^{\Lambda_{\mu \nu} \gamma^{\mu \nu}}$. Expanding $S(\Lambda)$ about the identity and only retaining terms up to the first order in the infinitesimals and expanding $\Lambda_{\mu \nu}$ to the first order in $\varepsilon_{\mu \nu}$

$$
\Lambda_{\mu \nu}=\delta_{\mu \nu}+\varepsilon_{\mu \nu}, \varepsilon_{i j}+\varepsilon_{j i}=0
$$

we get

$$
S(\Lambda)=1+\frac{1}{2} \varepsilon^{i j} \gamma_{i j}
$$


In order to determine the form of $\gamma_{i j}$, we substitute (3.66) and (3.67) into (3.63) to obtain

$$
\frac{1}{2} \varepsilon_{i j}\left[\gamma^{i j}, \gamma^{k}\right]=\eta^{k i} \varepsilon_{j i} \gamma^{j}
$$

Rewriting the rhs of (3.68) using the antisymmetry of $\varepsilon_{i j}$ as

$$
\eta^{k i} \varepsilon_{j i} \gamma^{j}=\frac{1}{2} \varepsilon_{i j}\left(\eta^{k i} \gamma^{j}-\eta^{k j} \gamma^{i}\right)
$$

yields

$$
\left[\gamma^{k}, \gamma^{i j}\right]=\eta^{k i} \gamma^{j}-\eta^{k j} \gamma^{i}
$$

Assuming the solution to have the form of an antisymmetric product of two matrices, we obtain the solution

$$
\gamma^{i j}:=\frac{1}{2}\left[\gamma^{i}, \gamma^{j}\right]
$$

If $\chi=\psi$, the group generator $S_{i j}$ appearing in (3.31) is identified with

$$
S_{i j} \equiv \gamma_{i j}=\frac{1}{2}\left(\gamma_{i} \gamma_{j}-\gamma_{j} \gamma_{i}\right)
$$

To be explicit, the Dirac field transforms under Lorentz transformations (LT) as

$$
\delta \psi(x)=\frac{1}{2} \varepsilon^{i j} \gamma_{i j} \psi(x)
$$

The Pauli conjugate of the Dirac field is denoted $\bar{\psi}$ and defined by

$$
\bar{\psi}(x):=i \psi^{\dagger}(x) \gamma_{0}, i \in \mathbb{C} .
$$

The conjugate field $\bar{\psi}$ transforms under LTs as,

$$
\delta \bar{\psi}=-\bar{\psi} \frac{1}{2} \varepsilon^{i j} \bar{\psi} \gamma_{i j}
$$

Under local LTs, $\varepsilon_{a b}(x)$ becomes a function of space-time. Now, unlike $\partial_{\mu} \psi(x)$, the derivative of $\psi^{\prime}\left(x^{\prime}\right)$ is no longer homogenous due to the occurrence of the term $\gamma^{a b}\left[\partial_{\mu} \varepsilon_{a b}(x)\right] \psi(x)$ in $\partial_{\mu} \psi^{\prime}\left(x^{\prime}\right)$, which is non-vanishing unless $\varepsilon_{a b}$ is constant. When going from locally flat to curved space-time, we must generalize $\partial_{\mu}$ to the covariant derivative $\nabla_{\mu}$ to compensate for this extra term, allowing to gauge the group of LTs. Thus, by using $\nabla_{\mu}$, we can preserve the invariance of the Lagrangian for arbitrary local LTs at each space-time point

$$
\nabla_{\mu} \psi^{\prime}\left(x^{\prime}\right)=S(\Lambda(x)) \nabla_{\mu} \psi(x)
$$

To determine the explicit form of the connection belonging to $\nabla_{\mu}$, we study the derivative of $S(\Lambda(x))$. The transformation $S(\Lambda(x))$ is given by

$$
S(\Lambda(x))=1+\frac{1}{2} \varepsilon_{a b}(x) \gamma^{a b}
$$

Since $\varepsilon_{a b}(x)$ is only a function of space-time for local Lorentz coordinates, we express this infinitesimal LT in terms of general coordinates only by shifting all space-time dependence of the local coordinates into tetrad fields as

$$
\varepsilon_{a b}(x)=V_{a}^{\lambda}(x) V_{b}^{\nu}(x) \varepsilon_{\lambda \nu}
$$

Substituting this expression for $\varepsilon_{a b}(x)$, we obtain

$$
\partial_{\mu} \varepsilon_{a b}(x)=\partial_{\mu}\left[V_{a}^{\lambda}(x) V_{b}^{\nu}(x) \varepsilon_{\lambda \nu}\right]
$$

However, since $\varepsilon_{\lambda \nu}$ has no space-time dependence, this reduces to

$$
\partial_{\mu} \varepsilon_{a b}(x)=V_{a}^{\lambda}(x) \partial_{\mu} V_{b \lambda}(x)-V_{b}^{\nu}(x) \partial_{\mu} V_{a \nu}(x) .
$$


Letting

$$
\omega_{\mu b a}:=V_{b}^{\nu}(x) \partial_{\mu} V_{a \nu}(x)
$$

the first and second terms in Eq. (3.80) become $V_{a}^{\lambda}(x) \partial_{\mu} V_{b \lambda}(x)=\frac{1}{2} \omega_{\mu a b}$ and $V_{b}^{\nu}(x) \partial_{\mu} V_{a \nu}(x)=\frac{1}{2} \omega_{\mu b a}$ respectively. Using the identification

$$
\partial_{\mu} \varepsilon_{a b}(x)=\omega_{\mu a b},
$$

we write

$$
\partial_{\mu} S(\Lambda(x))=-\frac{1}{2} \gamma^{a b} \omega_{\mu a b}
$$

According to (3.47), the covariant derivative of the Dirac spinor is

$$
\nabla_{\mu} \psi=\partial_{\mu} \psi+\frac{1}{2} A^{i j}{ }_{\mu} \gamma_{i j} \psi
$$

Correspondingly, the covariant derivative of $\bar{\psi}$ is given by

$$
\nabla_{\mu} \bar{\psi}=\partial_{\mu} \bar{\psi}-\frac{1}{2} A_{\mu}^{i j} \bar{\psi} \gamma_{i j}
$$

Using the covariant derivatives of $\psi$ and $\bar{\psi}$, we can show that

$$
\nabla_{\mu} v_{j}=\partial_{\mu} v_{j}-A_{j \mu}^{i} v_{i}
$$

The same covariant derivative should be used for any covariant vector $v_{k}$ under the Lorentz transformation. Since $\nabla_{\mu}\left(v_{i} v^{i}\right)=\partial_{\mu}\left(v_{i} v^{i}\right)$, the covariant derivative for a contravariant vector $v^{i}$ must be

$$
\nabla_{\mu} v^{i}=\partial_{\mu} v^{i}+A^{i}{ }_{j \mu} v^{j}
$$

Since the tetrad $V_{i}^{\mu}$ is a covariant vector under Lorentz transformations, its covariant derivative must transform according to the same rule. Using $\nabla_{a}=V_{a}^{\mu}(x) \nabla_{\mu}$, the covariant derivatives of a tetrad in local Lorentz coordinates read

$$
\nabla_{\nu} V_{i}^{\mu}=\partial_{\nu} V_{i}^{\mu}-A^{k}{ }_{i \nu} V_{k}^{\mu}, \nabla_{\nu} V_{\mu}^{i}=\partial_{\nu} V_{\mu}^{i}+A_{k \nu}^{i} V_{\mu}^{k}
$$

The inverse of $V_{i}^{\mu}$ is denoted by $V^{i}{ }_{\mu}$ and satisfies

$$
V_{\mu}^{i} V_{i}^{\nu}=\delta_{\mu}^{\nu}, \quad V_{\mu}^{i} V_{j}^{\mu}=\delta^{i}{ }_{j}
$$

To allow the transition to curved space-time, we take account of the general coordinates of objects that are covariant under local Poincaré transformations. Here we define the covariant derivative of a quantity $v^{\lambda}$ which behaves like a controvariant vector under the local Poincaré transformation. Namely

$$
D_{\nu} v^{\lambda} \equiv V_{i}^{\lambda} \nabla_{\nu} v^{i}=\partial_{\nu} v^{\lambda}+\Gamma_{\mu \nu}^{\lambda} v^{\mu}, \quad D_{\nu} v_{\mu} \equiv V_{\mu}^{i} \nabla_{\nu} v_{i}=\partial_{\nu} v_{\mu}-\Gamma_{\mu \nu}^{\lambda} v_{\lambda},
$$

where

$$
\Gamma_{\mu \nu}^{\lambda}:=V_{i}^{\lambda} \nabla_{\nu} V_{\mu}^{i} \equiv-V_{\mu}^{i} \nabla_{\nu} V_{i}^{\lambda}
$$

The definition of $\Gamma_{\mu \nu}^{\lambda}$ implies

$$
\begin{aligned}
& D_{\nu} V_{i}^{\lambda}=\nabla_{\nu} V_{i}^{\lambda}+\Gamma_{\mu \nu}^{\lambda} V_{i}^{\mu}=\partial_{\nu} V_{i}^{\lambda}-A_{i \nu}^{k} V_{k}^{\lambda}+\Gamma_{\mu \nu}^{\lambda} V_{i}^{\mu}=0, \\
& D_{\nu} V_{\mu}^{i}=\nabla_{\nu} V_{\mu}^{i}-\Gamma_{\mu \nu}^{\lambda} V_{\lambda}^{i}=\partial_{\nu} V_{\mu}^{i}+A_{k \nu}^{i} V_{\mu}^{k}-\Gamma_{\mu \nu}^{\lambda} V_{\lambda}^{i}=0 .
\end{aligned}
$$

From (3.92) we find,

$$
A_{k \nu}^{i}=V_{\lambda}^{i} \partial_{\nu} V_{k}^{\lambda}+\Gamma_{\mu \nu}^{\lambda} V_{\lambda}^{i} V_{k}^{\mu}=-V_{k}{ }^{\lambda} \partial_{\nu} V_{\lambda}^{i}+\Gamma_{\mu \nu}^{\lambda} V_{\lambda}^{i} V_{k}^{\mu} .
$$


or, equivalently, in terms of $\omega$ defined in (3.81),

$$
A_{k \nu}^{i}=\omega_{\nu k}^{i}+\Gamma_{\mu \nu}^{\lambda} V_{\lambda}^{i} V_{k}^{\mu}=-\omega_{k \nu}{ }^{i}+\Gamma_{\mu \nu}^{\lambda} V_{\lambda}^{i} V_{k}^{\mu} .
$$

Using this in (3.84), we may write

$$
\nabla_{\mu} \psi=\left(\partial_{\mu}-\Gamma_{\mu}\right) \psi
$$

where

$$
\Gamma_{\mu}=\frac{1}{4}\left(\omega^{i}{ }_{j \mu}-\Gamma_{\mu \nu}^{\lambda} V_{\lambda}^{i} V_{j}{ }^{\nu}\right) \gamma_{i}{ }^{j},
$$

which is known as the Fock-Ivanenko connection [139].

We now study the transformation properties of $A_{\mu a b}$. Recall $\omega_{\mu a b}=V_{a}{ }^{\lambda}(x) \partial_{\mu} V_{\beta \lambda}(x)$ and since $\partial_{\mu} \eta_{a b}=0$, we write

$$
\Lambda^{a}{ }_{\bar{a}} \eta_{a b} \partial_{\mu} \Lambda_{\bar{b}}^{b}=\Lambda^{a}{ }_{\bar{a}} \partial_{\mu} \Lambda_{a \bar{b}} .
$$

Note that barred indices are equivalent to the primed indices used above. Hence, the spin connection transforms as

$$
A_{\bar{a} \bar{b} \bar{c}}=\Lambda_{\bar{a}}^{a} \Lambda_{\bar{b}}^{b} \Lambda_{\bar{c}}^{c} A_{a b c}+\Lambda_{\bar{a}}^{a} \Lambda_{\bar{c}}^{c} V_{a}^{\mu}(x) \partial_{\mu} \Lambda_{\bar{b} c} .
$$

To determine the transformation properties of

$$
\Gamma_{a b c}=A_{a b c}-\left[V_{a}^{\mu}(x) \partial_{\mu} V_{b}^{\nu}(x)\right] V_{\nu c}(x),
$$

we consider the local LT of $\left[V_{a}^{\mu}(x) \partial_{\mu} V_{b}^{\nu}(x)\right] V_{\nu c}(x)$ which is,

$$
\left[V_{\bar{a}}^{\mu}(x) \partial_{\mu} V_{\bar{b}}^{\nu}\right] V_{\nu \bar{c}}(x)=\Lambda_{\bar{a}}{ }^{a} \Lambda_{\bar{b}}{ }^{b} \Lambda_{\bar{c}}{ }^{c}\left[A^{\nu}{ }_{a b} V_{\nu c}(x)\right]+\Lambda_{\bar{a}}{ }^{a} \Lambda_{\bar{c}}{ }^{c} V^{\mu}{ }_{a}(x) \partial_{\mu} \Lambda_{c \bar{b}} .
$$

From this result, we obtain the following transformation law,

$$
\Gamma_{\bar{a} \bar{b} \bar{c}}=\Lambda_{\bar{a}}^{a} \Lambda_{\bar{b}}^{b} \Lambda_{\bar{c}}^{c} \Gamma_{a b c}
$$

We now explore the consequence of the antisymmetry of $\omega_{a b c}$ in $b c$. Recalling the equation for $\Gamma_{a b c}$, exchanging $b$ and $c$ and adding the two equations, we obtain

$$
\Gamma_{a b c}+\Gamma_{a c b}=-V_{a}^{\mu}(x)\left[\left(\partial_{\mu} V_{b}^{\nu}(x)\right) V_{\nu c}(x)+\left(\partial_{\mu} V_{c}^{\nu}(x)\right) V_{\nu b}(x)\right] .
$$

We know however, that

$$
\partial_{\mu}\left[V_{b}^{\nu}(x) V_{\nu c}(x)\right]=V_{\nu c}(x) \partial_{\mu} V_{b}^{\nu}(x)+V_{\lambda b}(x) \partial_{\mu} V_{c}^{\lambda}(x)+V_{b}{ }^{\nu}(x) V_{c}^{\lambda}(x) \partial_{\mu} g_{\lambda \nu} .
$$

Letting $\lambda \rightarrow \nu$ and exchanging $b$ and $c$, we obtain

$$
\partial_{\mu}\left[V_{b}^{\nu}(x) V_{\nu c}(x)\right]=-V_{b}{ }^{\lambda}(x) V_{c}^{\nu}(x) \partial_{\mu} g_{\nu \lambda},
$$

so that, finally,

$$
\Gamma_{a b c}+\Gamma_{a c b}=V_{a}^{\mu}(x) V_{b}^{\lambda}(x) V_{c}{ }^{\nu}(x) \partial_{\mu} g_{\nu \lambda} .
$$

This, however, is equivalent to

$$
\Gamma_{\bar{a} \bar{b} \bar{c}}+\Gamma_{\bar{a}} \bar{c} \bar{b}=V_{\bar{a}}^{\bar{\mu}}(x) V_{\bar{b}}^{\bar{\lambda}}(x) V_{\bar{c}}^{\bar{\nu}}(x) \partial_{\bar{\mu}} g_{\bar{\nu} \bar{\lambda}}
$$

and then

$$
\Gamma_{\mu \lambda \nu}+\Gamma_{\mu \nu \lambda}=\partial_{\mu} g_{\nu \lambda},
$$

which we recognize as the general coordinate connection. It is known that the covariant derivative for general coordinates is

$$
\nabla_{\mu} A_{\nu}{ }^{\lambda}=\partial_{\mu} A_{\nu}{ }^{\lambda}+\Gamma_{\mu \sigma}^{\lambda} A_{\nu}{ }^{\sigma}-\Gamma_{\mu \nu}^{\sigma} A_{\sigma}{ }^{\lambda} .
$$


In a Riemannian manifold, the connection is symmetric under the exchange of $\mu \nu$, that is, $\Gamma_{\mu \nu}^{\lambda}=\Gamma_{\nu \mu}^{\lambda}$. Using the fact that the metric is a symmetric tensor we can now determine the form of the Christoffel connection by cyclically permuting the indices of the general coordinate connection equation (3.107) yielding

$$
\Gamma_{\mu \nu \lambda}=\frac{1}{2}\left(\partial_{\mu} g_{\nu \lambda}+\partial_{\nu} g_{\lambda \mu}-\partial_{\lambda} g_{\mu \nu}\right)
$$

Since $\Gamma_{\mu \nu \lambda}=\Gamma_{\nu \mu \lambda}$ is valid for general coordinate systems, it follows that a similar constraint must hold for local Lorentz transforming coordinates as well, so we expect $\Gamma_{a b c}=\Gamma_{b a c}$. Recalling the equation for $\Gamma_{a b c}$ and exchanging $a$ and $b$, we obtain

$$
\omega_{a b c}-\omega_{b a c}=V_{\nu c}(x)\left[V_{a}^{\mu}(x) \partial_{\mu} V_{b}^{\nu}(x)-V_{b}^{\mu}(x) \partial_{\mu} V_{a}^{\nu}(x)\right]
$$

We now define the objects of anholonomicity as

$$
\Omega_{c a b}:=V_{\nu c}(x)\left[V_{a}^{\mu}(x) \partial_{\mu} V_{b}^{\nu}(x)-V_{b}^{\mu}(x) \partial_{\mu} V_{a}^{\nu}(x)\right]
$$

Using $\Omega_{c a b}=-\Omega_{c b a}$, we permute indices in a similar manner as was done for the derivation of the Christoffel connection above yielding,

$$
\omega_{a b \mu}=\frac{1}{2}\left[\Omega_{c a b}+\Omega_{b c a}-\Omega_{a b c}\right] V_{\mu}^{c} \equiv \Delta_{a b \mu}
$$

For completeness, we determine the transformation law of the Christoffel connection. Making use of $\Gamma_{\mu \nu}^{\lambda} e_{\lambda}=\partial_{\mu} e_{\nu}$ where

$$
\partial_{\bar{\mu}} e_{\bar{\nu}}=X_{\bar{\mu}}^{\mu} X_{\bar{\nu}}^{\nu} \partial_{\mu} e_{\nu}+X_{\bar{\mu}}^{\mu}\left(\partial_{\mu} X_{\bar{\nu}}^{\nu}\right) e_{\nu}
$$

we can show

$$
\Gamma_{\bar{\mu}}^{\bar{\lambda}}=X_{\bar{\mu}}^{\mu} X_{\bar{\nu}}^{\nu} X_{\lambda}^{\bar{\lambda}} \Gamma_{\mu \nu}^{\lambda}+X_{\bar{\mu}}^{\mu} X_{\nu}^{\bar{\lambda}} X_{\mu \bar{\nu}}^{\nu}
$$

where

$$
X_{\mu \bar{\nu}}^{\nu} \equiv \partial_{\mu} \partial_{\bar{\nu}} x^{\nu}
$$

In the light of the above considerations, we may regard infinitesimal local gauge transformations as local rotations of basis vectors belonging to the tangent space [162, 398] of the manifold. For this reason, given a local frame on a tangent plane to the point $x$ on the base manifold, we can obtain all other frames on the same tangent plane by means of local rotations of the original basis vectors. Reversing this argument, we observe that by knowing all frames residing in the horizontal tangent space to a point $x$ on the base manifold enables us to deduce the corresponding gauge group of symmetry transformations. These arguments are completely general and can be adopted for any theory of gravity coming from gauge symmetry.

\section{F. Curvature, torsion and metric}

From the definition of the Fock-Ivanenko covariant derivative, we can find the second order covariant derivative

$$
\begin{aligned}
D_{\nu} D_{\mu} \psi= & \partial_{\nu} \partial_{\mu} \psi+\frac{1}{2} S_{c d}\left(\psi \partial_{\nu} A_{\mu}{ }^{c d}+A_{\mu}{ }^{c d} \partial_{\nu} \psi\right)+\Gamma_{\mu \nu}^{\rho} D_{\rho} \psi+\frac{1}{2} S_{e f} A_{\nu}{ }^{e f} \partial_{\mu} \psi \\
& +\frac{1}{4} S_{e f} S_{c d} A_{\nu}{ }^{e f} A_{\mu}{ }^{c d} \psi .
\end{aligned}
$$

Recalling $D_{\nu} V^{c \mu}=0$, we can solve for the spin connection in terms of the Christoffel connection

$$
A_{\mu}{ }^{c d}=-V_{\lambda}^{d} \partial_{\mu} V^{c \lambda}-\Gamma_{\mu}{ }^{c d} .
$$

The derivative of the spin connection is then

$$
\partial_{\mu} A_{\nu}^{c d}=-V_{\lambda}^{d} \partial_{\mu} \partial_{\nu} V^{c \lambda}-\left(\partial_{\nu} V^{c \lambda}\right) \partial_{\mu} V_{\lambda}{ }^{d}-\partial_{\mu} \Gamma_{\nu}^{c d} .
$$


Noting that the Christoffel connection is symmetric and partial derivatives commute, we find

$$
\left[D_{\mu}, D_{\nu}\right] \psi=\frac{1}{2} S_{c d}\left[\left(\partial_{\nu} A_{\mu}^{c d}-\partial_{\mu} A_{\nu}^{c d}\right) \psi\right]+\frac{1}{4} S_{e f} S_{c d}\left[\left(A_{\nu}^{e f} A_{\mu}^{c d}-A_{\mu}^{e f} A_{\nu}^{c d}\right) \psi\right]
$$

where

$$
\partial_{\nu} A_{\mu}^{c d}-\partial_{\mu} A_{\nu}^{c d}=\partial_{\mu} \Gamma_{\nu}^{c d}-\partial_{\nu} \Gamma_{\mu}^{c d}
$$

Relabeling running indices, we can write

$$
\frac{1}{4} S_{e f} S_{c d}\left(A_{\nu}^{e f} A_{\mu}^{c d}-A_{\mu}^{e f} A_{\nu}^{c d}\right) \psi=\frac{1}{4}\left[S_{c d}, S_{e f}\right] A_{\mu}^{e f} A_{\nu}^{c d} \psi
$$

Using $\left\{\gamma_{a}, \gamma_{b}\right\}=2 \eta_{a b}$ to deduce

$$
\left\{\gamma_{a}, \gamma_{b}\right\} \gamma_{c} \gamma_{d}=2 \eta_{a b} \gamma_{c} \gamma_{d}
$$

we find that the commutator of bi-spinors is given by

$$
\left[S_{c d}, S_{e f}\right]=\frac{1}{2}\left[\eta_{c e} \delta_{d}^{a} \delta_{f}^{b}-\eta_{d e} \delta_{c}^{a} \delta_{f}^{b}+\eta_{c f} \delta_{e}^{a} \delta_{d}^{b}-\eta_{d f} \delta_{e}^{a} \delta_{c}^{b}\right] S_{a b}
$$

Clearly the terms in brackets on the rhs of (3.123) are antisymmetric in $c d$ and $e f$ and also antisymmetric under the exchange of pairs of indices $c d$ and $e f$. Since the alternating spinor is antisymmetric in $a b$, so it must be the terms in brackets: this means that the commutator does not vanish. Hence, the term in brackets is totally antisymmetric under interchange of indices $a b, c d$ and $e f$ and exchange of these pairs of indices. We identify this as the structure constant of the Lorentz group [212]

$$
\left[\eta_{c e} \delta_{d}^{a} \delta_{f}^{b}-\eta_{d e} \delta_{c}^{a} \delta_{f}^{b}+\eta_{c f} \delta_{e}^{a} \delta_{d}^{b}-\eta_{d f} \delta_{e}^{a} \delta_{c}^{b}\right]=c_{[c d][e f]}^{[a b]}=c_{[c d][e f]}^{[a b]}
$$

with the aid of which we can write

$$
\frac{1}{4}\left[S_{c d}, S_{e f}\right] A_{\mu}^{e f} A_{\nu}^{c d} \psi=\frac{1}{2} S_{a b}\left[A^{a}{ }_{e \nu} A_{\mu}^{e b}-A_{e \nu}^{b} A_{\mu}^{a e}\right] \psi
$$

where

$$
A_{e \nu}^{a} A_{\mu}^{e b}-A_{e \nu}^{b} A_{\mu}^{a e}=\Gamma_{\nu e}^{a} \Gamma_{\mu}^{e b}-\Gamma_{\nu e}^{b} \Gamma_{\mu}^{e a}
$$

Combining these results, the commutator of two $\mu$-covariant differentiations gives

$$
\left[\nabla_{\mu}, \nabla_{\nu}\right] \chi=-\frac{1}{2} R_{\mu \nu}^{i j} S_{i j} \chi
$$

where

$$
R^{i}{ }_{j \mu \nu}=\partial_{\nu} A^{i}{ }_{j \mu}-\partial_{\mu} A^{i}{ }_{j \nu}+A_{k \nu}^{i} A^{k}{ }_{j \mu}-A_{k \mu}^{i} A^{k}{ }_{j \nu}
$$

Using the Jacobi identities for the commutator of covariant derivatives, it follows that the field strength $R^{i}{ }_{j \mu \nu}$ satisfies the Bianchi identity

$$
\nabla_{\lambda} R^{i}{ }_{j \mu \nu}+\nabla_{\mu} R_{j \nu \lambda}^{i}+\nabla_{\nu} R_{j \lambda \mu}^{i}=0
$$

Permuting indices, this can be put into the cyclic form

$$
\varepsilon^{\alpha \beta \rho \sigma} \nabla_{\beta} R_{\rho \sigma}^{i j}=0
$$

where $\varepsilon^{\alpha \beta \rho \sigma}$ is the Levi-Civita alternating symbol. Furthermore, $R^{i j}{ }_{\mu \nu}=\eta^{j k} R_{k \mu \nu}^{i}$ is antisymmetric with respect to both pairs of indices,

$$
R_{\mu \nu}^{i j}=-R_{\mu \nu}^{j i}=R_{\nu \mu}^{j i}=-R_{\nu \mu}^{i j} .
$$

This condition is known as the first curvature tensor identity. 
To determine the analogue of $\left[\nabla_{\mu}, \nabla_{\nu}\right] \chi$ in local coordinates, we start from $\nabla_{k} \psi=V_{k}^{\mu} \nabla_{\mu} \psi$. From $\nabla_{k} \psi$ we obtain,

$$
\nabla_{l} \nabla_{k} \psi=V_{l}^{\nu}\left(\nabla_{\nu} V_{k}^{\mu}\right) \nabla_{\mu} \psi+V_{l}^{\nu} V_{k}^{\mu} \nabla_{\nu} \nabla_{\mu} \psi \text {. }
$$

Permuting indices and recognizing

$$
V_{\mu}{ }^{a} \nabla_{\nu} V_{k}^{\mu}=-V_{k}{ }^{\mu} \nabla_{\nu} V_{\mu}^{a},
$$

(which follows from $\nabla_{\nu}\left(V_{\mu}^{a} V_{k}^{\mu}\right)=0$ ), we arrive at

$$
V_{l}^{\nu}\left(\nabla_{\nu} V_{k}^{\mu}\right) \nabla_{\mu} \psi-V_{k}^{\mu}\left(\nabla_{\mu} V_{l}^{\nu}\right) \nabla_{\nu} \psi=\left(V_{l}^{\mu} V_{k}^{\nu}-V_{k}^{\mu} V_{l}^{\nu}\right)\left(\nabla_{\nu} V_{\mu}{ }^{a}\right) \nabla_{a} \psi \text {. }
$$

Defining

$$
C^{a}{ }_{k l}:=\left(V_{k}^{\mu} V_{l}^{\nu}-V_{l}^{\mu} V_{k}^{\nu}\right) \nabla_{\nu} V_{\mu}^{a},
$$

the commutator of the $k$-covariant differentiations takes the final form [356]

$$
\left[\nabla_{k}, \nabla_{l}\right] \chi=-\frac{1}{2} R^{i j}{ }_{k l} S_{i j} \chi+C^{i}{ }_{k l} \nabla_{i} \chi
$$

where

$$
R^{i j}{ }_{k l}=V_{k}{ }^{\mu} V_{l}{ }^{\nu} R^{i j}{ }_{\mu \nu} .
$$

As done for $R_{j \mu \nu}^{i}$ using the Jacobi identities for the commutator of covariant derivatives, we find the Bianchi identity in Einstein-Cartan space-time [78, 314]

$$
\varepsilon^{\alpha \beta \rho \sigma} \nabla_{\beta} R_{\rho \sigma}^{i j}=\varepsilon^{\alpha \beta \rho \sigma} C_{\beta \rho}^{\lambda} R_{\sigma \lambda}^{i j}
$$

The second curvature identity

$$
R_{[\rho \sigma \lambda]}^{k}=2 \nabla_{[\rho} C_{\sigma \lambda]}^{k}-4 C_{[\rho \sigma}^{b} C_{\lambda] b}^{k}
$$

leads to,

$$
\varepsilon^{\alpha \beta \rho \sigma} \nabla_{\beta} C_{\rho \sigma}{ }^{k}=\varepsilon^{\alpha \beta \rho \sigma} R^{k}{ }_{j \rho \sigma} V_{\beta}^{j} .
$$

Notice that if

$$
\Gamma_{\mu \nu}^{\lambda}=V_{i}^{\lambda} \nabla_{\nu} V_{\mu}^{i}=-V_{\mu}^{i} \nabla_{\nu} V_{i}^{\lambda},
$$

then

$$
\Gamma_{\mu \nu}^{\lambda}-\Gamma_{\nu \mu}^{\lambda}=V_{i}^{\lambda}\left(\nabla_{\nu} V_{\mu}^{i}-\nabla_{\mu} V_{\nu}^{i}\right) .
$$

Contracting by $V_{k}^{\mu} V_{l}^{\nu}$, we obtain [356],

$$
C^{a}{ }_{k l}=V_{k}{ }^{\mu} V_{l}{ }^{\nu} V_{\lambda}{ }^{a}\left(\Gamma_{\mu \nu}^{\lambda}-\Gamma_{\nu \mu}^{\lambda}\right) .
$$

We therefore conclude that $C_{k l}^{a}$ is related to the antisymmetric part of the affine connection

$$
\Gamma_{[\mu \nu]}^{\lambda}=V_{\mu}{ }^{k} V_{\nu}{ }^{l} V_{a}{ }^{\lambda} C^{a}{ }_{k l} \equiv T_{\mu \nu}^{\lambda},
$$

which is usually interpreted as space-time torsion $T_{\mu \nu}^{\lambda}$. Considering $\Delta_{a b \mu}$ defined in (3.112), we see that the most general connection in the Poincaré gauge approach to gravitation is

$$
A_{a b \mu}=\Delta_{a b \mu}-K_{a b \mu}+\Gamma_{\nu \mu}^{\lambda} V_{a \lambda} V_{b}{ }^{\nu},
$$

where

$$
K_{a b c}=-\left(T_{\nu \mu}^{\lambda}-T_{\nu \mu}{ }^{\lambda}+T_{\mu}{ }_{\nu}^{\lambda}\right) V_{a \lambda} V_{b}{ }^{\nu} V_{c}{ }^{\mu},
$$


is the contorsion tensor. Now, the quantity $R_{\sigma \mu \nu}^{\rho}=V_{i}{ }^{\rho} R^{i}{ }_{\sigma \mu \nu}$ may be expressed as

$$
R_{\sigma \mu \nu}^{\rho}=\partial_{\nu} \Gamma_{\sigma \mu}^{\rho}-\partial_{\mu} \Gamma_{\sigma \nu}^{\rho}+\Gamma_{\lambda \nu}^{\rho} \Gamma_{\sigma \mu}^{\lambda}-\Gamma_{\lambda \mu}^{\rho} \Gamma_{\sigma \nu}^{\lambda}
$$

Therefore, we can regard $R^{\rho}{ }_{\sigma \mu \nu}$ as the curvature tensor with respect the affine connection $\Gamma^{\lambda}{ }_{\mu \nu}$. By using the inverse of the tetrad, we define the metric of the space-time manifold by

$$
g_{\mu \nu}=V_{\mu}^{i} V^{j}{ }_{\nu} \eta_{i j}
$$

From (3.92) and the fact that the Minkowski metric is constant, it is obvious that the metric so defined is covariantly constant, that is,

$$
D_{\lambda} g_{\mu \nu}=0
$$

The space-time thus specified by the local Poincaré transformation is said to be metric. It is not difficult to show that

$$
\sqrt{-g}=\left[\operatorname{det} V_{\mu}^{i}\right]=\left[\operatorname{det} V_{i}^{\mu}\right]^{-1}
$$

where $g=\operatorname{det} g_{\mu \nu}$. Hence we may take $\sqrt{-g}$ for the density function $h(x)$.

\section{G. The field equations of gravity}

Finally, we are able to deduce the field equations for the gravitational field. From the curvature tensor $R^{\rho}{ }_{\sigma \nu}$, given in (3.147), the Ricci tensor follows

$$
R_{\sigma \nu}=R_{\sigma \mu \nu}^{\mu}
$$

and the scalar curvature

$$
R=R_{\nu}^{\nu}=\stackrel{\mathrm{L}}{R}+\partial_{i} K_{a}{ }^{i a}-T_{a}{ }^{b c} K_{b c}{ }^{a}
$$

where $\stackrel{L}{R}$ denotes the usual Ricci scalar of GR. Using this scalar curvature $R$, we choose the Lagrangian density for free Einstein-Cartan gravity

$$
\mathcal{L}_{G}=\frac{1}{2 \kappa} \sqrt{-g}\left(\stackrel{\mathrm{L}}{R}+\partial_{i} K_{a}{ }^{i a}-T_{a}{ }^{b c} K_{b c}{ }^{a}-2 \Lambda\right),
$$

where $\kappa$ is a gravitational coupling constant, and $\Lambda$ is the cosmological constant. These considerations can be easily extended to any function of $\stackrel{\mathrm{L}}{R}$ as in [130]. Observe that the second term is a divergence and may be ignored. The field equation can be obtained from the total action,

$$
S=\int\left\{\mathcal{L}_{\text {field }}\left(\chi, \partial_{\mu} \chi, V_{i}{ }^{\mu}, A^{i j}{ }_{\mu}\right)+\mathcal{L}_{G}\right\} d^{4} x
$$

where the matter Lagrangian density is taken to be

$$
\mathcal{L}_{\text {field }}=\frac{1}{2}\left[\bar{\psi} \gamma^{a} D_{a} \psi-\left(D_{a} \bar{\psi}\right) \gamma^{a} \psi\right] .
$$

Modifying the connection to include Christoffel, spin connection and contorsion contributions so as to operate on general, spinorial arguments, we have

$$
\Gamma_{\mu}=\frac{1}{4} g_{\lambda \sigma}\left(\Delta_{\mu \rho}^{\sigma}-\stackrel{\mathrm{L}}{\Gamma}_{\rho \mu}^{\sigma}-K_{\rho \mu}^{\sigma}\right) \gamma^{\lambda \rho} .
$$

It is important to keep in mind that $\Delta^{\sigma}{ }_{\mu \rho}$ act only on multi-component spinor fields, while $\stackrel{\mathrm{L}}{\Gamma}{ }_{\rho \mu}$ act on vectors and arbitrary tensors. The gauge covariant derivative for a spinor and adjoint spinor is then given by

$$
D_{\mu} \psi=\left(\partial_{\mu}-\Gamma_{\mu}\right) \psi, \quad D_{\mu} \bar{\psi}=\partial_{\mu} \bar{\psi}-\bar{\psi} \Gamma_{\mu} .
$$


The variation of the field Lagrangian is

$$
\delta \mathcal{L}_{\text {field }}=\bar{\psi}\left(\delta \gamma^{\mu} D_{\mu}+\gamma^{\mu} \delta \Gamma_{\mu}\right) \psi
$$

We know that the Dirac gamma matrices are covariantly vanishing, so

$$
D_{\kappa} \gamma_{\iota}=\partial_{\kappa} \gamma_{\iota}-\Gamma_{\iota \kappa}^{\mu} \gamma_{\mu}+\left[\gamma_{\iota}, \widehat{\Gamma}_{\kappa}\right]=0
$$

The $4 \times 4$ matrices $\widehat{\Gamma}_{\kappa}$ are real matrices used to induce similarity transformations on quantities with spinor transformation [87] properties according to

$$
\gamma_{i}^{\prime}=\widehat{\Gamma}^{-1} \gamma_{i} \widehat{\Gamma}
$$

Solving for $\widehat{\Gamma}_{\kappa}$ leads to,

$$
\widehat{\Gamma}_{\kappa}=\frac{1}{8}\left[\left(\partial_{\kappa} \gamma_{\iota}\right) \gamma^{\iota}-\Gamma_{\iota \kappa}^{\mu} \gamma_{\mu} \gamma^{\iota}\right]
$$

Taking the variation of $\widehat{\Gamma}_{\kappa}$,

$$
\begin{aligned}
\delta \widehat{\Gamma}_{\kappa} & =\frac{1}{8}\left[\begin{array}{c}
\left(\partial_{\kappa} \delta \gamma_{\iota}\right) \gamma^{\iota}+\left(\partial_{\kappa} \gamma_{\iota}\right) \delta \gamma^{\iota}-\left(\delta \Gamma^{\mu}{ }_{\iota \kappa}\right) \gamma_{\mu} \gamma^{\iota} \\
-\Gamma^{\mu}{ }_{\iota \kappa}\left(\left(\delta \gamma_{\mu}\right) \gamma^{\iota}+\gamma_{\mu} \delta \gamma^{\prime}\right)
\end{array}\right] \\
& =\frac{1}{8}\left[\left(\partial_{\kappa} \delta \gamma_{\iota}\right) \gamma^{\iota}-\left(\delta \Gamma^{\mu}{ }_{\iota \kappa}\right) \gamma_{\mu} \gamma^{\iota}\right] .
\end{aligned}
$$

Since we require the anticommutator condition on the gamma matrices $\left\{\gamma^{\mu}, \gamma^{\nu}\right\}=2 g^{\mu \nu}$ to hold, the variation of the metric gives

$$
2 \delta g^{\mu \nu}=\left\{\delta \gamma^{\mu}, \gamma^{\nu}\right\}+\left\{\gamma^{\mu} \delta \gamma^{\nu}\right\}
$$

One solution to this equation is,

$$
\delta \gamma^{\nu}=\frac{1}{2} \gamma_{\sigma} \delta \gamma^{\sigma \nu}
$$

With the aid of this result, we can write

$$
\left(\partial_{\kappa} \delta \gamma_{\iota}\right) \gamma^{\iota}=\frac{1}{2} \partial_{\kappa}\left(\gamma^{\nu} \delta g_{\nu \iota}\right) \gamma^{\iota}
$$

Finally, exploiting the anti-symmetry in $\gamma_{\mu \nu}$ we obtain

$$
\delta \widehat{\Gamma}_{\kappa}=\frac{1}{8}\left[g_{\nu \sigma} \delta \Gamma_{\mu \kappa}{ }^{\sigma}-g_{\mu \sigma} \delta \Gamma_{\nu \kappa}^{\sigma}\right] \gamma^{\mu \nu} .
$$

The field Lagrangian defined in the Einstein-Cartan space-time can be written [78, 156, 312, 314, 504] explicitly in terms of its Lorentzian and contorsion components as

$$
\mathcal{L}_{\text {field }}=\frac{1}{2}\left[\left(\stackrel{\mathrm{L}}{D}_{\mu} \bar{\psi}\right) \gamma^{\mu} \psi-\bar{\psi} \gamma^{\mu} D_{\mu}^{\mathrm{L}} \psi\right]-\frac{\hbar c}{8} K_{\mu \alpha \beta} \bar{\psi}\left\{\gamma^{\mu}, \gamma^{\alpha \beta}\right\} \psi
$$

Using the following relations

$$
\left\{\begin{array}{c}
-\frac{1}{4} K_{\mu \alpha \beta} \bar{\psi}\left\{\gamma^{\mu}, \gamma^{\alpha \beta}\right\} \psi=\frac{1}{4} K_{\mu \alpha \beta} \bar{\psi} \gamma^{\beta \alpha} \gamma^{\mu} \psi-\frac{1}{4} K_{\mu \alpha \beta} \bar{\psi} \gamma^{\mu} \gamma^{\alpha \beta} \psi \\
\gamma^{\mu} \gamma^{\nu} \gamma^{\lambda} \varepsilon_{\mu \nu \lambda \sigma}=\left\{\gamma^{\mu}, \gamma^{\nu \lambda}\right\} \varepsilon_{\mu \nu \lambda \sigma}=3 ! \gamma_{\sigma} \gamma_{5}, \\
\left\{\gamma^{\mu}, \gamma^{\nu \lambda}\right\}=\gamma^{[\mu} \gamma^{\nu} \gamma^{\lambda]}
\end{array}\right.
$$

we obtain

$$
K_{\mu \alpha \beta} \bar{\psi}\left\{\gamma^{\mu}, \gamma^{\alpha \beta}\right\} \psi=\frac{1}{2 i} K_{\mu \alpha \beta} \varepsilon^{\alpha \beta \mu \nu}\left(\bar{\psi} \gamma_{5} \gamma_{\nu} \psi\right)
$$


Here we define the contorsion axial vector

$$
K_{\nu}:=\frac{1}{3 !} \varepsilon^{\alpha \beta \mu \nu} K_{\alpha \beta \mu} .
$$

Multiplying through by the axial current $j_{\nu}^{5}=\bar{\psi} \gamma_{5} \gamma_{\nu} \psi$, we obtain

$$
\left(\bar{\psi} \gamma_{5} \gamma_{\nu} \psi\right) \varepsilon^{\alpha \beta \mu \nu} K_{\mu \alpha \beta}=-6 i j_{\nu}^{5} K^{\nu} .
$$

Thus, the field Lagrangian density becomes

$$
\mathcal{L}_{\text {field }}=\frac{1}{2}\left[\left(\stackrel{\mathrm{L}}{D}_{\mu} \bar{\psi}\right) \gamma^{\mu} \psi-\bar{\psi} \gamma^{\mu}{\left.\stackrel{\mathrm{L}}{D_{\mu}} \psi\right]}+\frac{3 i \hbar c}{8} K_{\mu} j_{5}^{\mu} .\right.
$$

The total action reads

$$
\begin{aligned}
\delta I & =\delta \int \mathcal{L}_{G} \sqrt{-g} d^{4} x+\delta \int \mathcal{L}_{\text {field }} \sqrt{-g} d^{4} x \\
& =\int\left(\delta \mathcal{L}_{G}+\delta \mathcal{L}_{\text {field }}\right) \sqrt{-g} d^{4} x .
\end{aligned}
$$

Writing the metric in terms of the tetrads $g^{\mu \nu}=V_{i}^{\mu} V^{\nu i}$, we observe

$$
\delta \sqrt{-g}=-\frac{1}{2} \sqrt{-g}\left(\delta V_{i}^{\mu} V_{\mu}^{i}+V_{\nu i} \delta V^{\nu i}\right) .
$$

By using

$$
\delta V^{\nu i}=\delta\left(\eta^{i j} V_{j}^{\nu}\right)=\eta^{i j} \delta V_{j}^{\nu}
$$

we are able to deduce

$$
\delta \sqrt{-g}=-\sqrt{-g} V_{\mu}^{i} \delta V_{i}^{\mu} .
$$

For the variation of the Ricci tensor $R_{i \nu}=V_{i}{ }^{\mu} R_{\mu \nu}$ we have

$$
\delta \stackrel{\mathrm{L}}{R_{i \nu}}=\delta V_{i}^{\mu} \stackrel{\mathrm{L}}{R}_{\mu \nu}+V_{i}^{\mu} \delta \stackrel{\mathrm{L}}{R}_{\mu \nu} .
$$

In an inertial frame, the Ricci tensor reduces to

$$
\stackrel{\mathrm{L}}{R}_{\mu \nu}=\partial_{\nu} \stackrel{\mathrm{L}}{\Gamma}_{\beta \mu}^{\beta}-\partial_{\beta} \stackrel{\mathrm{L}}{\Gamma}_{\nu \mu}^{\beta},
$$

so that

$$
\delta \stackrel{\mathrm{L}}{R}_{i \nu}=\delta V_{i}^{\mu} \stackrel{\mathrm{L}}{R}_{\mu \nu}+V_{i}^{\mu}\left(\partial_{\nu} \delta \stackrel{\mathrm{L}}{\beta}_{\beta \mu}^{\beta}-\partial_{\beta} \delta \Gamma_{\nu \mu}^{\mathrm{L}}\right) .
$$

The second term can be converted into a surface term, so it may be ignored. Collecting our results, we have

$$
\left\{\begin{array}{c}
\delta g^{\mu \nu}=-g^{\mu \rho} g^{\nu \sigma} \delta g_{\rho \sigma}, \\
\delta \sqrt{-g}=-\frac{1}{2} \sqrt{-g} g_{\mu \nu} \delta g^{\mu \nu}=-\sqrt{-g} V_{\mu}{ }^{i} \delta V_{i}^{\mu}, \\
\delta R_{\mu \nu}=g_{\rho \mu}\left(\nabla_{\lambda} \delta \Gamma^{\lambda \rho}{ }_{\nu}-\nabla_{\nu} \delta \Gamma^{\lambda \rho}{ }_{\lambda}\right)+T_{\lambda \mu}{ }^{\rho} \delta \Gamma^{\lambda}{ }_{\rho \nu}, \delta R_{i \nu}=\delta V_{i}{ }^{\mu} \stackrel{\mathrm{L}}{R}_{\mu \nu} \\
\delta R=\stackrel{\mathrm{L}}{R}^{\mu \nu} \delta g_{\mu \nu}+g^{\mu \nu}\left(\nabla_{\lambda} \delta \Gamma^{\mathrm{L}}{ }_{\mu \nu}-\nabla_{\nu} \delta \Gamma^{\mathrm{L}}{ }_{\mu \lambda}\right)-T_{a}{ }^{b c} \delta K_{b c}{ }^{a} .
\end{array}\right.
$$

From the above results, we obtain

$$
\delta I_{G}=\frac{1}{16 \pi} \int\left[\begin{array}{c}
\left(R_{i}^{\mu}-\frac{1}{2} V_{i}^{\mu} R-V_{i}^{\mu} \Lambda\right) \delta V_{\mu}^{i}+2 g^{\rho \lambda} T_{\mu \lambda}{ }^{\sigma} \delta \Gamma_{\rho \sigma}^{\mu} \\
+g^{\mu \nu}\left(\nabla_{\lambda} \delta \Gamma_{\mu \nu}^{\lambda}{ }_{\mu \nu}{ }^{\mathrm{L}}{ }^{\lambda}{ }_{\mu \lambda}\right)^{\prime}
\end{array}\right] \sqrt{-g} d^{4} x
$$


The last term in the action can be converted into a surface term, so it may be ignored. Using the four-current $v^{\mu}$ introduced earlier, the action for the matter fields read [87]

$$
\begin{aligned}
\delta I_{\text {field }} & =\int\left[\bar{\psi} \delta \gamma^{\mu} \nabla_{\mu} \psi+\bar{\psi} \gamma^{\mu} \delta \widehat{\Gamma}_{\mu} \psi\right] \sqrt{-g} d^{4} x \\
& \left.=\int\left\{\begin{array}{c}
{\left[\frac{1}{2} g^{\mu \nu} \bar{\psi} \gamma_{i}\left(\nabla_{\nu} \psi\right)+T^{\mu}{ }_{\rho \sigma} T_{i}{ }^{\rho \sigma}-\delta_{i}^{\mu} T_{\lambda \rho \sigma} T^{\lambda \rho \sigma}\right] \delta V_{\mu}^{i}} \\
+\frac{1}{8}\left(g^{\rho \nu} v^{\mu}-g^{\rho \mu} v^{\nu}\right)\left(g_{\mu \sigma} \delta \Gamma^{{ }^{\sigma}}{ }_{\nu \rho}-g_{\nu \sigma} \delta \Gamma^{\sigma}{ }_{\mu \rho}\right.
\end{array}\right)\right\} \sqrt{-g} d^{4} x .
\end{aligned}
$$

Removing the derivatives of variations of the metric appearing in $\delta \Gamma^{\sigma}{ }_{\nu \rho}$ via partial integration, and equating to zero the coefficients of $\delta g^{\mu \nu}$ and $\delta T^{\sigma}{ }_{\nu \rho}$ in the variation of the action integral, we obtain

$$
\begin{aligned}
0= & \frac{1}{16 \pi}\left(R_{\mu \nu}-\frac{1}{2} g_{\mu \nu} R-g_{\mu \nu} \Lambda\right)+\left(\frac{1}{2} \bar{\psi} \gamma_{\nu} \nabla_{\mu} \psi-\frac{1}{4} \nabla_{\mu} v_{\nu}\right) \\
& +\nabla_{\sigma} T_{\mu \nu}{ }^{\sigma}+T_{\mu \rho \sigma} T_{\nu}{ }^{\rho \sigma}-g_{\mu \nu} T_{\lambda \rho \sigma} T^{\lambda \rho \sigma}
\end{aligned}
$$

and

$$
T_{\rho \sigma \lambda}=\kappa \tau_{\rho \sigma \lambda} .
$$

Eqs.(3.183) have the form of Einstein equations

$$
G_{\mu \nu}-g_{\mu \nu} \Lambda=\kappa \Sigma_{\mu \nu}
$$

where $\kappa=\frac{8 \pi G_{N}}{c^{4}}$ and the Einstein tensor and non-symmetric energy-momentum tensors are

$$
\begin{gathered}
G_{\mu \nu}=R_{\mu \nu}-\frac{1}{2} g_{\mu \nu} R, \\
\Sigma_{\mu \nu}=\Theta_{\mu \nu}+\mathfrak{T}_{\mu \nu},
\end{gathered}
$$

respectively 6 . Here we identify $\Theta_{\mu \nu}$ as the canonical energy-momentum

$$
\Theta^{\mu}{ }_{\nu}=\frac{\partial \mathcal{L}_{\text {field }}}{\partial\left(\nabla_{\mu} \chi\right)} \nabla_{\nu} \chi-\delta^{\mu}{ }_{\nu} \mathcal{L}_{\text {field }},
$$

while $\mathfrak{T}_{\mu \nu}$ is the stress-tensor form of the non-Riemannian manifold. For the case of spinor fields being considered here the explicit form of the energy-momentum components [503] are (after symmetrization of corresponding canonical source terms in the Einstein equation),

$$
\Theta_{\mu \nu}=-\left[\bar{\psi} \gamma_{\mu} \nabla_{\nu} \psi-\left(\nabla_{\nu} \bar{\psi}\right) \gamma_{\mu} \psi+\bar{\psi} \gamma_{\nu} \nabla_{\mu} \psi-\left(\nabla_{\mu} \bar{\psi}\right) \gamma_{\nu} \psi\right]
$$

and by using the second field equation (3.184), we determine

$$
\mathfrak{T}_{\mu \nu}=\nabla_{\sigma} T_{\mu \nu}{ }^{\sigma}+T_{\mu \rho \sigma} \tau_{\nu}{ }^{\rho \sigma}-g_{\mu \nu} T_{\lambda \rho \sigma} \tau^{\lambda \rho \sigma},
$$

where $\tau_{\mu \nu}{ }^{\sigma}$ is the so-called spin - energy potential [312, 314]

$$
\tau_{\mu \nu}{ }^{\sigma}:=\frac{\partial \mathcal{L}_{\text {field }}}{\partial\left(\nabla_{\sigma} \chi\right)} \gamma_{\mu \nu} \chi
$$

Explicitly, the spin energy potential reads $\tau^{\mu \nu \sigma}=\bar{\psi} \gamma^{[\mu} \gamma^{\nu} \gamma^{\sigma]} \psi$. The equation of motion obtained from the variation of the action with respect to $\bar{\psi}$ reads $[\underline{312}, \underline{314}]$

$$
\gamma^{\mu} \nabla_{\mu} \psi+\frac{3}{8} T_{\mu \nu \sigma} \gamma^{[\mu} \gamma^{\nu} \gamma^{\sigma]} \psi=0
$$

\footnotetext{
${ }^{6}$ We will deduce below these some equations in the framework of GR.
} 
It is interesting to observe that this generalized curved space-time Dirac equation can be recast into the nonlinear equation of the Heisenberg-Pauli type

$$
\gamma^{\mu} \nabla_{\mu} \psi+\frac{3}{8} \varepsilon\left(\bar{\psi} \gamma^{\mu} \gamma_{5} \psi\right) \gamma_{\mu} \gamma_{5} \psi=0
$$

Although the gravitational field equation is similar in form to the Einstein field equation, it differs from the original Einstein equations because the curvature tensor, containing space-time torsion, is non-Riemannian. Assuming that the Euler-Lagrange equations for the matter fields are satisfied, we obtain the following conservation laws for the angular - momentum and energy - momentum

$$
\begin{gathered}
V_{i}^{\mu} V_{j}^{\nu} \Sigma_{[\mu \nu]}=\nabla_{\nu} \tau_{i j}{ }^{\nu} \\
V_{\mu}{ }^{k} \nabla_{\nu} \Sigma_{\kappa}^{\nu}{ }_{\kappa}=\Sigma^{\nu}{ }_{\kappa} T_{\mu \nu}^{k}+\tau^{\nu}{ }_{i j} R^{i j}{ }_{\mu \nu} .
\end{gathered}
$$

\section{H. Concluding remarks}

In this section we have shown how all the necessary ingredients for a theory of gravitation can be obtained from a gauge theory of local Poincaré symmetry. In fact gauge fields can be obtained by requiring the invariance of the Lagrangian density under local Poincaré invariance. The resulting gravity theory describes a space endowed with non-vanishing curvature and torsion. The simplest prototype of this approach is the Einstein-Cartan Theory which is the lowest order gravitational action linear in the curvature scalar and quadratic in torsion. This scheme, as will see in the second part of this Report, can be extended to more general gravitational theories as the ETGs. The Dirac spinors can be introduced as matter sources which couple with gravity via the torsion stress-tensor $\mathfrak{T}_{\mu \nu}$ which is a component of the total energy- momentum $\Sigma_{\mu \nu}$.

In view of the structure of the generalized energy-momentum-tensor, we remark that the gravitational field equations here obtained are of Yang-Mills type. It is worth noticing that the torsion tensor plays the role of the Yang-Mills field strength. Beside this gauge approach, we want to show now that gravitational field can be recovered also by space-time deformations. In some sense, this approach is more general and it is directly related to one of the main features that will be widely adopted in ETGs: the conformal transformations.

\section{DEFORMATIONS AND CONFORMAL TRANSFORMATIONS}

To complete the above considerations, it is worth noticing that also space-time deformations can be related to the generation of gravitational field. In this section, we develop the space-time deformation formalism considering the main quantities which can be related to gravity. The issue to consider a general way to deform the space-time metrics is not new. It has been posed in different ways and is related to several physical problems ranging from the spontaneous symmetry breaking of unification theories up to gravitational waves, considered as space-time perturbations. In cosmology, for example, one faces the problem to describe an observationally lumpy Universe at small scales which becomes isotropic and homogeneous at very large scales according to the Cosmological Principle. In this context, it is crucial to find a way to connect background and locally perturbed metrics [241]. For example, McVittie [402] considered a metric which behaves as a Schwarzschild one at short ranges and as a Friedmann-Lemaitre-RobertsonWalker metric at very large scales. Gautreau [290] calculated the metric generated by a Schwarzschild mass embedded in a Friedmann cosmological fluid trying to address the same problem. On the other hand, the post-Newtonian parameterization, as a standard, can be considered as a deformation of a background, asymptotically flat Minkowski metric. In general, the deformation problem has been explicitly posed by Coll and collaborators [187, 188, 388] who conjectured the possibility to obtain any metric from the deformation of a space-time with constant curvature. The problem was solved only for 3-dimensional spaces but a straightforward extension should be to achieve the same result for space-times of any dimension.

In principle, new exact solutions of the Einstein field equations can be obtained by studying perturbations. In particular, dealing with perturbations as Lorentz matrices of scalar fields $\Phi_{C}^{A}$ reveals particularly useful. Firstly they transform as scalars with respect the coordinate transformations. Secondly, they are dimensionless and, in each point, the matrix $\Phi_{C}^{A}$ behaves as the element of a group. As we shall see below, such an approach can be related to the conformal transformations giving an "extended" interpretation and a straightforward physical meaning of them (see [16, 376] and references therein for a comprehensive review). Furthermore scalar fields related to space-time deformations have a straightforward physical interpretation which could contribute to explain several fundamental issues as the Higgs mechanism in unification theories, the inflation in cosmology and other pictures where scalar 
fields play a fundamental role in dynamics. In this section, we are going to discuss the properties of the deforming matrices $\Phi^{A}{ }_{C}$ and we will derive, the field equations for them, showing how them can parameterize the deformed metrics, according to the boundary and initial conditions and to the energy-momentum tensor. The layout is the following, we define the space-time perturbations in the framework of the metric formalism giving the notion of first and second deformation matrices. In particular, we discuss how deformation matrices can be split in their trace, traceless and skew parts. We derive the contributions of deformation to the geodesic equation and, starting from the Riemann curvature tensor, the general equation of deformations. We discuss the notion of linear perturbations under the standard of deformations. In particular, we recast the equation of propagating perturbations as the equation of propagating perturbations as the equation of gravitational waves and the transverse traceless gauge under the standard of deformations. After we discuss the action of deformations on the Killing vectors. The result consists in achieving a notion of approximate symmetry.

\section{A. Space-time deformations}

In order to start our considerations, let us take into account a metric $\mathbf{g}$ on a space-time manifold $\mathcal{M}$. We can decompose it by a co-tetrad field $\omega^{A}(x)$. Here capital Latin indices are for tetrad fields and we are assuming the most general context to develope our considerations. The metric is

$$
\mathbf{g}=\eta_{A B} \omega^{A} \omega^{B} .
$$

Let us define now a new tetrad field $\widetilde{\omega}=\Phi_{C}^{A}(x) \omega^{C}$, with $\Phi_{C}^{A}(x)$ a matrix of scalar fields. Finally we introduce a space-time $\widetilde{\mathcal{M}}$ with the metric $\widetilde{g}$ defined in the following way

$$
\widetilde{\mathbf{g}}=\eta_{A B} \Phi_{C}^{A} \Phi_{D}^{B} \omega^{C} \omega^{D}=\gamma_{C D}(x) \omega^{C} \omega^{D},
$$

where also $\gamma_{C D}(x)$ is a matrix of fields which are scalars with respect to the coordinate transformations.

If $\Phi_{C}^{A}(x)$ is a Lorentz matrix in any point of $\mathcal{M}$, then

$$
\widetilde{g} \equiv g
$$

otherwise we say that $\widetilde{g}$ is a deformation of $g$ and $\widetilde{\mathcal{M}}$ is a deformed $\mathcal{M}$. If all the functions of $\Phi^{A}{ }_{C}(x)$ are continuous, then there is a one - to - one correspondence between the points of $\mathcal{M}$ and the points of $\widetilde{\mathcal{M}}$.

In particular, if $\xi$ is a Killing vector for $g$ on $\mathcal{M}$, the corresponding vector $\widetilde{\xi}$ on $\widetilde{\mathcal{M}}$ could not necessarily be a Killing vector.

A particular subset of these deformation matrices is given by

$$
\Phi_{C}^{A}(x)=\Omega(x) \delta^{A}{ }_{C}
$$

which define conformal transformations of the metric,

$$
\widetilde{g}=\Omega^{2}(x) g .
$$

In this sense, the deformations defined by Eq. (4.2) can be regarded as a generalization of the conformal transformations which will be discussed in details in the next part of this Report.

We call the matrices $\Phi_{C}^{A}(x)$ first deformation matrices, while we can refer to

$$
\gamma_{C D}(x)=\eta_{A B} \Phi_{C}^{A}(x) \Phi^{B}{ }_{D}(x) .
$$

as the second deformation matrices, which, as seen above, are also matrices of scalar fields. They generalize the Minkowski matrix $\eta_{A B}$ with constant elements in the definition of the metric. A further restriction on the matrices $\Phi^{A}{ }_{C}$ comes from the theorem proved by Riemann by which an $n$-dimensional metric has $n(n-1) / 2$ degrees of freedom (see [188] for details). With this definitions in mind, let us consider the main properties of deforming matrices.

\section{B. Properties of deforming matrices}

Let us take into account a four dimensional space-time with Lorentzian signature. A family of matrices $\Phi_{C}^{A}(x)$ such that

$$
\Phi^{A}{ }_{C}(x) \in G L(4) \forall x,
$$


are defined on such a space-time.

These functions are not necessarily continuous and can connect space-times with different topologies. A singular scalar field introduces a deformed manifold $\widetilde{\mathcal{M}}$ with a space-time singularity.

As it is well known, the Lorentz matrices $\Lambda_{C}^{A}$ leave the Minkowski metric invariant and then

$$
\mathbf{g}=\eta_{E F} \Lambda^{E}{ }_{A} \Lambda^{F}{ }_{B} \Phi^{A}{ }_{C} \Phi^{B}{ }_{D} \omega^{C} \omega^{D}=\eta_{A B} \Phi^{A}{ }_{C} \Phi^{B}{ }_{D} \omega^{C} \omega^{D} .
$$

It follows that $\Phi_{C}^{A}$ give rise to right cosets of the Lorentz group, i.e. they are the elements of the quotient group $G L(4, \mathbf{R}) / S O(3,1)$. On the other hand, a right-multiplication of $\Phi^{A}{ }_{C}$ by a Lorentz matrix induces a different deformation matrix.

The inverse deformed metric is

$$
\widetilde{g}^{\alpha \beta}=\eta^{C D} \Phi^{-1}{ }_{C}^{A} \Phi^{-1}{ }_{D}^{B} e_{A}^{\alpha} e_{B}^{\beta}
$$

where $\Phi^{-1}{ }_{C}^{A} \Phi^{C}{ }_{B}=\Phi^{A}{ }_{C} \Phi^{-1}{ }_{B}{ }_{B}=\delta_{B}^{A}$.

Let us decompose now the matrix $\Phi_{A B}=\eta_{A C} \Phi^{C}{ }_{B}$ in its symmetric and antisymmetric parts

$$
\Phi_{A B}=\Phi_{(A B)}+\Phi_{[A B]}=\Omega \eta_{A B}+\Theta_{A B}+\varphi_{A B}
$$

where $\Omega=\Phi_{A}^{A}, \Theta_{A B}$ is the traceless symmetric part and $\varphi_{A B}$ is the skew symmetric part of the first deformation matrix respectively. Then standard conformal transformations are nothing else but deformations with $\Theta_{A B}=\varphi_{A B}=0$ [577.

Finding the inverse matrix $\Phi^{-1{ }^{A}}$ in terms of $\Omega, \Theta_{A B}$ and $\varphi_{A B}$ is not immediate, but as above, it can be split in the three terms

$$
\Phi^{-1^{A}}=\alpha \delta_{C}^{A}+\Psi_{C}^{A}+\Sigma_{C}^{A}
$$

where $\alpha, \Psi_{C}^{A}$ and $\Sigma_{C}^{A}$ are respectively the trace, the traceless symmetric part and the antisymmetric part of the inverse deformation matrix. The second deformation matrix, from the above decomposition, takes the form

$$
\gamma_{A B}=\eta_{C D}\left(\Omega \delta_{A}^{C}+\Theta_{A}^{C}+\varphi_{A}^{C}\right)\left(\Omega \delta_{B}^{D}+\Theta_{B}^{D}+\varphi_{B}^{D}\right)
$$

and then

$$
\begin{aligned}
\gamma_{A B}=\Omega^{2} \eta_{A B} & +2 \Omega \Theta_{A B}+\eta_{C D} \Theta^{C}{ }_{A} \Theta_{B}^{D}+\eta_{C D}\left(\Theta^{C}{ }_{A} \varphi^{D}{ }_{B}\right. \\
& \left.+\varphi^{C}{ }_{A} \Theta^{D}{ }_{B}\right)+\eta_{C D} \varphi^{C}{ }_{A} \varphi_{B}^{D} .
\end{aligned}
$$

In general, the deformed metric can be split as

$$
\tilde{g}_{\alpha \beta}=\Omega^{2} g_{\alpha \beta}+\gamma_{\alpha \beta}
$$

where

$$
\gamma_{\alpha \beta}=\left(2 \Omega \Theta_{A B}+\eta_{C D} \Theta^{C}{ }_{A} \Theta^{D}{ }_{B}+\eta_{C D}\left(\Theta^{C}{ }_{A} \varphi^{D}{ }_{B}+\varphi^{C}{ }_{A} \Theta^{D}{ }_{B}\right)+\eta_{C D} \varphi^{C}{ }_{A} \varphi^{D}{ }_{B}\right) \omega_{\alpha}^{A} \omega_{\beta}^{B}
$$

In particular, if $\Theta_{A B}=0$, the deformed metric simplifies to

$$
\widetilde{g}_{\alpha \beta}=\Omega^{2} g_{\alpha \beta}+\eta_{C D} \varphi_{A}^{C} \varphi^{D}{ }_{B} \omega^{A}{ }_{\alpha} \omega^{B}{ }_{\beta}
$$

and, if $\Omega=1$, the deformation of a metric consists in adding to the background metric a tensor $\gamma_{\alpha \beta}$. We have to remember that all these quantities are not independent as, by the theorem mentioned in [188], they have to form at most six independent functions in a four dimensional space-time.

Similarly the controvariant deformed metric can be always decomposed in the following way

$$
\widetilde{g}^{\alpha \beta}=\alpha^{2} g^{\alpha \beta}+\lambda^{\alpha \beta}
$$

Let us find the relation between $\gamma_{\alpha \beta}$ and $\lambda^{\alpha \beta}$. By using $\widetilde{g}_{\alpha \beta} \widetilde{g}^{\beta \gamma}=\delta_{\alpha}^{\gamma}$, we obtain

$$
\alpha^{2} \Omega^{2} \delta_{\alpha}^{\gamma}+\alpha^{2} \gamma_{\alpha}^{\gamma}+\Omega^{2} \lambda_{\alpha}^{\gamma}+\gamma_{\alpha \beta} \lambda^{\beta \gamma}=\delta_{\alpha}^{\gamma}
$$


if the deformations are conformal transformations, we have $\alpha=\Omega^{-1}$, so assuming such a condition, one obtain the following matrix equation

$$
\alpha^{2} \gamma_{\alpha}^{\gamma}+\Omega^{2} \lambda_{\alpha}^{\gamma}+\gamma_{\alpha \beta} \lambda^{\beta \gamma}=0
$$

and

$$
\left(\delta_{\alpha}^{\beta}+\Omega^{-2} \gamma_{\alpha}^{\beta}\right) \lambda_{\beta}^{\gamma}=-\Omega^{-4} \gamma_{\alpha}^{\gamma}
$$

and finally

$$
\lambda_{\beta}^{\gamma}=-\Omega^{-4}\left(\delta+\Omega^{-2} \gamma\right)_{\beta}^{-1 \alpha} \gamma_{\alpha}^{\gamma}
$$

where $\left(\delta+\Omega^{-2} \gamma\right)^{-1}$ is the inverse tensor of $\left(\delta_{\alpha}^{\beta}+\Omega^{-2} \gamma_{\alpha}^{\beta}\right)$.

To each matrix $\Phi^{A}{ }_{B}$, we can associate a $(1,1)$-tensor $\phi^{\alpha}{ }_{\beta}$ defined by

$$
\phi_{\beta}^{\alpha}=\Phi_{B}^{A} \omega_{\beta}^{B} e_{A}^{\alpha}
$$

such that

$$
\widetilde{g}_{\alpha \beta}=g_{\gamma \delta} \phi_{\alpha}^{\gamma} \phi_{\beta}^{\delta}
$$

which can be decomposed as in Eq. (4.16). Vice-versa from a $(1,1)$-tensor $\phi_{\beta}^{\alpha}$, we can define a matrix of scalar fields as

$$
\phi_{B}^{A}=\phi_{\beta}^{\alpha} \omega_{\alpha}^{A} e_{B}^{\beta}
$$

The Levi Civita connection corresponding to the metric (4.14) is related to the original connection by the relation

$$
\widetilde{\Gamma}_{\alpha \beta}^{\gamma}=\Gamma_{\alpha \beta}^{\gamma}+C_{\alpha \beta}^{\gamma}
$$

(see [577]), where

$$
C_{\alpha \beta}^{\gamma}=2 \widetilde{g}^{\gamma \delta} g_{d(\alpha} \nabla_{\beta)} \Omega-g_{\alpha \beta} \widetilde{g}^{\gamma \delta} \nabla_{\delta} \Omega+\frac{1}{2} \widetilde{g}^{\gamma \delta}\left(\nabla_{\alpha} \gamma_{\delta \beta}+\nabla_{\beta} \gamma_{\alpha \delta}-\nabla_{\delta} \gamma_{\alpha \beta}\right)
$$

Therefore, in a deformed space-time, the connection deformation acts like a force that deviates the test particles from the geodesic motion in the unperturbed space-time. As a matter of fact the geodesic equation for the deformed space-time

$$
\frac{d^{2} x^{\gamma}}{d \lambda^{2}}+\tilde{\Gamma}_{\alpha \beta}^{\gamma} \frac{d x^{\alpha}}{d \lambda} \frac{d x^{\beta}}{d \lambda}=0
$$

becomes

$$
\frac{d^{2} x^{\gamma}}{d \lambda^{2}}+\Gamma_{\alpha \beta}^{\gamma} \frac{d x^{\alpha}}{d \lambda} \frac{d x^{\beta}}{d \lambda}=-C_{\alpha \beta}^{\gamma} \frac{d x^{\alpha}}{d \lambda} \frac{d x^{\beta}}{d \lambda} .
$$

The deformed Riemann curvature tensor is then

$$
\widetilde{R}_{\alpha \beta \gamma}{ }^{\delta}=R_{\alpha \beta \gamma}{ }^{\delta}+\nabla_{\beta} C^{\delta}{ }_{\alpha \gamma}-\nabla_{\alpha} C_{\beta \gamma}^{\delta}+C_{\alpha \gamma}^{\epsilon} C_{\beta \epsilon}^{\delta}-C_{\beta \gamma}^{\epsilon} C_{\alpha \epsilon}^{\delta},
$$

while the deformed Ricci tensor obtained by contraction is

$$
\widetilde{R}_{\alpha \beta}=R_{\alpha \beta}+\nabla_{\delta} C_{\alpha \beta}^{\delta}-\nabla_{\alpha} C_{\delta \beta}^{\delta}+C_{\alpha \beta}^{\epsilon} C_{\delta \epsilon}^{\delta}-C_{\delta \beta}^{\epsilon} C_{\alpha \epsilon}^{\delta}
$$

and the curvature scalar

$$
\widetilde{R}=\widetilde{g}^{\alpha \beta} \widetilde{R}_{\alpha \beta}=\widetilde{g}^{\alpha \beta} R_{\alpha \beta}+\widetilde{g}^{\alpha \beta}\left[\nabla_{\delta} C_{\alpha \beta}^{\delta}-\nabla_{\alpha} C^{\delta}{ }_{\delta \beta}+C^{\epsilon \beta}{ }_{\alpha \beta} C_{\delta \epsilon}-C^{\epsilon \beta} C^{\delta \epsilon}\right]
$$


From the above curvature quantities, we obtain finally the equations for the deformations. In the vacuum case, we simply have

$$
\widetilde{R}_{\alpha \beta}=R_{\alpha \beta}+\nabla_{\delta} C_{\alpha \beta}^{\delta}-\nabla_{\alpha} C_{\delta \beta}^{\delta}+C_{a b}^{e} C_{d e}^{d}-C_{d b}^{e} C_{a e}^{d}=0
$$

where $R_{a b}$ must be regarded as a known function. In presence of matter, we consider the equations

$$
R_{\alpha \beta}+\nabla_{\delta} C_{\alpha \beta}^{\delta}-\nabla_{\alpha} C_{\delta \beta}^{\delta}+C_{\alpha \beta}^{\epsilon} C_{\delta \epsilon}^{\delta}-C_{\delta \beta}^{\epsilon} C_{\alpha \epsilon}^{\delta}=\kappa\left(\widetilde{T}_{\alpha \beta}-\frac{1}{2} \widetilde{g}_{\alpha \beta} \widetilde{T}\right)
$$

These last equations can be improved by considering the field equation:7

$$
R_{\alpha \beta}=\kappa\left(T_{\alpha \beta}-\frac{1}{2} g_{\alpha \beta} T\right)
$$

and then

$$
\nabla_{\delta} C_{\alpha \beta}^{\delta}-\nabla_{\alpha} C_{\delta \beta}^{\delta}+C_{\alpha \beta}^{\epsilon} C_{\delta \epsilon}^{\delta}-C_{\delta \beta}^{\epsilon} C_{\alpha \epsilon}^{\delta}=\kappa\left[\widetilde{T}_{\alpha \beta}-\frac{1}{2} \widetilde{g}_{\alpha \beta} \widetilde{T}-\left(T_{\alpha \beta}-\frac{1}{2} g_{\alpha \beta} T\right)\right]
$$

are the most general equations for deformations.

\section{The propagation of metric deformations}

Metric deformations can be used to describe perturbations. To this aim we can simply consider the deformations

$$
\Phi^{A}{ }_{B}=\delta_{B}^{A}+\varphi^{A}{ }_{B}
$$

with

$$
\left|\varphi_{B}^{A}\right| \ll 1,
$$

together with their derivatives

$$
\left|\partial \varphi_{B}^{A}\right| \ll 1 .
$$

With this approximation, immediately we find the inverse relation

$$
\left(\Phi^{-1}\right)_{B}^{A} \simeq \delta_{B}^{A}-\varphi^{A}{ }_{B} .
$$

As a remarkable example, we have that gravitational waves are generally described, in linear approximation, as perturbations of the Minkowski metric

$$
g_{\alpha \beta}=\eta_{\alpha \beta}+\gamma_{\alpha \beta} .
$$

In our case, we can extend in a covariant way such an approximation. If $\varphi_{A B}$ is an antisymmetric matrix, we have

$$
\widetilde{g}_{\alpha \beta}=g_{\alpha \beta}+\gamma_{\alpha \beta}
$$

where the first order terms in $\varphi^{A}{ }_{B}$ vanish and $\gamma_{\alpha \beta}$ is of second order

$$
\gamma_{\alpha \beta}=\eta_{A B} \varphi_{C}^{A} \varphi^{B}{ }_{D} \omega^{C}{ }_{\alpha} \omega^{D}{ }_{\beta} .
$$

Consequently

$$
\widetilde{g}^{\alpha \beta}=g^{\alpha \beta}+\gamma^{\alpha \beta}
$$

\footnotetext{
7 We will discuss these equations in the next section deriving them from variational principle.
} 
where

$$
\gamma^{\alpha \beta}=\eta^{A B}\left(\varphi^{-1}\right)^{C}{ }_{A}\left(\varphi^{-1}\right)^{D}{ }_{B} e_{C}^{\alpha} e_{D}^{\beta} .
$$

Let us consider the background metric $g_{a b}$, solution of the equations in the vacuum

$$
R_{\alpha \beta}=0 .
$$

We obtain the equation of perturbations considering only the linear terms in Eq. (4.32) and neglecting the contributions of quadratic terms. We find

$$
\widetilde{R}_{\alpha \beta}=\nabla_{\delta} C_{\alpha \beta}^{\delta}-\nabla_{\alpha} C_{\delta \beta}^{\delta}=0,
$$

and, by the explicit form of $C_{\alpha \beta}^{\delta}$, this equation becomes

$$
\left(\nabla_{\delta} \nabla_{\alpha} \gamma_{\beta}^{\delta}+\nabla_{\delta} \nabla_{\beta} \gamma_{\alpha}^{\delta}-\nabla_{\delta} \nabla^{\delta} \gamma_{\alpha \beta}\right)-\left(\nabla_{\alpha} \nabla_{\delta} \gamma_{\beta}^{\delta}+\nabla_{\alpha} \nabla_{\beta} \gamma_{\delta}^{\delta}-\nabla_{\alpha} \nabla^{\delta} \gamma_{\beta}^{\delta}\right)=0 .
$$

Imposing the transverse traceless gauge on $\gamma_{\alpha \beta}$, i.e. the standard gauge conditions

$$
\nabla^{\alpha} \gamma_{\alpha}=0
$$

and

$$
\gamma=\gamma_{\alpha}^{\alpha}=0
$$

Eq. (4.47) reduces to

$$
\nabla_{\beta} \nabla^{\beta} \gamma_{\alpha \gamma}-2 R_{\alpha \gamma}^{\beta}{ }^{\delta} \gamma_{\beta \delta}=0,
$$

see also [577]. In our context, this equation is a linearized equation for deformations and it is straightforward to consider perturbations and, in particular, gravitational waves, as small deformations of the metric. This result can be immediately translated into the above scalar field matrix equations. Note that such an equation can be applied to the conformal part of the deformation, when the general decomposition is considered.

As an example, let us take into account the deformation matrix equations applied to the Minkowski metric, when the deformation matrix assumes the form (4.36). In this case, the Eq. (4.47), become ordinary wave equations for $\gamma_{a b}$. Considering the deformation matrices, these equations become, for a tetrad field of constant vectors,

$$
\partial^{\delta} \partial_{\delta} \varphi^{C}{ }_{A} \varphi_{C B}+2 \partial_{\delta} \varphi^{C}{ }_{A} \partial^{\delta} \varphi_{C B}+\varphi^{C}{ }_{A} \partial^{\delta} \partial_{\delta} \varphi_{C B}=0 .
$$

The above gauge conditions are now

$$
\varphi_{A B} \varphi^{B A}=0
$$

and

$$
e_{D}^{\delta}\left[\partial_{\delta} \varphi_{C A} \varphi_{B}^{C}+\varphi_{C A} \partial_{\delta} \varphi_{B}^{C}\right]=0 .
$$

This result shows that the gravitational waves can be fully recovered starting from the scalar fields which describe the deformations of the metric. In other words, such scalar fields can assume the meaning of gravitational waves modes. We will discuss in details gravitational waves in the next section and in the third part of this Report.

\section{Approximate Killing vectors}

Another important issue which can be addressed starting from space-time deformations is related to the symmetries. In particular, they assume a fundamental role in describing when a symmetry is preserved or broken under the action of a given field. In GR, the Killing vectors are always related to the presence of given space-time symmetries [577].

Let us take an exact solution of the Einstein equations, which satisfies the Killing equation

$$
\left(L_{\xi} g\right)_{\alpha \beta}=0
$$


where $\xi$, being the generator of an infinitesimal coordinate transformation, is a Killing vector. If we take a deformation of the metric with the scalar matrix

$$
\Phi^{A}{ }_{B}=\delta^{A}{ }_{B}+\varphi^{A}{ }_{B}
$$

with

$$
\left|\varphi_{B}^{A}\right| \ll 1,
$$

and

$$
\left(L_{\xi} \widetilde{g}\right)_{\alpha \beta} \neq 0,
$$

being

$$
\left(L_{\xi} e^{A}\right)_{\alpha}=0,
$$

we have

$$
\left(L_{\xi} \varphi\right)^{A}{ }_{B}=\xi^{\alpha} \partial_{\alpha} \varphi^{A}{ }_{B} \neq 0 .
$$

If there is some region $\mathcal{D}$ of the deformed space-time $\mathcal{M}_{\text {deformed }}$ where

$$
\left|\left(L_{\xi} \varphi\right)^{A}{ }_{B}\right| \ll 1
$$

we say that $\xi$ is an approximate Killing vector on $\mathcal{D}$. In other words, these approximate Killing vectors allow to "control" the space-time symmetries under the action of a given deformation. We can calculate the modified connection $\Gamma_{\alpha \beta}^{\hat{\gamma}}$ in many alternative ways. Let us introduce the tetrad $e_{A}$ and cotetrad $\omega^{B}$ satisfying the orthogonality relation

$$
i_{e_{A}} \omega^{B}=\delta_{A}^{B}
$$

and the non-integrability condition (anholonomy)

$$
d \omega^{A}=\frac{1}{2} \Omega_{B C}^{A} \omega^{B} \wedge \omega^{C} .
$$

The corresponding connection is

$$
\Gamma_{B C}^{A}=\frac{1}{2}\left(\Omega_{B C}^{A}-\eta^{A A^{\prime}} \eta_{B B^{\prime}} \Omega_{A^{\prime} C}^{B^{\prime}}-\eta^{A A^{\prime}} \eta_{C C^{\prime}} \Omega_{A^{\prime} B}^{C^{\prime}}\right)
$$

If we deform the metric as in (4.2), we have two alternative ways to write this expression: either writing the "deformation" of the metric in the space of tetrads or "deforming" the tetrad field as in the following expression

$$
\hat{g}=\eta_{A B} \Phi_{C}^{A} \Phi^{B}{ }_{D} \omega^{C} \omega^{D}=\gamma_{A B} \omega^{A} \omega^{B}=\eta_{A B} \hat{\omega}^{A} \hat{\omega}^{B} .
$$

In the first case, the contribution of the Christoffel symbols, constructed by the metric $\gamma_{A B}$, appears

$$
\hat{\Gamma}_{B C}^{A}=\frac{1}{2}\left(\Omega_{B C}^{A}-\gamma^{A A^{\prime}} \gamma_{B B^{\prime}} \Omega_{A^{\prime} C}^{B^{\prime}}-\gamma^{A A^{\prime}} \gamma_{C C^{\prime}} \Omega_{A^{\prime} B}^{C^{\prime}}\right)+\frac{1}{2} \gamma^{A A^{\prime}}\left(i_{e_{C}} d \gamma_{B A^{\prime}}-i_{e_{B}} d \gamma_{C A^{\prime}}-i_{e_{A^{\prime}}} d \gamma_{B C}\right)
$$

In the second case, using (4.62), we can define the new anholonomy objects $\hat{C}_{B C}^{A}$.

$$
d \hat{\omega}^{A}=\frac{1}{2} \hat{\Omega}_{B C}^{A} \hat{\omega}^{B} \wedge \hat{\omega}^{C} .
$$

After some calculations, we have

$$
\hat{\Omega}_{B C}^{A}=\Phi_{E}^{A} \Phi_{B}^{-1 D} \Phi^{-1}{ }_{C} \Omega_{D F}^{E}+2 \Phi_{F}^{A} e_{G}^{a}\left(\Phi^{-1}{ }_{[B} \partial_{a} \Phi^{-1}{ }_{C]}\right)
$$

As we are assuming a constant metric in tetradic space, the deformed connection is

$$
\hat{\Gamma}_{B C}^{A}=\frac{1}{2}\left(\hat{\Omega}_{B C}^{A}-\eta^{A A^{\prime}} \eta_{B B^{\prime}} \hat{\Omega}_{A^{\prime} C}^{B^{\prime}}-\eta^{A A^{\prime}} \eta_{C C^{\prime}} \hat{\Omega}_{A^{\prime} B}^{C^{\prime}}\right) .
$$

Substituting (4.67) in (4.68), the final expression of $\hat{\Gamma}_{B C}^{A}$, as a function of $\Omega_{B C}^{A}, \Phi^{A}{ }_{B}, \Phi^{-1^{D}}{ }_{C}$ and $e_{G}^{\alpha}$ is

$$
\hat{\Gamma}_{A B C}=\Delta_{A B C}^{D E F}\left[\frac{1}{2} \eta_{D G} \Phi_{G^{\prime}}^{G} \Phi_{E}^{-1}{ }^{E^{\prime}} \Phi_{F}^{-1 F^{\prime}} \Omega_{E^{\prime} F^{\prime}}^{G^{\prime}}+\eta_{D K} \Phi^{K}{ }_{H} e_{G}^{\alpha} \Phi_{[E}^{-1}{ }_{[\alpha \mid} \partial_{\mid \alpha]} \Phi_{F]}^{-1}\right]
$$

where

$$
\Delta_{A B C}^{D E F}=\delta_{A}^{D} \delta_{C}^{E} \delta_{B}^{F}-\delta_{B}^{D} \delta_{C}^{E} \delta_{A}^{F}+\delta_{C}^{D} \delta_{A}^{E} \delta_{B}^{F} .
$$




\section{E. Discussion and conclusions}

In this section, we have proposed a novel definition of space-time metric deformations parameterizing them in terms of scalar field matrices. The main result is that deformations can be described as extended conformal transformations. This fact gives a straightforward physical interpretation of conformal transformations: conformally related metrics can be seen as the "background" and the "perturbed" metrics. In other words, the relations between the Jordan frame and the Einstein frame, which we will discuss for ETGs, can be directly interpreted through the action of the deformation matrices contributing to solve the issue of what the true physical frame is [16, 376].

Besides, space-time metric deformations can be immediately recast in terms of perturbation theory allowing a completely covariant approach to the problem of gravitational waves.

Results related to those presented here has been proposed in 187, 188]. There it is shown that any metric in a three dimensional manifold can be decomposed in the form

$$
\tilde{g}_{\alpha \beta}=\sigma(x) h_{\alpha \beta}+\epsilon s_{\alpha} s_{\beta}
$$

where $h_{\alpha \beta}$ is a metric with constant curvature, $\sigma(x)$ is a scalar function, $s_{a}$ is a three-vector and $\epsilon= \pm 1$. A relation has to be imposed between $\sigma$ and $s_{a}$ and then the metric can be defined, at most, by three independent functions.

In a subsequent paper [389], Llosa and Soler showed that (4.71) can be generalized to arbitrary dimensions by the form

$$
\widetilde{g}_{\alpha \beta}=\lambda(x) g_{\alpha \beta}+\mu(x) F_{\alpha \gamma} g^{\gamma \delta} F_{\alpha \beta}
$$

where $g_{a b}$ is a constant curvature metric, $F_{a b}$ is a two-form, $\lambda(x)$ and $\mu(x)$ are two scalar functions. These results are fully recovered and generalized from our approach as soon as the deformation of a constant metric is considered and suitable conditions on the tensor $\Theta_{A B}$ are imposed.

In general, we have shown that, when we turn to the tensor formalism, we can work with arbitrary metrics and arbitrary deforming $\gamma_{\alpha \beta}$ tensors. In principle, by arbitrary deformation matrices, not necessarily real, we can pass from a given metric to any other metric. As an example, a noteworthy result has been achieved by Newman and Janis [432]: They showed that, through a complex coordinate transformation, it is always possible to achieve a Kerr metric from a Schwarzschild one. In our language, this means that a space-time deformation allows to pass from a spherical symmetry to a cylindrical one. In Part 【II of this Report, we will give an example of this approach. Furthermore, it has been shown [37, 38] that three dimensional black hole solutions can be found by identifying 3-dimensional anti-de Sitter space on which acts a discrete subgroup of $S O(2,2)$.

In all these examples, the transformations which lead to the results are considered as "coordinate transformations". We think that this definition is a little bit misleading since one does not covariantly perform the same transformations on all the tensors defined on the manifold. On the other hand, our definition of metric deformations and deformed manifolds can be straightforwardly related to the standard notion of perturbations since, in principle, it works on a given region $\mathcal{D}$ of the deformed space-time (see, for example, [44, 423]).

Going back to the issue proposed in Sec. III]" What can generate gravity?", we can say that space-time deformations, as gauge invariance are intimately related to the gravitational field. In fact, deformation are fully connected to geometric nature of gravitational field but do not specify exactly a single theory. In other words GR is nothing else but a possible "realization" of gravity. This means that such an interaction can be described in a wider context than the Einstein theory. Before facing the ETGs, let us recall the main physical meanings related to GR.

\section{THE PHYSICAL MEANING OF GENERAL RELATIVITY}

GR, as we discussed above, is a theory of gravitation that was developed by Albert Einstein between 1907 and 1915. According to GR, the observed gravitational attraction between masses results from their warping of space and time. Up to the beginning of the 20th century, Newton's law of universal gravitation had been accepted for more than two hundred years as a valid description of the gravitational force between masses. In Newton's model, gravity is the result of an attractive force between massive objects. Although even Newton was bothered by the unknown nature of that force, the basic framework was extremely successful for describing motions. Experiments and observations show that Einstein's description of gravitation accounts for several effects that are unexplained by Newton's law, such as anomalies in the orbits of Mercury and other planets. GR also predicts novel effects of gravity, such as gravitational waves, gravitational lensing and an effect of gravity on time known as gravitational time dilation. Many of these predictions have been confirmed by experiments, while others are the subject of ongoing research. For example, although there is indirect evidence for gravitational waves, direct evidence of their existence is still being sought by several teams of scientists in experiments such as VIRGO, LIGO and GEO 600 [4, 7, 374, 512]. GR has developed 
as an essential tool in modern astrophysics. It provides the foundation for the current understanding of black holes, regions of space where gravitational attraction is so strong that light can not escape. Their strong gravity is thought to be responsible for the intense radiation emitted by certain types of astronomical objects (such as active galactic nuclei or quasars). GR is also part of the framework of the standard Big Bang model of cosmology. Although GR is not the only relativistic theory of gravity, it is the simplest theory that is consistent with the experimental data. Nevertheless, a number of open questions remain, the most fundamental of which is how GR can be reconciled with the laws of quantum physics to produce a complete and self-consistent theory of quantum gravity. In this section we discuss the physical foundation of GR outlining some of the issues that must be recovered in any relativistic theory of gravity, in particular the ETGs.

\section{A. The Equivalence Principle}

Equivalence principle is the physical foundation of any metric theory of gravity [113, $[586]$ and the starting point of our considerations.

The first formulation of Equivalence principle comes out from the theory of gravitation formulates by Galileo and Newton; it is called the Weak Equivalence Principle and it states that the "inertial mass" $m$ and the "gravitational mass" $M$ of any object are equivalent. In Newtonian physics, the "inertial mass" $m$ is a coefficient which appears in the second Newton law: $\vec{F}=m \vec{a}$ where $\vec{F}$ is the force exerted on a mass $m$ with acceleration $\vec{a}$; in Special Relativity (without gravitation) the "inertial mass" of a body appears to be proportional to the rest energy of the body: $E=m c^{2}$. Considering the Newtonian gravitational attraction, one introduces the "gravitational mass" $M$ : the gravitational attraction force between two bodies of "gravitational mass" $M$ and $M^{\prime}$ is $F=G_{N} M M^{\prime} / r^{2}$ where $G_{N}$ is the Newtonian gravitational constant and $r$ the distance between the two bodies. Various experiments [550] demonstrate that $m \equiv M$. The present accuracy of this relation in laboratory is of the order of $10^{-13}$; spatial projects are currently designed to achieve precision of $10^{-15}$ [324] and $10^{-18}$ [325].

The Weak Equivalence Principle statement implies that it is impossible to distinguish between the effects of a gravitational field from those experienced in uniformly accelerated frames, using the simple observation of the freefalling particles behavior. The Weak Equivalence Principle can be formulated again in the following statement [586]:

If an uncharged test body is placed at an initial event in space-time and given an initial velocity there, then its subsequent trajectory will be independent of its internal structure and composition.

A generalization of Weak Equivalence Principle claims that the Special Relativity is only locally valid. It has been achieved by Einstein after the formulation of Special Relativity theory where the concept of mass looses some of its uniqueness: the mass is reduced to a manifestation of energy and momentum. According to Einstein, it is impossible to distinguish between uniform acceleration and an external gravitational field, not only for free-falling particles but whatever is the experiment. This equivalence principle has been called Einstein Equivalence Principle ; its main statements are the following [586]:

- Weak Equivalence Principle is valid;

- the outcome of any local non-gravitational test experiment is independent of velocity of free-falling apparatus;

- the outcome of any local non-gravitational test experiment is independent of where and when in the Universe it is performed.

One defines as "local non-gravitational experiment" an experiment performed in a small-size 8 freely falling laboratory. From the Einstein Equivalence Principle, one gets that the gravitational interaction depends on the curvature of space-time, i.e. the postulates of any metric theory of gravity have to be satisfied [586]:

- space-time is endowed with a metric $g_{\mu \nu}$;

- the world lines of test bodies are geodesics of the metric;

- in local freely falling frames, called local Lorentz frames, the non-gravitational laws of physics are those of Special Relativity.

\footnotetext{
${ }^{8}$ In order to avoid the inhomogeneities.
} 
One of the predictions of this principle is the gravitational red-shift, experimentally verified by Pound and Rebka in 1960 [479]. It is worth stressing that gravitational interactions are specifically excluded from Weak Equivalence Principle and Einstein Equivalence Principle. In order to classify alternative theories of gravity, the Gravitational Weak Equivalence Principle and the Strong Equivalence Principle has to be introduced. The Strong Equivalence Principle extends the Einstein Equivalence Principle by including all the laws of physics in its terms [586]:

- Weak Equivalence Principle is valid for self-gravitating bodies as well as for test bodies (Gravitational Weak Equivalence Principle);

- the outcome of any local test experiment is independent of the velocity of the free-falling apparatus;

- the outcome of any local test experiment is independent of where and when in the Universe it is performed.

Therefore, the Strong Equivalence Principle contains the Einstein Equivalence Principle, when gravitational forces are ignored. Many authors claim that the only theory coherent with the Strong Equivalence Principle is GR. An extremely important issue related to the consistency of Equivalence Principle with respect to the Quantum Mechanics. Some phenomena, like neutrino oscillations could violate it if induced by the gravitational field. In Part III of this Report, we discuss possible neutrino oscillation effects induced by corrections to GR.

\section{B. The geodesic structure}

In GR the space-time metric is related to geodesic motion because the Equivalence Principle requires that the motion of a point-like body in free fall be described by the geodesic equation. The latter can be derived from the variational principle

$$
\delta S=\delta \int_{A}^{B} d s=0
$$

where $d s$ is the line element and $A$ and $B$ are the initial and final points along the space-time trajectory, respectively. It is instructive to deduce the geodesic equation in order to show how it is related to the metric structure (i.e. the casual structure) of GR.

The line element is written as

$$
d s=\left|g_{\alpha \beta} d x^{\alpha} d x^{\beta}\right|^{1 / 2}=\left|g_{\alpha \beta} \frac{d x^{\alpha}}{d s} \frac{d x^{\beta}}{d s}\right|^{1 / 2} d s,
$$

with $s$ playing the role of an affine parameter, and from which it follows that

$$
g_{\alpha \beta} u^{\alpha} u^{\beta}=-1,
$$

where $u^{\alpha}=\frac{d x^{\alpha}}{d s}$ is the four-velocity of the particle. Substitution into Eq. (5.1) yields

$$
\delta S=\delta \int_{A}^{B}\left|g_{\alpha \beta} \frac{d x^{\alpha}}{d s} \frac{d x^{\beta}}{d s}\right|^{1 / 2} d s=0 .
$$

By performing this variation, one obtains

$$
\delta S=\int_{A}^{B} \frac{1}{2 \sqrt{\left|g_{\alpha \beta} u^{\alpha} u^{\beta}\right|}}\left[g_{\alpha \beta, \lambda} \delta x^{\lambda} \frac{d x^{\alpha}}{d s} \frac{d x^{\beta}}{d s}+2 g_{\alpha \beta} \frac{d}{d s}\left(\delta x^{\alpha}\right) \frac{d x^{\beta}}{d s}\right] d s=0 .
$$

The second term in square brackets is $g_{\alpha \beta} \delta\left(\frac{d x^{\alpha}}{d s} \frac{d x^{\beta}}{d s}\right)$ as a consequence of the fact that $\delta(d s)=d(\delta s)$, hence

$$
g_{\alpha \beta} \delta\left(\frac{d x^{\alpha}}{d s} \frac{d x^{\beta}}{d s}\right)=g_{\alpha \beta} \frac{d x^{\alpha}}{d s} \delta\left(\frac{d x^{\beta}}{d s}\right)+g_{\alpha \beta} \frac{d x^{\beta}}{d s} \delta\left(\frac{d x^{\alpha}}{d s}\right)=2 g_{\alpha \beta} \frac{d x^{\beta}}{d s} \frac{d}{d s}\left(\delta x^{\alpha}\right) .
$$

Using $g_{\alpha \beta} u^{\alpha} u^{\beta}=-1$, it is

$$
\delta S=\int_{A}^{B} \frac{1}{2}\left[g_{\alpha \beta, \lambda} \delta x^{\lambda} \frac{d x^{\alpha}}{d s} \frac{d x^{\beta}}{d s}+2 g_{\alpha \beta} \frac{d x^{\beta}}{d s} \frac{d}{d s}\left(\delta x^{\alpha}\right)\right] d s=0
$$


and integration by parts of the second term yields

$$
\delta S=\int_{A}^{B} \frac{1}{2}\left(g_{\alpha \beta, \lambda} \delta x^{\lambda} \frac{d x^{\alpha}}{d s} \frac{d x^{\beta}}{d s}\right) d s+\left[g_{\alpha \beta} \frac{d x^{\beta}}{d s} \delta x^{\alpha}\right]_{A}^{B}-\int_{A}^{B} \frac{d}{d s}\left(g_{\alpha \beta} \frac{d x^{\beta}}{d s}\right) \delta x^{\alpha} d s=0 .
$$

By imposing that, at the endpoints, it is $\delta x^{\alpha}(A)=\delta x^{\alpha}(B)=0$, the second term vanishes and

$$
\delta S=\int_{A}^{B} \frac{1}{2}\left(g_{\alpha \beta, \lambda} \frac{d x^{\alpha}}{d s} \frac{d x^{\beta}}{d s} \delta x^{\lambda}\right) d s-\int_{A}^{B}\left(g_{\alpha \beta} \frac{d^{2} x^{\beta}}{d s^{2}}+g_{\alpha \beta, \lambda} \frac{d x^{\lambda}}{d s} \frac{d x^{\beta}}{d s}\right) \delta x^{\alpha} d s=0 .
$$

This equation can be written as

$$
\delta S=\int_{A}^{B}\left[\left(\frac{1}{2} g_{\alpha \beta, \lambda}-g_{\lambda \beta, \alpha}\right) \frac{d x^{\alpha}}{d s} \frac{d x^{\beta}}{d s}-g_{\lambda \beta} \frac{d^{2} x^{\beta}}{d s^{2}}\right] \delta x^{\lambda} d s=0 .
$$

This integral vanishes for all variations $\delta x^{\lambda}$ with fixed endpoints if

$$
g_{\lambda \beta} \frac{d^{2} x^{\beta}}{d s^{2}}=\left(\frac{1}{2} g_{\alpha \beta, \lambda}-g_{\lambda \beta, \alpha}\right) u^{\alpha} u^{\beta} .
$$

Since

$$
g_{\lambda \beta, \alpha} u^{\alpha} u^{\beta}=g_{\lambda \alpha, \beta} u^{\beta} u^{\alpha}=\frac{1}{2}\left(g_{\lambda \beta, \alpha}+g_{\lambda \alpha, \beta}\right) u^{\alpha} u^{\beta}
$$

whereas

$$
g_{\lambda \beta} \frac{d^{2} x^{\beta}}{d s^{2}}=\frac{1}{2}\left(g_{\alpha \beta, \lambda}-g_{\lambda \beta, \alpha}-g_{\lambda \alpha, \beta}\right) u^{\alpha} u^{\beta}
$$

we have

$$
\{\lambda, \alpha \beta\}=\frac{1}{2}\left(g_{\lambda \alpha, \beta}+g_{\lambda \beta, \alpha}-g_{\alpha \beta, \lambda}\right)
$$

and

$$
g_{\lambda \beta} \frac{d^{2} x^{\beta}}{d s^{2}}+\{\lambda, \alpha \beta\} u^{\alpha} u^{\beta}=0 .
$$

Multiplying by $g^{\lambda \tau}$ and remembering that

$$
g^{\lambda \tau} g_{\lambda \beta}=\delta_{\beta}^{\tau}, \quad g^{\lambda \tau}\{\lambda, \alpha \beta\}=\Gamma_{\alpha \beta}^{\tau},
$$

one has

$$
\frac{d^{2} x^{\tau}}{d s^{2}}+\Gamma_{\alpha \beta}^{\tau} u^{\alpha} u^{\beta}=0,
$$

which is the geodesic equation describing the free fall motion of a point-like body in the gravitational field represented by the Christoffel symbols $\Gamma_{\alpha \beta}^{\tau}$.

This result means that the connection is Levi-Civita and then $g$ and $\Gamma$ are intimately related in GR. This feature, as stressed by Palatini [464] and Einstein [235], shows that geodesic structure $(\Gamma)$ and casual structure $(g)$ are not independent. This is not true, in general, in ETGs as we will discuss in the next part of this Report.

\section{The Einstein field equations in metric and Palatini formalisms}

The Einstein field equations are a particular case of those deduced in previous section for the Einstein-Cartan theory. Here we report their derivation in details in view of the variational principles that we will adopt for ETGs. In other words, the field equations in the cases of non-minimal couplings and non-linear functions of curvature invariants. We illustrate the derivation of the Einstein field equations in vacuo as the starting point.

Let us consider

$$
\delta \int d \Omega \sqrt{-g} \mathcal{L}=0
$$


where $\sqrt{-g} d \Omega$ is the invariant volume element and $\mathcal{L}$ is the Lagrangian density. In fact, under the coordinate transformation $\bar{x}^{\alpha} \rightarrow x^{\alpha}=x^{\alpha}\left(\bar{x}^{\mu}\right)$, where $\bar{x}^{\mu}$ are the "initial" local coordinates, we have

$$
d \Omega=J d \bar{\Omega}, \quad J=\operatorname{det}\left(\frac{\partial x^{\alpha}}{\partial \bar{x}^{\mu}}\right)
$$

with $J$ the Jacobian determinant of the transformation. Moreover, we have

$$
\begin{aligned}
& \bar{g}_{\alpha \beta}=\operatorname{diag}(-1,1,1,1), \\
& \bar{g}_{\alpha \beta}=\frac{\partial x^{\mu}}{\partial \bar{x}^{\alpha}} \frac{\partial x^{\nu}}{\partial \bar{x}^{\beta}} g_{\mu \nu},
\end{aligned}
$$

$\bar{g}=-1=J^{2} g$ and, therefore,

$$
d \bar{\Omega}=\frac{d \Omega}{J}=\sqrt{-g} d \Omega
$$

Since we want the Euler-Lagrange equations deriving from the variational principle to be of second order, the Lagrangian must be quadratic in the first order derivatives of $g_{\mu \nu}$. These first order derivatives contain the Christoffel symbols, which are not coordinate-invariant. Then we have to choose for the Lagrangian density $\mathcal{L}$ expressions containing higher order derivatives and, a priori, this brings the danger that the field equations could become of order higher than second (we will discuss in detail this point for ETGs). The obvious choice of Hilbert and Einstein for the Lagrangian density $\mathcal{L}$ was the Ricci scalar curvature $R$. The variational principle is then

$$
\delta \int d \Omega \sqrt{-g} R=0
$$

The relations

$$
\delta g=g g^{\mu \nu} \delta g_{\mu \nu}=-g g_{\mu \nu} \delta g^{\mu \nu}
$$

yield

$$
\delta(\sqrt{-g})=-\frac{\delta g}{2 \sqrt{-g}}=-\frac{1}{2} \sqrt{-g} g_{\alpha \beta} \delta g^{\alpha \beta}
$$

from which it follows that

$$
\begin{aligned}
& \int\left[(\delta \sqrt{-g}) R+\sqrt{-g} R_{\mu \nu} \delta g^{\mu \nu}+\sqrt{-g} g^{\mu \nu} \delta R_{\mu \nu}\right] d \Omega= \\
& =\int \sqrt{-g} \delta g^{\mu \nu}\left[R_{\mu \nu}-\frac{1}{2} R g_{\mu \nu}\right] d \Omega+\int \sqrt{-g} g^{\mu \nu} \delta R_{\mu \nu} d \Omega=0 .
\end{aligned}
$$

The second integral can be evaluated in the local inertial frame, obtaining

$$
\begin{aligned}
& R_{\mu \nu}(0)=G_{\mu \nu, \alpha}^{\alpha}-G_{\mu \alpha, \nu}^{\alpha}, \\
& \delta R_{\mu \nu}(0)=\frac{\partial}{\partial x^{\alpha}}\left(\delta G_{\mu \nu}^{\alpha}\right)-\frac{\partial}{\partial x^{\nu}}\left(\delta G_{\mu \alpha}^{\alpha}\right), \\
& g^{\mu \nu}(0) \delta R_{\mu \nu}(0)=g^{\mu \nu}(0) \frac{\partial}{\partial x^{\alpha}}\left(\delta G_{\mu \nu}^{\alpha}\right)-g^{\mu \nu}(0) \frac{\partial}{\partial x^{\nu}}\left(\delta G_{\mu \alpha}^{\alpha}\right) \\
& =g^{\mu \nu}(0) \frac{\partial}{\partial x^{\rho}}\left(\delta G_{\mu \nu}^{\rho}\right)-g^{\mu \rho}(0) \frac{\partial}{\partial x^{\rho}}\left(\delta G_{\mu \alpha}^{\alpha}\right) \\
& =\frac{\partial}{\partial x^{\rho}}\left[g^{\mu \nu}(0) \delta G_{\mu \nu}^{\rho}-g^{\mu \rho}(0) \delta G_{\mu \alpha}^{\alpha}\right] .
\end{aligned}
$$

Then, we can write

$$
g^{\mu \nu}(0) \delta R_{\mu \nu}(0)=\frac{\partial W^{\rho}}{\partial x^{\rho}}, \quad W^{\rho}=g^{\mu \nu}(0) \delta G_{\mu \nu}^{\rho}-g^{\mu \rho}(0) \delta G_{\mu \alpha}^{\alpha}
$$


The second integral in Eq. (5.26) can be discarded since its argument is a pure divergence; in fact, in general coordinates it is

$$
\int \sqrt{-g} g^{\mu \nu} \delta R_{\mu \nu} d \Omega=\int \sqrt{-g} \frac{\partial W^{\rho}}{\partial x^{\rho}} d \Omega=\int \sqrt{-g} W_{; \rho}^{\rho} d \Omega=\int \frac{\partial}{\partial x^{\rho}}\left(\sqrt{-g} W^{\rho}\right) d \Omega=0
$$

and then

$$
\int \sqrt{-g} \delta g^{\mu \nu}\left(R_{\mu \nu}-\frac{1}{2} R g_{\mu \nu}\right) d \Omega=0
$$

from which we obtain the vacuum field equations of GR

$$
G_{\mu \nu} \equiv R_{\mu \nu}-\frac{1}{2} g_{\mu \nu} R=0
$$

as Euler-Lagrange equations of the Hilbert-Einstein action. Vice-versa, starting from Eq. (5.33) and retracing the previous steps in inverse order (i.e., integrating the Einstein equations), one can re-obtain the Hilbert-Einstein action (5.23), thus demonstrating the equivalence between this action and the field equations (5.33). Introducing matter fields as sources is straightforward, once we introduce suitable forms of stress-energy tensor. A suitable choice is the stress-energy tensor of a perfect fluid, that is

$$
T_{\mu \nu}^{(m)}=(P+\rho) u_{\mu} u_{\nu}+P g_{\mu \nu}
$$

where $u^{\mu}$ is the four-velocity of the fluid particles and $P$ and $\rho$ are the pressure and energy density of the fluid, respectively, the continuity equation requires $T_{\mu \nu}^{(m)}$ to be covariantly constant, i.e., to satisfy the conservation law

$$
\nabla^{\mu} T_{\mu \nu}^{(m)}=0
$$

where $\nabla_{\alpha}$ denotes the covariant derivative operator of the metric $g_{\mu \nu}$. In fact, $\nabla^{\mu} R_{\mu \nu}$ does not vanish, except in the special case $R \equiv 0$. Einstein and Hilbert independently concluded that the field equations had to be

$$
G_{\mu \nu}=\kappa T_{\mu \nu}^{(m)}
$$

where $G_{\mu \nu}$ is now called the Einstein tensor of $g_{\mu \nu}$. These equations satisfy the conservation law (5.35) since the relation

$$
\nabla^{\mu} G_{\mu \nu}=0
$$

holds as a contraction of the Bianchi identities that the curvature tensor of $g_{\mu \nu}$ has to satisfy [582].

The Lagrangian that, when varied, produces the field equations (5.36) is the sum of a "matter" Lagrangian density $\mathcal{L}^{(m)}$, the variational derivative of which is

$$
T_{\mu \nu}^{(m)}=-\frac{2}{\sqrt{-g}} \frac{\delta\left(\sqrt{-g} \mathcal{L}_{(m)}\right)}{\delta g^{\mu \nu}}
$$

and of the above gravitational (Hilbert-Einstein) Lagrangian

$$
L_{H E} \equiv \sqrt{-g} \mathcal{L}_{H E}=\sqrt{-g} R
$$

where $g$ is the determinant of the metric $g_{\mu \nu}$. Solving the Einstein field equations means to determine the form of the metric $g_{\mu \nu}$ starting from the distribution of matter-energy in a given region of space. In other words, this achievement fixes the (causal) metric structure of the space-time. This is the derivation of the Einstein field equations in the so called metric formalism.

However it is possible to obtain the field equations without resorting to a local inertial frame [414]. Following the Weyl attempt to unify Gravity with Electromagnetism [585], as discussed in Sec II] an affine connection defined over the space-time, can be assumed as a dynamical field non-trivially depending on a metric. As we have seen, Weyl's idea failed because of a wrong choice of the Lagrangian and few more issues, but it generated however a keypoint: connections may have a physically interesting dynamics.

Einstein soon showed a great interest in Weyl's idea. He too began to play with connections, in order to seek for a "geometrically" Unified Theory. But he never arrived to "dethronize" $g$ in the description of the gravitational field. He was not so happy with the fact that the gravitational field is not the fundamental object, but just a by-product of the metric; however, he never really changed his mind about the physical and mathematical role of $g$. 
In 1925 Einstein constructed a theory depending on a metric $g$ and a symmetric affine connection $\Gamma$ to be varied independently (the so-called "Palatini method", because of a misunderstanding with W. Pauli, see [268, 406]); he defined a Lagrangian theory in which the Palatini-Einstein gravitational Lagrangian is

$$
\sqrt{-g} \mathcal{L}_{\mathrm{PE}}(g, \Gamma, \partial \Gamma): \equiv \stackrel{\Gamma}{R} \sqrt{-g}
$$

The Lagrangian density $\mathcal{L}_{\mathrm{PE}}$ contains (first order) derivatives of $\Gamma$ but no derivatives of $g$. In other words, it is of order zero in the metric while it is first order in the connection. By a well-known feature of the Lagrangian formalism, this implies that a priori $g$ has no independent dynamics, being a sort of "Lagrange multiplier"; if we would assume that $\Gamma$ is frozen from the beginning, $g$ would be frozen too. On the other hand since $g$ has dynamics, this happens because $g$ is related to $\Gamma$, which is the true dynamical variable of this Lagrangian theory. This time is the metric $g$ to gain a dynamical meaning from $\Gamma$, that plays the role of a fundamental field.

In 4 dimension, there are $10+40$ independent variables and the field equations, in vacuum, are:

$$
\left\{\begin{aligned}
\stackrel{\Gamma}{R}_{(\mu \nu)}-\frac{1}{2} \stackrel{\Gamma}{R} g_{\mu \nu} & =0 \\
\stackrel{\Gamma}{\nabla}_{\alpha}\left(\sqrt{g} g^{\mu \nu}\right) & =0
\end{aligned}\right.
$$

where $\stackrel{\Gamma}{R}_{(\mu \nu)}$ is the symmetric part of $\stackrel{\Gamma}{R}_{\mu \nu}$ and $\nabla \Gamma$ denotes the covariant derivative with respect to $\Gamma$. The second field equation (5.41) constrains the connection $\Gamma$, which is a priori arbitrary, to coincide a posteriori with the Levi-Civita connection of the metric $g$ (Levi-Civita Theorem). By substituting this information into the first field equation (5.41), the vacuum Einstein equation for $g$ is obtained. In the "Palatini formalism", the metric $g$ determines rods, clocks and protractors, while the connection $\Gamma$ determines the free-fall, but since a posteriori the same result of GR is found, Einstein soon ceased to show a real interest in this formalism.

The situation does not change if matter is present through a matter Lagrangian $\mathcal{L}_{(m)}$ (independent of $\Gamma$ but just depending on $g$ and other external matter fields), that generates the above energy-momentum tensor $T_{\mu \nu}^{(m)}$. If the total Lagrangian is then assumed to be $\mathcal{L}_{\text {tot }}: \equiv \mathcal{L}_{\mathrm{PE}}+\mathcal{L}_{(m)}$, field equations (5.41) are replaced by

$$
\stackrel{\Gamma}{R}_{(\mu \nu)}-\frac{1}{2} \stackrel{\Gamma}{R} g_{\mu \nu}=\kappa T_{\mu \nu}
$$

and again (5.41) implies, a posteriori, that (5.42) reduces to the Einstein equations.

Let us remark that the dynamical coincidence between $\Gamma$ and the Levi-Civita connection of $g$ is entirely due to the particular Lagrangian considered by Einstein, which is the simplest, but not the only possible one. Furthermore, the Palatini method privileges the affine structure with respect to the metric structure. Notice that, in this case $(i . e$. in "Palatini formalism"), the relations

$$
\Gamma_{\mu \nu}^{\lambda}=\left\{\begin{array}{l}
\lambda \\
\mu \nu
\end{array}\right\}_{g}
$$

are field equations: the fact that $\Gamma$ is the Levi-Civita connection of $g$ is no longer an assumption a priori but it is the outcome of the field equations [14]. As soon as the Lagrangian is not the Palatini-Einstein but a more general one, the affine connection does not coincide, in general, with the Levi-Civita one. This fact gives rise to extremely interesting results. Below we will discuss this point considering the curvature quantities derived in the Palatini formalism as different from those derived in the metric approach.

\section{The Newtonian limit of General Relativity}

GR is not the only theory of gravitation and, several alternative theories of gravity have been investigated from the 60's, considering the space-time to be "special relativistic" at a background level and treating gravitation as a Lorentz-invariant field on the background.

Two different classes of experiments have been studied: the first ones testing the foundations of gravitation theory - among them the Equivalence Principle - the second one testing the metric theories of gravity where space-time is endowed with a metric tensor and where the Einstein Equivalence Principle is valid. However as discussed in the Introduction, for several fundamental reasons extra fields might be necessary to describe the gravitation, e.g. scalar fields or higher-order corrections in curvature invariants. 
Two sets of equations can be distinguished [550]. The first ones couple the gravitational fields to the nongravitational contents of the Universe, i.e. the matter distribution, the electromagnetic fields, etc... The second set of equations gives the evolution of non-gravitational fields. Within the framework of metric theories, these laws depend only on the metric: this is a consequence of the EEP and the so-called "minimal coupling". In most theories, including GR, the second set of equations is derived from the first one, which is the only fundamental one; however, in many situations, the two sets are decoupled. The gravitational field studied in these approaches (without cosmological considerations) is mainly due to the Sun and the Eddington-Robertson expansion gives the corresponding metric. Assuming spherical symmetry and a static gravitational field, one can prove that there exists a coordinate system such as

$$
d s^{2}=-A(r) d t^{2}+B(r) d r^{2}+r^{2}\left(\sin ^{2} \theta d \phi^{2}+d \theta^{2}\right)
$$

$d t$ being the proper time between two neighbouring events. The Newtonian gravitational field does not exceed $G_{N} M_{\odot} / R_{\odot} c^{2} \sim 2 \times 10^{-6}$, where $c$ is the speed of light, $M_{\odot}$ is the mass of the Sun and $R_{\odot}$ its radius. The metric is quasi-Minkowskian, $A(r)$ and $B(r)$ are dimensionless functions which depend only on $G_{N}, M, c$ and $r$. Indeed, the only pure number that can be built with these four quantities is $G_{N} M / r c^{2}$. The Eddington-Robertson metric is a Taylor expansion of $A$ and $B$ which gives

$$
d s^{2} \simeq-\left(1-2 \alpha \frac{G_{N} M}{r c^{2}}+2 \beta\left(\frac{G_{N} M}{r c^{2}}\right)^{2}+\ldots\right) d t^{2}+\left(1+2 \gamma \frac{G_{N} M}{r c^{2}}+\ldots\right) d r^{2}+r^{2}\left(\sin ^{2} \theta d \phi^{2}+d \theta^{2}\right)
$$

which in isotropic coordinates is

$$
d s^{2} \simeq-\left(1-2 \alpha \frac{G_{N} M}{r c^{2}}+2 \beta\left(\frac{G_{N} M}{r c^{2}}\right)^{2}+\ldots\right) d t^{2}+\left(1+2 \gamma \frac{G_{N} M}{r c^{2}}+\ldots\right)\left(d x^{2}+d y^{2}+d z^{2}\right) .
$$

where $r, \theta, \phi$ are related to $x, y, z$ in the usual manner:

$$
r=\sqrt{x^{2}+y^{2}+z^{2}}, \quad \theta=\tan ^{-1}\left(\frac{z}{\sqrt{x^{2}+y^{2}+z^{2}}}\right), \quad \phi=\tan ^{-1}\left(\frac{y}{x}\right)
$$

where $r$ is the new "isotropic" radial coordinate, not to be confused with the Schwarzschild $r$ (see [414] for a detailed discussion of this important issue). The coefficients $\alpha, \beta, \gamma$ are called the post- Newtonian parameters and their values depend on the considered theory of gravity: in GR, one has $\alpha=\beta=\gamma=1$. The post Newtonian parameters can be measured not only in the Solar System but also in relativistic binary neutron stars such as $P S R 1913+16$.

A generalization of the previous formalism is known as Parametrized Post-Newtonian (PPN) formalism. Comparing metric theories of gravity among them and with experimental results becomes particularly easy if the PPN approximation is used. The following requirements are needed:

- particles are moving slowly with respect to the speed of light;

- gravitational field is weak and considered as a perturbation of the flat space-time;

- gravitational field is also static, i.e. it does not change with time.

The PPN limit of metric theories of gravity is characterized by a set of 10 real-valued parameters; each metric theory of gravity corresponds to particular values of PPN parameters. The PPN framework has been used first for the analysis of Solar System gravitational experiments, then for the definition and the analysis of new tests of gravitation and finally for the analysis and the classification of alternative metric theories of gravity [586].

By the middle 1970's, the Solar System was no more considered as the unique testing ground of gravitation theories. Many alternative theories of gravity agree with GR in the Post-Newtonian limit and thus with Solar System experiments; nevertheless, they do not agree for other predictions (such as cosmology, neutron stars, black holes and gravitational radiation) for which the post-Newtonian limit is not adequate. In addition, the possibility that experimental probes, such as gravitational radiation detectors, would be available in the future to perform extra-Solar System tests led to the abandon of the Solar System as the only arena to test gravitation theories. 
The study of the binary pulsar PSR 1913+16, discovered by R. Hulse and J. Taylor 330], showed that this system combines large post-Newtonian gravitational effects, highly relativistic gravitational fields (associated with the pulsar) with the evidence of an emission of gravitational radiation (by the binary system itself). Relativistic gravitational effects allowed one to do accurate measurements of astrophysical parameters of the system, such as the mass of the two neutron stars. The measurement of the orbital period rate of change agreed with the prediction of the gravitational waves (GW) emission in the GR framework, in contradiction with the predictions of most alternative theories, even those with PPN limits identical to GR. However, the evidence was not conclusive to rule out other theories since several shortcomings remain, up to now, unexplained and, as we see below, other forms of gravitational radiation (e.g. polarizations and massive states) with respect to those predicted by GR.

\section{E. The Minkowskian limit of General Relativity}

Replacing the Newtonian limit by a less restrictive hypothesis leads to the weak field approximation: practically, the field is still weak, but it is allowed to change in time and there is no more restriction on the test particles motion. New phenomena are associated with this hypothesis like the emission of gravitational radiation and the deflection of light. This framework allows one to split the metric $g_{\mu \nu}$ into two parts: the flat Minkowski metric $\eta_{\mu \nu}=\operatorname{diag}(-1,1,1,1)$ plus a perturbative term $h_{\mu \nu}$, assumed to be small. This linearized version of GR describes the propagation of a symmetric tensor $h_{\mu \nu}$ on a flat background space-time. So, the metric reads

$$
g_{\mu \nu}=\eta_{\mu \nu}+h_{\mu \nu} \quad \text { with } \quad\left|h_{\mu \nu}\right| \ll 1 .
$$

As $h_{\mu \nu}$ is small, one can neglect terms higher than the first order in the perturbation $h_{\mu \nu}$; in particular, one can raise/lower indexes with $\eta_{\mu \nu}$ and $\eta^{\mu \nu}$ as the corrections are of higher order in the perturbation

$$
g^{\mu \nu}=\eta^{\mu \nu}-h_{\mu \nu} \quad \text { with } \quad h^{\mu \nu}=\eta^{\mu \rho} \eta^{\nu \sigma} h_{\rho \sigma} .
$$

The aim is to find the equations of motion to which the perturbations $h_{\mu \nu}$ obey by investigating the Einstein equations to the first order. Inserting the new metric (5.48) in the Einstein tensor, we obtain

$$
G_{\mu \nu}=\frac{1}{2}\left(\partial_{\sigma} \partial_{\nu} h_{\mu}^{\sigma}+\partial_{\sigma} \partial_{\mu} h_{\nu}^{\sigma}-\partial_{\mu} \partial_{\nu} h-h_{\mu \nu}-\eta_{\mu \nu} \partial_{\rho} \partial_{\sigma} h^{\rho \sigma}+\eta_{\mu \nu} h\right)
$$

where $h=\eta^{\mu \nu} h_{\mu \nu}=h_{\mu}^{\mu}$ is the trace of the perturbation and $=-\frac{1}{2} \partial_{t t}+\partial_{x x}+\partial_{y y}+\partial_{z z}$ is the d'Alembertian of the flat space-time, using from now on (unless otherwise stated) geometrical units for which $c=1$.

The stress-energy tensor is computed at the 0 -order in $h_{\mu \nu}$ : the energy and the momentum have to be small too, according to the weak field approximation and the lowest non-vanishing order in $T_{\mu \nu}$ is of the same order of magnitude as the perturbation. Therefore, the conservation law becomes $\partial^{\mu} T_{\mu \nu}=0$.

\section{Gravitational Waves}

GW are weak ripples in the curvature of space-time, produced by the motions of matter. They propagate at the speed of light. The linearized Einstein equations allow wave solutions, in a way similar to Electromagnetism. These $\mathrm{GW}$ are transverse to the propagation direction and show two independent polarization states.

The new metric (5.48) does not fix the space-time frame completely; two possible gauges can be applied in addition to simplify the Einstein equations. Using the Lorentz gauge $\partial_{\mu} h_{\lambda}^{\mu}-\frac{1}{2} \partial_{\lambda} h=0$, the Einstein are linearized and can be written as

$$
h_{\mu \nu}-\frac{1}{2} \eta_{\mu \nu} \square h=\frac{16 \pi G_{N}}{c^{4}} T_{\mu \nu}^{(m)} .
$$

where standard units have been restored. The trace-reversed perturbation is defined as

$$
\bar{h}_{\mu \nu}=h_{\mu \nu}-\frac{1}{2} \eta_{\mu \nu} h .
$$


One can choose a frame in which the harmonic gauge condition, $\partial_{\mu} \bar{h}_{\nu}^{\mu}=0$, is verified. Then, the Einstein field equations become

$$
\square \bar{h}_{\mu \nu}=\frac{16 \pi G_{N}}{c^{4}} T_{\mu \nu}^{(m)}
$$

and, in vacuum, one has simply

$$
\square \bar{h}_{\mu \nu}=0
$$

These equations are similar to of the Electromagnetism field equations and one can use the same method to solve them. Indeed, looking at the Einstein equations in vacuum, Eq. (5.54), one can note that they are in the form of a wave equation for $\bar{h}_{\mu \nu}$, the d'Alembertian reduces to the form $\square=-\frac{1}{2} \partial_{t t}+\nabla^{2}$. Therefore, in the absence of matter, one looks for plane waves solutions

$$
\bar{h}_{\mu \nu}=C_{\mu \nu} e^{i k_{\sigma} x^{\sigma}}
$$

where $C_{\mu \nu}$ is a constant and symmetric tensor of rank 2 and $k$ is a time-like vector, the wave vector. The plane wave in Eq. (5.55) is a solution of the linearized equations in vacuum, Eq. (5.54), if the wave vector $k$ is null, $i . e$. satisfies the condition $k^{\sigma} k_{\sigma}=0$ and shows that GW propagate to the speed of light.

The four conditions of the harmonic gauge $k_{\mu} C^{\mu \nu}=0$ lead to six independent components for the symmetric tensor $C^{\mu \nu}$. As there are still some unused degrees of freedom, one can make another gauge choice on the tensor $C_{\mu \nu}$ :

$$
\begin{array}{rlr}
C_{\mu}^{\mu}=0 & \text { Traceless; } \\
C^{0 \mu}=0 & \text { Transverse }
\end{array}
$$

One has, in this way, the so called Transverse-Traceless (TT) gauge. These five relations give four new constraints on $C^{\mu \nu}$ in addition to the harmonic gauge condition; therefore, only two independent components remain in $C^{\mu \nu}$. As the wave is traceless, one can check from Eq. (5.52) that $\bar{h}_{\mu \nu}^{T T}=h_{\mu \nu}^{T T}$. Therefore, the general form of the symmetric tensor $C_{\mu \nu}$ is finally

$$
C_{\mu \nu}=\left(\begin{array}{cccc}
0 & 0 & 0 & 0 \\
0 & C_{11} & C_{12} & 0 \\
0 & C_{12} & -C_{11} & 0 \\
0 & 0 & 0 & 0
\end{array}\right)
$$

Let us define $C_{11}=h_{+}$and $C_{12}=h_{\times}$; GW appears to have two polarized states, " +" and " $\times$", which modify the space-time curvature in different ways. In tensorial form, one can write

$$
h=\left[h_{+}\left(\overrightarrow{e_{1}} \otimes \overrightarrow{e_{1}}-\overrightarrow{e_{2}} \otimes \overrightarrow{e_{2}}\right)+2 h_{\times}\left(\overrightarrow{e_{1}} \otimes \overrightarrow{e_{2}}\right)\right] e^{i \omega(t-x / c)}
$$

Being $\vec{\xi}=\left(\xi^{1}, \xi^{2}, \xi^{3}\right)$ the separation between two free particles and taking into account the geodesic deviation [577] which describes the evolution of two free-falling particles, if the GW propagates in the direction $x^{3}$, only $\xi^{1}$ and $\xi^{2}$ are involved in its passage. Assuming a polarized GW, the integration of the geodesic deviation equation gives:

- Polarization " $+"\left(h_{\times}=0\right)$ :

$$
\left(\begin{array}{l}
\xi^{1}(t) \\
\xi^{2}(t)
\end{array}\right)=\left(\begin{array}{cc}
1+\frac{1}{2} h_{+} e^{i k^{\sigma} x_{\sigma}} & 0 \\
0 & 1-\frac{1}{2} h_{+} e^{i k^{\sigma} x_{\sigma}}
\end{array}\right)\left(\begin{array}{l}
\xi^{1}(0) \\
\xi^{2}(0)
\end{array}\right)
$$

- Polarization " $\times "\left(h_{+}=0\right)$ :

$$
\left(\begin{array}{l}
\xi^{1}(t) \\
\xi^{2}(t)
\end{array}\right)=\left(\begin{array}{cc}
1 & \frac{1}{2} h_{\times} e^{i k^{\sigma} x_{\sigma}} \\
\frac{1}{2} h_{\times} e^{i k^{\sigma} x_{\sigma}} & 1
\end{array}\right)\left(\begin{array}{l}
\xi^{1}(0) \\
\xi^{2}(0)
\end{array}\right)
$$


Let us consider now a test-mass ring (a massless and free-falling set of particles) interacting with GW, lying in an plane orthogonal to the direction of the wave propagation. Its oscillations depend on the GW polarization.

After having found a solution to Einstein equations in vacuum, let us solve now Eq. (5.53) with a non-zero source term. The solution is computed using the retarded Green function

$$
\bar{h}_{\mu \nu}(t, \vec{x})=\frac{4 G_{N}}{c^{4}} \int_{\vec{y} \epsilon \text { Source }} \frac{1}{|\vec{x}-\vec{y}|} T_{\mu \nu}^{(m)}\left(t_{r}, \vec{y}\right) d^{3} \vec{y},
$$

with $|\vec{x}-\vec{y}|=\sqrt{\delta_{i j}\left(x^{i}-y^{i}\right)\left(x^{j}-y^{j}\right)}$ (Euclidean distance) and $t_{r}=t-|\vec{x}-\vec{y}| / c$ (retarded time). Let us consider an isolated source with a density $\rho$ and a characteristic dimension $\delta R$, located at a distance $R$ from the observation point $\vec{x}$. One assumes $\delta R \ll R$ so, in particular, one gets $|\vec{x}-\vec{y}| \approx R$ and one can move this constant term outside the integral in Eq. (5.62). As the stress-energy tensor verifies the conservation of energy $\partial^{\mu} T_{\mu \nu}^{(m)}=0$, the harmonic gauge condition $\partial_{\mu} \bar{h}_{\nu}^{\mu}=0$ is also verified. Moreover, the radiation is mostly emitted at frequencies $\omega / 2 \pi$, so that $\frac{\delta R}{c} \ll \frac{1}{\omega}$. Then, it is possible to demonstrate that only the spatial coordinates of tensor $\bar{h}_{\mu \nu}$ are different from zero. The quadrupole momentum tensor $q_{i j}$ of the source energy density is defined as

$$
q_{i j}(t)=\int_{\vec{y} \in \text { source }} y_{i} y_{j} T_{00}^{(m)}(t, \vec{y}) d^{3} \vec{y} \quad \text { with } \quad T_{00}^{(m)} \approx \rho c^{2} .
$$

The metric perturbation is given by

$$
\bar{h}_{i j}(t, \mathbf{x})=\frac{2 G_{N}}{R c^{4}} \ddot{q}_{i j}\left(t_{r}\right) .
$$

So, GW, generated by an isolated non-relativistic object, are proportional to the second derivative of the quadrupolar momenta of the energy density. Eq. (5.64) shows that the metric perturbation amplitude $h$ varies as the inverse of distance to the source $R$; a faster decreasing with the distance, e.g. $1 / R^{2}$, would make vain the hope of any GW detection. Fortunately, GW detectors are sensitive to the amplitude of the signal.

The energy emitted by gravitational radiation is difficult to define. A way to overcome this difficulty is to define the stress-energy tensor by developing the metric $g_{\mu \nu}$ and the Einstein tensor $G_{\mu \nu}$ at the second order:

$$
\begin{aligned}
g_{\mu \nu} & =\eta_{\mu \nu}+h_{\mu \nu}+h_{\mu \nu}^{(2)}, \\
G_{\mu \nu} & =G_{\mu \nu}^{(1)}\left[\eta+h^{(2)}\right]+G_{\mu \nu}^{(2)}[\eta+h] .
\end{aligned}
$$

Einstein equations in the vacuum $G_{\mu \nu}=0$ can be written in the form

$$
G_{\mu \nu}^{(1)}\left[\eta+h^{(2)}\right]=\frac{8 \pi G_{N}}{c^{4}} t_{\mu \nu}
$$

with the definition

$$
t_{\mu \nu}=\frac{c^{4}}{8 \pi G_{N}} G_{\mu \nu}^{(2)}[\eta+h] .
$$

The Bianchi identity says that $\partial_{\mu} t^{\mu \nu}=0$, therefore $t_{\mu \nu}$ can be considered as the stress-energy tensor of a gravitational field, yet, it is only a pseudo-tensor [373]. One can compute the energy density $t_{00}$ by averaging over many cycles (because the energy is not localized) the GW energy:

$$
t_{00}=\frac{c^{2}}{16 \pi G}\left\langle{\dot{h_{+}}}^{2}+{\dot{h_{\times}}}^{2}\right\rangle .
$$

Then, the source luminosity is $L=r^{2} \int_{\mathrm{S}} c t_{00} d \Omega$, where the integration is performed on sphere $\mathrm{S}$ of radius $r$. Introducing the reduced quadrupolar momenta 


$$
Q_{i j}=q_{i j}-\frac{1}{3} \delta_{i j} \delta^{k l} q_{k l},
$$

one obtains the Einstein quadrupole formula

$$
L=\frac{G}{c^{5}}\left\langle\frac{d^{3} Q_{i j}}{d t^{3}} \frac{d^{3} Q_{i j}}{d t^{3}}\right\rangle .
$$

It is worth noticing that a GW emission requires a variation of the quadrupolar momentum, as shown already in Eq. (5.64). This typical feature of gravitational radiation, added to the weakness of the coupling constant between gravitation and matter, here $G_{N} / c^{5} \approx 10^{-53} \mathrm{~W}^{-1}$, explains why GW amplitudes are so small compared to those produced by electromagnetic radiation. In conclusion, the gravitational radiation is quadrupolar and a symmetric spherical body does not emit GWs because its reduced quadrupolar momenta are zero.

The corresponding quantum field is the graviton with zero mass and spin 2. Within the framework of more general theories, the gravitation can be described as a combination of states with a spin 2 and spin zero or as a particle with spin maximum 2. One can also imagine that the mass of graviton is not zero or that the state mass spectrum is complex. Presently, there is no observational reason to doubt that the present observational bounds on the mass of the graviton are severe.

Discovering GWs would open "a new window onto the Universe" [544]. It is clear that being sensitive to an additional radiation would lead to major discoveries like when the Universe became observed through radio, X or gamma waves. Then, it would allow physicists to test GR in the strong field regime, to check the gravity velocity (assumed to be the speed of light in the Einstein theory) or to verify that GWs only change distances perpendicular to their direction of propagation.

Alternative relativistic theories of gravity also predict the existence of GWs. However, many essential features of the radiation are different: the number of polarization states, the propagation speed, the efficiency of wave generation, the possible existence of massive states [404, 544].

In this section, we have outlined, with no claim to completeness, some physical issues which constitute the test-bed of GR and any alternative relativistic theory of gravity. As we shall see below, ETGs enlarge the possibilities of viable theories in the track of GR with the aim to address, partially, some of the open questions at infrared and ultra-violet scales. 


\section{PART II: EXTENDED THEORIES OF GRAVITY}

\section{INTRODUCTION}

As widely discussed above, due to the problems of Standard Cosmological Model, and, first of all, to the lack of a definitive Quantum Gravity Theory, alternative theories have been considered in order to attempt, at least, a semiclassical scheme where GR and its positive results could be recovered. One of the most fruitful approaches is that of Extended Theories of Gravity (ETGs) which have become a sort of paradigm in the study of gravitational interaction. They are based on corrections and enlargements of the Einstein theory. The paradigm consists, essentially, in adding higher-order curvature invariants and minimally or non-minimally coupled scalar fields into dynamics which come out from the effective action of Quantum Gravity [129, 444]. This approach is coherent to the fact that these generalized theories emerge, like Einstein's gravity, from the Gauge Theory, as we have seen above, and can be framed in a bundle structure as we will show below.

Other motivations to modify GR, as discussed, come from the issue of a full recovering of the Mach Principle which leads to assume a varying gravitational coupling. This principle states that the local inertial frame is determined by some average of the motion of distant astronomical objects [84]. This fact implies that the gravitational coupling can be scale-dependent and related to some scalar field. As a consequence, the concept of "inertia" and the Equivalence Principle have to be revised.

Besides, every unification scheme as Superstrings, Supergravity or Grand Unified Theories, takes into account effective actions where non-minimal couplings to the geometry or higher-order terms in the curvature invariants are present. Such contributions are due to one-loop or higher-loop corrections in the high-curvature regimes near the full (not yet available) Quantum Gravity regime [444]. Specifically, this scheme was adopted in order to deal with the quantization on curved space-times and the result was that the interactions among quantum scalar fields and background geometry or the gravitational self-interactions yield corrective terms in the Hilbert-Einstein Lagrangian [76. Moreover, it has been realized that such corrective terms are inescapable in order to obtain the effective action of Quantum Gravity at scales closed to the Planck one [572]. All these approaches are not the "full quantum gravity" but are needed as working schemes toward it. In the next subsection, we will discuss the Quantum Field Theory approach in curved space-time and the emergence of curvature corrections.

In summary, higher-order terms in curvature invariants (such as $R^{2}, R_{\mu \nu} R^{\mu \nu}, R^{\mu \nu \alpha \beta} R_{\mu \nu \alpha \beta} R \square R$, or $R \square{ }^{k} R$ ) or non-minimally coupled terms between scalar fields and geometry (such as $\phi^{2} R$ ) have to be added to the effective Lagrangian of gravitational field when quantum corrections are considered. For instance, one can notice that such terms occur in the effective Lagrangian of strings or in Kaluza-Klein theories, when the mechanism of dimensional reduction is used [287].

On the other hand, from a conceptual point of view, there are no a priori reason to restrict the gravitational Lagrangian to a linear function of the Ricci scalar $R$, minimally coupled with matter [129]. Furthermore, the idea that there are no "exact" laws of physics could be taken into serious account: in such a case, the effective Lagrangians of physical interactions are "stochastic" functions. This feature means that the local gauge invariances (i.e. conservation laws) are well approximated only in the low energy limit and the fundamental physical constants can vary [51].

Beside fundamental physics motivations, all these theories have acquired a huge interest in cosmology due to the fact that they "naturally" exhibit inflationary behaviours able to overcome the shortcomings of Cosmological Standard Model (based on GR). The related cosmological models seem realistic and capable of matching with the Cosmic Microwave Background Radiation observations [225, 369, 530]. Furthermore, it is possible to show that, via conformal transformations, the higher-order and non-minimally coupled terms always correspond to the Einstein gravity plus one or more than one minimally coupled scalar fields [8, 103, 297, 395, 542, 579].

More precisely, higher-order terms appear always as contributions of order two in the field equations in metric formalism. For example, a term like $R^{2}$ gives fourth order equations [495], $R \square R$ gives sixth order equations 8 , 23, 65, 96, 297], $R \square^{2} R$ gives eighth order equations [60] and so on. By a conformal transformation, any 2nd-order derivative term corresponds to a scalar field9: for example, fourth-order gravity gives Einstein plus one scalar field, sixth-order gravity gives Einstein plus two scalar fields and so on [297, 510].

Considering a mathematical point of view, the problem of reducing more general theories to Einstein standard form has been extensively treated; one can see that, through a "Legendre" transformation, higher-order theories, under suitable regularity conditions on the Lagrangian, take the form of the Einstein one in which a scalar field (or more than one) is the source of the gravitational field (see for example [129, 267, 405, 518]); on the other side, as discussed above, it has been studied the mathematical equivalence between models with variable gravitational coupling with the Einstein gravity through suitable conformal transformations (see [195, 196, 215, 216]).

\footnotetext{
9 The dynamics of such scalar fields is usually given by the corresponding Klein-Gordon Equation, which is second order.
} 
In any case, the debate on the physical meaning of conformal transformations is far to be solved [see [140, 376] and references therein for a comprehensive review]. Several authors claim for a true physical difference between Jordan frame (higher-order theories and/or variable gravitational coupling) since there are experimental and observational evidences which point out that the Jordan frame could be suitable to better match solutions with data. Others state that the true physical frame is the Einstein one according to the energy theorems [405]. However, the discussion is open and no definitive statement has been formulated up to now.

The problem should be faced from a more general point of view and the Palatini approach to gravity, introduced in Sec $\nabla C$ ] could be useful to this goal [97, 235, 268]. In [457], this approach is widely discussed for ETGs and several important applications are reported.

The fundamental idea of the Palatini formalism, as we have seen in Sec. V, is to consider the connection $\Gamma$, entering the definition of the Ricci tensor, to be independent of the metric $g$ defined on the space-time $\mathcal{M}$. The Palatini formulation for the standard Hilbert-Einstein theory results to be equivalent to the purely metric theory: this follows from the fact that the field equations for the connection $\Gamma$, firstly considered to be independent of the metric, give the Levi-Civita connection of the metric $g$. As a consequence, there is no reason to impose the Palatini variational principle in the standard Hilbert-Einstein theory instead of the metric variational principle.

However, the situation completely changes if we consider the ETGs, depending on functions of curvature invariants, as $f(R)$, or non-minimally coupled to some scalar field. In these cases, the Palatini and the metric variational principle provide different field equations and the theories thus derived differ [269, 405]. The relevance of Palatini approach, in this framework, has been recently proven in relation to cosmological applications [106, 141, 381, 382, 437, 444, 457, 573.

It has also been studied the crucial problem of the Newtonian potential in alternative theories of gravity and its relations with the conformal factor [413]. In [457] interesting revision of the literature on the Newtonian limit of Palatini theories is provided. From a physical point of view, considering the metric $g$ and the connection $\Gamma$ as independent fields means to decouple the metric structure of space-time and its geodesic structure (being, in general, the connection $\Gamma$ not the Levi-Civita connection of $g$ ). The chronological structure of space-time is governed by $g$ while the trajectories of particles, moving in the space-time, are governed by $\Gamma$.

This decoupling enriches the geometric structure of space-time and generalizes the purely metric formalism. This metric-affine structure of space-time is naturally translated, by means of the same (Palatini) field equations, into a bi-metric structure of space-time. Beside the physical metric $g$, another metric $h$ is involved. This new metric is related, in the case of $f(R)$-gravity, to the connection. As a matter of fact, the connection $\Gamma$ results to be the Levi-Civita connection of $h$ and thus provides the geodesic structure of space-time [16].

If we consider the case of non-minimally coupled interaction in the gravitational Lagrangian (scalar-tensor theories), the new metric $h$ is related to the non-minimal coupling. The new metric $h$ can be thus related to a different geometric and physical aspect of the gravitational theory. Thanks to the Palatini formalism, the non-minimal coupling and the scalar field, entering the evolution of the gravitational fields, are separated from the metric structure of space-time. The situation mixes when we consider the case of higher-order-scalar-tensor theories. Due to these features, the Palatini approach could greatly contribute to clarify the physical meaning of conformal transformation [16]. A part the issue of the physical frame, as we have said before, higher-order corrections in curvature invariants and non-minimal couplings emerge from the formulation of Quantum Field Theory in a curved space-time. In the next subsection, we will sketch this approach giving some fundamental motivations to extended GR.

\section{QUANTUM FIELD THEORY IN CURVED SPACE-TIME}

At small scales and high energies, an hydrodynamic description of matter as a perfect fluid is inadequate: a more accurate description requires quantum field theory formulated on a curved space, in the framework of either GR or another relativistic theory of gravity. Since, at scales comparable to the Compton wavelength of the relevant particles, matter must be quantized, one can employ a semiclassical description of gravitation in which the Einstein equations assume the form

$$
G_{\mu \nu} \equiv R_{\mu \nu}-\frac{1}{2} g_{\mu \nu} R=<T_{\mu \nu}>,
$$

where the usual Einstein tensor $G_{\mu \nu}$ appears on the left hand side whereas the right hand side contains the expectation value of a quantum stress-energy tensor sourcing the gravitational field. Here the coupling constant has been incorporated in the average process so we have not to distinguish between effective and bare couplings. More precisely, if $\mid \psi>$ is a quantum state describing the early Universe, then

$$
<T_{\mu \nu}>\equiv<\psi\left|\hat{T}_{\mu \nu}\right| \psi>,
$$


where $\hat{T}_{\mu \nu}$ is the quantum operator associated with the classical energy-momentum tensor of the matter field and the right hand side is an appropriately regularized expectation value. In general, a quantized matter field $\hat{\phi}$ is subject to self-interactions and it interacts also with other fields and with the gravitational background. Such interaction terms may be included in the definition of an effective potentia 10

$$
V_{\text {eff }}(\phi)=<a|\hat{\mathcal{H}}| a>
$$

with

$$
\phi=<a|\hat{\phi}| a>
$$

where $\mid a>$ represents a normalized state of the theory under consideration $($ i.e., $<a \mid a>=1)$ and $\hat{\mathcal{H}}$ is the Hamiltonian operator satisfying $\delta<a|\hat{\mathcal{H}}| a>=0$, where $\delta$ is the variation on the average of $\mathcal{H}$-eigenstates. This condition corresponds to energy conservation.

In a curved space-time, even in the absence of classical matter and radiation, quantum fluctuations of matter fields give non-vanishing contributions to $\left\langle T_{\mu \nu}\right\rangle$, an effect similar to the vacuum of QED [76, 468]. When matter fields are free, massless and conformally invariant, these corrections assume the form

$$
<T_{\mu \nu}>=k_{1}{ }^{(1)} H_{\mu \nu}+k_{3}{ }^{(3)} H_{\mu \nu} .
$$

Here $k_{1}$ and $k_{3}$ are numerical coefficients, while

$$
\begin{aligned}
& { }^{(1)} H_{\mu \nu}=2 R_{; \mu \nu}-2 g_{\mu \nu} \square R+2 R R_{-} \mu \nu-\frac{1}{2} g_{\mu \nu} R^{2}, \\
& { }^{(3)} H_{\mu \nu}=R_{\mu}^{\sigma} R_{\nu \sigma}-\frac{2}{3} R R_{\mu \nu}-\frac{1}{2} g_{\mu \nu} R^{\sigma \tau} R_{\sigma \tau}+\frac{1}{4} g_{\mu \nu} R^{2} .
\end{aligned}
$$

The divergence of the tensor ${ }^{(1)} H_{\mu \nu}$ vanishes,

$$
{ }^{(1)} H_{\mu ; \nu}^{\nu}=0 \text {. }
$$

This tensor can be obtained by varying a quadratic contribution to the local action,

$$
{ }^{(1)} H_{\mu \nu}=\frac{2}{\sqrt{-g}} \frac{\delta}{\delta g^{\mu \nu}}\left(\sqrt{-g} R^{2}\right) .
$$

In order to remove the infinities coming from $\left\langle T_{\mu \nu}\right\rangle$ and obtain a renormalizable theory, one has to introduce infinitely many counterterms in the Lagrangian density of gravity. One of these terms is $C R^{2} \sqrt{-g}$, where $C$ is a parameter that diverges logarithmically. Eq. (7.1) cannot be generated by a finite action because then the gravitational field would be completely renormalizable, i.e., it would suffice to eliminate a finite number of divergences to make gravity similar to QED. Instead, one can only construct a truncated quantum theory of gravity. The expansion in loops is done in terms of $\hbar$, so the truncated theory at the one-loop level contains all terms of order $\hbar$. In this sense, this is the first quantum correction to GR. It assumes that matter fields are free and, due to the Equivalence Principle, all forms of matter couple in the same way to gravity. It also implies an intrinsic non-linearity of gravity, so that a number of loops are needed in order to take into account self-interactions or mutual interactions between matter and gravitational fields. At the one-loop level, divergences can be removed by renormalizing the cosmological constant $\Lambda_{\text {eff }}$ and the gravitational constant $G_{e f f}$. The one-loop contributions to $\left\langle T_{\mu \nu}>\right.$ are the quantities ${ }^{(1)} H_{\mu \nu}$ and ${ }^{(3)} H_{\mu \nu}$ above. In addition, one has to consider

$$
{ }^{(2)} H_{\mu \nu}=2 R_{\mu ; \nu \sigma}^{\sigma}-\square R_{\mu \nu}-\frac{1}{2} g_{\mu \nu} \square R+R_{\mu}^{\sigma} R_{\sigma \nu}-\frac{1}{2} R^{\sigma \tau} R_{\sigma \tau} g_{\mu \nu}
$$

It is shown in Refs. [76, 468] that the relation

$$
{ }^{(2)} H_{\mu \nu}=\frac{1}{3}^{(1)} H_{\mu \nu}
$$

10 Hereafter, scalar fields and potentials are understood as their effective values, obtained averaging over quantum states. In this sense, classical fields and potentials are the expectation values of quantum fields and potentials. 
holds in conformally flat space-times. In this case, only the first and third terms of $H_{\mu \nu}$ are present in Eq. (7.5). Since one can add to the parameter $C$ in the Lagrangian term $C \sqrt{-q} R^{2}$ an arbitrary constant, the coefficient $k_{1}$ can assume any value — the latter should be determined experimentally [76, 468].

The tensor ${ }^{(3)} H_{\mu \nu}$ is conserved only in conformally flat space-times (for example, FLRW spaces) and it cannot be obtained by varying a local action. Finally, one has

$$
k_{3}=\frac{1}{1440 \pi^{2}}\left(N_{0}+\frac{11}{2} N_{1 / 2}+31 N_{1}\right),
$$

where the $N_{i}$ 's $(i=0,1 / 2,1)$ are determined by the number of quantum fields with spin $0,1 / 2$, and 1 . Vector fields contribute more to $k_{3}$ due to the larger coefficient 31 of $N_{1}$. These massless fields, as well as the spinorial ones, are described by conformally invariant equations and appear in $\left\langle T_{\mu \nu}\right\rangle$ in the form (7.5). The trace of the energymomentum tensor vanishes for conformally invariant classical fields while, owing to the term weighted by $k_{3}$, one finds that the expectation value of the tensor (7.5) has non-vanishing trace. This fact is at the origin of the so-called trace anomaly.

Let us discuss briefly how the conformal anomalies are generated when the origin of the tensor $T_{\mu \nu}$ is not classical, i.e., when quantum field theories are formulated in curved space-time. As we will see in more detail later, if a theory is conformally invariant, under the conformal transformation

$$
g_{\mu \nu}(x) \rightarrow \tilde{g}_{\mu \nu}(x) \equiv \Omega^{2}(x) g_{\mu \nu}(x) .
$$

the action in $(n+1)$ space-time dimensions satisfies the functional equation

$$
S\left[\tilde{g}_{\mu \nu}\right]=S\left[g_{\mu \nu}\right]+\int d^{n+1} x \frac{\delta S\left[\tilde{g}_{\mu \nu}\right]}{\delta \tilde{g}^{\rho \sigma}} \delta \tilde{g}^{\rho \sigma}
$$

where the use of

$$
\delta \tilde{g}^{\mu \nu}(x)=-2 \Omega^{-1}(x) \tilde{g}^{\mu \nu}(x) \delta \Omega(x),
$$

and of the classical variational principle

$$
T_{\mu \nu}^{(m)}=-\frac{2}{\sqrt{-g}} \frac{\delta S^{(m)}}{\delta g}
$$

yields

$$
S\left[\tilde{g}^{\mu \nu}\right]=S\left[g_{\mu \nu}\right]-\int d^{n+1} x \sqrt{-\tilde{g}} T_{\rho}^{\rho}\left(\tilde{g}_{\mu \nu}\right) \Omega^{-1} \delta \Omega .
$$

From this, it follows that

$$
T_{\rho}^{\rho}\left[g_{\mu \nu}(x)\right]=-\left.\frac{\Omega(x)}{\sqrt{-g}} \frac{\delta S\left[\tilde{g}^{\mu \nu}\right]}{\delta \Omega(x)}\right|_{\Omega=1} .
$$

Hence, if the classical action is invariant under conformal transformations, the trace of the energy-momentum tensor vanishes. At the quantum level this situation could not occur for the following reason. A conformal transformation is, essentially, a rescaling of lengths with a different rescaling factor at each space-time point $x$; the presence of a mass, and hence of a length scale, in the theory breaks conformal invariance and generates the trace anomaly. To preserve conformal invariance one has to consider massless fields, as done in (7.5). In this case one obtains the condition

$$
<T_{\rho}^{\rho}>=0 \text {, }
$$

which allows one to consider a conformally invariant theory. Note that gravity is not renormalizable in the usual way; because of this, divergences appear as soon as quantum effects are considered. A loop expansion yields

$$
<T_{\rho}^{\rho}>=<T_{\rho}^{\rho}>_{\text {div }}+<T_{\rho}^{\rho}>_{r e n}=0
$$

confirming the validity of Eq. (7.19). In this case conformal invariance is preserved only if the divergent part is equal (up to the sign) to the renormalized tensor. An anomalous trace term will appear on the right hand side of the field equations (7.1) which, at one-loop and in the zero mass limit of the fields, is given by

$$
<T_{\rho}^{\rho}>_{\text {div }}=\left[\tilde{k}_{1}\left(M-\frac{2}{3} \square R\right)+\tilde{k}_{3} \mathcal{G}\right]=-<T_{\rho}^{\rho}>_{r e n}
$$


for a four-dimensional theory. Here $\tilde{k}_{1}$ and $\tilde{k}_{3}$ are proportional to $k_{1}$ and $k_{3}$, while $M$ and $\mathcal{G}$ are obtained from ${ }^{(1)} H_{\mu \nu}$ and ${ }^{(3)} H_{\mu \nu}$ as

$$
\begin{aligned}
M & =R^{\alpha \beta \gamma \delta} R_{\alpha \beta \gamma \delta}-2 R^{\alpha \beta} R_{\alpha \beta}+\frac{1}{3} R^{2}, \\
\mathcal{G} & =R^{2}-4 R^{\alpha \beta} R_{\alpha \beta}+R^{\alpha \beta \gamma \delta} R_{\alpha \beta \gamma \delta} .
\end{aligned}
$$

$\mathcal{G}$ is the Gauss-Bonnet term. In four dimensions, the integral

$$
\int d^{4} x \sqrt{-g} \mathcal{G}
$$

is an invariant (Euler characteristic) which provides information about the topology of the space-time manifold on which the theory is formulated (Gauss-Bonnet theorem). In a FLRW background $M$ vanishes identically but $\mathcal{G}$ gives non-vanishing contributions to (7.5) even if the variation of (7.24) is zero (in four dimensions).

In general, by summing all the geometric terms deduced from the Riemann tensor and of the same order in $\left\langle T_{\rho}^{\rho}\right\rangle_{r e n}$, one derives the right hand side of (7.5). If the background metric is conformally flat, this can be expressed by means of eqs. (17.6) and (7.7). Then, one can conclude that the trace anomaly due to the geometric terms arises because the one-loop approach is an attempt to formulate quantum field theories on curved space-time 11 Cosmological models arising from (7.5) are studied in [33]. The masses of the matter fields and their mutual interactions can be neglected in the high curvature limit because $R>m^{2}$. The matter-graviton interactions generate non-minimal coupling terms in the effective Lagrangian. The one-loop contributions of such terms are comparable to the ones due to the trace anomaly and generate, from the conformal point of view, the same effects on gravity. The simplest effective Lagrangian that takes into account these corrections is

$$
\mathcal{L}_{N M C}=-\frac{1}{2} \nabla^{\alpha} \phi \nabla_{\alpha} \phi-V(\phi)-\frac{\xi}{2} R \phi^{2},
$$

where $\xi$ is a dimensionless coupling constant between the scalar and the gravitational fields. The scalar field stressenergy tensor will be modified accordingly but a conformal transformation can be found such that the modifications due to curvature terms can, at least formally, be cast in the form of a matter-curvature interaction. The same argument holds for the trace anomaly. Certain Grand-Unified theories lead to a polynomial coupling of the form $1+\xi \phi^{2}+\zeta \phi^{4}$ generalizing the one of (7.25), while an exponential coupling $\mathrm{e}^{-\alpha \varphi} R$ between a scalar field (dilaton) $\varphi$ and the Ricci scalar appears instead in the effective Lagrangian of string theories. The field equations obtained by varying the Lagrangian density $\sqrt{-g} \mathcal{L}_{N M C}$ are

$$
\begin{gathered}
\left(1-\kappa \xi \phi^{2}\right) G_{\mu \nu}=\kappa\left\{\nabla_{\mu} \phi \nabla_{\nu} \phi-\frac{1}{2} g_{\mu \nu} \nabla^{\alpha} \phi \nabla_{\alpha} \phi-V g_{\mu \nu} \xi\left[g_{\mu \nu} \square\left(\phi^{2}\right)-\nabla_{\mu} \nabla_{\nu}\left(\phi^{2}\right)\right]\right\}, \\
\square \phi-\frac{d V}{d \phi}-\xi R \phi=0 .
\end{gathered}
$$

The non-minimal coupling of the scalar field is reminiscent of that exhibited by the four-vector potential of curved space Maxwell theory. below. Motivation for the non-minimal coupling in the Lagrangian $\mathcal{L}_{N M C}$ comes from many directions. A nonzero $\xi$ is generated by first loop corrections even if it is absent in the classical action $75,76,276$, 277, 428, 467, 468]. Renormalization of a classical theory with $\xi=0$ shifts this coupling constant to a value which is typically small 17, 337 but can, however, affect drastically an inflationary cosmological scenario and determine its success or failure [1, 251, 252, 257, 281, 282]. A non-minimal coupling term is expected at high curvatures 276, 277], and it has been argued that classicalization of the Universe in quantum cosmology indeed requires $\xi \neq 0$ 451]. Moreover, non-minimal coupling can solve potential problems of primordial nucleosynthesis [164, 165] and the absence of pathologies in the propagation of $\phi$-waves seems to require conformal coupling for all non-gravitational scalar fields (250, 299, 300, 519], see also [210, 254) 12 The conformal value $\xi=1 / 6$ is also an infrared fixed point of the renormalization group in finite GUTs 81,82, , 90 95, 237, 424, 448, 486|. Non-minimally coupled scalar fields have been widely used in relation with specific inflationary scenarios [48, 246 249, 283, 284, 331, 362, 363, 377, 499]. The approach adopted was largely one in which $\xi$ is regarded as a free parameter to be used at will in order to fix

11 Eqs. 7.6 and 7.7 can include terms containing derivatives of the metric of order higher than fourth (fourth order corresponding to the $R^{2}$ term) if all possible Feynman diagrams are included. For example, corrections such as $R \square R$ or $R^{2} \square R$ can be present in ${ }^{(3)} H_{\mu \nu}$ implying equations of motion that contain sixth order derivatives of the metric. Also these terms can be treated by making use of conformal transformations [23].

12 Note, however, that the distinction between gravitational and non-gravitational fields becomes representation-dependent in ETGs, together with the various formulations of the EP. 
possible problems of specific inflationary scenarios; see [256, 257] for more general treatments. Geometric reheating of the Universe with strong coupling $\xi \gg 1$ has also been studied [551, 552] and non-minimally coupled scalar fields have been considered in relation with wormholes [192, 193, 308, black holes [321, 562, and boson stars 344, 387, 563. The value of the coupling $\xi$ is not, in general, a free parameter but it depends on the physical nature of the particular scalar field $\phi$ [276, 277, 319, 323, 466, 575] (see [251, 256, 257] for reviews of the available theoretical prescriptions for the value of $\xi)$.

To conclude, any attempt to formulate quantum field theory on a curved space-time necessarily leads to modifying the Hilbert-Einstein action. This means adding terms containing non-linear invariants of the curvature tensor or non-minimal couplings between matter and the curvature originating in the perturbative expansion. In cosmology, all these modifications may affect deeply inflationary scenarios originally proposed using minimally coupled scalars [251, 256]. Although rare and very speculative alternatives have been proposed to the inflationary paradigm, the latter is currently accepted by most authors as the "canonical" cure to the shortcomings of the Standard Big Bang Model, with the added bonus of providing a viable mechanism for the generation of density perturbations to seed the structures observed today in the Universe. However, the effects of non-minimal coupling on the inflationary paradigm need to be assessed carefully.

On the other hand, the vacuum energy of free quantized fields of very low masses can significantly alter also the recent expansion of the Universe as shown in [469, 470]. In fact, the effective action can be obtained from nonperturbative sums of scalar curvature terms in the propagator. As a result of non-perturbative quantum effects, the scalar curvature of the matter-dominated Universe stops decreasing and approaches a constant value. The Universe evolves from an open matter-dominated epoch to a mildly inflating de Sitter expansion. The Hubble constant, during the present de Sitter epoch, as well as the time at which the transition occurs from matter-dominated to de Sitter expansion, are determined by the mass of the field and by the present matter density. These models provide a theoretical explanation of the observed recent acceleration of the Universe, and gives a good fit to data from highredshift Type Ia Supernovae, with masses of about $10^{-33} \mathrm{eV}$, and a current ratio of matter density to critical density, $\Omega_{0}<0.4$. The age of the Universe then follows with no further free parameters in the theory, and turns out to be greater than 13 Gyr. The Universe is spatially open and consistent with the possibility of inflation in the very early Universe. Furthermore, such models arise from standard renormalizable theories of free quantum fields in curved space-time, and do not require a cosmological constant or the associated fine-tuning. In this perspective, ETGs represent also a valid alternative to Dark Energy models.

\section{VARIATIONAL PRINCIPLES AND FIELD EQUATIONS IN METRIC FORMALISM}

Having discussed the more general aspects of gravitation theory and briefly reviewed or mentioned some alternatives to GR, we will concentrate now on a number of specific gravitation theories that have received attention lately. We begin by devoting this section to the exploration of their theoretical aspects. In the following, their phenomenological aspects will be studied as well. The theories considered can come from an action as can many of the interesting theories of gravity. We concentrate on theories which include a scalar field as an extra field mediating the gravitational interaction (such as scalar-tensor theories), theories whose action includes higher order curvature invariants. We also extensively consider theories with a connection which is independent of the metric. The actions of these theories are presented and in many cases their resemblance with effective low-energy actions coming from more fundamental theories is briefly discussed. We also present the derivation of the field equations through the application of a suitable variational principle and analyse the basic characteristics of the theory, as expressed through the field equations.

\section{A. The Brans-Dicke gravity as the first extension}

The Brans-Dicke theory of gravity [86, 271, 345, 346] is the prototype of gravitational theories alternative to GR. The action in the Jordan frame (the set of variables $\left.\left(g_{\mu \nu}, \phi\right)\right)$ is

$$
S_{(B D)}=\frac{1}{16 \pi} \int d^{4} x \sqrt{-g}\left[\phi R-\frac{\omega}{\phi} g^{\mu \nu} \nabla_{\mu} \phi \nabla_{\nu} \phi-V(\phi)\right]+S^{(m)},
$$

where

$$
S^{(m)}=\int d^{4} x \sqrt{-g} \mathcal{L}^{(m)}
$$

is the action of ordinary matter and $\omega$ is the dimensionless Brans-Dicke parameter. The factor $\phi$ in the denominator of the kinetic term of $\phi$ in the action (8.1) is purely conventional and has the only purpose of making $\omega$ dimensionless. 
Matter does not couple directly to $\phi$, i.e., the Lagrangian density $\mathcal{L}^{(m)}$ is independent of $\phi$ ("minimal coupling" of matter). However, $\phi$ couples directly to the Ricci scalar. The gravitational field is described by both the metric tensor $g_{\mu \nu}$ and the Brans-Dicke scalar $\phi$ which, together with the matter variables, constitute the degrees of freedom of the theory. As usual for scalar fields, the potential $V(\phi)$ generalizes the cosmological constant and may reduce to a constant, or to a mass term 13

The original motivation for introducing Brans-Dicke theory was the implementation of Mach's Principle. This is achieved in Brans-Dicke theory by making the effective gravitational coupling strength $G_{\text {eff }} \sim \phi^{-1}$ depend on the space-time position and being governed by distant matter sources, as in Eq. (8.9) below. As already remarked, modern interest in Brans-Dicke and scalar-tensor theories is motivated by the fact that they are obtained as low-energy limits of string theories. The variation of the action (8.1) with respect to $g^{\mu \nu}$ and the well known properties [373]

$$
\begin{aligned}
\delta(\sqrt{-g}) & =-\frac{1}{2} \sqrt{-g} g_{\mu \nu} \delta g^{\mu \nu}, \\
\delta(\sqrt{-g} R) & =\sqrt{-g}\left(R_{\mu \nu}-\frac{1}{2} g_{\mu \nu} R\right) \delta g^{\mu \nu} \equiv \sqrt{-g} G_{\mu \nu} \delta g^{\mu \nu},
\end{aligned}
$$

yield the field equation

$$
G_{\mu \nu}=\frac{8 \pi}{\phi} T_{\mu \nu}^{(m)}+\frac{\omega}{\phi^{2}}\left(\nabla_{\mu} \phi \nabla_{\nu} \phi-\frac{1}{2} g_{\mu \nu} \nabla^{\alpha} \phi \nabla_{\alpha} \phi\right)+\frac{1}{\phi}\left(\nabla_{\mu} \nabla_{\nu} \phi-g_{\mu \nu} \square \phi\right)-\frac{V}{2 \phi} g_{\mu \nu},
$$

where

$$
T_{\mu \nu}^{(m)} \equiv \frac{-2}{\sqrt{-g}} \frac{\delta}{\delta g^{\mu \nu}}\left(\sqrt{-g} \mathcal{L}^{(m)}\right)
$$

is the energy-momentum tensor of ordinary matter. By varying the action with respect to $\phi$, one obtains

$$
\frac{2 \omega}{\phi} \square \phi+R-\frac{\omega}{\phi^{2}} \nabla^{\alpha} \phi \nabla_{\alpha} \phi-\frac{d V}{d \phi}=0 .
$$

Taking now the trace of Eq. (8.5),

$$
R=\frac{-8 \pi T^{(m)}}{\phi}+\frac{\omega}{\phi^{2}} \nabla^{\alpha} \phi \nabla_{\alpha} \phi+\frac{3 \square \phi}{\phi}+\frac{2 V}{\phi},
$$

and using the resulting Eq. (8.8) to eliminate $R$ from Eq. (8.7) leads to

$$
\square \phi=\frac{1}{2 \omega+3}\left(8 \pi T^{(m)}+\phi \frac{d V}{d \phi}-2 V\right) .
$$

According to this equation, the scalar $\phi$ is sourced by non-conformal matter (i.e., by matter with trace $T^{(m)} \neq 0$ ), however the scalar does not couple directly to $\mathcal{L}^{(m)}$ : the Brans-Dicke scalar $\phi$ reacts on ordinary matter only indirectly through the metric tensor $g_{\mu \nu}$, as dictated by Eq. (8.5). The term proportional to $\phi d V / d \phi-2 V$ on the right hand side of Eq. (8.9) vanishes if the potential has the form $V(\phi)=m^{2} \phi^{2} / 2$ familiar from the Klein-Gordon equation and from particle physics. The action (8.1) and the field equation (8.5) suggest that the field $\phi$ be identified with the inverse of the effective gravitational coupling

$$
G_{e f f}(\phi)=\frac{1}{\phi},
$$

a function of the space-time location. In order to guarantee a positive gravitational coupling, only the range of values $\phi>0$ corresponding to attractive gravity is considered. The dimensionless Brans-Dicke parameter $\omega$ is a free parameter of the theory: a value of $\omega$ of order unity would be natural in principle (and it does appear in the low-energy limit of the bosonic string theory). However, values of $\omega$ of this order of magnitude are excluded by Solar System experiments, for a massless or light field $\phi$ (i.e., one that has a range larger than the size of the Solar System).

\footnotetext{
13 Due to the particular equation (8.9) satisfied by the Brans-Dicke field $\phi$, its mass is not the coefficient of the quadratic term in the expansion of $V(\phi)$, as for minimally coupled scalar fields, but rather the quantity $m$ defined by $m^{2}=\frac{1}{2 \omega+3}\left(\phi \frac{d^{2} V}{d \phi^{2}}-\frac{d V}{d \phi}\right)[265]$.
} 
The larger the value of $\omega$, the closer Brans-Dicke gravity is to GR [582]; there are, however, exceptions such as vacuum Brans-Dicke solutions 14 and solutions sourced by conformal matter 24, 40, 253, 255, 257, 409, 489 491, 505]. The most stringent experimental limit, $\omega>40,000$, was set by the Cassini probe in 2003 [71].

Brans-Dicke theory with a free or light scalar field is viable in the limit of large $\omega$, but the large value of this parameter required to satisfy the experimental bounds is certainly fine-tuned and makes Brans-Dicke theory unappealing. However, this fine-tuning becomes unnecessary if the scalar field is given a sufficiently large mass and, therefore, a short range. This means that a self-interaction potential $V(\phi)$ has to be considered in discussing the limits on $\omega$ and this fact is an adjustment of the original Brans-Dicke theory [86].

\section{B. $f(R)$-gravity in metric formalism}

We now examine the variational principle and the field equations of another class of ETGs, $f(R)$-gravity in the metric formalism. The salient feature of these ETGs is that the field equations are of fourth order and, therefore, more complicated than those of GR (which is recovered as the special case $f(R)=R$ ). Due to their higher order, these field equations admit a much richer variety of solutions than the Einstein equations. For simplicity, we begin by discussing quadratic corrections to the Hilbert-Einstein theory, which provide interesting cosmology.

\section{The case of $f(R)=R+\alpha R^{2}$}

Quadratic corrections in the Ricci scalar motivated by attempts to renormalize GR, constitute a straightforward extension of GR and have been particularly relevant in cosmology since they allow a self-consistent inflationary model to be constructed [530]. We will use this model as an example before discussing general metric $f(R)$-gravity.

Let us begin by deriving the field equations for the Lagrangian density

$$
\mathcal{L}=R+\alpha R^{2}+2 \kappa \mathcal{L}^{(m)}
$$

from the variational principle $\delta \int d^{4} x \sqrt{-g} \mathcal{L}=0$. We consider vacuum first. The variation gives

$$
\int d^{4} x \sqrt{-g} G_{\alpha \beta} \delta g^{\alpha \beta}+\alpha \delta \int d^{4} x \sqrt{-g} R^{2}=0,
$$

in which the variation of $R \sqrt{-g}$ produces the Einstein tensor. We now compute the second term on the right hand side of Eq. 8.12). We have

$$
\delta \int d^{4} x \sqrt{-g} R^{2}=-\frac{1}{2} \int d^{4} x \sqrt{-g} g_{\alpha \beta} \delta g^{\alpha \beta} R^{2}+2 \int d^{4} x \sqrt{-g} R \delta R
$$

and

$$
\int d^{4} x \sqrt{-g} R \delta R=\int d^{4} x \sqrt{-g} R\left(\delta g^{\alpha \beta} R_{\alpha \beta}+g^{\alpha \beta} \delta R_{\alpha \beta}\right)
$$

By using the fact that

$$
g^{\alpha \beta} \delta R_{\alpha \beta}=\nabla_{\alpha} \nabla_{\beta} h^{\alpha \beta}-\square h,
$$

where

$$
h^{\alpha \beta} \equiv-\delta g^{\alpha \beta}, \quad h \equiv-g_{\alpha \beta} \delta g^{\alpha \beta},
$$

one has

$$
\int d^{4} x \sqrt{-g} R g^{\alpha \beta} \delta R_{\alpha \beta}=\int d^{4} x \sqrt{-g} R\left(\nabla_{\alpha} \nabla_{\beta} h^{\alpha \beta}-\square h\right) .
$$

Integrating by parts twice, the operators $\nabla_{\alpha} \nabla_{\beta}$ and $\square$ acting on $h^{\alpha \beta}$ and $h$, respectively, transfer their action onto $R$ and

$$
\int d^{4} x \sqrt{-g} R g^{\alpha \beta} \delta R_{\alpha \beta}=\int d^{4} x \sqrt{-g}\left(h^{\alpha \beta} \nabla_{\alpha} \nabla_{\beta} R-h \square R\right)
$$

14 One should keep in mind, however, that the limit of particular space-time solutions of the field equations of a gravitational theory should be taken in a coordinate-independent way [292, 462, 463]. 
Using Eq. (8.16), Eq. (8.18) becomes

$$
\int d^{4} x \sqrt{-g} R g^{\alpha \beta} \delta R_{\alpha \beta}=\int d^{4} x \sqrt{-g}\left(-\delta g^{\alpha \beta} \nabla_{\alpha} \nabla_{\beta} R+g_{\alpha \beta} \square R \delta g^{\alpha \beta}\right) .
$$

Upon substitution of Eq. (8.19) into Eq. (8.14), one obtains

$$
\int d^{4} x \sqrt{-g} R \delta R=\int d^{4} x \sqrt{-g}\left(R \delta g^{\alpha \beta} R_{\alpha \beta}-\delta g^{\alpha \beta} \nabla_{\alpha} \nabla_{\beta} R+g_{\alpha \beta} \square R \delta g^{\alpha \beta}\right)
$$

and Eq. 8.13) takes the form

$$
\begin{aligned}
\delta \int d^{4} x \sqrt{-g} R^{2} & =-\frac{1}{2} \int d^{4} x \sqrt{-g} g_{\alpha \beta} \delta g^{\alpha \beta} R^{2}+2 \int d^{4} x \sqrt{-g}\left(R \delta g^{\alpha \beta} R_{\alpha \beta}-\delta g^{\alpha \beta} \nabla_{\alpha} \nabla_{\beta} R+g_{\alpha \beta} \square R \delta g^{\alpha \beta}\right) \\
& =\int d^{4} x \sqrt{-g}\left(2 R R_{\alpha \beta}-\frac{1}{2} g_{\alpha \beta} R^{2}\right) \delta g^{\alpha \beta}+2 \int d^{4} x \sqrt{-g}\left(g_{\alpha \beta} \square R-\nabla_{\alpha} \nabla_{\beta} R\right) \delta g^{\alpha \beta} .
\end{aligned}
$$

Substituting this equation into Eq. (8.12) and including the matter part of the Lagrangian $\mathcal{L}^{(m)}$ which produces the energy-momentum tensor $T_{\mu \nu}^{(m)}$, the field equations

$$
G_{\alpha \beta}+\alpha\left[2 R\left(R_{\alpha \beta}-\frac{1}{4} g_{\alpha \beta} R\right)+2\left(g_{\alpha \beta} \square R-\nabla_{\alpha} \nabla_{\beta} R\right)\right]=\kappa T_{\alpha \beta}^{(m)}
$$

are obtained; they are fourth-order equations for the metric components. The trace of Eq. (8.22) is

$$
\square R-\frac{1}{6 \alpha}\left(R+\kappa T^{(m)}\right)=0,
$$

which shows that $\alpha$ must be positive. One can also define an angular frequency $\omega$ (equivalent to a mass $m$ ) so that

$$
\frac{1}{6 \alpha}=\omega^{2}=m^{2}
$$

Following this definition, Eq. (8.23) becomes

$$
\square R-m^{2}\left(R+\kappa T^{(m)}\right)=0 .
$$

Eq. 8.25 can be seen as an effective Klein-Gordon equation for the effective scalar field degree of freedom $R$ (sometimes called scalaron).

\section{2. $f(R)$-gravity: the general case}

Let us discuss now a generic analytica 15 function $f(R)$ in the metric formalism, beginning with the vacuum case, as described by the Lagrangian density $\sqrt{-g} \mathcal{L}=\sqrt{-g} f(R)$ obeying the variational principle $\delta \int d^{4} x \sqrt{-g} f(R)=0$. We have

$$
\begin{aligned}
& \delta \int d^{4} x \sqrt{-g} f(R)=\int d^{4} x[\delta(\sqrt{-g} f(R))+\sqrt{-g} \delta(f(R))] \\
& =\int d^{4} x \sqrt{-g}\left[f^{\prime}(R) R_{\mu \nu}-\frac{1}{2} g_{\mu \nu} f(R)\right] \delta g^{\mu \nu}+\int d^{4} x \sqrt{-g} f^{\prime}(R) g^{\mu \nu} \delta R_{\mu \nu},
\end{aligned}
$$

where the prime denotes differentiation with respect to $R$. We now compute these integrals in the local inertial frame. By using

$$
g^{\mu \nu} \delta R_{\mu \nu}=g^{\mu \nu} \partial_{\sigma}\left(\delta G_{\mu \nu}^{\sigma}\right)-g^{\mu \sigma} \partial_{\sigma}\left(\delta G_{\mu \nu}^{\nu}\right) \equiv \partial_{\sigma} W^{\sigma}
$$

\footnotetext{
15 This assumption is not, strictly speaking, necessary and is sometimes relaxed in the literature.
} 
where

$$
W^{\sigma} \equiv g^{\mu \nu} \delta G_{\mu \nu}^{\sigma}-g^{\mu \sigma} \delta G_{\mu \nu}^{\nu}
$$

the second integral in Eq. 8.26 can be written as

$$
\int d^{4} x \sqrt{-g} f^{\prime}(R) g^{\mu \nu} \delta R_{\mu \nu}=\int d^{4} x \sqrt{-g} f^{\prime}(R) \partial_{\sigma} W^{\sigma} .
$$

Integration by parts yields

$$
\int d^{4} x \sqrt{-g} f^{\prime}(R) g^{\mu \nu} \delta R_{\mu \nu}=\int d^{4} x \frac{\partial}{\partial x^{\sigma}}\left[\sqrt{-g} f^{\prime}(R) W^{\sigma}\right]-\int d^{4} x \partial_{\sigma}\left[\sqrt{-g} f^{\prime}(R)\right] W^{\sigma} .
$$

The first integrand is a total divergence and can be discarded by assuming that the fields vanish at infinity, obtaining

$$
\int d^{4} x \sqrt{-g} f^{\prime}(R) g^{\mu \nu} \delta R_{\mu \nu}=-\int d^{4} x \partial_{\sigma}\left[\sqrt{-g} f^{\prime}(R)\right] W^{\sigma}
$$

Let us calculate now the term $W^{\sigma}$ appearing in Eq. (8.31). We have

$$
\delta G_{\mu \nu}^{\sigma}=\delta\left[\frac{1}{2} g^{\sigma \alpha}\left(\partial_{\mu} g_{\alpha \nu}+\partial_{\nu} g_{\mu \alpha}-\partial_{\alpha} g_{\mu \nu}\right)\right]=\frac{1}{2} g^{\sigma \alpha}\left[\partial_{\mu}\left(\delta g_{\alpha \nu}\right)+\partial_{\nu}\left(\delta g_{\mu \alpha}\right)-\partial_{\alpha}\left(\delta g_{\mu \nu}\right)\right]
$$

since in the locally inertial frame considered here it is

$$
\partial_{\alpha} g_{\mu \nu}=\nabla_{\alpha} g_{\mu \nu}=0
$$

Similarly, it is

$$
\delta G_{\mu \nu}^{\nu}=\frac{1}{2} g^{\nu \alpha} \partial_{\mu}\left(\delta g_{\nu \alpha}\right)
$$

By combining Eqs. (8.33) and (8.34), one obtains

$$
\begin{aligned}
g^{\mu \nu} \delta G_{\mu \nu}^{\sigma} & =\frac{1}{2} g^{\mu \nu}\left[-\partial_{\mu}\left(g_{\alpha \nu} \delta g^{\alpha \sigma}\right)-\partial_{\nu}\left(g_{\mu \alpha} \delta g^{\sigma \alpha}\right)-g^{\sigma \alpha} \partial_{\alpha}\left(\delta g_{\mu \nu}\right)\right]=\frac{1}{2} \partial^{\sigma}\left(g_{\mu \nu} \delta g^{\mu \nu}\right)-\partial^{\mu}\left(g_{\alpha \mu} \delta g^{\nu \alpha}\right) \\
g^{\mu \sigma} \delta G_{\mu \nu}^{\nu} & =-\frac{1}{2} \partial^{\sigma}\left(g_{\nu \alpha} \delta g^{\nu \alpha}\right)
\end{aligned}
$$

from which it follows immediately that

$$
W^{\sigma}=\partial^{\sigma}\left(g_{\mu \nu} \delta g^{\mu \nu}\right)-\partial^{\mu}\left(g_{\mu \nu} \delta g^{\sigma \nu}\right)
$$

Using this equation one can write

$$
\int d^{4} x \sqrt{-g} f^{\prime}(R) g^{\mu \nu} \delta R_{\mu \nu}=\int d^{4} x \partial_{\sigma}\left[\sqrt{-g} f^{\prime}(R)\right]\left[\partial^{\mu}\left(g_{\mu \nu} \delta g^{\sigma \nu}\right)-\partial^{\sigma}\left(g_{\mu \nu} \delta g^{\mu \nu}\right)\right] .
$$

Integrating by parts and discarding total divergences, one obtains

$$
\int d^{4} x \sqrt{-g} f^{\prime}(R) g^{\mu \nu} \delta R_{\mu \nu}=\int d^{4} x g_{\mu \nu} \partial^{\sigma} \partial_{\sigma}\left[\sqrt{-g} f^{\prime}(R)\right] \delta g^{\mu \nu}-\int d^{4} x g_{\mu \nu} \partial^{\mu} \partial_{\sigma}\left[\sqrt{-g} f^{\prime}(R)\right] \delta g^{\sigma \nu} .
$$

The variation of the action is then

$$
\begin{aligned}
& \delta \int d^{4} x \sqrt{-g} f(R)=\int d^{4} x \sqrt{-g}\left[f^{\prime}(R) R_{\mu \nu}-\frac{1}{2} f(R) g_{\mu \nu}\right] \delta g^{\mu \nu} \\
& +\int d^{4} x\left[g_{\mu \nu} \partial^{\sigma} \partial_{\sigma}\left(\sqrt{-g} f^{\prime}(R)\right)-g_{\sigma \nu} \partial^{\mu} \partial_{\sigma}\left(\sqrt{-g} f^{\prime}(R)\right)\right] \delta g^{\mu \nu}
\end{aligned}
$$

The vanishing of the variation implies the fourth order vacuum field equations

$$
f^{\prime}(R) R_{\mu \nu}-\frac{f(R)}{2} g_{\mu \nu}=\nabla_{\mu} \nabla_{\nu} f^{\prime}(R)-g_{\mu \nu} \square f^{\prime}(R) .
$$


These equations can be re-arranged in the Einstein-like form

$$
f^{\prime}(R) R_{\mu \nu}-\frac{f^{\prime}(R)}{2} g_{\mu \nu} R+\frac{f^{\prime}(R)}{2} g_{\mu \nu} R-\frac{f(R)}{2} g_{\mu \nu}=\nabla_{\mu} \nabla_{\nu} f^{\prime}(R)-g_{\mu \nu} \square f^{\prime}(R),
$$

and then

$$
G_{\mu \nu}=\frac{1}{f^{\prime}(R)}\left\{\nabla_{\mu} \nabla_{\nu} f^{\prime}(R)-g_{\mu \nu} \square f^{\prime}(R)+g_{\mu \nu} \frac{\left[f(R)-f^{\prime}(R) R\right]}{2}\right\}
$$

The right hand side of Eq. (8.43) is then regarded as an effective stress-energy tensor, which we call curvature fluid energy-momentum tensor $T_{\mu \nu}^{(\text {curv })}$ sourcing the effective Einstein equations. Although this interpretation is questionable in principle because the field equations describe a theory different from GR, and one is forcing upon them the interpretation as effective Einstein equations, this approach is quite useful in practice.

\section{A more general class of Extended Theories of Gravity}

ETGs exhibit two main features: first, the geometry can couple non-minimally to some scalar field; second, derivatives of the metric components of order higher than second may appear. In the first case, we say that we have scalar-tensor theories of gravity, and in the second case we have higher order theories. Combinations of non-minimally coupled and higher order terms can also emerge in effective Lagrangians, producing mixed higher order/scalar-tensor gravity.

A general class of higher-order-scalar-tensor theories in four dimensions is 16 given by the action

$$
\mathcal{S}=\int d^{4} x \sqrt{-g}\left[F\left(R, \square R, \square^{2} R, . . \square^{k} R, \phi\right)-\frac{\epsilon}{2} g^{\mu \nu} \phi_{; \mu} \phi_{; \nu}+2 \kappa \mathcal{L}^{(m)}\right],
$$

where $F$ is an unspecified function of curvature invariants and of a scalar field $\phi$. The term $\mathcal{L}^{(m)}$, as above, is the minimally coupled ordinary matter contribution; $\epsilon$ is a constant which specifies the theory. Actually its values can be $\epsilon= \pm 1,0$ fixing the nature and the dynamics of the scalar field which can be a standard scalar field, a phantom field or a field without dynamics (see [258, 439, 492] for details). In the metric approach, the field equations are obtained by varying (8.44) with respect to $g_{\mu \nu}$. We get

$$
\begin{aligned}
G^{\mu \nu}= & \frac{1}{\mathcal{G}}\left[\kappa T^{\mu \nu}+\frac{1}{2} g^{\mu \nu}(F-\mathcal{G} R)+\left(g^{\mu \lambda} g^{\nu \sigma}-g^{\mu \nu} g^{\lambda \sigma}\right) \mathcal{G}_{; \lambda \sigma}\right. \\
& +\frac{1}{2} \sum_{i=1}^{k} \sum_{j=1}^{i}\left(g^{\mu \nu} g^{\lambda \sigma}+g^{\mu \lambda} g^{\nu \sigma}\right)\left(\square^{j-i}\right)_{; \sigma}\left(\square^{i-j} \frac{\partial F}{\partial \square^{i} R}\right)_{; \lambda} \\
& \left.-g^{\mu \nu} g^{\lambda \sigma}\left(\left(\square^{j-1} R\right)_{; \sigma} \square^{i-j} \frac{\partial F}{\partial \square^{i} R}\right)_{; \lambda}\right],
\end{aligned}
$$

where $G^{\mu \nu}$ is the above Einstein tensor and

$$
\mathcal{G} \equiv \sum_{j=0}^{n} \square^{j}\left(\frac{\partial F}{\partial \square^{j} R}\right) .
$$

The differential Eqs.(8.45) are of order $(2 k+4)$. The stress-energy tensor is due to the kinetic part of the scalar field and to the ordinary matter:

$$
T_{\mu \nu}=T_{\mu \nu}^{(m)}+\frac{\epsilon}{2}\left[\phi_{; \mu} \phi_{; \nu}-\frac{1}{2} \phi_{;}^{\alpha} \phi_{; \alpha}\right] .
$$

The (eventual) contribution of a potential $V(\phi)$ is contained in the definition of $F$. From now on, we shall indicate by a capital $F$ a Lagrangian density containing also the contribution of a potential $V(\phi)$ and by $F(\phi), f(R)$, or $f(R, \square R)$

${ }^{16}$ For the aims of this review, we do not need more complicated invariants like $R_{\mu \nu} R^{\mu \nu}, R_{\mu \nu \alpha \beta} R^{\mu \nu \alpha \beta}, C_{\mu \nu \alpha \beta} C^{\mu \nu \alpha \beta}$ which are also possible. We will consider such invariants for the discussion of new polarizations and gravitational modes in Part III. 
a function of such fields without potential. By varying with respect to the scalar field $\phi$, we obtain the Klein-Gordon equation

$$
\epsilon \square \phi=-\frac{\partial F}{\partial \phi}
$$

Several approaches can be used to deal with such equations. For example, as we said, by a conformal transformation, it is possible to reduce an ETG to a (multi) scalar-tensor theory of gravity [103, 196, 297, 480, 579]. The simplest extension of GR is achieved assuming, as discussed,

$$
F=f(R), \quad \epsilon=0,
$$

in the action (8.44). The standard Hilbert-Einstein action is, of course, recovered for $f(R)=R$. Varying with respect to $g_{\alpha \beta}$, we get Eq. (8.41) and, after some manipulations, Eq. 8.43) where the gravitational contribution due to higher-order terms can be simply reinterpreted as a stress-energy tensor contribution. This means that additional and higher-order terms in the gravitational action act, in principle, as a stress-energy tensor, related to the form of $f(R)$. Considering also the standard perfect-fluid matter contribution, we have

$$
G_{\alpha \beta}=\frac{1}{f^{\prime}(R)}\left\{\frac{1}{2} g_{\alpha \beta}\left[f(R)-R f^{\prime}(R)\right]+f^{\prime}(R)_{; \alpha \beta}-g_{\alpha \beta} \square f^{\prime}(R)\right\}+\frac{\kappa T_{\alpha \beta}^{(m)}}{f^{\prime}(R)}=T_{\alpha \beta}^{(c u r v)}+\frac{\kappa T_{\alpha \beta}^{(m)}}{f^{\prime}(R)},
$$

where $T_{\alpha \beta}^{(\text {curv })}$ is an effective stress-energy tensor constructed by the extra curvature terms. In the case of GR, $T_{\alpha \beta}^{(c u r v)}$ identically vanishes while the standard, minimal coupling is recovered for the matter contribution. The peculiar behaviour of $f(R)=R$ is due to the particular form of the Lagrangian itself which, even though it is a second order Lagrangian, can be non-covariantly rewritten as the sum of a first order Lagrangian plus a pure divergence term. The Hilbert-Einstein Lagrangian can be in fact recast as follows:

$$
L_{H E}=\mathcal{L}_{H E} \sqrt{-g}=\left[p^{\alpha \beta}\left(\Gamma_{\alpha \sigma}^{\rho} \Gamma_{\rho \beta}^{\sigma}-\Gamma_{\rho \sigma}^{\rho} \Gamma_{\alpha \beta}^{\sigma}\right)+\nabla_{\sigma}\left(p^{\alpha \beta} u_{\alpha \beta}^{\sigma}\right)\right]
$$

where:

$$
p^{\alpha \beta}=\sqrt{-g} g^{\alpha \beta}=\frac{\partial \mathcal{L}}{\partial R_{\alpha \beta}}
$$

$\Gamma$ is the Levi-Civita connection of $g$ and $u_{\alpha \beta}^{\sigma}$ is a quantity constructed out with the variation of $\Gamma$ [583]. Since $u_{\alpha \beta}^{\sigma}$ is not a tensor, the above expression is not covariant; however a standard procedure has been studied to recast covariance in the first order theories. This clearly shows that the field equations should consequently be second order and the Hilbert-Einstein Lagrangian is thus degenerate. From the action (8.44), it is possible to obtain another interesting case by choosing

$$
F=F(\phi) R-V(\phi), \quad \epsilon=-1
$$

In this case, we get

$$
\mathcal{S}=\int V(\phi)\left[F(\phi) R+\frac{1}{2} g^{\mu \nu} \phi_{; \mu} \phi_{; \nu}-V(\phi)\right]
$$

$V(\phi)$ and $F(\phi)$ are generic functions describing respectively the potential and the coupling of a scalar field $\phi$. The Brans-Dicke theory of gravity is a particular case of the action (8.54) for $V(\phi)=0$. The variation with respect to $g_{\mu \nu}$ gives the second-order field equations

$$
F(\phi) G_{\mu \nu}=F(\phi)\left[R_{\mu \nu}-\frac{1}{2} R_{\mu \nu}\right]=-\frac{1}{2} T_{\mu \nu}^{\phi}-g_{\mu \nu} \square_{g} F(\phi)+F(\phi)_{; \mu \nu},
$$

here $\square_{g}$ is the d'Alembert operator with respect to the metric $g$. The energy-momentum tensor relative to the scalar field is

$$
T_{\mu \nu}^{\phi}=\phi_{; \mu} \phi_{; \nu}-\frac{1}{2} g_{\mu \nu} \phi_{; \alpha} \phi_{;}^{\alpha}+g_{\mu \nu} V(\phi)
$$

The variation with respect to $\phi$ provides the Klein - Gordon equation, i.e. the field equation for the scalar field:

$$
\square_{g} \phi-R F_{\phi}(\phi)+V_{\phi}(\phi)=0
$$

where $F_{\phi}(\phi)=\frac{d F(\phi)}{d \phi}, V_{\phi}(\phi)=\frac{d V(\phi)}{d \phi}$. This last equation is equivalent to the Bianchi contracted identity [101]. Standard fluid matter can be treated as above. 


\section{THE PALATINI FORMALISM}

The fundamental idea of the Palatini formalism is to regard the (usually torsion-free) connection $\Gamma_{\nu \alpha}^{\mu}$ entering the definition of the Ricci tensor as a variable independent of the space-time metric $g_{\mu \nu}$. The Palatini formulationof GR is equivalent to the metric version of this theory as a consequence of the fact that the field equations for the connection $\Gamma_{\mu \nu}^{\alpha}$ give the Levi-Civita connection of the metric $g_{\mu \nu}$ [577]. As a consequence, there is no particular reason to impose the Palatini variational principle in GR instead of the metric variational principle.

As we said, the situation is different in ETGs depending on functions of curvature invariants or for gravity nonminimally coupled to a scalar field. In these cases, the Palatini and the metric variational principle yield different field equations and different physics [269, 405]. The Palatini approach in the context of ETGs has been the subject of much interest in cosmological applications 106, 381, 382, 444, 573. As discussed above, considering the metric $g_{\mu \nu}$ and the connection $\Gamma_{\mu \nu}^{\alpha}$ as independent fields amounts to decoupling the metric structure of space-time and its geodesic structure with the connection $\Gamma_{\mu \nu}^{\alpha}$ being distinct from the Levi-Civita connection of $g_{\alpha \beta}$. In principle, this decoupling enriches the geometric structure of space-time and generalizes the purely metric formalism. By means of the Palatini field equations, this dual structure of space-time is naturally translated into a bimetric structure of the theory: instead of a metric and an independent connection, the Palatini formalism can be seen as containing two independent metrics $g_{\mu \nu}$ and $h_{\mu \nu}=f^{\prime}(R) g_{\mu \nu}$. In Palatini $f(R)$-gravity the new metric $h_{\mu \nu}$ determining the geodesics is related to the connection $\Gamma_{\mu \nu}^{\alpha}$ by the fact that the latter turns out to be the Levi-Civita connection of $h_{\mu \nu}$. In scalar-tensor gravity, the second metric $h_{\mu \nu}$ is related to the non-minimal coupling of the Brans-Dicke-like scalar. In the Palatini formalism the non-minimal coupling and the scalar field are separated from the metric structure of space-time. Physical consequences of this fact are discussed in 21, 141]. However, also other geometrical invariants, besides $R$, can be considered in the Palatini formalism. In [386], microscopic and macroscopic behaviors of Palatini modified gravity theories are discussed, in particular a detailed study of $f\left(R, R^{\mu \nu} R_{\mu \nu}\right)$-models is reported. In [458] dynamical aspects of Palatini $f(R), f\left(R^{\mu \nu} R_{\mu \nu}\right)$, and $f\left(R, R^{\mu \nu} R_{\mu \nu}\right)$-theories are studied. Isotropic and anisotropic bouncing cosmologies in Palatini $f(R)$ and $f\left(R^{\mu \nu} R_{\mu \nu}\right)$-theories are discussed in [46]. A Lagrangian of the type $\mathcal{L}=R+\alpha R_{\mu \nu} R^{\mu \nu}$ is also studied in Palatini formalism in the classical paper [98]. Here, for the sake of simplicity, we will discuss only the cases of $f(R)$-gravity and non-minimally coupled theories in Palatini formalism.

\section{A. The Palatini approach and the conformal structure}

Let us work out examples showing the role of conformal transformations in the Palatini approach to ETGs [16], beginning with fourth order gravity in which the difference between metric and Palatini variational principles is evident. The Ricci scalar in $f(\mathcal{R})$ is $\mathcal{R} \equiv \mathcal{R}(g, \Gamma) \equiv g^{\alpha \beta} \mathcal{R}_{\alpha \beta}(\Gamma)$ and is a generalized Ricci scalar, whereas $\mathcal{R}_{\mu \nu}(\Gamma)$ is the Ricci tensor of a torsion-free connection $\Gamma_{\mu \nu}^{\alpha}$ which, a priori, has no relations with the space-time metric $g_{\mu \nu}$. The gravitational sector of the theory is described by the analytical function $f(\mathcal{R})$, while $\sqrt{-g}$ denotes the usual scalar density of weight 1 . The field equations derived with the Palatini variational principle are

$$
\begin{aligned}
& f^{\prime}(\mathcal{R}) \mathcal{R}_{(\mu \nu)}(\Gamma)-\frac{f(\mathcal{R})}{2} g_{\mu \nu}=T_{\mu \nu}^{(m)}, \\
& \nabla_{\alpha}^{\Gamma}\left[\sqrt{-g} f^{\prime}(\mathcal{R}) g^{\mu \nu}\right]=0,
\end{aligned}
$$

where $\nabla_{\mu}^{\Gamma}$ is the covariant derivative of the non-metric connection $\Gamma_{\mu \nu}^{\alpha}$, and we use units in which $8 \pi G=1$. It is important to stress that Eq. (9.2) is obtained under the assumption that the matter sector described by $\mathcal{L}^{(m)}$ is functionally independent of the (non-metric) connection $\Gamma_{\mu \nu}^{\alpha}$; however it may contain metric covariant derivatives $\stackrel{g}{\nabla}$ of the matter fields. This means that the matter stress-energy tensor $T_{\mu \nu}^{(m)}[g, \Psi]$ depends on the metric $g_{\mu \nu}$ and on the matter fields collectively denoted by $\Psi$, together with their covariant derivatives with respect to the Levi-Civita connection of $g_{\mu \nu}$. It is easy to see from Eq. (9.2) that $\sqrt{-g} f^{\prime}(\mathcal{R}) g^{\mu \nu}$ is a symmetric tensor density of weight 1 , which naturally leads to the introduction of a new metric $h_{\mu \nu}$ conformally related to $g_{\mu \nu}$ by [16, 269]

$$
\sqrt{-g} f^{\prime}(\mathcal{R}) g^{\mu \nu}=\sqrt{-h} h^{\mu \nu} .
$$

With this definition $\Gamma_{\mu \nu}^{\alpha}$ is the Levi-Civita connection of the metric $h_{\mu \nu}$, with the only restriction that the conformal factor $\sqrt{-g} f^{\prime}(\mathcal{R}) g^{\mu \nu}$ relating $g_{\mu \nu}$ and $h_{\mu \nu}$ be non-degenerate. In the case of the Hilbert-Einstein Lagrangian it is $f^{\prime}(\mathcal{R})=1$ and the statement is trivial.

The conformal transformation

$$
g_{\mu \nu} \longrightarrow h_{\mu \nu}=f^{\prime}(\mathcal{R}) g_{\mu \nu}
$$


implies that $\mathcal{R}_{(\mu \nu)}(\Gamma)=\mathcal{R}_{\mu \nu}(h)$. It is useful to consider the trace of the field equations (9.1)

$$
f^{\prime}(\mathcal{R}) \mathcal{R}-2 f(\mathcal{R})=g^{\alpha \beta} T_{\alpha \beta}^{(m)} \equiv T^{(m)},
$$

which controls the solutions of Eq. (9.2). We refer to this scalar equation as the structural equation of space-time. In vacuo and in the presence of conformally invariant matter with $T^{(m)}=0$, this scalar equation admits constant solutions. In these cases, Palatini $f(\mathcal{R})$-gravity reduces to GR with a cosmological constant [269, 496]. In the case of interaction with matter fields, the structural equation (9.4), if explicitly solvable, provides in principle an expression $\mathcal{R}=F\left(T^{(m)}\right)$ and, as a result, both $f(\mathcal{R})$ and $f^{\prime}(\mathcal{R})$ can be expressed in terms of $T^{(m)}$. This fact allows one to express, at least formally, $\mathcal{R}$ in terms of $T^{(m)}$, which has deep consequences for the description of physical systems, as we will see later. Matter rules the bimetric structure of space-time and, consequently, both the geodesic and metric structures which are intrinsically different. This behaviour generalizes the vacuum case.

Let us now extend the Palatini formalism to non-minimally coupled scalar-tensor theories, with the goal of understanding the bimetric structure of space-time in these theories and its possible geometric and physical interpretation. We denote by $S_{1}$ the action functional of Palatini scalar-tensor theories, while non-minimal interaction between scalartensor and $f(R)$ gravities will be considered later, calling $S_{2}$ the respective action. Then, we will finally consider the case of scalar fields $\phi$ non-minimally coupled to the gravitational fields $\left(g_{\mu \nu}, \Gamma_{\mu \nu}^{\alpha}\right)$, denoting by $S_{3}$ the corresponding action. In this case, the low curvature limit $\mathcal{R} \rightarrow 0$, which is relevant for the present epoch of the history of the Universe, is particularly significant.

The scalar-tensor action can be generalized, in order to better develop the Palatini approach, as

$$
S_{1}=\int d^{4} x \sqrt{-g}\left[F(\phi) \mathcal{R}-\frac{\epsilon}{2} \stackrel{g}{\nabla}_{\mu} \phi \stackrel{g}{\nabla}^{\mu} \phi-V(\phi)+\mathcal{L}^{(m)}(\Psi, \stackrel{g}{\nabla} \Psi)\right],
$$

with $\epsilon= \pm 1$ corresponding to an ordinary scalar or a phantom field, respectively. The field equations for the metric $g_{\mu \nu}$ and the connection $\Gamma_{\mu \nu}^{\alpha}$ are

$$
\begin{aligned}
& F(\phi)\left(\mathcal{R}_{(\mu \nu)}-\frac{1}{2} g_{\mu \nu} \mathcal{R}\right)=T_{\mu \nu}^{(\phi)}+T_{\mu \nu}^{(m)}, \\
& \nabla_{\alpha}^{\Gamma}\left[\sqrt{-g} F(\phi) g^{\mu \nu}\right]=0,
\end{aligned}
$$

where $\mathcal{R}_{(\mu \nu)}$ is defined by Eq. (9.1). The equation of motion of the matter fields is

$$
\begin{aligned}
& \epsilon \square \phi=V_{\phi}(\phi)+F_{\phi}(\phi) \mathcal{R}, \\
& \frac{\delta \mathcal{L}^{(m)}}{\delta \Psi}=0 .
\end{aligned}
$$

In this case, the structural equation of space-time implies that

$$
\mathcal{R}=-\frac{\left(T^{(\phi)}+T^{(m)}\right)}{F(\phi)}
$$

where we must require that $F(\phi)>0$. The bimetric structure of space-time is thus defined by the ansatz

$$
\sqrt{-g} F(\phi) g^{\mu \nu}=\sqrt{-h} h^{\mu \nu}
$$

so that $h_{\mu \nu}$ is conformal to $g_{\mu \nu}$,

$$
h_{\mu \nu}=F(\phi) g_{\mu \nu} .
$$

It follows from Eq. (9.11) that in vacuo $T^{(\phi)}=0$ and $T^{(m)}=0$ this theory is equivalent to vacuum GR. If $F(\phi)=F_{0}=$ const. we recover GR with a minimally coupled scalar field, which means that the Palatini approach intrinsically gives rise to the conformal structure (9.13) of the theory which is trivial in the Einsteinean, minimally coupled, case. As a further step, let us generalize the previous results to the case of non-minimal coupling in the framework of $f(R)$ theories. The action functional can be written as

$$
S_{2}=\int d^{4} x \sqrt{-g}\left[F(\phi) f(\mathcal{R})-\frac{\epsilon}{2} \stackrel{g}{\nabla}_{\mu} \phi \stackrel{g}{\nabla}^{\mu} \phi-V(\phi)+\mathcal{L}^{(m)}(\Psi, \stackrel{g}{\nabla} \Psi)\right]
$$


where $f(\mathcal{R})$ is, as usual, an analytical function of $\mathcal{R}$. The Palatini field equations for the gravitational sector are

$$
\begin{aligned}
& F(\phi)\left[f^{\prime}(\mathcal{R}) \mathcal{R}_{(\mu \nu)}-\frac{f(\mathcal{R})}{2} g_{\mu \nu}\right]=T_{\mu \nu}^{(\phi)}+T_{\mu \nu}^{(m)}, \\
& \nabla_{\alpha}^{\Gamma}\left[\sqrt{-g} F(\phi) f^{\prime}(\mathcal{R}) g^{\mu \nu}\right]=0 .
\end{aligned}
$$

The equations of motion for the scalar and matter fields are

$$
\begin{aligned}
& \epsilon \square \phi=V_{\phi}(\phi)+F_{\phi}(\phi) f(\mathcal{R}), \\
& \frac{\delta \mathcal{L}^{(m)}}{\delta \Psi}=0,
\end{aligned}
$$

in which the non-minimal interaction term enters the modified Klein-Gordon equations. In this case, the structural equation of space-time implies that

$$
f^{\prime}(\mathcal{R}) \mathcal{R}-2 f(\mathcal{R})=\frac{T^{(\phi)}+T^{(m)}}{F(\phi)} .
$$

The bimetric structure of space-time is given by

$$
\sqrt{-g} F(\phi) f^{\prime}(\mathcal{R}) g^{\mu \nu}=\sqrt{-h} h^{\mu \nu}
$$

with $g_{\mu \nu}$ and $h_{\mu \nu}$ again conformally related,

$$
h_{\mu \nu}=F(\phi) f^{\prime}(\mathcal{R}) g_{\mu \nu} .
$$

Once the structural equation is solved, the conformal factor depends on the values of the matter fields $(\phi, \Psi)$ or, more precisely, on the traces of their stress-energy tensors and the value of $\phi$. In vacuo, Eq. (9.19) implies that the theory reduces again to Einstein gravity as for minimally interacting $f(R)$ theories [269]. The validity of this property is related to the decoupling of the scalar field from the metric.

Finally, let us discuss the situation in which the gravitational Lagrangian is a general function of $\phi$ and $\mathcal{R}$, as in

$$
S_{3}=\int d^{4} x \sqrt{-g}\left[K(\phi, \mathcal{R})-\frac{\epsilon}{2} \stackrel{g}{\nabla}_{\mu} \phi \stackrel{g}{\nabla}^{\mu} \phi-V(\phi)+\mathcal{L}^{(m)}(\Psi, \stackrel{g}{\nabla} \Psi)\right],
$$

which yields the gravitational field equations

$$
\begin{aligned}
& \frac{\partial K(\phi, \mathcal{R})}{\partial \mathcal{R}} \mathcal{R}_{(\mu \nu)}-\frac{K(\phi, \mathcal{R})}{2} g_{\mu \nu}=T_{\mu \nu}^{(\phi)}+T_{\mu \nu}^{(m)}, \\
& \nabla_{\alpha}^{\Gamma}\left[\sqrt{-g} \frac{\partial K(\phi, \mathcal{R})}{\partial \mathcal{R}} g^{\mu \nu}\right]=0,
\end{aligned}
$$

while the scalar and matter fields obey

$$
\begin{aligned}
& \epsilon \square \phi=V_{\phi}(\phi)+\frac{\partial K(\phi, \mathcal{R})}{\partial \phi}, \\
& \frac{\delta \mathcal{L}^{(m)}}{\delta \Psi}=0 .
\end{aligned}
$$

The structural equation of space-time can be expressed as

$$
\frac{\partial K(\phi, \mathcal{R})}{\partial \mathcal{R}} \mathcal{R}-2 K(\phi, \mathcal{R})=T^{(\phi)}+T^{(m)}
$$

When solved, Eq. 9.27) provides again the form of the Ricci scalar in terms of the traces of the stress-energy tensors of matter and of the scalar field (with $K(\phi, \mathcal{R})>0$ ). The bimetric structure of space-time is defined by

$$
\sqrt{-g} \frac{\partial K(\phi, \mathcal{R})}{\partial \mathcal{R}} g^{\mu \nu}=\sqrt{-h} h^{\mu \nu}
$$


with

$$
h_{\mu \nu}=\frac{\partial K(\phi, \mathcal{R})}{\partial \mathcal{R}} g_{\mu \nu} .
$$

The conformal factor depends on the matter fields only through the traces of their stress-energy tensors. The conformal factor and the bimetric structure are ruled by these traces and by the value of the scalar field $\phi$. In this case, in general, one does not recover GR, as is evident from Eq. (9.27) in which the strong coupling between $\mathcal{R}$ and $\phi$ prevents, even in vacuo, the possibility of obtaining constant solutions.

Let us discuss the $R \rightarrow 0$ regime, a good approximation to the present epoch of the observed Universe. The linear expansion of the analytical function $K(\phi, \mathcal{R})$

$$
K(\phi, \mathcal{R})=K_{0}(\phi)+K_{1}(\phi) \mathcal{R}+\mathrm{O}\left(\mathcal{R}^{2}\right)
$$

with

$$
K_{0}(\phi)=\left.K(\phi, \mathcal{R})\right|_{\mathcal{R}=0}, \quad K_{1}(\phi)=\left.\left(\frac{\partial K(\phi, \mathcal{R})}{\partial \mathcal{R}}\right)\right|_{\mathcal{R}=0},
$$

can be substituted into Eqs. (9.27) and (9.29) obtaining, to first order, the structural equation and the bimetric structure. The structural equation yields

$$
\left.\mathcal{R}=\frac{-1}{K_{1}(\phi)}\left[T^{(\phi)}+T^{(m)}\right)+2 K_{0}(\phi)\right]
$$

and the value of the Ricci scalar is always determined, in the linear approximation, in terms of $T^{(\phi)}, T^{(m)}$, and $\phi$. The bimetric structure is, otherwise, simply defined by the first term of the Taylor expansion, which is

$$
h_{\mu \nu}=K_{1}(\phi) g_{\mu \nu}
$$

reproducing, as expected, the scalar-tensor case (9.13). Scalar-tensor theories can then be recovered as the linear approximation of a general theory in which gravity and the non-minimal couplings are arbitrary (cf. Eqs. (9.32) and (9.19)). This fact agrees with the above considerations when the Lagrangians of physical interactions can be considered as locally gauge-invariant stochastic functions [51]. Finally, there exist also bimetric theories which cannot be conformally related [586] and torsion will also appear in the most general framework [105, 317]. These more general theories will not be discussed here.

\section{B. Equivalence between $f(R)$ and scalar-tensor gravity}

Metric and Palatini $f(R)$ gravities are equivalent to scalar-tensor theories with the derivative of the function $f(R)$ playing the role of the Brans-Dicke scalar, as has been re-discovered several times [168, 318, [542, 579, 588]. We illustrate this equivalence beginning with the metric formalism.

\section{Equivalence between scalar-tensor and metric $f(R)$-gravity}

In metric $f(R)$-gravity, we introduce the scalar $\phi \equiv R$; then the action

$$
S=\frac{1}{2 \kappa} \int d^{4} x \sqrt{-g} f(R)+S^{(m)}
$$

is rewritten in the form [168, 318, 542, 579, 588]

$$
S=\frac{1}{2 \kappa} \int d^{4} x \sqrt{-g}[\psi(\phi) R-V(\phi)]+S^{(m)}
$$

when $f^{\prime \prime}(R) \neq 0$, where

$$
\psi=f^{\prime}(\phi), \quad V(\phi)=\phi f^{\prime}(\phi)-f(\phi) .
$$


It is trivial to see that the action (9.35) coincides with (9.34) if $\phi=R$. Vice-versa, let us vary the action (9.35) with respect to $\phi$, which leads to

$$
R \frac{d \psi}{d \phi}-\frac{d V}{d \phi}=(R-\phi) f^{\prime \prime}(R)=0 .
$$

Eq. (9.37) implies that $\phi=R$ when $f^{\prime \prime}(R) \neq 0$. The action (9.35) has the Brans-Dicke form

$$
S=\frac{1}{2 \kappa} \int d^{4} x \sqrt{-g}\left[\psi R-\frac{\omega}{2} \nabla^{\mu} \psi \nabla_{\mu} \psi-U(\psi)\right]+S^{(m)}
$$

with Brans-Dicke field $\psi$, Brans-Dicke parameter $\omega=0$, and potential $U(\psi)=V[\phi(\psi)]$. An $\omega=0$ Brans-Dicke theory was originally studied for the purpose of obtaining a Yukawa correction to the Newtonian potential in the weak-field limit [449] and called "O'Hanlon theory" or "massive dilaton gravity". The variation of the action 9.35) yields the field equations

$$
\begin{aligned}
& G_{\mu \nu}=\frac{\kappa}{\psi} T_{\mu \nu}^{(m)}-\frac{1}{2 \psi} U(\psi) g_{\mu \nu}+\frac{1}{\psi}\left(\nabla_{\mu} \nabla_{\nu} \psi-g_{\mu \nu} \square \psi\right), \\
& 3 \square \psi+2 U(\psi)-\psi \frac{d U}{d \psi}=\kappa T^{(m)} .
\end{aligned}
$$

2. Equivalence between scalar-tensor and Palatini $f(R)$ - gravity

Palatini $f(R)$-gravity is also equivalent to a special Brans-Dicke theory with a scalar field potential. The Palatini action

$$
S=\frac{1}{2 \kappa} \int d^{4} x \sqrt{-g} f(\mathcal{R})+S^{(m)}
$$

is equivalent to

$$
S=\frac{1}{2 \kappa} \int d^{4} x \sqrt{-g}\left[f(\chi)+f^{\prime}(\chi)(\mathcal{R}-\chi)\right]+S^{(m)} .
$$

It is straightforward to see that the variation of this action with respect to $\chi$ yields $\chi=\mathcal{R}$. We can now use the field $\phi \equiv f^{\prime}(\chi)$ and the fact that the curvature $\mathcal{R}$ is the (metric) Ricci curvature of the new metric $h_{\mu \nu}=f^{\prime}(\mathcal{R}) g_{\mu \nu}$ conformally related to $g_{\mu \nu}$, as already explained. Using now the well known transformation property of the Ricci scalar under conformal rescalings [538, 577]

$$
\mathcal{R}=R+\frac{3}{2 \phi} \nabla^{\alpha} \phi \nabla_{\alpha} \phi-\frac{3}{2} \square \phi
$$

and discarding a boundary term, the action (9.42) can be presented in the form

$$
S=\frac{1}{2 \kappa} \int d^{4} x \sqrt{-g}\left[\phi R+\frac{3}{2 \phi} \nabla^{\alpha} \phi \nabla_{\alpha} \phi-V(\phi)\right]+S^{(m)},
$$

where

$$
V(\phi)=\phi \chi(\phi)-f[\chi(\phi)] .
$$

This action is clearly that of a Brans-Dicke theory with Brans-Dicke parameter $\omega=-3 / 2$ and a potential. This theory has been studied occasionally in the literature [25, 194, 198, 209, 449, 450], but it turns out to be a pathological case [41, 43, 263, 273, 455].

A study of anisotropic singularities in non-minimally coupled modified gravity models is reported in [270].

\section{CONFORMAL TRANSFORMATIONS AND EXTENDED THEORIES OF GRAVITY}

We have already mentioned the Jordan and the Einstein frame on several occasions: it is now time to look in detail at the conformal transformations providing different representations of ETGs and a solution-generating technique. In this subsection we present of conformal transformations application of conformal transformations to Brans-Dicke gravity first, and then to more general scalar-tensor and $f(R)$ theories. 


\section{A. The case of Brans-Dicke gravity}

In Brans-Dicke theory the choice of conformal factor 216]

$$
\Omega=\sqrt{G \phi}
$$

in the conformal transformation $g_{\mu \nu} \rightarrow \tilde{g}_{\mu \nu}=\Omega^{2} g_{\mu \nu}$ brings the gravitational sector of the Brans-Dicke action

$$
S_{B D}=\int d^{4} x \sqrt{-g}\left[\phi R-\frac{\omega}{\phi} g^{\alpha \beta} \nabla_{\alpha} \phi \nabla_{\beta} \phi-V(\phi)\right]+S^{(m)}
$$

into the Einstein frame form. Then the scalar field redefinition

$$
\tilde{\phi}(\phi)=\sqrt{\frac{2 \omega+3}{16 \pi G}} \ln \left(\frac{\phi}{\phi_{0}}\right),
$$

with $\phi>0$ and $\omega>-3 / 2$ transforms the scalar field kinetic energy density into canonical form. In terms of the variables $\left(\tilde{g}_{\mu \nu}, \tilde{\phi}\right)$, the Brans-Dicke action assumes its Einstein frame form

$$
S_{B D}=\int d^{4} x\left\{\sqrt{-\tilde{g}}\left[\frac{\tilde{R}}{16 \pi G}-\frac{1}{2} \tilde{g}^{\alpha \beta} \tilde{\nabla}_{\alpha} \tilde{\phi} \tilde{\nabla}_{\beta} \tilde{\phi}-U(\tilde{\phi})\right]+\exp \left(-8 \sqrt{\frac{\pi G}{2 \omega+3}} \tilde{\phi}\right) \mathcal{L}^{(m)}[\tilde{g}]\right\}
$$

where $\tilde{\nabla}_{\alpha}$ is the covariant derivative operator of the rescaled metric $\tilde{g}_{\alpha \beta}$ and

$$
U(\tilde{\phi})=V[\phi(\tilde{\phi})] \exp \left(-8 \sqrt{\frac{\pi G}{2 \omega+3}} \tilde{\phi}\right)=\frac{V(\phi)}{(G \phi)^{2}}
$$

is the Einstein frame potential. The restriction of the parameter range to $\omega>-3 / 2$ is sometimes attributed to the need of guaranteeing that it is possible to perform the conformal transformation. However, one could take the absolute value $|2 \omega+3|$ there, but in actual fact $\omega$ cannot cross the barrier $-3 / 2$ : the $\omega=-3 / 2$ Brans-Dicke theory is pathological. With a special potential, $\omega=-3 / 2$ Brans-Dicke theory is equivalent to Palatini $f(R)$ gravity. The Jordan frame scalar has the dimensions of $G^{-1}$, while the Einstein frame scalar $\tilde{\phi}$ has the dimensions of $G^{-1 / 2}$ and is usually measured in Planck masses. In the GR limit $\phi \rightarrow$ const., the Jordan and the Einstein frames coincide. However an important remark is necessary at this point. Though the original Brans-Dicke theory, for which the potential $V(\phi)$ is set to zero, is pathological for $\omega=-3 / 2$, it is not necessarily true in general. In fact, as soon as the potential is different from zero, the pathology can be removed. In this general sense, some models within the $\omega=-3 / 2$ are pathological but not all of them. It is worth noticing that some successful applications to the early Universe of this type of theories exist in literature showing that not all $\omega=-3 / 2$ are pathological, see e.g. [47, 348, 349, 456].

It is also important to stress that the Einstein frame representation of Brans-Dicke theory with $\omega=-3 / 2$ or $\omega<-3 / 2$ can be perfectly formulate as long as one provides the suitable generalization of Eq. (10.3). In this sense the case $\omega=-3 / 2$ sets a frontier between standard scalar fields and phantom fields, because, for $\omega<-3 / 2$, the kinetic term of the scalar field has the "wrong" sign in front of it. A detailed discussion of this point is reported in [196].

The inspection of the action (10.4) often leads people to state that, in the Einstein frame, gravity is described by GR, but there are two important differences between Einstein frame Brans-Dicke gravity and Einstein's theory. First, the free scalar $\tilde{\phi}$ acting as a source of gravity on the right hand side of the field equations is always present, $i . e$. , in the Einstein frame solutions of the vacuum field equations $\tilde{R}_{\mu \nu}=0$ cannot be obtained as in vacuum GR because the scalar $\tilde{\phi}$ pervades the space-time manifold and cannot be removed. This persistence is a reminder of the cosmological origin of $\phi \sim G_{\text {eff }}^{-1}$ in the original (Jordan frame) Brans-Dicke theory [86]. The scalar $\tilde{\phi}$ is always present even if, formally, the gravitational field is only described by the metric tensor $\tilde{g}_{\alpha \beta}$ in the Einstein frame. The conformal transformation shifts the Jordan frame gravitational variable $\phi$ into Einstein frame matter $17 \tilde{\phi}$.

The second difference between GR and Einstein frame Brans-Dicke theory consists of the fact that the matter Lagrangian $\mathcal{L}^{(m)}$ is now multiplied by the exponential factor in Eq. (10.4). This factor is described as an anomalous coupling of matter to the scalar $\tilde{\phi}$ which has no counterpart in GR. It is because of this coupling that the matter

\footnotetext{
17 This property makes it clear that the distinction between gravitational and non-gravitational degrees of freedom depends on the conformal representation of a gravitational theory.
} 
energy-momentum tensor $\tilde{T}_{\alpha \beta}^{(m)}$ in the Einstein frame obeys $\tilde{\nabla}^{\beta} \tilde{T}_{\alpha \beta}^{(m)}=-\tilde{T}^{(m)} \tilde{\nabla}_{\alpha}(\ln \Omega)$ instead of the GR conservation equation $\nabla^{\beta} T_{\alpha \beta}^{(m)}=0$. The modified conservation equation implies changes to the geodesic equation and to the equation of geodesic deviation, and the violation of the Equivalence Principle in the Einstein frame. Under the conformal transformation, the matter energy-momentum tensor $T_{\mu \nu}^{(m)}$ scales as

$$
\tilde{T}_{(m)}^{\alpha \beta}=\Omega^{s} T_{(m)}^{\alpha \beta}, \quad \tilde{T}_{\alpha \beta}^{(m)}=\Omega^{s+4} T_{\alpha \beta}^{(m)},
$$

where $s$ is an appropriate conformal weight. The conservation equation $\nabla^{\beta} T_{\alpha \beta}^{(m)}=0$ transforms (in four space-time dimensions) as [577]

$$
\tilde{\nabla}_{\alpha}\left(\Omega^{s} T_{(m)}^{\alpha \beta}\right)=\Omega^{s} \nabla_{\alpha} T_{(m)}^{\alpha \beta}+(s+6) \Omega^{s-1} T_{(m)}^{\alpha \beta} \nabla_{a} \Omega-\Omega^{s-1} g^{\alpha \beta} T^{(m)} \nabla_{\alpha} \Omega .
$$

It is convenient to choose the conformal weight $s=-6$ which yields, consistently with $\tilde{T}^{(m)}=\Omega^{-4} T^{(m)}$,

$$
\tilde{T}^{(m)} \equiv \tilde{g}^{\alpha \beta} \tilde{T}_{\alpha \beta}^{(m)}=\Omega^{-4} T^{(m)},
$$

and $\tilde{T}^{(m)}$ vanishes if and only if $T^{(m)}=0$. Eq. (10.7) assumes the form

$$
\tilde{\nabla}_{\alpha} \tilde{T}_{(m)}^{\alpha \beta}=-\tilde{T}^{(m)} \tilde{g}^{\alpha \beta} \tilde{\nabla}_{\alpha}(\ln \Omega) .
$$

Since $\Omega=\sqrt{G \phi}$ it is

$$
\tilde{\nabla}_{\alpha} \tilde{T}_{(m)}^{\alpha \beta}=-\frac{1}{2 \phi} \tilde{T}^{(m)} \tilde{\nabla}^{\beta} \phi
$$

or, in terms of the Einstein frame scalar [580],

$$
\tilde{\nabla}_{\alpha} \tilde{T}_{(m)}^{\alpha \beta}=-\sqrt{\frac{4 \pi G}{2 \omega+3}} \tilde{T}^{(m)} \tilde{\nabla}^{\beta} \tilde{\phi} .
$$

The geodesic equation receives corrections as a consequence of Eq. (10.11). Consider a dust fluid with energymomentum tensor

$$
\tilde{T}_{\alpha \beta}^{(m)}=\tilde{\rho}^{(m)} \tilde{u}_{\alpha} \tilde{u}_{\beta}
$$

Eq. (10.11) yields

$$
\tilde{u}_{\alpha} \tilde{u}_{\beta} \tilde{\nabla}^{\beta} \tilde{\rho}^{(m)}+\tilde{\rho}^{(m)} \tilde{u}_{\alpha} \tilde{\nabla}^{\beta} \tilde{u}_{\beta}+\tilde{\rho}^{(m)} \tilde{u}_{\gamma} \tilde{\nabla}^{\gamma} \tilde{u}_{\alpha}=\sqrt{\frac{4 \pi G}{2 \omega+3}} \tilde{\rho}^{(m)} \tilde{\nabla}_{\alpha} \tilde{\phi} .
$$

Using an affine parameter $\lambda$ along the fluid worldlines with tangent $\tilde{u}^{\mu}$, Eq. (10.13) becomes

$$
\tilde{u}_{\alpha}\left(\frac{d \tilde{\rho}^{(m)}}{d \lambda}+\tilde{\rho}^{(m)} \tilde{\nabla}^{\gamma} \tilde{u}_{\gamma}\right)+\tilde{\rho}^{(m)}\left(\frac{d \tilde{u}_{a}}{d \lambda}-\sqrt{\frac{4 \pi G}{2 \omega+3}} \tilde{\nabla}_{\alpha} \phi\right)=0 .
$$

This equations splits into the two equations

$$
\frac{d \tilde{\rho}^{(m)}}{d \lambda}+\tilde{\rho}^{(m)} \tilde{\nabla}^{\gamma} \tilde{u}_{\gamma}=0
$$

and

$$
\frac{d \tilde{u}^{\alpha}}{d \lambda}=\sqrt{\frac{4 \pi G}{2 \omega+3}} \tilde{\nabla}^{\alpha} \tilde{\phi}
$$

The geodesic equation is then modified in the Einstein frame as [172, 173, 580]

$$
\frac{d^{2} x^{\mu}}{d \lambda^{2}}+\tilde{\Gamma}_{\nu \sigma}^{\mu} \frac{d x^{\nu}}{d \lambda} \frac{d x^{\sigma}}{d \lambda}=\sqrt{\frac{4 \pi G}{2 \omega+3}} \tilde{\nabla}^{\mu} \tilde{\phi}
$$

The correction on the right hand side is often described as a fifth force proportional to the gradient $\tilde{\nabla}^{\mu} \tilde{\phi}$ that couples universally to all massive test particles. The Weak Equivalence Principle (universality of free fall) is violated by this fifth force because of the space-time dependence of $\tilde{\nabla}^{\mu} \tilde{\phi}$. Due to this coupling, scalar-tensor theories in the Einstein 
frame appear to be non-metric theories. On the other hand, it is well known that all metric theories of gravity satisfy the Weak Equivalence Principle [586], and the (non-)metricity becomes a statement on whether a theory satisfies or not it. Therefore, the metric character of ETGs, and whether they satisfy or not the Equivalence Principle, become properties dependent on the conformal frame representation. This fact leaves the foundation of relativistic gravity on a rather shaky ground, which is a problem especially when trying to isolate the fundamental properties of classical gravity which should be preserved in approaches to quantum or emergent gravity.

As expected, the equation of null geodesics is left unaffected by the conformal transformation: null geodesics receive no fifth force correction in the Einstein frame. This invariance is consistent with the fact that the equation of null geodesics can be derived from the Maxwell equations in the high frequency limit of the geometric optics approximation, in conjunction with the fact that Maxwell's equations are conformally invariant in a four-dimensional manifold. A more direct way of looking at conformal invariance for null geodesics is by noting that the electromagnetic field stressenergy tensor has vanishing trace $T=0$ and the corresponding conservation equation $\nabla^{\beta} T_{\alpha \beta}=0$ is unaffected by the conformal rescaling $g_{\mu \nu} \rightarrow \tilde{g}_{\mu \nu}=\Omega^{2} g_{\mu \nu}$, together with the geodesic equation for a null dust described by Eq. (10.12) when $\tilde{u}_{\mu} \tilde{u}^{\mu}=0$.

A correction to the timelike geodesic equation similar to the one discovered in Brans-Dicke theory appears in the low-energy limit of string theory [289, 541], in which the dilaton replaces the Brans-Dicke field and a similar coupling violates the Equivalence Principle [172, 173, 197, 541]. The violation is kept small in order not to violate the Solar System bounds [586]. In the low-energy limit of string theory the dilaton couples with different strengths to bodies of different nuclear composition which carry a dilatonic charge $q$, contrary to the Brans-Dicke field which couples universally to all forms of non-conformal matter. The formal substitution of the dilatonic charge $q$ with the factor $2 \sqrt{\pi G /(2 \omega+3)}$ allows a parallel between the two theories, but in string theory it may be possible to eliminate the coupling by setting the dilatonic charge $q$ to zero in certain cases, whereas the coupling of the Einstein frame Brans-Dicke scalar cannot be eliminated.

\section{B. Scalar-tensor theories}

More general scalar-tensor theories are described by the Jordan frame action

$$
S_{S T}=\int d^{4} x \sqrt{-g}\left\{\frac{1}{16 \pi G}\left[\phi R-\frac{\omega(\phi)}{\phi} g^{\alpha \beta} \nabla_{\alpha} \phi \nabla_{\beta} \phi\right]-V(\phi)+\alpha_{m} \mathcal{L}^{(m)}\right\},
$$

where $\alpha_{m}$ is the coupling constant of ordinary matter. The conformal factor is still given by Eq. (10.1) while the Einstein frame scalar field is defined by the differential relation

$$
d \tilde{\phi}=\sqrt{\frac{2 \omega(\phi)+3}{16 \pi G}} \frac{d \phi}{\phi} .
$$

The Einstein frame scalar-tensor action is

$$
S_{S T}=\int d^{4} x \sqrt{-\tilde{g}}\left[\frac{\tilde{R}}{16 \pi G}-\frac{1}{2} \tilde{g}^{\alpha \beta} \tilde{\nabla}_{\alpha} \tilde{\phi} \tilde{\nabla}_{\beta} \tilde{\phi}-U(\tilde{\phi})+\tilde{\alpha}_{m}(\phi) \mathcal{L}^{(m)}\right]
$$

with scalar field potential

$$
U(\tilde{\phi})=\frac{V[\phi(\tilde{\phi})]}{(G \phi)^{2}}
$$

and coupling

$$
\tilde{\alpha}_{m}(\tilde{\phi})=\frac{\alpha_{m}}{(G \phi)^{2}} .
$$

Again, Eq. (10.20) can be seen as the action for GR with a canonical scalar field which has positive-definite kinetic energy density, but with the important difference that the matter Lagrangian density is multiplied by the factor $\Omega^{-4}=(G \phi)^{-2}$, which can be interpreted as a variation of the coupling constant $\alpha_{m}$ with space and/or time. Again, this matter- $\tilde{\phi}$ coupling is responsible for the non-conservation of $\tilde{T}_{\mu \nu}^{(m)}$ as in Eq. (10.11), and for violating the Equivalence Principle. The conformal transformation technique has been used as a tool for generating exact solutions of a scalartensor theory beginning from known solutions of GR [53, 63, 309, 391, 556 561], and for deriving approximate solutions 
of the linearized theory [49]. This solution-generating technique is most convenient for solutions with vanishing potential: in fact, when a potential $V(\phi)$ is present, solutions that correspond to a physically well motivated potential in one frame generate, via the conformal mapping, solutions in the other frame which rarely correspond to a physical potential. Consider, for example, Brans-Dicke theory with a mass term $V(\phi)=m^{2} \phi^{2} / 2$ in the Jordan frame. The Einstein frame potential is

$$
U=\frac{1}{2}\left(\frac{m}{G}\right)^{2}
$$

i.e., a cosmological constant. A given functional form of $V(\phi)$ in the Jordan frame corresponds to a very different form of $U(\tilde{\phi})$ in the Einstein frame. Reversing the problem, which Jordan frame potential $V(\phi)$ produces a mass term $U(\tilde{\phi})=m^{2} \tilde{\phi}^{2} / 2$ in the Einstein frame? Eqs. (10.3) and (10.21) yield the answer

$$
V(\phi)=U(\tilde{\phi}) \phi^{2}=\frac{m^{2} G}{32 \pi}(2 \omega+3)\left[\phi \ln \left(\frac{\phi}{\phi_{1}}\right)\right]^{2},
$$

with $\phi_{1}$ is a constant. It would be difficult to motivate this potential from a known theory of particle physics. As a conclusion, it is legitimate to use exact solutions in the Einstein frame to generate solutions in the Jordan frame, but this procedure usually produces solutions of limited physical interest.

In $D>2$ space-time dimensions, the scalar-tensor theory described by the action

$$
S_{S T}^{(D)}=\int d^{D} x \sqrt{-g}\left[f(\phi) R-\omega(\phi) \nabla^{\alpha} \phi \nabla_{\alpha} \phi-V(\phi)+\alpha_{m} \mathcal{L}^{(m)}\right],
$$

can be conformally transformed according to

$$
g_{\alpha \beta} \longrightarrow \tilde{g}_{\alpha \beta}=f(\phi)^{\frac{2}{D-2}} g_{\alpha \beta},
$$

and

$$
d \tilde{\phi}=\frac{d \phi}{f(\phi)} \sqrt{f(\phi)+\frac{D-1}{D-2}\left(\frac{d f}{d \phi}\right)^{2}}
$$

producing the new scalar field potential in the Einstein frame

$$
\begin{aligned}
& U(\tilde{\phi})=\frac{V[\phi(\tilde{\phi})]}{f(\phi)^{\frac{D}{D-2}}} . \\
& \text { C. } \quad f(R, \phi) \text {-gravity }
\end{aligned}
$$

The action of generalized scalar-tensor gravity

$$
S=\frac{1}{16 \pi} \int d^{4} x \sqrt{-g}\left[f(\phi, R)-\frac{\epsilon}{2} g^{\alpha \beta} \nabla_{\alpha} \phi \nabla_{\beta} \phi\right]
$$

is mapped into its Einstein frame form by a conformal transformation which was rediscovered many times in particular realizations [50, 190, 297, 395, 508, 531, 542, 579, 588]. The conformal factor is

$$
\Omega=\left[16 \pi G\left|\frac{\partial f}{\partial R}\right|+\text { constant }\right]^{1 / 2}
$$

and, together with the scalar field redefinition

$$
\tilde{\phi}=\frac{1}{\sqrt{8 \pi G}} \sqrt{\frac{3}{2}} \ln \left[\sqrt{32 \pi} G\left|\frac{\partial f}{\partial R}\right|\right],
$$

allows the action (10.29) to be rewritten in the Einstein frame form

$$
S=\alpha \int d^{4} x \sqrt{-\tilde{g}}\left\{\frac{\tilde{R}}{16 \pi G}-\frac{1}{2} \tilde{g}^{\alpha \beta} \tilde{\nabla}_{\alpha} \tilde{\phi} \tilde{\nabla}_{\beta} \tilde{\phi}-\frac{\epsilon \alpha}{2} \exp \left[-\sqrt{\frac{16 \pi G}{3}} \tilde{\phi}\right]-U(\phi, \tilde{\phi})\right\},
$$


where 332,395 ]

$$
\alpha=\operatorname{sign}\left(\frac{\partial f}{\partial R}\right)
$$

and

$$
\begin{aligned}
& U(\phi, \tilde{\phi})=\alpha \mathrm{e}^{-8 \sqrt{\frac{\pi G}{3}} \tilde{\phi}}\left[\frac{\alpha R(\phi, \tilde{\phi})}{16 \pi G} \exp \left(\sqrt{\frac{16 \pi G}{3}} \tilde{\phi}\right)-F(\phi, \tilde{\phi})\right], \\
& F(\phi, \tilde{\phi})=f[\phi, R(\phi, \tilde{\phi})] .
\end{aligned}
$$

This action describes a non-linear $\sigma$-model with canonical gravity and two scalar fields $\phi$ and $\tilde{\phi}$ which reduce to a single one if $f(\phi, \tilde{\phi})$ is linear in $R$. In this case the Einstein frame action is

$$
S=\frac{|f|}{f} \int d^{4} x \sqrt{-\tilde{g}}\left[\frac{\tilde{R}}{16 \pi G}-\frac{1}{2} \tilde{g}^{\alpha \beta} \tilde{\nabla}_{\alpha} \tilde{\phi} \tilde{\nabla}_{\beta} \tilde{\phi}-U(\tilde{\phi})\right],
$$

where

$$
\begin{aligned}
\tilde{\phi} & =\frac{1}{\sqrt{8 \pi G}} \int d \phi\left[\frac{2 \epsilon f(\phi)+6(d f / d \phi)^{2}}{4 f^{2}(\phi)}\right]^{1 / 2}, \\
U(\tilde{\phi}) & =\frac{\operatorname{sign}(f) V(\phi)}{(16 \pi G f)^{2}}
\end{aligned}
$$

in which $\phi=\phi(\tilde{\phi})$.

\section{The interpretation of conformal frames}

Some considerations are in order at this point. The conformal transformation from the Jordan to the Einstein frame is a mathematical map which allows one to study several aspects of scalar-tensor gravity, $f(R)$-gravity and, in general, any ETG. However, having now available both the Jordan and the Einstein conformal frames (and infinitely many other conformal frames could be defined by choosing the conformal factor $\Omega$ arbitrarily), one wonders whether the two frames are also physically equivalent or only mathematically related. In other words, the problem is whether the physical meaning of the theory is "preserved" or not by the use of conformal transformations. One has now the metric $g_{\mu \nu}$ and its conformal cousin $\tilde{g}_{\mu \nu}$ and the question has been posed of which one is the "physical metric", $i . e$. , the metric from which curvature, geometry, and physical effects should be calculated and compared with experiment [141]. The issue of "which frame is the physical one" has been debated for a long time and it regularly resurfaces in the literature, with authors arguing in favor of one frame against the other, and others supporting the view that the two frames are physically equivalent and that the issue is a pseudo-problem. Many errors in the literature over the years, from advocates of both points of view, have contributed to confusion.

The first to approach this issue seems to have been Fierz (see 305]) but the first popular argument is due to Dicke, who presented it in the paper introducing the conformal transformation for Brans-Dicke theory [216]. Dicke's argument is that physics must be invariant under a rescaling of units and the conformal transformation is merely a local rescaling: units are not changed rigidly over the entire space-time manifold, but by amounts which are different at different space-time points. In Dicke's view, the two frames are equivalent provided that the units of mass, length, and time, and quantities derived from them scale with appropriate powers of the conformal factor in the Einstein frame [216].

With this view in mind, it is not difficult to see why many authors consider the issue of which conformal frame is physical a pseudo-problem. In principle, it is difficult to object to this argument, but there are two difficulties:

1. even though Dicke's argument is clear in principle, its application to practical situations is a different matter. The view that the two conformal frames are merely different representations of the same theory, similar to different gauges of a gauge theory, should be checked explicitly using the equations describing the physics. "Physical equivalence" is a vague concept because one can consider many different matter (or test) fields in 
curved space-time and different types of physics, or different physical aspects of a problem. When checking explicitly the physical equivalence between the two frames, one has to specify which physical field, or physical process is considered and the equations describing it. The equivalence could then be shown explicitly, but there is no proof that holds for all of physics, for example for Klein-Gordon fields, spinors, for cosmology, black holes, etc. While physical equivalence has been proved for various physical aspects, no proof comprehensive of all physical fields and different physical applications exists.

2. Dicke's argument is purely classical. In cosmology, black hole physics, and ETGs quantum fields in curved space play a significant role and the equivalence of conformal frames is not clear at all at the quantum level. Of course, not much is known about this equivalence in quantum gravity due to the lack of a definitive quantum gravity theory, but when the metric $g_{\mu \nu}$ is quantized in full quantum gravity approaches, inequivalent quantum theories are found [32, 240, 274, 305]. One can consider the semiclassical regime in which gravity is classical and the matter fields are quantized: again, one would expect the conformal frames to be inequivalent because the conformal transformation can be seen as a Legendre transformation [405], similar to the Legendre transformation of the classical mechanics of point particles which switches from the canonical Lagrangian coordinates $q$ to the variables $\{q, p\}$ of the Hamiltonian formalism. Now, it is well known that Hamiltonians that are classically equivalent become inequivalent when quantized, producing different energy spectra and scattering amplitudes [100, 204, 294]. However, the conformal equivalence between Jordan and Einstein frame seems to hold to some extent at the semiclassical level 274] 18 Again, only a particular kind of physics has been considered and one cannot make statements about all possible physical situations.

Unfortunately, the scaling of units in the Einstein frame is often forgotten, producing results that either do not make sense or are partially or totally incorrect, or sometimes the error is inconsequential 19 reinforcing the opposite view that the two frames are completely equivalent. While Dicke's explanation is very appealing and several claims supporting the view that the two frames are inequivalent turned out to be incorrect because they simply neglected the scaling of units in the Einstein frame, one should not forget that Dicke's argument is not inclusive of all areas of physics and it is better to check explicitly that the physics of a certain field does not depend on the conformal representation and not make sweeping statements. Certain points have been raised in the literature which either constitute a problem for Dicke's view, or, at least, indicate that this viewpoint cannot be applied blindly, including the following.

- Massive test particles follow timelike geodesics in the Jordan frame, while they deviate from geodesic motion in the Einstein frame due to a force proportional to the gradient of the scalar field (equivalently, of the conformal factor or of the varying mass unit [261]). Hence, the Weak Equivalence Principle is satisfied in the Jordan frame but not in the Einstein frame due to the coupling of the scalar field to ordinary matter, or to the variation of units. Since the Equivalence Principle is the foundation of relativistic gravity, this aspect is important and there are two ways to look at it. One can cherish the view that the two conformal frames are equivalent also with respect to the Equivalence Principle, which implies that the latter is formulated in a way that depends on the conformal frame representation. Or, one could view the violation of the Weak Equivalence Principle in the Einstein frame more pragmatically by saying that "physical equivalence" of the two frames is a vague term which must be defined precisely and this concept cannot be used blindly, in fact the Equivalence Principle of standard textbooks holds only in one frame but not in the other. This fact could be used as an argument against the physical equivalence of the frames.

- The Brans-Dicke-like scalar field easily violates all of the energy conditions in the Jordan frame, but satisfies them in the Einstein frame. While this fact does not eliminate singularities in one frame leaving them in the other 261] (i.e., the two frames are equivalent with respect to the presence of singularities), one cannot say that the two frames are "equivalent" with respect to the energy conditions. This difficulty arises because part of the matter sector of the theory, in the Einstein frame, comes from the conformal factor; in other words, the conformal transformation mixes matter and geometric degrees of freedom, which is the source of many interpretational problems [61, 102]. Thus, even if the theory turns out to be independent of the conformal representation, its interpretation is not.

18 A common argument among particle physicists relies on the equivalence theorem of Lagrangian field theory stating that the $S$-matrix is invariant under local (nonlinear) field redefinitions [79, 171, 185, 229, [351, [371, 498]. Since the conformal transformation is, essentially, a field redefinition, it would seem that quantum physics is invariant under change of the conformal frame. However, the field theory in which the equivalence theorem is derived applies to gravity only in the perturbative regime in which the fields deviate slightly from Minkowski space. In this regime, tree level quantities can be calculated in any conformal frame with the same result, but in the non-perturbative regime field theory and the equivalence theorem do not apply.

19 Dicke himself applied the conformal transformation and the scaling of units incorrectly [217] in GR cosmology (see Ref. [155, 261]). 


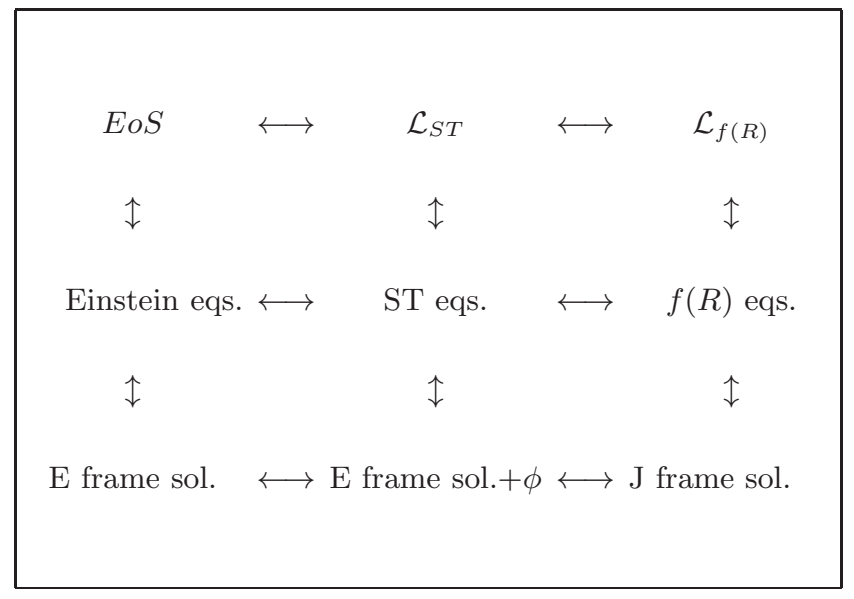

Table I. Three approaches (EoS, scalar-tensor and $f(R)$ ) compared at the level of Lagrangians, field equations, and their solutions. Mathematical equivalence of the three levels does not automatically imply physical equivalence of the solutions.

- There are studies of FLRW cosmology in which the Universe accelerates in one frame but not in the other. From the pragmatic point of view of an astronomer attempting to fit observational data (for example, type Ia supernovae data to a model of the present acceleration of the Universe), the two frames certainly do not appear to be "physically equivalent" [117, 150].

To approach correctly the problem of physical equivalence under conformal transformations, one can compare physics in different conformal frames at the level of the Lagrangian, of the field equations, and of their solutions. This comparison may not always be easy but, in certain cases, it is extremely useful to discriminate between frames. It has been adopted, for example, in Ref. [118], to compare cosmological models in the Einstein and the Jordan frame. Specifically, it has been shown that solutions of $f(R)$ and scalar-tensor gravity cannot be assumed to be physically equivalent to those in the Einstein frame when matter fields are given by generalized Equations of State (EoS). The situation is summarized in Table 【.

In these, and in other situations, one must specify precisely what "physical equivalence" means. In certain situations physical equivalence is demonstrated simply by taking into account the coupling of the Brans-Dicke-like scalar field to matter and the varying units in the Einstein frame, but in other cases the physical equivalence is not obvious and it does not seem to hold. At the very least, this equivalence, if it is valid at all, must be defined in precise terms and discussed in ways that are far from obvious. For this reason, it would be too simplistic to dismiss the issue of the conformal frame entirely as a pseudo-problem that has been solved for all physical situations of interest. It is fair to say that there have been surprises and non-trivial difficulties have been uncovered.

\section{EXTENDED THEORIES WITH TORSION}

In this section, we want to face the problem to study $f(R)$-gravity considering also torsion. Torsion theories have been taken into account firstly by Cartan and then where introduced by Sciama and Kibble in order to deal with spin in GR (see [317] for a review). Being the spin as fundamental as the mass of the particles, torsion was introduced in order to complete the following scheme: the mass (energy) as the source of curvature and the spin as the source of torsion.

Up to some time ago, torsion did not seem to produce models with observable effects since phenomena implying spin and gravity were considered to be significant only in the very early Universe. After, it has been proven that spin is not the only source of torsion. As a matter of fact, torsion field can be decomposed in three irreducible tensors, with different properties. In [105], a systematic classification of these different types of torsion and their possible sources is discussed. This means that a wide class of torsion models could be investigate independently of spin as their source.

In principle, torsion could be constrained at every astrophysical scale and, as recently discussed, data coming from Gravity Probe B could contribute to this goal also at Solar System level [400].

In above section, a systematic discussion of metric-affine $f(R)$-gravity has been pursued. Here, following the same philosophy, we want to show that, starting from a generic $f(R)$ theory, the curvature and the torsion can give rise to an effective curvature-torsion stress-energy tensor capable, in principle, to address the problem of the Dark Side of 
the Universe in a very general geometric scheme [128]. We do not consider the possible microscopic distribution of spin but a general torsion vector field in $f(R)$-gravity.

\section{A. The $f(R)$-field equations with torsion}

Let us discuss the main features of a $f(R)$-gravity considering the most general case in which torsion is present in a $\mathcal{U}_{4}$ manifold 20 [126. In a metric-affine formulation, the metric $g$ and the connection $\Gamma$ can be, in general, considered independent fields. More precisely, the dynamical fields are pairs $(g, \Gamma)$ consisting of a pseudo-Riemannian metric $g$ and a metric compatible linear connection $\Gamma$ on the space-time manifold $M$. The corresponding field equations are derived by varying separately with respect to the metric and the connection the action functional

$$
\mathcal{S}(g, \Gamma)=\int \sqrt{-g} f(\mathcal{R}) d s
$$

where $f$ is a real function, $\mathcal{R}(g, \Gamma)=g^{\mu \nu} \mathcal{R}_{\mu \nu}$ (with $\mathcal{R}_{\mu \nu}:=\mathcal{R}_{\mu \sigma \nu}^{\sigma}$ ) is the scalar curvature associated with the connection $\Gamma$ and $d s:=d x^{1} \wedge \cdots \wedge d x^{4}$. We use the index notation

$$
\mathcal{R}_{\gamma \mu \nu}^{\sigma}=\frac{\partial \Gamma_{\nu \gamma}^{\sigma}}{\partial x^{\mu}}-\frac{\partial \Gamma_{\mu \gamma}^{\sigma}}{\partial x^{\nu}}+\Gamma_{\mu \phi}^{\sigma} \Gamma_{\nu \gamma}^{\phi}-\Gamma_{\nu \phi}^{\sigma} \Gamma_{\mu \gamma}^{\phi}
$$

for the curvature tensor and

$$
\nabla_{\frac{\partial}{\partial x^{\mu}}} \frac{\partial}{\partial x^{\nu}}=\Gamma_{\mu \nu}^{\sigma} \frac{\partial}{\partial x^{\sigma}}
$$

for the connection coefficients. In order to evaluate the variation $\delta \mathcal{S}$ under arbitrary deformations of the connection, we recall that, given a metric tensor $g_{\mu \nu}$, every metric connection $\Gamma$ may be expressed as

$$
\Gamma_{\mu \nu}^{\sigma}=\tilde{\Gamma}_{\mu \nu}^{\sigma}-K_{\mu \nu}^{\sigma}
$$

where (in the holonomic basis $\left.\left\{\frac{\partial}{\partial x^{\mu}}, d x^{\mu}\right\}\right) \tilde{\Gamma}_{\mu \nu}^{\sigma}$ denote the coefficients of the Levi-Civita connection associated with the metric $g_{\mu \nu}$, while $K_{\mu \nu}{ }^{\sigma}$ indicate the components of a tensor satisfying the antisymmetry property $K_{\mu}{ }^{\nu \sigma}=-K_{\mu}{ }^{\sigma \nu}$. This last condition ensures the metric compatibility of the connection $\Gamma$. In view of this, we can identify the actual degrees of freedom of the theory with the (independent) components of the metric $g$ and the tensor $K$. Moreover, it is easily seen that the curvature and the contracted curvature tensors associated with every connection (11.4) can be expressed respectively as

$$
\mathcal{R}_{\mu \eta \nu}^{\sigma}=\tilde{R}_{\mu \eta \nu}^{\sigma}+\tilde{\nabla}_{\nu} K_{\eta \mu}{ }^{\sigma}-\tilde{\nabla}_{\eta} K_{\nu \mu}{ }^{\sigma}+K_{\nu \mu}{ }^{\phi} K_{\eta \phi}{ }^{\sigma}-K_{\eta \mu}{ }^{\phi} K_{\nu \phi}{ }^{\sigma},
$$

and

$$
\mathcal{R}_{\mu \nu}=\mathcal{R}_{\mu \sigma \nu}^{\sigma}=\tilde{R}_{\mu \nu}+\tilde{\nabla}_{\nu} K_{\sigma \mu}{ }^{\sigma}-\tilde{\nabla}_{\sigma} K_{\nu \mu}{ }^{\sigma}+K_{\nu \mu}{ }^{\phi} K_{\sigma \phi}{ }^{\sigma}-K_{\sigma \mu}{ }^{\phi} K_{\nu \phi}{ }^{\sigma},
$$

where $\tilde{R}_{\mu \eta \nu}^{\sigma}$ and $\tilde{R}_{\mu \nu}=\tilde{R}_{\mu \sigma \nu}^{\sigma}$ are respectively the Riemann and the Ricci tensors of the Levi-Civita connection $\tilde{\Gamma}$ associated with the given metric $g$, and $\tilde{\nabla}$ indicates the Levi-Civita covariant derivative.

Making use of the identities (11.5b), the action functional (11.1) can be written in the equivalent form

$$
\mathcal{S}(g, \Gamma)=\int \sqrt{-g} f\left(g^{\mu \nu}\left(\tilde{R}_{\mu \nu}+\tilde{\nabla}_{\nu} K_{\sigma \mu}^{\sigma}-\tilde{\nabla}_{\sigma} K_{\mu \nu}^{\sigma}+K_{\nu \mu}^{\phi} K_{\sigma \phi}^{\sigma}-K_{\sigma \mu}^{\phi} K_{\nu \phi}^{\sigma}\right)\right) d s
$$

more suitable for variations in the connection. Taking the metric $g$ fixed, we have the identifications $\delta \Gamma_{\mu \nu} \sigma=\delta K_{\mu \nu}{ }^{\sigma}$ and then the variation

$$
\delta \mathcal{S}=\int \sqrt{-g} f^{\prime}(\mathcal{R}) g^{\mu \nu}\left(\tilde{\nabla}_{\nu} \delta K_{\sigma \mu}^{\sigma}-\tilde{\nabla}_{\sigma} \delta K_{\nu \mu}{ }^{\sigma}+\delta K_{\nu \mu}{ }^{\phi} K_{\sigma \phi}{ }^{\sigma}+K_{\nu \mu}{ }^{\phi} \delta K_{\sigma \phi}{ }^{\sigma}-\delta K_{\sigma \mu}{ }^{\phi} K_{\nu \phi}{ }^{\sigma}-K_{\sigma \mu}{ }^{\phi} \delta K_{\nu \phi}{ }^{\sigma}\right) d s .
$$

${ }^{20}$ We indicate with $\mathcal{V}_{4}$ a $4 D$ pseudo-Riemaniann manifold without torsion and with $\mathcal{U}_{4}$ a $4 D$ manifold with torsion. Some of the notions already introduced in Sec. III will be adopted here. 
Using the divergence theorem, taking the antisymmetry properties of $K$ into account and renaming finally some indexes, we get the expression

$$
\delta \mathcal{S}=\int \sqrt{-g}\left[-\frac{\partial f^{\prime}}{\partial x^{\mu}} \delta_{\nu}^{\sigma}+\frac{\partial f^{\prime}}{\partial x^{\nu}} \delta_{\mu}^{\sigma}+f^{\prime} K_{\phi \nu}^{\phi} \delta_{\mu}^{\sigma}-f^{\prime} K_{\phi \mu}^{\phi} \delta_{\nu}^{\sigma}-f^{\prime} K_{\mu \nu}{ }^{\sigma}+f^{\prime} K_{\nu \mu}{ }^{\sigma}\right] \delta K_{\sigma}{ }^{\mu \nu} d s .
$$

The requirement $\delta \mathcal{S}=0$ yields therefore a first set of field equations given by

$$
K_{\phi \nu}^{\phi} \delta_{\mu}^{\sigma}-K_{\phi \mu}^{\phi} \delta_{\nu}^{\sigma}-K_{\mu \nu}^{\sigma}+K_{\nu \mu}^{\sigma}=\frac{1}{f^{\prime}} \frac{\partial f^{\prime}}{\partial x^{\phi}}\left(\delta_{\mu}^{\phi} \delta_{\nu}^{\sigma}-\delta_{\nu}^{\phi} \delta_{\mu}^{\sigma}\right)
$$

Considering that the torsion coefficients of the connection $\Gamma$ are $T_{\mu \nu}{ }^{\sigma}:=\Gamma_{\mu \nu}{ }^{\sigma}-\Gamma_{\nu \mu}{ }^{\sigma}=-K_{\mu \nu}{ }^{\sigma}+K_{\nu \mu}{ }^{\sigma}$ and thus (due to antisymmetry) $T_{\phi \mu}^{\phi}=-K_{\phi \mu}^{\phi}$, eqs. (11.9) can be rewritten as

$$
T_{\mu \nu}^{\sigma}+T_{\nu \phi}^{\phi} \delta_{\mu}^{\sigma}-T_{\mu \phi}^{\phi} \delta_{\nu}^{\sigma}=\frac{1}{f^{\prime}} \frac{\partial f^{\prime}}{\partial x^{\phi}}\left(\delta_{\mu}^{\phi} \delta_{\nu}^{\sigma}-\delta_{\nu}^{\phi} \delta_{\mu}^{\sigma}\right)
$$

or, equivalently, as

$$
T_{\mu \nu}^{\sigma}=-\frac{1}{2 f^{\prime}} \frac{\partial f^{\prime}}{\partial x^{\phi}}\left(\delta_{\mu}^{\phi} \delta_{\nu}^{\sigma}-\delta_{\nu}^{\phi} \delta_{\mu}^{\sigma}\right)
$$

In order to study the variation $\delta \mathcal{S}$ under arbitrary deformations of the metric, it is convenient to resort to the representation (11.1). Indeed, from the latter, we have directly

$$
\delta \mathcal{S}=\int \sqrt{-g}\left[f^{\prime}(\mathcal{R}) \mathcal{R}_{\mu \nu}-\frac{1}{2} f(\mathcal{R}) g_{\mu \nu}\right] \delta g^{\mu \nu} d s,
$$

thus getting the second set of field equations

$$
f^{\prime}(\mathcal{R}) \mathcal{R}_{(\mu \nu)}-\frac{1}{2} f(\mathcal{R}) g_{\mu \nu}=0
$$

Of course, one can obtain the same equations (11.13) starting from the representation (11.6) instead of (11.1). In that case, the calculations are just longer. As a remark concerning Eqs. (11.13), it is worth noticing that any connection satisfying Eqs. (11.4) and (11.11) gives rise to a contracted curvature tensor $\mathcal{R}_{\mu \nu}$ automatically symmetric. Indeed, since the tensor $K$ coincides necessarily with the contorsion tensor, namely

$$
K_{\mu \nu}^{\sigma}=\frac{1}{2}\left(-T_{\mu \nu}^{\sigma}+T_{\nu}^{\sigma}{ }_{\mu}-T_{\mu \nu}^{\sigma}\right)
$$

from Eqs. 11.11 we have

$$
K_{\mu \nu}^{\sigma}=\frac{1}{3}\left(T_{\nu} \delta_{\mu}^{\sigma}-T_{\phi} g^{\phi \sigma} g_{\mu \nu}\right)
$$

being

$$
T_{\mu}:=T_{\mu \sigma}^{\sigma}=-\frac{3}{2 f^{\prime}} \frac{\partial f^{\prime}}{\partial x^{\mu}} .
$$

Inserting Eq. (11.15) in Eq (11.5b), the contracted curvature tensor can be represented as

$$
\mathcal{R}_{\mu \nu}=\tilde{R}_{\mu \nu}+\frac{2}{3} \tilde{\nabla}_{\nu} T_{\mu}+\frac{1}{3} \tilde{\nabla}_{\sigma} T^{\sigma} g_{\mu \nu}+\frac{2}{9} T_{\mu} T_{\nu}-\frac{2}{9} T_{\sigma} T^{\sigma} g_{\mu \nu} .
$$

The last expression, together with Eqs. (11.16), entails the symmetry of the indexes $\mu$ and $\nu$. Therefore, in Eq. (11.13) we can omit the symmetrization symbol and write

$$
f^{\prime}(\mathcal{R}) \mathcal{R}_{\mu \nu}-\frac{1}{2} f(\mathcal{R}) g_{\mu \nu}=0
$$

Now, considering the trace of the equation (11.18), we get

$$
f^{\prime}(\mathcal{R}) \mathcal{R}-2 f(\mathcal{R})=0 .
$$


The latter is an identity automatically satisfied by all possible values of $\mathcal{R}$ only in the special case $f(\mathcal{R})=\alpha \mathcal{R}^{2}$. In all other cases, Eq. (11.19) represents a constraint on the scalar curvature $\mathcal{R}$. As a conclusion follows that, if $f(\mathcal{R}) \neq \alpha \mathcal{R}^{2}$, the scalar curvature $\mathcal{R}$ has to be constant (at least on connected domains) and coincides with a given solution value of (11.19). In such a circumstance, Eqs.(11.11) imply that the torsion $T_{\mu \nu}{ }^{\sigma}$ has to be zero and the theory reduces to a $f(\mathcal{R})$-theory without torsion. In particular, we notice that in the case $f(\mathcal{R})=\mathcal{R}$, eq. (11.19) yields $\mathcal{R}=0$ and therefore Eqs. (11.18) are equivalent to Einstein's equations in empty space $\mathcal{R}_{\mu \nu}=0$. On the other hand, if we assume $f(\mathcal{R})=\alpha \mathcal{R}^{2}$, we can have non-vanishing torsion. In this case, by replacing Eq. (11.19) in Eqs. (11.11) and (11.18), we obtain field equations of the form

$$
\begin{gathered}
\mathcal{R}_{\mu \nu}-\frac{1}{4} \mathcal{R} g_{\mu \nu}=0 \\
T_{\mu \nu}{ }^{\sigma}=-\frac{1}{2 \mathcal{R}} \frac{\partial \mathcal{R}}{\partial x^{\mu}} \delta_{\nu}^{\sigma}+\frac{1}{2 \mathcal{R}} \frac{\partial \mathcal{R}}{\partial x^{\nu}} \delta_{\mu}^{\sigma} .
\end{gathered}
$$

Finally, making use of Eq. (11.17) and the consequent relation

$$
\mathcal{R}=\tilde{R}+2 \tilde{\nabla}_{\sigma} T^{\sigma}-\frac{2}{3} T_{\sigma} T^{\sigma}
$$

in Eqs. (11.20), we can separately point out the contribution due to the metric and that due to the torsion. In fact, directly from eqs. (11.20a) we have

$$
\tilde{R}_{\mu \nu}-\frac{1}{4} \tilde{R} g_{\mu \nu}=-\frac{2}{3} \tilde{\nabla}_{\nu} T_{\mu}+\frac{1}{6} \tilde{\nabla}_{\sigma} T^{\sigma} g_{\mu \nu}-\frac{2}{9} T_{\mu} T_{\nu}+\frac{1}{18} T_{\sigma} T^{\sigma} g_{\mu \nu},
$$

while from the "trace" $T_{\mu}:=T_{\nu \sigma}{ }^{\sigma}=-\frac{3}{2 \mathcal{R}} \frac{\partial \mathcal{R}}{\partial x^{\mu}}$ of Eqs. (11.20b), we derive

$$
\frac{\partial}{\partial x^{\mu}}\left(\tilde{R}+2 \tilde{\nabla}_{\sigma} T^{\sigma}-\frac{2}{3} T_{\sigma} T^{\sigma}\right)=-\frac{2}{3}\left(\tilde{R}+2 \tilde{\nabla}_{\sigma} T^{\sigma}-\frac{2}{3} T_{\sigma} T^{\sigma}\right) T_{\mu},
$$

Eqs. (11.22) and (11.23) are the coupled field equations in vacuum for metric and torsion in the $f(\mathcal{R})=\alpha \mathcal{R}^{2}$ gravitational theory.

The presence of matter is embodied in the action functional (11.1) by adding to the gravitational Lagrangian a suitable material Lagrangian density $\mathcal{L}_{m}$, namely

$$
\mathcal{S}(g, \Gamma)=\int\left(\sqrt{-g} f(\mathcal{R})+\mathcal{L}_{m}\right) d s .
$$

Throughout this Review Paper we shall consider material Lagrangian density $\mathcal{L}_{m}$ not containing terms depending on torsion degrees of freedom. The physical meaning of this assumption will be discussed later. In this case, the field equations take the form

$$
\begin{gathered}
f^{\prime}(\mathcal{R}) \mathcal{R}_{\mu \nu}-\frac{1}{2} f(\mathcal{R}) g_{\mu \nu}=\Sigma_{\mu \nu}, \\
T_{\mu \nu}^{\sigma}=-\frac{1}{2 f^{\prime}(\mathcal{R})} \frac{\partial f^{\prime}(\mathcal{R})}{\partial x^{p}}\left(\delta_{i}^{\phi} \delta_{\nu}^{\sigma}-\delta_{\nu}^{\phi} \delta_{\mu}^{\sigma}\right),
\end{gathered}
$$

where $\Sigma_{\mu \nu}:=-\frac{1}{\sqrt{-g}} \frac{\delta \mathcal{L}_{m}}{\delta g^{\mu \nu}}$ plays the role of the energy-momentum tensor. As in Sec. III, the notation for the stressenergy tensor has been changed in order to avoid confusion. Also here we are adopting physical units. From the trace of Eq. (11.25a), we obtain a fundamental relation between the curvature scalar $\mathcal{R}$ and the trace $\Sigma:=g^{\mu \nu} \Sigma_{\mu \nu}$, which is

$$
f^{\prime}(\mathcal{R}) \mathcal{R}-2 f(\mathcal{R})=\Sigma,
$$

(see also [129] and references therein). In what follows, we shall systematically suppose that the relation (11.26) is invertible and that $\Sigma \neq$ const, thus allowing to express the curvature scalar $R$ as a suitable function of $\Sigma$, namely

$$
\mathcal{R}=F(\Sigma) .
$$


With this assumption in mind, using Eqs. (11.26) and (11.27) we can rewrite equations (11.25a) and (11.25b) in the form

$$
\begin{gathered}
\mathcal{R}_{\mu \nu}-\frac{1}{2} \mathcal{R} g_{\mu \nu}=\frac{1}{f^{\prime}(F(\Sigma))}\left(\Sigma_{\mu \nu}-\frac{1}{4} \Sigma g_{\mu \nu}\right)-\frac{1}{4} F(\Sigma) g_{\mu \nu} \\
T_{\mu \nu}{ }^{\sigma}=-\frac{1}{2 f^{\prime}(F(\Sigma))} \frac{\partial f^{\prime}(F(\Sigma))}{\partial x^{\phi}}\left(\delta_{\mu}^{\phi} \delta_{\nu}^{\sigma}-\delta_{\nu}^{\phi} \delta_{\mu}^{\sigma}\right) .
\end{gathered}
$$

Moreover, making use of Eqs. (11.17) and (11.21), in Eq. (11.28a) we can decompose the contracted curvature tensor and the curvature scalar in their Christoffel and torsion dependent terms, thus getting an Einstein-like equation of the form

$$
\tilde{R}_{\mu \nu}-\frac{1}{2} \tilde{R} g_{\mu \nu}=\frac{1}{f^{\prime}(F(\Sigma))}\left(\Sigma_{\mu \nu}-\frac{1}{4} \Sigma g_{\mu \nu}\right)-\frac{1}{4} F(\Sigma) g_{\mu \nu}-\frac{2}{3} \tilde{\nabla}_{\nu} T_{\nu}+\frac{2}{3} \tilde{\nabla}_{\sigma} T^{\sigma} g_{\mu \nu}-\frac{2}{9} T_{\mu} T_{\nu}-\frac{1}{9} T_{\sigma} T^{h} g_{\mu \nu}
$$

Now, setting

$$
\varphi:=f^{\prime}(F(\Sigma))
$$

from the trace of Eqs. (11.28b), we obtain

$$
T_{\mu}:=T_{\mu \sigma}{ }^{\sigma}=-\frac{3}{2 \varphi} \frac{\partial \varphi}{\partial x^{\mu}}
$$

Therefore, substituting in Eqs. (11.29), we end up with the final equations

$$
\tilde{R}_{\mu \nu}-\frac{1}{2} \tilde{R} g_{\mu \nu}=\frac{1}{\varphi} \Sigma_{\mu \nu}+\frac{1}{\varphi^{2}}\left(-\frac{3}{2} \frac{\partial \varphi}{\partial x^{\mu}} \frac{\partial \varphi}{\partial x^{\nu}}+\varphi \tilde{\nabla}_{\mu} \frac{\partial \varphi}{\partial x^{\mu}}+\frac{3}{4} \frac{\partial \varphi}{\partial x^{\sigma}} \frac{\partial \varphi}{\partial x^{\gamma}} g^{\sigma \gamma} g_{\mu \nu}-\varphi \tilde{\nabla}^{\sigma} \frac{\partial \varphi}{\partial x^{\sigma}} g_{\mu \nu}-V(\varphi) g_{\mu \nu}\right)
$$

where we defined the effective potential

$$
V(\varphi):=\frac{1}{4}\left[\varphi F^{-1}\left(\left(f^{\prime}\right)^{-1}(\varphi)\right)+\varphi^{2}\left(f^{\prime}\right)^{-1}(\varphi)\right]
$$

Eqs. (11.32) may be difficult to solve, neverthless we can simplify this task finding solutions for a conformally related metric. Indeed, performing a conformal transformation of the kind $\bar{g}_{\mu \nu}=\varphi g_{\mu \nu}$, eqs. (11.32) may be rewritten in the easier form (see, for example, [129, 291, 453])

$$
\bar{R}_{\mu \nu}-\frac{1}{2} \bar{R} \bar{g}_{\mu \nu}=\frac{1}{\varphi} \Sigma_{\mu \nu}-\frac{1}{\varphi^{3}} V(\varphi) \bar{g}_{\mu \nu}
$$

where $\bar{R}_{\mu \nu}$ and $\bar{R}$ are respectively the Ricci tensor and the Ricci scalar curvature associated with the conformal metric $\bar{g}_{\mu \nu}$. Concerning the connection $\Gamma$, solution of the variational problem $\delta \mathcal{S}=0$, from eqs. (11.4), (11.15) and (11.31), one gets the explicit expression

$$
\Gamma_{\mu \nu}^{\sigma}=\tilde{\Gamma}_{\mu \nu}^{\sigma}+\frac{1}{2 \varphi} \frac{\partial \varphi}{\partial x^{\nu}} \delta_{\mu}^{\sigma}-\frac{1}{2 \varphi} \frac{\partial \varphi}{\partial x^{\phi}} g^{\phi \sigma} g_{\mu \nu}
$$

We can now compare these results with those obtained for $f(R)$ theories in the Palatini formalism [16, 453, 522, 573]. If both the theories (with torsion and Palatini-like) are considered as "metric", in the sense that the dynamical connection $\Gamma$ is not coupled with matter $\left(\frac{\delta \mathcal{L}_{m}}{\delta \Gamma}=0\right)$ and it does not define parallel transport and covariant derivative in space-time, then the two approaches are completely equivalent. Indeed, in the "metric" framework, the true connection of space-time is the Levi-Civita one associated with the metric $g$ and the role played by the dynamical connection $\Gamma$ is just to generate the right Einstein-like equations of the theory. Now, surprisingly enough, our field equations (11.32) are identical to the Einstein-like equations derived within the Palatini formalism [452]. On the other hand, if the theories are genuinely metric-affine, then they are different even though the condition $\frac{\delta \mathcal{L}_{m}}{\delta \Gamma}=0$ holds. In order to stress this point, we recall that in a metric-affine theory the role of dynamical connection is not only that of generating Einstein-like field equations but also defining parallel transport and covariant derivative in space-time. 
Therefore, different connections imply different space-time properties. This means that the geodesic structure and the causal structures could not obviously coincide. For a discussion on this point see [16]. Furthermore, it can be easily shown that the dynamical connection (11.35) differs from that derived within the Palatini formalism. Indeed the latter results to be the Levi-Civita connection $\bar{\Gamma}$ associated with the conformal metric $\bar{g}=\varphi g$ [453, 522], while clearly (11.35) is not. More precisely, Eq. (11.35) is related to $\bar{\Gamma}$ by the projective transformation

$$
\bar{\Gamma}_{\mu \nu}^{\sigma}=\Gamma_{\mu \nu}{ }^{\sigma}+\frac{1}{2 \varphi} \frac{\partial \varphi}{\partial x^{\mu}} \delta_{\nu}^{h}
$$

which is not allowed in the present theory because, for a fixed metric $g$, the connection (11.36) is no longer metric compatible.

To conclude, we notice that Eqs. (11.34) are deducible from an Einstein-Hilbert like action functional only under restrictive conditions. More precisely, let us suppose that the material Lagrangian depends only on the components of the metric and not on its derivatives as well as that the trace $\Sigma=\Sigma_{\mu \nu} g^{\mu \nu}$ is independent of the metric and its derivatives. Then, from the identities

$$
\sqrt{-\bar{g}}=\varphi^{2} \sqrt{-g}, \quad \frac{\partial}{\partial g^{\mu \nu}}=\frac{1}{\varphi} \frac{\partial}{\partial \bar{g}^{\mu \nu}} \quad \text { and } \quad \Sigma_{\mu \nu}=-\frac{1}{\sqrt{-g}} \frac{\delta \mathcal{L}_{m}}{\delta g^{\mu \nu}}=-\frac{1}{\sqrt{-g}} \frac{\partial \mathcal{L}_{m}}{\partial g^{\mu \nu}}
$$

we have the following relation

$$
\Sigma_{\mu \nu}=-\varphi \frac{1}{\sqrt{-\bar{g}}} \frac{\partial \mathcal{L}_{m}}{\partial \bar{g}^{\mu \nu}}:=\varphi \bar{\Sigma}_{\mu \nu}
$$

In view of this, and being $\varphi=\varphi(\Sigma)$, it is easily seen that Eqs. (11.34) may be derived by varying with respect to $\bar{g}^{\mu \nu}$ the action functional

$$
\overline{\mathcal{S}}(\bar{g})=\int\left[\sqrt{-\bar{g}}\left(\bar{R}-\frac{2}{\varphi^{3}} V(\varphi)\right)+\mathcal{L}_{m}\right] d s
$$

Therefore, under the stated assumptions, $f(\mathcal{R})$-gravity with torsion in the metric framework is conformally equivalent to an Einstein-Hilbert like theory.

\section{B. Equivalence of $f(R)$-gravity with torsion and scalar-tensor theories}

The above considerations directly lead to study the relations between $f(R)$-gravity with torsion and scalar-tensor theories with the aim to investigate their possible equivalence. To this end, we recall that the action functional of a (purely metric) scalar-tensor theory is

$$
\mathcal{S}(g, \varphi)=\int\left[\sqrt{-g}\left(\varphi \tilde{R}-\frac{\omega_{0}}{\varphi} \varphi_{\mu} \varphi^{\mu}-U(\varphi)\right)+\mathcal{L}_{m}\right] d s
$$

where $\varphi$ is the scalar field which, depending on the sign of the kinetic term, could assume also the role of a phantom field [258], $\varphi_{\mu}:=\frac{\partial \varphi}{\partial x^{\mu}}$ and $U(\varphi)$ is the potential of $\varphi$. For $U(\varphi)=0$ such a theory reduces to the standard Brans-Dicke theory [86]. The matter Lagrangian $\mathcal{L}_{m}\left(g_{\mu \nu}, \psi\right)$ is a function of the metric and some matter fields $\psi ; \omega_{0}$ is the so called Brans-Dicke parameter. The field equations derived by varying with respect to the metric and the scalar field are

$$
\tilde{R}_{\mu \nu}-\frac{1}{2} \tilde{R} g_{\mu \nu}=\frac{1}{\varphi} \Sigma_{\mu \nu}+\frac{\omega_{0}}{\varphi^{2}}\left(\varphi_{\mu} \varphi_{\nu}-\frac{1}{2} \varphi_{\sigma} \varphi^{\sigma} g_{\mu \nu}\right)+\frac{1}{\varphi}\left(\tilde{\nabla}_{j} \varphi_{\mu}-\tilde{\nabla}_{\sigma} \varphi^{\sigma} g_{\mu \nu}\right)-\frac{U}{2 \varphi} g_{\mu \nu}
$$

and

$$
\frac{2 \omega_{0}}{\varphi} \tilde{\nabla}_{\sigma} \varphi^{\sigma}+\tilde{R}-\frac{\omega_{0}}{\varphi^{2}} \varphi_{\sigma} \varphi^{\sigma}-U^{\prime}=0
$$

where $\Sigma_{\mu \nu}:=-\frac{1}{\sqrt{-g}} \frac{\delta \mathcal{L}^{(m)}}{\delta g^{\mu \nu}}$ and $U^{\prime}:=\frac{d U}{d \varphi}$. 
Taking the trace of Eq. (11.41) and using it to replace $\tilde{R}$ in Eq. (11.42), one obtains the equation

$$
\left(2 \omega_{0}+3\right) \tilde{\nabla}_{\sigma} \varphi^{\sigma}=\Sigma+\varphi U^{\prime}-2 U .
$$

By a direct comparison, it is immediately seen that for $\omega_{0}=-\frac{3}{2}$ and $U(\varphi)=\frac{2}{\varphi} V(\varphi)$ (where $V(\varphi)$ is defined as in Eq. (11.33), Eqs. (11.41) become formally identical to the Einstein-like equations (11.32) for a $f(R)$ theory with torsion. Moreover, in such a circumstance, Eq. (11.43) reduces to the algebraic equation

$$
\Sigma+\varphi U^{\prime}-2 U=0,
$$

relating the matter trace $\Sigma$ to the scalar field $\varphi$, exactly as it happens for $f(R)$-gravity. In particular, it is a straightforward matter to verify that (under the condition $f^{\prime \prime} \neq 0$ ) Eq. (11.44) expresses exactly the inverse relation of (11.30), namely

$$
\Sigma=F^{-1}\left(\left(f^{\prime}\right)^{-1}(\varphi)\right) \quad \Leftrightarrow \quad \varphi=f^{\prime}(F(\Sigma))
$$

being $F^{-1}(X)=f^{\prime}(X) X-2 f(X)$. In fact we have

$$
U(\varphi)=\frac{2}{\varphi} V(\varphi)=\frac{1}{2}\left[F^{-1}\left(\left(f^{\prime}\right)^{-1}(\varphi)\right)+\varphi\left(f^{\prime}\right)^{-4}(\varphi)\right]=\left[\varphi\left(f^{\prime}\right)^{-1}(\varphi)-f\left(\left(f^{\prime}\right)^{-1}(\varphi)\right)\right],
$$

so that

$$
U^{\prime}(\varphi)=\left(f^{\prime}\right)^{-1}(\varphi)+\frac{\varphi}{f^{\prime \prime}\left(\left(f^{\prime}\right)^{-1}(\varphi)\right)}-\frac{\varphi}{f^{\prime \prime}\left(\left(f^{\prime}\right)^{-4}(\varphi)\right)}=\left(f^{\prime}\right)^{-1}(\varphi)
$$

and then

$$
\Sigma=-\varphi U^{\prime}(\varphi)+2 U(\varphi)=f^{\prime}\left(\left(f^{\prime}\right)^{-1}(\varphi)\right)\left(f^{\prime}\right)^{-1}(\varphi)-2 f\left(\left(f^{\prime}\right)^{-1}(\varphi)\right)=F^{-1}\left(\left(f^{\prime}\right)^{-1}(\varphi)\right)
$$

As a conclusion follows that, in the "metric" interpretation, $f(R)$ theories with torsion are equivalent to $\omega_{0}=-\frac{3}{9}$ Brans-Dicke theories. Of course, the above statement is not true if we regard $f(R)$ theories as genuinely metric-affine ones. Nevertheless, also in this case it is possible to prove the equivalence between $f(R)$ theories with torsion and a certain class of Brans-Dicke theories, namely $\omega_{0}=0$ Brans-Dicke theories with torsion [291]. In this regard, let us consider the action functional

$$
\mathcal{S}(g, \Gamma, \varphi)=\int\left[\sqrt{-g}(\varphi \mathcal{R}-U(\varphi))+\mathcal{L}_{m}\right] d s,
$$

where the dynamical fields are respectively a metric $g_{\mu \nu}$, a metric connection $\Gamma_{\mu \nu}^{\gamma}$ and a scalar field $\varphi$. As mentioned above, the action (11.49) describes a Brans-Dicke theory with torsion and parameter $\omega_{0}=0$. The variation with respect to $\varphi$ yields the field equation

$$
\mathcal{R}=U^{\prime}(\varphi)
$$

To evaluate the variations with respect to the metric and the connection we may repeat exactly the same arguments stated in the previous discussion for $f(R)$-gravity. Omitting for brevity the straightforward details, the resulting field equations are

$$
T_{\mu \nu}{ }^{\sigma}=-\frac{1}{2 \varphi} \frac{\partial \varphi}{\partial x^{\phi}}\left(\delta_{\mu}^{\phi} \delta_{\nu}^{\sigma}-\delta_{\nu}^{\phi} \delta_{\mu}^{\sigma}\right)
$$

and

$$
\mathcal{R}_{\mu \nu}-\frac{1}{2} \mathcal{R} g_{\mu \nu}=\frac{1}{\varphi} \Sigma_{\mu \nu}-\frac{1}{2 \varphi} U(\varphi) g_{\mu \nu},
$$

Inserting the content of Eq. (11.50) in the trace of Eq. (11.52)

$$
\frac{1}{\varphi} \Sigma-\frac{2}{\varphi} U(\varphi)+\mathcal{R}=0
$$


we obtain again an algebraic relation between $\Sigma$ and $\varphi$ identical to Eq. (11.44). Therefore, choosing as above the potential $U(\varphi)=\frac{2}{\varphi} V(\varphi)$, from (11.44) we get $\varphi=f^{\prime}\left(F(\Sigma)\right.$ ). In view of this, decomposing $\mathcal{R}_{\mu \nu}$ and $\mathcal{R}$ in their Christoffel and torsion dependent terms, eqs. (11.51) and (11.52) become identical to eq. (11.31) and (11.32) respectively. As mentioned previously, this fact shows the equivalence between $f(R)$ theories and $\omega_{0}=0$ - Brans-Dicke theories with torsion, in the metric-affine framework. These considerations can be extremely useful in order to give a geometrical characterization to the Brans-Dicke scalar field.

In summary, torsion field plays a fundamental role in clarifying the relations between the Palatini and the metric approaches: it gives further degrees of freedom which contribute, together with curvature degrees of freedom, to the dynamics. A goal could be to achieve a self-consistent theory where unknown ingredients as dark energy and dark matter (up to now not detected at a fundamental level) could be completely "geometrized" [126, 128]. Torsion field assumes a relevant role in presence of standard matter since it allows to establish a definite equivalence between scalar-tensor theories and $f(R)$-gravity, also in relation to conformal transformations.

\section{THE $\mathcal{J}$-BUNDLES FRAMEWORK}

In some papers 175, 176, 567], a new geometric approach for Gauge Theories and GR, in the tetrad-affine formulation, has been proposed. It is called the $\mathcal{J}$-bundles framework (from now on the $\mathcal{J}$-bundles).

The starting point for the construction of such a framework is that several field Lagrangians have corresponding Lagrangian densities which depend on the fields derivatives only through suitable antisymmetric combinations. This is the case of the Einstein-Hilbert Lagrangian which, in the tetrad-affine formulation, depends on the antisymmetric derivatives of the spin-connection through the curvature.

In view of this fact, the basic idea developed in [175, 176, 567] consists in defining a suitable quotient space of the first jet-bundle, making equivalent two sections which have a first order contact with respect to the exterior differentiation (or, equivalently, with respect to the exterior covariant differentiation), instead of the whole set of derivatives. The resulting fiber coordinates of the so defined new spaces are exactly the antisymmetric combinations appearing in the Lagrangian densities.

For GR, it has been shown that the fiber coordinates of the quotient space can be identified with the components of the torsion and curvature tensors and the approach results particularly useful in the gauge treatment of gravity (see [340] for a general discussion).

The aim of this section is to extend the mathematical machinery developed in [567] to the $f(R)$-theories of gravity with torsion [126] in order to recast such theories in the $\mathcal{J}$-bundle formalism. As we will see, such an approach is particularly useful to put in evidence the peculiar geometric structures of the theories, as symmetries and conservation laws.

Let $\mathcal{M}$ be a 4-dimensional orientable space-time manifold, with a metric tensor $g$ of signature $\eta=(1,3)=$ $(-1,1,1,1)$. Let us denote by $\mathcal{E}$ the co-frame bundle of $\mathcal{M}$. Moreover, let $P \rightarrow \mathcal{M}$ be a principal fiber bundle over $\mathcal{M}$, with structural group $G=S O(1,3)$. We denote by $\mathcal{C}:=J_{1}(P) / S O(1,3)$ the space of principal connections over $P$. We refer $\mathcal{E}$ and $\mathcal{C}$ to local coordinates $x^{i}, e_{i}^{\mu}(i, \mu=1, \ldots, 4)$ and $x^{i}, \omega_{i}{ }^{\mu \nu}(\mu<\nu)$ respectively. In this section, we shall adopt the standard notation of $\mathcal{J}$-bundle formalism [175, 176].

The configuration space of the theory is the fiber product $\mathcal{E} \times \mathcal{M} \mathcal{C}(\mathcal{E} \times \mathcal{E}$ for short $)$ over $\mathcal{M}$. The dynamical fields are (local) sections of $\mathcal{E} \times \mathcal{E}$, namely pairs formed by a (local) tetrad field $e(x)=e_{i}^{\mu}(x) d x^{i}$ and a principal connection 1-form $\omega(x)=\omega_{i}{ }^{\mu \nu}(x) d x^{i}$. We notice that the connection $\omega(x)$ is automatically metric-compatible with the metric $g(x)=\eta_{\mu \nu} e^{\mu}(x) \otimes e^{\nu}(x)\left(\eta_{\mu \nu}:=\operatorname{diag}(-1,1,1,1)\right)$, induced on $\mathcal{M}$ by the tetrad field $e^{\mu}(x)$ itself. We consider the first $\mathcal{J}$-bundle $\mathcal{J}(\mathcal{E} \times \mathcal{C})$ (see [567]) associated with the fibration $\mathcal{E} \times \mathcal{C} \rightarrow \mathcal{M}$. It is built similarly to an ordinary $\mathcal{J}$-bundle, but the first order contact between sections is calculated with respect to exterior (or exterior covariant) differentials. $\mathcal{J}$-bundles have been recently used to provide new geometric formulations of gauge theories and GR [175, 176, 567,571 .

For convenience of the reader, we briefly recall the construction of the bundle $\mathcal{J}(\mathcal{E} \times \mathcal{C})$. Let $J_{1}(\mathcal{E} \times \mathcal{C})$ be the first $\mathcal{J}$-bundle associated with $\mathcal{E} \times \mathcal{C} \rightarrow \mathcal{M}$, referred to local jet-coordinates $x^{i}, e_{i}^{\mu}, \omega_{i}{ }^{\mu \nu}, e_{i j}^{\mu}, \omega_{i j}{ }^{\mu \nu}$. We introduce on $J_{1}(\mathcal{E} \times \mathcal{C})$ the following equivalence relation. Let $z=\left(x^{i}, e_{i}^{\mu}, \omega_{i}{ }^{\mu \nu}, e_{i j}^{\mu}, \omega_{i j}{ }^{\mu \nu}\right)$ and $\hat{z}=\left(x^{i}, \hat{e}_{i}^{\mu}, \hat{\omega}_{i}{ }^{\mu \nu}, \hat{e}_{i j}^{\mu}, \hat{\omega}_{i j}{ }^{\mu \nu}\right)$ be two elements of $J_{1}(\mathcal{E} \times \mathcal{C})$, having the same projection $x$ over $\mathcal{M}$. Denoting by $\left\{e^{\mu}(x), \omega^{\mu \nu}(x)\right\}$ and $\left\{\hat{e}^{\mu}(x), \hat{\omega}^{\mu \nu}(x)\right\}$ two different sections of the bundle $\mathcal{E} \times \mathcal{C} \rightarrow \mathcal{M}$, respectively chosen among the representatives of the equivalence classes $z$ and $\hat{z}$, we say that $z$ is equivalent to $\hat{z}$ if and only if

$$
e^{\mu}(x)=\hat{e}^{\mu}(x), \quad \omega^{\mu \nu}(x)=\hat{\omega}^{\mu \nu}(x),
$$


and

$$
d e^{\mu}(x)=d \hat{e}^{\mu}(x), \quad D \omega^{\mu \nu}(x)=D \hat{\omega}^{\mu \nu}(x),
$$

where $D$ is the covariant differential induced by the connection. In local coordinates, it is easily seen that $z \sim \hat{z}$ if and only if the following identities hold

$$
\begin{gathered}
e_{i}^{\mu}=\hat{e}_{i}^{\mu}, \quad \omega_{i}^{\mu \nu}=\hat{\omega}_{i}{ }^{\mu \nu}, \\
\left(e_{i j}^{\mu}-e_{j i}^{\mu}\right)=\left(\hat{e}_{i j}^{\mu}-\hat{e}_{j i}^{\mu}\right), \quad\left(\omega_{i j}{ }^{\mu \nu}-\omega_{j i}{ }^{\mu \nu}\right)=\left(\hat{\omega}_{i j}{ }^{\mu \nu}-\hat{\omega}_{j i}{ }^{\mu \nu}\right) .
\end{gathered}
$$

We denote by $\mathcal{J}(\mathcal{E} \times \mathcal{C})$ the quotient space $J_{1}(\mathcal{E} \times \mathcal{C}) / \sim$ and by $\rho: J_{1}(\mathcal{E} \times \mathcal{C}) \rightarrow \mathcal{J}(\mathcal{E} \times \mathcal{C})$ the corresponding canonical projection. A system of local fiber coordinates on the bundle $\mathcal{J}(\mathcal{E} \times \mathcal{C})$ is provided by $x^{i}, e_{i}^{\mu}, \omega_{i}{ }^{\mu \nu}, E_{i j}^{\mu}:=$ $\frac{1}{2}\left(e_{i j}^{\mu}-e_{j i}^{\mu}\right), \Omega_{i j}{ }^{\mu \nu}:=\frac{1}{2}\left(\omega_{i j}^{\mu \nu}-\omega_{j i}{ }^{\mu \nu}\right)(i<j)$.

The geometry of $\mathcal{J}$-bundles has been thoroughly examined in Refs. [175, 176, 567]. As a matter of fact, the quotient projection $\rho$ endows the bundle $\mathcal{J}(\mathcal{E} \times \mathcal{C})$ with most of the standard features of jet-bundles geometry $(\mathcal{J}$-extension of sections, contact forms, $\mathcal{J}$-prolongation of morphisms and vector fields), which are needed to implement variational calculus on $\mathcal{J}(\mathcal{E} \times \mathcal{C})$. Referring the reader to [175, 176, 567] for a detailed discussion on $\mathcal{J}$-bundles geometry, the relevant fact we need to recall here is that the components of the torsion and curvature tensors can be chosen as fiber $\mathcal{J}$-coordinates on $\mathcal{J}(\mathcal{E} \times \mathcal{C})$. In fact, the following relations

$$
\begin{gathered}
T_{i j}^{\mu}=2 E_{j i}^{\mu}+\omega_{i}{ }^{\mu}{ }_{\lambda} e_{j}^{\lambda}-\omega_{j}{ }^{\mu}{ }_{\lambda} e_{i}^{\lambda}, \\
\mathcal{R}_{i j}{ }^{\mu \nu}=2 \Omega_{j i}{ }^{\mu \nu}+\omega_{i}{ }^{\mu}{ }_{\lambda} \omega_{j}{ }^{\lambda \nu}-\omega_{j}{ }^{\mu}{ }_{\lambda} \omega_{i}{ }^{\lambda \nu},
\end{gathered}
$$

can be regarded as fiber coordinate transformations on $\mathcal{J}(\mathcal{E} \times \mathcal{C})$, allowing to refer the bundle $\mathcal{J}(\mathcal{E} \times \mathcal{C})$ to local coordinates $x^{i}, e_{i}^{\mu}, \omega_{i}{ }^{\mu \nu}, T_{i j}^{\mu}(i<j), \mathcal{R}_{i j}{ }^{\mu \nu}(i<j, \mu<\nu)$. In such coordinates, local sections $\gamma: \mathcal{M} \rightarrow \mathcal{J}(\mathcal{E} \times \mathcal{C})$ are expressed as

$$
\gamma: x \rightarrow\left(x^{i}, e_{i}^{\mu}(x), \omega_{i}^{\mu \nu}(x), T_{i j}^{\mu}(x), \mathcal{R}_{i j}{ }^{\mu \nu}(x)\right) .
$$

In particular, a section $\gamma$ is said holonomic if it is the $\mathcal{J}$-extension $\gamma=\mathcal{J} \sigma$ of a section $\sigma: \mathcal{M} \rightarrow \mathcal{E} \times \mathcal{C}$. In local coordinates, a section is holonomic if it satisfies the relations [567.

$$
\begin{gathered}
T_{i j}^{\mu}(x)=\frac{\partial e_{j}^{\mu}(x)}{\partial x^{i}}-\frac{\partial e_{i}^{\mu}(x)}{\partial x^{j}}+\omega_{i}{ }^{\mu}{ }_{\lambda}(x) e_{j}^{\lambda}(x)-\omega_{j}{ }^{\mu}{ }_{\lambda}(x) e_{i}^{\lambda}(x), \\
\mathcal{R}_{i j}{ }^{\mu \nu}(x)=\frac{\partial \omega_{j}{ }^{\mu \nu}(x)}{\partial x^{i}}-\frac{\partial \omega_{i}{ }^{\mu \nu}(x)}{\partial x^{j}}+\omega_{i}{ }^{\mu}{ }_{\lambda}(x) \omega_{j}{ }^{\lambda \nu}(x)-\omega_{j}{ }^{\mu}{ }_{\lambda}(x) \omega_{i}{ }^{\lambda \nu}(x),
\end{gathered}
$$

namely if the quantities $T_{i j}^{\mu}(x)$ and $\mathcal{R}_{i j}{ }^{\mu \nu}(x)$ are the components of torsion and curvature tensors associated with the tetrad $e_{i}^{\mu}(x)$ and the connection $\omega_{i}{ }^{\mu \nu}(x)$, in turn, represents the section $\sigma$. We also recall that the bundle $\mathcal{J}(\mathcal{E} \times \mathcal{C})$ is endowed with a suitable contact bundle. The latter is locally spanned by the following 2-forms

$$
\begin{gathered}
\theta^{\mu}=d e_{i}^{\mu} \wedge d x^{i}+E_{i j}^{\mu} d x^{i} \wedge d x^{j}, \\
\theta^{\mu \nu}=d \omega_{i}{ }^{\mu \nu} \wedge d x^{i}+\Omega_{i j}{ }^{\mu \nu} d x^{i} \wedge d x^{j} .
\end{gathered}
$$

It is easily seen that a section $\gamma: \mathcal{M} \rightarrow \mathcal{J}(\mathcal{E} \times \mathcal{C})$ is holonomic if and only if it satisfies the condition $\gamma^{*}\left(\theta^{\mu}\right)=$ $\gamma^{*}\left(\theta^{\mu \nu}\right)=0 \forall \mu, \nu=1, \ldots, 4$. Moreover, in the local coordinates $\{x, e, \omega, T, \mathcal{R}\}$, the 2 -forms (12.6) can be expressed as

$$
\theta^{\mu}=\tau^{\mu}-T^{\mu} \quad \text { and } \quad \theta^{\mu \nu}=\rho^{\mu \nu}-\mathcal{R}^{\mu \nu},
$$

being $\tau^{\mu}=d e_{i}^{\mu} \wedge d x^{i}+\omega_{j}{ }^{\mu}{ }_{\nu} e_{i}^{\nu} d x^{j} \wedge d x^{i}, T^{\mu}=\frac{1}{2} T_{i j}^{\mu} d x^{i} \wedge d x^{j}, \rho^{\mu \nu}=d \omega_{i}{ }^{\mu \nu} \wedge d x^{i}+\frac{1}{2}\left(\omega_{j}{ }^{\mu}{ }_{\lambda} \omega_{i}{ }^{\lambda \nu}-\omega_{j}{ }^{\nu}{ }_{\lambda} \omega_{i}{ }^{\lambda \mu}\right) d x^{j} \wedge d x^{i}$ and $\mathcal{R}^{\mu \nu}=\frac{1}{2} \mathcal{R}_{i j}{ }^{\mu \nu} d x^{i} \wedge d x^{j}$. 


\section{A. The field equations in the $\mathcal{J}$-bundle formalism}

We call a Lagrangian on $\mathcal{J}$ any horizontal 4-form, locally expressed as

$$
L=\mathcal{L}\left(x^{i}, e_{i}^{\mu}, \omega_{i}^{\mu \nu}, T_{i j}^{\mu}, \mathcal{R}_{i j}^{\mu \nu}\right) d s .
$$

Associated with any of such a Lagrangian there is a corresponding Poincaré-Cartan 4-form, having local expression (see [567])

$$
\Theta=\mathcal{L} d s-\frac{1}{2} \frac{\partial \mathcal{L}}{\partial T_{h k}^{\alpha}} \theta^{\alpha} \wedge d s_{h k}-\frac{1}{4} \frac{\partial \mathcal{L}}{\partial \mathcal{R}_{h k}^{\alpha \beta}} \theta^{\alpha \beta} \wedge d s_{h k}
$$

where $\left.\left.d s_{h k}:=\frac{\partial}{\partial x^{h}}\right\rfloor \frac{\partial}{\partial x^{k}}\right\rfloor d s$. Taking the identities $d x^{t} \wedge d s_{i j}=-\delta_{j}^{t} d s_{i}+\delta_{i}^{t} d s_{j}$ and $d x^{p} \wedge d x^{t} \wedge d s_{i j}=$ $-\left(\delta_{i}^{p} \delta_{j}^{t}-\delta_{j}^{p} \delta_{i}^{t}\right) d s$ into account, it is easily seen that the 4-form (12.9) may be expressed as

$$
\Theta=\mathcal{L} d s-\frac{\partial \mathcal{L}}{\partial T_{h k}{ }^{\alpha}}\left(d e_{h}^{\alpha} \wedge d s_{k}-\omega_{h}{ }^{\alpha}{ }_{\nu} e_{k}^{\nu} d s+\frac{1}{2} T_{h k}^{\alpha} d s\right)-\frac{1}{2} \frac{\partial \mathcal{L}}{\partial \mathcal{R}_{h k}{ }^{\alpha \beta}}\left(d \omega_{h}{ }^{\alpha \beta} \wedge d s_{k}-\omega_{h}{ }^{\alpha}{ }_{\lambda} \omega_{k}^{\lambda \beta} d s+\frac{1}{2} \mathcal{R}_{h k}{ }^{\alpha \beta} d s\right)
$$

The field equations are derived from the variational principle

$$
\mathcal{S}(\sigma)=\int \mathcal{J} \sigma^{*}(\Theta)=\int \mathcal{J} \sigma^{*}(\mathcal{L} d s)
$$

where $\sigma: \mathcal{M} \rightarrow \mathcal{E} \times \mathcal{C}$ denotes any section and $\mathcal{J} \sigma: \mathcal{M} \rightarrow \mathcal{J}$ its $\mathcal{J}$-extension satisfying Eqs. (12.5). Referring the reader to [567] for a detailed discussion, we recall here that the corresponding Euler-Lagrange equations can be expressed as

$$
\left.\mathcal{J} \sigma^{*}(\mathcal{J}(X)\rfloor d \Theta\right)=0
$$

for all $\mathcal{J}$-prolongable vector fields $\mathrm{X}$ on $\mathcal{E} \times \mathcal{C}$. Moreover, we notice that the expression of $\mathcal{J}$-prolongable vector fields and their $\mathcal{J}$-prolongations, involved in Eq. (12.12), is not needed here. In order to make explicit Eq. (12.12), we calculate the differential of the form (12.10), that is

$$
\begin{aligned}
& d \Theta=d \mathcal{L} \wedge d s-d\left(\frac{\partial \mathcal{L}}{\partial T_{h k}^{\alpha}}\right) \wedge\left(d e_{h}^{\alpha} \wedge d s_{k}-\omega_{h}{ }^{\alpha}{ }_{\nu} e_{k}^{\nu} d s+\frac{1}{2} T_{h k}^{\alpha} d s\right) \\
& -\frac{\partial \mathcal{L}}{\partial T_{h k}^{\alpha}}\left(-e_{k}^{\nu} d \omega_{h}{ }^{\alpha}{ }_{\nu} \wedge d s-\omega_{h}{ }^{\alpha}{ }_{\nu} d e_{k}^{\nu} \wedge d s+\frac{1}{2} d T_{h k}^{\alpha} \wedge d s\right) \\
& -\frac{1}{2} d\left(\frac{\partial \mathcal{L}}{\partial \mathcal{R}_{h k}{ }^{\alpha \beta}}\right) \wedge\left(d \omega_{h}{ }^{\alpha \beta} \wedge d s_{k}-\omega_{h}{ }^{\alpha}{ }_{\lambda} \omega_{k}{ }^{\lambda \beta} d s+\frac{1}{2} \mathcal{R}_{h k}{ }^{\alpha \beta} d s\right) \\
& -\frac{1}{2} \frac{\partial \mathcal{L}}{\partial \mathcal{R}_{h k}{ }^{\alpha \beta}}\left(-2 \omega_{h}{ }^{\alpha}{ }_{\lambda} d \omega_{k}{ }^{\lambda \beta} \wedge d s+\frac{1}{2} d \mathcal{R}_{h k}{ }^{\alpha \beta} \wedge d s\right)= \\
& \frac{\partial \mathcal{L}}{\partial e_{q}^{\mu}} d e_{q}^{\mu} \wedge d s+\frac{1}{2} \frac{\partial \mathcal{L}}{\partial \omega_{h}^{\alpha \beta}} d \omega_{h}^{\alpha \beta} \wedge d s \\
& -d\left(\frac{\partial \mathcal{L}}{\partial T_{h k}^{\alpha}}\right) \wedge\left(d e_{h}^{\alpha} \wedge d s_{k}-\omega_{h}{ }^{\alpha}{ }_{\nu} e_{k}^{\nu} d s+\frac{1}{2} T_{h k}^{\alpha} d s\right) \\
& +\frac{\partial \mathcal{L}}{\partial T_{h k}^{\alpha}}\left(e_{k}^{\nu} d \omega_{h}{ }^{\alpha}{ }_{\nu} \wedge d s+\omega_{h}{ }^{\alpha}{ }_{\nu} d e_{k}^{\nu} \wedge d s\right)+\frac{\partial \mathcal{L}}{\partial \mathcal{R}_{h k}{ }^{\alpha \beta}} \omega_{h}{ }^{\alpha}{ }_{\lambda} d \omega_{k}{ }^{\lambda \beta} \wedge d s \\
& -\frac{1}{2} d\left(\frac{\partial \mathcal{L}}{\partial \mathcal{R}_{h k}{ }^{\alpha \beta}}\right) \wedge\left(d \omega_{h}{ }^{\alpha \beta} \wedge d s_{k}-\omega_{h}{ }^{\alpha}{ }_{\lambda} \omega_{k}{ }^{\lambda \beta} d s+\frac{1}{2} \mathcal{R}_{h k}{ }^{\alpha \beta} d s\right) .
\end{aligned}
$$

Choosing infinitesimal deformations $X$ of the special form

$$
X=G_{q}^{\mu}(x) \frac{\partial}{\partial e_{q}^{\mu}}+\frac{1}{2} G_{q}^{\mu \nu}(x) \frac{\partial}{\partial \omega_{q}^{\mu \nu}},
$$


we have then [567]

$$
\begin{aligned}
& \mathcal{J}(X)\lrcorner d \Theta=\left[\frac{\partial \mathcal{L}}{\partial e_{q}^{\mu}} d s+d\left(\frac{\partial \mathcal{L}}{\partial T_{q k}^{\mu}}\right) \wedge d s_{k}+\frac{\partial \mathcal{L}}{\partial T_{h q}^{\alpha}} \omega_{h}{ }^{\alpha}{ }_{\mu} d s\right] G_{q}^{\mu} \\
& +\left[\frac{1}{2} \frac{\partial \mathcal{L}}{\partial \omega_{q}{ }^{\mu \nu}} d s+\frac{\partial \mathcal{L}}{\partial T_{q k}^{\mu}} e_{k}^{\sigma} \eta_{\sigma \nu} d s+\frac{1}{2} d\left(\frac{\partial \mathcal{L}}{\partial \mathcal{R}_{q k}{ }^{\mu \nu}}\right) \wedge d s_{k}+\frac{\partial \mathcal{L}}{\partial \mathcal{R}_{k q}{ }^{\alpha \nu}} \omega_{k}{ }^{\alpha}{ }_{\mu} d s\right] G_{q}^{\mu \nu} \\
& -\mathcal{J}(X)\lrcorner d\left(\frac{\partial \mathcal{L}}{\partial T_{h k}^{\alpha}}\right) \wedge\left(d e_{h}^{\alpha} \wedge d s_{k}-\omega_{h}{ }^{\alpha}{ }_{\nu} e_{k}^{\nu} d s+\frac{1}{2} T_{h k}^{\alpha} d s\right) \\
& \left.-\frac{1}{2} \mathcal{J}(X)\right\lrcorner d\left(\frac{\partial \mathcal{L}}{\partial \mathcal{R}_{h k}{ }^{\alpha \beta}}\right) \wedge\left(d \omega_{h}{ }^{\alpha \beta} \wedge d s_{k}-\omega_{h}{ }^{\alpha}{ }_{\lambda} \omega_{k}{ }^{\lambda \beta} d s+\frac{1}{2} \mathcal{R}_{h k}{ }^{\alpha \beta} d s\right) .
\end{aligned}
$$

Due to the arbitrariness of $X$ and the holonomy of the $\mathcal{J}$-extension $\mathcal{J} \sigma$ (compare with Eqs. (12.5)), the requirement (12.12) yields two sets of final field equations

$$
\mathcal{J} \sigma^{*}\left(\frac{\partial \mathcal{L}}{\partial e_{q}^{\mu}}+\frac{\partial \mathcal{L}}{\partial T_{k q}^{\alpha}} \omega_{k}{ }^{\alpha}{ }_{\mu}\right)-\frac{\partial}{\partial x^{k}}\left(\mathcal{J} \sigma^{*}\left(\frac{\partial \mathcal{L}}{\partial T_{k q}^{\mu}}\right)\right)=0
$$

and

$$
\begin{aligned}
\mathcal{J} \sigma^{*}\left(\frac{\partial \mathcal{L}}{\partial \omega_{q}{ }^{\mu \nu}}-\frac{\partial \mathcal{L}}{\partial T_{k q}^{\mu}} e_{k}^{\sigma} \eta_{\sigma \nu}+\frac{\partial \mathcal{L}}{\partial T_{k q}^{\nu}} e_{k}^{\sigma} \eta_{\sigma \mu}\right. & \left.+\frac{\partial \mathcal{L}}{\partial \mathcal{R}_{k q}{ }^{\alpha \nu}} \omega_{k}{ }^{\alpha}{ }_{\mu}+\frac{\partial \mathcal{L}}{\partial \mathcal{R}_{k q}{ }^{\mu \alpha}} \omega_{k}{ }^{\alpha}{ }_{\nu}\right) \\
& -\frac{\partial}{\partial x^{k}}\left(\mathcal{J} \sigma^{*}\left(\frac{\partial \mathcal{L}}{\partial \mathcal{R}_{k q}{ }^{\mu \nu}}\right)\right)=0 .
\end{aligned}
$$

To conclude, it is worth noticing that all the restrictions about the vector fields $\mathcal{J}(X)$ in Eq. (12.12) may be removed. In fact, it is easily seen that Eq. (12.12) automatically implies

$$
\left.\mathcal{J} \sigma^{*}(X\lrcorner d \Theta\right)=0, \quad \forall X \in D^{1}(\mathcal{J}(\mathcal{E} \times \mathcal{C})) .
$$

\section{B. $\quad f(R)$-gravity within the $\mathcal{J}$-bundle framework}

Let us now apply the above formalism to the $f(R)$ theories of gravity. The Lagrangian densities which we are going to consider are of the specific kind $\mathcal{L}=e f(\mathcal{R})$, with $e=\operatorname{det}\left(e_{i}^{\mu}\right)$ and $\mathcal{R}=\mathcal{R}_{i j}{ }^{\mu \nu} e_{\mu}^{i} e_{\nu}^{j}$. Therefore, taking the identities $\frac{\partial e}{\partial e_{i}^{\mu}}=e e_{\mu}^{i}$ and $\frac{\partial e_{\nu}^{j}}{\partial e_{i}^{\mu}}=-e_{\nu}^{i} e_{\mu}^{j}$ into account, we have

$$
\begin{gathered}
\frac{\partial \mathcal{L}}{\partial e_{i}^{\mu}}=e e_{\mu}^{i} f(\mathcal{R})-2 e f^{\prime}(\mathcal{R}) \mathcal{R}_{\mu \sigma}{ }^{\lambda \sigma} e_{\lambda}^{i}, \\
\frac{\partial \mathcal{L}}{\partial \mathcal{R}_{k i}{ }^{\mu \nu}}=2 e f^{\prime}(\mathcal{R})\left[e_{\mu}^{k} e_{\nu}^{i}-e_{\mu}^{i} e_{\nu}^{k}\right],
\end{gathered}
$$

In view of this, Eqs. (12.16) become

$$
e_{\mu}^{i} f(\mathcal{R})-2 f^{\prime}(\mathcal{R}) \mathcal{R}_{\mu \sigma}{ }^{\lambda \sigma} e_{\lambda}^{i}=0
$$

and

$$
\frac{\partial}{\partial x^{k}}\left[2 e f^{\prime}(\mathcal{R})\left(e_{\mu}^{k} e_{\nu}^{i}-e_{\mu}^{i} e_{\nu}^{k}\right)\right]-\omega_{k}^{\lambda}{ }_{\mu}\left[2 e f^{\prime}(\mathcal{R})\left(e_{\lambda}^{k} e_{\nu}^{i}-e_{\lambda}^{i} e_{\nu}^{k}\right)\right]-\omega_{k}{ }^{\lambda}{ }_{\nu}\left[2 e f^{\prime}(\mathcal{R})\left(e_{\mu}^{k} e_{\lambda}^{i}-e_{\mu}^{i} e_{\lambda}^{k}\right)\right]=0 .
$$

After some calculations, Eqs. 12.19b may be rewritten in the form

$$
e f^{\prime \prime}(\mathcal{R}) \frac{\partial \mathcal{R}}{\partial x^{t}} e_{s}^{\alpha}-e f^{\prime \prime}(\mathcal{R}) \frac{\partial \mathcal{R}}{\partial x^{s}} e_{t}^{\alpha}-e f^{\prime}(\mathcal{R})\left(T_{t s}^{\alpha}-T_{t \sigma}^{\sigma} e_{s}^{\alpha}+T_{s \sigma}^{\sigma} e_{t}^{\alpha}\right)=0
$$


where $T_{t s}^{\alpha}=\frac{\partial e_{s}^{\alpha}}{\partial x^{t}}-\frac{\partial e_{t}^{\alpha}}{\partial x^{s}}+\omega_{t}{ }^{\alpha}{ }_{\lambda} e_{s}^{\lambda}-\omega_{s}{ }^{\alpha}{ }_{\lambda} e_{t}^{\lambda}$ are the torsion coefficients of the connection $\omega_{i}{ }^{\mu \nu}(x)$. Recalling the relationships $\mathcal{R}^{h}{ }_{k i j}=\mathcal{R}_{i j}{ }^{\mu \sigma} \eta_{\sigma \nu} e_{\mu}^{h} e_{k}^{\nu}$ and $T_{i j}{ }^{h}=T_{i j}^{\mu} e_{\mu}^{h}$ among the quantities related to the spin connection $\omega$ and the associated linear connection $\Gamma$, that is $\Gamma_{i j}{ }^{h}=e_{\mu}^{h}\left(\frac{\partial e_{j}^{\mu}}{\partial x^{i}}+\omega_{i}{ }^{\mu}{ }_{\nu} e_{j}^{\nu}\right)$, it is straightforward to see that Eqs. (12.19a) and (12.20) are equivalent to eqs. (11.6) obtained in the metric-affine formalism. At this point, the same considerations made in [126] hold. In particular, let us take into account the trace of the equation (12.19a), namely

$$
2 f(\mathcal{R})-f^{\prime}(\mathcal{R}) \mathcal{R}=0 .
$$

This is identically satisfied by all possible values of $\mathcal{R}$ only in the special case $f(\mathcal{R})=k \mathcal{R}^{2}$. In all the other cases, equation (12.21) represents a constraint on the scalar curvature $\mathcal{R}$. As a conclusion, it follows that, if $f(\mathcal{R}) \neq k \mathcal{R}^{2}$, the scalar curvature $\mathcal{R}$ has to be a constant (at least on connected domains) and coincides with a given solution value of (12.21). In such a circumstance, equations (12.20) imply that the torsion $T_{i j}^{\alpha}$ has to be zero and the theory reduces to a $f(R)$-theory without torsion, thus leading to Einstein equations with a cosmological constant.

In particular, we it is worth noticing that:

- in the case $f(\mathcal{R})=\mathcal{R}$, Eq. (12.21) yields $\mathcal{R}=0$ and therefore Eqs. (12.19a) are equivalent to Einstein's equations in empty space;

- if we assume $f(\mathcal{R})=k \mathcal{R}^{2}$, by replacing eq. (12.21) into Eq. (12.19), we obtain final field equations of the form

$$
\begin{gathered}
\frac{1}{4} e_{\mu}^{i} \mathcal{R}-\mathcal{R}_{\mu}{ }^{\lambda} e_{\lambda}^{i}=0 \\
\frac{1}{\mathcal{R}} \frac{\partial \mathcal{R}}{\partial x^{t}} e_{s}^{\alpha}-\frac{1}{\mathcal{R}} \frac{\partial \mathcal{R}}{\partial x^{s}} e_{t}^{\alpha}-\left(T_{t s}^{\alpha}-T_{t \sigma}^{\sigma} e_{s}^{\alpha}+T_{s \sigma}^{\sigma} e_{t}^{\alpha}\right)=0 .
\end{gathered}
$$

After some straightforward calculations, Eq. (12.22b can be put in normal form with respect to the torsion, namely

$$
T_{t s}^{\alpha}=-\frac{1}{2 R} \frac{\partial \mathcal{R}}{\partial x^{t}} e_{s}^{\alpha}+\frac{1}{2 \mathcal{R}} \frac{\partial \mathcal{R}}{\partial x^{s}} e_{t}^{\alpha} .
$$

\section{Symmetries and conserved quantities}

The Poincaré-Cartan formulation (12.17) of the field equations turns out to be especially useful in the study of symmetries and conserved quantities. To see this point, we recall the following [567]

Definition XII.1. A vector field $Z$ on $\mathcal{J}(\mathcal{E} \times \mathcal{C})$ is called a generalized infinitesimal Lagrangian symmetry if it satisfies the requirement

$$
L_{Z}(\mathcal{L} d s)=d \alpha,
$$

for some 3 -form $\alpha$ on $\mathcal{J}(\mathcal{E} \times \mathcal{C})$.

Definition XII.2. A vector field $Z$ on $\mathcal{J}(\mathcal{E} \times \mathcal{C})$ is called a Noether vector field if it satisfies the condition

$$
L_{Z} \Theta=\omega+d \alpha,
$$

where $\omega$ is a 4-form belonging to the ideal generated by the contact forms and $\alpha$ is any 3 -form on $\mathcal{J}(\mathcal{E} \times \mathcal{C})$.

Proposition XII.1. If a generalized infinitesimal Lagrangian symmetry $Z$ is a $\mathcal{J}$-prolongation, then it is a Noether vector field.

Proposition XII.2. If a Noether vector field $Z$ is a $\mathcal{J}$-prolongation, then it is an infinitesimal dynamical symmetry.

We can associate with any Noether vector field $Z$ a corresponding conserved current. In fact, given $Z$ satisfying Eq. 12.25) and a critical section $\sigma: \mathcal{M} \rightarrow \mathcal{E} \times \mathcal{C}$ we have

$$
\left.\left.d \mathcal{J} \sigma^{*}(Z\rfloor \Theta-\alpha\right)=\mathcal{J} \sigma^{*}(\omega-Z\rfloor d \Theta\right)=0,
$$


showing that the current $\left.\mathcal{J} \sigma^{*}(Z\lrcorner \Theta-\alpha\right)$ is conserved on shell.

As it is well known, diffeomorphisms and Lorentz transformations (for tetrad and connection) have to be dynamical symmetries for the theory: let us prove it.

To start with, let $Y=\xi^{i} \frac{\partial}{\partial x^{i}}$ be the generator of a (local) one parameter group of diffeomorphisms on $\mathcal{M}$. The vector field $Y$ may be "lifted" to a vector field $X$ on $\mathcal{E} \times \mathcal{C}$ by setting

$$
X=\xi^{i} \frac{\partial}{\partial x^{i}}-\frac{\partial \xi^{k}}{\partial x^{q}} e_{k}^{\mu} \frac{\partial}{\partial e_{k}^{\mu}}-\frac{1}{2} \frac{\partial \xi^{k}}{\partial x^{q}} \omega_{k}^{\mu \nu} \frac{\partial}{\partial \omega_{q}^{\mu \nu}} .
$$

The vector fields (12.27) are $\mathcal{J}$-prolongable and their $\mathcal{J}$-prolongations are expressed as [567]

$$
\mathcal{J}(X)=\xi^{i} \frac{\partial}{\partial x^{i}}-\frac{\partial \xi^{k}}{\partial x^{q}} e_{k}^{\mu} \frac{\partial}{\partial e_{k}^{\mu}}-\frac{1}{2} \frac{\partial \xi^{k}}{\partial x^{q}} \omega_{k}{ }^{\mu \nu} \frac{\partial}{\partial \omega_{q}{ }^{\mu \nu}}+T_{j k}^{\mu} \frac{\partial \xi^{k}}{\partial x^{i}} \frac{\partial}{\partial T_{i j}^{\mu}}+\frac{1}{2} \mathcal{R}_{j k}{ }^{\mu \nu} \frac{\partial \xi^{k}}{\partial x^{i}} \frac{\partial}{\partial \mathcal{R}_{i j}{ }^{\mu \nu}} .
$$

A direct calculation shows that the vector fields (12.28) satisfy $L_{\mathcal{J}(X)}(\operatorname{ef}(\mathcal{R}) d s)=0$, so proving that they are infinitesimal Lagrangian symmetries for generic $f(R)$-models. Due to Propositions (XII.1) and (XII.2), we conclude that the vector fields (12.28) are Noether vector fields and thus infinitesimal dynamical symmetries. There are no associated conserved quantities, the inner product $\mathcal{J}(X)\lrcorner \Theta$ consisting in an exact term plus a term vanishing identically when pulled-back under critical section. We have indeed

$$
\begin{array}{r}
\left.\mathcal{J}(X)\lrcorner \Theta=e \xi^{j}\left(f(\mathcal{R}) \delta_{j}^{k}-2 f^{\prime}(\mathcal{R}) \mathcal{R}_{j}^{k}\right) d s_{k}-\frac{1}{4} \theta^{\alpha \beta} \wedge(\mathcal{J}(X)\lrcorner \frac{\partial \mathcal{L}}{\partial \mathcal{R}_{h k}{ }^{\alpha \beta}} d s_{h k}\right)+ \\
\frac{1}{4} d\left(\xi^{j} \omega_{j}^{\alpha \beta} \frac{\partial \mathcal{L}}{\partial \mathcal{R}_{h k}{ }^{\alpha \beta}} d s_{h k}\right)-\frac{1}{4} \xi^{j} \omega_{j}{ }^{\alpha \beta} D\left(\frac{\partial \mathcal{L}}{\partial \mathcal{R}_{h k}{ }^{\alpha \beta}}\right) \wedge d s_{h k},
\end{array}
$$

where $D\left(\frac{\partial \mathcal{L}}{\partial \mathcal{R}_{h k}{ }^{\alpha \beta}}\right)=d\left(\frac{\partial \mathcal{L}}{\partial \mathcal{R}_{h k}{ }^{\alpha \beta}}\right)-\frac{\partial \mathcal{L}}{\partial \mathcal{R}_{h k}{ }^{\lambda \beta}} \omega_{i}{ }^{\lambda}{ }_{\alpha} d x^{i}-\frac{\partial \mathcal{L}}{\partial \mathcal{R}_{h k}{ }^{\alpha \lambda}} \omega_{i}{ }^{\lambda}{ }_{\beta} d x^{i}$.

Infinitesimal Lorentz transformations are represented by vector fields on $\mathcal{J}(\mathcal{E} \times \mathcal{C})$ of the form

$$
Y=A_{\sigma}^{\gamma} e_{q}^{\sigma} \frac{\partial}{\partial e_{q}^{\gamma}}-\frac{1}{2} D_{q} A^{\mu \nu} \frac{\partial}{\partial \omega_{q}^{\mu \nu}},
$$

where $A^{\mu \nu}(x)=-A^{\nu \mu}(x)$ is a tensor-valued function on $\mathcal{M}$ in the Lie algebra of $S O(3,1)$, and

$$
D_{q} A^{\mu \nu}=\frac{\partial A^{\mu \nu}}{\partial x^{q}}+\omega_{q}^{\mu}{ }_{\sigma} A^{\sigma \nu}+\omega_{q}{ }^{\nu}{ }_{\sigma} A^{\mu \sigma} .
$$

As above, the vector fields (12.30) are $\mathcal{J}$-prolongable and their $\mathcal{J}$-prolongations are expressed as 567]

$$
\mathcal{J}(Y)=A^{\gamma}{ }_{\sigma} e_{q}^{\sigma} \frac{\partial}{\partial e_{q}^{\gamma}}-\frac{1}{2} D_{q} A^{\mu \nu} \frac{\partial}{\partial \omega_{q}{ }^{\mu \nu}}+\frac{1}{2} A^{\mu}{ }_{\sigma} T_{i j}^{\sigma} \frac{\partial}{\partial T_{i j}^{\mu}}+\frac{1}{2} A^{\mu}{ }_{\sigma} \mathcal{R}_{i j}{ }^{\sigma \nu} \frac{\partial}{\partial \mathcal{R}_{i j}{ }^{\mu \nu}} .
$$

It is straightforward to verify that the vector fields (12.31) obey the condition $L_{\mathcal{J}(Y)}(\operatorname{ef}(\mathcal{R}) d s)=0$. Once again, the conclusion follows that they are infinitesimal dynamical symmetries. Moreover, it is easily seen that

$$
\mathcal{J}(Y)\lrcorner \Theta=-\frac{1}{4} d\left(A^{\alpha \beta} \frac{\partial \mathcal{L}}{\partial \mathcal{R}_{h k}{ }^{\alpha \beta}} d s_{h k}\right)+\frac{1}{4} A^{\alpha \beta} D\left(\frac{\partial \mathcal{L}}{\partial \mathcal{R}_{h k}{ }^{\alpha \beta}}\right) \wedge d s_{h k} .
$$

Therefore, as above, since the inner product $\mathcal{J}(X)\lrcorner \Theta$ consists in an exact term plus a term vanishing identically when pulled-back under critical section, there are no associated conserved quantities.

\section{D. $f(R)$-gravity in presence of matter}

In presence of matter, the configuration space-time of the theory results to be the fiber product $\mathcal{E} \times \mathcal{C} \times \mathcal{M} F$ over $\mathcal{M}$, between $\mathcal{E} \times \mathcal{C}$ and the bundle $F \rightarrow \mathcal{M}$ where the matter fields $\psi^{A}$ take their values. 
The field equations are derived from a variational problem built on the manifold $\mathcal{J} \times{ }_{\mathcal{M}} J_{1}(F)$, where $J_{1}(F)$ indicates the standard first $\mathcal{J}$-bundle associated with the fibration $F \rightarrow \mathcal{M}$. The total Lagrangian density of the theory is obtained by adding to the gravitational one a suitable matter Lagrangian density $\mathcal{L}^{(m)}$. Throughout this section, we shall consider matter Lagrangian densities of the kind $\mathcal{L}^{(m)}=\mathcal{L}^{(m)}(e, \omega, \psi, \partial \psi)$. The corresponding PoincaréCartan form is given by the sum $\Theta+\theta_{m}$, where $\theta_{m}=\mathcal{L}^{(m)} d s+\frac{\partial \mathcal{L}^{(m)}}{\partial \psi_{i}^{A}} \theta^{A} \wedge d s_{i}$ is the standard Poincaré-Cartan form associated with the matter density $\mathcal{L}^{(m)}$, being $\theta^{A}=d \psi^{A}-\psi_{i}^{A} d s_{i}$ the usual contact 1 -forms of the bundle $J_{1}(F)$. In such a circumstance, the Euler-Lagrange equations (12.16) assume the local expression

$$
f^{\prime}(\mathcal{R}) \mathcal{R}_{\mu \sigma}^{\lambda \sigma} e_{\lambda}^{i}-\frac{1}{2} e_{\mu}^{i} f(R)=\Sigma_{\mu}^{i}
$$

and

$$
f^{\prime}(R)\left(T_{t s}^{\alpha}-T_{t \sigma}^{\sigma} e_{s}^{\alpha}+T_{s \sigma}^{\sigma} e_{t}^{\alpha}\right)=\frac{\partial f^{\prime}(\mathcal{R})}{\partial x^{t}} e_{s}^{\alpha}-\frac{\partial f^{\prime}(\mathcal{R})}{\partial x^{s}} e_{t}^{\alpha}+S_{t s}^{\alpha}
$$

where $\Sigma_{\mu}^{i}:=\frac{1}{2 e} \frac{\partial \mathcal{L}_{m}}{\partial e_{i}^{\mu}}$ and $S_{t s}^{\alpha}:=-\frac{1}{2 e} \frac{\partial \mathcal{L}_{m}}{\partial \omega_{i}{ }^{\mu \nu}} e_{t}^{\mu} e_{s}^{\nu} e_{i}^{\alpha}$ play the role of energy-momentum and spin density tensors respectively. We are adopting physical units. In particular, from Eqs. 12.33b), we obtain

$$
f^{\prime} T_{t \sigma}^{\sigma}=-\frac{3}{2} \frac{\partial f^{\prime}}{\partial x^{t}}-\frac{1}{2} S_{t \sigma}^{\sigma}
$$

Then, substituting Eqs. (12.34) into Eqs. (12.33b), we find the expression for the torsion

$$
T_{t s}^{\alpha}=-\frac{1}{2 f^{\prime}}\left(\frac{\partial f^{\prime}}{\partial x^{p}}+S_{p \sigma}^{\sigma}\right)\left(\delta_{t}^{p} e_{s}^{\alpha}-\delta_{s}^{p} e_{t}^{\alpha}\right)+\frac{1}{f^{\prime}} S_{t s}^{\alpha}
$$

Eqs. (12.35) tell us that, in presence of $\omega$-dependent matter, there are two sources of torsion: the spin density $S_{t s}^{\alpha}$ and the nonlinearity of the gravitational Lagrangian. It is important to stress that this feature is not present in standard GR. Now, by considering the trace of Eqs. [12.33a) we obtain a relation between the scalar curvature $R$ and the trace $\Sigma$ of the energy-momentum tensor given by

$$
f^{\prime}(\mathcal{R}) \mathcal{R}-2 f(\mathcal{R})=\Sigma
$$

When the trace $\Sigma$ is allowed to assume only a constant value, the present theory amounts to an Einstein-like (if $S_{t s}^{\alpha}=0$,i.e. $\omega$-independent matter) or an Einstein-Cartan-like theory (if $S_{t s}^{\alpha} \neq 0$, i.e. $\omega$-dependent matter) with cosmological constant. In fact, in such a circumstance, Eq. (12.36) implies that the scalar curvature $\mathcal{R}$ also is constant. As a consequence, Eqs. (12.33a) and (12.35) can be expressed as

$$
\begin{gathered}
\mathcal{R}_{\mu}^{i}-\frac{1}{2}(\mathcal{R}+\Lambda) e_{\mu}^{i}=k \Sigma_{\mu}^{i}, \\
T_{t s}^{\alpha}=\frac{k}{2}\left(2 S_{t s}^{\alpha}-S_{t \sigma}^{\sigma} e_{s}^{\alpha}+S_{s \sigma}^{\sigma} e_{t}^{\alpha}\right),
\end{gathered}
$$

where $\Lambda=k f(\mathcal{R})-\mathcal{R}$ and $k=\frac{1}{f^{\prime}(\mathcal{R})}, \mathcal{R}$ being the constant value determined by Eq.(12.36), provided that $f^{\prime}(\mathcal{R}) \neq 0$. As in the previous section, this result holds with the exception of the particular case $\Sigma=0$ and $f(\mathcal{R})=\alpha \mathcal{R}^{2}$. Indeed, under these conditions, Eq. (12.36) is a trivial identity which imposes no restriction on the scalar curvature $\mathcal{R}$. From now on, we shall suppose that $\Sigma$ is not forced to be a constant when the matter field equations are satisfied. Besides, we shall suppose that the relation (12.36) is invertible so that the scalar curvature can be thought as a suitable function of $\Sigma$, namely

$$
\mathcal{R}=F(\Sigma)
$$

With this assumption in mind, defining the tensors $\mathcal{R}_{j}^{i}:=\mathcal{R}_{\mu \sigma}{ }^{\lambda \sigma} e_{\lambda}^{i} e_{j}^{\mu}, \Sigma_{j}^{i}:=\Sigma_{\mu}^{i} e_{j}^{\mu}, T_{i j}{ }^{h}:=T_{i j}^{\alpha} e_{\alpha}^{h}$ and $S_{i j}{ }^{h}:=S_{i j}^{\alpha} e_{\alpha}^{h}$, we rewrite Eqs. (12.33a) and (12.35) in the equivalent form, in strict analogy to the results of the previous section 21

$$
\mathcal{R}_{i j}-\frac{1}{2} \mathcal{R} g_{i j}=\frac{1}{f^{\prime}(F(\Sigma))}\left(\Sigma_{i j}-\frac{1}{4} \Sigma g_{i j}\right)-\frac{1}{4} F(\Sigma) g_{i j}
$$

\footnotetext{
21 The Latin indices have here the same meaning of Greek indices of previous section.
} 


$$
T_{i j}{ }^{h}=-\frac{1}{2 f^{\prime}(F(\Sigma))}\left(\frac{\partial f^{\prime}(F(\Sigma))}{\partial x^{p}}+S_{p \sigma}^{\sigma}\right)\left(\delta_{i}^{p} \delta_{j}^{h}-\delta_{j}^{p} \delta_{i}^{h}\right)+\frac{1}{f^{\prime}(F(\Sigma))} S_{i j}{ }^{h},
$$

In Eqs. 12.39a one has to distinguish the order of the indexes since, in general, the tensors $\mathcal{R}_{i j}$ and $\Sigma_{i j}$ are not symmetric.

Moreover, following [126], in the lhs of Eqs. [12.39a), we can distinguish the contribution due to the Christoffel terms from that due to the torsion dependent terms. To see this point, from Eqs. (11.4) and (11.5b), we first get the following representation for the contracted curvature tensor

$$
\mathcal{R}_{i j}=\tilde{R}_{i j}+\tilde{\nabla}_{j} K_{h i}{ }^{h}-\tilde{\nabla}_{h} K_{j i}{ }^{h}+K_{j i}{ }^{p} K_{h p}{ }^{h}-K_{h i}{ }^{p} K_{j p}{ }^{h},
$$

where $\tilde{R}_{i j}$ is the Ricci tensor of the Levi-Civita connection $\tilde{\Gamma}$ associated with the metric $g_{i j}=\eta_{\mu \nu} e_{i}^{\mu} e_{j}^{\nu}$, and $\tilde{\nabla}$ denotes the Levi-Civita covariant derivative. Then, recalling the expression of the contortion tensor [315]

$$
K_{i j}{ }^{h}=\frac{1}{2}\left(-T_{i j}{ }^{h}+T_{j}{ }^{h}-T^{h}{ }_{i j}\right),
$$

and using the second set of field equations (12.39b), we obtain the following representations

$$
\begin{gathered}
K_{i j}{ }^{h}=\hat{K}_{i j}{ }^{h}+\hat{S}_{i j}{ }^{h}, \\
\hat{S}_{i j}{ }^{h}:=\frac{1}{2 f^{\prime}}\left(-S_{i j}{ }^{h}+S_{j}{ }^{h}{ }_{i}-S_{i j}^{h}\right), \\
\hat{K}_{i j}{ }^{h}:=-\hat{T}_{j} \delta_{i}^{h}+\hat{T}_{p} g^{p h} g_{i j}, \\
\hat{T}_{j}:=\frac{1}{2 f^{\prime}}\left(\frac{\partial f^{\prime}}{\partial x^{j}}+S_{j \sigma}^{\sigma}\right) .
\end{gathered}
$$

Inserting eqs. (12.42) in eq. (12.40) we end up with the final expression for $\mathcal{R}_{i j}$

$$
\begin{aligned}
\mathcal{R}_{i j}= & \tilde{R}_{i j}+\tilde{\nabla}_{j} \hat{K}_{h i}{ }^{h}+\tilde{\nabla}_{j} \hat{S}_{h i}{ }^{h}-\tilde{\nabla}_{h} \hat{K}_{j i}{ }^{h}-\tilde{\nabla}_{h} \hat{S}_{j i}{ }^{h}+\hat{K}_{j i}{ }^{p} \hat{K}_{h p}{ }^{h}+\hat{K}_{j i}{ }^{p} \hat{S}_{h p}{ }^{h} \\
& +\hat{S}_{j i}{ }^{p} \hat{K}_{h p}{ }^{h}+\hat{S}_{j i}{ }^{p} \hat{S}_{h p}{ }^{h}-\hat{K}_{h i}{ }^{p} \hat{K}_{j p}{ }^{h}-\hat{K}_{h i}{ }^{p} \hat{S}_{j p}{ }^{h}-\hat{S}_{h i}{ }^{p} \hat{K}_{j p}{ }^{h}-\hat{S}_{h i}{ }^{p} \hat{S}_{j p}{ }^{h} .
\end{aligned}
$$

The last step is the substitution of Eqs. (12.43) into Eqs. 12.39a). Explicit examples of the described procedure are given in the next subsections. We will discuss specific cases of fields coupled with gravity acting as matter sources.

\section{The case of Dirac fields}

As a first example of matter, we consider the case of Dirac fields $\psi$. The matter Lagrangian density is given by

$$
\mathcal{L}_{D}=e\left[\frac{i}{2}\left(\bar{\psi} \gamma^{i} D_{i} \psi-D_{i} \bar{\psi} \gamma^{i} \psi\right)-m \bar{\psi} \psi\right]
$$

where $D_{i} \psi=\frac{\partial \psi}{\partial x^{i}}+\omega_{i}{ }^{\mu \nu} S_{\mu \nu} \psi$ and $D_{i} \bar{\psi}=\frac{\partial \bar{\psi}}{\partial x^{i}}-\bar{\psi} \omega_{i}{ }^{\mu \nu} S_{\mu \nu}$ are the covariant derivatives of the Dirac fields, $S_{\mu \nu}=\frac{1}{8}\left[\gamma_{\mu}, \gamma_{\nu}\right], \gamma^{i}=\gamma^{\mu} e_{\mu}^{i} ; \gamma^{\mu}$ denotes the Dirac matrices. The field equations for the Dirac fields are

$$
i \gamma^{h} D_{h} \psi-m \psi=0, \quad i D_{h} \bar{\psi} \gamma^{h}+m \bar{\psi}=0
$$

Since the Lagrangian (12.44) vanishes for $\psi$ and $\bar{\psi}$ satisfying the equation (12.45), the corresponding energy-momentum and spin density tensors are expressed, respectively, as

$$
\Sigma_{i j}=\frac{i}{4}\left[\bar{\psi} \gamma_{i} D_{j} \psi-\left(D_{j} \bar{\psi}\right) \gamma_{i} \psi\right]
$$


and

$$
S_{i j}^{h}=-\frac{i}{2} \bar{\psi}\left\{\gamma^{h}, S_{i j}\right\} \psi
$$

with $S_{i j}=\frac{1}{8}\left[\gamma_{i}, \gamma_{j}\right]$. Now, using the properties of the Dirac matrices, it is easily seen that $\left\{\gamma^{h}, S^{i j}\right\}=\frac{1}{2} \gamma^{[i} \gamma^{j} \gamma^{h]}$. This fact implies the total antisymmetry of the spin density tensor $S_{i j}{ }^{h}$. As a consequence, the contracted curvature and scalar curvature assume the simplified expressions (compare with Eq. (12.43)

$$
\mathcal{R}_{i j}=\tilde{R}_{i j}-2 \tilde{\nabla}_{j} \hat{T}_{i}-\tilde{\nabla}_{h} \hat{T}^{h} g_{i j}+2 \hat{T}_{i} \hat{T}_{j}-2 \hat{T}_{h} \hat{T}^{h} g_{i j}-\tilde{\nabla}_{h} \hat{S}_{j i}{ }^{h}-\hat{S}_{h i}{ }^{p} \hat{S}_{j p}{ }^{h}
$$

and

$$
\mathcal{R}=\tilde{R}-6 \tilde{\nabla}_{i} \hat{T}^{i}-6 \hat{T}_{i} \hat{T}^{i}-\hat{S}_{h i}{ }^{p} \hat{S}_{p}^{i}{ }^{h}
$$

where now $\hat{T}_{i}=\frac{1}{2 f^{\prime}} \frac{\partial f^{\prime}}{\partial x^{i}}$ and $\hat{S}_{i j}{ }^{h}:=-\frac{1}{2 f^{\prime}} S_{i j}{ }^{h}$. Inserting eqs. (12.48) into eqs. 112.39a) and using of the above expression for $\hat{T}_{i}$, we obtain the final Einstein-like equations

$$
\begin{array}{r}
\tilde{R}_{i j}-\frac{1}{2} \tilde{R} g_{i j}=\frac{1}{\varphi} \Sigma_{i j}+\frac{1}{\varphi^{2}}\left(-\frac{3}{2} \frac{\partial \varphi}{\partial x^{i}} \frac{\partial \varphi}{\partial x^{j}}+\varphi \tilde{\nabla}_{j} \frac{\partial \varphi}{\partial x^{i}}+\frac{3}{4} \frac{\partial \varphi}{\partial x^{h}} \frac{\partial \varphi}{\partial x^{k}} g^{h k} g_{i j}\right. \\
\left.-\varphi \tilde{\nabla}^{h} \frac{\partial \varphi}{\partial x^{h}} g_{i j}-V(\varphi) g_{i j}\right)+\tilde{\nabla}_{h} \hat{S}_{j i}{ }^{h}+\hat{S}_{h i}{ }^{p} \hat{S}_{j p}{ }^{h}-\frac{1}{2} \hat{S}_{h q}{ }^{p} \hat{S}^{q}{ }_{p}{ }^{h} g_{i j}
\end{array}
$$

where we have defined the scalar field

$$
\varphi:=f^{\prime}(F(\Sigma))
$$

and the effective potential

$$
V(\varphi):=\frac{1}{4}\left[\varphi F^{-1}\left(\left(f^{\prime}\right)^{-1}(\varphi)\right)+\varphi^{2}\left(f^{\prime}\right)^{-1}(\varphi)\right]
$$

To conclude, we notice that Eqs. (12.49) can be simplified by performing a conformal transformation. Indeed, setting $\bar{g}_{i j}:=\varphi \eta_{\mu \nu} e_{i}^{\mu} e_{j}^{\nu}$, Eqs. (12.49) can be rewritten in the easier form

$$
\bar{R}_{i j}-\frac{1}{2} \bar{R} \bar{g}_{i j}=\frac{1}{\varphi} \Sigma_{i j}-\frac{1}{\varphi^{3}} V(\varphi) \bar{g}_{i j}+\tilde{\nabla}_{h} \hat{S}_{j i}{ }^{h}+\hat{S}_{h i}{ }^{p} \hat{S}_{j p}{ }^{h}-\frac{1}{2 \varphi} \hat{S}_{h q}{ }^{p} \hat{S}^{q}{ }_{p}{ }^{h} g_{i j}
$$

where $\bar{R}_{i j}$ and $\bar{R}$ are respectively the Ricci tensor and the Ricci scalar curvature associated with the conformal metric $\bar{g}_{i j}$.

\section{The case of Yang-Mills fields}

As it has been shown in some previous works [175, 176, 567, 568], also gauge theories can be formulated within the framework of $\mathcal{J}$-bundles. Therefore, we can describe $f(R)$-gravity coupled with Yang-Mills fields in the new geometric setting.

To see this point, let $Q \rightarrow \mathcal{M}$ be a principal fiber bundle over space-time, with structural group a semi-simple Lie group $G$. We consider the affine bundle $J_{1} Q / G \rightarrow \mathcal{M}$ (the space of principal connections of $Q \rightarrow \mathcal{M}$ ) and refer it to local coordinates $x^{i}, a_{i}^{A}, A=1, \ldots, r=\operatorname{dim} G$.

In a combined theory of $f(R)$-gravity and Yang-Mills fields, additional dynamical fields are principal connections of $Q$ represented by sections of the bundle $J_{1} Q / G \rightarrow \mathcal{M}$. The extended configuration space of the theory is then the fiber product $\mathcal{E} \times \mathcal{C} \times J_{1} Q / G \rightarrow \mathcal{M}$ over $\mathcal{M}$.

Following the approach illustrated above, we may construct the quotient space $\mathcal{J}\left(\mathcal{E} \times \mathcal{C} \times J_{1} Q / G\right.$ ) (see [567] for details). As additional $\mathcal{J}$-coordinates the latter admits the components $F_{i j}^{A}$ of the curvature tensors of the principal connections of $Q \rightarrow \mathcal{M}$. Holonomic sections of the bundle $\mathcal{J}\left(\mathcal{E} \times \mathcal{C} \times J_{1} Q / G\right)$ are of the form (12.5) together with

$$
F_{i j}^{A}(x)=\frac{\partial a_{j}^{A}(x)}{\partial x^{i}}-\frac{\partial a_{i}^{A}(x)}{\partial x^{j}}+a_{j}^{B}(x) a_{i}^{C}(x) C_{C B}^{A}
$$


where $C_{C B}^{A}$ are the structure coefficients of the Lie algebra of $G$.

The Poincaré-Cartan 4-form associated with a Lagrangian on $\mathcal{J}\left(\mathcal{E} \times \mathcal{C} \times J_{1} Q / G\right)$ of the form $L=\mathcal{L}\left(x^{i}, e_{i}^{\mu}, a_{i}^{A}, \mathcal{R}_{i j}{ }^{\mu \nu}, F_{i j}^{A}\right) d s$ is

$$
\Theta=\mathcal{L} d s-\frac{1}{4} \frac{\partial \mathcal{L}}{\partial \mathcal{R}_{h k}{ }^{\alpha \beta}} \theta^{\alpha \beta} \wedge d s_{h k}-\frac{1}{2} \frac{\partial \mathcal{L}}{\partial F_{h k}^{A}} \theta^{A} \wedge d s_{h k}
$$

where $\theta^{A}=\Phi^{A}-F^{A}$, being $F^{A}:=\frac{1}{2} F_{i j}^{A} d x^{i} \wedge d x^{j}$ and $\Phi^{A}:=d a_{i}^{A} \wedge d x^{i}+\frac{1}{2} a_{i}^{B} a_{j}^{C} C_{C B}^{A} d x^{j} \wedge d x^{i}$.

Variational field equations are still of the form

$$
\left.\mathcal{J} \sigma^{*}(\mathcal{J}(X)\lrcorner d \Theta\right)=0
$$

for all $\mathcal{J}$-prolongable vector fields $\mathrm{X}$ on $\mathcal{E} \times \mathcal{C} \times J_{1} Q / G$. Due to the arbitrariness of the infinitesimal deformations $X$, Eq. (12.55) splits into three sets of final equations, respectively given by Eqs. (12.16) together with

$$
\mathcal{J} \sigma^{*}\left(\frac{\partial \mathcal{L}}{\partial a_{i}^{A}}-D_{k} \frac{\partial \mathcal{L}}{\partial F_{k j}^{A}}\right)=0
$$

In particular, if the Lagrangian density is

$$
\mathcal{L}=e\left(f(R)-\frac{1}{4} F_{i j}^{A} F_{p q}^{B} \gamma_{A B} \eta^{\mu \nu} e_{\mu}^{p} e_{\nu}^{i} \eta^{\lambda \sigma} e_{\lambda}^{q} e_{\sigma}^{j}\right)
$$

expressing $f(R)$-gravity coupled with a free Yang-Mills field, Eqs. (12.16) and (12.56) assume the explicit form

$$
\begin{gathered}
e_{\mu}^{i} f(\mathcal{R})-2 f^{\prime}(\mathcal{R}) \mathcal{R}_{\mu \sigma}{ }^{\lambda \sigma} e_{\lambda}^{i}=\frac{1}{4} F_{j k}^{A} F_{A}^{j k} e_{\mu}^{i}-F_{j}^{A i} F_{A k}^{j} e_{\mu}^{k} \\
\frac{\partial}{\partial x^{k}}\left[2 e f^{\prime}(\mathcal{R})\left(e_{\mu}^{k} e_{\nu}^{i}-e_{\mu}^{i} e_{\nu}^{k}\right)\right]-\omega_{k}{ }^{\lambda}{ }_{\mu}\left[2 e f^{\prime}(\mathcal{R})\left(e_{\lambda}^{k} e_{\nu}^{i}-e_{\lambda}^{i} e_{\nu}^{k}\right)\right]-\omega_{k}{ }^{\lambda}{ }_{\nu}\left[2 e f^{\prime}(\mathcal{R})\left(e_{\mu}^{k} e_{\lambda}^{i}-e_{\mu}^{i} e_{\lambda}^{k}\right)\right]=0 \\
D_{k}\left(e F_{A}^{i k}\right)=0
\end{gathered}
$$

where $D_{k}\left(e F_{A}^{i k}\right)=\frac{\partial\left(e F_{A}^{i k}\right)}{\partial x^{k}}-a_{k}^{B}\left(e F_{C}^{i k}\right) C_{B A}^{C}$.

Since the trace of the energy-impulse tensor $T_{\mu}^{i}:=\frac{1}{4} F_{j k}^{A} F_{A}^{j k} e_{\mu}^{i}-F_{j}^{A i} F_{A k}^{j} e_{\mu}^{k}$ vanishes identically, we have again:

- if $f(\mathcal{R})=k \mathcal{R}$ we recover the Einstein-Yang-Mills theory;

- if $f(\mathcal{R}) \neq k \mathcal{R}^{2}$ the torsion is necessarily zero and we recover a $f(\mathcal{R})$-theory without torsion coupled with a Yang-Mills field;

- We can have non-vanishing torsion only in the case $f(\mathcal{R})=k \mathcal{R}^{2}$.

\section{The case of spin fluid matter}

As a last example of matter source, we consider the case of a semiclassical spin fluid. This is characterized by an energy-momentum tensor of the form

$$
\Sigma^{i j}=(\rho+p) u^{i} u^{j}+p g^{i j}
$$

and a spin density tensor given by

$$
S_{i j}{ }^{h}=S_{i j} u^{h}
$$

where $u^{i}$ and $S_{i j}$ denote, respectively, the 4-velocity and the spin density of the fluid (see, for example, 313] and references therein). However, the constraint $u^{i} u_{i}=-1$ must hold. Other models of spin fluids are possible, where, 
due to the treatment of spin as a thermodynamical variable, different expressions for the energy-momentum tensor may be taken into account 206, 288, 482].

The 4-velocity and the spin density satisfy by the so called convective condition

$$
S_{i j} u^{j}=0
$$

It is easily seen that the relations (12.60) imply the identities

$$
\hat{S}_{i}{ }^{i h}=-\hat{S}_{i}{ }^{h i}
$$

obtained inserting Eq. (12.59b) in Eq. (12.42b) and using (12.60). Making use of (12.61) as well as of Eqs. (12.42b), (12.43), (12.59b) and (12.60), we can express the Ricci curvature tensor and scalar respectively as

$$
\begin{array}{r}
\mathcal{R}_{i j}=\tilde{R}_{i j}-2 \tilde{\nabla}_{j} \hat{T}_{i}-\tilde{\nabla}_{h} \hat{T}^{h} g_{i j}+2 \hat{T}_{i} \hat{T}_{j}-2 \hat{T}_{h} \hat{T}^{h} g_{i j}-\frac{1}{f^{\prime}} \hat{T}_{h} S^{h}{ }_{j} u_{i} \\
-\frac{1}{2 f^{\prime}} \tilde{\nabla}_{h}\left(S_{j i} u^{h}+S_{i}{ }^{h} u_{j}-S^{h}{ }_{j} u_{i}\right)+\frac{1}{4\left(f^{\prime}\right)^{2}} S^{p q} S_{p q} u_{i} u_{j}
\end{array}
$$

and

$$
\mathcal{R}=\tilde{R}-6 \tilde{\nabla}_{i} \hat{T}^{i}-6 \hat{T}_{i} \hat{T}^{i}-\frac{1}{4\left(f^{\prime}\right)^{2}} S^{p q} S_{p q}
$$

where $\hat{T}_{i}=\frac{1}{2 f^{\prime}} \frac{\partial f^{\prime}}{\partial x^{i}}$. In view of this, substituting Eqs. (12.62) in Eqs. (12.39a), and using the definitions (12.50) and (12.51), we obtain Einstein-like equations of the form

$$
\begin{array}{r}
\tilde{R}_{i j}-\frac{1}{2} \tilde{R} g_{i j}=\frac{1}{\varphi} \Sigma_{i j}+\frac{1}{\varphi^{2}}\left(-\frac{3}{2} \frac{\partial \varphi}{\partial x^{i}} \frac{\partial \varphi}{\partial x^{j}}+\varphi \tilde{\nabla}_{j} \frac{\partial \varphi}{\partial x^{i}}+\frac{3}{4} \frac{\partial \varphi}{\partial x^{h}} \frac{\partial \varphi}{\partial x^{k}} g^{h k} g_{i j}\right. \\
\left.-\varphi \tilde{\nabla}^{h} \frac{\partial \varphi}{\partial x^{h}} g_{i j}-V(\varphi) g_{i j}\right)+\frac{1}{\varphi} \hat{T}_{h} S^{h}{ }_{j} u_{i}+\frac{1}{2 \varphi} \tilde{\nabla}_{h}\left(S_{j i} u^{h}+S_{i}{ }^{h} u_{j}-S^{h}{ }_{j} u_{i}\right) \\
-\frac{1}{4 \varphi^{2}} S^{p q} S_{p q} u_{i} u_{j}-\frac{1}{8 \varphi^{2}} S^{p q} S_{p q} g_{i j}
\end{array}
$$

Eqs. (12.63) are the "microscopic" field equations for $f(R)$-gravity with torsion, coupled with a semiclassical spin fluid. In this form, all the source contributions are put in evidence and their role is clearly defined into dynamics.

\section{E. Equivalence with scalar-tensor theories}

The results of the last subsection lead to take into account the analogies between $f(R)$-gravity with torsion and scalar-tensor theories with torsion, as discussed, for example, in [291, 537]. To this end, let us consider a Lagrangian density of the form

$$
\mathcal{L}=\varphi e \mathcal{R}-e U(\varphi)+\mathcal{L}_{m}
$$

where $\varphi$ is a scalar field, $U(\varphi)$ is a suitable potential and $\mathcal{L}_{m}$ is a matter Lagrangian density.

The Euler-Lagrange equations (12.16) applied to the Lagrangian density (12.64), yield the corresponding field equations

$$
\begin{gathered}
\mathcal{R}_{\mu \sigma}{ }^{\lambda \sigma} e_{\lambda}^{i}-\frac{1}{2} \mathcal{R} e_{\mu}^{i}=\frac{1}{\varphi} \Sigma_{\mu}^{i}-\frac{1}{2 \varphi} U(\varphi) e_{\mu}^{i} \\
\varphi\left(T_{t s}^{\alpha}-T_{t \sigma}^{\sigma} e_{s}^{\alpha}+T_{s \sigma}^{\sigma} e_{t}^{\alpha}\right)=\frac{\partial \varphi}{\partial x^{t}} e_{s}^{\alpha}-\frac{\partial \varphi}{\partial x^{s}} e_{t}^{\alpha}+S_{t s}^{\alpha}
\end{gathered}
$$

while the Euler-Lagrange equation for the scalar field is given by

$$
\mathcal{R}=U^{\prime}(\varphi)
$$


Inserting Eq. (12.65c) in the trace of Eqs. (12.65a), we obtain an algebraic relation between the matter trace $\Sigma$ and the scalar field $\varphi$ expressed as

$$
\Sigma-2 U(\varphi)+\varphi U^{\prime}(\varphi)=0
$$

Now, under the conditions $U(\varphi)=\frac{2}{\varphi} V(\varphi)$, where $V(\varphi)$ is defined as in Eq. (12.51) and $f^{\prime \prime} \neq 0$. It is easily seen that the relation (12.66) represents exactly the inverse of (12.50). In fact, from the definition of the effective potential (12.51) and the expression $F^{-1}(X)=f^{\prime}(X) X-2 f(X)$ we have

$$
U(\varphi)=\frac{2}{\varphi} V(\varphi)=\frac{1}{2}\left[F^{-1}\left(\left(f^{\prime}\right)^{-1}(\varphi)\right)+\varphi\left(f^{\prime}\right)^{-1}(\varphi)\right]=\left[\varphi\left(f^{\prime}\right)^{-1}(\varphi)-f\left(\left(f^{\prime}\right)^{-1}(\varphi)\right)\right]
$$

so that

$$
U^{\prime}(\varphi)=\left(f^{\prime}\right)^{-1}(\varphi)+\frac{\varphi}{f^{\prime \prime}\left(\left(f^{\prime}\right)^{-1}(\varphi)\right)}-\frac{\varphi}{f^{\prime \prime}\left(\left(f^{\prime}\right)^{-1}(\varphi)\right)}=\left(f^{\prime}\right)^{-1}(\varphi)
$$

and then

$$
\Sigma=-\varphi U^{\prime}(\varphi)+2 U(\varphi)=f^{\prime}\left(\left(f^{\prime}\right)^{-1}(\varphi)\right)\left(f^{\prime}\right)^{-1}(\varphi)-2 f\left(\left(f^{\prime}\right)^{-1}(\varphi)\right)=F^{-1}\left(\left(f^{\prime}\right)^{-1}(\varphi)\right)
$$

In view of the latter relation, Eqs. (12.65) result to be equivalent to Eqs. (12.38) and (12.39). This fact proves the equivalence between $f(R)$-gravity and scalar-tensor theories with torsion obtained also in the $\mathcal{J}$-bundles framework.

\section{F. Discussion}

In this Section, we have discussed the $f(R)$-theories of gravity with torsion in the $\mathcal{J}$-bundles framework.

This formalism gives rise to a new geometric picture which allows to put in evidence several features of the theories, in particular their symmetries and conservation laws. In particular, due to the fact that the components of the torsion and curvature tensors can be chosen as fiber $\mathcal{J}$-coordinates on $\mathcal{J}(\mathcal{E} \times \mathcal{C})$, the field equations can be easily obtained in suitable forms where the role of the geometry and the sources is clearly defined.

Furthermore, such a representation allows to classify the couplings with respect to the various matter fields whose global effect is that to enlarge and characterize the $\mathcal{J}$-bundle.

We have given specific examples of couplings taking into account Dirac fields, Yang-Mills fields and spin fluids. In any case, the $\mathcal{J}$-vector fields allow to write the $f(R)$-field equations in such a way that curvature, torsion and matter components have a clear and distinct role into dynamics. This distinction emerges since we have adopted here a tetrad-affine formallism.

\section{THE HAMILTONIAN FORMULATION}

Here we want to face the problem to study $f(R)$-gravity at a fundamental level. In particular, in the framework of the Arnowitt-Deser-Misner $(\mathcal{A D} \mathcal{M})$ formalism [30], we want to investigate the possibility to find out cosmological terms as eigenvalues of generalized $f(R)$-Hamiltonians in a Sturm-Liouville-like problem 22. This issue is particularly relevant from several viewpoints. First of all, our aim is to show that vacuum energy of gravitational field is not a particular feature of GR where the cosmological constant has to be added by hand into dynamics. At a classical level, it is well known that $f(R)$-gravity, for the Ricci scalar $R$ equal to a constant, exhibit several de Sitter solutions [51] but a definite discussion, at a fundamental level, considering cosmological terms as eigenvalues of such theories is lacking. Besides, the computation of the Casimir energy, the seeking for zero point energy in different background 23 give a track to achieve one-loop energy regularization and renormalization for this kind of theories [182, 238]. On the other hand, these issues can be considered in a multigravity approach to space-time foam if the $\mathcal{N}$ space-times constituting the foam are supposed to evolve, in general, with different curvature laws and ground states (cosmological constants) [286].

\footnotetext{
22 See Ref. [285], for the application of the Sturm-Liouville problem in the case of $f(R)=R$, even in presence of a massive graviton.

23 For different $f(R)$, we expect different zero point energies and, obviously, different vacuum states.
} 


\section{A. The Hamiltonian constraint of General Relativity}

Let us briefly report how to compute the Hamiltonian constraint for GR considering the standard Hilbert-Einstein theory $f(R)=R$ and the Arnowitt-Deser-Misner (ADM) $3+1$ decomposition [30]. In terms of these variables, the line element is 24

$$
d s^{2}=g_{\mu \nu}(x) d x^{\mu} d x^{\nu}=\left(-N^{2}+N_{i} N^{i}\right) d t^{2}+2 N_{j} d t d x^{j}+g_{i j} d x^{i} d x^{j}
$$

$N$ is the lapse function, while $N_{i}$ the shift function. In terms of these variables, the gravitational Lagrangian, with the boundary terms neglected, can be written as

$$
\mathcal{L}\left[N, N_{i}, g_{i j}\right]=\sqrt{-g} R=\frac{N \sqrt{{ }^{3} g}}{2 \kappa}\left[K_{i j} K^{i j}-K^{2}+\left({ }^{3} R-2 \Lambda_{c}\right)\right]
$$

where $K_{i j}$ is the second fundamental form, $K=g^{i j} K_{i j}$ is the trace, ${ }^{3} R$ is the three dimensional scalar curvature and $\sqrt{{ }^{3} g}$ is the three dimensional determinant of the metric. The conjugate momentum is simply

$$
\pi^{i j}=\frac{\delta \mathcal{L}}{\delta\left(\partial_{t} g_{i j}\right)}=\left({ }^{3} g^{i j} K-K^{i j}\right) \frac{\sqrt{{ }^{3} g}}{2 \kappa} .
$$

By a Legendre transformation, we calculate the Hamiltonian

$$
H=\int d^{3} x\left[N \mathcal{H}+N_{i} \mathcal{H}^{i}\right],
$$

where

$$
\mathcal{H}=(2 \kappa) G_{i j k l} \pi^{i j} \pi^{k l}-\frac{\sqrt{{ }^{3} g}}{2 \kappa}\left({ }^{3} R-2 \Lambda_{c}\right)
$$

and

$$
\mathcal{H}^{i}=-2 \nabla_{j} \pi^{j i}
$$

where $\Lambda_{c}$ is the bare cosmological constant. The equations of motion lead to two classical constraints

$$
\left\{\begin{array}{c}
\mathcal{H}=0 \\
\mathcal{H}^{i}=0
\end{array}\right.
$$

representing invariance under time re-parameterization and invariance under diffeomorphism, respectively. $G_{i j k l}$ is the supermetric defined as

$$
G_{i j k l}=\frac{1}{2 \sqrt{g}}\left(g_{i k} g_{j l}+g_{i l} g_{j k}-g_{i j} g_{k l}\right) .
$$

When $\mathcal{H}$ and $\mathcal{H}^{i}$ are considered as operators acting on some wave function, we have

$$
\mathcal{H} \Psi\left[g_{i j}\right]=0
$$

and

$$
\mathcal{H}_{i} \Psi\left[g_{i j}\right]=0 \text {. }
$$

Eq. (13.8) is the Wheeler-De Witt equation (WDW) 213]. Eqs.(13.8) and (13.9) describe the wave function of the Universe $\Psi\left[g_{i j}\right]$. The WDW equation represents invariance under time re-parameterization in an operatorial form. This standard lore can be applied to a generic $f(R)$ theory of gravity with the aim to achieve a cosmological term as an eigenvalue of the WDW equation.

\footnotetext{
${ }^{24}$ Here we adopt again the standard notations and the signature used throughout this Report.
} 


\section{B. The Hamiltonian constraint for $f(R)$-gravity}

Let us consider now the Lagrangian density describing a generic $f(R)$ theory of gravity, namely

$$
\mathcal{L}=\sqrt{-g}\left(f(R)-2 \Lambda_{c}\right), \quad \text { with } f^{\prime \prime} \neq 0,
$$

where $f(R)$ is an arbitrary smooth function of the scalar curvature and primes denote differentiation with respect to the scalar curvature. A cosmological term is added also in this case for the sake of generality. Obviously $f^{\prime \prime}=0$ corresponds to GR. The generalized Hamiltonian density for the $f(R)$ theory assumes the form 25

$$
\mathcal{H}=\frac{1}{2 \kappa}\left[\frac{\mathcal{P}}{6}\left({ }^{(3)} R-2 \Lambda_{c}-3 K_{i j} K^{i j}+K^{2}\right)+V(\mathcal{P})-\frac{1}{3} g^{i j} \mathcal{P}_{\mid i j}-2 p^{i j} K_{i j}\right],
$$

where

$$
V(\mathcal{P})=\sqrt{g}\left[R f^{\prime}(R)-f(R)\right]
$$

Henceforth, the superscript 3 indicating the spatial part of the metric will be omitted on the metric itself. When $f(R)=R, V(\mathcal{P})=0$ as it should be. Since

$$
\mathcal{P}^{i j}=-2 \sqrt{g} g^{i j} f^{\prime}(R) \quad \Longrightarrow \quad \mathcal{P}=-6 \sqrt{g} f^{\prime}(R)
$$

we have

$$
\mathcal{H}=\frac{1}{2 \kappa}\left[-\sqrt{g} f^{\prime}(R)\left({ }^{(3)} R-2 \Lambda_{c}-3 K_{i j} K^{i j}+K^{2}\right)+V(\mathcal{P})+2 g^{i j}\left(\sqrt{g} f^{\prime}(R)\right)_{\mid i j}-2 p^{i j} K_{i j}\right] .
$$

With the help of Eq.(13.2), Eq.(13.14) becomes

$$
\mathcal{H}=f^{\prime}(R)\left[(2 \kappa) G_{i j k l} \pi^{i j} \pi^{k l}-\frac{\sqrt{g}}{2 \kappa}\left({ }^{(3)} R-2 \Lambda_{c}\right)\right]+\frac{1}{2 \kappa}\left[\sqrt{g} f^{\prime}(R)\left(2 K_{i j} K^{i j}\right)+V(\mathcal{P})+2 g^{i j}\left(\sqrt{g} f^{\prime}(R)\right)_{\mid i j}-2 p^{i j} K_{i j}\right] .
$$

However

$$
p^{i j}=\sqrt{g} K^{i j}
$$

then we obtain

$$
\mathcal{H}=f^{\prime}(R)\left[(2 \kappa) G_{i j k l} \pi^{i j} \pi^{k l}-\frac{\sqrt{g}}{2 \kappa}\left({ }^{(3)} R-2 \Lambda_{c}\right)\right]+\frac{1}{2 \kappa}\left[2 \sqrt{g} K_{i j} K^{i j}\left(f^{\prime}(R)-1\right)+V(\mathcal{P})+2 g^{i j}\left(\sqrt{g} f^{\prime}(R)\right)_{\mid i j}\right]
$$

and transforming into canonical momenta, one gets

$$
\begin{aligned}
\mathcal{H}=f^{\prime}(R)\left[(2 \kappa) G_{i j k l} \pi^{i j} \pi^{k l}-\frac{\sqrt{g}}{2 \kappa}\left({ }^{(3)} R-2 \Lambda_{c}\right)\right]+ & 2(2 \kappa)\left[G_{i j k l} \pi^{i j} \pi^{k l}+\frac{\pi}{4}^{2}\right]\left(f^{\prime}(R)-1\right)+ \\
& +\frac{1}{2 \kappa}\left[V(\mathcal{P})+2 g^{i j}\left(\sqrt{g} f^{\prime}(R)\right)_{\mid i j}\right]
\end{aligned}
$$

By imposing the Hamiltonian constraint, we obtain

$$
\begin{gathered}
f^{\prime}(R)\left[(2 \kappa) G_{i j k l} \pi^{i j} \pi^{k l}-\frac{\sqrt{g}}{2 \kappa}(3) R\right]+ \\
+2(2 \kappa)\left[G_{i j k l} \pi^{i j} \pi^{k l}+\frac{\pi^{2}}{4}\right]\left(f^{\prime}(R)-1\right)+\frac{1}{2 \kappa}\left[V(\mathcal{P})+2 g^{i j}\left(\sqrt{g} f^{\prime}(R)\right)_{\mid i j}\right]=-f^{\prime}(R) \sqrt{g} \frac{\Lambda_{c}}{\kappa}
\end{gathered}
$$

\footnotetext{
${ }^{25}$ See also Ref. 481] for technical details.
} 
If we assume that $f^{\prime}(R) \neq 0$ the previous expression becomes

$$
\begin{aligned}
{\left[(2 \kappa) G_{i j k l} \pi^{i j} \pi^{k l}-\frac{\sqrt{g}}{2 \kappa}{ }^{(3)} R\right]+(2 \kappa)\left[G_{i j k l} \pi^{i j} \pi^{k l}+\frac{\pi}{4}^{2}\right] \frac{2\left(f^{\prime}(R)-1\right)}{f^{\prime}(R)} } & +\frac{1}{2 \kappa f^{\prime}(R)}\left[V(\mathcal{P})+2 g^{i j}\left(\sqrt{g} f^{\prime}(R)\right)_{\mid i j}\right]= \\
& =-\sqrt{g} \frac{\Lambda_{c}}{\kappa}
\end{aligned}
$$

Now, we integrate over the hypersurface $\Sigma$ to obtain

$$
\begin{aligned}
\int_{\Sigma} d^{3} x\{ & {\left.\left[(2 \kappa) G_{i j k l} \pi^{i j} \pi^{k l}-\frac{\sqrt{g}}{2 \kappa}{ }^{(3)} R\right]+(2 \kappa)\left[G_{i j k l} \pi^{i j} \pi^{k l}+\frac{\pi}{4}^{2}\right] \frac{2\left(f^{\prime}(R)-1\right)}{f^{\prime}(R)}\right\} } \\
& +\int_{\Sigma} d^{3} x \frac{1}{2 \kappa f^{\prime}(R)}\left[V(\mathcal{P})+2 g^{i j}\left(\sqrt{g} f^{\prime}(R)\right)_{\mid i j}\right]=-\frac{\Lambda_{c}}{\kappa} \int_{\Sigma} d^{3} x \sqrt{g} .
\end{aligned}
$$

The term

$$
\frac{1}{\kappa} \int_{\Sigma} d^{3} x \frac{1}{f^{\prime}(R)} g^{i j}\left(\sqrt{g} f^{\prime}(R)\right)_{\mid i j}
$$

appears to be a three-divergence and therefore will not contribute to the computation. The remaining equation simplifies into

$$
\int_{\Sigma} d^{3} x\left\{\left[(2 \kappa) G_{i j k l} \pi^{i j} \pi^{k l}-\frac{\sqrt{g}}{2 \kappa}{ }^{(3)} R\right]+(2 \kappa)\left[G_{i j k l} \pi^{i j} \pi^{k l}+\frac{\pi}{4}^{2}\right] \frac{2\left(f^{\prime}(R)-1\right)}{f^{\prime}(R)}+\frac{V(\mathcal{P})}{2 \kappa f^{\prime}(R)}\right\}=-\frac{\Lambda_{c}}{\kappa} \int_{\Sigma} d^{3} x \sqrt{g} .
$$

By a canonical procedure of quantization, we want to obtain the vacuum state of a generic $f(R)$ theory.

\section{The cosmological constant as an eigenvalue}

The standard WDW equation (13.8) can be cast into the form of an eigenvalue equation

$$
\hat{\Lambda}_{\Sigma} \Psi\left[g_{i j}\right]=\Lambda(\vec{x}) \Psi\left[g_{i j}\right]
$$

where

$$
\hat{\Lambda}_{\Sigma}=(2 \kappa) G_{i j k l} \pi^{i j} \pi^{k l}-\frac{\sqrt{g}_{3}}{2 \kappa} R
$$

If we multiply Eq.(13.26) by $\Psi^{*}\left[g_{i j}\right]$ and we functionally integrate over the three spatial metric $g_{i j}$, we get

$$
\int \mathcal{D}\left[g_{i j}\right] \Psi^{*}\left[g_{i j}\right] \hat{\Lambda}_{\Sigma} \Psi\left[g_{i j}\right]=\int \mathcal{D}\left[g_{i j}\right] \Lambda(\vec{x}) \Psi^{*}\left[g_{i j}\right] \Psi\left[g_{i j}\right]
$$

and after integrating over the hypersurface $\Sigma$, one can formally re-write the modified WDW equation as

$$
\frac{1}{V} \frac{\int \mathcal{D}\left[g_{i j}\right] \Psi^{*}\left[g_{i j}\right] \int_{\Sigma} d^{3} x \hat{\Lambda}_{\Sigma} \Psi\left[g_{i j}\right]}{\int \mathcal{D}\left[g_{i j}\right] \Psi^{*}\left[g_{i j}\right] \Psi\left[g_{i j}\right]}=\frac{1}{V} \frac{\left\langle\Psi\left|\int_{\Sigma} d^{3} x \hat{\Lambda}_{\Sigma}\right| \Psi\right\rangle}{\langle\Psi \mid \Psi\rangle}=-\frac{\Lambda_{c}}{\kappa},
$$

where the explicit expression of $\Lambda(\vec{x})$ has been used and we have defined the volume of the hypersurface $\Sigma$ as

$$
V=\int_{\Sigma} d^{3} x \sqrt{g}
$$

The formal eigenvalue equation (13.29) is a simple manipulation of Eq.(13.8). We can gain more information considering a separation of the spatial part of the metric into a background term, $\bar{g}_{i j}$, and a quantum fluctuation, $h_{i j}$,

$$
g_{i j}=\bar{g}_{i j}+h_{i j}
$$


Thus Eq.13.29) becomes

$$
\frac{\left\langle\Psi\left|\int_{\Sigma} d^{3} x\left[\hat{\Lambda}_{\Sigma}^{(0)}+\hat{\Lambda}_{\Sigma}^{(1)}+\hat{\Lambda}_{\Sigma}^{(2)}+\ldots\right]\right| \Psi\right\rangle}{\langle\Psi \mid \Psi\rangle}=-\frac{\Lambda_{c}}{\kappa} \Psi\left[g_{i j}\right]
$$

where $\hat{\Lambda}_{\Sigma}^{(i)}$ represents the $i^{\text {th }}$ order of perturbation in $h_{i j}$. By observing that the kinetic part of $\hat{\Lambda}_{\Sigma}$ is quadratic in the momenta, we only need to expand the three-scalar curvature $\int d^{3} x \sqrt{g} R^{(3)}$ up to the quadratic order and we get

$$
\begin{gathered}
\int_{\Sigma} d^{3} x \sqrt{\bar{g}}\left[-\frac{1}{4} h \triangle h+\frac{1}{4} h^{l i} \triangle h_{l i}-\frac{1}{2} h^{i j} \nabla_{l} \nabla_{i} h_{j}^{l}+\right. \\
\left.+\frac{1}{2} h \nabla_{l} \nabla_{i} h^{l i}-\frac{1}{2} h^{i j} R_{i a} h_{j}^{a}+\frac{1}{2} h R_{i j} h^{i j}+\frac{1}{4} h\left(R^{(0)}\right) h\right]
\end{gathered}
$$

where $h$ is the trace of $h_{i j}$ and $R^{(0)}$ is the three dimensional scalar curvature. By repeating the same procedure for the generalized WDW equation Eq.113.25, we obtain

$$
\frac{1}{V} \frac{\left\langle\Psi\left|\int_{\Sigma} d^{3} x\left[\hat{\Lambda}_{\Sigma}^{(2)}\right]\right| \Psi\right\rangle}{\langle\Psi \mid \Psi\rangle}+\frac{2 \kappa}{V} \frac{2\left(f^{\prime}(R)-1\right)}{f^{\prime}(R)} \frac{\left\langle\Psi\left|\int_{\Sigma} d^{3} x\left[G_{i j k l} \pi^{i j} \pi^{k l}+\frac{\pi}{4}^{2}\right]\right| \Psi\right\rangle}{\langle\Psi \mid \Psi\rangle}+\frac{1}{V} \frac{\left\langle\Psi\left|\int_{\Sigma} d^{3} x \frac{V(\mathcal{P})}{2 \kappa f^{\prime}(R)}\right| \Psi\right\rangle}{\langle\Psi \mid \Psi\rangle}=-\frac{\Lambda_{c}}{\kappa}
$$

From Eq.(13.34), we can define a "modified" $\hat{\Lambda}_{\Sigma}^{(2)}$ operator which includes $f^{\prime}(R)$. Thus, we obtain

$$
\frac{\left\langle\Psi\left|\int_{\Sigma} d^{3} x\left[\hat{\Lambda}_{\Sigma, f(R)}^{(2)}\right]\right| \Psi\right\rangle}{\langle\Psi \mid \Psi\rangle}+\frac{2 \kappa}{V} \frac{2\left(f^{\prime}(R)-1\right)}{f^{\prime}(R)} \frac{\left\langle\Psi\left|\int_{\Sigma} d^{3} x\left[\frac{\pi}{4}^{2}\right]\right| \Psi\right\rangle}{\langle\Psi \mid \Psi\rangle}+\frac{1}{V} \frac{\left\langle\Psi\left|\int_{\Sigma} d^{3} x \frac{V(\mathcal{P})}{2 \kappa f^{\prime}(R)}\right| \Psi\right\rangle}{\langle\Psi \mid \Psi\rangle}=-\frac{\Lambda_{c}}{\kappa},
$$

where

$$
\hat{\Lambda}_{\Sigma, f(R)}^{(2)}=(2 \kappa) h(R) G_{i j k l} \pi^{i j} \pi^{k l}-\frac{\sqrt{g}}{2 \kappa}{ }^{3} R^{l i n}
$$

with

$$
h(R)=1+\frac{2\left[f^{\prime}(R)-1\right]}{f^{\prime}(R)}
$$

and where ${ }^{3} R^{\text {lin }}$ is the linearized scalar curvature whose expression is shown in square brackets of Eq. (13.33). Note that when $f(R)=R$, consistently it is $h(R)=1$. From Eq.(13.35), we redefine $\Lambda_{c}$

$$
\Lambda_{c}^{\prime}=\Lambda_{c}+\frac{1}{2 V} \frac{\left\langle\Psi\left|\int_{\Sigma} d^{3} x \frac{V(\mathcal{P})}{f^{\prime}(R)}\right| \Psi\right\rangle}{\langle\Psi \mid \Psi\rangle}=\Lambda_{c}+\frac{1}{2 V} \int_{\Sigma} d^{3} x \sqrt{g} \frac{R f^{\prime}(R)-f(R)}{f^{\prime}(R)}
$$

where we have explicitly used the definition of $V(\mathcal{P})$. In order to make explicit calculations, we need an orthogonal decomposition for both $\pi_{i j}$ and $h_{i j}$ to disentangle gauge modes from physical deformations. We define the inner product

$$
\langle h, k\rangle:=\int_{\Sigma} \sqrt{g} G^{i j k l} h_{i j}(x) k_{k l}(x) d^{3} x,
$$

by means of the inverse WDW metric $G_{i j k l}$, to have a metric on the space of deformations, i.e. a quadratic form on the tangent space at $h_{i j}$, with

$$
G^{i j k l}=\frac{1}{2}\left(g^{i k} g^{j l}+g^{i l} g^{j k}-2 g^{i j} g^{k l}\right)
$$

The inverse metric is defined on cotangent space and it assumes the form 


$$
\langle p, q\rangle:=\int_{\Sigma} \sqrt{g} G_{i j k l} p^{i j}(x) q^{k l}(x) d^{3} x
$$

so that

$$
G^{i j n m} G_{n m k l}=\frac{1}{2}\left(\delta_{k}^{i} \delta_{l}^{j}+\delta_{l}^{i} \delta_{k}^{j}\right) .
$$

Note that in this scheme the "inverse metric" is actually the WDW metric defined on phase space. The desired decomposition on the tangent space of 3-metric deformations [66, 411, 564, 593] is:

$$
h_{i j}=\frac{1}{3} h g_{i j}+(L \xi)_{i j}+h_{i j}^{\perp}
$$

where the operator $L$ maps $\xi_{i}$ into symmetric tracefree tensors

$$
(L \xi)_{i j}=\nabla_{i} \xi_{j}+\nabla_{j} \xi_{i}-\frac{2}{3} g_{i j}(\nabla \cdot \xi) .
$$

Thus the inner product between three-geometries becomes

$$
\begin{gathered}
\langle h, h\rangle:=\int_{\Sigma} \sqrt{g} G^{i j k l} h_{i j}(x) h_{k l}(x) d^{3} x= \\
\int_{\Sigma} \sqrt{g}\left[-\frac{2}{3} h^{2}+(L \xi)^{i j}(L \xi)_{i j}+h^{i j \perp} h_{i j}^{\perp}\right] .
\end{gathered}
$$

With the orthogonal decomposition in hand we can define the trial wave functional as

$$
\Psi\left[h_{i j}(\vec{x})\right]=\mathcal{N} \Psi\left[h_{i j}^{\perp}(\vec{x})\right] \Psi\left[h_{i j}^{\|}(\vec{x})\right] \Psi\left[h_{i j}^{\text {trace }}(\vec{x})\right],
$$

where

$$
\begin{gathered}
\Psi\left[h_{i j}^{\perp}(\vec{x})\right]=\exp \left\{-\frac{1}{4}\left\langle h K^{-1} h\right\rangle_{x, y}^{\perp}\right\} \\
\Psi\left[h_{i j}^{\|}(\vec{x})\right]=\exp \left\{-\frac{1}{4}\left\langle(L \xi) K^{-1}(L \xi)\right\rangle_{x, y}^{\|}\right\} . \\
\Psi\left[h_{i j}^{\text {trace }}(\vec{x})\right]=\exp \left\{-\frac{1}{4}\left\langle h K^{-1} h\right\rangle_{x, y}^{\text {Trace }}\right\}
\end{gathered}
$$

The symbol " $\perp$ " denotes the transverse-traceless tensor (TT) (spin 2) of the perturbation, while the symbol "||" denotes the longitudinal part (spin 1) of the perturbation. Finally, the symbol "trace" denotes the scalar part of the perturbation. $\mathcal{N}$ is a normalization factor, $\langle\cdot, \cdot\rangle_{x, y}$ denotes space integration and $K^{-1}$ is the inverse "propagator". We will fix our attention to the TT tensor sector of the perturbation representing the graviton. Therefore, representation (13.46) reduces to

$$
\Psi\left[h_{i j}(\vec{x})\right]=\mathcal{N} \exp \left\{-\frac{1}{4}\left\langle h K^{-1} h\right\rangle_{x, y}^{\perp}\right\} .
$$

Actually there is no reason to neglect longitudinal and trace perturbations. However, following the analysis of Refs. 304, 411, 574] on the perturbation decomposition, we can discover that the relevant components can be restricted to the TT modes and to the trace modes. Moreover, for certain backgrounds, TT tensors can be a source of instability as shown in Refs. [304, 574, 590]. Even the trace part can be regarded as a source of instability. Indeed this is usually termed conformal instability. The appearance of an instability on the TT modes is known as non-conformal instability. This means that does not exist a gauge choice that can eliminate negative modes. To proceed with Eq.(13.32), we 
need to know the action of some basic operators on $\Psi\left[h_{i j}\right]$. The action of the operator $h_{i j}$ on $|\Psi\rangle=\Psi\left[h_{i j}\right]$ is realized by 353 ]

$$
h_{i j}(x)|\Psi\rangle=h_{i j}(\vec{x}) \Psi\left[h_{i j}\right] .
$$

The action of the operator $\pi_{i j}$ on $|\Psi\rangle$, in general, is

$$
\pi_{i j}(x)|\Psi\rangle=-i \frac{\delta}{\delta h_{i j}(\vec{x})} \Psi\left[h_{i j}\right]
$$

while the inner product is defined by the functional integration:

$$
\left\langle\Psi_{1} \mid \Psi_{2}\right\rangle=\int\left[\mathcal{D} h_{i j}\right] \Psi_{1}^{*}\left[h_{i j}\right] \Psi_{2}\left[h_{k l}\right]
$$

We demand that

$$
\frac{1}{V} \frac{\left\langle\Psi\left|\int_{\Sigma} d^{3} x \hat{\Lambda}_{\Sigma, f(R)}^{(2)}\right| \Psi\right\rangle}{\langle\Psi \mid \Psi\rangle}=\frac{1}{V} \frac{\int \mathcal{D}\left[g_{i j}\right] \Psi^{*}\left[h_{i j}\right] \int_{\Sigma} d^{3} x \hat{\Lambda}_{\Sigma, f(R)}^{(2)} \Psi\left[h_{i j}\right]}{\int \mathcal{D}\left[g_{i j}\right] \Psi^{*}\left[h_{i j}\right] \Psi\left[h_{i j}\right]}
$$

be stationary against arbitrary variations of $\Psi\left[h_{i j}\right]$. Note that Eq. (13.52) can be considered as the variational analog of a Sturm-Liouville problem with the cosmological constant regarded as the associated eigenvalue. Therefore the solution of Eq.(13.29) corresponds to the minimum of Eq.(13.52). The form of $\left\langle\Psi\left|\hat{\Lambda}_{\Sigma}\right| \Psi\right\rangle$ can be computed with the help of the wave functional (13.48) and with the help of

$$
\frac{\left\langle\Psi\left|h_{i j}(\vec{x})\right| \Psi\right\rangle}{\langle\Psi \mid \Psi\rangle}=0
$$

and

$$
\frac{\left\langle\Psi\left|h_{i j}(\vec{x}) h_{k l}(\vec{y})\right| \Psi\right\rangle}{\langle\Psi \mid \Psi\rangle}=K_{i j k l}(\vec{x}, \vec{y}) .
$$

Extracting the TT tensor contribution, we get

$$
\hat{\Lambda}_{\Sigma, f(R)}^{(2), \perp}=\frac{1}{4 V} \int_{\Sigma} d^{3} x \sqrt{\bar{g}} G^{i j k l}\left[(2 \kappa) h(R) K^{-1 \perp}(x, x)_{i j k l}+\frac{1}{(2 \kappa)}\left(\triangle_{2}\right)_{j}^{a} K^{\perp}(x, x)_{i a k l}\right] .
$$

The propagator $K^{\perp}(x, x)_{i a k l}$ can be represented as

$$
K^{\perp}(\vec{x}, \vec{y})_{i a k l}:=\sum_{\tau} \frac{h_{i a}^{(\tau) \perp}(\vec{x}) h_{k l}^{(\tau) \perp}(\vec{y})}{2 \lambda(\tau)},
$$

where $h_{i a}^{(\tau) \perp}(\vec{x})$ are the eigenfunctions of $\triangle_{2} . \tau$ denotes a complete set of indices and $\lambda(\tau)$ are a set of variational parameters to be determined by the minimization of Eq.(13.55). The expectation value of $\hat{\Lambda}_{\Sigma} \frac{1}{\Sigma}$ is easily obtained by inserting the form of the propagator into Eq.13.55

$$
-\frac{\Lambda_{c}^{\prime}\left(\lambda_{i}\right)}{\kappa}=\frac{1}{4} \sum_{\tau} \sum_{i=1}^{2}\left[(2 \kappa) h(R) \lambda_{i}(\tau)+\frac{\omega_{i}^{2}(\tau)}{(2 \kappa) \lambda_{i}(\tau)}\right] .
$$

By minimizing with respect to the variational function $\lambda_{i}(\tau)$, we obtain the total one loop energy density for TT tensors

$$
\Lambda_{c}^{\prime}\left(\lambda_{i}\right)=-\kappa \sqrt{h(R)} \frac{1}{4} \sum_{\tau}\left[\sqrt{\omega_{1}^{2}(\tau)}+\sqrt{\omega_{2}^{2}(\tau)}\right],
$$

where $\Lambda_{c}^{\prime}$ is expressed by the Eq. (13.38). The above expression makes sense only for $\omega_{i}^{2}(\tau)>0$. It is the main formal result. It is true for generic $f(R)$ functions since $h(R)$ explicitly appears in it. 


\section{The transverse traceless (TT) spin 2 operator for the Schwarzschild metric and the WKB approximation}

The above considerations can be specified choosing a given metric. For example, the quantity $\Lambda_{c}^{\prime}$ can be calculated for a Schwarzschild metric in the WKB approximation. Apparently, there is no a strong motivation to consider a Schwarzschild metric as a probe for a cosmological problem. Nevertheless, every quantum field induces a "cosmological term" by means of vacuum expectation values and the variational approach we have considered is particularly easy to use for a spherically symmetric metric. The Schwarzschild metric is the simplest sourceless solution of the Einstein field equations which can be used to compute a cosmological constant spectrum. Of course, also Minkowski space can be put in the form of a spherically symmetric metric, but in that case there is no gravity at all. The other solution need a source which is not considered in the present Review Paper. In this sense, the computation is a real vacuum contribution to the cosmological term. The spin-two operator for the Schwarzschild metric is defined by

$$
\left(\triangle_{2} h^{T T}\right)_{i}^{j}:=-\left(\triangle_{T} h^{T T}\right)_{i}^{j}+2\left(R h^{T T}\right)_{i}^{j}
$$

where the transverse-traceless (TT) tensor for the quantum fluctuation is obtained by the following decomposition

$$
h_{i}^{j}=h_{i}^{j}-\frac{1}{3} \delta_{i}^{j} h+\frac{1}{3} \delta_{i}^{j} h=\left(h^{T}\right)_{i}^{j}+\frac{1}{3} \delta_{i}^{j} h .
$$

This implies that $\left(h^{T}\right)_{i}^{j} \delta_{j}^{i}=0$. The transversality condition is applied on $\left(h^{T}\right)_{i}^{j}$ and becomes $\nabla_{j}\left(h^{T}\right)_{i}^{j}=0$. Thus

$$
-\left(\triangle_{T} h^{T T}\right)_{i}^{j}=-\triangle_{S}\left(h^{T T}\right)_{i}^{j}+\frac{6}{r^{2}}\left(1-\frac{2 M G}{r}\right),
$$

where $\triangle_{S}$ is the scalar curved Laplacian, whose form is

$$
\triangle_{S}=\left(1-\frac{2 M G}{r}\right) \frac{d^{2}}{d r^{2}}+\left(\frac{2 r-3 M G}{r^{2}}\right) \frac{d}{d r}-\frac{L^{2}}{r^{2}}
$$

and $R_{j}^{a}$ is the mixed Ricci tensor whose components are:

$$
R_{i}^{a}=\left\{-\frac{2 M G}{r^{3}}, \frac{M G}{r^{3}}, \frac{M G}{r^{3}}\right\},
$$

This implies that the scalar curvature is traceless. We are therefore led to study the following eigenvalue equation

$$
\left(\triangle_{2} h^{T T}\right)_{i}^{j}=\omega^{2} h_{j}^{i}
$$

where $\omega^{2}$ is the eigenvalue of the corresponding equation. In doing so, we follow Regge and Wheeler in analyzing the equation as modes of definite frequency, angular momentum and parity [483]. In particular, our choice for the three-dimensional gravitational perturbation is represented by its even-parity form

$$
\left(h^{\text {even }}\right)_{j}^{i}(r, \vartheta, \phi)=\operatorname{diag}[H(r), K(r), L(r)] Y_{l m}(\vartheta, \phi)
$$

with

$$
\left\{\begin{array}{l}
H(r)=h_{1}^{1}(r)-\frac{1}{3} h(r) \\
K(r)=h_{2}^{2}(r)-\frac{1}{3} h(r) \\
L(r)=h_{3}^{3}(r)-\frac{1}{3} h(r)
\end{array}\right.
$$

From the transversality condition, we obtain $h_{2}^{2}(r)=h_{3}^{3}(r)$. Then $K(r)=L(r)$. For a generic value of the angular momentum $L$, representation (13.65) joined to Eq.13.61) lead to the following system of PDE's

$$
\left\{\begin{array}{l}
\left(-\triangle_{S}+\frac{6}{r^{2}}\left(1-\frac{2 M G}{r}\right)-\frac{4 M G}{r^{3}}\right) H(r)=\omega_{1, l}^{2} H(r) \\
\left(-\triangle_{S}+\frac{6}{r^{2}}\left(1-\frac{2 M G}{r}\right)+\frac{2 M G}{r^{3}}\right) K(r)=\omega_{2, l}^{2} K(r)
\end{array} .\right.
$$

Defining the "reduced" fields 


$$
H(r)=\frac{f_{1}(r)}{r} ; \quad K(r)=\frac{f_{2}(r)}{r},
$$

and passing to the proper geodesic distance from the throat of the bridge

$$
d x= \pm \frac{d r}{\sqrt{1-\frac{2 M G}{r}}},
$$

the system (13.67) becomes

$$
\left\{\begin{array}{l}
{\left[-\frac{d^{2}}{d x^{2}}+V_{1}(r)\right] f_{1}(x)=\omega_{1, l}^{2} f_{1}(x)} \\
{\left[-\frac{d^{2}}{d x^{2}}+V_{2}(r)\right] f_{2}(x)=\omega_{2, l}^{2} f_{2}(x)}
\end{array}\right.
$$

with

$$
\left\{\begin{array}{c}
V_{1}(r)=\frac{l(l+1)}{r^{2}}+U_{1}(r)+m_{g}^{2} \\
V_{2}(r)=\frac{l(l+1)}{r^{2}}+U_{2}(r)+m_{g}^{2}
\end{array},\right.
$$

where we have defined $r \equiv r(x)$ and

$$
\left\{\begin{array}{l}
U_{1}(r)=\left[\frac{6}{r^{2}}\left(1-\frac{2 M G}{r}\right)-\frac{3 M G}{r^{3}}\right] \\
U_{2}(r)=\left[\frac{6}{r^{2}}\left(1-\frac{2 M G}{r}\right)+\frac{3 M G}{r^{3}}\right]
\end{array} .\right.
$$

Note that

$$
\left\{\begin{array}{c}
U_{1}(r) \geq 0 \quad \text { when } r \geq \frac{5 M G}{2} \\
U_{1}(r)<0 \quad \text { when } 2 M G \leq r<\frac{5 M G}{2} \\
U_{2}(r)>0 \forall r \in[2 M G,+\infty)
\end{array} .\right.
$$

In order to use the WKB approximation, we define two r-dependent radial wave numbers $k_{1}\left(r, l, \omega_{1, n l}\right)$ and $k_{2}\left(r, l, \omega_{2, n l}\right)$

$$
\left\{\begin{array}{l}
k_{1}^{2}\left(r, l, \omega_{1, n l}\right)=\omega_{1, n l}^{2}-\frac{l(l+1)}{r^{2}}-m_{1}^{2}(r) \\
k_{2}^{2}\left(r, l, \omega_{2, n l}\right)=\omega_{2, n l}^{2}-\frac{l(l+1)}{r^{2}}-m_{2}^{2}(r)
\end{array}\right.
$$

where we have defined two r-dependent effective masses $m_{1}^{2}(r)$ and $m_{2}^{2}(r)$. The WKB approximation we will use to evaluate Eq. (13.58) is equivalent to the scattering phase shift method and to the entropy computation in the brick wall model. We begin by counting the number of modes with frequency less than $\omega_{i}, i=1,2$. This is given approximately by

$$
\tilde{g}\left(\omega_{i}\right)=\int \nu_{i}\left(l, \omega_{i}\right)(2 l+1),
$$

where $\nu_{i}\left(l, \omega_{i}\right), i=1,2$ is the number of nodes in the mode with $\left(l, \omega_{i}\right)$, such that $(r \equiv r(x))$

$$
\nu_{i}\left(l, \omega_{i}\right)=\frac{1}{2 \pi} \int_{-\infty}^{+\infty} d x \sqrt{k_{i}^{2}\left(r, l, \omega_{i}\right)} .
$$

Here it is understood that the integration with respect to $x$ and $l$ is taken over those values which satisfy $k_{i}^{2}\left(r, l, \omega_{i}\right) \geq 0$, $i=1,2$. With the help of Eqs.(13.75 13.76), we obtain the one loop total energy for TT tensors which is

$$
\frac{1}{8 \pi} \sum_{i=1}^{2} \int_{-\infty}^{+\infty} d x\left[\int_{0}^{+\infty} \omega_{i} \frac{d \tilde{g}\left(\omega_{i}\right)}{d \omega_{i}} d \omega_{i}\right] .
$$


By extracting the energy density contributing to the cosmological constant, we get

$$
\Lambda_{c}^{\prime}=\Lambda_{c, 1}^{\prime}+\Lambda_{c, 2}^{\prime}=\rho_{1}+\rho_{2}=-\sqrt{h(R)} \frac{\kappa}{16 \pi^{2}}\left\{\int_{0}^{+\infty} \omega_{1}^{2} \sqrt{\omega_{1}^{2}-m_{1}^{2}(r)} d \omega_{1}+\int_{0}^{+\infty} \omega_{2}^{2} \sqrt{\omega_{2}^{2}-m_{2}^{2}(r)} d \omega_{2}\right\},
$$

where we have included an additional $4 \pi$ coming from the angular integration.

\section{E. One-loop energy Regularization and Renormalization}

In this subsection, we will use the zeta function regularization method to compute the energy densities $\rho_{1}$ and $\rho_{2}$ (see Appendix B). Note that this procedure is completely equivalent to the subtraction procedure of the Casimir energy computation where the zero point energy (ZPE) in different backgrounds with the same asymptotic properties is involved. To this purpose, we introduce the additional mass parameter $\mu$ in order to restore the correct dimension for the regularized quantities. Such an arbitrary mass scale emerges unavoidably in any regularization scheme. Then we have

$$
\rho_{i}(\varepsilon)=-\sqrt{h(R)} \frac{\kappa}{16 \pi^{2}} \mu^{2 \varepsilon} \int_{0}^{+\infty} d \omega_{i} \frac{\omega_{i}^{2}}{\left(\omega_{i}^{2}-m_{i}^{2}(r)\right)^{\varepsilon-\frac{1}{2}}},
$$

where

$$
\left\{\begin{array}{l}
\rho_{1}(\varepsilon)=-\sqrt{h(R)} \frac{\kappa}{16 \pi^{2}} \int_{0}^{+\infty} \omega_{1}^{2} \sqrt{\omega_{1}^{2}-m_{1}^{2}(r)} d \omega_{1} \\
\rho_{2}(\varepsilon)=-\sqrt{h(R)} \frac{\kappa}{16 \pi^{2}} \int_{0}^{+\infty} \omega_{2}^{2} \sqrt{\omega_{2}^{2}-m_{2}^{2}(r)} d \omega_{2}
\end{array} .\right.
$$

The integration has to be meant in the range where $\omega_{i}^{2}-m_{i}^{2}(r) \geq 26$. One gets

$$
\rho_{i}(\varepsilon)=\sqrt{h(R)} \kappa \frac{m_{i}^{4}(r)}{256 \pi^{2}}\left[\frac{1}{\varepsilon}+\ln \left(\frac{\mu^{2}}{m_{i}^{2}(r)}\right)+2 \ln 2-\frac{1}{2}\right],
$$

$i=1,2$. In order to renormalize the divergent ZPE, we write

$$
\Lambda_{c}^{\prime}=8 \pi G\left[\rho_{1}(\varepsilon)+\rho_{2}(\varepsilon)+\rho_{1}(\mu)+\rho_{2}(\mu)\right],
$$

where we have separated the divergent part from the finite part. For practical purposes, it is useful to divide $\Lambda_{c}^{\prime}$ with the factor $\sqrt{h(R)}$. To this aim, we define

$$
\frac{\Lambda_{c}^{\prime}}{\sqrt{h(R)}}=\left[\Lambda_{c}+\frac{1}{2 V} \int_{\Sigma} d^{3} x \sqrt{g} \frac{R f^{\prime}(R)-f(R)}{f^{\prime}(R)}\right] \frac{1}{\sqrt{h(R)}}
$$

and we extract the divergent part of $\Lambda$, in the limit $\varepsilon \rightarrow 0$, by setting

$$
\Lambda^{\text {div }}=8 \pi G\left[\rho_{1}(\varepsilon)+\rho_{2}(\varepsilon)\right]=\frac{G}{32 \pi \varepsilon}\left[m_{1}^{4}(r)+m_{2}^{4}(r)\right] .
$$

Thus, the renormalization is performed via the absorption of the divergent part into the re-definition of the bare classical cosmological constant $\Lambda_{c}$, that is

$$
\Lambda_{c} \rightarrow \Lambda_{0}+\sqrt{h(R)} \Lambda^{d i v}
$$

The remaining finite value for the cosmological constant read:27

$$
\frac{\Lambda_{0}^{\prime}(\mu)}{8 \pi G}=\rho_{1}(\mu)+\rho_{2}(\mu)=\frac{1}{256 \pi^{2}}\left\{m_{1}^{4}(r)\left[\ln \left(\frac{\mu^{2}}{\left|m_{1}^{2}(r)\right|}\right)+2 \ln 2-\frac{1}{2}\right]+\right.
$$

\footnotetext{
26 Details of the calculation can be found in the Appendix B.

27 Since $m_{1}^{2}(r)$ can change in sign, when we integrate over $\omega_{1}$ we can use either $I_{+}$or $I_{-}$. This leads to the appearance of the absolute value.
} 


$$
\left.+m_{2}^{4}(r)\left[\ln \left(\frac{\mu^{2}}{m_{2}^{2}(r)}\right)+2 \ln 2-\frac{1}{2}\right]\right\}=\rho_{\text {eff }}^{T T}(\mu, r)
$$

where

$$
\Lambda_{0}^{\prime}(\mu)=\frac{1}{\sqrt{h(R)}}\left[\Lambda_{0}(\mu)+\frac{1}{2 V} \int_{\Sigma} d^{3} x \sqrt{g} \frac{R f^{\prime}(R)-f(R)}{f^{\prime}(R)}\right]
$$

is the modified cosmological constant. The quantity in Eq.13.86) depends on the arbitrary mass scale $\mu$. It is appropriate to use the renormalization group equation to eliminate such a dependence. To this aim, we impose that [473]

$$
\frac{1}{8 \pi G} \mu \frac{\partial \Lambda_{0}^{\prime}(\mu)}{\partial \mu}=\mu \frac{d}{d \mu} \rho_{e f f}^{T T}(\mu, r) .
$$

Solving it, we find that the renormalized constant $\Lambda_{0}$ should be treated as a running one in the sense that it varies, provided that the scale $\mu$ is changing

$$
\Lambda_{0}^{\prime}(\mu, r)=\Lambda_{0}^{\prime}\left(\mu_{0}, r\right)+\frac{G}{16 \pi}\left[m_{1}^{4}(r)+m_{2}^{4}(r)\right] \ln \frac{\mu}{\mu_{0}} .
$$

Substituting Eq.(13.89) into Eq.(13.86) we find

$$
\frac{\Lambda_{0}^{\prime}\left(\mu_{0}, r\right)}{8 \pi G}=-\frac{1}{256 \pi^{2}}\left\{m_{1}^{4}(r)\left[\ln \left(\frac{\left|m_{1}^{2}(r)\right|}{\mu_{0}^{2}}\right)-2 \ln 2+\frac{1}{2}\right]+m_{2}^{4}(r)\left[\ln \left(\frac{m_{2}^{2}(r)}{\mu_{0}^{2}}\right)-2 \ln 2+\frac{1}{2}\right]\right\} .
$$

It is worth remarking that while $m_{2}^{2}(r)$ is constant in sign, $m_{1}^{2}(r)$ is not. Indeed, for the critical value $\bar{r}=5 M G / 2$, $m_{1}^{2}(\bar{r})=m_{g}^{2}$ and in the range $(2 M G, 5 M G / 2)$ for some values of $m_{g}^{2}, m_{1}^{2}(\bar{r})$ can be negative. It is interesting therefore concentrate in this range. To further proceed, we observe that $m_{1}^{2}(r)$ and $m_{2}^{2}(r)$ can be recast into a more suggestive and useful form, namely

$$
\left\{\begin{array}{l}
m_{1}^{2}(r)=U_{1}(r)=m_{1}^{2}(r, M)-m_{2}^{2}(r, M) \\
m_{2}^{2}(r)=U_{2}(r)=m_{1}^{2}(r, M)+m_{2}^{2}(r, M)
\end{array}\right.
$$

where $m_{1}^{2}(r, M) \rightarrow 0$ when $r \rightarrow \infty$ or $r \rightarrow 2 M G$ and $m_{2}^{2}(r, M)=3 M G / r^{3}$. Nevertheless, in the above mentioned range $m_{1}^{2}(r, M)$ is negligible when compared with $m_{2}^{2}(r, M)$. So, in a first approximation we can write

$$
\left\{\begin{array}{c}
m_{1}^{2}(r) \simeq-m_{2}^{2}\left(r_{0}, M\right)=-m_{0}^{2}(M) \\
m_{2}^{2}(r) \simeq m_{2}^{2}\left(r_{0}, M\right)=m_{0}^{2}(M)
\end{array}\right.
$$

where we have defined a parameter $r_{0}>2 M G$ and $m_{0}^{2}(M)=3 M G / r_{0}^{3}$. The main reason for introducing a new parameter resides in the fluctuation of the horizon that forbids any kind of approach. Of course the quantum fluctuation must obey the uncertainty relations. Thus Eq.(13.90) becomes

$$
\frac{\Lambda_{0}^{\prime}\left(\mu_{0}, r\right)}{8 \pi G}=-\frac{m_{0}^{4}(M)}{128 \pi^{2}}\left[\ln \left(\frac{m_{0}^{2}(M)}{4 \mu_{0}^{2}}\right)+\frac{1}{2}\right] .
$$

Now, we compute the maximum of $\Lambda_{0}^{\prime}$, by setting

$$
x=\frac{m_{0}^{2}(M)}{4 \mu_{0}^{2}} .
$$

Thus $\Lambda_{0}^{\prime}$ becomes

$$
\Lambda_{0}^{\prime}\left(\mu_{0}, x\right)=-\frac{G \mu_{0}^{4}}{\pi} x^{2}\left[\ln (x)+\frac{1}{2}\right]
$$


As a function of $x, \Lambda_{0}\left(\mu_{0}, x\right)$ vanishes for $x=0$ and $x=\exp \left(-\frac{1}{2}\right)$ and when $x \in\left[0, \exp \left(-\frac{1}{2}\right)\right], \Lambda_{0}^{\prime}\left(\mu_{0}, x\right) \geq 0$. It has a maximum for

$$
\bar{x}=\frac{1}{e} \quad \Longleftrightarrow \quad m_{0}^{2}(M)=\frac{4 \mu_{0}^{2}}{e}
$$

and its value is

$$
\Lambda_{0}^{\prime}\left(\mu_{0}, \bar{x}\right)=\frac{G \mu_{0}^{4}}{2 \pi e^{2}} \quad \text { or } \quad \frac{1}{\sqrt{h(R)}}\left[\Lambda_{0}\left(\mu_{0}, \bar{x}\right)+\frac{1}{2 V} \int_{\Sigma} d^{3} x \sqrt{g} \frac{R f^{\prime}(R)-f(R)}{f^{\prime}(R)}\right]=\frac{G \mu_{0}^{4}}{2 \pi e^{2}} .
$$

Isolating $\Lambda_{0}\left(\mu_{0}, \bar{x}\right)$, we get

$$
\Lambda_{0}\left(\mu_{0}, \bar{x}\right)=\sqrt{h(R)} \frac{G \mu_{0}^{4}}{2 \pi e^{2}}-\frac{1}{2 V} \int_{\Sigma} d^{3} x \sqrt{g} \frac{R f^{\prime}(R)-f(R)}{f^{\prime}(R)} .
$$

Note that $\Lambda_{0}\left(\mu_{0}, \bar{x}\right)$ can be set to zero when

$$
\sqrt{h(R)} \frac{G \mu_{0}^{4}}{2 \pi e^{2}}=\frac{1}{2 V} \int_{\Sigma} d^{3} x \sqrt{g} \frac{R f^{\prime}(R)-f(R)}{f^{\prime}(R)} .
$$

Let us see what happens when $f(R)=\exp (-\alpha R)$. This choice is simply suggested by the regularity of the function at every scale and by the fact that any power of $R$, considered as a correction to GR, is included. In this case, Eq. (13.99) becomes

$$
\sqrt{\frac{3 \alpha \exp (-\alpha R)+2}{\alpha \exp (-\alpha R)}} \frac{G \mu_{0}^{4}}{\pi e^{2}}=\frac{1}{\alpha V} \int_{\Sigma} d^{3} x \sqrt{g}(1+\alpha R) .
$$

For Schwarzschild, it is $R=0$, then

$$
\frac{G \mu_{0}^{4}}{\pi e^{2}}=\sqrt{\frac{1}{(3 \alpha+2) \alpha}} .
$$

By setting $\alpha=G$, we have the relation

$$
\mu_{0}^{4}=\frac{\pi e^{2}}{G} \sqrt{\frac{1}{(3 G+2) G}} .
$$

Note that in any case, the maximum of $\Lambda$ corresponds to the minimum of the energy density.

\section{F. Concluding remarks}

Despite of its successes, GR can only be considered as a step toward a much more complete and comprehensive structure due to a large number of weaknesses as discussed in this Report. Among them, the issue to find out the fundamental gravitational vacuum state is one of the main problem to achieve a definite Quantum Gravity theory which, till now, is lacking. However, several semiclassical approaches have been proposed and, from several points of view, it is clear that the former Hilbert-Einstein scheme has to be enlarged. The $f(R)$ theories of gravity are a minimal but well founded extension of GR where the form of the function $f(R)$ is not supposed "a priori" but is reconstructed by the observed dynamics at galactic and cosmological scales [115, 119]. Also if they seem a viable scheme from cosmology and astrophysics viewpoints, their theoretical foundation has to be sought at a fundamental level. In particular, one has to face the possibility to encompass the $f(R)$-gravity in the general framework of Quantum Field Theory on curved space-time. Here, we have dealt with the problem to find out vacuum states for $f(R)$-gravity via the $(3+1)$ ADM formalism. Analogously to GR, we have constructed the Hamiltonian constraint of a generic $f(R)$ theory and then achieved a canonical quantization giving the $f(R)$-WDW equation. In this context, the cosmological constant (vacuum state) emerges as a WDW eigenvalue. The related wave functional can be split by an orthogonal decomposition and then, constructing the transverse-traceless propagator, it is possible to obtain, after a variational minimization, the total one-loop energy density for the TT tensors. Such a quantity explicitly depends on the form of $f(R)$. As an application, we derived the energy density contributions to the cosmological constant for a TT 
spin 2 operator in the Schwarzschild metric and in the WKB approximation. The one-loop energy regularization and renormalization are achieved by the zeta function regularization method. The resulting renormalized $\Lambda_{0}$ is a running constant which can be set to zero depending on the value of an arbitrary mass scale parameter $\mu$. As explicit calculation, we find out the value of such a parameter for a theory of the form $f(R)=\exp (-\alpha R)$ in the Schwarzschild metric. This case can be used for several applications at cosmological and astrophysical scales. In particular, truncated versions of such an exponential function, power law $f(R)$, have been used for galactic dynamics [111, 119, 121]. In those cases, a corrected Newtonian potential, derived from the $f(R)$ Schwarzschild solution, has been used to fit, with great accuracy, data from low surface brightness galaxies without using dark matter haloes. This approach has allowed to fix a suitable mass scale comparable with the core size of galactic systems. Such a mass can be directly related to the above parameter $\alpha$ depending on the core radius $r_{c}$ (see also [121]).

In summary, the application of Quantum Field Theory methods to $f(R)$-gravity seems a viable scheme and gives positive results toward the issue to select vacuum states (eigenvalues) which can be interpreted as the cosmological constant. However, further studies are needed in order to generalize such results to other metrics and other ETGs.

\section{THE INITIAL VALUE PROBLEM}

Another important problem is the initial value formulation. There are several criteria for the viability of a theory of gravity, and one of them is certainly the property of having a well-posed initial value problem in order to guarantee that the theory has predictive power [577]. The Cauchy problem of theories described by quadratic actions in curvature invariants was studied in [436, 542] and found to be well-posed (in four space-time dimensions, the Gauss-Bonnet identity allows one to drop the Kretschmann scalar $R_{\mu \nu \rho \sigma} R^{\mu \nu \rho \sigma}$ from the action). Ref. 372] discusses the initial value problem of metric and Palatini $f(R)$-gravity with a general (i.e., not restricted to the quadratic form considered in [436, 542]) function $f(R)$ but dropping $R_{\mu \nu} R^{\mu \nu}$ and $R_{\mu \nu \rho \sigma} R^{\mu \nu \rho \sigma}$. It is worth noticing that in this paper, the Cauchy problem for the Palatini approach of $f(R)$-gravity is ill-formulated and ill-posed leading to the wrong result that the ADM formulation is inadequate for the Initial Value Problem. This shortcoming has recently been removed in [459], where the Hamiltonian formulation of $f(R)$-gravity, both in metric and in Palatini formalism, is fully consistent with the well formulation of the Cauchy problem and there is no reason to believe that it is not well-posed in general.

It is useful to begin with the Cauchy problem of scalar-tensor gravity, which was studied in [497], and then to use the results and the equivalence of metric and Palatini $f(R)$-gravity with an $\omega=0,-3 / 2$ Brans-Dicke theory.

Let us provide some terminology [517]: the system of $3+1$ equations of motion of GR or ETGs is well-formulated if it can be recast as a system of only first order equations in time and space in the scalar field variables. The goal is to write this system in the full first order form

$$
\partial_{t} \vec{u}+M^{i} \nabla_{i} \vec{u}=\vec{S}(\vec{u})
$$

where $\vec{u}$ denotes the fundamental variables $h_{i j}, K_{i j}$, etc. of the usual $3+1$ ADM splitting, $M^{i}$ is called the characteristic matrix of the system, and $\vec{S}(\vec{u})$ describes source terms and contains only the fundamental variables but not their derivatives. The initial value formulation is then said to be well-posed if the system of partial differential equations is symmetric hyperbolic (i.e., $M^{i}$ is symmetric) and strongly hyperbolic (i.e., $s_{i} M^{i}$ has a real set of eigenvalues and a complete set of eigenvectors for any one-form $s_{i}$, and obeys some boundedness conditions).

For a physical theory to be viable, it must admit an appropriate initial value formulation to guarantee its predictability [191]. This means that, starting from suitably prescribed initial data, the subsequent dynamical evolution of the physical system is completely and uniquely determined. In this case, the problem is said to be well-formulated. For example, in classical mechanics, given the initial positions and velocities of the particles (or of the constituents) composing a physical system with a finite number of degrees of freedom and knowing the interactions between them, if the system evolves without external interferences the dynamical evolution is determined. This is true also for field theories, for example, for the Maxwell field. However, even if the initial value problem is well-formulated, the theory must possess additional properties in order to be viable. First, small changes of the initial data must produce only small perturbations in the subsequent dynamics over reasonably short time scales, in other words the evolution equations should exhibit a continuous dependence on the initial data in order to be predictive. Second, for hyperbolic equations, changes in the initial data must preserve the causal structure of the theory. If both these requirements are satisfied, the initial value problem of the theory is also well-posed. GR has been shown to admit a well-formulated and well-posed initial value problem in vacuo and in the presence of "reasonable" forms of matter (perfect fluids, minimally coupled scalar fields, etc.) but, for other relativistic field theories, the initial value formulation must be studied carefully. One needs to satisfy constraints between the initial data and perform wise gauge choices in order to cast the field equations in a form suitable to correctly formulate the Cauchy problem. The consequence of wellposedness is that GR is a "stable" theory with a robust causal structure in which singularities can be classified (for 
a detailed discussion see Refs. [538, 577]). Here we focus on whether the initial value problem of ETGs (including scalar-tensor and $f(R)$ theories in both the metric and metric-affine formulation) is well-formulated. It is not a priori obvious that standard GR methods are suitable for the discussion of the Cauchy problem in every ETG and it is doubtful that the full Cauchy problem can be properly addressed using only the results available in the literature for the fourth order theories described by a quadratic Lagrangian [225, 542]. $f(R)$-gravity, like GR, is a gauge theory with constrained dynamics and establishing results on the initial value formulation relies on solving the constraints on the initial data and on finding suitable gauges, coordinate choices in which the Cauchy problem can be demonstrated to well-formulated and, possibly, well-posed. In [436, 542] the initial value problem is studied for quadratic Lagrangians in the metric approach with the conclusion that it is well-posed. The Cauchy problem for generic $f(R)$ models is studied below in the metric and Palatini approaches with the result that the problem is well-formulated for the metric theory in the presence of "reasonable" matter and well-posed in vacuo. It is shown below that the Cauchy problem of metric-affine $f(R)$-gravity is well-formulated and well-posed in vacuo, while it can be at least well-formulated for various forms of matter including perfect fluids, Klein-Gordon, and Yang-Mills fields. We use the $3+1$ ADM formulation and the Gaussian normal coordinates approach, both of which prove useful in the discussion of whether the Cauchy problem is well-formulated. Of course, in order to prove the complete viability of a theory, also well-posedness has to be demonstrated.

\section{A. The Cauchy problem of scalar-tensor gravity}

Early work on the initial value problem of scalar-tensor gravity includes Refs. [181, 436, 542]. Noakes [436] proved well-posedness of the Cauchy problem for a non-minimally coupled scalar field $\phi$ with vacuum action

$$
S_{N M C}=\int d^{4} x \sqrt{-g}\left[\left(\frac{1}{2 \kappa}-\xi \phi^{2}\right) R-\frac{1}{2} \nabla^{\mu} \phi \nabla_{\mu} \phi-V(\phi)\right] .
$$

Cocke and Cohen 181] used Gaussian normal coordinates to study the Cauchy problem of Brans-Dicke theory without potential $V(\phi)$. A systematic approach to the Cauchy problem of scalar-tensor theories of the form

$$
S=\int d^{4} x \sqrt{-g}\left[\frac{f(\phi) R}{2 \kappa}-\frac{1}{2} \nabla^{\alpha} \phi \nabla_{\alpha} \phi-V(\phi)\right]+S^{(m)}
$$

independent of particular coordinate choices was proposed by Salgado [497], obtaining the result that the Cauchy problem is well-posed in vacuo and well-formulated otherwise. Slightly more general scalar-tensor theories of the form

$$
S=\int d^{4} x \sqrt{-g}\left[\frac{f(\phi) R}{2 \kappa}-\frac{\omega(\phi)}{2} \nabla^{\mu} \phi \nabla_{\mu} \phi-V(\phi)\right]+S^{(m)},
$$

containing the additional coupling function $\omega(\phi)$ were studied in [372]. In the notation of Ref. [497], and setting $\kappa=1$ in this section, the field equations are

$$
\begin{aligned}
G_{\mu \nu} & =\frac{1}{f}\left[f^{\prime \prime}\left(\nabla_{\mu} \phi \nabla_{\nu} \phi-g_{\mu \nu} \nabla^{\alpha} \phi \nabla_{\alpha} \phi\right)+f^{\prime}\left(\nabla_{\mu} \nabla_{\nu} \phi-g_{\mu \nu} \square \phi\right)\right] \\
& +\frac{1}{f}\left[\omega\left(\nabla_{\mu} \phi \nabla_{\nu} \phi-\frac{1}{2} g_{\mu \nu} \nabla^{\alpha} \phi \nabla_{\alpha} \phi\right)-V(\phi) g_{\mu \nu}+T_{\mu \nu}^{(m)}\right], \\
\omega \square \phi & +\frac{f^{\prime}}{2} R-V^{\prime}(\phi)+\frac{\omega^{\prime}}{2} \nabla^{\alpha} \phi \nabla_{\alpha} \phi=0,
\end{aligned}
$$

where a prime denotes differentiation with respect to $\phi$. Eq. (14.5) is in the form of an effective Einstein equation 497]

$$
G_{\mu \nu}=T_{\mu \nu}^{(e f f)}=\frac{1}{f(\phi)}\left(T_{\mu \nu}^{(f)}+T_{\mu \nu}^{(\phi)}+T_{\mu \nu}^{(m)}\right)
$$

where

$$
T_{\mu \nu}^{(f)}=f^{\prime \prime}(\phi)\left(\nabla_{\mu} \phi \nabla_{\nu} \phi-g_{\mu \nu} \nabla^{\alpha} \phi \nabla_{\alpha} \phi\right)+f^{\prime}(\phi)\left(\nabla_{\mu} \nabla_{\nu} \phi-g_{\mu \nu} \square \phi\right),
$$

and

$$
T_{\mu \nu}^{(\phi)}=\omega(\phi)\left(\nabla_{\mu} \phi \nabla_{\nu} \phi-\frac{1}{2} g_{\mu \nu} \nabla^{\alpha} \phi \nabla_{\alpha} \phi\right)-V(\phi) g_{\mu \nu}
$$


has canonical structure. The trace of Eq. (14.7) yields

$$
\square \phi=\frac{\frac{f^{\prime} T^{(m)}}{2}-2 f^{\prime} V(\phi)+f V^{\prime}(\phi)+\left[-\frac{\omega^{\prime} f}{2}-\frac{f^{\prime}}{2}\left(\omega+3 f^{\prime \prime}\right)\right] \nabla^{\alpha} \phi \nabla_{\alpha} \phi}{f\left[\omega+\frac{3\left(f^{\prime}\right)^{2}}{2 f}\right]} .
$$

One then proceeds in the usual $3+1 \mathrm{ADM}$ formulation of the theory in terms of lapse, shift, extrinsic curvature, and gradient of $\phi$ [485, 497, 577]. It is assumed that a time function $t$ is defined so that the space-time $\left(M, g_{\mu \nu}, \phi\right)$ is foliated by a family of hypersurfaces $\Sigma_{t}$ of constant $t$ with unit timelike normal $n^{\mu}$. The three-dimensional metric is $h_{\mu \nu} \equiv g_{\mu \nu}+n_{\mu} n_{\nu}, h^{\mu}{ }_{\nu}$ is the projection operator on $\Sigma_{t}$, and $n^{\mu}$ and $h_{\mu}{ }^{\nu}$ satisfy

$$
n^{\mu} n_{\mu}=-1, \quad h_{\mu \nu} n^{\mu}=h_{\mu \nu} n^{\nu}=0, \quad h_{\mu}{ }^{\nu} h_{\nu \rho}=h_{\mu \rho} .
$$

The metric decomposition in terms of lapse function $N$ and shift vector $N^{\mu}$ is

$$
d s^{2}=-\left(N^{2}-N^{i} N_{i}\right) d t^{2}-2 N_{i} d t d x^{i}+h_{i j} d x^{i} d x^{j},
$$

where $i, j, k$ are spatial indices assuming the values 1,2 , and $3, N>0, n_{\mu}=-N \nabla_{\mu} t$, and

$$
N^{\mu}=-h^{\mu}{ }_{\nu} t^{\nu}
$$

and where the time flow vector $t^{\mu}$ obeys

$$
\begin{aligned}
& t^{\mu} \nabla_{\mu} t=1, \\
& t^{\mu}=-N^{\mu}+N n^{\mu} .
\end{aligned}
$$

As a consequence, $N=-n_{\mu} t^{\mu}$ and $N^{\mu} n_{\mu}=0$. The extrinsic curvature of the hypersurfaces $\Sigma_{t}$ is

$$
K_{\mu \nu}=-h_{\mu}{ }^{\rho} h_{\nu}{ }^{\sigma} \nabla_{\rho} n_{\sigma}
$$

and the three-dimensional covariant derivative of $h_{\mu \nu}$ on $\Sigma_{t}$ is given by

$$
D_{i}^{(3)} T^{\mu_{1} \ldots}{ }_{\nu_{1} \ldots}=h^{\mu_{1}}{ }_{\rho_{1}} \ldots h^{\sigma_{1}}{ }_{\nu_{1}} \ldots h^{f}{ }_{i} \nabla_{f}^{(3)} T^{\rho_{1} \ldots}{ }_{\sigma_{1} \ldots}
$$

for any three-tensor ${ }^{(3)} T^{\mu_{1} \ldots}{ }_{\nu_{1} \ldots}$, with $D_{i} h_{\mu \nu}=0$. The spatial gradient of the scalar field is

$$
Q_{\mu} \equiv D_{\mu} \phi
$$

its momentum is

$$
\Pi=\mathcal{L}_{n} \phi=n^{\mu} \nabla_{\mu} \phi
$$

and

$$
\begin{gathered}
K_{i j}=-\nabla_{i} n_{j}=-\frac{1}{2 N}\left(\frac{\partial h_{i j}}{\partial t}+D_{i} N_{j}+D_{j} N_{i}\right), \\
\Pi=\frac{1}{N}\left(\partial_{t} \phi+N^{\alpha} Q_{\alpha}\right),
\end{gathered}
$$

while

$$
\partial_{t} Q_{i}+N^{l} \partial_{l} Q_{i}+Q_{l} \partial_{i} N^{l}=D_{i}(N \Pi) .
$$

The ADM decomposition of the effective energy-momentum tensor $T_{\mu \nu}^{(e f f)}$ is then

$$
T_{\mu \nu}^{(e f f)}=\frac{1}{f}\left(S_{\mu \nu}+J_{\mu} n_{\nu}+J_{\nu} n_{\mu}+E n_{\mu} n_{\nu}\right)
$$

where

$$
S_{\mu \nu} \equiv h_{\mu}{ }^{\rho} h_{\nu}{ }^{\sigma} T_{\rho \sigma}^{(e f f)}=\frac{1}{f}\left(S_{\mu \nu}^{(f)}+S_{\mu \nu}^{(\phi)}+S_{\mu \nu}^{(m)}\right)
$$




$$
\begin{gathered}
J_{\mu} \equiv-h_{\mu}{ }^{\rho} T_{\rho \sigma}^{(e f f)} n^{\sigma}=\frac{1}{f}\left(J_{\mu}^{(f)}+J_{\mu}^{(\phi)}+J_{\mu}^{(m)}\right), \\
E \equiv n^{\mu} n^{\nu} T_{\mu \nu}^{(e f f)}=\frac{1}{f}\left(E^{(f)}+E^{(\phi)}+E^{(m)}\right),
\end{gathered}
$$

and $T^{(e f f)}=S-E$, where $T^{(e f f)} \equiv T^{(e f f)^{\mu}}{ }_{\mu}$ and $S \equiv S^{\mu}{ }_{\mu}$. Using the Gauss-Codazzi equations [577], the effective Einstein equations projected tangentially and orthogonally to $\Sigma_{t}$ yield the Hamiltonian constraint [497, 577]

$$
{ }^{(3)} R+K^{2}-K_{i j} K^{i j}=2 E,
$$

the momentum constraint

$$
D_{l} K_{i}^{l}-D_{i} K=J_{i}
$$

and the dynamical equations

$$
\begin{gathered}
\partial_{t} K^{i}{ }_{j}+N^{l} \partial_{l} K^{i}{ }_{j}+K^{i}{ }_{l} \partial_{j} N^{l}-K^{l}{ }_{j} \partial_{l} N^{i}+D^{i} D_{j} N \\
{ }_{-}^{(3)} R_{j}^{i} N-N K K^{i}{ }_{j}=\frac{N}{2}\left[(S-E) \delta_{j}^{i}-2 S_{j}^{i}\right],
\end{gathered}
$$

where $K \equiv K^{i}{ }_{i}$. The trace of eq. (14.29) leads to

$$
\partial_{t} K+N^{l} \partial_{l} K+{ }^{(3)} \Delta N-N K_{i j} K^{i j}=\frac{N}{2}(S+E),
$$

where ${ }^{(3)} \Delta \equiv D^{i} D_{i}$. The second order derivatives of $\phi$ are in principle troublesome because they could make the initial value problem ill-formulated, but they can be eliminated in most cases [497]. The $f$ - and $\phi$-quantities of eqs. (14.24)-(14.26) turn out to be

$$
\begin{gathered}
E^{(f)}=f^{\prime}\left(D^{\alpha} Q_{\alpha}+K \Pi\right)+f^{\prime \prime} Q^{2}, \\
J_{\mu}^{(f)}=-f^{\prime}\left(K_{\mu}^{\rho} Q_{\rho}+D_{\mu} \Pi\right)-f^{\prime \prime} \Pi Q_{\mu}, \\
S_{\mu \nu}^{(f)}=f^{\prime}\left(D_{\mu} Q_{\nu}+\Pi K_{\mu \nu}-h_{\mu \nu} \square \phi\right)-f^{\prime \prime}\left[h_{\mu \nu}\left(Q^{2}-\Pi^{2}\right)-Q_{\mu} Q_{\nu}\right],
\end{gathered}
$$

where $Q^{2} \equiv Q^{\alpha} Q_{\alpha}$. The quantities

$$
\begin{gathered}
S^{(f)}=f^{\prime}\left(D_{\alpha} Q^{\alpha}+K \Pi-3 \square \phi\right)+f^{\prime \prime}\left(3 \Pi^{2}-2 Q^{2}\right), \\
S^{(f)}-E^{(f)}=-3 f^{\prime} \square \phi-3 f^{\prime \prime}\left(Q^{2}-\Pi^{2}\right),
\end{gathered}
$$

are also useful [497], and the introduction of $\omega$ and $\omega^{\prime}$ yields the further quantities

$$
\begin{gathered}
E^{(\phi)}=\frac{\omega}{2}\left(\Pi^{2}+Q^{2}\right)+V(\phi), \\
J_{\mu}^{(\phi)}=-\omega \Pi Q_{\mu}, \\
S_{\mu \nu}^{(\phi)}=\omega Q_{\mu} Q_{\nu}-h_{\mu \nu}\left[\frac{\omega}{2}\left(Q^{2}-\Pi^{2}\right)+V(\phi)\right],
\end{gathered}
$$

while

$$
S^{(\phi)}=\frac{\omega}{2}\left(3 \Pi^{2}-Q^{2}\right)-3 V(\phi)
$$

and

$$
S^{(\phi)}-E^{(\phi)}=\omega\left(\Pi^{2}-Q^{2}\right)-4 V(\phi) .
$$


Finally, the quantities appearing on the right hand sides of the $3+1$ field equations are

$$
\begin{aligned}
E & =\frac{1}{f}\left[f^{\prime}\left(D^{\alpha} Q_{\alpha}+K \Pi\right)+\left(f^{\prime \prime}+\frac{\omega}{2}\right) Q^{2}+\frac{\omega}{2} \Pi^{2}+V(\phi)+E^{(m)}\right], \\
J_{\mu} & =\frac{1}{f}\left[-f^{\prime}\left(K_{\mu}{ }^{\alpha} Q_{\alpha}+D_{\mu} \Pi\right)-\left(f^{\prime \prime}+\omega\right) \Pi Q_{\mu}+J_{\mu}^{(m)}\right], \\
S_{\mu \nu} & =\frac{1}{f}\left\{f^{\prime}\left(D_{\mu} Q_{\nu}+\Pi K_{\mu \nu}\right)-h_{\mu \nu}\left[\left(f^{\prime \prime}+\frac{\omega}{2}\right)\left(Q^{2}-\Pi^{2}\right)+V(\phi)+f^{\prime} \square \phi\right]\right\} \\
& +\frac{1}{f}\left[\left(\omega+f^{\prime \prime}\right) Q_{\mu} Q_{\nu}+S_{\mu \nu}^{(m)}\right],
\end{aligned}
$$

while

$$
\begin{aligned}
S & =\frac{1}{f}\left[f^{\prime}\left(D^{\alpha} Q_{\alpha}+\Pi K\right)-3 V(\phi)-3 f^{\prime} \square \phi\right] \\
& -\frac{1}{f}\left[\left(2 f^{\prime \prime}+\frac{\omega}{2}\right) Q^{2}-3\left(f^{\prime \prime}+\frac{\omega}{2}\right) \Pi^{2}+S^{(m)}\right], \\
S-E & =\frac{1}{f}\left[\left(3 f^{\prime \prime}+\omega\right)\left(\Pi^{2}-Q^{2}\right)-4 V(\phi)-3 f^{\prime} \square \phi+S^{(m)}-E^{(m)}\right], \\
S+E & =\frac{1}{f}\left[2 f^{\prime}\left(D^{\alpha} Q_{\alpha}+K \Pi\right)-f^{\prime \prime} Q^{2}+\left(3 f^{\prime \prime}+2 \omega\right) \Pi^{2}\right] \\
& +\frac{1}{f}\left(-2 V(\phi)-3 f^{\prime} \square \phi+S^{(m)}+E^{(m)}\right) .
\end{aligned}
$$

The Hamiltonian and the momentum constraints assume the form

$$
\begin{aligned}
&{ }^{(3)} R+K^{2}-K_{i j} K^{i j}-\frac{2}{f}\left[f^{\prime}\left(D_{\alpha} Q^{\alpha}+K \Pi\right)+\frac{\omega}{2} \Pi^{2}+\frac{Q^{2}}{2}\left(\omega+2 f^{\prime \prime}\right)\right] \\
&=\frac{2}{f}\left(E^{(m)}+V(\phi)\right), \\
& D_{l} K^{l}{ }_{i}-D_{i} K+\frac{1}{f}\left[f^{\prime}\left(K_{i}{ }^{\alpha} Q_{\alpha}+D_{i} \Pi\right)+\left(\omega+f^{\prime \prime}\right) \Pi Q_{i}\right]=\frac{J_{i}^{(m)}}{f},
\end{aligned}
$$

respectively, and the dynamical equation (14.29) is written as

$$
\begin{aligned}
& \partial_{t} K^{i}{ }_{j}+N^{l} \partial_{l} K^{i}{ }_{j}+K^{i}{ }_{l} \partial_{j} N^{l}-K_{j}{ }^{l} \partial_{l} N^{i}+D^{i} D_{j} N-{ }^{(3)} R_{j}^{i} N-N K K_{j}{ }_{j} \\
& +\frac{N}{2 f}\left[f^{\prime \prime}\left(Q^{2}-\Pi^{2}\right)+2 V(\phi)+f^{\prime} \square \phi\right] \delta_{j}^{i}+\frac{N f^{\prime}}{f}\left(D^{i} Q_{j}+\Pi K^{i}{ }_{j}\right) \\
& +\frac{N}{f}\left(\omega+f^{\prime \prime}\right) Q^{i} Q_{j}=\frac{N}{2 f}\left[\left(S^{(m)}-E^{(m)}\right) \delta_{j}^{i}-2 S^{(m) i}{ }_{j}\right] .
\end{aligned}
$$

The trace of this equation is

$$
\begin{aligned}
& \partial_{t} K+N^{l} \partial_{l} K+{ }^{(3)} \Delta N-N K_{i j} K^{i j}-\frac{N f^{\prime}}{f}\left(D^{\alpha} Q_{\alpha}+\Pi K\right) \\
& +\frac{N}{2 f}\left[f^{\prime \prime} Q^{2}-\left(2 \omega+3 f^{\prime \prime}\right) \Pi^{2}\right]=\frac{N}{2 f}\left(-2 V(\phi)-3 f^{\prime} \square \phi+S^{(m)}+E^{(m)}\right)
\end{aligned}
$$


where [497]

$$
\begin{aligned}
& \mathcal{L}_{n} \Pi-\Pi K-Q^{\alpha} D_{\alpha}(\ln N)-D_{\alpha} Q^{\alpha}=-\square \phi \\
& =-\frac{1}{f\left[\omega+\frac{3\left(f^{\prime}\right)^{2}}{2 f}\right]}\left(\frac{f^{\prime} T^{(m)}}{2}-2 f^{\prime} V(\phi)+f V^{\prime}(\phi)\right) \\
& -\frac{1}{f\left[\omega+\frac{3\left(f^{\prime}\right)^{2}}{2 f}\right]}\left\{\left[\frac{-\omega^{\prime} f}{2}-\left(\omega+3 f^{\prime \prime}\right) \frac{f^{\prime}}{2}\right] \nabla^{\alpha} \phi \nabla_{\alpha} \phi\right\} .
\end{aligned}
$$

The initial data in vacuo $\left(h_{i j}, K_{i j}, \phi, Q_{i}, \Pi\right)$ on an initial hypersurface $\Sigma_{0}$ must satisfy the constraints (14.47) and (14.48), in addition to

$$
\begin{aligned}
& Q_{i}-D_{i} \phi=0, \\
& D_{i} Q_{j}=D_{j} Q_{i} .
\end{aligned}
$$

When matter is present, the additional variables $E^{(m)}, J_{\mu}^{(m)}, S_{\mu \nu}^{(m)}$ must be assigned on the initial hypersurface. Prescribing lapse $N$ and shift $N^{\mu}$ is equivalent to fixing a gauge. The differential system (14.47)-(14.50) contains only first-order derivatives in both space and time once the d'Alembertian $\square \phi$ is written in terms of $\phi, \nabla^{\mu} \phi \nabla_{\mu} \phi, f$, and its derivatives by means of Eq. (14.51) [372, 459, 497]. Following [497], the reduction to a first-order system indicates that the Cauchy problem is well-posed in vacuo and well-formulated in the presence of matter.

\section{B. The initial value problem of $f(R)$-gravity in the ADM formulation}

In order to deal with the Initial Value Problem for $f(R)$-gravity, let us consider the analogy of such a theory with scalar tensor gravity. Remembering the results of the previous subsection, metric $f(R)$-gravity models are equivalent to Brans-Dicke gravity with $\omega=0$, while Palatini $f(R)$-gravity models are equivalent to Brans-Dicke gravity with $\omega=-3 / 2$. Following the discussion in [459], the Hamiltonian formulation of Brans-Dicke theories with a non-trivial potential can be carried out paying special attention to the case $\omega=-3 / 2$. The scalar field, in this case, presents a degenerate momentum which is proportional to a linear combination of the momenta of the induced metric $h_{\mu \nu}$. This degeneracy requires, following Dirac's algorithm for constrained systems, the introduction of a new constraint in the Hamiltonian. Consistency of the evolution of that constraint leads to a secondary constraint which establishes an algebraic relation between the scalar field and the trace of the energy-momentum tensor of the matter. In [459], the constraint and evolution equations are written in a way that allows a comparison between the general case $\omega \neq-3 / 2$ and $\omega=-3 / 2$. The resulting constraint and evolution equations of the case $\omega=-3 / 2$ do not contain any higher-order time derivative of the matter fields, and only spatial derivatives of the scalar field appear up to the second-order. This implies that the spatial profiles of the matter sources must satisfy stronger differentiability requirements than in GR. This property can be interpreted as a requirement due to the existence of a conformal geometry related with the matter fields. By comparing the constraint and evolution equations of the theory with those of GR, the initial value Cauchy problem is well-formulated because of the intrinsic geometry of space-time is determined uniquely by an initial choice of $h_{\mu \nu}$ and $\Pi^{\mu \nu}$, plus the corresponding positions and momenta of matter on the initial Cauchy surface. This result indicates that also the well-posedness of the problem is possible in contrast with the results in [372].

\section{The Gaussian normal coordinates approach in General Relativity}

A different approach to the Initial Value Problem uses Gaussian normal coordinates (also called synchronous coordinates) instead of the ADM decomposition. Before discussing ETGs, we recall the initial value formulation of GR in these coordinates, which is well-formulated and well-posed, as shown in [577]. We adopt the formalism developed in Ref. [538].

Let us consider a system of Gaussian normal coordinates [577], in which the metric tensor has components $g_{00}=-1$ and $g_{0 i}=0$. These coordinates serve the purpose of splitting the space-time manifold $\mathcal{M}$ into a spatial hypersurface $\Sigma_{3}$ of constant time from the orthogonal time direction.

\footnotetext{
28 Various gauge conditions employed in the literature are surveyed in [228, 497].
} 
Given a second rank symmetric tensor $W_{\mu \nu}$ on the globally hyperbolic space-time $\left(\mathcal{M}, g_{\mu \nu}\right)$, we define its (symmetric) conjugate tensor

$$
W_{\mu \nu}^{*}=W_{\mu \nu}-\frac{W}{2} g_{\mu \nu}
$$

where | denotes the covariant derivative with respect to the Levi-Civita connection induced by $g_{\mu \nu}$ and $W^{\prime} \equiv W^{\mu \nu} g_{\mu \nu}$ is the trace of $W_{\mu \nu}$. If $V_{0}$ is a space-time domain in $\mathcal{M}$ in which $g_{00} \neq 0$ and $\Sigma_{3}$ is the three-surface of equation $x^{0}=0$, then the following statements are equivalent:

1. $W_{\mu \nu}=0$ in $V_{0}$;

2. $W_{i j}^{*}=0$ and $W_{0 \alpha}=0$ in $V_{0}$;

3. $W_{i j}^{*}=0$ and $W_{\nu \mid \mu}^{\mu}=0$ in $V_{0}$ with $W_{o \mu}=0$ in $\Sigma_{3}$.

Let us consider the Einstein equations $G_{\mu \nu}=\kappa T_{\mu \nu}^{(m)}$ and the contracted Bianchi identities $\nabla^{\nu} T_{\mu \nu}^{(m)}=0$; introducing the tensor

$$
W_{\mu \nu} \equiv G_{\mu \nu}-\kappa T_{\mu \nu}^{(m)}
$$

the conjugate tensor is

$$
W_{\mu \nu}^{*}=R_{\mu \nu}-\kappa T_{\mu \nu}^{*}
$$

and the Einstein equations are

$$
W_{\mu \nu}=0 .
$$

These are 10 equations for the 20 unknown functions $g_{\mu \nu}$ and $T_{\mu \nu}^{(m)}$. We assign the 10 functions $g_{0 \mu}$ and $T_{i j}^{(m)}$; the remaining 10 functions $g_{i j}$ and $T_{0 \mu}^{(m)}$ are determined by Eq. (14.57). These functions can be expressed in the equivalent form

$$
R_{i j}-\kappa T_{i j}^{*}=0, \quad W_{\nu \mid \mu}^{\mu}=T_{\nu \mid \mu}^{\mu}=0,
$$

with the condition

$$
G_{0 \mu}-\kappa T_{0 \mu}^{(m)}=0
$$

on the hypersurface $x^{0}=0$. Eqs. (14.58) can be rewritten as

$$
\begin{array}{r}
g_{i j, 00}=2 \bar{R}_{i j}-\frac{A}{2} g_{i j, 0}+g^{l m} g_{i l, 0} g_{j m, 0}+2 \kappa T_{i j}^{*}, \\
T_{0 \nu, 0}^{(m)}=-T_{\nu, 0}^{(m)}=T_{\nu, i}^{(m)^{i}}+\Gamma_{i \mu}{ }_{i \mu} T_{\nu}^{(m)}{ }_{\nu}^{\mu}-\Gamma_{i \nu}{ }^{\mu} T_{\nu}^{(m)^{i}},
\end{array}
$$

where $\bar{R}_{i j}$ is the intrinsic Ricci tensor of the hypersurface $x^{0}=0, \Gamma_{\mu \nu}^{\rho}$ is the Levi-Civita connection of the metric $g_{\mu \nu}$, and

$$
A \equiv g^{i j} g_{i j, 0} .
$$

In the same way, the constraint equation (14.59) becomes

$$
\begin{gathered}
A_{, i}-D^{j} g_{i j, 0}+2 \kappa T_{0 i}^{(m)}=0, \\
\tilde{R}-\frac{A^{2}}{4}+\frac{B}{4}+2 \kappa T_{00}=0,
\end{gathered}
$$

where $\bar{R}$ is the intrinsic Ricci scalar of the hypersurface $x^{0}=0, D_{i}$ denotes the covariant derivative operator on this hypersurface associated with the Levi-Civita connection of the intrinsic metric $\left.g_{i j}\right|_{\Sigma_{0}}$ and

$$
B=g^{i j} g^{l m} g_{i l, 0} g_{i m, 0} .
$$


Let us assign now the Cauchy data

$$
g_{i j}, \quad g_{i j, 0}, \quad T_{\mu 0}^{(m)}
$$

on the hypersurface $x^{0}=0$; they must satisfy the constraint equations (14.63), (14.64), (14.60), and eq. (14.61) gives the quantities

$$
g_{i j, 00}, \quad T_{0 \mu, 0}^{(m)},
$$

as functions of the Cauchy data. By differentiating Eqs. (14.60) and 14.61), it is straightforward to obtain time derivatives of higher order as functions of the initial data. This procedure allows one to locally reconstruct the solution of the field equations as a power series of $x^{0}$. The initial three-surface $\Sigma_{3}$ is then a Cauchy hypersurface for the globally hyperbolic space-time $\left(\mathcal{M}, g_{\mu \nu}\right)$ and the initial value problem is well-formulated in GR. Our goal is now to extend these results to $f(R)$-gravity in the metric-affine formalism.

\section{The Cauchy problem of $f(R)$-gravity in Gaussian normal coordinates}

\section{The vacuum case}

In the metric-affine formulation of $f(R)$-gravity the independent variables are $\left(g_{\mu \nu}, \Gamma_{\mu \nu}^{\alpha}\right)$, where $g_{\mu \nu}$ is the metric and $\Gamma_{\mu \nu}^{\alpha}$ is the linear connection. In vacuo, the field equations are obtained by varying the action

$$
S[g, \Gamma]=\int d^{4} x \sqrt{-g} f(\mathcal{R})
$$

with respect to the metric and the connection, where $\mathcal{R}(g, \Gamma)=g^{\mu \nu} \mathcal{R}_{\mu \nu}$ is the scalar curvature of the connection $\Gamma_{\mu \nu}^{\alpha}$ and $\mathcal{R}_{\mu \nu}$ is the Ricci tensor constructed with this connection. The metric connection $\Gamma_{\mu \nu}^{\alpha}$ can have a non-vanishing torsion while, in the Palatini approach, $\Gamma_{\mu \nu}^{\alpha}$ is a non-metric but torsion-free connection [126].

In vacuo, the field equations of $f(R)$-gravity with torsion are 126 - 128$]$

$$
\begin{aligned}
& f^{\prime}(\mathcal{R}) \mathcal{R}_{\mu \nu}-\frac{f(\mathcal{R})}{2} g_{\mu \nu}=0, \\
& T_{\mu \nu}{ }^{\alpha}=-\frac{1}{2 f^{\prime}} \frac{\partial f^{\prime}}{\partial x^{\rho}}\left(\delta_{\mu}^{\rho} \delta_{\nu}^{\sigma}-\delta_{\nu}^{\rho} \delta_{\mu}^{\sigma}\right),
\end{aligned}
$$

while the field equations of $f(R)$-gravity à la Palatini are [406, 407, 452]

$$
\begin{aligned}
& f^{\prime}(\mathcal{R}) \mathcal{R}_{\mu \nu}-\frac{f(\mathcal{R})}{2} g_{\mu \nu}=0, \\
& \nabla_{\mu}\left[f^{\prime}(\mathcal{R}) g_{\mu \nu}\right]=0 .
\end{aligned}
$$

In both cases, the trace of the field equations (14.69) and (14.71) yields

$$
f^{\prime}(\mathcal{R}) \mathcal{R}-2 f(\mathcal{R})=0 .
$$

When this equation admits solutions, the scalar curvature $\mathcal{R}$ is a constant; then eqs. (14.70) and (14.72) imply that both connections coincide with the Levi-Civita connection of the metric $g_{\mu \nu}$ which solves the field equations and both theories reduce to GR with a cosmological constant, for which the Cauchy problem is well-formulated and well-posed [577].

\section{The case with matter}

Let us allow now a perfect fluid and study the Cauchy problem of $f(R)$-gravity. We discuss simultaneously the Palatini approach and a non-vanishing torsion, but we assume that the matter Lagrangian does not couple explicitly 
to the connection. Then the field equations are

$$
\begin{aligned}
& f^{\prime}(\mathcal{R}) \mathcal{R}_{\mu \nu}-\frac{f(\mathcal{R})}{2} g_{\mu \nu}=T_{\mu \nu}^{(m)} \\
& T_{\mu \nu}{ }^{\alpha}=-\frac{1}{2 f^{\prime}(\mathcal{R})} \frac{\partial f^{\prime}(\mathcal{R})}{\partial x^{\rho}}\left(\delta_{\mu}^{\rho} \delta_{\nu}^{\sigma}-\delta_{\nu}^{\rho} \delta_{\mu}^{\alpha}\right)
\end{aligned}
$$

in the case of $f(R)$-gravity with torsion, and

$$
\begin{aligned}
& f^{\prime}(\mathcal{R}) \mathcal{R}_{\mu \nu}-\frac{f(\mathcal{R})}{2} g_{\mu \nu}=T_{\mu \nu}^{(m)}, \\
& \nabla_{\alpha}\left[f^{\prime}(\mathcal{R}) g_{\mu \nu}\right]=0,
\end{aligned}
$$

for Palatini $f(R)$-gravity, where $T_{\mu \nu}^{(m)} \equiv-\frac{2}{\sqrt{-g}} \frac{\delta\left(\sqrt{-g} \mathcal{L}^{(m)}\right)}{\delta g^{\mu \nu}}$ is the matter energy-momentum tensor. The trace of eqs. (14.74) and (14.76) yields the relation between $\mathcal{R}$ and $T^{(m)} \equiv g^{\mu \nu} T_{\mu \nu}^{(m)}$

$$
f^{\prime}(\mathcal{R}) \mathcal{R}-2 f(\mathcal{R})=T^{(m)} .
$$

When $T^{(m)}=$ const. the theory reduces to GR with a cosmological constant and the initial value problem is identical to the vacuum case. Assuming that the relation (14.78) is invertible and $T^{(m)} \neq$ const., the Ricci scalar can be expressed as a function of $T^{(m)}$

$$
\mathcal{R}=F\left(T^{(m)}\right)
$$

It is then easy to show that the field equations of both the Palatini and the metric-affine theory with torsion can be expressed in the form [126, 127, 452]

$$
\begin{aligned}
\mathcal{R}_{\mu \nu}-\frac{1}{2} g_{\mu \nu} \mathcal{R} & =\frac{1}{\varphi} T_{\mu \nu}^{(m)}+\frac{1}{\varphi^{2}}\left[-\frac{3}{2} \frac{\partial \varphi}{\partial x^{\mu}} \frac{\partial \varphi}{\partial x^{\nu}}+\varphi \tilde{\nabla}_{\nu} \frac{\partial \varphi}{\partial x^{\mu}}\right. \\
& \left.+\frac{3}{4} \frac{\partial \varphi}{\partial x^{\alpha}} \frac{\partial \varphi}{\partial x^{\beta}} g^{\alpha \beta} g_{\mu \nu}-\varphi \tilde{\nabla}^{\alpha} \frac{\partial \varphi}{\partial x^{\alpha}} g_{\mu \nu}-V(\varphi) g_{\mu \nu}\right]
\end{aligned}
$$

where

$$
V(\varphi) \equiv \frac{1}{4}\left[\varphi F^{-1}\left(\left(f^{\prime}\right)^{-1}(\varphi)\right)+\varphi^{2}\left(f^{\prime}\right)^{-1}(\varphi)\right],
$$

is an effective potential for the scalar field

$$
\varphi \equiv f^{\prime}\left(F\left(T^{(m)}\right)\right)
$$

By performing the conformal transformation $g_{\mu \nu} \longrightarrow \tilde{g}_{\mu \nu}=\varphi g_{\mu \nu}$, Eq. (14.80) assumes the simpler form [126, 291, 452]

$$
\tilde{R}_{\mu \nu}-\frac{1}{2} \tilde{g}_{\mu \nu} \tilde{R}=\frac{1}{\varphi} T_{\mu \nu}^{(m)}-\frac{1}{\varphi^{3}} V(\varphi) \tilde{g}_{\mu \nu}
$$

where $\tilde{R}_{\mu \nu}$ and $\tilde{R}$ are the Ricci tensor and the Ricci scalar of the conformal metric $\tilde{g}_{\mu \nu}$, respectively.

The connection $\Gamma_{\mu \nu}{ }^{\alpha}$, solution of the field equations with torsion, is

$$
\Gamma_{\mu \nu}^{\alpha}=\tilde{\Gamma}_{\mu \nu}^{\alpha}+\frac{1}{2 \varphi} \frac{\partial \varphi}{\partial x^{\nu}} \delta_{\mu}^{\alpha}-\frac{1}{2 \varphi} \frac{\partial \varphi}{\partial x^{\rho}} g^{\rho \alpha} g_{\mu \nu}
$$

where $\tilde{\Gamma}_{\mu \nu}{ }^{\alpha}$ is the Levi-Civita connection of the metric $g_{\mu \nu}$ while $\tilde{\Gamma}_{\mu \nu}^{\alpha}$, solution of the Palatini field equations, coincides with the Levi-Civita connection of the conformal metric $\tilde{g}_{\mu \nu} . \Gamma_{\mu \nu}{ }^{\alpha}$ and $\tilde{\Gamma}_{\mu \nu}^{\alpha}$ satisfy the relation

$$
\tilde{\Gamma}_{\mu \nu}^{\alpha}=\Gamma_{\mu \nu}^{\alpha}+\frac{1}{2 \varphi} \frac{\partial \varphi}{\partial x^{\mu}} \delta_{\nu}^{\alpha}
$$


and the Levi-Civita connections induced by the metrics $g_{\mu \nu}$ and $\tilde{g}_{\mu \nu}$ are related by the identity

$$
\tilde{\Gamma}_{\mu \nu}^{\alpha}=\Gamma_{\mu \nu}^{\alpha}+\frac{1}{2 \varphi} \frac{\partial \varphi}{\partial x^{\nu}} \delta_{\mu}^{\alpha}-\frac{1}{2 \varphi} \frac{\partial \varphi}{\partial x^{\rho}} g^{\rho \alpha} g_{\mu \nu}+\frac{1}{2 \varphi} \frac{\partial \varphi}{\partial x^{\mu}} \delta_{\nu}^{\alpha} .
$$

The field equations (14.80) have to be considered together with the matter field equations and it must be kept in mind that the conservation equations for both the metric-affine theories (with torsion and $a$ la Palatini) coincide with the standard conservation laws of GR [145]

$$
\tilde{\nabla}_{\nu} T^{\mu \nu}=0
$$

It is straightforward to show that eq. (14.87) is equivalent to the conservation law

$$
\tilde{\nabla}_{\nu} T^{\mu \nu}=0
$$

where

$$
T_{\mu \nu}=\frac{1}{\varphi} T_{\mu \nu}^{(m)}-\frac{1}{\varphi^{3}} V(\varphi) \tilde{g}_{\mu \nu}
$$

for the conformally transformed theories (14.83). In fact, by an explicit calculation of the divergence $\tilde{\nabla}_{\nu} T^{\mu \nu}$ where the relations (14.86) have been used, we obtain

$$
\tilde{\nabla}^{\nu} T_{\mu \nu}=\frac{1}{\varphi^{2}} \tilde{\nabla}^{\nu} T_{\mu \nu}^{(m)}+\frac{1}{\varphi^{3}} \frac{\partial \varphi}{\partial x^{\mu}}\left[-\frac{T^{(m)}}{2}+\frac{3 V(\varphi)}{\varphi}-V^{\prime}(\varphi)\right] .
$$

The constraint equations (14.87) and (14.88) are then mathematically equivalent in view of the relation

$$
T^{(m)}-\frac{6 V(\varphi)}{\varphi}+2 V^{\prime}(\varphi)=0
$$

which is equivalent to the definition $\varphi=f^{\prime}\left(F\left(T^{(m)}\right)\right)[126]$.

With these results in mind, the Cauchy problem for Eq. (14.80) and the related equations of motion for matter can be approached by discussing the equivalent initial value problem of the conformally transformed theories. Using, as in GR, Gaussian normal coordinates and beginning with Eqs. (14.83) and (14.88), it is easy to conclude that the Cauchy problem is well-formulated also in this case.

In general, the equations of motion for matter imply the Levi-Civita connection of the metric $g_{\mu \nu}$ and not the connection induced from the conformal metric $\tilde{g}_{\mu \nu}$. Thanks to Eq. (14.86), this is not a problem since the connection $\tilde{\Gamma}_{\mu \nu}^{\alpha}$ can be expressed in terms of $\Gamma_{\mu \nu}{ }^{\alpha}$ and the scalar field $\varphi$ which, on the other hand, is a function of the matter fields. As a result, we could obtain slightly more complicated equations implying further constraints on the initial data but, in any case, the same equations can always be rewritten in "normal form" with respect to the maximal order time derivatives of the matter fields, determining a well-formulated Cauchy problem [577].

As an example, let us examine in detail the perfect fluid case with barotropic equation of state $P=P(\rho)$. The corresponding energy-momentum tensor is

$$
T_{\mu \nu}^{(m)}=(P+\rho) u_{\mu} u_{\nu}+P g_{\mu \nu},
$$

and satisfies Eq. (14.87) with the normalization

$$
g_{\mu \nu} u^{\mu} u^{\nu}=-1
$$

of the fluid four-velocity. Eq. (14.87) gives

$$
\left(\rho+P u^{\nu}\right)_{\mid \nu} u_{\mu}+(\rho+P) u_{\mu \mid \nu} u^{\nu}+\frac{\partial P}{\partial x^{\mu}}=0 .
$$

Contraction with $u^{\alpha}$ yields

$$
\left(\rho u^{\nu}\right)_{\mid \nu}=-P u_{\mid \nu}^{\nu}
$$

while, substituting Eq. (14.95) into Eq. (14.94) for $\alpha=1,2$, and 3, we obtain

$$
(\rho+P) u^{\nu} u_{\mid \nu}^{i}=-\frac{\partial P}{\partial x^{\nu}}\left(u^{i} u^{\nu}+g^{i \nu}\right) .
$$


Eqs. (14.93), (14.95), and (14.96) involve the metric $g_{\mu \nu}$ and its first derivatives; using the relation (14.86) we can rewrite them in terms of the conformal metric $\tilde{g}_{\mu \nu}$, the scalar $\varphi=\varphi(\rho)$, and their first derivatives, obtaining

$$
\begin{aligned}
& \frac{1}{\varphi} \tilde{g}_{u} u^{\mu} u^{\nu}=-1 \\
& \frac{\partial}{\partial x^{\nu}}\left(\rho V^{\nu}\right)+\tilde{\Gamma}_{\nu \sigma}^{\nu} \rho u^{\sigma}-\frac{2}{\varphi} \frac{\partial \varphi}{\partial x^{\sigma}} \rho u^{\sigma} \\
= & -P\left(\frac{\partial u^{\nu}}{\partial x^{\nu}}+\tilde{\Gamma}_{\nu \sigma}^{\nu} u^{\sigma}-\frac{2}{\varphi} \frac{\partial \varphi}{\partial x^{\sigma}} u^{\sigma}\right), \\
& (\rho+P) u^{\nu}\left[\frac{\partial u^{i}}{\partial x^{\nu}}+\tilde{\Gamma}_{\nu \sigma}^{i} u^{\sigma}+\frac{1}{2 \varphi}\left(-\frac{\partial \varphi}{\partial x^{\sigma}} \delta_{\nu}^{i}+\frac{\partial \varphi}{\partial x^{\nu}} \delta_{\sigma}^{i}-\frac{\partial \varphi}{\partial x^{\rho}} g^{\rho i} g_{\nu \sigma}\right) u^{\sigma}\right] \\
= & -\frac{\partial P}{\partial x^{\nu}}\left(u^{i} u^{\nu}+g^{i \nu}\right) .
\end{aligned}
$$

In Gaussian normal coordinates in which $\tilde{g}_{00}=-1$ (assuming $\varphi>0$ ) and $\tilde{g}_{0 i}=0$, Eq. (14.97) yields the expression of $u^{0}$ in terms of the remaining components $u^{i}$. Eqs. (14.97) and (14.98) can be regarded as linear equations for the functions $\partial u^{i} / \partial x^{0}$ and $\partial \rho / \partial x^{0}$. The explicit solution of these equations, in terms of the unknown functions, could originate further constraints on the initial data and on the form of the function $f(\mathcal{R})$. In Gaussian normal coordinates, Eqs. (14.97) and (14.98) allow one to obtain $\partial u^{i} / \partial x^{0}$ and $\partial \rho / \partial x^{0}$ as functions of the initial data $\tilde{g}_{i j}, \partial \tilde{g}_{i j} / \partial x^{0}, u_{i}$, and $\rho$ allowing the equations of motion of matter to be cast in normal form, hence the Cauchy problem is well-formulated.

Consider, as another example, the initial value formulation of $f(R)$-gravity coupled with Yang-Mills fields, in particular with the electromagnetic field. Also in this case, the problem is well-formulated. In fact, the stress-energy tensor of a Yang-Mills field has vanishing trace. Using Eq. (14.78), it is easy to prove that the Ricci scalar is constant and then, using Eqs. (14.75) and (14.77) one concludes that the connection coincides with the Levi-Civita connection of $g_{\mu \nu}$. In this situation, both theories ( $\grave{a} l a$ Palatini and with torsion) reduce to GR with a cosmological constant and the Cauchy problem is well-formulated (this conclusions was already reached for the Maxwell field). Moreover, the initial value problem is well-posed for any theory in which the trace of the matter energy-momentum tensor is constant, which is reduced to Einstein gravity with a cosmological constant. The Cauchy problem for perfect fluid and scalar field sources is discussed in [144, 145].

To conclude, we have shown that the initial value problem for ETGs can be at least well-formulated, passing another test for the viability of these theories. Well-posedness is also necessary in order to achieve a complete control of the dynamics but it depends on the specific matter fields adopted and the discussion becomes specific to them.

Since ETGs, like GR, are gauge theories, the choice of suitable coordinates may be crucial to show that the Cauchy problem is formulated correctly. We have discussed the two approaches using the $3+1$ ADM decomposition and Gaussian normal coordinates, which can be defined when the covariant derivative operator $\nabla_{\mu}$ arises from a metric. These coordinates are useful for calculations on a given non-null surface $\Sigma_{3}$, i.e., a three-dimensional embedded submanifold of the four-dimensional manifold $\mathcal{M}$. Gaussian normal coordinates allow one to define uniquely timelike geodesics orthogonal to $\Sigma_{3}$ and to formulate correctly the conditions for the validity of the Cauchy-Kowalewski theorem 577].

In the metric-affine formalism a given $f(R)$ theory in vacuo is equivalent to GR plus a cosmological constant, hence the initial value problem is well-formulated and well-posed. The same conclusion holds with matter sources whenever the trace of the energy-momentum tensor is constant. As shown in [126 128], by introducing matter fields in the Palatini and in the metric-affine approach with torsion, one can define $\mathcal{R}=F\left(T^{(m)}\right)$ and then the scalar field $\varphi \equiv f^{\prime}\left(F\left(T^{(m)}\right)\right)$, which allows one to reduce the theory to scalar-tensor gravity and to relate the form of $f(R)$ to the trace of the matter energy-momentum tensor. In this case, it is always possible to show that the initial value problem is well-formulated according to the results in [459]. Moreover, matter fields could induce further constraints on the Cauchy hypersurface $x^{0}=0$ which, if suitably defined, lead to the normal form of the equations of motion for the matter sources. This is one of the main requirements for a well-formulated initial value problem. However different sources of the gravitational field, such as perfect fluids, Yang-Mills, and Klein-Gordon fields, could generate different constraints on the initial hypersurface $\Sigma_{3}$. These constraints could also imply restrictions on the possible form of $f(R)$. In conclusion, as in GR, the choice of gauge is essential for a correct formulation of the initial value problem, while the source fields have to be discussed carefully.

As final consideration, we can say that through the Lagrangian formulation, we have obtained the field equations of various theories of gravity. We have seen how the metric and Palatini variations produce different field equations in ETGs, contrary to what happens in GR. Conformal transformations have been applied to ETGs, and an overview 
of the Cauchy problem has been given. It is beginning to be clear that several aspects of a gravitational theory need to be taken into account before the latter can be claimed to be viable. 


\section{PART III: APPLICATIONS}

In Part III of this Report we will discuss some remarkable applications which, beside cosmology and astrophysics, could constitute test-bed probes for ETGs. In particular, we will take into account the problems of spherical and axial symmetry in $f(R)$-gravity which give rise to solutions more general than the standard ones in GR. For example the Jebsen-Birkhoff theorem does not always hold and this fact could have remarcable physical consequences on self-gravitating systems [122, 131].

Furthermore we consider the post-Newtonian limit showing that the recovering of Newtonian potential is nothing else but a particular case of GR while, in general, Yukawa-like terms come out from the weak field approximation. Such a new feature, beside addressing the problem of dark matter in galaxies and clusters of galaxies [135], could give rise to new interesting phenomenology like neutrino oscillations induced by gravity.

Moreover the post-Minkowskian limit will be studied. In theories alternative to GR, it is straightforward to show that gravitational radiation presents further modes and polarizations which cannot be simply ignored if the problem of gravitational waves has to be seriously addressed.

\section{SPHERICAL AND AXIAL SYMMETRY}

In all areas of physics and mathematics it is common to search for insight into a theory by finding exact solutions of its fundamental equations and by studying these solutions in detail. This goal is particularly difficult in nonlinear theories and the usual approach consists of assuming particular symmetries and searching for solutions with these symmetries. Stripped of inessential features and simplified in this way, the search for exact solutions becomes easier. In a sense, this approach betrays a reductionist point of view but, pragmatically, it is often crucial to gain an understanding of the theory that cannot be obtained otherwise and that no physicist or mathematician would want to renounce to. In this section we discuss exact solutions of ETGs with spherical symmetry. Finally, the section ends with a discussion from spherical to axially symmetric solutions. An example is given.

\section{A. Spherically symmetric solutions in $f(R)$-gravity}

The physically relevant spherically symmetric solutions of GR include the asymptotically flat Schwarzschild solution describing an isolated body, which was derived in the very early days of GR. Other relevant solutions include the Schwarzschild-de Sitter (or Kottler) metric representing a black hole embedded in a de Sitter Universe, and the Lemaitre-Tolman-Bondi class of solutions describing spherical objects embedded in a dust-dominated cosmological background [85, 378, 533, 548], to which one should add the McVittie metric and its generalizations [402] (see [366] for a survey of inhomogeneous solutions including spherically symmetric ones, and [533] for exact solutions of GR). Spherical solutions representing black holes have been instrumental in the development of black hole mechanics and thermodynamics [577, 578].

\section{Generalities of spherical symmetry}

The classical tests of GR pertain to the realm of spherically symmetric solutions and the weak-field limit [586]. One of the fundamental properties of a gravitational theory is the possibility of asymptotic flatness, i.e., space-time being Minkowskian far away from a localized distribution of mass-energy. Alternative gravitational theories may or may not exhibit this physical property which allows for a consistent comparison with GR. This point is sometimes forgotten in the study of the weak-field limit of alternative theories of gravity and can be discussed in general by considering the meaning of spherical solutions in ETGs when the standard results of GR are recovered in the limits $r \rightarrow \infty$ and $f(R) \rightarrow R$. Spherical solutions can be classified using the Ricci curvature $R$ as

- solutions with $R=0$,

- solutions with constant Ricci scalar $R=R_{0} \neq 0$,

- solutions with Ricci scalar $R(r)$ depending only on the radial coordinate $r$, and

- solutions with time-dependent $R(t, r)$. 
In the first three cases the Jebsen-Birkhoff theorem is valid [311], meaning that stationary spherically symmetric solutions are necessarily static. However, as shown in the following, this theorem does not hold for every situation in $f(R)$-gravity because temporal evolution can emerge already in perturbation theory at some order of approximation.

A crucial role for the existence of exact spherical solutions is played by the relation between the metric potentials and by the relations between the latter and the Ricci scalar. The relation between the metric potentials and $R$ can be regarded as a constraint which assumes the form of a Bernoulli equation [132]. In principle, spherically symmetric solutions can be obtained for any analytic function $f(R)$ by solving this Bernoulli equation, for both the case of constant Ricci scalar and $R=R(r)$. These spherically symmetric solutions can be used as backgrounds to test how generic $f(R)$-gravity may deviate from GR. Theories that imply $f(R) \rightarrow R$ in the weak-field limit are particularly interesting. In such cases, the comparison with GR is straightforward and the experimental results evading the GR constraints can be framed in a self-consistent picture [70]. Finally, a perturbation approach can be developed to obtain spherical solutions at zero order, after which first order solutions are searched for. This scheme is iterative and can, in principle, be extended to any order in the perturbations. It is crucial to consider $f(R)$ theories which can be Taylor-expanded about a constant value $R_{0}$ of the curvature scalar $R$.

\section{The Ricci scalar in spherical symmetry}

By imposing that the space-time metric is spherically symmetric,

$$
d s^{2}=-A(t, r) d t^{2}+B(t, r) d r^{2}+r^{2} d \Omega_{2}^{2},
$$

the Ricci scalar can be expressed as

$$
\begin{array}{r}
R(t, r)=\left\{B\left(\dot{A} \dot{B}-A^{\prime 2}\right) r^{2}+A\left[r\left(\dot{B}^{2}-A^{\prime} B^{\prime}\right)+2 B\left(2 A^{\prime}+r A^{\prime \prime}-r \ddot{B}\right)\right]\right. \\
\left.-4 A^{2}\left[B^{2}-B+r B^{\prime}\right]\right\}\left(2 r^{2} A^{2} B^{2}\right)^{-1},
\end{array}
$$

where a prime and an overdot denote differentiation with respect to $r$ and $t$, respectively. If the metric (15.1) is time-independent, i.e., $A(t, r)=a(r)$ and $B(t, r)=b(r)$, then Eq. (15.2) assumes the simple form

$$
\begin{aligned}
R(r) & =\left\{a(r)\left[2 b(r)\left(2 a^{\prime}(r)+r a^{\prime \prime}(r)\right)-r a^{\prime}(r) b^{\prime}(r)\right]-b(r) a^{\prime}(r)^{2} r^{2}\right. \\
& \left.-4 a^{2}(r)\left(b(r)^{2}-b(r)+r b^{\prime}(r)\right)\right\}\left(2 r^{2} a^{2}(r) b^{2}(r)\right)^{-1}
\end{aligned}
$$

One can see Eq. (15.3) as a constraint on the functions $a(r)$ and $b(r)$ once a specific form of the Ricci scalar is given. Eq. (15.3) reduces to the Bernoulli equation of index two [132]

$$
b^{\prime}(r)+h(r) b(r)+l(r) b^{2}(r)=0
$$

for the metric component $b(r)$, i.e.,

$$
\begin{aligned}
b^{\prime}(r) & +\left\{\frac{r^{2} a^{\prime}(r)^{2}-4 a(r)^{2}-2 r a(r)\left[2 a(r)^{\prime}+r a(r)^{\prime \prime}\right]}{r a(r)\left[4 a(r)+r a^{\prime}(r)\right]}\right\} b(r) \\
& +\left\{\frac{2 a(r)}{r}\left[\frac{2+r^{2} R(r)}{4 a(r)+r a^{\prime}(r)}\right]\right\} b(r)^{2}=0 .
\end{aligned}
$$

The general solution of Eq. (15.5) is

$$
b(r)=\frac{\exp \left[-\int d r h(r)\right]}{K+\int d r l(r) \exp \left[-\int d r h(r)\right]},
$$

where $K$ is an integration constant and $h(r)$ and $l(r)$ are the coefficients of the linear and quadratic terms in $b(r)$, respectively. Inspection of this Bernoulli equation reveals that solutions corresponding to $l(r)=0$ exist, which have a Ricci curvature scaling as $R \sim-2 / r^{2}$ as spatial infinity is approached. No real solutions exist if $h(r)$ vanishes identically. The limit $r \rightarrow+\infty$ deserves special care: in order for the gravitational potential $b(r)$ to have the correct 
Minkowskian limit, both functions $h(r)$ and $l(r)$ must go to zero provided that the quantity $r^{2} R(r)$ is constant. This fact implies that $b^{\prime}(r)=0$, and, finally, also the metric potential $b(r)$ has the correct Minkowskian limit.

If asymptotic flatness of the metric is imposed, the Ricci curvature must scale as $r^{-n}$ when $r \rightarrow+\infty$, where $n \geqslant 2$ is an integer,

$$
r^{2} R(r) \simeq r^{-n} \quad \text { as } r \rightarrow+\infty .
$$

Any other behavior of the Ricci scalar would compromise asymptotic flatness, as can be seen from Eq. (15.5). In fact, let us consider the simplest spherically symmetric case in which

$$
d s^{2}=-a(r) d t^{2}+\frac{d r^{2}}{a(r)}+r^{2} d \Omega_{2}^{2} .
$$

The Bernoulli equation (15.5) is easily integrated and the most general metric potential $a(r)$ compatible with the constraint (15.3) is

$$
a(r)=1+\frac{k_{1}}{r}+\frac{k_{2}}{r^{2}}+\frac{1}{r^{2}} \int d r\left[\int r^{2} R(r) d r\right],
$$

where $k_{1}$ and $k_{2}$ are integration constants. The Minkowskian limit $a(r) \rightarrow 1$ as $r \rightarrow \infty$ is obtained only if the condition (15.7) is satisfied, otherwise the gravitational potential diverges.

\section{Spherical symmetry in $f(R)$-gravity}

Let us specialize now to metric $f(R)$ theories by considering an analytic function $f(R)$, the fourth order field equations

$$
f^{\prime}(R) R_{\mu \nu}-\frac{1}{2} f(R) g_{\mu \nu}-f^{\prime}(R)_{; \mu \nu}+g_{\mu \nu} \square f^{\prime}(R)=\kappa T_{\mu \nu},
$$

and the corresponding trace equation

$$
3 \square f^{\prime}(R)+f^{\prime}(R) R-2 f(R)=\kappa T .
$$

By rewriting Eq. (15.10) as

$$
\begin{aligned}
G_{\mu \nu} & =T_{\mu \nu}^{(\text {curv })}+T_{\mu \nu}^{(m)}, \\
T_{\mu \nu}^{(\text {curv })} & =\frac{1}{f^{\prime}(R)}\left\{g_{\mu \nu}\left[f(R)-R f^{\prime}(R)\right]+f^{\prime}(R)^{; \rho \sigma}\left(g_{\mu \rho} g_{\nu \sigma}-g_{\rho \sigma} g_{\mu \nu}\right)\right\}
\end{aligned}
$$

matter enters Eq. (15.12) through the modified stress-energy tensor

$$
T_{\mu \nu}^{(m)}=\frac{\kappa T_{\mu \nu}}{f^{\prime}(R)} .
$$

The most general spherically symmetric metric can be written as

$$
d s^{2}=-m_{1}\left(t^{\prime}, r^{\prime}\right) d t^{\prime 2}+m_{2}\left(t^{\prime}, r^{\prime}\right) d r^{2}+m_{3}\left(t^{\prime}, r^{\prime}\right) d t^{\prime} d r^{\prime}+m_{4}\left(t^{\prime}, r^{\prime}\right) d \Omega_{2}^{2},
$$

where $m_{i}$ are functions of the radius $r^{\prime}$ and of the time $t^{\prime}$. A coordinate transformation $t=U_{1}\left(t^{\prime}, r^{\prime}\right), r=U_{2}\left(t^{\prime}, r^{\prime}\right)$ diagonalizes the metric (15.15) and introduces the areal radius $r$ such that $m_{4}\left(t^{\prime}, r^{\prime}\right)=r^{2}$, giving

$$
d s^{2}=-A(t, r) d t^{2}+B(t, r) d r^{2}+r^{2} d \Omega_{2}^{2},
$$

hence Eq. (15.16) can be taken as the most general torsion-free Lorentzian spherically symmetric metric without loss of generality. The field equations (15.10) and (15.11) for this metric reduce to

$$
f^{\prime}(R) R_{\mu \nu}-\frac{1}{2} f(R) g_{\mu \nu}+\mathcal{H}_{\mu \nu}=\kappa T_{\mu \nu}
$$




$$
g^{\sigma \tau} H_{\sigma \tau}=f^{\prime}(R) R-2 f(R)+\mathcal{H}=\kappa T
$$

where

$$
\begin{aligned}
\mathcal{H}_{\mu \nu} & =-f^{\prime \prime}(R)\left\{R_{, \mu \nu}-\Gamma_{\mu \nu}^{t} R_{, t}-\Gamma_{\mu \nu}^{r} R_{, r}-g_{\mu \nu}\left[\left(g_{, t}^{t t}+g^{t t} \ln \sqrt{-g}_{, t}\right) R_{, t}\right.\right. \\
& \left.\left.+\left(g^{r r}{ }_{, r}+g^{r r} \ln \sqrt{-g}_{, r}\right) R_{, r}+g^{t t} R_{, t t}+g^{r r} R_{, r r}\right]\right\} \\
& -f^{\prime \prime \prime}(R)\left[R_{, \mu} R_{, \nu}-g_{\mu \nu}\left(g^{t t} R_{, t}^{2}+g^{r r} R_{, r}^{2}\right)\right], \\
\mathcal{H} & =g^{\sigma \tau} \mathcal{H}_{\sigma \tau}=3 f^{\prime \prime}(R)\left[\left(g^{t t}{ }_{, t}+g^{t t} \ln \sqrt{-g}_{, t}\right) R_{, t}+\left(g^{r r}{ }_{, r}+g^{r r} \ln \sqrt{-g}_{, r}\right) R_{, r}\right. \\
& \left.+g^{t t} R_{, t t}+g^{r r} R_{, r r}\right]+3 f^{\prime \prime \prime}(R)\left[g^{t t} R_{, t}{ }^{2}+g^{r r} R_{, r}{ }^{2}\right] .
\end{aligned}
$$

In these equations the derivatives of $f(R)$ with respect to $R$ are distinct from the time and spatial derivatives of $R$, a feature which will allow us to better understand the dynamical behavior of the solutions.

\section{Solutions with constant Ricci scalar}

Let us assume that the Ricci scalar is constant, $R=R_{0}$. The field equations (15.17) and (15.18) with $\mathcal{H}_{\mu \nu}=0$ are

$$
\begin{gathered}
f_{0}^{\prime} R_{\mu \nu}-\frac{1}{2} f_{0} g_{\mu \nu}=\kappa T_{\mu \nu}^{(m)}, \\
f_{0}^{\prime} R_{0}-2 f_{0}=\kappa T^{(m)},
\end{gathered}
$$

where $f_{0} \equiv f\left(R_{0}\right)$ and $f_{0}^{\prime} \equiv f^{\prime}\left(R_{0}\right)$, and they can be rewritten as

$$
\begin{gathered}
R_{\mu \nu}+\lambda g_{\mu \nu}=q \kappa T_{\mu \nu}^{(m)} \\
R_{0}=q \kappa T-4 \lambda
\end{gathered}
$$

where $\lambda=-\frac{f_{0}}{2 f_{0}^{\prime}}$ and $q^{-1}=f_{0}^{\prime}$. We restrict to Lagrangians which reduce to the Hilbert-Einstein one as $R \rightarrow 0$ and do not contain a cosmological constant $\Lambda$,

$$
f(R) \simeq R \quad \text { as } R \rightarrow 0
$$

Then, the trace equation (15.22) indicates that in vacuo $\left(T_{\mu \nu}^{(m)}=0\right)$ one obtains a class of solutions with constant Ricci curvature $R=R_{0}$. In particular, there exist solutions with $R_{0}=0$.

Let us suppose now that the above Lagrangian density reduces to a constant for small curvature values, $\lim _{R \rightarrow 0} f=$ $\Lambda$. Interesting features emerge again from the trace equation: using Eq. (15.22) and the definition of $f(R)$, it is seen that zero curvature solutions do not exist in this case because

$$
\Psi^{\prime} R-2 \Psi-\Psi_{0} R-2 \Lambda=\kappa T^{(m)} .
$$

Contrary to GR, even in absence of matter there are no Ricci-flat solution of the field equations since the higher order derivatives give constant curvature solutions corresponding to a sort of effective cosmological constant. In fact, in GR, solutions with non-vanishing constant curvature occur only in the presence of matter because of the proportionality of the Ricci scalar to the trace of the matter energy-momentum tensor. A similar situation can be obtained in the presence of a cosmological constant $\Lambda$. The difference between GR and higher order gravity is that the Schwarzschildde Sitter solution is not necessarily generated by a $\Lambda$-term, while the effect of an "effective" cosmological constant can be achieved by the higher order derivative contributions, as discussed extensively in [51, 124, 419, 420, 461]. 
Let us consider now the problem of finding the general solution of Eqs. (15.21) and (15.22) for the spherically symmetric metric (15.16). The substitution of this metric into the $(t, r)$ component of (15.21) yields $\frac{\dot{B}(t, r)}{r B(t, r)}=0$, which means that $B(t, r)$ must be time-independent, $B(t, r)=b(r)$. On the other hand, the $(\theta, \theta)$ component of Eq. (15.21) yields $\frac{A^{\prime}(t, r)}{A(t, r)}=\zeta(r)$, where $\zeta(r)$ is a time-independent function and

$$
A(t, r)=\tilde{a}(t) \exp \left[\int \zeta(r) d r\right]=\tilde{a}(t) \frac{b}{r^{2}} \exp \left[\int d r \frac{\left[2-r^{2}(2 \lambda+2 q \kappa p)\right] b(r)}{r}\right]
$$

where $P$ is the pressure of a perfect fluid with stress-energy tensor

$$
T_{\mu \nu}^{(m)}=(P+\rho) u_{\mu} u_{\nu}+P g_{\mu \nu} .
$$

The function $A(t, r)$ is separable, $A(t, r)=\tilde{a}(t) a(r)$, and the line element (15.16) becomes

$$
d s^{2}=-\tilde{a}(t) a(r) d t^{2}+b(r) d r^{2}+r^{2} d \Omega_{2}^{2}
$$

and is rewritten as

$$
d s^{2}=-a(r) d \tilde{t}^{2}+b(r) d r^{2}+r^{2} d \Omega_{2}^{2}
$$

by redefining the time coordinate $t \rightarrow \tilde{t}$ as $d \tilde{t}=\sqrt{\tilde{a}(t)} d t$. From now on, the tilde will be dropped from this time coordinate.

To summarize, in a space-time with constant scalar curvature, any spherically symmetric background is necessarily static or, the Jebsen-Birkhoff theorem holds for $f(R)$-gravity with constant curvature (cf. Ref. [311]).

A remark is in order at this point. We have assumed a space-time with constant Ricci scalar and deduced conditions on the form of the gravitational potentials. The inverse problem can also be considered: whenever the gravitational potential $a(t, r)$ is a separable function and $b(t, r)$ is time-independent, using the definition of the Ricci scalar, it is $R=R_{0}=$ const. and at the same time the solutions of the field equations will be static if spherical symmetry is invoked. For a complete analysis of this problem, one should take into account the remaining field equations contained in (15.23) and (15.24) which have to be satisfied by taking into account the expression of the Ricci scalar (15.3). One must then solve the system

$$
\begin{aligned}
& R_{t t}+\lambda a(r)-q \kappa[\rho+P(1-a(r))]=0, \\
& R_{r r}-\lambda b(r)-q \kappa P b(r)=0, \\
& R_{0}-q \kappa(\rho-3 P)+4 \lambda=0, \\
& R(a(r), b(r))=R_{0},
\end{aligned}
$$

which takes the form

$$
\begin{aligned}
& \mathrm{e}^{\int \frac{2-r^{2}(2 \lambda+2 q \kappa P) b(r)}{r} d r}\left\{\left[r^{2}(2 \lambda+2 q \kappa P-2)\right]^{2} b(r)^{4}-4 b(r)^{3}\right. \\
& -3 r\left[r^{2}(2 \lambda+2 q \kappa P)-2\right] b^{\prime}(r) b(r)^{2} \\
& \left.+2 r\left[b^{\prime}(r)+r b^{\prime \prime}(r)\right] b(r)-2 r^{2} b^{\prime}(r)^{2}\right\}-4 r^{4} q \kappa(P+\rho) b(r)^{2}=0, \\
& \left\{3 r\left[r^{2}(2 \lambda+2 q \kappa P) b^{\prime}(r)-2\right]-8\right\} b(r)^{2}-4\left[r^{2}(2 \lambda+2 q \kappa P)-3\right] b(r)^{3} \\
& -\left[r^{2}(2 \lambda+2 q \kappa P)-2\right] b(r)^{4}+2 r^{2} b^{\prime}(r)^{2}-2 r b(r)\left[r b(r)^{\prime \prime}-3 b^{\prime}(r)\right]=0, \\
& \left\{r^{2}[4 \lambda+2 q \kappa(P-\rho)]-8\right\} b(r)^{3}-\left\{3 r\left[r^{2}(2 \lambda+2 q \kappa P)-2\right] b^{\prime}(r)-4\right\} b(r)^{2} \\
& +\left[r^{2}(2 \lambda+2 q \kappa P)-2\right] b(r)^{4}-2 r^{2} b^{\prime}(r)^{2}+2 r\left[r b^{\prime \prime}(r)-b^{\prime}(r)\right] b(r)=0
\end{aligned}
$$


where, using Eq. (15.3), the only unknown potential is now $b(r)$. A general solution is found for the particular equation of state $P=-\rho$ :

$$
d s^{2}=-\left(1+\frac{k_{1}}{r}+\frac{2 q \kappa \rho-2 \lambda}{6} r^{2}\right) d t^{2}+\frac{d r^{2}}{1+\frac{k_{1}}{r}+\frac{q \kappa \rho-\lambda}{3} r^{2}}+r^{2} d \Omega_{2}^{2} .
$$

In the case of constant Ricci scalar $R=R_{0}$, all $f(R)$ theories admit solutions with de Sitter-like behavior even in the weak-field limit. This is one of the reasons why dark energy can be replaced by $f(R)$-gravity [51, 106, 108, 109, 115, 154, 158, 437, 438.

Let us consider now $f(R)$-gravity with an analytic Lagrangian function $f(R)$, which we write as

$$
f(R)=\Lambda+\Psi_{0} R+\Psi(R),
$$

where $\Psi_{0}$ is a constant, $\Lambda$ plays the role of the cosmological constant, and $\Psi(R)$ is an analytic function of $R$ satisfying the condition

$$
\lim _{R \rightarrow 0} \frac{\Psi(R)}{R^{2}}=\Psi_{1}
$$

with $\Psi_{1}$ another constant. By neglecting the cosmological constant $\Lambda$ and setting $\Psi_{0}$ to zero, a new class of theories is obtained which, in the limit $R \rightarrow 0$, does not reproduce GR (Eq. (15.40) implies that $f(R) \sim R^{2}$ as $R \rightarrow 0$ ). In this case, analyzing the complete set of equations (15.21) and (15.22), one can observe that both zero and constant (but non-vanishing) curvature solutions are possible. In particular, if $R=R_{0}=0$ the field equations are solved for all forms of the gravitational potentials appearing in the spherically symmetric background (15.30), provided that the Bernoulli equation (15.5) relating these functions is satisfied for $R(r)=0$. The solutions are thus defined by the relation

$$
b(r)=\frac{\exp \left[-\int d r h(r)\right]}{K+4 \int \frac{d r a(r) \exp \left[-\int d r h(r)\right]}{r\left[a(r)+r a^{\prime}(r)\right]}} .
$$

Table I provides examples of $f(R)$ theories admitting solutions with constant but non-zero values of $R$ or null $R$. Each model admits Schwarzschild and Schwarzschild-de Sitter solutions, in addition to the class of solutions given by (15.41).

\section{Solutions with Ricci scalar depending on the radial coordinate}

Thus far, we have discussed the behavior of $f(R)$-gravity searching for spherically symmetric solutions with constant Ricci curvature. In GR this situation is well known to give rise to the Schwarzschild $(R=0)$ and the Schwarzschild-de Sitter $\left(R=R_{0} \neq 0\right)$ solutions. The search for spherically symmetric solutions can be generalized to $f(R)$-gravity by allowing the Ricci scalar to depend on the radial coordinate. This approach is interesting because, in general, higher order theories of gravity admit naturally this kind of solution, with several examples reported in the literature [119, 121, 124, 419, 420, 534]. In the following we approach the problem from a general point of view.

If we choose the Ricci scalar as a generic function $R(r)$ of the radial coordinate, it is possible to show that also in this case the solution of the field equations (15.17) and (15.18) is time-independent (if $T_{\mu \nu}^{(m)}=0$ ). In other words, the Jebsen-Birkhoff theorem holds. As in GR, it is crucial to study the off-diagonal $(t, r)$ component of (15.17) which, for a generic $f(R)$, reads

$$
\frac{d}{d r}\left[r^{2} f^{\prime}(R)\right] \dot{B}(t, r)=0
$$

and two possibilities can occur. First, one can choose $\dot{B}(t, r) \neq 0$, implying that $f^{\prime}(R) \sim 1 / r^{2}$. In this case the remaining field equation is not satisfied and there is incompatibility. The only possible solution is then given by $\dot{B}(t, r)=0$ and $B(t, r)=b(r)$. The $(\theta, \theta)$ equation is then used to determine that the potential $A(t, r)$ can be factorized with respect to time, the solutions are of the type (15.29), and the metric can be recast in the stationary spherically symmetric form (15.30) by a suitable coordinate transformation.

Even the more general radial-dependent case admits time-independent solutions. From the trace equation and the $(\theta, \theta)$ equation, the relation

$$
a(r)=b(r) \frac{\mathrm{e}^{\frac{2}{3} \int \frac{\left(R f^{\prime}-2 f\right) b(r)}{R^{\prime} f^{\prime \prime}} d r}}{r^{4} R^{\prime 2} f^{\prime \prime 2}}
$$




\begin{tabular}{|c|c|}
\hline$f(\mathbf{R})$ theory & Field equations \\
\hline$R$ & $R_{\mu \nu}=0$ \\
\hline$\xi_{1} R+\xi_{2} R^{n}$ & $\begin{cases}R_{\mu \nu}=0 & \text { with } R=0, \quad \xi_{1} \neq 0 \\
R_{\mu \nu}+\lambda g_{\mu \nu}=0 & \text { with } R=\left[\frac{\xi_{1}}{(n-2) \xi_{2}}\right]^{\frac{1}{n-1}}, \quad \xi_{1} \neq 0, \quad n \neq 2 \\
0=0 & \text { with } R=0, \quad \xi_{1}=0 \\
R_{\mu \nu}+\lambda g_{\mu \nu}=0 & \text { with } R=R_{0}, \quad \xi_{1}=0, n=2\end{cases}$ \\
\hline$\xi_{1} R+\xi_{2} R^{-m}$ & $R_{\mu \nu}+\lambda g_{\mu \nu}=0$ with $R=\left[-\frac{(m+2) \xi_{2}}{\xi_{1}}\right]^{\frac{1}{m+1}}$ \\
\hline$\xi_{1} R+\xi_{2} R^{n}+\xi_{3} R^{-m}$ & $\begin{array}{c}R_{\mu \nu}+\lambda g_{\mu \nu}=0, \text { with } R=R_{0} \text { so that } \\
\xi_{1} R_{0}^{m+1}+(2-n) \xi_{2} R_{0}^{n+m}+(m+2) \xi_{3}=0\end{array}$ \\
\hline$\frac{R}{\xi_{1}+R}$ & $\begin{cases}R_{\mu \nu}=0 & \text { with } R=0 \\
R_{\mu \nu}+\lambda g_{\mu \nu}=0 & \text { with } R=-\frac{\xi_{1}}{2}\end{cases}$ \\
\hline$\frac{1}{\xi_{1}+R}$ & $R_{\mu \nu}+\lambda g_{\mu \nu}=0$ with $R=-\frac{2 \xi_{1}}{3}$ \\
\hline
\end{tabular}

Table II. Examples of $f(R)$ models admitting constant or zero scalar curvature solutions. The powers $n$ and $m$ are integers while the $\xi_{i}$ are real constants.

(with $f^{\prime \prime}>0$ ) linking $a(r)$ and $b(r)$ can be obtained, in addition to [419, 420]

$$
b(r)=\frac{6\left[f^{\prime}\left(r R^{\prime} f^{\prime \prime}\right)^{\prime}-r R^{\prime 2} f^{\prime \prime 2}\right]}{r f\left(r R^{\prime} f^{\prime \prime}-4 f^{\prime}\right)+2 f^{\prime}\left[r R\left(f^{\prime}-r R^{\prime} f^{\prime \prime}\right)-3 R^{\prime} f^{\prime \prime}\right]} .
$$

Again, three more equations have to be satisfied in order to completely solve the system (respectively the $(t, t)$ and $(r, r)$ components of the field equations plus the Ricci scalar constraint), while the only unknown functions are $f(R)$ and the Ricci scalar $R(r)$.

If we now consider a fourth order theory described by $f(R)=R+\Phi(R)$ with $\Phi(R) \ll R$ we are able to satisfy the complete set of equations up to third order in $\Phi$. In particular, we can solve the full set of equations; the relations (15.43) and (15.44) will provide the general solution depending only on the forms of the functions $\Phi(R)$ and $R(r)$, i.e.,

$$
\begin{aligned}
& a(r)=b(r) \frac{\mathrm{e}^{-\frac{2}{3} \int \frac{\left[R+\left(2 \Phi-R \Phi^{\prime}\right)\right] b(r)}{R^{\prime} \Phi^{\prime \prime}} d r}}{r^{4} R^{\prime 2} \Phi^{\prime 2}}, \\
& b(r)=-\frac{3\left(r R^{\prime} \Phi^{\prime \prime}\right)_{, r}}{r R} .
\end{aligned}
$$

Once the radial dependence of the scalar curvature is obtained, Eq. (15.45) allows one to obtain the solution of the field equations and the gravitational potential related to the function $a(r)$. The physical relevance of this potential can be assessed by comparison with astrophysical data (e.g., [348]). 


\section{B. The Noether approach and spherical symmetry}

Exact spherically symmetric solutions with constant Ricci scalar in $f(R)$-gravity can be found using the Noether symmetry 124]. To begin, one needs to derive a point-like Lagrangian from the action of modified gravity by imposing spherical symmetry, while enforcing the constancy of the Ricci scalar by means of a suitable Lagrange multiplier. With the previous considerations in mind, a spherically symmetric space-time is described by the line element

$$
d s^{2}=-A(r) d t^{2}+B(r) d r^{2}+M(r) d \Omega_{2}^{2} .
$$

The Schwarzschild solution of GR is obtained if $M(r)=r^{2}$ and $A(r)=B^{-1}(r)=1-2 M / r$, with $r$ an areal radius. In the presence of spherical symmetry the action

$$
S=\int d r L\left(A, A^{\prime}, B, B^{\prime}, M, M^{\prime}, R, R^{\prime}\right)
$$

contains only a finite number of degrees of freedom, the Ricci scalar $R$ and the potentials $A, B$, and $M$ defining the configuration space. The point-like Lagrangian is obtained by writing the action as

$$
S=\int d^{4} x \sqrt{-g}[f(R)-\lambda(R-\bar{R})],
$$

where $\lambda$ is a Lagrangian multiplier and $\bar{R}$ is the Ricci scalar of the metric (15.47)

$$
\begin{aligned}
\bar{R} & =\frac{A^{\prime \prime}}{A B}+\frac{2 M^{\prime \prime}}{B M}+\frac{A^{\prime} M^{\prime}}{A B M}-\frac{A^{\prime 2}}{2 A^{2} B}-\frac{M^{\prime 2}}{2 B M^{2}}-\frac{A^{\prime} B^{\prime}}{2 A B^{2}}-\frac{B^{\prime} M^{\prime}}{B^{2} M}-\frac{2}{M} \\
& \equiv R^{*}+\frac{A^{\prime \prime}}{A B}+\frac{2 M^{\prime \prime}}{B M},
\end{aligned}
$$

where $R^{*}$ collects the terms containing first order derivatives. The Lagrange multiplier $\lambda$ is obtained by varying the action (15.49) with respect to $R$, which yields $\lambda=f_{R}(R)$. By expressing the metric determinant $g$ and $\bar{R}$ as functions of $A, B$, and $M, \mathrm{Eq}$. 115.49) gives

$$
\begin{aligned}
S & =\int d r \sqrt{A B} M\left[f-f_{R}\left(R-R^{*}-\frac{A^{\prime \prime}}{A B}-\frac{2 M^{\prime \prime}}{B M}\right)\right] \\
& =\int d r\left\{\sqrt{A B} M\left[f-f_{R}\left(R-R^{*}\right)\right]-\left(\frac{f_{R} M}{\sqrt{A B}}\right)^{\prime} A^{\prime}-\left(\frac{2 \sqrt{A}}{\sqrt{B}} f_{R}\right)^{\prime} M^{\prime}\right\} .
\end{aligned}
$$

The last two integrals differ by a total divergence which can be discarded, and the point-like Lagrangian becomes

$$
\begin{aligned}
L & =-\frac{\sqrt{A} f_{R}}{2 M \sqrt{B}}\left(M^{\prime}\right)^{2}-\frac{f_{R}}{\sqrt{A B}} A^{\prime} M^{\prime}-\frac{M f_{R R}}{\sqrt{A B}} A^{\prime} R^{\prime} \\
& -\frac{2 \sqrt{A} f_{R R}}{\sqrt{B}} R^{\prime} M^{\prime}-\sqrt{A B}\left[(2+M R) f_{R}-M f\right] .
\end{aligned}
$$

The canonical Lagrangian (15.52) is written in compact form using matrix notation as

$$
L=\underline{q}^{\prime} \hat{T} \underline{q}^{\prime}+V
$$

where $\underline{q}=(A, B, M, R)$ and $\underline{q}^{\prime}=\left(A^{\prime}, B^{\prime}, M^{\prime}, R^{\prime}\right)$ are the generalized Lagrangian coordinates and velocities. The index " $t$ " deñotes the transposed vector. The kinetic tensor is

$$
\hat{T}_{i j}=\frac{\partial^{2} L}{\partial q_{i}^{\prime} \partial q_{j}^{\prime}}
$$

and $V(q)$ is the potential energy depending only on the generalized coordinates. The Euler-Lagrange equations are

$$
\begin{aligned}
& \frac{d}{d r}\left(\nabla_{q^{\prime}} L\right)-\nabla_{q} L=2 \frac{d}{d r}\left(\hat{T} \underline{q}^{\prime}\right)-\nabla_{q} V-\underline{q}^{\prime t}\left(\nabla_{q} \hat{T}\right) \underline{q}^{\prime} \\
& =2 \hat{T} \underline{q}^{\prime \prime}+2\left(\underline{q}^{\prime} \cdot \nabla_{q} \hat{T}\right) \underline{q}^{\prime}-\nabla_{q} V-\underline{q}^{\prime t}\left(\nabla_{q} \hat{T}\right) \underline{q}^{\prime}=0 .
\end{aligned}
$$


$R$ obeys a constraint relating the Lagrangian coordinates. The Hessian determinant of $(\underline{15.52}),\left\|\frac{\partial^{2} L}{\partial q_{i}^{\prime} \partial q_{j}^{\prime}}\right\|$, vanishes because the point-like Lagrangian does not depend on the generalized velocity $B^{\prime}$. The metric component $B$ does not contribute to the dynamics, but its equation of motion has to be taken into account as a further constraint. The definition of the energy

$$
E_{L}=\underline{q}^{\prime} \cdot \nabla_{q^{\prime}} L-L
$$

coincides with the Euler-Lagrangian equation for the component $B$ of the generalized coordinate $\underline{q}$. Then, the Lagrangian (15.52) contains only three degrees of freedom and not four, as expected a priori. Now, since the equation of motion for $B$ does not contain the derivative $B^{\prime}$, it can be solved explicitly in term of $B$ as a function of the other Lagrangian coordinates:

$$
B=\frac{2 M^{2} f_{R R} A^{\prime} R^{\prime}+2 M f_{R} A^{\prime} M^{\prime}+4 A M f_{R R} M^{\prime} R^{\prime}+A f_{R} M^{\prime 2}}{2 A M\left[(2+M R) f_{R}-M f\right]} .
$$

By inserting Eq. (15.57) into the Lagrangian (15.52), we obtain a non-vanishing Hessian matrix removing the singular dynamics. The new Lagrangian read 29

$$
L^{*}=\sqrt{\mathbf{L}}
$$

with

$$
\begin{aligned}
\mathbf{L}= & \underline{q^{\prime}} \hat{\hat{\mathbf{L}}} \underline{q^{\prime}}=\frac{\left[(2+M R) f_{R}-f M\right]}{M} \\
& \cdot\left[2 M^{2} f_{R R} A^{\prime} R^{\prime}+2 M M^{\prime}\left(f_{R} A^{\prime}+2 A f_{R R} R^{\prime}\right)+A f_{R} M^{\prime 2}\right] .
\end{aligned}
$$

Since $\frac{\partial \mathbf{L}}{\partial r}=0, \mathbf{L}$ is canonical ( $\mathbf{L}$ is a quadratic form in the generalized velocities $A^{\prime}, M^{\prime}$ and $R^{\prime}$ and coincides with the Hamiltonian), hence $\mathbf{L}$ can be regarded as the new Lagrangian with three degrees of freedom. It is crucial that the Hessian determinant

$$
\left\|\frac{\partial^{2} \mathbf{L}}{\partial q_{i}^{\prime} \partial q_{j}^{\prime}}\right\|=3 A M\left[(2+M R) f_{R}-M f\right]^{3} f_{R} f_{R R}^{2}
$$

now does not vanish. It is assumed that $(2+M R) f_{R}-M f \neq 0$, otherwise the above definitions of $B$, and $\mathbf{L}$ (Eqs. (15.57) and (15.59) ) are meaningless. Moreover, it is assumed that $f_{R R} \neq 0$ allows for a wide class of fourth order gravity models. The GR case $f(R)=R$ is special: the GR point-like Lagrangian requires a further reduction of the number of degrees of freedom and the previous results cannot be applied directly. Eq. (15.52) yields

$$
L_{G R}=-\frac{\sqrt{A}}{2 M \sqrt{B}}\left(M^{\prime}\right)^{2}-\frac{1}{\sqrt{A B}} A^{\prime} M^{\prime}-2 \sqrt{A B},
$$

which, through the Euler-Lagrange equations, provides the standard GR equations for the Schwarzschild metric. The absence of the generalized velocity $B^{\prime}$ in Eq. (15.61) is evident. Again, the Hessian determinant vanishes. Nevertheless, considering again the constraint (15.57) for $B$, it is possible to obtain a Lagrangian with non-vanishing Hessian. In particular, it is

$$
\begin{aligned}
& B_{G R}=\frac{\left(M^{\prime}\right)^{2}}{4 M}+\frac{A^{\prime} M^{\prime}}{2 A}, \\
& L_{G R}^{*}=\sqrt{\mathbf{L}_{G R}}=\sqrt{\frac{M^{\prime}\left(2 M A^{\prime}+A M^{\prime}\right)}{M}},
\end{aligned}
$$

\footnotetext{
${ }^{29}$ Lowering the dimension of the configuration space through the substitution 15.57 leaves the dynamics unaffected because $B$ is nondynamical. In fact, if Eq. (15.57) is introduced into the set of dynamical equations (15.52), these coincide with the equation derived from 15.59.
} 
and the Hessian determinant is

$$
\left\|\frac{\partial^{2} \mathbf{L}_{G R}}{\partial q_{i}^{\prime} \partial q_{j}^{\prime}}\right\|=-1,
$$

a non-vanishing sub-matrix of the $f(R)$ Hessian matrix.

The Euler-Lagrange equations derived from Eqs. (15.62) and (15.63) yield the vacuum solutions of GR

$$
A=k_{4}-\frac{k_{3}}{r+k_{1}}, \quad B=\frac{k_{2} k_{4}}{A}, \quad M=k_{2}\left(r+k_{1}\right)^{2} .
$$

In particular, the standard form of the Schwarzschild solution is recovered for $k_{1}=0, k_{2}=1, k_{3}=2 G M / c^{2}$, and $k_{4}=1$.

Table III summarizes the field equations associated with the point-like Lagrangians and their relation with respect to the ones of the standard approach.

\begin{tabular}{|ccc|}
\hline Field equations approach & & Point-like Lagrangian approach \\
$\delta \int d^{4} x \sqrt{-g} f=0$ & $\leftrightarrows$ & $\delta \int d r L=0$ \\
$\downarrow$ & $\downarrow$ & $\downarrow$ \\
$H_{\mu \nu}=\partial_{\rho}\left[\frac{\partial(\sqrt{-g} f)}{\partial \rho g^{\mu \nu}}\right]-\frac{\partial(\sqrt{-g} f)}{\partial g^{\mu \nu}}=0$ & & $\frac{d}{d r}\left(\nabla_{q^{\prime}} L\right)-\nabla_{q} L=0$ \\
$H=g^{\mu \nu} H_{\mu \nu}=0$ & $\leftrightarrows$ & \\
$\downarrow$ & & \\
$H_{00}=0$ & $\leftrightarrows$ & $\frac{d}{d r}\left(\frac{\partial L}{\partial A^{\prime}}\right)-\frac{\partial L}{\partial A}=0$ \\
$H_{r r}=0$ & $\leftrightarrows$ & $\frac{d}{d r}\left(\frac{\partial L}{\partial B^{\prime}}\right)-\frac{\partial L}{\partial B} \propto E_{L}=0$ \\
$H_{\theta \theta}=\csc ^{2} \theta H_{\phi \phi}=0$ & $\leftrightarrows$ & $\frac{d}{d r}\left(\frac{\partial L}{\partial M^{\prime}}\right)-\frac{\partial L}{\partial M}=0$ \\
$B_{q^{\prime}} L-L$ \\
$A^{-1} H_{r r}-2 M^{-1} \csc ^{2} \theta H_{\phi \phi}=0$ & $\leftrightarrows$ a combination of the above equations \\
\hline
\end{tabular}

Table III. The field equations approach and the point-like Lagrangian approach differ because spherical symmetry can be imposed either in the field equations after standard variation with respect to the metric, or directly into the Lagrangian, which then becomes point-like. The energy $E_{L}$ corresponds to the $(0,0)$ component of $H_{\mu \nu}$. The absence of $B^{\prime}$ in the Lagrangian implies the proportionality between the constraint equation for $B$ and the energy function $E_{L}$. As a consequence, there are only three independent equations and three unknown functions. The $(\theta, \theta)$ component corresponds to the field equation for $M$. $H_{\mu \nu}$ is given in Appendix C.

In spherical symmetry, the areal radius $r$ plays the role of an affine parameter. Then, the configuration space is $\mathcal{Q}=(A, M, R)$ and the tangent space is $\mathcal{T} \mathcal{Q}=\left(A, A^{\prime}, M, M^{\prime}, R, R^{\prime}\right)$. According to the Noether theorem, the existence of a symmetry for the dynamics described by the Lagrangian (15.59) implies the existence of a conserved quantity. The Lie differentiation of Eq. (15.59) yield 30

$$
\mathcal{L}_{\mathbf{X}} \mathbf{L}=\underline{\alpha} \cdot \nabla_{q} \mathbf{L}+\underline{\alpha}^{\prime} \cdot \nabla_{q^{\prime}} \mathbf{L}=\underline{q}^{\prime t}\left[\underline{\alpha} \cdot \nabla_{q} \hat{\mathbf{L}}+2\left(\nabla_{q} \alpha\right)^{t} \hat{\mathbf{L}}\right] \underline{q}^{\prime} .
$$

\footnotetext{
${ }^{30}$ From now on, $\underline{q}$ denotes the vector $(A, M, R)$.
} 
This Lie derivative vanishes if the functions $\underline{\alpha}$ satisfy the system

$$
\alpha_{i} \frac{\partial \hat{\mathbf{L}}_{k m}}{\partial q_{i}}+2 \frac{\partial \alpha_{i}}{\partial q_{k}} \hat{\mathbf{L}}_{i m}=0
$$

Solving the system (15.67) means finding the functions $\alpha_{i}$ which identify the Noether vector. However the system (15.67) depends implicitly on the form of the function $f(R)$ and, by solving it, one obtains the forms of the function $f(R)$ which are compatible with spherical symmetry. Alternatively, by choosing the form of $f(R)$, (15.67) can be solved explicitly. As an example, the system (15.67) is satisfied if we choose

$$
f(R)=f_{0} R^{s}, \quad \underline{\alpha}=\left(\alpha_{1}, \alpha_{2}, \alpha_{3}\right)=((3-2 s) k A,-k M, k R)
$$

with $s$ a real number, $k$ an integration constant, and $f_{0}$ a dimensional coupling constant 31 This means that for $f(R)=R^{s}$ there exist at least one Noether symmetry and a related conserved quantity

$$
\begin{aligned}
\Sigma_{0} & =\underline{\alpha} \cdot \nabla_{q^{\prime}} \mathbf{L} \\
& =2 s k M R^{2 s-3}[2 s+(s-1) M R]\left[(s-2) R A^{\prime}-\left(2 s^{2}-3 s+1\right) A R^{\prime}\right] .
\end{aligned}
$$

A physical interpretation of $\Sigma_{0}$ is possible in GR. In this case, obtained for $s=1$, the above procedure must be applied to the Lagrangian (15.63), obtaining the solution

$$
\underline{\alpha}_{G R}=(-k A, k M) .
$$

The functions $A$ and $M$ provide the Schwarzschild solution (15.65), and then the constant of motion takes the form

$$
\Sigma_{0}=\frac{2 G M}{c^{2}}
$$

in standard units; the conserved quantity is the Schwarzschild radius (or the mass of the gravitating system).

Another solution can be found for constant Ricci scalar $R=R_{0}$ [419], for which the field equations reduce to

$$
R_{\mu \nu}+k_{0} g_{\mu \nu}=0
$$

where $k_{0}=-\frac{1}{2} f\left(R_{0}\right) / f_{R}\left(R_{0}\right)$. The general solution is

$$
A(r)=\frac{1}{B(r)}=1+\frac{k_{0}}{r}+\frac{R_{0}}{12} r^{2}, \quad M=r^{2}
$$

which includes the special case

$$
A(r)=\frac{1}{B(r)}=1+\frac{k_{0}}{r}, \quad M=r^{2}, \quad R=0 .
$$

The solution (15.73) is the well known Schwarzschild-de Sitter metric.

In the general case $f(R)=R^{s}$, the Lagrangian (15.59) becomes

$$
\begin{aligned}
\mathbf{L} & =\frac{s R^{2 s-3}[2 s+(s-1) M R]}{M} \\
& \cdot\left[2(s-1) M^{2} A^{\prime} R^{\prime}+2 M R M^{\prime} A^{\prime}+4(s-1) A M M^{\prime} R^{\prime}+A R M^{\prime 2}\right]
\end{aligned}
$$

and the expression (15.57) of $B$ is

$$
B=\frac{s\left[2(s-1) M^{2} A^{\prime} R^{\prime}+2 M R M^{\prime} A^{\prime}+4(s-1) A M M^{\prime} R^{\prime}+A R M^{\prime 2}\right]}{2 A M R[2 s+(s-1) M R]},
$$

\footnotetext{
31 The dimensions are given by $R^{1-s}$ in term of the Ricci scalar. For simplicity, $f_{0}$ is set to unity in the following.
} 
and GR is recovered for $s=1$.

Using the constant of motion (15.69), one can solve for A, obtaining

$$
A=R^{\frac{2 s^{2}-3 s+1}{s-2}}\left\{k_{1}+\Sigma_{0} \int \frac{R^{\frac{4 s^{2}-9 s+5}{2-s}} d r}{2 k s(s-2) M[2 s+(s-1) M R]}\right\}
$$

for $s \neq 2$, where $k_{1}$ is an integration constant. For $s=2$ one finds

$$
A=-\frac{\Sigma_{0}}{12 k r^{2}\left(4+r^{2} R\right) R R^{\prime}} .
$$

These relations allow one to find general solutions of the field equations regulating the function $R(r)$. For example, the solution corresponding to

$$
s=5 / 4, \quad M=r^{2}, \quad R=5 r^{-2},
$$

is the spherically symmetric metric given by

$$
d s^{2}=-\frac{1}{\sqrt{5}}\left(k_{2}+k_{1} r\right) d t^{2}+\frac{1}{2}\left(\frac{1}{1+\frac{k_{2}}{k_{1} r}}\right) d r^{2}+r^{2} d \Omega_{2}^{2}
$$

with $k_{2}=\frac{32 \Sigma_{0}}{225 k}$. The value of $s$ for this solution is ruled out by Solar System experiments [54, 178, 179].

To summarize, the Noether symmetry approach provides a general method to find spherically symmetric exact solutions of ETGs, and of metric $f(R)$-gravity in particular. The procedure consists of $i$ ) obtaining the point-like $f(R)$ Lagrangian with spherical symmetry; ii) writing the Euler-Lagrange equations; iii) searching for a Noether vector field; and $i v$ ) reducing the dynamics and then integrating the equations of motion using the constants of motion. Vice-versa, this approach also allows one to select families of $f(R)$ models with spherical symmetry. The method can be generalized. If a symmetry exists, the Noether approach allows transformations of variables to cyclic ones, reducing the dynamics to obtain exact solutions. For example, since we know that $f(R)=R^{s}$-gravity admits a constant of motion, the Noether symmetry suggests the coordinate transformation

$$
\mathbf{L}\left(A, M, R, A^{\prime}, M^{\prime}, R^{\prime}\right) \rightarrow \widetilde{\mathbf{L}}\left(\widetilde{M}, \widetilde{R}, \widetilde{A}^{\prime}, \widetilde{M}^{\prime}, \bar{R}^{\prime}\right),
$$

for the Lagrangian (15.59), where the conserved quantity corresponds to the cyclic variable $\widetilde{A}$. In the presence of multiple symmetries one can find multiple cyclic variables. If three Noether symmetries exist, the Lagrangian $\mathbf{L}$ can be mapped into a Lagrangian with three cyclic coordinates $\widetilde{A}=\widetilde{A}(\underline{q}), \widetilde{M}=\widetilde{M}(\underline{q})$ and $\widetilde{R}=\widetilde{R}(\underline{q})$ which are functions of the old generalized coordinates. These new functions must satisfy the system

$$
\begin{aligned}
& (3-2 s) A \frac{\partial \widetilde{A}}{\partial A}-M \frac{\partial \widetilde{A}}{\partial M}+R \frac{\partial \widetilde{A}}{\partial R}=1, \\
& (3-2 s) A \frac{\partial \widetilde{q}_{i}}{\partial A}-M \frac{\partial \widetilde{q}_{i}}{\partial M}+R \frac{\partial \widetilde{q}_{i}}{\partial R}=0,
\end{aligned}
$$

with $i=2,3$ (we have set $k=1$ ). A solution of (15.83) for $s \neq 3 / 2$ is

$$
\begin{aligned}
& \widetilde{A}=\frac{\ln A}{(3-2 s)}+F_{A}\left(A^{\frac{\eta_{A}}{3-2 s}} M^{\eta_{A}} A^{\frac{\xi_{A}}{2 s-3}} M^{\xi_{A}}\right), \\
& \widetilde{q}_{i}=F_{i}\left(A^{\frac{\eta_{i}}{3-2 s}} M^{\eta_{i}}, A^{\frac{\xi_{i}}{2 s-3}} M^{\xi_{i}}\right)
\end{aligned}
$$

while, if $s=3 / 2$,

$$
\begin{aligned}
\widetilde{A} & =-\ln M+F_{A}(A) G_{A}(M R), \\
\widetilde{q}_{i} & =F_{i}(A) G_{i}(M R),
\end{aligned}
$$

where $F_{A}, F_{i}, G_{A}$ and $G_{i}$ are arbitrary functions and $\eta_{A}, \eta_{i}, \xi_{A}$, and $\xi_{i}$ are integration constants.

The considerations of this section make it clear once again that the Jebsen-Birkhoff theorem does not hold, in general, for metric $f(R)$-gravity. 


\section{From spherical to axial symmetry in $f(R)$-gravity}

Here, we want to seek for a general metod to find out axially symmetric solutions by performing a complex coordinate transformation on the spherical metrics. Since the discovery of the Kerr solution [352], many attempts have been made to find a physically reasonable interior matter distribution that may be considered as its source. For a review on these approaches see [365, 401]. Though much progress has been made, results have been generally disappointing. As far as we know, nobody has obtained a physically satisfactory interior solution. This seems surprising given the success of matching internal spherically symmetric solutions to the Schwarzschild metric. The problem is that the loss of a degree of symmetry makes the derivation of analytic results much more difficult. Severe restrictions are placed on the interior metric by maintaining that it must be joined smoothly to the external axially symmetric metric. Further restrictions are placed on the interior solutions to ensure that they correspond to physical objects.

Furthermore since the axially symmetric metric has no radiation field associated with it, its source should be also non-radiating. This places even further constraints on the structure of the interior solution [533]. Given the strenuous nature of these limiting conditions, it is not surprising to learn that no satisfactory solution to the problem of finding sources for the Kerr metric has been obtained. In general, the failure is due to internal structures whose physical properties are unknown. This shortcoming makes hard to find consistent boundary conditions.

Newman and Janis showed that it is possible to obtain an axially symmetric solution (like the Kerr metric) by making an elementary complex transformation on the Schwarzschild solution [429]. This same method has been used to obtain a new stationary and axially symmetric solution known as the Kerr-Newman metric [430]. The KerrNewman space-time is associated to the exterior geometry of a rotating massive and charged black-hole. For a review on the Newman-Janis method to obtain both the Kerr and Kerr-Newman metrics see [218].

By means of very elegant mathematical arguments, Schiffer et al. [507] have given a rigorous proof to show how the Kerr metric can be derived starting from a complex transformation on the Schwarzschild solution. We will not go into the details of this demonstration, but point out that the proof relies on two main assumptions. The first is that the metric belongs to the same algebraic class of the Kerr-Newman solution, namely the Kerr-Schild class 202]. The second assumption is that the metric corresponds to an empty solution of the Einstein field equations. In the case we are going to study, these assumptions are not considered and hence the proof in [507] is not applicable. It is clear, by the generation of the Kerr-Newman metric, that all the components of the stress-energy tensor need to be non-zero for the Newman-Janis method to be successful. In fact, Gürses and Gürsey, in 1975 [306], showed that if a metric can be written in the Kerr-Schild form, then a complex transformation "is allowed in General Relativity." Here, we will show that such a transformation can be extended to $f(R)$-gravity.

We now show how it is possible to obtain an axially symmetric solution starting from a spherically symmetric one, using a method developed by Newman and Janis in GR [429, 430]. This method can be applied to a static spherically symmetric metric adopted as a "seed" metric. In principle, the procedure could be applied whenever Noether symmetries are present. We apply this procedure to solutions of metric $f(R)$-gravity [149].

In general, the approach is not straightforward since, if $f(R) \neq R$, the field equations are of fourth order and the relevant existence theorems and boundary conditions are different from those of GR. However, the existence of a Noether symmetry guarantees the consistency of the chosen $f(R)$ model with the field equations.

Let us consider a spherically symmetric metric of the suitable form

$$
d s^{2}=-\mathrm{e}^{2 \phi(r)} d t^{2}+\mathrm{e}^{2 \lambda(r)} d r^{2}+r^{2} d \Omega_{2}^{2} .
$$

Following Newman and Janis, the line element (15.88) can be written in Eddington-Finkelstein coordinates $(u, r, \theta, \varphi)$, i.e., the $g_{r r}$ component is eliminated by the coordinate change and a cross term is introduced [544]. We set $d t=$ $d u+F(r) d r$ with $F(r)= \pm \mathrm{e}^{\lambda(r)-\phi(r)}$, turning the line element (15.88) into

$$
d s^{2}=-\mathrm{e}^{2 \phi(r)} d u^{2} \mp 2 \mathrm{e}^{\lambda(r)-\phi(r)} d u d r+r^{2} d \Omega_{2}^{2} .
$$

The surface $u=$ costant is a light cone with vertex in $r=0$. The inverse metric tensor in null coordinates is

$$
g^{\mu \nu}=\left(\begin{array}{cccc}
0 & \mp \mathrm{e}^{-\lambda(r)-\phi(r)} & 0 & 0 \\
\mp \mathrm{e}^{-\lambda(r)-\phi(r)} & \mathrm{e}^{-2 \lambda(r)} & 0 & 0 \\
0 & 0 & \frac{1}{r^{2}} & 0 \\
0 & 0 & 0 & \frac{1}{r^{2} \sin ^{2} \theta}
\end{array}\right) .
$$


The matrix (15.90) can be written in terms of a null tetrad as

$$
g^{\mu \nu}=-l^{\mu} n^{\nu}-l^{\nu} n^{\mu}+m^{\mu} \bar{m}^{\nu}+m^{\nu} \bar{m}^{\mu},
$$

where $l^{\mu}, n^{\mu}, m^{\mu}$, and $\bar{m}^{\mu}$ satisfy the conditions

$$
l_{\mu} l^{\mu}=m_{\mu} m^{\mu}=n_{\mu} n^{\mu}=0, \quad l_{\mu} n^{\mu}=-m_{\mu} \bar{m}^{\mu}=-1, \quad l_{\mu} m^{\mu}=n_{\mu} m^{\mu}=0,
$$

and where an overbar denotes complex conjugation. At any spatial point, the tetrad can be chosen in the following manner: $l^{\mu}$ is the outward null vector tangent to the light cone, $n^{\mu}$ is the inward null vector pointing toward the origin, and $m^{\mu}$ and $\bar{m}^{\mu}$ are vectors tangent to the two-dimensional sphere defined by constant $r$ and $u$. For the space-time (15.90), the null tetrad can be chosen as

$$
\begin{aligned}
l^{\mu} & =\delta_{1}^{\mu}, \\
n^{\mu} & =-\frac{1}{2} \mathrm{e}^{-2 \lambda(r)} \delta_{1}^{\mu}+\mathrm{e}^{-\lambda(r)-\phi(r)} \delta_{0}^{\mu}, \\
m^{\mu} & =\frac{1}{\sqrt{2} r}\left(\delta_{2}^{\mu}+\frac{i}{\sin \theta} \delta_{3}^{\mu}\right), \\
\bar{m}^{\mu} & =\frac{1}{\sqrt{2} r}\left(\delta_{2}^{\mu}-\frac{i}{\sin \theta} \delta_{3}^{\mu}\right) .
\end{aligned}
$$

Now we extend the set of coordinates $x^{\mu}=(u, r, \theta, \phi)$ by promoting the real radius to the role of a complex variable. The null tetrad then becomes 32

$$
\begin{aligned}
l^{\mu} & =\delta_{1}^{\mu}, \\
n^{\mu} & =-\frac{1}{2} \mathrm{e}^{-2 \lambda(r, \bar{r})} \delta_{1}^{\mu}+\mathrm{e}^{-\lambda(r, \bar{r})-\phi(r, \bar{r})} \delta_{0}^{\mu}, \\
m^{\mu} & =\frac{1}{\sqrt{2} \bar{r}}\left(\delta_{2}^{\mu}+\frac{i}{\sin \theta} \delta_{3}^{\mu}\right), \\
\bar{m}^{\mu} & =\frac{1}{\sqrt{2} r}\left(\delta_{2}^{\mu}-\frac{i}{\sin \theta} \delta_{3}^{\mu}\right) .
\end{aligned}
$$

A new metric is obtained by performing the complex coordinate transformation

$$
x^{\mu} \longrightarrow \tilde{x}^{\mu}=x^{\mu}+i y^{\mu}\left(x^{\sigma}\right),
$$

where $y^{\mu}\left(x^{\sigma}\right)$ are analytic functions of the real coordinates $x^{\sigma}$, and simultaneously letting the null tetrad $Z_{a}^{\mu} \equiv$ $\left(l^{\mu}, n^{\mu}, m^{\mu}, \bar{m}^{\mu}\right)$ with $a=1,2,3,4$, undergo the transformation

$$
Z_{a}^{\mu} \longrightarrow \tilde{Z}_{a}^{\mu}\left(\tilde{x}^{\sigma}, \overline{\tilde{x}}^{\sigma}\right)=Z_{a}^{\rho} \frac{\partial \tilde{x}^{\mu}}{\partial x^{\rho}} .
$$

Obviously, one has to recover the old tetrad and metric as soon as $\tilde{x}^{\sigma}=\overline{\tilde{x}}^{\sigma}$. In summary, the effect of the "tilde transformation" (15.101) is to generate a new metric whose components are real functions of complex variables,

$$
g_{\mu \nu} \longrightarrow \tilde{g}_{\mu \nu}: \tilde{\mathbf{x}} \times \tilde{\mathbf{x}} \mapsto \mathbb{R}
$$

with

$$
\left.\tilde{Z}_{a}^{\mu}\left(\tilde{x}^{\sigma}, \overline{\tilde{x}}^{\sigma}\right)\right|_{\mathbf{x}=\tilde{\mathbf{x}}}=Z_{a}^{\mu}\left(x^{\sigma}\right)
$$

${ }^{32}$ A certain degree of arbitrariness is present in the complexification of the functions $\lambda$ and $\phi$. Obviously, we must recover the metric 15.90 as soon as $r=\bar{r}$. 
For our purposes, we can make the choice

$$
\tilde{x}^{\mu}=x^{\mu}+i a\left(\delta_{1}^{\mu}-\delta_{0}^{\mu}\right) \cos \theta \longrightarrow\left\{\begin{array}{l}
\tilde{u}=u+i a \cos \theta, \\
\tilde{r}=r-i a \cos \theta, \\
\tilde{\theta}=\theta, \\
\tilde{\phi}=\phi,
\end{array}\right.
$$

where $a$ is a constant and, with the choice $\tilde{r}=\overline{\tilde{r}}$, the null vectors (15.97) reduce to

$$
\begin{aligned}
\tilde{l}^{\mu} & =\delta_{1}^{\mu}, \\
\tilde{n}^{\mu} & =-\frac{1}{2} \mathrm{e}^{-2 \lambda(\tilde{r}, \theta)} \delta_{1}^{\mu}+\mathrm{e}^{-\lambda(\tilde{r}, \theta)-\phi(\tilde{r}, \theta)} \delta_{0}^{\mu}, \\
\tilde{m}^{\mu} & =\frac{1}{\sqrt{2}(\tilde{r}-i a \cos \theta)}\left[i a\left(\delta_{0}^{\mu}-\delta_{1}^{\mu}\right) \sin \theta+\delta_{2}^{\mu}+\frac{i}{\sin \theta} \delta_{3}^{\mu}\right], \\
\overline{\tilde{m}}^{\mu} & =\frac{1}{\sqrt{2}(\tilde{r}+i a \cos \theta)}\left[-i a\left(\delta_{0}^{\mu}-\delta_{1}^{\mu}\right) \sin \theta+\delta_{2}^{\mu}-\frac{i}{\sin \theta} \delta_{3}^{\mu}\right] .
\end{aligned}
$$

A new metric is recovered from the transformed null tetrad via Eq. (15.91). With the null vectors (15.106) - (15.109) and the transformation (15.105), the new metric in coordinates $\tilde{x}^{\mu}=(\tilde{u}, \tilde{r}, \theta, \phi)$ is

$$
\tilde{g}^{\mu \nu}=\left(\begin{array}{cccc}
\frac{a^{2} \sin ^{2} \theta}{\Sigma^{2}} & -\mathrm{e}^{-\lambda(\tilde{r}, \theta)-\phi(\tilde{r}, \theta)}-\frac{a^{2} \sin ^{2} \theta}{\Sigma^{2}} & 0 & \frac{a}{\Sigma^{2}} \\
\cdot & \mathrm{e}^{-2 \lambda(\tilde{r}, \theta)}+\frac{a^{2} \sin ^{2} \theta}{\Sigma^{2}} & 0 & -\frac{a}{\Sigma^{2}} \\
\cdot & \frac{1}{\Sigma^{2}} & 0 \\
\cdot & \cdot & & \frac{1}{\Sigma^{2} \sin ^{2} \theta}
\end{array}\right)
$$

where $\Sigma=\sqrt{\tilde{r}^{2}+a^{2} \cos ^{2} \theta}$. The covariant metric $\tilde{g}_{\mu \nu}$ is

$$
\left(\begin{array}{ccc}
-\mathrm{e}^{2 \phi(\tilde{r}, \theta)}-\mathrm{e}^{\lambda(\tilde{r}, \theta)-\phi(\tilde{r}, \theta)} & 0 & -a \mathrm{e}^{\phi(\tilde{r}, \theta)}\left[\mathrm{e}^{\lambda(\tilde{r}, \theta)}-\mathrm{e}^{\phi(\tilde{r}, \theta)}\right] \sin ^{2} \theta \\
\cdot & 0 & a \mathrm{e}^{\phi(\tilde{r}, \theta)-\lambda(\tilde{r}, \theta)} \sin ^{2} \theta \\
\cdot & \Sigma^{2} & 0 \\
\cdot & \cdot & {\left[\Sigma^{2}+a^{2} \sin ^{2} \theta \mathrm{e}^{\phi(\tilde{r}, \theta)}\left(2 \mathrm{e}^{\lambda(\tilde{r}, \theta)}-\mathrm{e}^{\phi(\tilde{r}, \theta)}\right)\right] \sin ^{2} \theta}
\end{array}\right)
$$

The dots in the matrix denote symmetric entries satisfying the metric symmetry $g^{\mu \nu}=g^{\nu \mu}$. The form of this metric gives the general result of the Newman-Janis algorithm starting from any spherical seed metric.

The metric (15.111) can be simplified by a further gauge transformation so that the only off-diagonal component is $g_{\phi t}$. This procedure makes it easier to compare with the standard Boyer-Lindquist form of the Kerr metric [544] and to interpret physical properties such as frame dragging. The coordinates $\tilde{u}$ and $\varphi$ can be redefined in such a way that the metric in the new coordinates has the properties described above. Explicitly, using

$$
d \tilde{u}=d t+g(\tilde{r}) d \tilde{r}
$$

and

$$
d \phi=d \phi+h(\tilde{r}) d \tilde{r},
$$


where

$$
\begin{aligned}
& g(\tilde{r})=-\frac{\mathrm{e}^{\lambda(\tilde{r}, \theta)}\left(\Sigma^{2}+a^{2} \sin ^{2} \theta \mathrm{e}^{\lambda(\tilde{r}, \theta)+\phi(\tilde{r}, \theta)}\right)}{\mathrm{e}^{\phi(\tilde{r}, \theta)}\left(\Sigma^{2}+a^{2} \sin ^{2} \theta \mathrm{e}^{2 \lambda(\tilde{r}, \theta)}\right)}, \\
& h(\tilde{r})=-\frac{a \mathrm{e}^{2 \lambda(\tilde{r}, \theta)}}{\Sigma^{2}+a^{2} \sin ^{2} \theta \mathrm{e}^{2 \lambda(\tilde{r}, \theta)}},
\end{aligned}
$$

after algebraic manipulations the covariant metric (15.111) becomes, in coordinates $(t, \tilde{r}, \theta, \phi)$,

$$
\left(\begin{array}{cccc}
\mathrm{e}^{2 \phi} & 0 & 0 & a \mathrm{e}^{\phi}\left[\mathrm{e}^{\lambda}-\mathrm{e}^{\phi}\right] \sin ^{2} \theta \\
\cdot & -\frac{\Sigma^{2}}{\left(\Sigma^{2} \mathrm{e}^{-2 \lambda}+a^{2} \sin ^{2} \theta\right)} & 0 & 0 \\
\cdot & \cdot & -\Sigma^{2} & 0 \\
\cdot & \cdot & \cdot & -\left[\Sigma^{2}+a^{2} \sin ^{2} \theta \mathrm{e}^{\phi}\left(2 \mathrm{e}^{\lambda}-\mathrm{e}^{\phi}\right)\right] \sin ^{2} \theta
\end{array}\right),
$$

where $\phi=\phi(\tilde{r}, \theta)$ and $\lambda=\lambda(\tilde{r}, \theta)$. This metric represents the complete family of metrics that may be obtained by performing the Newman-Janis algorithm on any static spherically symmetric seed metric, written in Boyer-Lindquist coordinates. These transformations require that $\Sigma^{2}+a^{2} \sin ^{2} \theta \mathrm{e}^{2 \lambda(\tilde{r}, \theta)} \neq 0$, where $\mathrm{e}^{2 \lambda(\tilde{r}, \theta)}>0$. We now show that this approach can be used to derive axially symmetric solutions also in $f(R)$-gravity.

Begin with the spherically symmetric solution (15.80), that we rewrite as

$$
d s^{2}=-(\alpha+\beta r) d t^{2}+\frac{\beta r}{2(\alpha+\beta r)} d r^{2}+r^{2} d \Omega_{2}^{2},
$$

where $\alpha$ is a combination of $\Sigma_{0}, k$, and $\beta=k_{1}$ obtained with the Noether approach. The metric tensor in EddingtonFinkelstein coordinates $(u, r, \theta, \phi)$ of the form (15.90) is

$$
g^{\mu \nu}=\left(\begin{array}{ccc}
0-\sqrt{\frac{2}{\beta r}} & 0 & 0 \\
\cdot 2+\frac{2 \alpha}{\beta r} & 0 & 0 \\
\cdot & \frac{1}{r^{2}} & 0 \\
\cdot & \cdot & \frac{1}{r^{2} \sin ^{2} \theta}
\end{array}\right) .
$$

The complex null tetrad (15.97)-(15.100) is now

$$
\begin{aligned}
l^{\mu} & =\delta_{1}^{\mu}, \\
n^{\mu} & =-\left[1+\frac{\alpha}{\beta}\left(\frac{1}{\bar{r}}+\frac{1}{r}\right)\right] \delta_{1}^{\mu}+\sqrt{\frac{2}{\beta}} \frac{1}{(\bar{r} r)^{1 / 4}} \delta_{0}^{\mu}, \\
m^{\mu} & =\frac{1}{\sqrt{2} \bar{r}}\left(\delta_{2}^{\mu}+\frac{i}{\sin \theta} \delta_{3}^{\mu}\right) .
\end{aligned}
$$

By computing the complex coordinate transformation (15.105), the null tetrad becomes

$$
\begin{aligned}
\tilde{l}^{\mu} & =\delta_{1}^{\mu}, \\
\tilde{n}^{\mu} & =-\left[1+\frac{\alpha}{\beta} \frac{\operatorname{Re}\{\tilde{r}\}}{\Sigma^{2}}\right] \delta_{1}^{\mu}+\sqrt{\frac{2}{\beta \Sigma}} \delta_{0}^{\mu}, \\
\tilde{m}^{\mu} & =\frac{1}{\sqrt{2}(\tilde{r}+i a \cos \theta)}\left[i a\left(\delta_{0}^{\mu}-\delta_{1}^{\mu}\right) \sin \theta+\delta_{2}^{\mu}+\frac{i}{\sin \theta} \delta_{3}^{\mu}\right] .
\end{aligned}
$$


By performing the same procedure as in GR, one derives an axially symmetric metric of the form (15.116) but starting from the spherically symmetric covariant metric (15.117),

$$
g_{\mu \nu}=\left(\begin{array}{cccc}
-\frac{r(\alpha+\beta r)+a^{2} \beta \cos ^{2} \theta}{\Sigma} & 0 & 0 & \frac{\sigma_{1}}{2 \Sigma} \\
\cdot & \frac{\beta \Sigma^{2}}{2 \alpha r+\beta\left(a^{2}+r^{2}+\Sigma^{2}\right)} & 0 & 0 \\
\cdot & \cdot & \Sigma^{2} & 0 \\
\cdot & \cdot & \cdot\left(\Sigma^{2}-\frac{\sigma_{2}}{\Sigma}\right) \sin ^{2} \theta
\end{array}\right) .
$$

where

$$
\begin{aligned}
& \sigma_{1}=a\left(2 \alpha r+2 \beta \Sigma^{2}-\sqrt{2 \beta} \Sigma^{3 / 2}\right) \sin ^{2} \theta, \\
& \sigma_{2}=a^{2}\left(\alpha r+\beta \Sigma^{2}-\sqrt{2 \beta} \Sigma^{3 / 2}\right) \sin ^{2} \theta .
\end{aligned}
$$

By setting $a=0$, the metric (15.117) is immediately recovered.

The method illustrated by this example is general and can be extended to any spherically symmetric solution of $f(R)$-gravity [149]. The key point of the method is to find out a suitable complex transformation which, from a physical viewpoint, corresponds to the fact that we are reducing the number of independent Killing vectors. From a mathematical viewpoint, it is useful since allows to overcome the problem of a direct search for axially symmetric solutions that, in $f(R)$-gravity, could be extremely cumbersome due to the fourth-order field equations. However, other generating techniques exist and all of them should be explored in order to completely extend solutions of GR to $f(R)$-gravity. They can be more general and solid than the Newman-Janis approach. A good source for references and basic features of generating techniques is reference [533]. In particular, the paper by Talbot [539], considering the Newman-Penrose approach to twisting degenerate metrics, provides some theoretical justification for the scope and limitations of adopting the "complex trick". As reported in Chap. 21 of [533], several techniques can be pursued to achieve axially symmetric solutions which can be particularly useful to deal with non-empty space-times ( in particular when perfect fluids are the sources of the field equations) and to deal, in general, with problems related to Einstein-Maxwell field equations. We have to stress that the utility of generating techniques is not simply to obtain a new metric, but a metric of a new space-time with specific properties as the transformation properties of the energy-momentum tensor and Killing vectors. In its original application, the Newman-Janis procedure transforms an Einstein-Maxwell solution (Reissner-Nordstrom) into another Einstein-Maxwell solution (Kerr-Newman). As a particular case (setting the charge to zero) it is possible to achieve the transformation between two vacuum solutions (Schwarzschild and Kerr). Also in case of $f(R)$-gravity, new features emerge by adopting such a technique. In particular, it is worth studying how certain features of spherically simmetric metrics, derived in $f(R)$-gravity, result transformed in the new axially symmetric solutions. For example, considering the $f(R)$ spherically symmetric solution studied here, the Ricci scalar evolves as $r^{-2}$ and then the asymptotic flatness is recovered. Let us consider now the axially symmetric metric achieved by the Newman-Janis method. The parameter $a \neq 0$ indicates that the spherical symmetry $(a=0)$ is broken. Such a parameter can be immediately related to the presence of an axis of symmetry and then to the fact that a Killing vector, related to the angle $\theta$, has been lost. To conclude, we can say that once the vacuum case is discussed, more general spherical metrics can be transformed in new axially symmetric metrics adopting more general techniques [533].

\section{THE POST- NEWTONIAN LIMIT}

\section{A. The weak field and small velocities approximations}

At shorter (Galactic and Solar System) spatial scales, ETGs exhibit gravitational potentials with non-Newtonian corrections [196, 525, 586, 587]. This feature was discovered long ago [535], and recent interest arises from the possibility of explaining the flatness of the rotation curves of spiral galaxies without huge amounts of dark matter. In particular, the rotation curves of a wide sample of low surface brightness spiral galaxies can be fitted successfully by the corrected potentials [119, 121], and this possibility may be extended to other types of galaxies [280]. 
One could attempt to investigate other issues such as, for example, the Pioneer anomaly [26, 27] with the same approach [70]. A systematic analysis of ETGs at scales much smaller than the Hubble radius is then necessary. In this section we discuss the weak-field limit of $f(R)$-gravity without specifying the form of the theory and highlighting the differences and similarities with the post-Newtonian and post-Minkowskian limits of GR. The literature contains conflicting claims 6. 36, 54, 114, 120, 131 133, 161, 178, 179, 214, 230, 328, 335, 419, 422, 426, 427, 453, 494, 513, 522, 526, 594], and clarity is needed in order to compare theory and experiment . Based on the scalar-tensor representation of $f(R)$-gravity with $33 \omega=0$, Chiba [168] originally suggested that all $f(R)$ theories are ruled out because of the experimental limit $|\omega|>40,000$ [1] ]. While this constraint can be circumvented by giving the scalar degree of freedom a large mass and, therefore, making it short-ranged, it seemed that its range must be at least comparable with the Hubble radius in order to affect the dynamics of the Universe. This conclusion is incorrect because of the so called chameleon mechanism and the weak-field limit is subtler than it appears [133]. Solar System experiments constrain the PPN parameter $\gamma$, and then the Brans-Dicke parameter $\omega$, only when the range of the scalar degree of freedom is comparable to, or larger than the spatial scale of the experiment (for the Cassini experiment providing the lower bound on $\omega$ [71], this is the size of the Solar System) [580]. If the mass of this scalar is large, the parameter $\gamma$ is close to unity. However, the scalar does not have a fixed range but, rather, its mass depends on the energy density of its environment, so that this field becomes short-ranged and is undetectable at small (Solar System) scales, while its range is cosmological at cosmological densities $\rho$. This chameleon mechanism is widely used in quintessence models of dark energy [354, 355].

Again, a direct approach independent of the equivalence of metric $f(R)$ and scalar-tensor gravity is more convincing, and was first formulated for the prototype model $f(R)=R-\mu^{4} / R$ (which, at the time, was already ruled out by the Dolgov-Kawasaki instability [221, 259]) in [244]. The weak-field limit for a general function $f(R)$ was presented in 170,454$]$.

Weak-field experiments such as light bending, the perihelion shift of planets, and frame-dragging experiments are valuable tests of ETGs. There are sufficient theoretical predictions to state that certain higher order theories of gravity can be compatible with Newtonian and post-Newtonian experiments [15, 69, 114, 168, 170, 177, 178, 205, 244, 260, 392, 425, 443, 522], as can be shown also by using the scalar-tensor representation of $f(R)$-gravity.

In the following we outline a formalism addressing the weak-field and small velocity limit of fourth order gravity allowing a Jordan frame systematic discussion of these limits and of spherically symmetric solutions [136]. This discussion is valid also for general higher order theories containing the invariants $R_{\mu \nu} R^{\mu \nu}$ or $R_{\alpha \beta \mu \nu} R^{\alpha \beta \mu \nu}[138$. The non-Newtonian corrections in the gravitational potentials could potentially explain known astrophysical phenomenology.

A preliminary step consists of concentrating on the vacuum case and then building a Newtonian and post-Newtonian formalism for $f(R)$ theories in the presence of matter. It is possible to estimate the post-Newtonian parameter $\gamma$ by considering second order solutions for the metric components in vacuo. For completeness, we treat the problem also by imposing the harmonic gauge on the field equations.

\section{B. General remarks on Newtonian and post-Newtonian approximations}

Certain general features must be taken into account when performing the Newtonian and post-Newtonian limits of a relativistic theory of gravity. For a virialized system of particles of total mass $\bar{M}$ interacting gravitationally, the kinetic energy $\bar{M}(\bar{v})^{2} / 2$ is approximately of the same order of magnitude as the potential energy $U=G \bar{M}{ }^{2} / \bar{r}$, where $\bar{r}$ and $\bar{v}$ are typical average values of the separations and velocities of these particles. As a consequence, it is

$$
\overline{v^{2}} \sim \frac{G \bar{M}}{\bar{r}}
$$

(for instance, in Newtonian mechanics, a test particle in a circular orbit of radius $r$ about a spherically distributed mass $M$ has velocity $v$ given by $\left.v^{2}=G M / r\right)$. The post-Newtonian approximation can be described as a method for obtaining the motion of the system beyond first (i.e., Newtonian) order with respect to the quantities $G \bar{M} / \bar{r}$ and $(\bar{v})^{2}$, which are assumed to be small with respect to the square of the speed of light $c^{2}$ (this approximation is an expansion in inverse powers of $c$ ).

Typical values of the Newtonian gravitational potential $U$ in the Solar System are nowhere larger than $10^{-5}($ the quantity $U / c^{2}$ is dimensionless). Planetary velocities satisfy the condition $(\bar{v})^{2} \lesssim U$, whil 34 the matter pressure $P$

33 Although some caution about the equivalence with scalar-tensor theory in the Newtonian and the GR limits is necessary [260, 348], the equivalence holds in the post-Newtonian limit [262].

${ }^{34}$ Here the velocity $v$ is expressed in units of $c$. 
inside the Sun and the planets is much smaller than the energy density $\rho U$ of matter $35 P / \rho \lesssim U$. Furthermore, one must consider that other forms of energy in the Solar System (stresses, radiation, thermal energy, etc.) have small magnitudes and their specific energy density $\Pi$ (the ratio of the energy density to the rest mass density) is related to $U$ by $\Pi \lesssim U$ ( $\Pi$ is approximately $10^{-5}$ in the Sun and $10^{-9}$ in the Earth [586, 587]). One can consider that these quantities, as functions of velocity, give only second order contributions,

$$
U \sim v^{2} \sim \frac{P}{\rho} \sim \Pi \sim \mathcal{O}(2),
$$

therefore the velocity $v$ contributes to order $\mathcal{O}(1), U^{2}$ to order $\mathcal{O}(4), U v$ to order $\mathcal{O}(3), U \Pi$ is of order $\mathcal{O}(4)$, etc. In this approximation, one has

$$
\frac{\partial}{\partial x^{0}} \sim \mathbf{v} \cdot \nabla
$$

and

$$
\frac{\left|\partial / \partial x^{0}\right|}{|\nabla|} \sim \mathcal{O}(1) .
$$

Massive test particles move along geodesics given by the equation

$$
\frac{d^{2} x^{\mu}}{d s^{2}}+\Gamma_{\sigma \tau}^{\mu} \frac{d x^{\sigma}}{d s} \frac{d x^{\tau}}{d s}=0
$$

or

$$
\frac{d^{2} x^{i}}{d x^{02}}=-\Gamma_{00}^{i}-2 \Gamma_{0 m}^{i} \frac{d x^{m}}{d x^{0}}-\Gamma_{m n}^{i} \frac{d x^{m}}{d x^{0}} \frac{d x^{n}}{d x^{0}}+\left(\Gamma_{00}^{0}+2 \Gamma_{0 m}^{0} \frac{d x^{m}}{d x^{0}}+2 \Gamma_{m n}^{0} \frac{d x^{m}}{d x^{0}} \frac{d x^{n}}{d x^{0}}\right) \frac{d x^{i}}{d x^{0}} .
$$

In the small velocity approximation and retaining only first order terms in the deviations of $g_{\mu \nu}$ from the Minkowski metric $\eta_{\mu \nu}$, the particle equations of motion reduce to the Newtonian result

$$
\frac{d^{2} x^{i}}{d\left(x^{0}\right)^{2}} \simeq-\Gamma_{00}^{i} \simeq-\frac{1}{2} \frac{\partial g_{00}}{\partial x^{i}}
$$

The quantity $\left(1+g_{00}\right)$ is of order $G \bar{M} / \bar{r}$, hence the Newtonian approximation gives $\frac{d^{2} x^{i}}{d\left(x^{0}\right)^{2}}$ to order $G \bar{M} / \bar{r}^{2}$, that is, to order $(\bar{v})^{2} / r$. As a consequence, the post-Newtonian approximation requires one to compute $\frac{d^{2} x^{i}}{d\left(x^{0}\right)^{2}}$ to order $(\bar{v})^{4} / \bar{r}$. According to the Equivalence Principle and the local flatness of the space-time manifold, it is possible to find a coordinate system in which the metric tensor is nearly equal to $\eta_{\mu \nu}$, with the correction expanded in powers of $G \bar{M} / \bar{r} \sim(\bar{v})^{2}$

$$
\begin{aligned}
& g_{00}\left(x^{0}, \mathbf{x}\right)=-1+g_{00}^{(2)}\left(x^{0}, \mathbf{x}\right)+g_{00}^{(4)}\left(x^{0}, \mathbf{x}\right)+\mathcal{O}(6) \\
& g_{0 i}\left(x^{0}, \mathbf{x}\right)=g_{0 i}^{(3)}\left(x^{0}, \mathbf{x}\right)+\mathcal{O}(5) \\
& g_{i j}\left(x^{0}, \mathbf{x}\right)=\delta_{i j}+g_{i j}^{(2)}\left(x^{0}, \mathbf{x}\right)+\mathcal{O}(4)
\end{aligned}
$$

and with inverse metric

$$
\begin{aligned}
& g^{00}\left(x^{0}, \mathbf{x}\right)=-1+g^{(2) 00}\left(x^{0}, \mathbf{x}\right)+g^{(4) 00}\left(x^{0}, \mathbf{x}\right)+\mathcal{O}(6) \\
& g^{0 i}\left(x^{0}, \mathbf{x}\right)=g^{(3) 0 i}\left(x^{0}, \mathbf{x}\right)+\mathcal{O}(5) \\
& g^{i j}\left(x^{0}, \mathbf{x}\right)=\delta^{i j}+g^{(2) i j}\left(x^{0}, \mathbf{x}\right)+\mathcal{O}(4)
\end{aligned}
$$

\footnotetext{
35 Typical values of $P / \rho$ are $10^{-5}$ in the Sun and $10^{-10}$ in the Earth [586, 587].
} 
When computing the connection coefficients $\Gamma_{\alpha \beta}^{\mu}$, one must take into account the fact that the space and time scales in the gravitational system are set by $\bar{r}$ and $\bar{r} / \bar{v}$, respectively, hence spatial and time derivatives are of order

$$
\frac{\partial}{\partial x^{i}} \sim \frac{1}{\bar{r}}, \quad \frac{\partial}{\partial x^{0}} \sim \frac{\bar{v}}{\bar{r}} .
$$

Using the approximations (16.8)-(16.13), we have

$$
\begin{aligned}
& \Gamma^{(3)}{ }_{00}^{0}=\frac{1}{2} g_{00}^{(2), 0}, \\
& \Gamma_{00}^{(2)}{ }_{00}^{i}=\frac{1}{2} g_{00}^{(2), i}, \\
& \Gamma^{(2)}{ }_{j k}^{i}=\frac{1}{2}\left(g_{j k}^{(2)}{ }_{j k}^{i}-g_{j, k}^{(2)}-g_{k, j}^{(2)}\right), \\
& \Gamma_{i j}^{(3)}=\frac{1}{2}\left(g^{(3)}{ }_{i, j}^{0}+g^{(3)}{ }_{j, i}^{0}-g^{(3)}{ }_{i j}^{, 0}\right), \\
& \Gamma_{0 j}^{(3)}{ }_{0 j}^{i}=\frac{1}{2}\left(g^{(3)}{ }_{0 j}^{i}-g_{0, j}^{(3)}{ }_{0, j}-g_{j, 0}^{(2)}{ }_{j,}\right), \\
& \Gamma_{0 i}^{(4)}=\frac{1}{2}\left(g_{0, i}^{(4)}+g^{0}(2) 00 g_{00, i}^{(2)}\right), \\
& \Gamma_{00}^{(4)}{ }_{00}^{i}=\frac{1}{2}\left(g_{00}^{(4)}{ }_{00}^{i}+g^{(2) i m} g_{00, m}^{(2)}-2 g_{0,0}^{(3)^{i}}\right) \text {, } \\
& \Gamma^{(2)}{ }_{0 i}^{0}=\frac{1}{2} g^{(2)}{ }_{0, i}^{0} .
\end{aligned}
$$

The only non-vanishing components of the Ricci tensor are

$$
\begin{aligned}
& R_{00}^{(2)}=\frac{1}{2} \nabla^{2} g_{00}^{(2)}, \\
& R_{00}^{(4)}=\frac{1}{2} \nabla^{2} g_{00}^{(4)}-\frac{1}{2} g_{, m}^{(2)}{ }_{, m}^{m n} g_{00, n}^{(2)}-\frac{1}{2} g^{(2)^{m n}} g_{00, m n}^{(2)} \\
& +\frac{1}{2} g_{m, 00}^{(2)^{m}}-\frac{1}{4} g_{0}^{(2)}{ }_{0}^{0, m} g_{00, m}^{(2)}-\frac{1}{4} g_{m}^{(2)}{ }_{m}^{m, n} g_{00, n}^{(2)}-g_{0, m 0}^{(3)}{ }_{0,}^{m} \text {, } \\
& R_{0 i}^{(3)}=\frac{1}{2} \nabla^{2} g_{0 i}^{(3)}-\frac{1}{2} g_{i, m 0}^{(2)}-\frac{1}{2} g_{0, m i}^{(3)}+\frac{1}{2} g_{m, 0 i}^{(2)}{ }_{m,}^{m}, \\
& R_{i j}^{(2)}=\frac{1}{2} \nabla^{2} g_{i j}^{(2)}-\frac{1}{2} g_{i, m j}^{(2)}-\frac{1}{2} g_{j, m i}^{(2)}-\frac{1}{2} g_{0, i j}^{(2)} \\
& +\frac{1}{2} g_{m, i j}^{(2)^{m}} \text {. }
\end{aligned}
$$

In the harmonic gauge $g^{\rho \sigma} \Gamma_{\rho \sigma}^{\mu}=0$ these expressions become

$$
\begin{aligned}
R_{00}^{(2)} & =\frac{1}{2} \nabla^{2} g_{00}^{(2)}, \\
R_{00}^{(4)} & =\frac{1}{2} \nabla^{2} g_{00}^{(4)}-\frac{1}{2} g^{(2)}{ }_{00, m n}^{(2)}-\frac{1}{2} g_{0,00}^{(2)_{0}^{0}}-\frac{1}{2}\left|\vec{\nabla} \vec{\nabla}_{\eta} g_{00}^{(2)}\right|^{2}, \\
R_{0 i}^{(3)} & =\frac{1}{2} \nabla^{2} g_{0 i}^{(3)}, \\
R_{i j}^{(2)} & =\frac{1}{2} \nabla^{2} g_{i j}^{(2)},
\end{aligned}
$$


where $\nabla^{2}$ and $\vec{\nabla}$ denote the Laplacian and the gradient in flat space, respectively. The Ricci scalar in this gauge is

$$
\begin{aligned}
R^{(2)} & =R_{0}^{(2)}{ }_{0}^{0}-R_{m}^{(2)}{ }_{m}^{m}=\frac{1}{2} \nabla^{2} g_{0}^{(2)}-\frac{1}{2} \nabla^{2} g_{m}^{(2)}, \\
R^{(4)} & =R_{0}^{(4)}{ }_{0}^{0}+g^{(2)^{00}} R_{00}^{(2)}+g^{(2)^{m n}} R_{m n}^{(2)} \\
& =\frac{1}{2} \nabla^{2} g^{(4)^{0}}-\frac{1}{2} g_{0,0}^{(2)}-\frac{1}{2} g^{(2)^{m n}}\left(g_{0, m n}^{(2)_{0}^{0}}-\nabla^{2} g_{m n}^{(2)}\right)-\frac{1}{2}\left|\vec{\nabla} g^{(2)_{0}^{0}}\right|^{2} \\
& +\frac{1}{2} g^{(2)^{00}} \nabla^{2} g_{00}^{(2)} .
\end{aligned}
$$

The inverse of the metric tensor is defined by $g^{\alpha \rho} g_{\rho \beta}=\delta_{\beta}^{\alpha}$. The relations between terms of order higher than first are

$$
\begin{aligned}
& g^{(2) 00}\left(x^{0}, \mathbf{x}\right)=-g_{00}^{(2)}\left(x^{0}, \mathbf{x}\right), \\
& g^{(4) 00}\left(x^{0}, \mathbf{x}\right)=g_{00}^{(2)}\left(x^{0}, \mathbf{x}\right)^{2}-g_{00}^{(4)}\left(x^{0}, \mathbf{x}\right), \\
& g^{(3) 0 i}\left(x^{0}, \mathbf{x}\right)=g_{0 i}^{(3)}\left(x^{0}, \mathbf{x}\right), \\
& g^{(2) i j}\left(x^{0}, \mathbf{x}\right)=-g_{i j}^{(2)}\left(x^{0}, \mathbf{x}\right) .
\end{aligned}
$$

Finally, the Lagrangian of a particle in the gravitational field is proportional to the invariant distance $d s$,

$$
\begin{aligned}
L & =\left(g_{\rho \sigma} \frac{d x^{\rho}}{d x^{0}} \frac{d x^{\sigma}}{d x^{0}}\right)^{1 / 2}=\left(g_{00}+2 g_{0 m} v^{m}+g_{m n} v^{m} v^{n}\right)^{1 / 2} \\
& =\left(1+g_{00}^{(2)}+g_{00}^{(4)}+2 g_{0 m}^{(3)} v^{m}-\mathbf{v}^{2}+g_{m n}^{(2)} v^{m} v^{n}\right)^{1 / 2} .
\end{aligned}
$$

To second order, this expression reduces to the Newtonian test particle Lagrangian $L_{\text {Newt }}=\left(1+g_{00}^{(2)}-\mathbf{v}^{2}\right)^{1 / 2}$, where $v^{2}=\frac{d x^{m}}{d x^{0}} \frac{d x_{m}}{d x^{0}}$. Post-Newtonian physics involves terms of order higher than fourth in the Lagrangian.

Since the odd-order perturbation terms $\mathcal{O}(1)$ or $\mathcal{O}(3)$ contain odd powers of the velocity $\mathbf{v}$ or of time derivatives, they are related to the dissipation or absorption of energy by the system. Mass-energy conservation prevents losses of energy and mass and, as a consequence, in the Newtonian limit it prevents terms of order $\mathcal{O}(1)$ and $\mathcal{O}(3)$ to appear in the Lagrangian. When contributions of order higher than $\mathcal{O}(4)$ are included, different theories produce different predictions. For example, due to the conservation of post-Newtonian energy, GR forbids terms of order $\mathcal{O}(5)$, while terms of order $\mathcal{O}(7)$ can appear and are related to the energy lost due to gravitational radiation.

\section{Corrections to the Newtonian potential}

Let us apply the formalism of the previous section to the weak-field and small velocity regime of metric $f(R)$-gravity. Assuming spherical symmetry in vacuum and specifying with " $t$ " the time index 36 , we have

$$
\begin{aligned}
& g_{t t}(t, r)=A(t, r) \simeq-1+g_{t t}^{(2)}(t, r)+g_{t t}^{(4)}(t, r), \\
& g_{r r}(t, r)=B(t, r) \simeq 1+g_{r r}^{(2)}(t, r), \\
& g_{\theta \theta}(t, r)=r^{2} \\
& g_{\phi \phi}(t, r)=r^{2} \sin ^{2} \theta
\end{aligned}
$$

${ }^{36}$ However, as mentioned in previous sections, Solar System tests are analyzed using isotropic coordinates [14 4 . The transformations from isotropic to Schwarzschild coordinates are non-trivial, as shown in Sec. V D. In the following discussion, we will take into account this issue and consider consistent orders of approximation for the metric. 
while the inverse metric components are

$$
\begin{aligned}
& g^{t t}=A(t, r)^{-1} \simeq-1-g_{t t}^{(2)}+g_{t t}^{(2)}-g_{t t}^{(4)}, \\
& g^{r r}=B(t, r)^{-1} \simeq 1-g_{r r}^{(2)},
\end{aligned}
$$

the metric determinant is

$$
g \simeq r^{4} \sin ^{2} \theta\left[-1+\left(g_{r r}^{(2)}-g_{t t}^{(2)}\right)+\left(g_{t t}^{(2)} g_{r r}^{(2)}-g_{t t}^{(4)}\right)\right],
$$

and the Christoffel symbols are given by

$$
\begin{aligned}
& \Gamma_{t t}^{(3)}=\frac{g_{t t, t}^{(2)}}{2}, \quad \Gamma_{t t}^{(2)^{r}}+\Gamma_{t t}^{(4)}=\frac{g_{t t, r}^{(2)}}{2}+\frac{g_{r r}^{(2)} g_{t t, r}^{(2)}+g_{t t, r}^{(4)}}{2}, \\
& \Gamma_{t r}^{(3)}=-\frac{g_{r r, t}^{(2)}}{2}, \quad \Gamma_{t r}^{(2)}+\Gamma_{t r}^{t}=\frac{g_{t t, r}^{(2)}}{2}+\frac{g_{t t, r}^{(4)}-g_{t t}^{(2)} g_{t t, r}^{(2)}}{2}, \\
& \Gamma_{r r}^{(3)}=-\frac{g_{r r, t}^{(2)}}{2}, \quad \Gamma_{r r}^{(2)^{r}}+\Gamma_{r r}^{(4)^{r}}=-\frac{g_{r r, r}^{(2)}}{2}-\frac{g_{r r}^{(2)} g_{r r, r}^{(2)}}{2}, \\
& \Gamma_{\phi \phi}^{r}=\sin ^{2} \theta \Gamma_{\theta \theta}^{r}, \quad \Gamma_{\theta \theta}^{(0)^{r}}+\Gamma^{(2)^{r}}{ }_{\theta \theta}^{r}+\Gamma^{(4)^{r}}=-r-r g_{r r}^{(2)}-r g_{r r}^{(2)}{ }^{2} .
\end{aligned}
$$

The only non-vanishing components of the Ricci tensor are

$$
\begin{aligned}
& R_{t t}=R_{t t}^{(2)}+R_{t t}^{(4)}, \\
& R_{t r}=R_{t r}^{(3)}, \\
& R_{r r}=R_{r r}^{(2)}, \\
& R_{\theta \theta}=R_{\theta \theta}^{(2)}, \\
& R_{\phi \phi}=R_{\theta \theta}^{(2)} \sin ^{2} \theta,
\end{aligned}
$$

where

$$
\begin{aligned}
R_{t t}^{(2)} & =\frac{r g_{t t, r r}^{(2)}+2 g_{t t, r}^{(2)}}{2 r} \\
R_{t t}^{(4)} & =\left[-r\left(g_{t t, r}^{(2)}\right)^{2}+4 g_{t t, r}^{(4)}+r g_{t t, r}^{(2)} g_{r r, r}^{(2)}+2 g_{r r}^{(2)}\left(2 g_{t t, r}^{(2)}+r g_{t t, r r}^{(2)}\right)\right. \\
& \left.+2 r g_{t t, r r}^{(4)}+2 r g_{r r, t t}^{(2)}\right](4 r)^{-1} \\
R_{t r}^{(3)} & =-\frac{g_{r r, t}^{(2)}}{r} \\
R_{r r}^{(2)} & =-\frac{r g_{t t, r r}^{(2)}+2 g_{r r, r}^{(2)}}{2 r} \\
R_{\theta \theta}^{(2)} & =-\frac{2 g_{r r}^{(2)}+r\left(g_{t t, r}^{(2)}+g_{r r, r}^{(2)}\right)}{2}
\end{aligned}
$$

and the post-Newtonian Ricci scalar is

$$
R \simeq R^{(2)}+R^{(4)}
$$


with

$$
\begin{aligned}
R^{(2)} & =\frac{2 g_{r r}^{(2)}+r\left(2 g_{t t, r}^{(2)}+2 g_{r r, r}^{(2)}+r g_{t t, r r}^{(2)}\right)}{r^{2}}, \\
R^{(4)} & =\left\{4 g_{r r}^{(2)}{ }^{2}+2 r g_{r r}^{(2)}\left(2 g_{t t, r}^{(2)}+4 g_{r r, r}^{(2)}+r g_{t t, r r}^{(2)}\right)\right. \\
& \left.+r\left[-r g_{t t, r}^{(2)}{ }^{2}+4 g_{t t, r}^{(4)}+r g_{t t, r}^{(2)} g_{r r, r}^{(2)}-2 g_{t t}^{(2)}\left(2 g_{t t, r}^{(2)}+r g_{t t, r r}^{(2)}\right)+2 r g_{t t, r r}^{(4)}+2 r g_{r r, t t}^{(2)}\right]\right\} \\
& \cdot\left(2 r^{2}\right)^{-1} .
\end{aligned}
$$

We restrict the discussion to functions $f(R)$ which are analytic at the value $R_{0}$ of the Ricci curvature 37

$$
f(R)=\sum_{n=0}^{+\infty} \frac{f^{n}\left(R_{0}\right)}{n !}\left(R-R_{0}\right)^{n}=f_{0}+f_{1} R+f_{2} R^{2}+f_{3} R^{3}+\ldots,
$$

where in the last equality we have assumed $R_{0}=0$. The coefficient $f_{1}$ must be positive in order to have a positive gravitational coupling. The post-Newtonian formalism consists of using this expansion in the field equations, which are expanded to orders $\mathcal{O}(0), \mathcal{O}(2)$, and $\mathcal{O}(4)$, and then solved.

The substitution of Eq. (16.63) in the vacuum field equations and their expansion to orders $\mathcal{O}(0), \mathcal{O}(2)$, and $\mathcal{O}(4)$ yield

$$
\begin{aligned}
& \mathcal{H}_{\mu \nu}^{(0)}=0, \quad \mathcal{H}^{(0)}=0, \\
& \mathcal{H}_{\mu \nu}^{(2)}=0, \quad \mathcal{H}^{(2)}=0, \\
& \mathcal{H}_{\mu \nu}^{(3)}=0, \quad \mathcal{H}^{(3)}=0, \\
& \mathcal{H}_{\mu \nu}^{(4)}=0, \quad \mathcal{H}^{(4)}=0,
\end{aligned}
$$

where the notations of Eqs. (15.19) and (15.19) have been adopted. The order $\mathcal{O}(0)$ approximation gives

$$
f_{0}=0,
$$

a trivial consequence of the assumption (16.8)-(16.10) that space-time is asymptotically Minkowskian. If the Lagrangian is expandable around the zero value of the Ricci scalar $\left(R_{0}=0\right)$, the cosmological constant must vanish in vacuo.

If we now consider the second order approximation, the system (16.64)-(16.67) in vacuo yields

$$
\begin{aligned}
& f_{1} r R^{(2)}-2 f_{1} g_{t t, r}^{(2)}+8 f_{2} R_{, r}^{(2)}-f_{1} r g_{t t, r r}^{(2)}+4 f_{2} r R^{(2)}=0 \\
& f_{1} r R^{(2)}-2 f_{1} g_{r r, r}^{(2)}+8 f_{2} R_{, r}^{(2)}-f_{1} r g_{t t, r r}^{(2)}=0 \\
& 2 f_{1} g_{r r}^{(2)}-r\left(f_{1} r R^{(2)}-f_{1} g_{t t, r}^{(2)}-f_{1} g_{r r, r}^{(2)}+4 f_{2} R_{, r}^{(2)}+4 f_{2} r R_{, r r}^{(2)}\right)=0 \\
& f_{1} r R^{(2)}+6 f_{2}\left(2 R_{, r}^{(2)}+r R_{, r r}^{(2)}\right)=0 \\
& 2 g_{r r}^{(2)}+r\left(2 g_{t t, r}^{(2)}-r R^{(2)}+2 g_{r r, r}^{(2)}+r g_{t t, r r}^{(2)}\right)=0 .
\end{aligned}
$$

\footnotetext{
37 At least, the non-analytic part of $f(R)$ (if it is allowed to exist) must go to zero faster than $R^{3}$ as $R \rightarrow 0$.
} 
The trace equation (16.72), in particular, is a differential equation for the Ricci scalar which allows one to solve the system (16.69)-16.73) to order $\mathcal{O}(2)$ as

$$
\begin{aligned}
& g_{t t}^{(2)}=-\delta_{0}+\frac{\delta_{1}(t)}{3 \xi r} \mathrm{e}^{-r \sqrt{-\xi}}-\frac{\delta_{2}(t)}{6(-\xi)^{3 / 2} r} \mathrm{e}^{r \sqrt{-\xi}} \\
& g_{r r}^{(2)}=-\frac{\delta_{1}(t)(r \sqrt{-\xi}+1)}{3 \xi r} \mathrm{e}^{-r \sqrt{-\xi}}+\frac{\delta_{2}(t)(\xi r+\sqrt{-\xi})}{6 \xi^{2} r} \mathrm{e}^{r \sqrt{-\xi}} \\
& R^{(2)}=\frac{\delta_{1}(t)}{r} \mathrm{e}^{-r \sqrt{-\xi}}-\frac{\delta_{2}(t) \sqrt{-\xi}}{2 \xi r} \mathrm{e}^{r \sqrt{-\xi}}
\end{aligned}
$$

where

$$
\xi=\frac{f_{1}}{6 f_{2}}
$$

and $f_{1}$ and $f_{2}$ are expansion coefficients of $f(R)$. The integration constant $\delta_{0}$ is dimensionless, while the two arbitrary functions of time $\delta_{1}(t)$ and $\delta_{2}(t)$ have the dimensions of an inverse length and an inverse length squared, respectively, and $\xi$ has the dimensions on an inverse length squared. These functions are completely arbitrary because the differential system (16.69)-16.73) contains only spatial derivatives. The additive quantity $\delta_{0}$ can be set to zero.

The gravitational potential for a generic analytic $f(R)$ can now be obtained. Eqs. (16.74) - (16.76) provide the second order solution in term of the metric expansion (see the definition (16.38)-(16.41) ) but, as said above, this term coincides with the gravitational potential at the Newtonian order, $g_{t t}=-1-2 \phi=-1+g_{t t}^{(2)}$. The gravitational potential of a fourth order theory of gravity analytic in $R$ is

$$
\phi^{(F O G)}=\frac{K_{1}}{3 \xi r} \mathrm{e}^{-r \sqrt{-\xi}}+\frac{K_{2}}{6(-\xi)^{3 / 2} r} \mathrm{e}^{r \sqrt{-\xi}}
$$

with $K_{1}=\delta_{1}(t)$ and $K_{2}=\delta_{2}(t)$.

For a given $f(R)$ theory, the structure of the potential is determined by the parameter $\xi$, which depends on the first and second derivatives of $f(R)$ at $R_{0}$. The potential (16.78) is valid for non-vanishing $f_{2}$, since we manipulated Eqs. (16.69) - 16.73) dividing by $f_{2}$. The Newtonian limit of GR cannot be obtained directly from the solution (16.78) but requires the field equations (16.69) - 16.73) once the appropriate expressions in terms of the constants $f_{i}$ are derived.

The solution (16.78) must be discussed in relation to the sign of the term under square root in the exponents. If this sign is positive (which means that $f_{1}$ and $f_{2}$ have opposite signature), the solutions (16.74) - (16.76) and (16.78) can be rewritten introducing the scale parameter $l=|\xi|^{-1 / 2}$. In particular, considering $\delta_{0}=0$, the functions $\delta_{i}(t)$ as constants, $k_{1}=l \delta_{1}(t) / 3$ and $k_{2}(t)=l^{2} \delta_{2}(t) / 6$ and introducing a radial coordinate $\tilde{r}$ in units of $l$, we have

$$
\begin{aligned}
g_{t t}^{(2)} & =-\delta_{0}-\frac{\delta_{1}(t) l}{3} \frac{\mathrm{e}^{-r / l}}{r / l}-\frac{\delta_{2}(t) l^{2}}{6} \frac{\mathrm{e}^{r / l}}{r / l}=\frac{k_{1}}{\tilde{r}} \mathrm{e}^{-\tilde{r}}+\frac{k_{2}}{\tilde{r}} \mathrm{e}^{\tilde{r}}, \\
g_{r r}^{(2)} & =\frac{\delta_{1}(t) l}{3} \frac{(r / l+1)}{r / l} \mathrm{e}^{-r / l}-\frac{\delta_{2}(t) l^{2}}{6} \frac{(r / l-1)}{r / l} \mathrm{e}^{r / l} \\
& =-k_{1} \frac{(\tilde{r}+1) \mathrm{e}^{-\tilde{r}}}{\tilde{r}}+k_{2} \frac{(\tilde{r}-1) \mathrm{e}^{\tilde{r}}}{\tilde{r}}, \\
R^{(2)} & =\frac{\delta_{1}(t)}{l} \frac{\mathrm{e}^{-r / l}}{r / l}+\frac{\delta_{2}(t)}{2} \frac{\mathrm{e}^{r / l}}{r / l}=\frac{3}{l^{2}}\left(k_{1} \frac{\mathrm{e}^{-\tilde{r}}}{\tilde{r}}+k_{2} \frac{\mathrm{e}^{\tilde{r}}}{\tilde{r}}\right) .
\end{aligned}
$$

The gravitational potential can then be rewritten as

$$
\phi^{(F O G)}=\frac{k_{1}}{\tilde{r}} \mathrm{e}^{-\tilde{r}}+\frac{k_{2}}{\tilde{r}} \mathrm{e}^{\tilde{r}}
$$

which is analogous to the result or Ref. [535] derived for the theory $R+\alpha R^{2}+\beta R_{\mu \nu} R^{\mu \nu}$ and consistent 38 with Refs. [480, 509] discussing higher order Lagrangians such as $f(R, \square R)=R+\sum_{k=0}^{p} a_{k} R \square^{k} R$. In this last case, it was

${ }^{38}$ In a spatially homogeneous and isotropic space-time manifold, the higher order curvature invariants $R_{\mu \nu} R^{\mu \nu}$ and $R_{\alpha \beta \mu \nu} R^{\alpha \beta \mu \nu}$ can be written in terms of $R^{2}$. 
demonstrated that the number of Yukawa corrections to the gravitational potential is related to the order of the theory [480]. However, it is straightforward to show [138] that the usual form Newton plus Yukawa is recovered in Eq. (16.82) using a coordinate change, and Eq. (16.82) assumes the form

$$
\phi^{(F O G)}=-\left(\frac{G M}{f_{1} r}+\frac{\delta_{1}(t)}{6 \xi r} \mathrm{e}^{-r \sqrt{-\xi}}\right),
$$

where $\delta_{1}(t)$ is again an arbitrary function of time and the parameters depend on the Taylor coefficients. An effective Newton constant $G_{\text {eff }}=G / f_{1}$ and a range $l=|\xi|^{-1 / 2}$ emerge, and depend on the form of the function $f(R)$.

The inspection of Eqs. (16.74)-(16.76) and (16.79)-(16.81) reveals that the Newtonian limit of an analytic $f(R)$ theory depends only on the first and second coefficients of the Taylor expansion of $f(R)$. The gravitational potential is always characterized by two Yukawa corrections determined only by the first two terms of the Taylor expansion.

The diverging contribution, arising from the exponentially growing mode, has to be analyzed carefully and, in particular, the physical relevance of this term must be evaluated in relation to the length scale $(-\xi)^{-1 / 2}$. For $r \gg(-\xi)^{-1 / 2}$, the weak-field approximation turns out to be unphysical and (16.74)-116.76) no longer holds. One can obtain a modified gravitational potential which can work as a standard Newtonian one in the appropriate limit and provides interesting behavior at larger scales, even in the presence of the growing mode, once the constants in eq. (16.78) have been suitably adjusted. Once the growing exponential term is discarded, this potential reproduces the Yukawa-like potential phenomenologically introduced in order to explain the flat rotation curves of spiral galaxies without dark matter [501].

Yukawa-like corrections to the gravitational potential have been suggested in several contexts, for example, in a model describing the gravitational interaction between dark matter and baryons. In this model the interaction suppressed at small (subgalactic) scales is described by a Yukawa contribution to the standard Newtonian potential. This behaviour is suggested by observations of the inner rotation curves of low-mass galaxies and provides a natural scenario in which to interpret the cuspy profile of dark matter halos arising in $N$-body simulations [477].

The result outlined here is consistent with other calculations. Since an exponential potential is expanded in a power-law series, it is not surprising to find a power-law correction to the Newtonian potential [119, 121] when a less rigorous approach is considered in order to calculate the weak-field limit of a generic $f(R)$ theory, and perturbative calculations will provide effective potentials which can be recovered by means of an appropriate approximation from the general case (16.82).

Let us consider now a negative sign of $\xi$, when the two Yukawa corrections in (16.79)-(16.81) are complex. Using the form of $g_{t t}$, the gravitational potential (16.82) is

$$
\phi^{(F O G)}=\frac{k_{1}}{\tilde{r}} \exp (-i \tilde{r})+\frac{k_{2}}{\tilde{r}} \exp (i \tilde{r}),
$$

which can be recast as

$$
\phi^{(F O G)}=\frac{1}{\tilde{r}}\left[\left(k_{1}+k_{2}\right) \cos \tilde{r}+i\left(k_{2}-k_{1}\right) \sin \tilde{r}\right] .
$$

This gravitational potential, which could a priori be discarded as physically irrelevant, satisfies the Helmholtz equation $\nabla^{2} \phi+\vec{k}^{2} \phi=4 \pi G \rho$, where $\rho$ is a real function acting both as matter and antimatter density. As discussed in [55, 56], $\operatorname{Re}\left\{\phi^{(F O G)}\right\}$ can be seen as a classically modified Newtonian potential corrected by a Yukawa factor while $\operatorname{Im}\left\{\phi^{(F O G)}\right\}$ could have implications for quantum mechanics. This term can provide an astrophysical origin for the puzzling decay $K_{L} \rightarrow \pi^{+} \pi^{-}$, whose phase is related to an imaginary potential in the kaon mass matrix. Of course, these considerations are purely speculative but it could be interesting to pursue them.

Let us consider now third order contributions in the system (16.64)-116.67); at this order the off-diagonal equation

$$
f_{1} g_{r r, t}^{(2)}+2 f_{2} r R_{, t r}^{(2)}=0
$$

relating the time derivatives of $R$ and $g_{r r}^{(2)}$ must be taken into account. If the Ricci scalar depends on time, also the metric components and the gravitational potential do. This result agrees with the analysis of [136] in terms of the dynamical evolution of $R$ and demonstrating that a time-independent Ricci scalar implies static spherically symmetric solutions, which is confirmed (and explained) by eq. (16.86). In conjunction with Eqs. (16.79)-(16.81), eq. (16.86) suggests that if one considers the problem to lower (second) order, the background metric can have static solutions according to the Jebsen-Birkhoff theorem, but this is no longer true when higher orders are considered. The validity of the Jebsen-Birkhoff theorem in higher order theories of gravity depends on the approximation order considered. This theorem holds in metric $f(R)$-gravity only when the Ricci scalar is time-independent, and to second order in a $v / c$ expansion of the metric coefficients. According to Eqs. (16.74)-(16.76) and (16.78), it is only in the limit of 
small velocities and weak fields that the gravitational potential is effectively time-independent. But, contrary to GR, in metric $f(R)$-gravity a spherically symmetric background can have time-dependent evolution.

The next step is the order $\mathcal{O}(4)$ analysis of the system (16.64) 16.67) providing the solutions in terms of $g_{t t}^{(4)}$, the order necessary to compute the post-Newtonian parameters. Unfortunately, at this order the system is much more complicated and a general solution is not possible. One sees from Eqs. (16.64)-116.67) that the general solution is characterized only by the first three orders of the $f(R)$ expansion, in agreement with the $f(R)$ reconstruction using the post-Newtonian parameters in the scalar-tensor representation [114, 120]. Although a complete description is difficult, an estimate of the post-Newtonian parameter $\gamma$ can be obtained from the order $\mathcal{O}(2)$ evaluation of the metric coefficients in vacuo. Since (16.74)-116.76) suggest a non-Newtonian gravitational potential as a general solution of analytic $f(R)$-gravity, there is no reason to ask for a post-Newtonian description of these theories. In fact, as said earlier, the post-Newtonian analysis presupposes to evaluate deviations from the Newtonian potential at a higher than second order approximation in $v / c$. Thus, if the gravitational potential deduced from a given $f(R)$ theory is a general function of the radial coordinate displaying a Newtonian behaviour only in a certain regime (or in a given range of the radial coordinate), it would be meaningless to develop a general post-Newtonian formalism as in GR [446, 586, 587]. Of course, by a proper expansion of the gravitational potential for small values of the radial coordinate, and only in this limit, one can develop an analog of the post-Newtonian limit for these theories.

In order to estimate the post-Newtonian parameter $\gamma$, one proceeds by expanding $g_{t t}$ and $g_{r r}$, obtained to second order in (16.79)-(16.81), with respect to the dimensionless coordinate $\tilde{r}$, obtaining

$$
\begin{aligned}
& g_{t t}^{(2)}=k_{2}-k_{1}+\frac{k_{1}+k_{2}}{\tilde{r}}+\frac{k_{1}+k_{2}}{2} \tilde{r}+\mathcal{O}(2), \\
& g_{r r}^{(2)}=-\frac{k_{1}+k_{2}}{\tilde{r}}+\frac{k_{1}+k_{2}}{2} \tilde{r}+\mathcal{O}(2),
\end{aligned}
$$

where $k_{1}+k_{2}=G M$ and $k_{1}=k_{2}$ in the standard case. When $\tilde{r} \rightarrow 0$ (i.e., when $r \ll \sqrt{-\xi}$ ) the linear and successive order terms are small and the first (Newtonian) term dominates. Since the post-Newtonian parameter $\gamma$ is related to the coefficients of the $1 / r$ terms in $g_{t t}$ and $g_{r r}$, one can estimate this quantity by comparing the coefficients of the Newtonian terms relative to both expressions in (16.88). Since $\gamma=1$ in GR, the difference between these two coefficients gives the effective deviation from the GR value.

A generic fourth order gravity theory provides a post-Newtonian parameter $\gamma$ consistent with the GR prescription if $k_{1}=k_{2}$. Conversely, deviations from this behavior can be accommodated by tuning the relation between the two integration constants $k_{1}$ and $k_{2}$. This is equivalent to adjusting the form of the $f(R)$ theory to obtain the correct GR limit first, and then the Newtonian potential. This result agrees with recovering the GR behavior from generic $f(R)$ theories in the post-Newtonian limit [524, 594]. This is particularly true when the $f(R)$ Lagrangian behaves, in the weak-field and small velocity regime, as the Hilbert-Einstein Lagrangian. If deviations from this regime are observed, an $f(R)$ Lagrangian which is a third order polynomial in the Ricci scalar can be more appropriate [120].

The degeneracy in the integration constants can be partially broken once a complete post-Newtonian parameterization is developed in the presence of matter. Then, the integration constants are constrained by the Boltzmann-Vlasov equation describing conservation of matter at small scales [74].

So far, no specific gauge choice has been made, however particular gauges can be considered to simplify the calculations. A natural choice consists of the conditions (16.27) (16.30), which coincide with the standard postNewtonian gauge

$$
\begin{aligned}
& h_{j k}{ }^{, k}-\frac{1}{2} h_{, j}=\mathcal{O}(4), \\
& h_{0 k}{ }^{, k}-\frac{1}{2} h_{k, 0}^{k}=\mathcal{O}(5),
\end{aligned}
$$

where $h_{\mu \nu} \equiv g_{\mu \nu}-\eta_{\mu \nu}$. In this gauge the Ricci tensor becomes 39

$$
\begin{aligned}
& R_{\left.t t\right|_{h g}}=R_{\left.t t\right|_{h g}}^{(2)}+R_{\left.t t\right|_{h g}}^{(4)}, \\
& R_{\left.r r\right|_{h g}}=R_{\left.r r\right|_{h g}}^{(2)},
\end{aligned}
$$

\footnotetext{
39 We denote harmonic gauge quantities with the subscript $h g$.
} 
where

$$
\begin{aligned}
R_{\left.t t\right|_{h g}}^{(2)} & =\frac{r g_{t t, r r}^{(2)}+2 g_{t t, r}^{(2)}}{2 r}, \\
R_{\left.t t\right|_{h g}}^{(4)} & =\frac{r g_{t t, r r}^{(4)}+2 g_{t t, r}^{(4)}+r\left(g_{r r}^{(2)} g_{t t, r r}^{(2)}-g_{t t, t t}^{(2)}-g_{t t, r r}^{(2)}{ }^{2}\right)}{2 r}, \\
R_{\left.r r\right|_{h g}}^{(2)} & =\frac{r g_{r r, r r}^{(2)}+2 g_{r r, r}^{(2)}}{2 r} \\
R_{\left.\theta \theta\right|_{h g}}^{(2)} & =R_{\left.\phi \phi\right|_{h g}}^{(2)}=0,
\end{aligned}
$$

while the Ricci scalar to order $\mathcal{O}(2)$ and $\mathcal{O}(4)$ is

$$
\begin{aligned}
R_{\mid h g}^{(2)} & =\frac{r g_{t t, r r}^{(2)}+2 g_{t t, r}^{(2)}-r g_{r r, r r}^{(2)}-2 g_{r r, r}^{(2)}}{2 r} \\
R_{\mid h g}^{(4)} & =\left[r g_{t t, r r}^{(4)}+2 g_{t t, r}^{(4)}+r\left(g_{r r}^{(2)} g_{t t, r r}^{(2)}-g_{t t, t t}^{(2)}-g_{t t, r r}^{(2)}{ }^{2}\right)-g_{t t}^{(2)}\left(r g_{t t, r r}^{(2)}+2 g_{t t, r}^{(2)}\right)\right. \\
& \left.-g_{r r}^{(2)}\left(r g_{r r, r r}^{(2)}+2 g_{r r, r}^{(2)}\right)\right](2 r)^{-1} .
\end{aligned}
$$

The gauge choice does not affect the connection coefficients. The solution of the system (16.64)-(16.67) in this gauge is

$$
\begin{aligned}
& g_{t t_{\left.\right|_{h g}}}(t, r)=-1+\frac{k_{1}}{r}+\frac{k_{2}}{r^{2}}+\frac{k_{3} \log r}{r}, \\
& g_{r r_{\left.\right|_{h g}}}(t, r)=1+\frac{k_{4}}{r},
\end{aligned}
$$

where the constants $k_{1}$ and $k_{4}$ pertain to the order $\mathcal{O}(2)$, while $k_{2}$ and $k_{3}$ pertain to the order $\mathcal{O}(4)$. The Ricci scalar vanishes to orders $\mathcal{O}(2)$ and $\mathcal{O}(4)$.

Using Eqs. (16.100) and (16.101), it is easy to check that the GR prescriptions are immediately recovered for $k_{1}=k_{4}$ and $k_{2}=k_{3}=0$. The $g_{r r}$ component contains only the second order term, as required by a GR-like behavior, while the $g_{t t}$ component exhibits also the fourth order corrections which determine the second post-Newtonian parameter $\beta$ [586, 587. A full post-Newtonian formalism requires the consideration of matter in the system (16.64)-(16.67): the presence of matter links the second and fourth order contributions in the metric coefficients [586, 587]. A relevant application of these considerations is the following.

\section{An example: Neutrino oscillation phase dynamically induced by non-Newtonian corrections}

Neutrinos are elementary particles that travel at the speed of light (or close to it if massive), are electrically neutral and are capable of passing through ordinary matter with minimal interaction. Due to these properties, they can be investigated, in principle, at all length scales, ranging from nuclei [484], to molecular structures [186], up to galaxies [110, 582] and to the whole Universe [80]. They results from radioactive decays or nuclear reactions such as those that take place in the Sun, stars or nuclear reactors. In particular, they are generated when cosmic rays hit atoms. Current evidences of dark matter and dark energy can be related to the issue that neutrinos have masses and that mass eigenstates mix and/or superimpose [45, 410, 434]. The observation of such a mixing is related to suitable constraints. Such constraints should work on observables sensitive to the effective neutrino mass as the mass in Tritium-beta decay, the sum of neutrinos masses in cosmology and the effective Majorana neutrino mass in neutrinoless double-beta decay 275 .

A key role is played by the neutrino oscillations that allow the transition among the three types or "flavor" eigenstates, that is the electron, muon, and tauon neutrinos. It is well known that such a problem is still open and the research of new effects, in which the oscillations could manifest is one of the main goal of modern physics. For this reason, the quantum mechanical phase of neutrinos, propagating in gravitational field, has been discussed by several authors, also in view of the astrophysical consequences. More controversial is the debate concerning the redshift of 
flavor oscillation clocks, in the framework of the weak gravitational field of a star [10]. It has also been suggested that the gravitational oscillation phase might have a significant effect in supernova explosions due to the extremely large fluxes of neutrinos produced with different energies, corresponding to the flavor states. This result has been confirmed in [303], and it has been also derived under the assumption that the radial momentum of neutrinos is constant along the trajectory of the neutrino itself [364]. Besides, neutrino oscillations, in particular the gravitational part of the oscillation phase, could straightforwardly come into the debate to select what is the correct theory of gravity [104, 586]. Further gravitational interaction lengths, emerging from ETGs, could be related to neutrino oscillation phase. On the other hand, the experimental identification of such a gravitational phase could be a formidable probe both for confirming or ruling out such theories at a fundamental level.

Let us start our discussion considering how the gravitational field contributes to the neutrino oscillations. The approach has been firstly developed in [10] and we will outline the main results reported there. If $R_{A}$ is the size of a physical region where neutrinos are generated, a neutrino energy eigenstate $E_{\nu}$ can be denoted by $\left|\nu_{l}, R_{A}\right\rangle$ (where 1 $=e, \mu, \tau$ represents the weak flavor eigenstates). The three neutrino mass eigenstates can be represented by $\left|\nu_{i}\right\rangle$ with $i=1,2,3$ corresponding to the masses $m_{1}, m_{2}, m_{3}$. The mixing between mass and flavor eigenstates is achieved by the unitary transformation

$$
\left|\nu_{l}^{\prime}, R_{A}\right\rangle=\sum_{i=1,2,3} U_{l i}\left|\nu_{i}\right\rangle
$$

where

$$
U(\theta, \beta, \psi)=\left(\begin{array}{ccc}
c_{\theta} c_{\beta} & s_{\theta} c_{\beta} & s_{\beta} \\
-c_{\theta} s_{\beta} s_{\psi}-s_{\theta} c_{\psi} & c_{\theta} c_{\psi}-s_{\theta} s_{\beta} s_{\psi} & c_{\beta} s_{\psi} \\
-c_{\theta} s_{\beta} c_{\psi}-s_{\theta} s_{\psi} & -s_{\theta} s_{\beta} c_{\psi}-c_{\theta} s_{\psi} & c_{\beta} c_{\psi}
\end{array}\right)
$$

is a $3 \times 3$ unitary matrix parametrized by the three mixing angles $\eta=\theta, \beta, \psi$ with $c_{\eta}=\cos \eta$ and $s_{\eta}=\sin \eta$. At time $t=t_{B}>t_{A}$, the weak flavor eigenstates can be detected in a region $R_{B}$ and, in general, the evolution is given by

$$
\left|\nu_{l}, R_{B}\right\rangle=\exp \left(-\frac{i}{\hbar} \int_{t_{A}}^{t_{B}} \mathcal{H} d t+\frac{i}{\hbar} \int_{r_{A}}^{r_{B}} \vec{P} \cdot d \vec{x}\right)\left|\nu_{l}, R_{A}\right\rangle
$$

where $\mathcal{H}$ is the Hamiltonian operator associated to the system representing the time translation operator and $\vec{P}$ is the momentum operator representing the spatial translation operator. The phase change in Eq.(16.104) is the argument of the exponential function. It can be recast in the form

$$
\phi_{\nu}=\frac{1}{\hbar} \int_{r_{A}}^{r_{B}}\left[E \frac{d t}{d r}-p_{r}\right] d r .
$$

The covariant formulation is

$$
\phi_{\nu}=\frac{1}{\hbar} \int_{r_{A}}^{r_{B}} m d s=\frac{1}{\hbar} \int_{r_{A}}^{r_{B}} p_{\mu} d x^{\mu}
$$

where $p_{\mu}=m g_{\mu \nu} \frac{d x^{\nu}}{d s}$ is the 4-momentum of the particle. The effect of gravitational field is given by $g_{\mu \nu}$ and, in general, the neutrino oscillation probability from a state $\left|\nu_{l}, R_{A}\right\rangle$ to another state $\left|\nu_{l}, R_{B}\right\rangle$ is given by

$$
\begin{aligned}
\mathcal{P}\left[\left|\nu_{l}, R_{A}\right\rangle \rightarrow\left|\nu_{l^{\prime}}, R_{B}\right\rangle\right]= & \delta_{l l^{\prime}}-4 U_{l^{\prime} 1} U_{l 1} U_{l^{\prime} 2} U_{l 2} \sin ^{2}\left[\phi_{0}^{21}+\right. \\
& \left.+\phi_{G}^{21}\right]-4 U_{l^{\prime} 1} U_{l 1} U_{l^{\prime} 3} U_{l 3} \cdot \\
& \cdot \sin ^{2}\left[\phi_{0}^{31}+\phi_{G}^{31}\right]-4 U_{l^{\prime} 1} U_{l 1} U_{l^{\prime} 3} . \\
& \cdot U_{l 3} \sin ^{2}\left[\phi_{0}^{31}+\phi_{G}^{31}\right]
\end{aligned}
$$

where $\phi_{0}^{i j}$ are the usual kinematic phase while $\phi_{G}^{i j}$ are the gravitational contributions. It can be shown that, in a flat space-time, the $\phi_{G}^{i j}$ contributions are zero. In fact, a particle passing nearby a point mass feels a Schwarzschild geometry so the trajectories is

$$
d x \simeq\left[1-\frac{2 G_{N} M}{c^{2} r}\right] c d t
$$


If the effects of gravitational field are vanishing, Eq. (16.107) becomes $d x \simeq c d t$. Considering two generic neutrino mass eingestates in a Schwarzshild geometry, the standard phase of neutrino oscillation is

$$
\phi_{0}=\frac{\Delta m^{2} c^{3}}{4 E \hbar}\left(r_{B}-r_{A}\right)
$$

while the total gravitational phase shift is

$$
\phi_{\text {grav }}=\frac{G_{N} \triangle m^{2} M c}{4 \hbar E} \log \frac{r_{B}}{r_{A}}
$$

as shown in [10], where $\triangle m^{2}$ is the mass squared difference, $\triangle m^{2}=\left|m_{2}^{2}-m_{1}^{2}\right|, E$ the neutrino energy, $r_{A}$ and $r_{B}$ the point where neutrinos are created and detected, respectively. Nevertheless, assuming that the neutrino energy is constant along the trajectory, the term (16.109) could be cancelled out at typical astrophysical scales 72].

With this considerations in mind, let us take into account how possible corrections to the Newtonian potential could affect this result. We will follow the discussion developed above for the post-Newtonian limit of $f(R)$-gravity. We do not want to impose a particular forms for $f(R)$-model but only consider analytic Taylor expansion where the cosmological term and terms higher than second are discarded. The Lagrangian is then

$$
f(R) \sim a_{1} R+a_{2} R^{2}+\ldots
$$

where the parameters $a_{1,2}$ specifies the particular models. Adopting the argument of previous subsection, Eq. (16.83) gives

$$
\Phi(r)=-\frac{3 G_{N} M}{4 a_{1} r}\left(1+\frac{1}{3} e^{-\frac{r}{L}}\right)=\Phi(r)_{N e w t o n}+\Phi(r)_{Y u k a w a}
$$

where

$$
L \equiv L\left(a_{1}, a_{2}\right)=\left(-\frac{6 a_{2}}{a_{1}}\right)^{1 / 2} .
$$

$L$ is an interaction gravitational length due to the correction to the Newtonian potential. However, as soon as $a_{1}=3 / 4$, $a_{2}=0$ and $\Phi(r)_{Y u k a w a} \rightarrow 0$, the standard Newtonian limit of GR is fully recovered (for a discussion on this point, see [135]).

Now we calculate the gravitational phase shift of neutrino oscillation in $f(R)$-gravity using the potential (16.111) in the Eq. (16.105). We obtain the general expression

$$
\phi_{\text {grav }}=\frac{\Delta m^{2} M c}{4 \hbar E}\left(\frac{3 G_{N}}{4 a_{1}}\right) \int_{r_{A}}^{r_{B}}\left(\frac{1}{r}+\frac{1}{3 r} e^{-\frac{r}{L}}\right),
$$

from which we have the following result

$$
\phi_{\text {grav }}=\frac{\Delta m^{2} M c}{4 \hbar E}\left(\frac{3 G_{N}}{4 a_{1}}\right)\left[\log \frac{r_{B}}{r_{A}}+\sum_{n=1}^{\infty}(-1)^{n}\left(\frac{\left(\frac{r_{B}-r_{A}}{L}\right)^{n}}{n \cdot n !}\right)\right]=\phi_{\text {Newton }}+\phi_{Y u k a w a}
$$

where $\phi_{\text {grav }}$ is the total gravitational phase shift in Eq. (16.109) and

$$
\phi_{Y u k a w a}=\frac{\Delta m^{2} M c}{4 \hbar E}\left(\frac{3 G_{N}}{4 a_{1}}\right)\left[\sum_{n=1}^{\infty}(-1)^{n}\left(\frac{\left(\frac{r_{B}-r_{A}}{L}\right)^{n}}{n \cdot n !}\right)\right]
$$

The Yukawa term disappears in standard Einstein gravity, that is for $f(R)=R$. Note that the series in above equation is absolutely convergent. If we consider Solar neutrinos, we can use the following values: $M_{\sim} \sim M_{\odot} \sim 1.9891 \times 10^{30} \mathrm{Kg}$, $r_{A} \sim r_{\oplus} \sim 6.3 \times 10^{3} \mathrm{Km}$, and $r_{B} \sim r_{A}+D$, where $D \sim 1.5 \times 10^{8} \mathrm{Km}$ is the Sun-Earth distance. In order to estimate the phases differences (16.108), (16.109) and (16.115), we introduce the ratio $Q_{\text {grav }}$ defined as

$$
Q_{\text {grav }}=\frac{\phi_{N e w t o n}}{\phi_{0}} \sim \frac{G_{N} M \log \frac{r_{B}}{r_{A}}}{c^{2}\left(r_{B}-r_{A}\right)} \sim 10^{-7}
$$


and the ratio $Q_{Y u k a w a}$ defined as

$$
Q_{Y u k a w a}=\frac{\phi_{Y u k a w a}}{\phi_{0}} \sim \frac{G_{N} M}{c^{2}\left(r_{B}-r_{A}\right)} \sum_{n=1}^{\infty}(-1)^{n}\left(\frac{\left(\frac{r_{B}-r_{A}}{L}\right)^{n}}{n \cdot n !}\right),
$$

where we have assumed that $3 / 4 a_{1} \sim 1$. Note that both $Q_{\text {Newton }}$ and $Q_{Y \text { ukawa }}$ do not depend on the squared-mass difference $\Delta m^{2}$ and on the neutrino energy $E$. The ratio $Q_{Y \text { ukawa }}$ can be calculated for different values of the interaction lenght $L$. For example from Eq. (16.117), after summing the series, we obtain the results:

$$
\begin{gathered}
L \sim 1.5 \cdot 10^{7} \mathrm{Km} \Longrightarrow Q_{\text {Yukawa }} \sim-2.9 \cdot 10^{-8}, \\
L \sim 1.5 \cdot 10^{8} \mathrm{Km} \Longrightarrow Q_{\text {Yukawa }} \sim-8 \cdot 10^{-9} .
\end{gathered}
$$

In this way the values of $Q_{Y \text { ukawa }}$ can be seen as corrections to the standard gravitational phase shift of neutrino oscillations depending on the particular choice of $L$ and so, through Eq.(16.112), directly on the particular $f(R)$-model considered.

We remark that the calculated correction to gravitational phase shift in Eq. 116.115) depends on the interaction lenght $L$ defined in Eq. (16.112). This is directly related to the $f(R)$-gravity model through the coefficients $a_{1}$ and $a_{2}$ in Eq. (16.110). This fact could be used as an experimental test to probe a given gravity theory through the neutrino oscillation induced by means of the gravitational field itself. On the other hand, interpreting $L$ as the characteristic wavelenght of the neutrino interaction with the gravitational field, the gravitational phase correction could be used as a method to constrain the mass of electronic neutrinos travelling from the Sun to the Earth surface, or, eventually, also from other neutrinos sources as Supernovae or neutron stars.

\section{THE POST- MINKOWSKIAN LIMIT}

\section{A. The weak field limit in Minkowski space-time}

We have developed a general analytic procedure to deduce the Newtonian and post-Newtonian limits of $f(R)$ gravity outside matter sources. Now we discuss a different limit of these theories, obtained when the small velocity assumption is relaxed and only the weak-field approximation is retained. Again, we assume spherical symmetry of the metric, considering gravitational potentials $A$ and $B$ of the form

$$
\begin{aligned}
& A(t, r)=-1+a(t, r), \\
& B(t, r)=1+b(t, r),
\end{aligned}
$$

with $|a(t, r)|,|b(t, r)| \ll 1$. Let us perturb the field equations considering again the Taylor expansion (16.63). In vacuo and to first order in $a$ and $b$, one obtains

$$
\begin{aligned}
& f_{0}=0 \\
& f_{1}\left(R_{\mu \nu}^{(1)}-\frac{1}{2} g_{\mu \nu}^{(0)} R^{(1)}\right)+\mathcal{H}_{\mu \nu}^{(1)}=0,
\end{aligned}
$$

where

$$
\begin{aligned}
\mathcal{H}_{\mu \nu}^{(1)} & =-f_{2}\left[R_{, \mu \nu}^{(1)}-\Gamma_{\mu \nu}^{(0)}{ }_{\mu \nu}^{\rho} R_{, \rho}^{(1)}-g_{\mu \nu}^{(0)}\left(g_{, \rho}^{(0) \rho \sigma} R_{, \sigma}^{(1)}+g^{(0) \rho \sigma} R_{, \rho \sigma}^{(1)}\right.\right. \\
& \left.\left.+g^{(0) \rho \sigma} \ln \sqrt{-g}_{, \rho}^{(0)} R_{, \sigma}^{(1)}\right)\right] .
\end{aligned}
$$

In this approximation the Ricci scalar vanishes and the derivatives are evaluated at $R=0$. Let us consider now the large $r$ limit far from the sources of the gravitational field: Eqs. (17.3) and (17.4) become

$$
\begin{aligned}
& \frac{\partial^{2} a(t, r)}{\partial r^{2}}-\frac{\partial^{2} b(t, r)}{\partial t^{2}}=0, \\
& f_{1}[a(t, r)-b(t, r)]-8 f_{2}\left[\frac{\partial^{2} b(t, r)}{\partial r^{2}}+\frac{\partial^{2} a(t, r)}{\partial t^{2}}-2 \frac{\partial^{2} b(t, r)}{\partial t^{2}}\right]=\Psi(t),
\end{aligned}
$$


where $\Psi(t)$ is a generic time-dependent function. Eqs. (17.6) and (17.7) are coupled wave equations for $a(t, r)$ and $b(t, r)$, therefore we search for a wave-like solution

$$
\begin{aligned}
& a(t, r)=\int \frac{d \omega d k}{2 \pi} \tilde{a}(\omega, k) \mathrm{e}^{i(k r \omega t)}, \\
& b(t, r)=\int \frac{d \omega d k}{2 \pi} \tilde{b}(\omega, k) \mathrm{e}^{i(k r \omega t)},
\end{aligned}
$$

where $k \equiv|\vec{k}|$, and we substitute these into Eqs. (17.6) and (17.7), setting $\Psi(t)=0$. Eqs. (17.6) and (17.7) are satisfied if

$$
\begin{aligned}
& \tilde{a}(\omega, k)=\tilde{b}(\omega, k), \quad \omega= \pm k, \\
& \tilde{a}(\omega, k)=\left(1-\frac{3 \xi}{4 k^{2}}\right) \tilde{b}(\omega, k), \quad \omega= \pm \sqrt{k^{2}-\frac{3 \xi}{4}}
\end{aligned}
$$

where, as before, $\xi=f_{1} / 6 f_{2}$. In particular, for $f_{1}=0$ or $f_{2}=0$, one obtains solutions with dispersion relation $\omega= \pm k$. For $f_{i} \neq 0(i=1,2)$, the dispersion relation suggests that massive modes are present. In particular, for $\xi<0$, the mass of the scalar graviton is $m_{\text {grav }}=-3 \xi / 4$ and, accordingly, it is obtained for a modified real gravitational potential. A non-Newtonian gravitational potential describes a massive degree of freedom in the particle spectrum of the gravity sector with interesting perspectives for the detection and production of gravitational waves [123]. The presence of massive modes in higher order gravity is well known [535].

If $\xi>0$, the solution

$$
\begin{aligned}
& a(\tilde{t}, \tilde{r})=\left(a_{0}+a_{1} \tilde{r}\right) \mathrm{e}^{ \pm \frac{\sqrt{3}}{2} \tilde{t}} \\
& b(\tilde{t}, \tilde{r})=\left(b_{0}+b_{1} \tilde{t}\right) \cos \left(\frac{\sqrt{3}}{2} \tilde{r}\right)+\left(b_{0}^{\prime}+b_{1}^{\prime} \tilde{t}\right) \sin \left(\frac{\sqrt{3}}{2} \tilde{r}\right)+b_{0}^{\prime \prime}+b_{1}^{\prime \prime} \tilde{t}
\end{aligned}
$$

with $a_{0}, a_{1}, b_{0}, b_{1}, b_{0}^{\prime}, b_{1}^{\prime}, b_{0}^{\prime \prime}, b_{1}^{\prime \prime}$ constants is admitted. The variables $\tilde{r}$ and $\tilde{t}$ are expressed in units of $\xi^{-1 / 2}$. In the post-Minkowskian approximation, as expected, the gravitational field propagates via wave-like solutions. The gravitational wave content of fourth order gravity originates new phenomenology (massive modes) to be taken into account by the gravitational wave community. These massive degrees of freedom could also constitute a potential candidate for cold dark matter [224].

\section{B. The $f(R)$-gravity energy-momentum pseudo-tensor and gravitational radiation}

As we have seen, higher order theories of gravity introduce extra degrees of freedom which can be described by writing the field equations as effective Einstein equations and introducing an additional curvature "effective source" in their right hand side. This quantity behaves as an effective energy-momentum tensor contributing to the energy loss of a system due to the emission of gravitational radiation. The procedure to calculate the stress-energy pseudo-tensor of gravitational waves in GR can be extended to more general theories and this quantity can be obtained by varying the gravitational Lagrangian. In GR this quantity is known as the Landau-Lifshitz pseudo-tensor [373].

Let us consider $f(R)$-gravity, for which

$$
\begin{aligned}
\delta \int d^{4} x \sqrt{-g} f(R) & =\delta \int d^{4} x \mathcal{L}\left(g_{\mu \nu}, g_{\mu \nu, \rho}, g_{\mu \nu, \rho \sigma}\right) \\
& \approx \int d^{4} x\left[\frac{\partial \mathcal{L}}{\partial g_{\rho \sigma}}-\partial_{\lambda}\left(\frac{\partial \mathcal{L}}{\partial g_{\rho \sigma, \lambda}}\right)+\partial_{\lambda \xi}^{2}\left(\frac{\partial \mathcal{L}}{\partial g_{\rho \sigma, \lambda \xi}}\right)\right] \delta g_{\rho \sigma} \\
& \equiv \int d^{4} x \sqrt{-g} \mathcal{H}^{\rho \sigma} \delta g_{\rho \sigma}=0 .
\end{aligned}
$$

The Euler-Lagrange equations

$$
\frac{\partial \mathcal{L}}{\partial g_{\rho \sigma}}-\partial_{\lambda}\left(\frac{\partial \mathcal{L}}{\partial g_{\rho \sigma, \lambda}}\right)+\partial_{\lambda \xi}^{2}\left(\frac{\partial \mathcal{L}}{\partial g_{\rho \sigma, \lambda \xi}}\right)=0
$$


coincide with the vacuum field equations. Even in the case of more general theories, it is possible to define the energy-momentum pseudo-tensor

$$
t_{\alpha}^{\lambda}=\frac{1}{\sqrt{-g}}\left\{\left[\frac{\partial \mathcal{L}}{\partial g_{\rho \sigma, \lambda}}-\partial_{\xi}\left(\frac{\partial \mathcal{L}}{\partial g_{\rho \sigma, \lambda \xi}}\right)\right] g_{\rho \sigma, \alpha}+\frac{\partial \mathcal{L}}{\partial g_{\rho \sigma, \lambda \xi}} g_{\rho \sigma, \xi \alpha}-\delta_{\alpha}^{\lambda} \mathcal{L}\right\} .
$$

This quantity, together with the matter energy-momentum tensor $T_{\mu \nu}^{(m)}$, satisfies a conservation law as required by the contracted Bianchi identities in conjunction with the effective Einstein equations. In fact, in the presence of matter one has $\mathcal{H}_{\mu \nu}=\frac{\kappa}{2} T_{\mu \nu}^{(m)}$ and

$$
\left(\sqrt{-g} t_{\alpha}^{\lambda}\right)_{, \lambda}=-\sqrt{-g} H^{\rho \sigma} g_{\rho \sigma, \alpha}=-\frac{\kappa}{2} \sqrt{-g} T_{(m)}^{\rho \sigma} g_{\rho \sigma, \alpha}=-\kappa\left(\sqrt{-g} T_{(m)_{\alpha}}^{\lambda}\right)_{, \lambda} ;
$$

as a consequence,

$$
\left[\sqrt{-g}\left(t_{\alpha}^{\lambda}+\kappa T_{\alpha}^{(m)^{\lambda}}\right)\right]_{, \lambda}=0
$$

which is the conservation law given by the contracted Bianchi identities. We can now write the expression of the energy-momentum pseudo-tensor $t_{\alpha}^{\lambda}$ in terms of $f(R)$ and its derivatives

$$
\begin{aligned}
t_{\alpha}^{\lambda} & =f^{\prime}\left\{\left[\frac{\partial R}{\partial g_{\rho \sigma, \lambda}}-\frac{1}{\sqrt{-g}} \partial_{\xi}\left(\sqrt{-g} \frac{\partial R}{\partial g_{\rho \sigma, \lambda \xi}}\right)\right] g_{\rho \sigma, \alpha}+\frac{\partial R}{\partial g_{\rho \sigma, \lambda \xi}} g_{\rho \sigma, \xi \alpha}\right\} \\
& -f^{\prime \prime} R_{, \xi} \frac{\partial R}{\partial g_{\rho \sigma, \lambda \xi}} g_{\rho \sigma, \alpha}-\delta_{\alpha}^{\lambda} f .
\end{aligned}
$$

$t_{\alpha}^{\lambda}$ is a non-covariant quantity in GR while its generalization to fourth order gravity turns out to be covariant. This expression reduces to the Landau-Lifshitz pseudo-tensor of GR in the limit $f(R) \rightarrow R$, in which

$$
t_{\left.\alpha\right|_{\mathrm{GR}}}^{\lambda}=\frac{1}{\sqrt{-g}}\left(\frac{\partial \mathcal{L}_{\mathrm{GR}}}{\partial g_{\rho \sigma, \lambda}} g_{\rho \sigma, \alpha}-\delta_{\alpha}^{\lambda} \mathcal{L}_{\mathrm{GR}}\right)
$$

and where the GR Lagrangian has been considered in its effective form containing the symmetric part of the Ricci tensor which leads to the equations of motion

$$
\mathcal{L}_{\mathrm{GR}}=\sqrt{-g} g^{\mu \nu}\left(\Gamma_{\mu \sigma}^{\rho} \Gamma_{\rho \nu}^{\sigma}-\Gamma_{\mu \nu}^{\sigma} \Gamma_{\sigma \rho}^{\rho}\right) .
$$

The definitions of energy-momentum pseudo-tensor in GR and in $f(R)$-gravity are different. The difference is due to the fact that terms of order higher than second are present in $f(R)$-gravity and they cannot be discarded as boundary terms following integration by parts, as is done in GR. The effective Lagrangian of GR turns out to be the symmetric part of the Ricci scalar since the second order terms appearing in the expression of $R$ can be removed integrating by parts. An analytic $f(R)$ Lagrangian can be rewritten, to linear order, as $f \sim f_{0}^{\prime} R+\mathcal{F}(R)$, where the function $\mathcal{F}$ is such that $\mathcal{F}(R) \approx R^{2}$ as $R \rightarrow 0$. As a consequence, one can rewrite $t_{\alpha}^{\lambda}$ as

$$
\begin{aligned}
t_{\alpha}^{\lambda} & =f_{0}^{\prime} t_{\left.\alpha\right|_{\mathrm{GR}}}^{\lambda}+\mathcal{F}^{\prime}\left\{\left[\frac{\partial R}{\partial g_{\rho \sigma, \lambda}}-\frac{1}{\sqrt{-g}} \partial_{\xi}\left(\sqrt{-g} \frac{\partial R}{\partial g_{\rho \sigma, \lambda \xi}}\right)\right] g_{\rho \sigma, \alpha}\right. \\
& \left.+\frac{\partial R}{\partial g_{\rho \sigma, \lambda \xi}} g_{\rho \sigma, \xi \alpha}\right\}-\mathcal{F}^{\prime \prime} R_{, \xi} \frac{\partial R}{\partial g_{\rho \sigma, \lambda \xi}} g_{\rho \sigma, \alpha}-\delta_{\alpha}^{\lambda} \mathcal{F} .
\end{aligned}
$$

The general expression of the Ricci scalar obtained by splitting its linear $\left(R^{*}\right)$ and quadratic $(\bar{R})$ parts in the metric perturbations is

$$
R=g^{\mu \nu}\left(\Gamma_{\mu \nu, \rho}^{\rho}-\Gamma_{\mu \rho, \nu}^{\rho}\right)+g^{\mu \nu}\left(\Gamma_{\sigma \rho}^{\rho} \Gamma_{\mu \nu}^{\sigma}-\Gamma_{\rho \mu}^{\sigma} \Gamma_{\nu \sigma}^{\rho}\right)=R^{*}+\bar{R}
$$

(note that $\mathcal{L}_{\mathrm{GR}}=-\sqrt{-g} \bar{R}$ ). In the GR case $t_{\left.\alpha\right|_{\mathrm{GR}}}^{\lambda}$, the first non-vanishing term of the Landau-Lifshitz pseudo-tensor is of order $h^{2}$ [336, 373]. A similar result can be obtained in $f(R)$-gravity: using Eq. (17.22) one obtains that, to 
lowest order,

$$
\begin{aligned}
t_{\alpha}^{\lambda} & \sim t_{\alpha \mid h^{2}}^{\lambda}=f_{0}^{\prime} t_{\left.\alpha\right|_{\mathrm{GR}}}^{\lambda}+f_{0}^{\prime \prime} R^{*}\left[\left(-\partial_{\xi} \frac{\partial R^{*}}{\partial g_{\rho \sigma, \lambda \xi}}\right) g_{\rho \sigma, \alpha}+\frac{\partial R^{*}}{\partial g_{\rho \sigma, \lambda \xi}} g_{\rho \sigma, \xi \alpha}\right] \\
& -f_{0}^{\prime \prime} R_{, \xi}^{*} \frac{\partial R^{*}}{\partial g_{\rho \sigma, \lambda \xi}} g_{\rho \sigma, \alpha}-\frac{f_{0}^{\prime \prime}}{2} \delta_{\alpha}^{\lambda} R^{* 2} \\
& =f_{0}^{\prime} t_{\left.\alpha\right|_{\mathrm{GR}}}^{\lambda}+f_{0}^{\prime \prime}\left[R^{*}\left(\frac{\partial R^{*}}{\partial g_{\rho \sigma, \lambda \xi}} g_{\rho \sigma, \xi \alpha}-\frac{1}{2} R^{*} \delta_{\alpha}^{\lambda}\right)\right. \\
& \left.-\partial_{\xi}\left(R^{*} \frac{\partial R^{*}}{\partial g_{\rho \sigma, \lambda \xi}}\right) g_{\rho \sigma, \alpha}\right] .
\end{aligned}
$$

Using the perturbed metric we have $R^{*} \sim R^{(1)}$, where $R^{(1)}$ is defined by

$$
\begin{aligned}
& R_{\mu \nu}^{(1)}=h_{(\mu, \nu) \sigma}^{\sigma}-\frac{1}{2} \square h_{\mu \nu}-\frac{1}{2} h_{, \mu \nu}, \\
& R^{(1)}=h_{\sigma \tau}{ }^{, \sigma \tau}-\square h
\end{aligned}
$$

with $h \equiv h_{\sigma}^{\sigma}$. In terms of $h$ and $\eta_{\mu \nu}$, one obtains

$$
\begin{aligned}
& \frac{\partial R^{*}}{\partial g_{\rho \sigma, \lambda \xi}} \sim \frac{\partial R^{(1)}}{\partial h_{\rho \sigma, \lambda \xi}}=\eta^{\rho \lambda} \eta^{\sigma \xi}-\eta^{\lambda \xi} \eta^{\rho \sigma}, \\
& \frac{\partial R^{*}}{\partial g_{\rho \sigma, \lambda \xi}} g_{\rho \sigma, \xi \alpha} \sim h_{, \xi \alpha}^{\lambda \xi}-h_{\alpha}^{, \lambda} .
\end{aligned}
$$

The first significant term in Eq. (17.24) is of second order in the perturbations. We can now write the explicit expression of the pseudo-tensor in terms of the perturbation $h$,

$$
\begin{aligned}
t_{\alpha}^{\lambda} & \sim f_{0}^{\prime} t_{\left.\alpha\right|_{\mathrm{GR}}}^{\lambda}+f_{0}^{\prime \prime}\left\{\left(h_{, \rho \sigma}^{\rho \sigma}-\square h\right)\left[h_{, \xi \alpha}^{\lambda \xi}-h_{\alpha}^{, \lambda}-\frac{1}{2} \delta_{\alpha}^{\lambda}\left(h_{, \rho \sigma}^{\rho \sigma}-\square h\right)\right]\right. \\
& \left.-h_{, \rho \sigma \xi}^{\rho \sigma} h_{, \alpha}^{\lambda \xi}+h_{, \rho \sigma}^{\rho \sigma}{ }_{, \alpha}^{\lambda} h_{, \alpha}+h_{, \alpha}^{\lambda \xi} \square h_{, \xi}-\square h^{, \lambda} h_{, \alpha}\right\} .
\end{aligned}
$$

This expression can be put in compact form using the metric perturbation $\tilde{h}_{\mu \nu}$ as

$$
t_{\left.\alpha\right|_{f}}^{\lambda}=\frac{1}{2}\left[\frac{1}{2} \tilde{h}_{\alpha}^{, \lambda} \square \tilde{h}-\frac{1}{2} \tilde{h}_{, \alpha} \square \tilde{h}^{, \lambda}-\tilde{h}_{\sigma, \alpha}^{\lambda} \square \tilde{h}^{, \sigma}-\frac{1}{4}(\square \tilde{h})^{2} \delta_{\alpha}^{\lambda}\right] .
$$

The energy-momentum pseudo-tensor of the gravitational field describing the energy transport during propagation has a natural generalization to $f(R)$-gravity. Here we have adopted the Landau-Lifshitz construct, but many other pseudo-tensors can be used [421, 532]. The general definition of $t_{\alpha}^{\lambda}$ obtained above consists of the sum of a GR contribution plus a term characteristic of $f(R)$-gravity,

$$
t_{\alpha}^{\lambda}=f_{0}^{\prime} t_{\left.\alpha\right|_{\mathrm{GR}}}^{\lambda}+f_{0}^{\prime \prime} t_{\left.\alpha\right|_{f}}^{\lambda}
$$

In the limit $f(R) \rightarrow R$ one obtains $t_{\alpha}^{\lambda}=t_{\left.\alpha\right|_{\mathrm{GR}}}^{\lambda}$. Massive gravitational modes are contained in $t_{\left.\alpha\right|_{f}}^{\lambda}$ since $\square \tilde{h}$ can be considered as an effective scalar field degree of freedom evolving in a potential and $t_{\alpha}^{\lambda}$ describes the transport of energy and momentum.

\section{Ghosts, massless and massive gravitational modes}

Detecting new gravitational modes could be a crucial experiment able to discriminate among theories since these modes would constitute evidence that GR must be enlarged or modified [64, 142]. In general, field equations containing higher order terms describe, in addition to the massless spin two field (the standard graviton of GR), also spin zero and spin two massive modes, the latter possibly being ghosts. This result is general and can be obtained by means of a straightforward generalization of the above discussion for $f(R)$-gravity. 
Let us generalize the Hilbert-Einstein action by adding curvature invariants different from the Ricci scalar,

$$
S=\int d^{4} x \sqrt{-g} f(R, P, Q),
$$

where

$$
\begin{aligned}
& P \equiv R_{\mu \nu} R^{\mu \nu}, \\
& Q \equiv R_{\mu \nu \rho \sigma} R^{\mu \nu \rho \sigma} .
\end{aligned}
$$

By varying the action (17.32) with respect to $g^{\mu \nu}$, one obtains the field equations 157]

$$
\begin{aligned}
F G_{\mu \nu} & =\frac{1}{2} g_{\mu \nu}(f-R F)-\left(g_{\mu \nu} \square-\nabla_{\mu} \nabla_{\nu}\right) F \\
& -2\left(f_{P} R_{\mu}^{\alpha} R_{\alpha \nu}+f_{Q} R_{\alpha \beta \gamma \mu} R_{\nu}^{\alpha \beta \gamma}\right) \\
& -g_{\mu \nu} \nabla_{\alpha} \nabla_{\beta}\left(f_{P} R^{\alpha \beta}\right)-\square\left(f_{P} R_{\mu \nu}\right) \\
& +2 \nabla_{\alpha} \nabla_{\beta}\left(f_{P} R_{(\mu}^{\alpha} \delta_{\nu)}^{\beta}+2 f_{Q} R_{(\mu \nu)}^{\alpha}{ }^{\beta}\right),
\end{aligned}
$$

where

$$
F \equiv \frac{\partial f}{\partial R}, \quad f_{P} \equiv \frac{\partial f}{\partial P}, \quad f_{Q} \equiv \frac{\partial f}{\partial Q}
$$

The trace of Eq. (17.35) yields

$$
\begin{aligned}
& \square\left(F+\frac{f_{P}}{3} R\right) \\
& =\frac{1}{3}\left\{2 f-R F-2 \nabla_{\alpha} \nabla_{\beta}\left[\left(f_{P}+2 f_{Q}\right) R^{\alpha \beta}\right]-2\left(f_{P} P+f_{Q} Q\right)\right\} .
\end{aligned}
$$

Expanding the third term on the right hand side of (17.37) and using the contracted Bianchi identities, one obtains

$$
\begin{aligned}
& \square\left(F+\frac{2}{3}\left(f_{P}+f_{Q}\right) R\right) \\
= & \frac{1}{3}\left[2 f-R F-2 R^{\mu \nu} \nabla_{\mu} \nabla_{\nu}\left(f_{P}+2 f_{Q}\right)-R \square\left(f_{P}+2 f_{Q}\right)\right. \\
- & \left.2\left(f_{P} P+f_{Q} Q\right)\right] .
\end{aligned}
$$

By defining

$$
\Phi \equiv F+\frac{2}{3}\left(f_{P}+f_{Q}\right) R
$$

and

$$
\frac{d V}{d \Phi} \equiv \frac{1}{3}\left[2 f-R F-2 R^{\mu \nu} \nabla_{\mu} \nabla_{\nu}\left(f_{P}+2 f_{Q}\right)-R \square\left(f_{P}+2 f_{Q}\right)-2\left(f_{P} P+f_{Q} Q\right)\right],
$$

the Klein-Gordon equation

$$
\square \Phi-\frac{d V}{d \Phi}=0
$$


is obtained. In order to find the modes of the gravity waves of this theory, we linearize around the Minkowski background,

$$
\begin{gathered}
g_{\mu \nu}=\eta_{\mu \nu}+h_{\mu \nu}, \\
\Phi=\Phi_{0}+\delta \Phi ;
\end{gathered}
$$

then Eq. (17.39) yields

$$
\delta \Phi=\delta F+\frac{2}{3}\left(\delta f_{P}+\delta f_{Q}\right) R_{0}+\frac{2}{3}\left(f_{P 0}+f_{Q 0}\right) \delta R
$$

where $R_{0} \equiv R\left(\eta_{\mu \nu}\right)=0$ and, similarly, $f_{P 0}=\left.\frac{\partial f}{\partial P}\right|_{\eta_{\mu \nu}}$, which is either constant or zero (a zero subscript denoting quantities evaluated with the Minkowski metric). $\delta R$ denotes the first order perturbation of the Ricci scalar which, together with the perturbed parts of the Riemann and Ricci tensors, is given by

$$
\begin{aligned}
\delta R_{\mu \nu \rho \sigma} & =\frac{1}{2}\left(\partial_{\rho} \partial_{\nu} h_{\mu \sigma}+\partial_{\sigma} \partial_{\mu} h_{\nu \rho}-\partial_{\sigma} \partial_{\nu} h_{\mu \rho}-\partial_{\rho} \partial_{\mu} h_{\nu \sigma}\right), \\
\delta R_{\mu \nu} & =\frac{1}{2}\left(\partial_{\sigma} \partial_{\nu} h_{\mu}^{\sigma}+\partial_{\sigma} \partial_{\mu} h_{\nu}^{\sigma}-\partial_{\mu} \partial_{\nu} h-\square h_{\mu \nu}\right), \\
\delta R & =\partial_{\mu} \partial_{\nu} h^{\mu \nu}-\square h,
\end{aligned}
$$

where $h \equiv \eta^{\mu \nu} h_{\mu \nu}$. The first term of Eq. (17.44) is

$$
\delta F=\left.\frac{\partial F}{\partial R}\right|_{0} \delta R+\left.\frac{\partial F}{\partial P}\right|_{0} \delta P+\left.\frac{\partial F}{\partial Q}\right|_{0} \delta Q
$$

however since $\delta P$ and $\delta Q$ are second order, it is $\delta F \simeq F_{, R 0} \delta R$ and

$$
\delta \Phi=\left[F_{, R 0}+\frac{2}{3}\left(f_{P 0}+f_{Q 0}\right)\right] \delta R .
$$

Eq. (17.38) then yields the Klein-Gordon equation for the scalar perturbation $\delta \Phi$

$$
\begin{aligned}
\square \delta \Phi & =\frac{1}{3} \frac{F_{0}}{F_{, R 0}+\frac{2}{3}\left(f_{P 0}+f_{Q 0}\right)} \delta \Phi \\
& -\frac{2}{3} \delta R^{\alpha \beta} \partial_{\alpha} \partial_{\beta}\left(f_{P 0}+2 f_{Q 0}\right)-\frac{1}{3} \delta R \square\left(f_{P 0}+2 f_{Q 0}\right) \\
& =m_{s}^{2} \delta \Phi .
\end{aligned}
$$

The second line of Eq. (17.50) vanishes because $f_{P 0}$ and $f_{Q 0}$ are constant and the scalar mass is defined as

$$
m_{s}^{2} \equiv \frac{F_{0}}{3 F_{, R 0}+2\left(f_{P 0}+f_{Q 0}\right)} .
$$

Perturbing the field equations (17.35), one obtains

$$
\begin{aligned}
& F_{0}\left(\delta R_{\mu \nu}-\frac{1}{2} \eta_{\mu \nu} \delta R\right) \\
= & -\left(\eta_{\mu \nu} \square-\partial_{\mu} \partial_{\nu}\right)\left[\delta \Phi-\frac{2}{3}\left(f_{P 0}+f_{Q 0}\right) \delta R\right] \\
- & \eta_{\mu \nu} \partial_{\alpha} \partial_{\beta}\left(f_{P 0} \delta R^{\alpha \beta}\right)-\square\left(f_{P 0} \delta R_{\mu \nu}\right) \\
+ & 2 \partial_{\alpha} \partial_{\beta}\left(f_{P 0} \delta R_{(\mu}^{\alpha} \delta_{\nu)}^{\beta}+2 f_{Q 0} \delta R_{(\mu \nu)}^{\alpha}\right) .
\end{aligned}
$$


It is convenient to work in Fourier space so that, for example, $\partial_{\gamma} h_{\mu \nu} \rightarrow i k_{\gamma} h_{\mu \nu}$ and $\square h_{\mu \nu} \rightarrow-k^{2} h_{\mu \nu}$, where now $k^{2} \equiv k^{\mu} k_{\mu}$. Then, Eq. (17.52) becomes

$$
\begin{aligned}
& F_{0}\left(\delta R_{\mu \nu}-\frac{1}{2} \eta_{\mu \nu} \delta R\right) \\
= & \left(\eta_{\mu \nu} k^{2}-k_{\mu} k_{\nu}\right)\left[\delta \Phi-\frac{2}{3}\left(f_{P 0}+f_{Q 0}\right) \delta R\right] \\
+ & \eta_{\mu \nu} k_{a} k_{b}\left(f_{P 0} \delta R^{a b}\right)+k^{2}\left(f_{P 0} \delta R_{\mu \nu}\right) \\
- & 2 k_{\alpha} k_{\beta}\left(f_{P 0} \delta R_{(\mu}^{\alpha} \delta_{\nu)}^{\beta}\right)-4 k_{\alpha} k_{\beta}\left(f_{Q 0} \delta R_{(\mu \nu)}^{\alpha}{ }^{\beta}\right) .
\end{aligned}
$$

We rewrite the metric perturbation as

$$
h_{\mu \nu}=\bar{h}_{\mu \nu}-\frac{\bar{h}}{2} \eta_{\mu \nu}+\eta_{\mu \nu} h_{f}
$$

and use the gauge freedom to demand that the usual conditions $\partial_{\mu} \bar{h}^{\mu \nu}=0$ and $\bar{h}=0$ hold. The first condition implies that $k_{\mu} \bar{h}^{\mu \nu}=0$, while the second one gives

$$
\begin{aligned}
h_{\mu \nu} & =\bar{h}_{\mu \nu}+\eta_{\mu \nu} h_{f}, \\
h & =4 h_{f} .
\end{aligned}
$$

With these conditions in mind, we have

$$
\begin{aligned}
& \delta R_{\mu \nu}=\frac{1}{2}\left(2 k_{\mu} k_{\nu} h_{f}+k^{2} \eta_{\mu \nu} h_{f}+k^{2} \bar{h}_{\mu \nu}\right), \\
& \delta R=3 k^{2} h_{f}, \\
& k_{\alpha} k_{\beta} \delta R_{(\mu \nu)}^{\alpha}=-\frac{1}{2}\left[\left(k^{4} \eta_{\mu \nu}-k^{2} k_{\mu} k_{\nu}\right) h_{f}+k^{4} \bar{h}_{\mu \nu}\right], \\
& k_{\alpha} k_{\beta} \delta R_{(\mu}^{\alpha} \delta_{\nu)}^{\beta}=\frac{3}{2} k^{2} k_{\mu} k_{\nu} h_{f} .
\end{aligned}
$$

Using Eqs. (17.54)-117.60) in Eq. (17.53), a little algebra yields

$$
\begin{aligned}
& \frac{1}{2}\left(k^{2}-k^{4} \frac{f_{P 0}+4 f_{Q 0}}{F_{0}}\right) \bar{h}_{\mu \nu} \\
& =\left(\eta_{\mu \nu} k^{2}-k_{\mu} k_{\nu}\right) \frac{\delta \Phi}{F_{0}}+\left(\eta_{\mu \nu} k^{2}-k_{\mu} k_{\nu}\right) h_{f} .
\end{aligned}
$$

Defining now $h_{f} \equiv-\delta \Phi / F_{0}$, we find the perturbation equation

$$
k^{2}\left(1+\frac{k^{2}}{m_{\text {spin } 2}^{2}}\right) \bar{h}_{\mu \nu}=0,
$$

where

$$
m_{\text {spin } 2}^{2} \equiv-\frac{F_{0}}{f_{P 0}+4 f_{Q 0}}
$$

while Eq. (17.50) gives

$$
\square h_{f}=m_{s}^{2} h_{f} .
$$


It is easy to see from Eq. (17.62) that we have a modified dispersion relation corresponding to a massless spin two field $\left(k^{2}=0\right)$ and a massive spin two ghost mode with

$$
k^{2}=\frac{2 F_{0}}{f_{P 0}+4 f_{Q 0}} \equiv-m_{\text {spin } 2}^{2}
$$

with mass $m_{\text {spin } 2}^{2}$. In fact, the propagator of $\bar{h}_{\mu \nu}$ can be rewritten as

$$
G(k) \propto \frac{1}{k^{2}}-\frac{1}{k^{2}+m_{\text {spin } 2}^{2}} .
$$

The negative sign of the second term indicates its ghost nature, which agrees with the results found in the literature for this class of theories [62, 169, 183, 440, 447, 535]. As a check, we can see that for the Gauss-Bonnet Lagrangian density $\mathcal{G}=Q-4 P+R^{2}$, we have $f_{P 0}=-4$ and $f_{Q 0}=1$, then Eq. (17.62) simplifies to $k^{2} \bar{h}_{\mu \nu}=0$ and in this case we have no ghosts, as expected.

The solution of Eqs. (17.62) and (17.64) can be expanded in plane waves as

$$
\begin{gathered}
\bar{h}_{\mu \nu}=A_{\mu \nu}(\vec{p}) \exp \left(i k^{\alpha} x_{\alpha}\right)+\text { c.c. }, \\
h_{f}=a(\vec{p}) \exp \left(i q^{\alpha} x_{\alpha}\right)+\text { c.c. },
\end{gathered}
$$

where

$$
\begin{aligned}
& k^{\alpha} \equiv\left(\omega_{m_{\text {spin } 2}}, \vec{p}\right), \quad \omega_{m_{\text {spin } 2}}=\sqrt{m_{\text {spin } 2}^{2}+p^{2}} \\
& q^{\alpha} \equiv\left(\omega_{m_{s}}, \vec{p}\right), \quad \omega_{m_{s}}=\sqrt{m_{s}^{2}+p^{2}}
\end{aligned}
$$

and where $m_{\text {spin } 2}$ is zero (respectively, non-zero) in the case of massless (respectively, massive) spin two modes and the polarization tensor $A_{\mu \nu}(\vec{p})$ is given by Eqs. (21)-(23) of Ref. [555]. In Eqs. (17.62) and (17.67), the equation and the solution for the standard waves of GR [544] have been obtained while Eqs. (17.64) and (17.68) are the equation and the solution for the massive mode, respectively (see also Ref. [134]).

The fact that the dispersion law for the modes of the massive field $h_{f}$ is not linear has to be emphasized. The velocity of every "ordinary" (i.e., arising from GR) mode $\bar{h}_{\mu \nu}$ is the light speed $c$, but the dispersion law (17.70) for the modes of $h_{f}$ is that of a massive field which can be discussed like a wave packet [134]. The group velocity of a wave packet of $h_{f}$ centered in $\vec{p}$ is

$$
\overrightarrow{v_{g}}=\frac{\vec{p}}{\omega}
$$

which is exactly the velocity of a massive particle with mass $m$ and momentum $\vec{p}$. From Eqs. (17.70) and (17.71), it is easy to obtain

$$
v_{g}=\frac{\sqrt{\omega^{2}-m^{2}}}{\omega} .
$$

In order for the wave packet to have constant speed, it must be 134]

$$
m=\sqrt{\left(1-v_{g}^{2}\right)} \omega .
$$

Before proceeding, we discuss the phenomenological constraints on the mass of the gravitational wave field. For frequencies in the range relevant for space-based and terrestrial gravitational antennas, i.e., $10^{-4} \mathrm{~Hz} \leq f \leq 10 \mathrm{kHz}$ [2, 7, 28, 326, 514, 540, 589], a strong constraint is available. For a massive gravitational wave it is

$$
\omega=\sqrt{m^{2}+p^{2}}
$$

and then

$$
0 \mathrm{eV} \leq m \leq 10^{-11} \mathrm{eV}
$$

A stronger bound comes from cosmology and Solar System tests, which provide

$$
0 \mathrm{eV} \leq m \leq 10^{-33} \mathrm{eV} .
$$

The effects of these light scalars can be discussed as those of a coherent gravitational wave. 


\section{New polarization states of gravitational radiation}

Looking at Eq. (17.50) we see that we can have a $k^{2}=0$ mode corresponding to a massless spin two field with two independent polarizations plus a scalar mode while, if $k^{2} \neq 0$, we have a massive spin two ghost mode ("poltergeist") and there are five independent polarization tensors plus a scalar mode. First, let us consider the case in which the spin two field is massless.

Taking $\vec{p}$ in the $z$-direction, a gauge in which only $A_{11}, A_{22}$, and $A_{12}=A_{21}$ are different from zero can be chosen. The condition $\bar{h}=0$ gives $A_{11}=-A_{22}$. In this frame, we can take the polarization bases 40

$$
\begin{gathered}
e_{\mu \nu}^{(+)}=\frac{1}{\sqrt{2}}\left(\begin{array}{ccc}
1 & 0 & 0 \\
0 & -1 & 0 \\
0 & 0 & 0
\end{array}\right), \quad e_{\mu \nu}^{(\times)}=\frac{1}{\sqrt{2}}\left(\begin{array}{lll}
0 & 1 & 0 \\
1 & 0 & 0 \\
0 & 0 & 0
\end{array}\right), \\
e_{\mu \nu}^{(s)}=\frac{1}{\sqrt{2}}\left(\begin{array}{lll}
0 & 0 & 0 \\
0 & 0 & 0 \\
0 & 0 & 1
\end{array}\right) .
\end{gathered}
$$

Substituting these expressions into Eq. (17.54), it follows that

$$
\begin{aligned}
h_{\mu \nu}(t, z) & =A^{+}(t-z) e_{\mu \nu}^{(+)}+A^{\times}(t-z) e_{\mu \nu}^{(\times)} \\
& +h_{s}\left(t-v_{g} z\right) e_{\mu \nu}^{s} .
\end{aligned}
$$

The terms $A^{+}(t-z) e_{\mu \nu}^{(+)}$and $A^{\times}(t-z) e_{\mu \nu}^{(\times)}$describe the two standard polarizations of gravitational waves which arise in GR, while the term $h_{s}\left(t-v_{g} z\right) \eta_{\mu \nu}$ is the massive field arising from the generic $f(R)$ theory.

When the spin two field is massive, the bases of the six polarizations are defined by

$$
\begin{aligned}
& e_{\mu \nu}^{(+)}=\frac{1}{\sqrt{2}}\left(\begin{array}{ccc}
1 & 0 & 0 \\
0 & -1 & 0 \\
0 & 0 & 0
\end{array}\right), \quad e_{\mu \nu}^{(\times)}=\frac{1}{\sqrt{2}}\left(\begin{array}{lll}
0 & 1 & 0 \\
1 & 0 & 0 \\
0 & 0 & 0
\end{array}\right), \\
& e_{\mu \nu}^{(B)}=\frac{1}{\sqrt{2}}\left(\begin{array}{ccc}
0 & 0 & 1 \\
0 & 0 & 0 \\
1 & 0 & 0
\end{array}\right), \quad e_{\mu \nu}^{(C)}=\frac{1}{\sqrt{2}}\left(\begin{array}{lll}
0 & 0 & 0 \\
0 & 0 & 1 \\
0 & 1 & 0
\end{array}\right), \\
& e_{\mu \nu}^{(D)}=\frac{\sqrt{2}}{3}\left(\begin{array}{ccc}
\frac{1}{2} & 0 & 0 \\
0 & \frac{1}{2} & 0 \\
0 & 0 & -1
\end{array}\right), \quad e_{\mu \nu}^{(s)}=\frac{1}{\sqrt{2}}\left(\begin{array}{lll}
0 & 0 & 0 \\
0 & 0 & 0 \\
0 & 0 & 1
\end{array}\right),
\end{aligned}
$$

and the amplitude can be written in terms of the six polarization states as

$$
\begin{aligned}
h_{\mu \nu}(t, z) & =A^{+}\left(t-v_{g_{s 2}} z\right) e_{\mu \nu}^{(+)}+A^{\times}\left(t-v_{g_{s 2}} z\right) e_{\mu \nu}^{(\times)} \\
& +B^{B}\left(t-v_{g_{s 2}} z\right) e_{\mu \nu}^{(B)}+C^{C}\left(t-v_{g_{s 2}} z\right) e_{\mu \nu}^{(C)} \\
& +D^{D}\left(t-v_{g_{s 2}} z\right) e_{\mu \nu}^{(D)}+h_{s}\left(t-v_{g} z\right) e_{\mu \nu}^{s}
\end{aligned}
$$

\footnotetext{
40 These polarizations are defined in the physical three-space. The polarization vectors are orthogonal to each another and are normalized according to $e_{\mu \sigma} e^{\sigma \nu}=2 \delta_{\mu}^{\nu}$. The other modes are not traceless, in contrast to the ordinary "plus" and "cross" polarization modes of GR.
} 

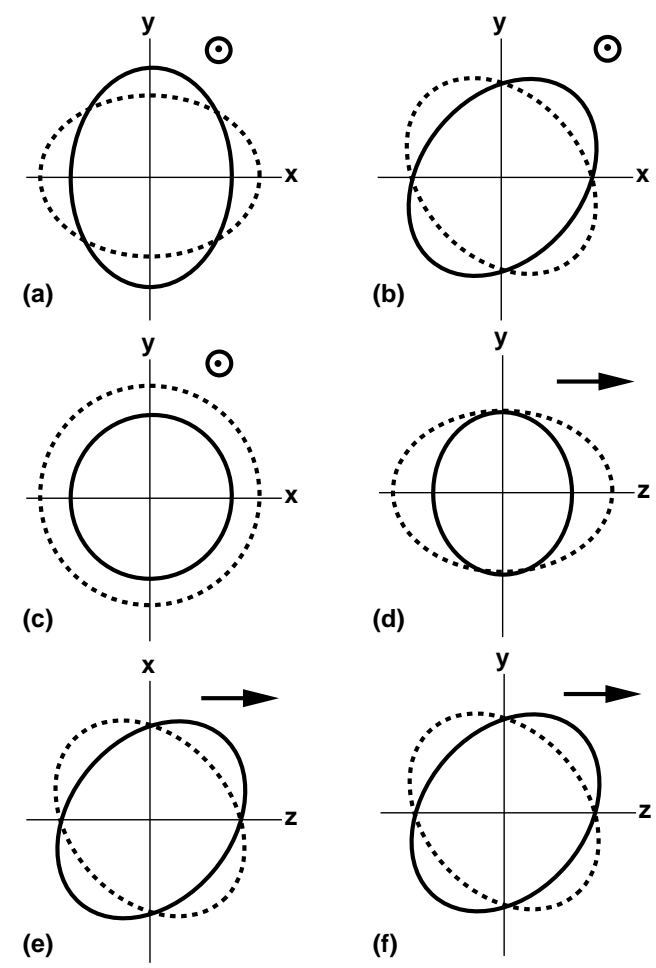

Figure 1. The six polarization modes of gravitational waves. We illustrate the displacement induced at phases spaced by $\pi$ radians by each mode on a circle of test particles at rest before the wave impinges upon them. The wave propagates out of the plane of the page in (a), (b), and (c) and into this plane in (d), (e), and (f). While (a) and (b) describe the "plus" and "cross" modes, respectively, (c) corresponds to the scalar mode, and (d), (e), and (f) to the D, B, and C modes.

where

$$
v_{g_{s 2}}=\frac{\sqrt{\omega^{2}-m_{s 2}^{2}}}{\omega}
$$

is the group velocity of the massive spin two field. The first two polarizations are the same as in the massless case, inducing tidal deformations of the $(x, y)$ plane. Fig. 1 illustrates how each gravitational wave polarization affects test masses arranged on a circle before the wave impinges on them.

From a purely quantum-mechanical point of view, the presence of the ghost mode may seem as a pathology of the theory. There are several reasons why this mode is problematic in the particle interpretation of the metric perturbations. The ghost mode can be viewed as either a particle state with positive energy and negative probability density, or as a positive probability density state with negative energy. In the first case, allowing the presence of such a particle will induce violations of unitarity, while the negative energy scenario leads to a theory without ground state and the system becomes unstable. Vacuum can decay into pairs of ordinary and ghost gravitons leading to a catastrophic instability.

A way out of these problems consists of imposing a very weak coupling of the ghost with the other particles in the theory, such that the decay rate of the vacuum becomes comparable to the inverse of the Hubble time. The present vacuum state will then appear to be sufficiently stable. This is not a viable option in our theory because the ghost state appears in the gravitational sector, which is bound to couple to all forms of matter present and it seems physically and mathematically unlikely for the ghost graviton to couple differently than the ordinary massless graviton does.

Another possibility consists of assuming that this picture does not hold up to arbitrarily high energies and that at some cutoff scale $M_{\text {cutoff }}$ the theory gets modified appropriately to ensure a ghost-free behavior and a stable ground state. This can happen, for example, if we assume that Lorentz-invariance is violated at $M_{\text {cutoff }}$, thereby restricting any potentially harmful decay [243]. However, there is no guarantee that modified gravities like the one investigated here are valid to arbitrarily high energies. Such models are plagued at the quantum level by the same problems of ordinary GR, i.e., they are not renormalizable. It is, therefore, not necessary for them to be considered as genuine candidates for a quantum gravity theory and the corresponding ghost particle interpretation becomes ambiguous. At 
the classical level, the perturbation $h_{\mu \nu}$ should be viewed as nothing more than a tensor representing the stretching of space-time away from flatness. A ghost mode then makes sense as just another way of propagating this perturbation of the space-time geometry, one which, in the propagator, carries a sign opposite to that of an ordinary massive graviton. Viewed in this way, the presence of the massive ghost graviton will induce on an interferometer the same effects as an ordinary massive graviton transmitting the perturbation, but with the opposite sign of the displacement. Tidal stretching of the polarization plane by a polarized wave will turn into shrinking and vice-versa. Eventually, the signal will be a superposition of the displacements coming from the ordinary massless spin two graviton and the massive ghost. Since these two modes induce competing effects, their superposition will lead to a less pronounced signal than the one expected were the ghost mode absent, setting less stringent constraints on the theory. However, the presence of the new modes will also affect the total energy density carried by the gravitational waves and this may also appear as a candidate signal in stochastic gravitational wave backgrounds.

\section{The detector response}

Let us consider now the possible response of a detector in the presence of gravitational waves coming from a definite direction. The detector output depends on the gravitational wave amplitude, which is determined by specific theoretical models. However, one can study the detector response to each gravitational wave polarization without specifying a priori the theoretical model. Following Refs. 3, 35, 83, 272, 375, 403, 566], the angular pattern function of a detector of gravitational waves is given by

$$
\begin{aligned}
& F_{A}(\hat{\Omega})=\mathbf{D}: \mathbf{e}_{A}(\hat{\Omega}), \\
& \mathbf{D}=\frac{1}{2}(\hat{\mathbf{u}} \otimes \hat{\mathbf{u}}-\hat{\mathbf{v}} \otimes \hat{\mathbf{v}}),
\end{aligned}
$$

where $A=+, \times, B, C, D, s$ and : denotes a contraction between tensors. $\mathbf{D}$ is the detector tensor representing the response of a laser-interferometric detector. It maps the metric perturbation in a signal on the detector. The vectors $\hat{\mathbf{u}}$ and $\hat{\mathbf{v}}$ are unitary and orthogonal to each other, they are directed to each detector arm, and they form an orthonormal coordinate basis together with the unit vector $\hat{\mathbf{w}}$ (see Fig. 2 ). $\hat{\Omega}$ is the unit vector directed along the direction of propagation of the gravitational wave. Eq. (17.85) holds only when the arm length of the detector is much smaller than the gravitational wave wavelength, a condition satisfied by ground-based laser interferometers but not by space interferometers such as LISA. A standard orthonormal coordinate system for the detector is

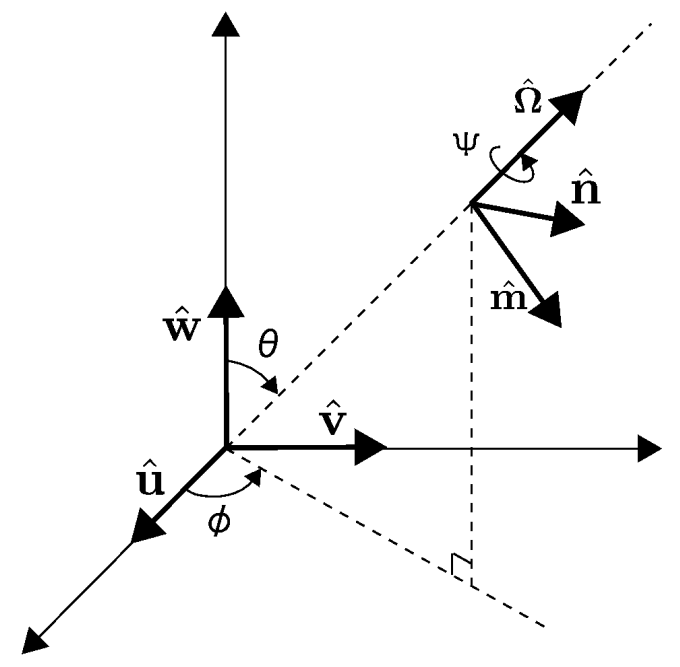

Figure 2. The coordinate systems used to calculate the polarization tensors and a view of the coordinate transformation. 


$$
\begin{gathered}
\hat{\mathbf{u}}=(1,0,0), \\
\hat{\mathbf{v}}=(0,1,0), \\
\hat{\mathbf{w}}=(0,0,1),
\end{gathered}
$$

and the coordinate system for the gravitational wave, rotated by $(\theta, \phi)$, is given by

$$
\begin{aligned}
\hat{\mathbf{u}}^{\prime} & =(\cos \theta \cos \phi, \cos \theta \sin \phi,-\sin \theta), \\
\hat{\mathbf{v}}^{\prime} & =(-\sin \phi, \cos \phi, 0), \\
\hat{\mathbf{w}}^{\prime} & =(\sin \theta \cos \phi, \sin \theta \sin \phi, \cos \theta) .
\end{aligned}
$$

A rotation by the angle $\psi$ around the direction of propagation of the gravitational wave gives the most general choice of coordinates, that is

$$
\begin{aligned}
& \hat{\mathbf{m}}=\hat{\mathbf{u}}^{\prime} \cos \psi+\hat{\mathbf{v}}^{\prime} \sin \psi, \\
& \hat{\mathbf{n}}=-\hat{\mathbf{v}}^{\prime} \sin \psi+\hat{\mathbf{u}}^{\prime} \cos \psi, \\
& \hat{\Omega}=\hat{\mathbf{w}}^{\prime} .
\end{aligned}
$$

The coordinates $(\hat{\mathbf{u}}, \hat{\mathbf{v}}, \hat{\mathbf{w}})$ are related to $(\hat{\mathbf{m}}, \hat{\mathbf{n}}, \hat{\Omega})$ by the rotation angles $(\phi, \theta, \psi)$, as shown in Fig. 2 Using the vectors $\hat{\mathbf{m}}, \hat{\mathbf{n}}$, and $\hat{\Omega}$, the polarization tensors are

$$
\begin{aligned}
& \mathbf{e}_{+}=\frac{1}{\sqrt{2}}(\hat{\mathbf{m}} \otimes \hat{\mathbf{m}}-\hat{\mathbf{n}} \otimes \hat{\mathbf{n}}), \\
& \mathbf{e}_{\times}=\frac{1}{\sqrt{2}}(\hat{\mathbf{m}} \otimes \hat{\mathbf{n}}+\hat{\mathbf{n}} \otimes \hat{\mathbf{m}}), \\
& \mathbf{e}_{B}=\frac{1}{\sqrt{2}}(\hat{\mathbf{m}} \otimes \hat{\Omega}+\hat{\Omega} \otimes \hat{\mathbf{m}}), \\
& \mathbf{e}_{C}=\frac{1}{\sqrt{2}}(\hat{\mathbf{n}} \otimes \hat{\Omega}+\hat{\Omega} \otimes \hat{\mathbf{n}}) . \\
& \mathbf{e}_{D}=\frac{\sqrt{3}}{2}\left(\frac{\hat{\mathbf{m}}}{\mathbf{2}} \otimes \frac{\hat{\mathbf{m}}}{\mathbf{2}}+\frac{\hat{\mathbf{n}}}{\mathbf{2}} \otimes \frac{\hat{\mathbf{n}}}{\mathbf{2}}+\hat{\Omega} \otimes \hat{\Omega}\right), \\
& \mathbf{e}_{s}=\frac{1}{\sqrt{2}}(\hat{\Omega} \otimes \hat{\Omega}) .
\end{aligned}
$$


Taking into account Eqs. (17.85) and (17.86), the angular patterns for each polarization are

$$
\begin{aligned}
F_{+}(\theta, \phi, \psi) & =\frac{1}{\sqrt{2}}\left(1+\cos ^{2} \theta\right) \cos 2 \phi \cos 2 \psi \\
& -\cos \theta \sin 2 \phi \sin 2 \psi, \\
F_{\times}(\theta, \phi, \psi) & =-\frac{1}{\sqrt{2}}\left(1+\cos ^{2} \theta\right) \cos 2 \phi \sin 2 \psi \\
& -\cos \theta \sin 2 \phi \cos 2 \psi, \\
F_{B}(\theta, \phi, \psi) & =\sin \theta(\cos \theta \cos 2 \phi \cos \psi-\sin 2 \phi \sin \psi), \\
F_{C}(\theta, \phi, \psi) & =\sin \theta(\cos \theta \cos 2 \phi \sin \psi+\sin 2 \phi \cos \psi), \\
F_{D}(\theta, \phi) & =\frac{\sqrt{3}}{32} \cos 2 \phi\left[6 \sin ^{2} \theta+(\cos 2 \theta+3) \cos 2 \psi\right], \\
F_{s}(\theta, \phi) & =\frac{1}{\sqrt{2}} \sin ^{2} \theta \cos 2 \phi .
\end{aligned}
$$

The angular pattern functions for each polarization are plotted in Fig. 3. Even if we have considered a different model, these results are consistent, for example, with those of Refs. 3, 219, 220, 272, 435, 547].

Another area of research which we do not discuss here consists of the study of the stochastic background of gravitational waves which may contain the possible signature of extra gravitational wave modes and be relevant for the detectability of these contributions to gravitational radiation.

The above analysis covers extended gravity models with a generic class of higher order Lagrangian densities and Lagrangian terms of the form $f(R, P, Q)$. We have linearized the field equations of these theories around a Minkowski background and found that, in addition to a massless spin two field, the theory contains also spin zero and two massive modes with the latter being, in general, ghosts. If the interferometer is directionally sensitive and we also know the orientation of the source (and, of course, if the source is coherent) the discussion is straightforward. In this case, the massive mode coming from the simplest extension of GR, i.e., $f(R)$ gravity, would induce longitudinal displacements along the direction of propagation of the wave, which should be detectable, and only the scalar mode would be the detectable truly new signal [134]. But, even in this case, there could be a second scalar mode inducing a similar effect and representing a massive ghost, although with a negative sign.

For the situation considered here, massive modes are certainly of interest for the LISA space interferometer. It is in principle possible that massive gravitational wave modes could be produced in more significant quantities in cosmological or early astrophysical processes in alternative theories of gravity, a possibility which is still largely unexplored. This situation should be kept in mind when looking for a signature capable of distinguishing these theories from GR, and it seems to deserve further investigation.

\section{Concluding remarks}

The weak-field limit of ETGs shows new aspects of gravitation which are not present in GR. The Newtonian and post-Newtonian limits give weak-field potentials which are not of the standard Newtonian form. The corrections, in general, are Yukawa-like terms which could explain in a very natural fashion several astrophysical and cosmological observations [11, 119, 135].

The post-Minkowskian limit of ETGs exhibits new gravitational field modes which can easily be interpreted as massive gravitons.

The study of generation, propagation, and detection of GWs in the weak-field limit of a given relativistic theory of gravity is an important part of astrophysics. Primordial gravitational waves generated during the early epochs of the Universe (especially during inflation) would allow, when detected, to rule out or constrain certain theories and investigate others. The detection of GWs of astrophysical or cosmological origin can hardly be over emphasized because it would open a new branch of astronomy providing information which is not accessible with visible, infrared, optical, X-ray, or $\gamma$-ray astronomy. In fact, GWs can be generated in regions deep inside supernovae, near black hole 


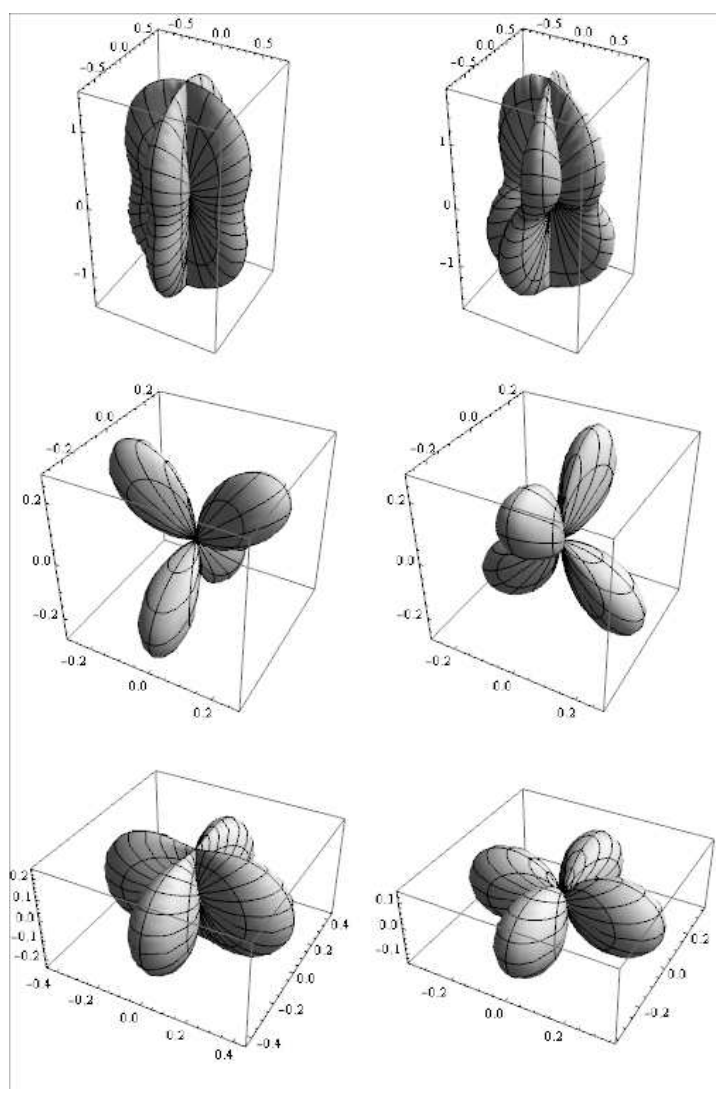

Figure 3. Angular pattern functions of an interferometric detector for the various polarizations. From left to right and from top to bottom, one sees constant level surfaces corresponding to the "plus", "cross", B, C, D, and scalar modes.

horizons, or very early in the history of the Universe when the latter is completely opaque to photons. The study of relativistic astrophysics related to GWs in ETGs is a broad and complex subject for which could open new interesting scenarios.

\section{CONCLUSIONS AND PERSPECTIVES}

In this Report, we have outlined the theoretical foundations of Extended Theories of Gravity, a new approach aimed to address and solve shortcomings and inconsistencies of General Relativity. These problems essentially come out at infra-red and ultra-violet scales, that is at cosmological and astrophysical scales from one side and at quantum scales from the other side. The issue is that to overcome the lack of explanation (at fundamental level) of cosmic dark sector and the lack of a self-consistent theory of quantum gravity, people are driving new patterns with two objectives: $i$ ) maintaining the good results of GR by extending its approach; $i i$ ) avoiding the introduction of new ingredients in the cosmic pie since no final evidence exists, up to now, for them. This could appear nothing else but a trial and error approach if a robust theoretical structure is missing.

We have shown, that ETGs can be traced back within the fold of gauge theories like GR. In fact gauge invariants, local gauge transformations, and symmetries plays the same fundamental role as in GR. As final result, GR is nothing else but a particular case of a more extended class of theories were higher-order curvature invariants and non-minimal couplings have to be considered.

Besides, space-time deformations play an interesting role in this line of thinking since it is clear that they are related not only with GR but with large families of theories. However the well founded results of GR must be recovered in any case. This means that the Equivalence Principle, the geodesic and causal structures, the post-Newtonian dynamics and the post-Minkowskian limit have to be constistently addressed by any ETGs. In our opinion, a part the urgency of a full quantization of gravity, this issues are the test-bed of any theory of gravity. In any case such features could result very different with respect to the Einstein theory but they have to be recovered as soon as ETGs $\longrightarrow$ GR. The first need of "corrections" to GR emerges when Quantum Field Theory is formulated on curved space-time. 
Normalization and regularization processes lead to non-minimal couplings and higher-order corrections in curvature invariants. These new terms do not give rise to finite renormalizable series but ensure regular perturbative behaviours at least at one-loop level. Starting from these considerations, it straightforward to observe that any unification theory predicts effective actions where higher-order curvature terms or non-minimal coupling are present. On the other hand, at larger scales, the Mach Principle asks for non-minimal couplings and cosmological issues as inflation and dark energy can be addressed in the realm of these enlarged schemes. In this sense, ETGs have become a paradigm in modern theoretical physics. In the track of GR, variational principles and field equations can be obtained for any ETGs. However the field equations can result of order higher than second and very difficult to handle. Instead of GR, metric structure and geodesic structure cannot coincide and then dynamics is described by the metric field $g$ and the connection field $\Gamma$. In this case, we deal with the Palatini or metric-affine formalism. The standard metric formalism is recovered by observing that the Palatini approaches endowed with a bi-metric structure is strictly connected to the conformal structure. However we have to point out that, in theories like $f\left(R, R_{\mu \nu} R^{\mu \nu}\right)$ as those discussed in [46, 386, 458], the bi-metric structure is much more general than the conformal structure. Specifically, it can be discussed in the most general frame of deformations considered in Sec IV.

At this point, it is worth stressing that the whole geometric budget of a generic relativistic theory of gravity could include also torsion beside curvature. It is well known that such an ingredient can represent the geometric counterpart of spin as mass-energy is the source of curvature. We discussed in detail the role of torsion in $f(R)$-gravity showing the straightforward relation with the metric-affine formalism and scalar tensor theories. In a genuine gauge-formalism, torsion, curvature, and matter can be represented in the $\mathcal{J}$-bundle framework. This picture is particularly relevant if we want to put in evidence symmetries and conserved quantities. We have taken into account the case of $f(R)$-gravity with different matter fields acting as source in the field equations.

Also the Hamiltonian representation can give an interesting insight for ETGs. For example, the cosmological constant can be recovered as an eigenvalue of the $f(R)$-Hamiltonian constructed by the ADM formalism. It is straightforward to achieve one-loop energy regularization and renormalization by this approach.

One the most important issues related to ETGs is the Initial Value Problem. In order to construct a self-consistent theory of physics, as GR or Electromagnetism, this problem needs to be well-formulated and well-posed. We have discussed the Cauchy problem for scalar tensor and $f(R)$-gravity adopting the ADM formulation and the Gaussian normal coordinates approach. It results that well-formulation and well-position are always possible in metric formalism. In the metric-affine approach, the well-formulation is recovered while the well-position strictly depends on the matter-field acting as source. The Initial Value Poblem is still an open question that needs to be fully addressed for the self-consistency of ETGs. However, as recently reported in [459], the ADM methods results extremely suitable to investigate such a problem in the Palatini formalism indicating that not only the well-formulation but also the well-position is at hand.

One of the crucial achievement of a give theory of physics is the possibility to get exact solutions. In this case, one has the full control of initial conditions and dynamics. Among the applications of ETGs, we have discussed the spherical and axial symmetry with the aim to get exact solutions. It is interesting to see that while spherical solutions are essentially Schwarzschild-like in GR (with constant or null curvature), in $f(R)$-gravity we have several possibilities essentially related to the fact that also curvature dependent on radial coordinate has to be considered. We have developed various techniques to get exact spherical solutions and have shown how it is possible to generate axially symmetric solutions, starting from spherical ones, by the Newman-Janis algorithm.

The post-Newtonian limit of ETGs gives rise to several results that cannot be found in standard GR. The main achievement is the fact that Newtonian potential has to be corrected thanks to the higher-order and non-minimal coupling terms in the field equations. The corrections are essentially Yukawa-like and the physical implication is that new characteristic lengths emerge beside the standard Schwarzschild radius related to the gravitational mass. This feature can have dramatic consequences in astrophysics and cosmology as discussed in [121, 129, 135]. An implication could emerge also for quantum effects like neutrino oscillations when considered in presence of gravitational fields [151].

Also the post-Minkowskian limit of ETGs deserves an accurate consideration, in particular with respect to the problem of gravitational radiation. In fact, new polarizations emerge as soon as the gravitational action is not the Hilbert-Einstein one: in general, massive, massless, and ghost modes have to be taken into account, while only massless modes and two polarizations are present in GR. This result implies a revision of the physics of GWs. In fact, detectors should be designed and calibrated also in view of these possibilities. On the other hand, the detection of gravitational massive modes (or modes different from those predicted by GR) could be the final "experimentum crucis" for ETGs.

In conclusion, we can say that such theories seem a reliable approach to address the gravity problem at various scales also if no final result is available up to day. In this Report we have investigated the theoretical foundations of ETGs in order to show that they are not only phenomenological toy models but satisfy the issues of modern gauge theories. The validity of this approach could be definitely confirmed or ruled out by forthcoming experiments (e.g. at LHC see [152]) or by fine and comprehensive astrophysical observations. 


\section{ACKNOWLEDGMENTS}

We are indebted to many collaborators and colleagues for discussions, results and remarks reported in this work. In particular we thank G. Basini, T. Bernal, C. Bogdanos, M. Bouhmadi López, S. Carloni, V.F. Cardone, R. Cianci, F. Darabi, A. De Felice, M. Demianski, R. de Ritis, P.K.S. Dunsby, E. Elizalde, L. Fabbri, V. Faraoni, L. Fatibene, M. Francaviglia, R. Garattini, X. Hernandez, J. C. Hidalgo, G. Lambiase, S. Mendoza, S. Mercadante, D.F. Mota, S. Nesseris, S. Nojiri, S.D. Odintsov, G. J. Olmo, L. Perivolaropoulos, E. Piedipalumbo, C. Rubano, D. Saez-Gomez, V. Salzano, P. Scudellaro, A. Stabile, C. Stornaiolo, A. Troisi, S. Tsujikawa, D. Vernieri, S. Vignolo. We wish to thank Prof. R. Petronzio for his kind invitation to write this Report. We are supported by Istituto Nazionale di Fisica Nucleare (INFN) and by the Università di Napoli "Federico II". 
APPENDIX A. NOTATIONS

Mathematical symbols

\begin{tabular}{|c|c|}
\hline$\partial_{\mu}=\frac{\partial}{\partial x^{\mu}}$ & Partial derivative with respect to $\left\{x_{\mu}\right\}$ \\
\hline$\left\{e_{\mu}\right\}:$ & Set with elements $e_{\mu}$ \\
\hline$\nabla_{\mu}=\partial_{\mu}+\Gamma_{\mu}:$ & Gauge covariant derivative operator \\
\hline$\Gamma_{\mu}:$ & Gauge potential 1-form \\
\hline$d:$ & Exterior derivative operator \\
\hline$\langle V \mid e\rangle:$ & Inner multiplication between vector $e$ and 1 -form $V$ \\
\hline$[A, B]:$ & Commutator of operators $A$ and $B$ \\
\hline$\{A, B\}:$ & Anti-commutator of operators $A$ and $B$ \\
\hline$\wedge:$ & Exterior multiplication operator \\
\hline$\rtimes:$ & Semi-direct product \\
\hline $\mathcal{M}:$ & differential manifold \\
\hline $\mathcal{U}_{4}:$ & $4 D$-manifold with torsion \\
\hline $\mathcal{V}_{4}:$ & $4 D$ pseudo-Riemaniann manifold without torsion \\
\hline $\mathcal{E}:$ & co-frame bundle of $\mathcal{M}$ \\
\hline $\mathcal{J}:$ & jet-bundle \\
\hline$\times:$ & Direct product \\
\hline$\times_{\mathcal{M}}:$ & Fibered product over manifold $\mathcal{M}$ \\
\hline$\rfloor:$ & Inner product \\
\hline$\oplus:$ & Direct sum \\
\hline$\otimes:$ & Tensor product \\
\hline$A \cup B:$ & Union of $A$ and $B$ \\
\hline$A \cap B:$ & Intersection of $A$ and $B$ \\
\hline$\Theta(\bar{\Theta}):$ & Right (left) invariant Maurer-Cartan 1-form \\
\hline$\circ:$ & Group (element) composition operator \\
\hline$\eta_{\alpha \beta}=\operatorname{diag}(-1,1$ & Lorentz group metric (flat metric) \\
\hline$G L(4, \mathbb{R}):$ & Group of real $4 \times 4$ invertible matrices \\
\hline$S O(3,1):$ & Lorentz group \\
\hline$P(3,1):$ & Poincaré group \\
\hline $\mathfrak{g}:$ & Lie algebra of group $G$ \\
\hline$g \in G:$ & Element $g$ of $G$ \\
\hline$\{\mathcal{U}\} \subset \mathcal{M}:$ & Set $\mathcal{U}$ is a subset of $\mathcal{M}$ \\
\hline G : & Algebra generator of group $G$ \\
\hline$\rho(\mathbf{G}):$ & Representation of $G$-algebra \\
\hline${ }^{*} A:$ & Dual of $A$ with respect to (coordinate) basis indices \\
\hline$\epsilon_{a_{1} \ldots a_{n}}$ or $\varepsilon_{a_{1} \ldots a_{n}}:$ & Levi-Civita totally skew tensor density \\
\hline$\eta_{a_{1} \ldots a_{n}}:$ & Eta basis volume $n$-form density \\
\hline$\sigma^{*}:$ & Pullback by local section $\sigma$ \\
\hline$L_{h *}:$ & Differential (pushforward) map induced by $L_{h}$ \\
\hline$T_{\left(a_{1} \ldots a_{n}\right)}:$ & Symmetrization of indices \\
\hline$T_{\left[a_{1} \ldots a_{n}\right]}:$ & Antisymmetrization of indices \\
\hline$T(\mathcal{M}):$ & Tangent space to manifold $\mathcal{M}$ \\
\hline$f: A \rightarrow B:$ & Map $f$ taking elements $\{a\} \in A$ to $\{b\} \in B$ \\
\hline
\end{tabular}


Convention for the gravitational field

$\begin{array}{ll}\text { Standard gravitational coupling } & \kappa=\frac{8 \pi G_{N}}{c^{4}} \\ \text { Index ranges } & \alpha, \beta=0,1,2,3 ; i, j=1,2,3 \\ \text { Coordinates } & x^{\alpha}=\left(x^{0}, x^{1}, x^{2}, x^{3}\right)=\left(c t, x^{1}, x^{2}, x^{3}\right) \\ \text { Vectors } & \underline{\mathrm{v}}=\left(v^{1}, v^{2}, v^{3}\right) ; \nabla=\left(\partial / \partial x^{1}, \partial / \partial x^{2}, \partial / \partial x^{3}\right) \\ \text { Symmetrization } & T_{(\alpha|\beta \ldots \gamma| \delta)}=\frac{1}{2}\left(T_{\alpha \beta \ldots \gamma \delta}+T_{\delta \beta \ldots \gamma \alpha}\right) \\ \text { Kronecker } & \delta_{\beta}^{\alpha}=1 \text { if } \alpha=\beta, 0 \text { else } \\ \text { Connection } & \Gamma_{\mu \nu}^{\alpha}=\frac{1}{2} g^{\alpha \sigma}\left(g_{\mu \sigma, \nu}+g_{\nu \sigma, \mu}-g_{\mu \nu, \sigma}\right) \\ \text { Riemann tensor } & R_{\beta \mu \nu}^{\alpha}=\Gamma_{\beta \nu, \mu}^{\alpha}-\Gamma_{\beta \mu, \nu}^{\alpha}+\Gamma_{\beta \nu}^{\sigma} \Gamma_{\sigma \mu}^{\alpha}-\Gamma_{\beta \mu}^{\sigma} \Gamma_{\sigma \nu}^{\alpha} \\ \text { Ricci tensor } & R_{\mu \nu}=R_{\mu \sigma \nu}^{\sigma} \\ & \end{array}$

\section{APPENDIX B. ZETA FUNCTION REGULARIZATION}

In this appendix, we report details on computation leading to expression (13.79). We begin with the following integral

$$
\rho(\varepsilon)=\left\{\begin{array}{l}
I_{+}=\mu^{2 \varepsilon} \int_{0}^{+\infty} d \omega \frac{\omega^{2}}{\left(\omega^{2}+m^{2}(r)\right)^{\varepsilon-\frac{1}{2}}} \\
I_{-}=\mu^{2 \varepsilon} \int_{0}^{+\infty} d \omega \frac{\omega^{2}}{\left(\omega^{2}-m^{2}(r)\right)^{\varepsilon-\frac{1}{2}}}
\end{array}\right.
$$

with $m^{2}(r)>0$.

\section{$I_{+}$computation}

If we define $t=\omega / \sqrt{m^{2}(r)}$, the integral $I_{+}$in Eq. (18.1) becomes

$$
\begin{aligned}
\rho(\varepsilon) & =\mu^{2 \varepsilon} m^{4-2 \varepsilon}(r) \int_{0}^{+\infty} d t \frac{t^{2}}{\left(t^{2}+1\right)^{\varepsilon-\frac{1}{2}}} \\
& =\frac{1}{2} \mu^{2 \varepsilon} m^{4-2 \varepsilon}(r) B\left(\frac{3}{2}, \varepsilon-2\right) \\
& \frac{1}{2} \mu^{2 \varepsilon} m^{4-2 \varepsilon}(r) \frac{\Gamma\left(\frac{3}{2}\right) \Gamma(\varepsilon-2)}{\Gamma\left(\varepsilon-\frac{1}{2}\right)} \\
= & \frac{\sqrt{\pi}}{4} m^{4}(r)\left(\frac{\mu^{2}}{m^{2}(r)}\right)^{\varepsilon} \frac{\Gamma(\varepsilon-2)}{\Gamma\left(\varepsilon-\frac{1}{2}\right)}
\end{aligned}
$$

where we have used the following identities involving the beta function

$$
B(x, y)=2 \int_{0}^{+\infty} d t \frac{t^{2 x-1}}{\left(t^{2}+1\right)^{x+y}} \quad \operatorname{Re} x>0, \operatorname{Re} y>0
$$

related to the gamma function by means of

$$
B(x, y)=\frac{\Gamma(x) \Gamma(y)}{\Gamma(x+y)}
$$


Taking into account the following relations for the $\Gamma$-function

$$
\begin{gathered}
\Gamma(\varepsilon-2)=\frac{\Gamma(1+\varepsilon)}{\varepsilon(\varepsilon-1)(\varepsilon-2)} \\
\Gamma\left(\varepsilon-\frac{1}{2}\right)=\frac{\Gamma\left(\varepsilon+\frac{1}{2}\right)}{\varepsilon-\frac{1}{2}}
\end{gathered}
$$

and the expansion for small $\varepsilon$

$$
\begin{array}{cc}
\Gamma(1+\varepsilon)= & 1-\gamma \varepsilon+O\left(\varepsilon^{2}\right) \\
\Gamma\left(\varepsilon+\frac{1}{2}\right)=\Gamma\left(\frac{1}{2}\right)-\varepsilon \Gamma\left(\frac{1}{2}\right)(\gamma+2 \ln 2)+O\left(\varepsilon^{2}\right), \\
x^{\varepsilon}= & 1+\varepsilon \ln x+O\left(\varepsilon^{2}\right)
\end{array}
$$

where $\gamma$ is the Euler's constant, we find

$$
\rho(\varepsilon)=-\frac{m^{4}(r)}{16}\left[\frac{1}{\varepsilon}+\ln \left(\frac{\mu^{2}}{m^{2}(r)}\right)+2 \ln 2-\frac{1}{2}\right] .
$$

\section{$I_{-}$computation}

If we define $t=\omega / \sqrt{m^{2}(r)}$, the integral $I_{-}$in Eq. (18.1) becomes

$$
\begin{gathered}
\rho(\varepsilon)=\mu^{2 \varepsilon} m^{4-2 \varepsilon}(r) \int_{0}^{+\infty} d t \frac{t^{2}}{\left(t^{2}-1\right)^{\varepsilon-\frac{1}{2}}} \\
=\frac{1}{2} \mu^{2 \varepsilon} m^{4-2 \varepsilon}(r) B\left(\varepsilon-2, \frac{3}{2}-\varepsilon\right) \\
\frac{1}{2} \mu^{2 \varepsilon} m^{4-2 \varepsilon}(r) \frac{\Gamma\left(\frac{3}{2}-\varepsilon\right) \Gamma(\varepsilon-2)}{\Gamma\left(-\frac{1}{2}\right)} \\
=-\frac{1}{4 \sqrt{\pi}} m^{4}(r)\left(\frac{\mu^{2}}{m^{2}(r)}\right)^{\varepsilon} \Gamma\left(\frac{3}{2}-\varepsilon\right) \Gamma(\varepsilon-2),
\end{gathered}
$$

where we have used the following identity involving the beta function

$$
\begin{gathered}
\frac{1}{p} B\left(1-\nu-\frac{\mu}{p}, \nu\right)=\int_{1}^{+\infty} d t t^{\mu-1}\left(t^{p}-1\right)^{\nu-1} \\
p>0, \operatorname{Re} \nu>0, \operatorname{Re} \mu<p-p \operatorname{Re} \nu
\end{gathered}
$$

and the reflection formula

$$
\Gamma(z) \Gamma(1-z)=-z \Gamma(-z) \Gamma(z)
$$

From the first of Eqs. 18.5) and from the expansion for small $\varepsilon$

$$
\begin{gathered}
\Gamma\left(\frac{3}{2}-\varepsilon\right)=\Gamma\left(\frac{3}{2}\right)(1-\varepsilon(-\gamma-2 \ln 2+2))+O\left(\varepsilon^{2}\right) \\
x^{\varepsilon}=1+\varepsilon \ln x+O\left(\varepsilon^{2}\right),
\end{gathered}
$$

we find

$$
\rho(\varepsilon)=-\frac{m^{4}(r)}{16}\left[\frac{1}{\varepsilon}+\ln \left(\frac{\mu^{2}}{m^{2}(r)}\right)+2 \ln 2-\frac{1}{2}\right] .
$$




\section{APPENDIX C. THE FIELD EQUATIONS AND THE NOETHER VECTOR FOR SPHERICALLY} SYMMETRIC $f(R)$-GRAVITY

The field equations of metric $f(R)$-gravity with spherical symmetry are

$$
\begin{aligned}
H_{00} & =2 A^{2} B^{2} M f+\left\{B M A^{\prime 2}-A\left[2 B A^{\prime} M^{\prime}+M\left(2 B A^{\prime \prime}-A^{\prime} B^{\prime}\right)\right]\right\} f_{R} \\
& +\left(-2 A^{2} M B^{\prime} R^{\prime}+4 A^{2} B M^{\prime} R^{\prime}+4 A^{2} B M R^{\prime \prime}\right) f_{R R} \\
& +4 A^{2} B M R^{\prime 2} f_{R R R}=0 \\
H_{r r} & =2 A^{2} B^{2} M^{2} f+\left(B M^{2} A^{\prime 2}+A M^{2} A^{\prime} B^{\prime}+2 A^{2} M B^{\prime} M^{\prime}+2 A^{2} B M^{\prime 2}\right. \\
& \left.-2 A B M^{2} A^{\prime \prime}-4 A^{2} B M M^{\prime \prime}\right) f_{R}+\left(2 A B M^{2} A^{\prime} R^{\prime}\right. \\
& \left.+4 A^{2} B M M^{\prime} R^{\prime}\right) f_{R R}=0,
\end{aligned}
$$

$$
\begin{aligned}
H_{\theta \theta} & =2 A B^{2} M f+\left(4 A B^{2}-B A^{\prime} M^{\prime}+A B^{\prime} M^{\prime}-2 A B M^{\prime \prime}\right) f_{R} \\
& +\left(2 B M A^{\prime} R^{\prime}-2 A M B^{\prime} R^{\prime}+2 A B M^{\prime} R^{\prime}+4 A B M R^{\prime \prime}\right) f_{R R} \\
& +4 A B M R^{\prime 2} f_{R R R}=0 \\
& =H_{\varphi \varphi}=\sin ^{2} \theta H_{\theta \theta}=0 .
\end{aligned}
$$

The trace of the field equations is

$$
\begin{aligned}
H & =g^{\mu \nu} H_{\mu \nu}=4 A B^{2} M f-2 A B^{2} M R f_{R}+3\left(B M A^{\prime} R^{\prime}-A M B^{\prime} R^{\prime}\right. \\
& \left.+2 A B M^{\prime} R^{\prime}+2 A B M R^{\prime \prime}\right) f_{R R}+6 A B M R^{\prime 2} f_{R R R}=0
\end{aligned}
$$

The system (15.67) is derived from the condition for the existence of a Noether symmetry $\mathcal{L}_{\mathbf{X}} L=0$. Considering the configuration space $\underline{q}=(A, M, R)$ and defining the Noether vector $\underline{\alpha}=\left(\alpha_{1}, \alpha_{2}, \alpha_{3}\right)$, the system (15.67) assumes the 
explicit form

$$
\begin{aligned}
& \xi\left(\frac{\partial \alpha_{2}}{\partial A} f_{R}+M \frac{\partial \alpha_{3}}{\partial A} f_{R R}\right)=0, \\
& \frac{A}{M}\left[(2+M R) \alpha_{3} f_{R R}-\frac{2 \alpha_{2}}{M} f_{R}\right] f_{R} \\
+ & \xi\left[\left(\frac{\alpha_{1}}{M}+2 \frac{\partial \alpha_{1}}{\partial M}+\frac{2 A}{M} \frac{\partial \alpha_{2}}{\partial M}\right) f_{R}+A\left(\frac{\alpha_{3}}{M}+4 \frac{\partial \alpha_{3}}{\partial M}\right) f_{R R}\right]=0, \\
& \xi\left(M \frac{\partial \alpha_{1}}{\partial R}+2 A \frac{\partial \alpha_{2}}{\partial R}\right) f_{R R}=0, \\
& \alpha_{2}\left(f-R f_{R}\right) f_{R}-\xi\left[\left(\alpha_{3}+M \frac{\partial \alpha_{3}}{\partial M}+2 A \frac{\partial \alpha_{3}}{\partial A}\right) f_{R R}\right. \\
& \left.+\left(\frac{\partial \alpha_{2}}{\partial M}+\frac{\partial \alpha_{1}}{\partial A}+\frac{A}{M} \frac{\partial \alpha_{2}}{\partial A}\right) f_{R}\right]=0, \\
& {\left[M(2+M R) \alpha_{3} f_{R R}-2 \alpha_{2} f_{R}\right] f_{R R}+\xi\left[f_{R} \frac{\partial \alpha_{2}}{\partial R}\right.} \\
& \left.+\left(2 \alpha_{2}+M \frac{\partial \alpha_{1}}{\partial A}+2 A \frac{\partial \alpha_{2}}{\partial A}+M \frac{\partial \alpha_{3}}{\partial R}\right) \alpha_{3} f_{R R}+M f_{R R R}\right]=0 \\
2 A & {\left[(2+M R) \alpha_{3} f_{R R}-\left(f-R f_{R}\right) \alpha_{2}\right] f_{R R}+\xi\left[\left(\frac{\partial \alpha_{1}}{\partial R}+\frac{A}{M} \frac{\partial \alpha_{2}}{\partial R}\right) f_{R}\right.} \\
+ & \left.\left(2 \alpha_{1}+2 A \frac{\partial \alpha_{3}}{\partial R}+M \frac{\partial \alpha_{1}}{\partial M}+2 A \frac{\partial \alpha_{2}}{\partial M}\right) f_{R R}+2 A \alpha_{3} f_{R R R}\right]=0,
\end{aligned}
$$

with the condition $\xi=(2+M R) f_{R}-M f \neq 0$ to guarantee the non-vanishing of the Hessian of the Lagrangian (15.59).

[1] L.F. Abbott, Nucl. Phys. B 185, 233, (1981).

[2] B. Abbott et al., Phys. Rev. D 72, 042002, (2005).

[3] B. Abbott et al., Phys. Rev. D 76, 082003, (2007).

[4] B. Abbott et al. (LIGO Scientific Collaboration), Class. Quantum Grav. 27, 173001, (2010).

[5] J.C. Adams, On the Perturbations of Uranus Cambridge University Press, Cambridge, UK (1846).

[6] A.J. Accioly, S. Ragusa, E.C. de Rey Neto, H. Mukai, Nuovo Cimento B 114, 595, (1999).

[7] F. Acernese et al., Class. Quantum Grav. 24, S381, (2007).

[8] F. Adams, K. Freese, A. Guth, Phys. Rev. D 43, 965, (1991).

[9] Y. Aharonov, D. Bohm, Phys Rev 123, 1511, (1961).

[10] D.V. Ahluwalia, C. Burgard, Gen. Relativ. Grav. 28, 1161 (1996).

[11] H.G. Alexander, The Leibniz-Clarke correspondence, Manchester University Press, Manchester, UK (1998) .

[12] M.M. Ali, A.S. Majumdar, D. Home and A.K. Pan, Class. Quant. Grav. 23, 6493, (2006).

[13] S.A. Ali and S. Capozziello, Int. Jou. Geom. Meth. Mod. Phys. 4, 1041,(2007).

[14] G. Allemandi, A. Borowiec, M. Francaviglia, Phys. Rev. D 70, 103503, (2004).

[15] G. Allemandi, M. Francaviglia, M.L. Ruggiero, A. Tartaglia, Gen. Relat. Gravit. 37, 1891, (2005).

[16] G. Allemandi, M. Capone, S. Capozziello, M. Francaviglia, Gen. Rel. Grav. 38, 33, (2006).

[17] B. Allen, Nucl. Phys. B 226, 232, (1983).

[18] B. Allen, Phys. Rev. D 30, 1153, (1984).

[19] G. Amelino-Camelia, Mod. Phys. Lett. A 17, 899, (2002).

[20] L. Amendola, S. Tsujikawa, Dark energy theory and observations, Cambridge Univ. Press., Cambridge, UK (2010).

[21] L. Amendola, K. Enqvist, T. Koivisto, Phys. Rev. D 83, 044016, (2011).

[22] L. Amendola, S. Capozziello, M. Litterio, F. Occhionero, Phys. Rev. D 45, 417, (1992).

[23] L. Amendola, A. Battaglia-Mayer, S. Capozziello, S. Gottlöber, V. Müller, F. Occhionero, H.J. Schmidt, Class. Quantum Grav. 10, L43, (1993).

[24] L.A. Anchordoqui, S.E. Perez-Bergliaffa, M.L. Trobo, G.S. Birman, Phys. Rev. D 57, 829, (1998). 
[25] J.L. Anderson, Phys. Rev. D 3, 1689, (1971).

[26] J.D. Anderson et al., Phys. Rev. Lett. 81, 2858, (1998).

[27] J.D. Anderson et al., Phys. Rev. D 65, 082004, (2002).

[28] M. Ando et al., Class. Quantum Grav. 19, 1615, (2002).

[29] V.I. Arnold, Mathematical Methods of Classical Mechanics, Springer-Verlag, Berlin, (1978).

[30] R. Arnowitt, S. Deser, C.W. Misner, in Gravitation: An Introduction to Current Research, ed. by L. Witten Wiley, New York, (1962).

[31] M. Artigas, W.R. Shea, Galileo in Rome: The Rise and Fall of a Troublesome Genius, Oxford University Press, Oxford, (2004).

[32] A. Ashtekar, A. Corichi, Class. Quantum Grav. 20, 4473, (2003).

[33] J. Audretsch, Phys. Rev. D 27, 2872, (1983).

[34] J. Audretsch and C. Lämmerzhal, J. Math. Phys. 32, 2099 (1991).

[35] D. Babusci, L. Baciotti, F. Fucito, A. Nagar, Phys. Rev. D 64, 062001, (2001).

[36] S. Baghram, M. Farhang, S. Rahvar, Phys. Rev. D 75, 044024, (2007).

[37] M. Banados, C. Teitelboim and J. Zanelli, Phys. Rev. Lett. 69, 1849, (1992).

[38] M. Banados, M. Henneaux, C. Teitelboim and J. Zanelli, Phys. Rev. D 48 1506, (1993).

[39] E.C. Banks, Ernst Mach's World Elements, Kluwer Academic Publishers, Dordrecht, The Netherlands (2003).

[40] N. Banerjee, S. Sen, Phys. Rev. D 56, 1334, (1997).

[41] E. Barausse, T.P. Sotiriou, J. Miller, Class. Quantum Grav. 25, 062001, (2008).

[42] E. Barausse, T.P. Sotiriou, J. Miller, Class. Quantum Grav. 25, 105008, (2008).

[43] E. Barausse, T.P. Sotiriou, J. Miller, EAS Publ. Ser. 30, 189, (2008).

[44] J. M. Bardeen,Phys. Rev. D 22, 1882, (1980).

[45] G. Barenboim, N.E. Mavromatos, Phys.Rev. D 70, 093015, (2004).

[46] C. Barragan, G.J. Olmo Phys. Rev. D 82, 084015, (2010).

[47] C. Barragan, G.J. Olmo, H. Sanchis-Alepuz Phys. Rev. D 80, 024016 (2009).

[48] A. Barroso, J. Casasayas, P. Crawford, P. Moniz, A. Nunes, Phys. Lett. B 275, 264, (1992).

[49] A. Barros, C. Romero, Phys. Lett. A 245, 31, (1998).

[50] J.D. Barrow, S. Cotsakis, Phys. Lett. B 214, 515, (1988).

[51] J.D. Barrow, A.C. Ottewill, J. Phys. A 16, 2757 (1983).

[52] J. D. Barrow and M. Madsen, Nucl. Phys. B 323, 242 (1989).

[53] J.D. Barrow, K. Maeda, Nucl. Phys. B 341, 294, (1990).

[54] J.D. Barrow, T. Clifton, Class. Quantum Grav. 23, L1, (2006).

[55] D.F. Bartlett, Am. J. Phys. 62, 8, (1994).

[56] D.F. Bartlett, Int. J. Mod. Phys. A 16S1B, 680, (2001).

[57] G. Basini , S. Capozziello, Gen. Rel. Grav. 35, 2217, (2003).

[58] G. Basini and S. Capozziello, Mod. Phys. Lett. A 20, 251, (2005).

[59] B.A. Bassett, M. Kunz, D. Parkinson, C. Ungarelli, Phys. Rev. D 68, 043504, (2003).

[60] A. Battaglia-Mayer, H.J. Schmidt, Class. Quantum Grav. 10, 2441, (1993).

[61] D. Bazeia, B. Carneiro da Cunha, R. Menezes, A.Yu. Petrov, Phys. Lett. B 649,445, (2007).

[62] D. Bazeia, R. Menezes, A. Yu. Petrov, Eur. Phys. J. C 58, 171, (2008).

[63] V.A. Belinsky, I.M. Khalatnikov, Sov. Phys. JETP 36, 591, (1973).

[64] S. Bellucci, S. Capozziello, M. De Laurentis, V. Faraoni, Phys. Rev. D 79, 104004, (2009).

[65] A. Berkin, K. Maeda, Phys. Lett. B 245, 348, (1990).

[66] M. Berger and D. Ebin, J. Diff. Geom. 3, 379, (1969).

[67] P. G. Bergmann, Introduction to the Theory of Relativity, Prentice-Hill, New York, (1964).

[68] P. G. Bergmann, Physics Today, 44, (1979).

[69] Ch. P. L. Berry, J. R. Gair, Phys. Rev. D 83, 104022, (2011).

[70] O. Bertolami, C.G. Böhmer, F.S.N. Lobo, Phys. Rev. D 75, 104016, (2007).

[71] B. Bertotti, L. Iess, P. Tortora, Nature 425, 374, (2003).

[72] T. Bhattacharya, S. Habib, E. Mottola, Phys. Rev D 59, 067301, (1999).

[73] N. Bilić, B. Guberina, R. Horvat, H. Nikolić, H. Stefancić, Phys. Lett. B 657, 232, (2007).

[74] J. Binney, S. Tremaine, Galactic Dynamics, Princeton University Press, Princeton (1987).

[75] N.D. Birrell, P.C.W. Davies, Phys. Rev. D 22, 322, (1980).

[76] N.D. Birrell, P.C.W. Davies, Quantum Fields in Curved Space, Cambridge University Press, Cambridge, UK (1982).

[77] J.D. Bjorken and S. Drell, Relativistic Quantum Fields, mcGraw-Hill, New York, (1965).

[78] M. Blagojevic Three Lectures on Poincaré Gauge Theory, SFIN A1 (2003) 147.

[79] A. Blasi, N. Maggiore, S.P. Sorella, L.C.Q. Vilar, Phys. Rev. D 59, 121701, (1999).

[80] M. Blasone, A. Capolupo, S. Capozziello, S. Carloni, G. Vitiello, Phys. Lett. A, 323,182, (2004).

[81] A. Bonanno, Phys. Rev. D 52, 969, (1995).

[82] A. Bonanno, D. Zappalá, Phys. Rev. D 55, 6135, (1997).

[83] N. Bonasia, M. Gasperini, Phys. Rev. D 71, 104020, (2005).

[84] H. Bondi, Cosmology, Cambridge Univ. Press, Cambridge, UK (1952).

[85] H. Bondi, Mon. Not. R. Astron. Soc. 107, 410 (1947).

[86] C.H. Brans, R.H. Dicke, Phys. Rev. 124, 925, (1961). 
[87] D.R. Brill et al. Rev. Mod. Phys. 29, 465, (1957).

[88] D.R. Brill, R.H. Gowdy, Rep. Prog. Phys. 33, 413, (1970).

[89] T. Bringmann, C. Kiefer, D. and Polarski, Phys. Rev. D, 65, 024008, (2002).

[90] I.L. Buchbinder, Fortschr. Phys. 34, 605, (1986).

[91] I.L. Buchbinder, S.D. Odintsov, Sov. J. Nucl. Phys. 40, 848, (1984).

[92] I.L. Buchbinder, S.D. Odintsov, Lett. Nuovo Cimento 42, 379, (1985).

[93] I.L. Buchbinder, S.D. Odintsov, I. Shapiro, in Group-Theoretical Methods in Physics, ed. by M. Markov, Nauka, Moscow, (1986).

[94] I.L. Buchbinder, S.D. Odintsov, I. Shapiro, Effective Action in Quantum Gravity IOP Publishing, Bristol, (1992).

[95] I.L. Buchbinder, S.D. Odintsov, I. Lichzier, Class. Quantum Grav. 6, 605, (1989).

[96] H.A. Buchdahl, Acta Math. 85, 63, (1951).

[97] H.A. Buchdahl, J. Phys. A 12, 8, 1229, (1979).

[98] H.A. Buchdahl, J. Phys. A 12, 8, 1235, (1979).

[99] A. Burkert, Astroph. Journ. 447, L25, (1995).

[100] F. Calogero, A. Degasperis, Am. J. Phys. 72, 1202, (2004).

[101] S. Capozziello and R. de Ritis, Class. Quant.Grav 11, 107, (1994).

[102] S. Capozziello, R. de Ritis, A.A. Marino, Class. Quantum Grav. 14, 3243, (1997).

[103] S. Capozziello, R. de Ritis, A.A. Marino, Gen. Relativ. Grav. 30, 1247, (1998).

[104] S. Capozziello and G. Lambiase, Mod. Phys. Lett. A 14, 2193, (1999).

[105] S. Capozziello, G. Lambiase and C. Stornaiolo, Ann. Phys. (Leipzig) 10 , 713, (2001).

[106] S. Capozziello, Int. J. Mod. Phys. D 11, 483, (2002).

[107] S. Capozziello, V.F. Cardone, E. Piedipalumbo , M. Sereno , A. Troisi, Int. J. Mod. Phys. D 12, 38,1, (2003).

[108] S. Capozziello, V.F. Cardone, S. Carloni, A. Troisi, Int. J. Mod. Phys. D 12, 1969, (2003).

[109] S. Capozziello, S. Carloni, A. Troisi, Rec. Res. Dev. Astron. Astrophys. 1, 625, (2003).

[110] S. Capozziello, G.Lambiase, G. Iovane, Mod. Phys. Lett. A 18, 905, (2003).

[111] S. Capozziello, V.F. Cardone, S. Carloni, A. Troisi, Phys. Lett. A 326, 292, (2004).

[112] S. Capozziello, F.V. Cardone, M. Funaro, S. Andreon, Phys. Rev. D 70, 123501, (2004).

[113] S. Capozziello, In: Quantum Gravity Research Trends, Editor: Albert Reimer, 227, Nova Science Publishers, Inc. (2005).

[114] S. Capozziello, A. Troisi, Phys. Rev. D, 72, 044022, (2005).

[115] S. Capozziello, V.F. Cardone, A. Troisi, Phys. Rev. D, 71, 043503, (2005).

[116] S. Capozziello, V.F. Cardone, E. Elizalde, S. Nojiri, S.D. Odintsov, Phys. Rev. D 73, 043512, (2006).

[117] S. Capozziello, S. Nojiri, S.D. Odintsov, A. Troisi, Phys. Lett. B 639, 135, (2006).

[118] S. Capozziello, S. Nojiri, S.D. Odintsov, Phys. Lett. B 634, 93, (2006).

[119] S. Capozziello, V.F. Cardone, A. Troisi, Jou. Cosm. and Astrop. Phys. 0608, 001, (2006).

[120] S. Capozziello, A. Stabile, A. Troisi, Mod. Phys. Lett. A 21, 2291, (2006).

[121] S. Capozziello, V.F. Cardone, A. Troisi, Mont. Not. R. Astr. Soc. 375, 1423, (2007).

[122] S. Capozziello, A. Stabile, A. Troisi, Phys. Rev. D 76, 104019, (2007).

[123] S. Capozziello, C. Corda. M. De Laurentis, Mod. Phys. Lett. A 22, 1097, (2007).

[124] S. Capozziello, A. Stabile, A. Troisi, Class. Quant. Grav. 24, 2153, (2007).

[125] S. Capozziello, P. K. S. Dunsby, E. Piedipalumbo, and C. Rubano, Astr. 85 Astrop. 472, (1), 51, (2007).

[126] S. Capozziello, R. Cianci, C. Stornaiolo and S. Vignolo, Class. Quantum. Grav. 24, 6417, (2007).

[127] S. Capozziello, R. Cianci, C. Stornaiolo, S. Vignolo, Int. J. Geom. Meth. Mod. Phys. 5, 765, (2008).

[128] S. Capozziello, R. Cianci, C. Stornaiolo and S. Vignolo, Physica Scripta, 78, 065010, (2008).

[129] S. Capozziello and M. Francaviglia, Gen. Rel. Grav.: Special Issue on Dark Energy 40, 357, (2007).

[130] S. Capozziello, R. Cianci, C. Stornaiolo, S. Vignolo, Int.J.Geom.Meth.Mod.Phys. 5, 765, (2008).

[131] S. Capozziello, A. Stabile, A. Troisi, Phys. Rev. D 76, 104019, (2008).

[132] S. Capozziello, A. Stabile, A. Troisi, Class. Quantum Grav. 25, 085004, (2008).

[133] S. Capozziello, S. Tsujikawa, Phys. Rev. D 77, 107501, (2008).

[134] S. Capozziello, C. Corda, M. De Laurentis, Phys. Lett. B 699, 255, (2008).

[135] S. Capozziello , E. De Filippis , V. Salzano , Mon. Not. R. Ast. Soc. 394, 947, (2009).

[136] S. Capozziello, A. Stabile, A. Troisi, Mod. Phys. Lett. A 24, 659, (2009).

[137] S. Capozziello, M . De Laurentis Int.J.Geom.Meth.Mod.Phys. 6, 1, (2009).

[138] S. Capozziello, A. Stabile, Class. Quantum Grav. 26, 085019, (2009).

[139] S. Capozziello, M. De Laurentis, Found Phys 40 867, (2010).

[140] Capozziello S., De Laurentis M., Faraoni V., The Special Issue in Cosmology, The Open Astronomy Journal 2, 1874, (2009).

[141] S. Capozziello, M. De Laurentis, M. Francaviglia, S. Mercadante S., Foundations of Physics 39, 1161, (2009).

[142] S. Capozziello, E. Elizalde, S. Nojiri, S.D. Odintsov, Phys. Lett. B 671, 193, (2009).

[143] S. Capozziello, S. Vignolo, Class. Quantum Grav. 26, 168001, (2009).

[144] S. Capozziello, S. Vignolo, Class. Quantum Grav. 26, 175013, (2009).

[145] S. Capozziello, S. Vignolo, Int. J. Geom. Meth. Mod. Phys. 6, 985, (2009).

[146] S. Capozziello, V. Salzano, Advances in Astronomy, special issue "Dark Sides of Our Universe", 217420, 34, (2009).

[147] S. Capozziello, V. Faraoni, Beyond Einstein Gravity: A Survey Of Gravitational Theories For Cosmology And Astrophysics, Fundamental Theories of Physics 170, Springer, New York (2011). 
[148] S. Capozziello and M. De Laurentis, Invariance Principles and Extended Gravity: Theory and Probes, Nova Science Publishers, New York (2011).

[149] S. Capozziello, M. De Laurentis, A. Stabile, Class. Quantum Grav. 27, 165008, (2010).

[150] S. Capozziello, P. Martin-Moruno, C. Rubano, Phys. Lett. B 689, 117 (2010).

[151] S. Capozziello, M. De Laurentis, D. Vernieri, Mod. Phys. Lett. A, 25, 1163, (2010).

[152] S. Capozziello, G. Basini, M. De Laurentis, Eur. Phys. J. C 71, 1679, (2011).

[153] V.F. Cardone, A. Troisi, S. Capozziello, Phys. Rev. D 69, 083517, (2004).

[154] S. Carloni, P.K.S. Dunsby, S. Capozziello, A. Troisi, Class. Quantum Grav. 22, 4839, (2005).

[155] M. Carrera, D. Giulini, Rev. Mod. Phys. 82, 169, (2010).

[156] S. Carroll et al., Phys. Rev. D, 50 3867, (1994).

[157] S.M. Carroll, A. De Felice, V. Duvvuri, D.A. Easson, M. Trodden, M.S. Turner, Phys. Rev. D 71, 063513, (2005).

[158] S.M. Carroll, V. Duvvuri, M. Trodden, M.S. Turner, Phys. Rev. D 70, 043528, (2004).

[159] E. Cartan, Ann. Ec. Norm. 42, 17, (1925).

[160] M. Castagnino and A. Ordonez, Rend. Mat. Serie VII, 9, 299, (1989).

[161] J.A.R. Cembranos, Phys. Rev. D 73, 064029, (2006).

[162] L. N. Chang et al., Phys. Rev. D 13, 235, (1976).

[163] S. Chandrasekhar The mathematical theory of black holes, Oxford University Press, USA (1983).

[164] X. Chen, in Proceedings COSMO 2000, 4th International Workshop on Particle Physics and the Early Universe, Cheju Island, Korea, 2000, ed. by J.E. Kim, P. Ko, K. Lee (World Scientific, Singapore, 2001).

[165] X. Chen, R.J. Scherrer, G. Steigman, Phys. Rev. D 63, 123504, (2001).

[166] G. Chen, B. Ratra, Astrophys. J. 582, 586, (2003).

[167] G. Chen, B. Rathra, Ap. J. 582, 586, (2003).

[168] T. Chiba, Phys. Lett. B 575, 1, (2003).

[169] T. Chiba, J. Cosmol. Astropart. Phys. 0503, 008, (2005).

[170] T. Chiba, T.L. Smith, A. Erickcek, Phys. Rev. D 75, 124014, (2007).

[171] J.S. Chisolm, Nucl. Phys. 26, 469, (1961).

[172] Y.M. Cho, Phys. Rev. Lett. 68, 3133, (1992).

[173] Y.M. Cho, Class. Quantum Grav. 14, 2963, (1997).

[174] Y. Choque-Bruhat, C. de Witt-Morette and M. Dillard-Bleick, Analysis, Manifolds and Physics, revised edition, NorthHolland, New-York (1982).

[175] R. Cianci, S. Vignolo and D. Bruno, J. Phys. A: Math. Gen. 36, 8341, (2003).

[176] R. Cianci, S. Vignolo and D. Bruno, J. Phys. A: Math. Gen. 37, 2519, (2004).

[177] T. Clifton, Phys. Rev. D 77, 024041, (2008).

[178] T. Clifton, J.D. Barrow, Phys. Rev. D 72, 103005, (2005).

[179] T. Clifton, J.D. Barrow, Class. Quantum Grav. 23, 2951, (2006).

[180] T. Clifton, P.G. Ferreira, A. Padilla, C. Skordis e-Print: arXiv:1106.2476 [astro-ph.CO]

[181] J. Cocke, J.M. Cohen, J. Math. Phys. 9, 971, (1968).

[182] G. Cognola, E. Elizalde, S. Nojiri, S.D. Odintsov, S. Zerbini, JCAP 0502, 010, (2005).

[183] G. Cognola , E. Elizalde, S. Nojiri, S.D. Odintsov, S. Zerbini, Phys.Rev. D 73, 084007, (2006).

[184] R. A. Coleman and H. Korte, J. Math. Phys. 25, 3513, (1984).

[185] S. Coleman, J. Wess, B. Zumino, Phys. Rev. 177, 2239, (1969).

[186] J.I Collar, Phys. Rev. Lett. 76, 999, (1996).

[187] B. Coll , A universal law of gravitational deformation for General Relativity, Proc. of the Spanish Relativistic Meeting, EREs, Salamanca Spain, (1998).

[188] B. Coll, J. Llosa, D. Soler, Gen. Rel. Grav. 34, 269, (2002).

[189] E.J. Copeland, M. Sami, S. Tsujikawa, Int. J. Mod. Phys. D 15, 1753, (2006).

[190] S. Cotsakis, P.J. Saich, Class. Quantum Grav. 11, 383, (1994).

[191] R. Courant, D. Hilbert, Methods of Mathematical Physics, Interscience, New York, (1962).

[192] D.H. Coule, K. Maeda, Class. Quantum Grav. 7, 955, (1990).

[193] D.H. Coule, Class. Quantum Grav. 9, 2353, (1992).

[194] M.P. Dabrowski, T. Denkiewicz, D. Blaschke, Ann. Phys. (Leipzig) 16, 237, (2007).

[195] T. Damour, G.W. Gibbons, C. Gundlach,Phys. Rev. Lett. 64, 123, (1990).

[196] T. Damour, G. Esposito-Farèse, Class. Quantum Grav. 9, 2093, (1992).

[197] T. Damour, A.M. Polyakov, Nucl. Phys. B 423, 532, (1994).

[198] A. Davidson, Class. Quantum Grav. 22, 1119, (2005).

[199] F. Daxecker, The Physicist and Astronomer, Christoph Scheiner: Biography, Letters, Works. Veroffentlichungen der Universitat Innsbruck 246 (2004).

[200] P. de Bernardis et al., Nature 404, 955, (2000).

[201] W.J.G. de Blok, A. Bosma, Astron. Astroph., 385, 816, (2002).

[202] G. C. Debney, R. P. Kerr and A. Schild, J. Math. Phys. 10, 1842, (1969).

[203] A. De Felice, S.Tsujikawa, Living Rev. Rel. 13, 3, (2010).

[204] A. Degasperis, S.N.M. Ruijsenaars, Ann. Phys. (N.Y.) 293, 92, (2001).

[205] M. De Laurentis, S. Capozziello, to appear in Astropart. Phys. DOI:10.1016/j.astropartphys.2011.08.006.

[206] R. de Ritis, M. Lavorgna, G. Platania and C. Stornaiolo, Phys. Rev. D 28, 713, (1984). 
[207] S. Drake, Discoveries and Opinions of Galileo. (1610 Letter to the Grand Duchess Christina), Anchor Books, (1957).

[208] S. Drake, Galileo at work: his scientific biography, The University of Chicago Press, Chicago, (1978).

[209] S. Deser, Ann. Phys. (NY) 59, 248 (1970).

[210] S. Deser, R.I. Nepomechie, Ann. Phys. (NY) 154, 396, (1984).

[211] B.S. De Witt, in Battelle Rencontres, ed. by C. DeWitt, J.A. Wheeler (Benjamin, New York, 1968).

[212] B.S. De Witt, Dynamical theory of groups and ...elds (Les Houches Lectures 1963), Relativity, Groups and Topology, Gordon and Breach Science Publishers, New York (1965).

[213] B.S. De Witt, Phys. Rev. 160, 1113, (1967).

[214] R. Dick, Gen. Relat. Gravit. 36, 217, (2004).

[215] R.H. Dicke, The many faces of Mach in Gravitation and Relativity eds. H.Y. Chiu and W.F. Hofmann, Benjamin, New York, (1964).

[216] R.H. Dicke, Phys. Rev. D 125, 2163, (1962).

[217] R.H. Dicke, P.J.E. Peebles, Phys. Rev. Lett. 12, 435, (1964).

[218] R. D'Inverno, Introducing Einstein's Relativity Oxford University Press, New York, (1992).

[219] D. di Serafino, S. Gomez, L. Milano, F. Riccio, G. Toraldo, Jou. of Global Optimization 48, 41 (2010).

[220] D. di Serafino, G. Liuzzi, V. Piccialli, F. Riccio and G. Toraldo Jou. of Global Optimization, doi: 10.1007/s10898-0109525-9.

[221] A.D. Dolgov, M. Kawasaki Phys. Lett. B 573, 1, (2003).

[222] S. Drake, Galileo's Discovery of the Law of Free Fall. Scientific American, 228, 5, 84, (1973).

[223] W. Dreschler and M. E. Mayer, Fiber Bundle Thechniques in Gauge Theories, Springer-Verlag, Heidelberg, (1977).

[224] S.L. Dubovsky, P.G. Tiyakov, I.I. Tkachev, Phys. Rev. Lett. 94, 181102, (2005).

[225] J.P. Duruisseau , R. Kerner, Gen. Rel. Grav. 15, 797, (1983).

[226] J.P. Duruisseau and R. Kerner,Gen. Relativ. Grav. 15, 797, (1983).

[227] G.R. Dvali, G. Gabadadze, M. Porrati, Phys. Lett. B 485, 208, (2000).

[228] E. Dyer, K. Hinterbichler, Phys. Rev. D 79, 024028, (2009).

[229] F.J. Dyson, Phys. Rev. 73, 929, (1948).

[230] D.A. Easson, Int. J. Mod. Phys. A 19, 5343, (2004).

[231] J. Ehlers and A. Schild, Comm. Math. Phys. 32, 119, (1973).

[232] J. Ehlers, F.A.E. Pirani and A. Schild, in General Relativity, Papers in Honor of J.L. Synge, edited by O'Raifeartaigh, Oxford University Press, Oxford, (1972).

[233] A. Einstein, Ann. der Physik 49, 769, (1916).

[234] A. Einstein The Meaning of Relativity, Princeton University, New Jersey, (1955).

[235] A. Einstein, Sitzung-ber. Preuss. Akad. Wiss., 414, (1925).

[236] J. Eisberg, Eddington's stellar models and early twentieth century astrophysics, Ph.D. Thesis, Harvard University, (1991).

[237] E. Elizalde, S.D. Odintsov, Phys. Lett. B 333, 33,1, (1994).

[238] E. Elizalde, S.D. Odintsov, A. Romeo, A.A. Bytsenko, S. Zerbini Zeta regularization techniques with applications, World Scientific, Singapore (1994).

[239] E Elizalde, S Nojiri and S D Odintsov, Phys. Rev. D 59, 061501, (1999).

[240] E. Elizalde, S. Naftulin, S.D. Odintsov, Int. J. Mod. Phys. A 9, 933, (1994).

[241] G.F.R. Ellis, Gen. Rel. and Grav., GR10 Conf. Rep. Ed. B. Bertotti, Reidel, Dordrecht 215, (1984).

[242] L.P. Eisenhart, Riemannian Geometry Princeton Univ. Press, Princeton, (1955).

[243] R. Emparan, J. Garriga, J. High Energy Phys. 0603, 028, (2006).

[244] A.L. Erickcek, T.L. Smith, M. Kamionkowski, Phys. Rev. D 74, 121501, (2006).

[245] H. Ewen and H.-J. Schmidt, J. Math. Phys. 30, 1480, (1989).

[246] R. Fakir, W.G. Unruh, Phys. Rev. D 41, 3012, (1990).

[247] R. Fakir, W.G. Unruh, Phys. Rev. D 41, 1792, (1990).

[248] R. Fakir, S. Habib, W.G. Unruh, Astrophys. J. 394, 396, (1992).

[249] R. Fakir, S. Habib, Mod. Phys. Lett. A 8, 2827, (1993).

[250] V. Faraoni, S. Sonego, in Proceedings of the 5th Canadian Conference on General Relativity and Relativistic Astrophysics, Waterloo, Canada 1993, ed. by R.B. Mann, R.G. McLenaghan ,Singapore: World Scientific, 386 (1994).

[251] V. Faraoni, Phys. Rev. D 53, 6813, (1996).

[252] V. Faraoni, Proceedings of the 7th Canadian Conference on General Relativity and Relativistic Astrophysics, Calgary, Canada (1997) .

[253] V. Faraoni, Phys. Lett. A 245, 26, (1998).

[254] V. Faraoni, E. Gunzig, Int. J. Mod. Phys. D 8, 177, (1998).

[255] V. Faraoni, Phys. Rev. D 59, 084021, (1999).

[256] V. Faraoni, Int. J. Theor. Phys. 40, 2259, (2001).

[257] V. Faraoni, Cosmology in Scalar-Tensor Gravity,Kluwer Academic, Dordrecht, (2004).

[258] V. Faraoni, Class. Quantum Grav. 22, 32352, (2005).

[259] V. Faraoni, Phys. Rev. D 74, 104017, (2006).

[260] V. Faraoni, Phys. Rev. D 74, 023529, (2006).

[261] V. Faraoni, S. Nadeau, Phys. Rev. D 75, 023501, (2007).

[262] V. Faraoni, Phys. Rev. D 75, 067302, (2007).

[263] V. Faraoni, Phys. Lett. B 665, 135, (2008). 
[264] V. Faraoni, Class. Quantum Grav. 26, 168002, (2009).

[265] V. Faraoni, Class. Quantum Grav. 26, 145014, (2009).

[266] V. Faraoni and T.P. Sotiriou Rev. Mod. Phys. 82, 451, (2010).

[267] M. Ferraris, M. Francaviglia, G. Magnano, Class. Quantum Grav. 5, L95, (1988).

[268] M. Ferraris, M. Francaviglia, C. Reina, Gen. Relat. Gravit. 14, 243, (1982).

[269] M. Ferraris, M. Francaviglia, I. Volovich, Class. Quantum Grav. 11, 1505, (1994).

[270] M. Ferraz Figuero, A. Saa, Phys. Rev. D, 80, 063504, (2009).

[271] M. Fierz, Helv. Phys. Acta 29, 128, (1956).

[272] L.S. Finn, P.J. Sutton, Phys. Rev. D 65, 044022, (2002).

[273] E.E. Flanagan, Phys. Rev. Lett. 92, 071101, (2004).

[274] E.E. Flanagan, Class. Quantum Grav. 21, 3817, (2004).

[275] G.L. Fogli, E. Lisi, A. Marrone, A. Melchiorri, A. Palazzo, P. Serra, J. Silk, Phys. Rev .D 70, 113003, (2004).

[276] L.H. Ford, D.J. Toms, Phys. Rev. D 25, 1510, (1982).

[277] L.H. Ford, Phys. Rev. D 32, 1409, (1987).

[278] P.W. France, Am. Juor. Phys., 44, 798, (1976).

[279] K. Freese, M. Lewis, Phys. Lett. B 540, 1, (2002).

[280] C. Frigerio Martins, P. Salucci, Mon. Not. R. Astron. Soc. 381, 1103, (2007).

[281] T. Futamase, K. Maeda, Phys. Rev. D 39, 399, (1989).

[282] T. Futamase, T. Rothman, R. Matzner, Phys. Rev. D 39, 405, (1989).

[283] T. Futamase, M. Tanaka, Phys. Rev. D 60, 063511, (1999).

[284] J. Garcia-Bellido, A. Linde, Phys. Rev. D 52, 6730, (1995).

[285] R. Garattini, TSPU Vestnik 44 N7, 72 (2004);

R. Garattini, J. Phys. Conf. Ser. 33215 (2006);

R. Garattini, J. Phys. A 39 6393, (2006).

[286] R. Garattini, Int. J. Mod. Phys. D 4, 635 (2002);

R. Garattini, J. Phys. A 40, 7055 (2007).

[287] M. Gasperini, G. Veneziano, Phys. Lett. B 277, 256, (1992).

[288] M. Gasperini,Phys. Rev. Let. 56, 2873,(1986).

[289] M. Gasperini, Phys. Lett. B 470, 67, (1999).

[290] R. Gautreau, Phys. Rev. D 29, 198, (1984).

[291] G. Germán, Phys. Rev. D., 32, 3307, (1985).

[292] R. Geroch, Comm. Math. Phys. 13, 180, (1969).

[293] R. Geroch and P. S. Jang, J. Math. Phys. 16, 65, (1975).

[294] E.N. Glass, J.J.G. Scanio, Am. J. Phys. 45, 344, (1977).

[295] J. Gleick, Isaac Newton. Pantheon Publisher, New York (2003).

[296] H. Goldstein Classical Mechanics, Addison-Wesley, Reading, Mass, (1959).

[297] S. Gottlöber, H.-J. Schmidt, A.A. Starobinsky, Class. Quantum Grav. 7, 893, (1990).

[298] M.B. Green, J.H Schwarz ., E. Witten , Superstring Theory, Cambridge Univ. Press, Cambridge, (1987).

[299] A.A. Grib, E.A. Poberii, Helv. Phys. Acta 68, 380, (1995).

[300] A.A. Grib, W.A. Rodrigues, Gravit. Cosmol. 1, 273, (1995).

[301] G. Grignani et. al., Phys. Rev. D 45, 2719, (1992).

[302] M. Grosser, The Discovery of Neptune, Harvard University Press. (1962).

[303] Y. Grossman, H.J. Lipkin, Phys. Rev. D 55, 2760, (1997).

[304] D.J. Gross, M.J. Perry and L.G. Yaffe, Phys. Rev. D 25, 330, (1982) .

[305] D. Grumiller, W. Kummer, D.V. Vassilevich, J. High Energy Phys. 7, 009, (2003).

[306] M. Gürses and F. Gürsey, J. Math. Phys. 16, 2385, (1975).

[307] G. S. Hall and D. P. Lonie, J. Phys. A 39 2995, (2006).

[308] J.J. Halliwell, R. Laflamme, Class. Quantum Grav. 6, 1839, (1989).

[309] E.R. Harrison, Phys. Rev. D 6, 2077, (1972).

[310] S. Hawking, On the Shoulders of Giants: The Great Works of Physics and Astronomy. Running Press, London, UK (2002).

[311] S.W. Hawking, G.F.R. Ellis, The Large Scale Structure of Space-Time, Cambridge University Press, Cambridge, UK (1973).

[312] F. W. Hehl et al.J. Math. Phys. 12 1334, (1971) .

[313] F. W. Hehl, P. von der Heyde and G. D. Kerlick, Phys. Rev. D., 10,1066, (1974).

[314] F. W. Hehl, P. von der Heyde, G. D. Kerlick and J. M. Nester, Rev. Mod. Phys. 48, 393, (1976).

[315] F. W. Hehl and G. D. Kerling, Gen. Rel. Grav. 9, 691, (1978).

[316] F. W. Hehl, E. A. Lord and L. L. Smalley, Gen. Rel. Grav. 13, 1037, (1981).

[317] F. W. Hehl, J. D. McCrea, E. W. Mielke and Y. Ne'eman, Phys. Rept. 258, 1, (1995).

[318] P.W. Higgs, Nuovo Cimento 11, 816, (1959).

[319] C.T. Hill, D.S. Salopek, Ann. Phys. (NY) 213, 21, (1992).

[320] G. Hinshaw et al., Astrophys. J. (Suppl.) 148, 135, (2003).

[321] W.A. Hiscock, Class. Quantum Grav. 7, L35, (1990).

[322] C.J. Hogan, Rev. Mod. Phys., 72, 1149, (2000). 
[323] Y. Hosotani, Phys. Rev. D 32, 1949, (1985).

[324] http://www.onera.fr/prix-en/brun2002/edmond-brun2002.html

[325] http://astro.estec.esa.nl/astrogen/COSPAR/step/

[326] http://www.lisa.esa.int

[327] http:// lhc.web.cern.ch/lhc/

[328] W. Hu, I. Sawicki, Phys. Rev. D 76, 064004, (2007).

[329] S. Huerfano, S. Sahu, M. Socolovsky, Int. J. of Pure and Applied Math. 49 153, (2008).

[330] R.A. Hulse and J.H. Taylor, Ap. J. 195, L51, (1975).

[331] J.C. Hwang, H. Noh, Phys. Rev. Lett. 81, 5274, (1998).

[332] J.C. Hwang, Class. Quantum Grav. 7, 1613, (1990).

[333] E.L. Ince, Ordinary Differential Equations, Dover, New York (1956).

[334] A. Inomata et al., Phys. Rev. D 19, 1665, (1979).

[335] L. Iorio, Adv. High Energy Phys. 2009, 90731, (2007).

[336] R.A. Isaacson, Phys. Rev. 166, 1272 , (1968).

[337] J. Ishikawa, Phys. Rev. D 28, 2445, (1983).

[338] C.J. Isham, in Quantum Gravity 2: A Second Oxford Symposium, edited by C.J. Isham, R. Penrose, D.W. Sciama, Clarendon Press, Oxford, (1981).

[339] C. Itzykson, J.C. Zuber, Quantum Field Theory, McGraw-Hill, New York, (1980).

[340] D. Ivanenko and G. A. Sardanashvily, Phys. Rep. 94,1, (1983).

[341] E. A. Ivanov and J. Niederle, Phys. Rev. D 25 976, (1982).

[342] E. A. Ivanov and J. Niederle, Phys. Rev. D 25, 988, (1982).

[343] J.D. Jackson, Classical Electrodynamics, J.Wiley, New York (1962).

[344] P. Jetzer, Phys. Rep. 220, 163 , (1993).

[345] P. Jordan, Naturwiss. 26, 417, (1938).

[346] P. Jordan, Schwerkraft und Weltfall, Grundlagen der Theoretische Kosmologie Vieweg \& Sohns, Braunschweig, (1952).

[347] M. Kaku, Quantum Field Theory, Oxford Univ. Press, Oxford, 1993.

[348] K. Kainulainen, J. Piilonen, V. Reijonen, D. Sunhede, Phys. Rev. D 76, 024020, (2007).

[349] K. Kainulainen, V. Reijonen and D. Sunhede Phys. Rev. D 76, 043503 (2007).

[350] A. Kamenshchik, U. Moschella, V. Pasquier, Phys. Lett. B 511, 265, (2001).

[351] S. Kamefuchi, L. O'Raifeartaigh, A. Salam, Nucl. Phys. 28, 529, (1961).

[352] R. P. Kerr, Phys. Rev. Lett. 11, 237, (1963).

[353] A. K. Kerman and D. Vautherin, Ann. Phys., 192, 408, (1989);

[354] J. Khoury, A. Weltman, Phys. Rev. Lett. 93, 171104, (2004).

[355] J. Khoury, A. Weltman, Phys. Rev. D 69, 044026, (2004).

[356] T.W. Kibble J. Math. Phys. 2, 212, (1961).

[357] C. W. Kilmister, Eddington's search for a fundamental theory : a key to the Universe (Cambridge, 1994).

[358] D. Kimberly, and J. Magueijo, COSMOLOGY AND GRAVITATION: XIth Brazilian School of Cosmology and Gravitation. AIP Conf. Proc. 782, 241, (2005).

[359] O. Klein, New Theories in Physics, 77, Intern.Inst. of Intellectual Co-operation, League of Nations, (1938).

[360] R.A. Knop et al., Astrophys. J. 598, 102, (2003).

[361] T. Koivisto, Class. Quant. Grav. 23, 4289, (2006).

[362] E. Komatsu, T. Futamase, Phys. Rev. D 58, 023004, (1998).

[363] E. Komatsu, T. Futamase, Phys. Rev. D 59, 064029, (1999).

[364] K. Konno, M. Kasai, Progr. Theor. Phys 100, 1145, (1998).

[365] A. Kransinski, Ann. Phys. 112, 22, (1978).

[366] A. Krasinski, Inhomogeneous Cosmological Models, Cambridge University Press, Cambridge, UK (1997).

[367] L.M. Krauss, M. White, Astrophys. J. 397, 357 (1992).

[368] G. Kunstatter, Gen. Rel. Grav. 12, 373, (1979).

[369] D. La, P.J Steinhardt, Phys. Rev. Lett. 62, 376, (1989).

[370] O. Lahav, et al., Mon. Not. R. Ast. Soc. 333, 961, (2002).

[371] Y.M. Lam, Phys. Rev. D 7, 2943, (1973).

[372] N. Lanahan-Tremblay, V. Faraoni, Class. Quantum Grav. 24, 5667, (2007).

[373] L.D. Landau, E.M. Lifschitz, The Classical Theory of Fields, Pergamon Press, Oxford, (1989).

[374] R. N. Lang, S. A. Hughes, Class. Quantum Grav. 26, 094035, (2009).

[375] P. Leaci et al., Class. Quantum Grav. 25, 195018, (2008).

[376] N. Lanahan-Trembley and V. Faraoni, Class. Quantum Grav., Vol. 24, 5667, (2007).

[377] J. Lee et al., Phys. Rev. D 61, 027301, (1999).

[378] G. Lemaître, Ann. Soc. Sci. Brussels A 53, 51 (1933), reprinted in Gen. Relat. Gravit. 29, 641 (1997).

[379] J. Lense, H. Thirring, Physikalische Zeitschrift, 19, 156, (1918).

[380] Levi-Civita T., The Absolute Differential Calculus, Blackie and Son, London, 1929.

[381] B. Li, M.C. Chu, Phys. Rev. D 74, 104010, (2006).

[382] B. Li, K.C. Chan, M.C. Chu, Phys. Rev. D 76, 024002, (2007).

[383] Li B., Barrow J.D., Mota D.F., Phys. Rev. D,76, 044027, (2007).

[384] B. Li, J.D. Barrow, Phys. Rev. D 75, 084010, (2007). 
[385] B. Li, J.D. Barrow, D.F. Mota, Phys. Rev. D 76, 104047, (2007).

[386] B. Li, D.F. Mota, D.J. Shaw, Phys. Rev. D 78, 064018 (2008).

[387] A.R. Liddle, M.S. Madsen, Int. J. Mod. Phys. D 1, 101, (1992).

[388] J. Llosa, D. Soler, Class. Quant. Grav. 21, 3067, (2004).

[389] J. Llosa and D. Soler, Class. Quant. Grav. 22, 893, (2005).

[390] F.S.N. Lobo, arXiv:0807.1640v1 [gr-qc] (2008).

[391] D. Lorentz-Petzold, in Lecture Notes in Physics 105, ed. by C. Hoenselaers, W. Dietz, Springer, Berlin, (1984).

[392] M. Lubini, C. Tortora, J. Näf, Ph. Jetzer, S. Capozziello, arXiv:1104.2851v1 [gr-qc] (2011).

[393] A. Lue, R. Scoccimarro, G. Starkman, Phys. Rev. D 69, 044005, (2004).

[394] E. Mach, Die Mechanik in ihrer Entwicklung historisch-kritisch dargestellt, F.A. Brockhaus, Princeton University Press, Princeton, (1912).

[395] K. Maeda, Phys. Rev. D 39, 3159, (1989).

[396] P.D. Mannheim, D. Kazanas, Astrophys. J. 342, 635 (1989).

[397] F. Mansouri et al. Phys. Rev. D 13, 3192, (1976).

[398] F. Mansouri, Phys. Rev. D 16 2456, (1977).

[399] F. Mansouri, Phys. Rev. Lett. 42, 1021, (1979).

[400] Y. Mao, M. Tegmark, A. Guth, S. Cabi, Phys. Rev. D 76, 104029, (2007).

[401] D. McManus, Class. Quant. Grav. 8, 863, (1991).

[402] G.C. McVittie, Mon. Not. R. Astron. Soc. 93, 325, (1933).

[403] M. Maggiore, Phys. Rep. 331, 283, (2000).

[404] M. Maggiore, Gravitational Wawes: Theory and Experiments, Oxford Univ. Press, Oxford (2007).

[405] G. Magnano, L.M. Sokolowski, Phys. Rev. D 50, 5039, (1994).

[406] G. Magnano, M. Ferraris, and M. Francaviglia, Gen. Rel. Grav. 19, 465, (1987).

[407] G. Magnano, M. Ferraris, M. Francaviglia, Class. Quantum Grav. 7, 557, (1990).

[408] G. Marmo, E.J. Saletan, A. Simoni and B. Vitale, Dynamical Systems. A Differential Geometric Approach to Symmetry and Reduction, Wiley, New York (1985).

[409] T. Matsuda, Prog. Theor. Phys. 47, 738, (1962).

[410] N.E. Mavromatos, Braz.J.Phys., 35, 284, (2005).

[411] P. O. Mazur and E. Mottola, Nucl. Phys. B 341, 187, (1990).

[412] S.S. McGaugh, Astroph. Journ., 632, 859, (2005).

[413] X. Meng, P. Wang, Gen. Rel. Grav. 36 (8), 1947 (2004);

X. Meng, P. Wang,,Gen. Rel. Grav. 36, 2673, (2004).

[414] C.W. Misner, K.S. Thorne, J.A. Wheeler, Gravitation, ed. W.H. Freeman, San Francisco (1973).

[415] C.W. Misner, in Relativity, ed. by M. Carmeli, L. Fickler, L. Witten, Plenum, San Francisco, (1970).

[416] C.W. Misner, in Magic Without Magic, ed. by J. Klauder, Freeman, San Francisco, (1972).

[417] G. Morandi, C. Ferrario C., G. Lo Vecchio, G. Marmo, C. Rubano Phys. Rep. 188, 149, (1990).

[418] K. Moriyasu, Gauge Theory, World Scientific Publishing, Singapore, (1983).

[419] T. Multamaki, I. Vilja, Phys. Rev. D 74, 064022, (2006).

[420] T. Multamaki, I. Vilja, Phys. Rev. D 76, 064021, (2007).

[421] T. Multamaki, A. Putaja, E.C. Vagenas, I. Vilja, Class. Quantum Grav. 25, 075017, (2008).

[422] T. Multamaki, I. Vilja, Phys. Lett. B 659, 843, (2008).

[423] V.F. Mukhanov, H.A. Feldman and R.H. Brandenberger, Phys. Rept. 215, 203, (1992).

[424] T.S. Muta, S.D. Odintsov, Mod. Phys. Lett. A 6, 3641, (1991).

[425] J. Näf, Ph. Jetzer, Phys. Rev. D 84, 024027 (2011).

[426] I. Navarro, K. van Acoleyen, Phys. Lett. B 622, 1, (2005).

[427] I. Navarro, K. Van Acoleyen, J. Cosmol. Astropart. Phys. 0603, 008, (2006).

[428] B. Nelson, P. Panangaden, Phys. Rev. D 25, 1019, (1982).

[429] E.T. Newman, A.I. Janis, J. Math. Phys. 6, 915, (1965).

[430] E. T. Newman, E. Couch, K. Chinnapared, A. Exton, A. Prakash and R. Torrence, J. Math. Phys. 6, 918, (1965).

[431] S. Newcomb, The Elements of the Four Inner Planets and the Fundamental Constants of Astronomy, American Ephemeris (1897).

[432] E.T. Newman and A.I. Janis, J. Math. Phys. 6, 915, (1965).

[433] P. van Nieuwenhuizen Phys. Rep 68, 189, (1981).

[434] Th. M. Nieuwenhuizen, Euro. Phys. Lett. 86, 59001, (2009).

[435] A. Nishizawa, A. Taruya, K. Hayama, S. Kawamura, M. Sakagami, Phys. Rev. D 79, 082002, (2009).

[436] D.R. Noakes, J. Math. Phys. 24, 1846, (1983).

[437] S. Nojiri, S.D. Odintsov, Phys. Rev. D 68, 123512, (2003).

[438] S. Nojiri, S.D. Odintsov, Phys. Lett. B 576, 5, (2003).

[439] S. Nojiri, S.D. Odintsov, S. Tsujikawa, Phys.Rev. D 71, 063004, (2005).

[440] S. Nojiri, S.D. Odintsov, M. Sasaki, Phys.Rev. D 71, 123509, (2005).

[441] S. Nojiri, S.D. Odintsov, Gen.Rel.Grav. 38, 1285, (2006).

[442] S. Nojiri, S.D. Odintsov Phys.Rev. D 74, 086005, (2006).

[443] S. Nojiri, S.D. Odintsov, Phys. Lett. B 657, 245, (2007).

[444] S. Nojiri, S.D. Odintsov, Int. J. Geom. Meth. Mod. Phys. 4, 115, (2007). 
[445] S. Nojiri, S.D. Odintsov, Physics Reports 505, 59, (2011).

[446] K. Nordtvedt, Phys. Rev. 169, 1017, (1968).

[447] A. Nunez, S. Solganik, Phys. Lett. B 608, 189 (2005).

[448] S.D. Odintsov, Fortschr. Phys. 39, 621, (1991).

[449] J. O'Hanlon, J. Phys. A 5, 803, (1972).

[450] J. O'Hanlon, B. Tupper, Nuovo Cimento B 7, 305, (1972).

[451] T. Okamura, in Proceedings of the Asia-Pacific Conference on Gravitation and Cosmology, Seoul, Korea 1996, ed. by Y.M. Cho, C.H. Lee, S.-W. Kim, World Scientific, Singapore, (1998).

[452] G. J. Olmo, Phys. Rev. D 72, 083505, (2005).

[453] G.J. Olmo, Phys. Rev. Lett. 95, 26110, (2005).

[454] G.J. Olmo, Phys. Rev. D 75, 023511, (2007).

[455] G.J. Olmo, Phys. Rev. D 77, 084021, (2008).

[456] G.J. Olmo, Phys. Rev. D 78, 104026, (2008).

[457] G.J. Olmo, Int. J. Mod. Phys. D 20, 413, (2011).

[458] G.J. Olmo, H. Sanchis-Alepuz, S. Tripathi, Phys. Rev. D 80, 024013, (2009).

[459] G.J. Olmo, H. Sanchis-Alepuz, Phys. Rev. D 83, 104036, (2011).

[460] T. Padmanabhan, Phys. Rev. D 66, 021301, (2002).

[461] T. Padmanabhan, Phys. Repts. 380, 235, (2003).

[462] F.M. Paiva, C. Romero, Gen. Relat. Gravit. 25, 1305, (1993).

[463] F.M. Paiva, M. Reboucas, M. MacCallum, Class. Quantum Grav. 10, 1165, (1993).

[464] A. Palatini, Rend. Circ. Mat. Palermo 43, 203, (1919).

[465] A. Papapetrou and J. Stackel, Gen. Rel. Grav. 9, 1075, (1978).

[466] L. Parker, D.J. Toms, Phys. Rev. D 32, 1409, (1985).

[467] L. Parker, D.J. Toms, Phys. Rev. D 29, 1584, (1985).

[468] L. Parker and D.J. Toms, Quantum Field Theory in Curved Space-Time: Quantized Fields and Gravity, Cambridge University Pres, Cambridge, UK (2009).

[469] L. Parker, A. Raval Phys.Rev. D 60, 063512 (1999).

[470] L. Parker, W. Komp, and D. A. T. Vanzella ApJ, 588, 663 (2003).

[471] R. Penrose, Gen. Rel.Grav. 28, 581, (1996).

[472] I. Percival, and W. Strunz. Proc. R. Soc. Lond. A, 453, 431,(1997).

[473] J.Perez-Mercader and S.D. Odintsov, Int. J. Mod. Phys. D 1, 401, (1992).

[474] S. Perlmutter et al., Astrophys. J. 517, 565, (1999).

[475] A. Peters, K.Y. Chung and S. Chu, Metrologia, 38, 25, (2001).

[476] H. Pfister, Gen. Relativ. Grav. 39, 11, 1735, (2007).

[477] F. Piazza, C. Marinoni, Phys. Rev. Lett. 91, 141301, (2003).

[478] S. Podariu, R.A. Daly, M.P. Mory, B. Ratra, Astrophys. J. 584, 577, (2003).

[479] R.V. Pound and G.A. Rebka, Phys. Rev. Lett. 4, 337, (1960).

[480] I. Quandt, H.J. Schmidt, Astron. Nachr. 312, 97, (1991).

[481] L. Querella, Variational Principles and Cosmological Models in Higher-Order Gravity - Ph.D. Thesis. gr-qc/9902044.

[482] J.R. Ray and L.L. Smalley, Phys. Rev. D 27, 1383, (1983) .

[483] T. Regge and J.A. Wheeler, Phys. Rev. 108, 1063, (1957).

[484] F. Reines, C.L. Cowan, Phys. Rev. 92, 830, (1953).

[485] O.A. Reula, Living Rev. Relativity 1, 3, (1998).

[486] M. Reuter, Phys. Rev. D 49, 6379,(1994).

[487] A.G. Riess et al., Astron. J. 116, 1009, (1998).

[488] A.G. Riess et al., Astrophys. J. 607, 665, (2004).

[489] C. Romero, A. Barros, Astr. Sp. Sci. 192, 263, (1992).

[490] C. Romero, A. Barros, Gen. Relat. Gravit. 25, 1305, (1993).

[491] C. Romero, A. Barros, Phys. Lett. A 173, 243, (1993).

[492] C. Rubano and P. Scudellaro, Gen. Relat. Gravit. 37, 521, (2005).

[493] G.F. Rubilar, Class. Quant. Grav. 15, 239, (1998).

[494] M.L. Ruggiero, L. Iorio, J. Cosmol. Astropart. Phys. 0701, 010, (2007).

[495] T.V. Ruzmaikina, A.A. Ruzmaikin , JETP, 30, 372, (1970).

[496] V. Sahni, A.A. Starobinsky, Int. J. Mod. Phys. D 9, 373, (2000).

[497] M. Salgado, Class. Quantum Grav. 23, 4719, (2006).

[498] A. Salam, J. Strathdee, Phys. Rev. D 2, 2869, (1970).

[499] D.S. Salopek, J.R. Bond, J.M. Bardeen, Phys. Rev. D 40, 1753, (1989).

[500] V.D. Sandberg, Phys. Rev. D 12, 3013, (1975).

[501] R.H. Sanders, Ann. Rev. Astron. Astrophys. 2, 1, (1990).

[502] G. Sardanashvily, arXiv: gr-qc/0201074 (2002).

[503] J. Schwinger, Phys. Rev. 130, 800, (1963).

[504] I.L. Shapiro, Phys.Rept. 357,113, (2002).

[505] M.A. Scheel, S.L. Shapiro, S.A. Teukolsky, Phys. Rev. D 51, 4208, (1995). 
[506] D.W. Sciama, On the analog between charge and spin in General Relativity, in Recent Developments in General Relativity, Festschrift for Leopold Infeld, Pergamon Press, New York, (1962).

[507] M.M. Schiffer, R. J. Adler, J. Mark and C. Sheffield, J. Math. Phys. 14, 52, (1973).

[508] H.J. Schmidt, Astr. Nachr. 308, 183, (1987).

[509] H.J. Schmidt, Int. J. Geom. Meth. Mod. Phys. 4, 209, (2007).

[510] H.J. Schmidt, Class. Quant. Grav. 7, 1023, (1990).

[511] E. Schrödinger, Space-Time Structure, Cambridge Univ. Press, Cambridge, (1960).

[512] S. Sengupta Anand for the LIGO Scientific Collaboration and the Virgo Collaboration, LIGO-Virgo searches for gravitational waves from coalescing binaries: a status update, Proceedings of the 8th E. Amaldi Conference on Gravitational Waves (Amaldi8), New York, 2009; J. Phys.: Conf. Series.

[513] C.G. Shao, R.G. Cai, B. Wang, R.K. Su, Phys. Lett. B 633, 164 (2006).

[514] D. Sigg et al., www.ligo.org/pdfpublic/P050036.pdf

[515] T. P. Singh, Bulg. J. Phys. 33, 217, (2006).

[516] P. Schneider, J. Ehlers, E.E. Falco, Gravitational Lenses, Springer, Berlin, (1992).

[517] P. Solín, Partial Differential Equations and The Finite Element Method, Wiley, New York, (2006).

[518] L.M. Sokolowski, Class. Quantum Grav. 6, 2045, (1989).

[519] S. Sonego, V. Faraoni, Class. Quantum Grav. 10, 1185, (1993).

[520] S. Sonego, Phys. Lett. A 208, 1, (1995).

[521] S. Sonego and H. Westman, Class. Quant. Grav. 21, 433, (2004).

[522] T.P. Sotiriou, Gen. Relat. Gravit. 38, 1407, (2006).

[523] T.P. Sotiriou, Class. Quant. Grav. 23, 5117, (2006).

[524] T.P. Sotiriou, E. Barausse, Phys. Rev. D 75, 084007, (2007).

[525] T.P. Sotiriou, V. Faraoni, Rev. Mod. Phys. 82, 451, (2010).

[526] M.E. Soussa, R.P. Woodard, Gen. Relat. Gravit. 36, 855, (2004).

[527] D.N. Spergel et al., Astrophys. J. (Suppl.) 148, 175, (2003).

[528] D.N. Spergel et al. [WMAP Collaboration], Astrophys. J. (Suppl.) 170, 377, (2007).

[529] A. A. Starobinsky, JETP Lett., 30, 682, (1979).

[530] A.A. Starobinsky, Phys. Lett. B 91, 99, (1980).

[531] A.A. Starobinsky, in Proceedings of the 4th Seminar on Quantum Gravity, ed. by M.A. Markov, V.P. Frolov, World Scientific, Singapore, (1987).

[532] L. C. Stein, N. Yunes, Phys. Rev. D 83, 064038 (2011).

[533] H. Stephani, D. Kramer, M. MacCallum, C. Hoenselaers, E. Herlt, Exact Solutions of the Einstein Field Equations , Cambridge University Press, Cambridge, UK (2003).

[534] K.S. Stelle, Phys. Rev. D 16, 953, (1977).

[535] K.S. Stelle, Gen. Relat. Gravit. 9, 343, (1978).

[536] R. Stompor et al., Astrophys. J. (Lett.) 561, L7, (2001).

[537] K. Sung-Won, Phys. Rev. D, 34, 1011,(1986).

[538] J.L. Synge, Relativity: The General Theory, Amsterdam: North Holland, (1955).

[539] C.J. Talbot, Commun. Math. Phys. 13, 45, (1969).

[540] D. Tatsumi, Y. Tsunesada, Class. Quantum Grav. 21, S451 (2004).

[541] T.R. Taylor, G. Veneziano, Phys. Lett. B 213, 450, (1988).

[542] P. Teyssandier, P. Tourrenc, J. Math. Phys. 24, 2793, (1983).

[543] K.S. Thorne and C.M. Will, Astrophys. J. 163, 595, (1971).

[544] K.S. Thorne, The theory of gravitational radion: an introductory review, in Gravitational Radiation, pp 1-57, ed. N. Deruelle and T. Piran, North-Holland, Amsterdam (1983).

[545] H. Thirring Physikalische Zeitschrift, 19, 204, (1918).

[546] H. Thirring Physikalische Zeitschrift, 22, 29, (1921).

[547] M.E. Tobar, T. Suzuki, K. Kuroda, Phys. Rev. D 79, 082002, (2009).

[548] R.C. Tolman, Proc. Nat. Acad. Sci. U.S.A. 20, 169, (1934).

[549] J.L. Tonry et al., Astrophys. J. 594, 1, (2003).

[550] Ph. Tourrenc, General Relativity and Gravitational Waves in Proc. of the Int. Summer School on Experimental Physics of Grav. Waves, September 6-18, 1999, Urbino (Italy), Eds. M. Barone et al., World Scientific, Singapore (2000).

[551] S. Tsujikawa, K. Maeda, T. Torii, Phys. Rev. D 61, 103501, (2000).

[552] S. Tsujikawa, B. Bassett, Phys. Lett. B 536, 9, (2002)

[553] R. Utiyama, Phys. Rev. 101, 1597, (1956).

[554] R. Utiyama and B. S. De Witt, J. Math. Phys. 3, 608, (1962).

[555] H. Van Dam, M.J.G. Veltman, Nucl. Phys. B 22, 397, (1970).

[556] N. Van den Bergh, Gen. Relat. Gravit. 12, 863, (1980).

[557] N. Van den Bergh, Gen. Relat. Gravit. 14, 17, (1982).

[558] N. Van den Bergh, Gen. Relat. Gravit. 15, 441, (1983).

[559] N. Van den Bergh, Gen. Relat. Gravit. 15, 449, (1983).

[560] N. Van den Bergh, Gen. Relat. Gravit. 15, 1043, (1983).

[561] N. Van den Bergh, Gen. Relat. Gravit. 16, 2191, (1983).

[562] J.J. Van der Bij, E. Radu, Nucl. Phys. B 585, 637, (2000). 
[563] J.J. Van der Bij, M. Gleiser, Phys. Lett. B 194, 482, (1987).

[564] D.V. Vassilevich, Int. J. Mod. Phys. A 8, 1637, (1993).

[565] D.V. Vassilevich, Phys. Rept. 388, 279, (2003).

[566] A. Viceré, Phys. Rev. D 66, 062002, (2002).

[567] S. Vignolo and R. Cianci, J. Math. Phys 45, 4448 (2004).

[568] S. Vignolo, R. Cianci and D. Bruno, Int. J. Geom. Methods Mod. Phys. 2,1115, (2005) .

[569] S. Vignolo, R. Cianci and D. Bruno, CQG 22, 4063, (2005) .

[570] S. Vignolo, R. Cianci and D. Bruno, Int. J. Geom. Methods Mod. Phys. 3 ,1493, (2006) .

[571] S. Vignolo and E. Massa, Class. Quantum Grav. 23, 6781, (2006) .

[572] G.A. Vilkovisky, Class. Quant. Grav. 9, $895,(1992)$.

[573] D.N. Vollik, Phys. Rev. D 68, 063510, (2003).

[574] M.S. Volkov and A. Wipf, Nucl. Phys. B 582, 313, (2000).

[575] M.B. Voloshin, A.D. Dolgov, Sov. J. Nucl. Phys. 35, 120, (1982).

[576] H. Von Kluber Vistas in Astronomy 3, 47,(1960).

[577] R.M. Wald, General Relativity, Chicago University Press, Chicago, (1984).

[578] R.M. Wald, Quantum Field Theory in Curved Spacetime and Black Hole Thermodynamics, Chicago University Press, Chicago, (1995).

[579] D. Wands, Class. Quantum Grav. 11, 269, (1994).

[580] R.V. Wagoner, Phys. Rev. D 1, 3209, (1970).

[581] J.A. Wheeler, Rev. Mod. Phys. 29, 463, (1957).

[582] S. Weinberg, Gravitation and Cosmology, Wiley, New York, (1972).

[583] S. Weinberg Rev. Mod. Phys. 46, 255, (1974).

[584] R.S. Westfall, Never at Rest, Cambridge University Press, Cambridge, UK (1980).

[585] H. Weyl, Ann. der Physik 364, 101, (1919).

[586] C.M. Will, Theory and Experiment in Gravitational Physics, 2nd ed. Cambridge University Press, Cambridge, UK (1993).

[587] C.M. Will, Living Rev. Relativity 9, 3, (2006).

[588] B. Whitt, Phys. Lett. B 145, 176 (1984).

[589] B. Willke et al., Class. Quantum Grav. 23, S207, (2006).

[590] E. Witten, Nucl Phys B 195, 481,(1982).

[591] C.N. Yang, R.L. Mills, Phys. Rev. 96, 191, (1954).

[592] C.N. Yang Physics Today 42, (1980).

[593] J.W. York Jr., J. Math. Phys., 14, 4 (1973).

[594] P. Zhang, Phys. Rev. D 76, 024007, (2007). 